\title{
Prediction of Combustion Stability and Flashback in Turbines with High-Hydrogen Fuel
}

\author{
Final Technical Report \\ $4 / 01 / 2007$ thru $03 / 31 / 2012$ \\ Principal Authors: Tim Lieuwen, Dom Santavicca, and Vigor Yang \\ Date Report was Issued: JUNE, 2012
}

DOE Award Number: DE-FG26-07NT43069

DOE Program Manager: Mark Freeman

Lead University: Georgia Institute of Technology, School of Aerospace Engineering 270 Ferst Drive

Atlanta, GA 30332-0150

Voice: (404) 894-3041

Fax: (404) 894-2760

tim.lieuwen@aerospace.gatech.edu 


\section{$\underline{\text { Disclaimer }}$}

"This report was prepared as an account of work sponsored by an agency of the United States Government. Neither the United States Government nor any agency thereof, nor any of their employees, makes any warranty, express or implied, or assumes any legal liability or responsibility for the accuracy, completeness, or usefulness of any information, apparatus, product, or process disclosed, or represents that its use would not infringe privately owned rights. Reference herein to any specific commercial product, process, or service by trade name, trademark, manufacturer, or otherwise does not necessarily constitute or imply its endorsement, recommendation, or favoring by the United States Government or any agency thereof. The views and opinions of authors expressed herein do not necessarily state or reflect those of the United States Government or any agency thereof."

\section{$\underline{\text { Abstract }}$}

During the duration of this sponsorship, we broadened our understanding of combustion instabilities through both analytical and experimental work. Predictive models were developed for flame response to transverse acoustic instabilities and for quantifying how a turbulent flame responds to velocity and fuel/air ratio forcing. Analysis was performed on the key instability mechanisms controlling heat release response for flames over a wide range of instability frequencies. Importantly, work was done closely with industrial partners to transition existing models into internal instability prediction codes. Experimentally, the forced response of hydrogen-enriched natural gas/air premixed and partially premixed flames were measured. The response of a lean premixed flame was investigated, subjected to velocity, equivalence ratio, and both forcing mechanisms simultaneously. In addition, important physical mechanisms controlling the response of partially premixed flames to inlet velocity and equivalence ratio oscillations were analyzed. This final technical report summarizes our findings and major publications stemming from this program. 


\section{Table of Contents}

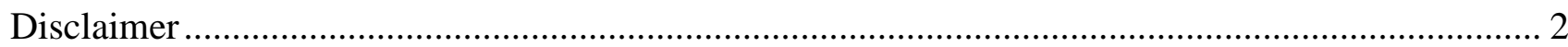

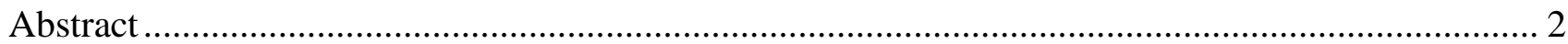

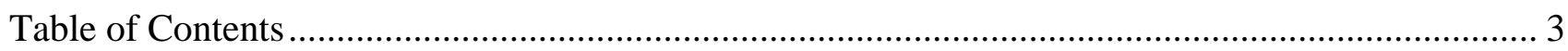

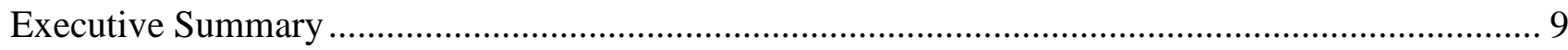

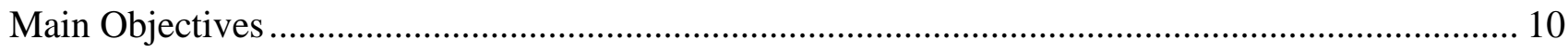

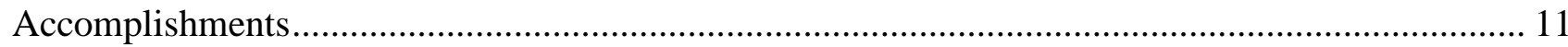

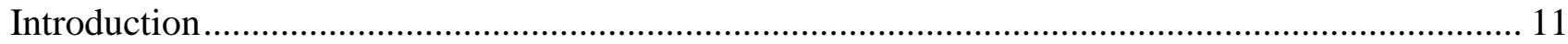

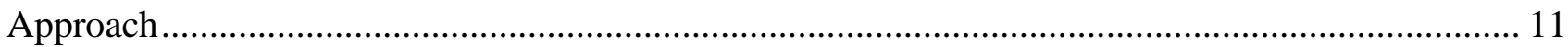

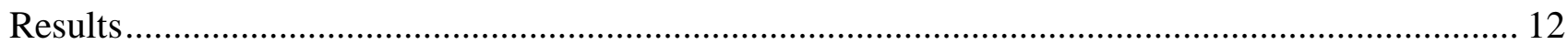

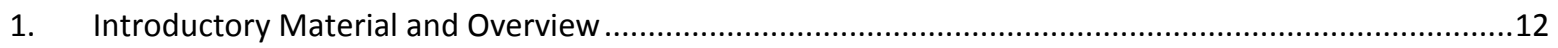

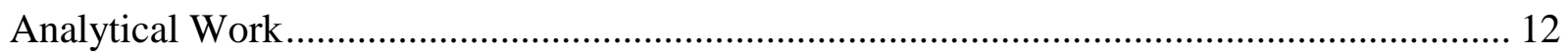

2. Modeling Flame Response to Fuel/Air Ratio Oscillations .................................................................13

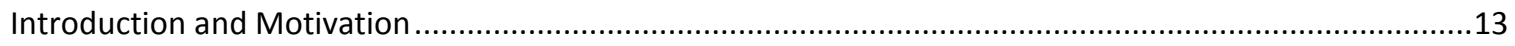

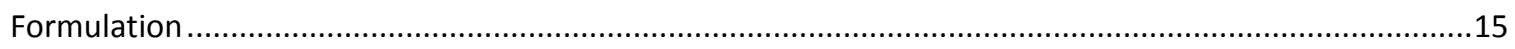

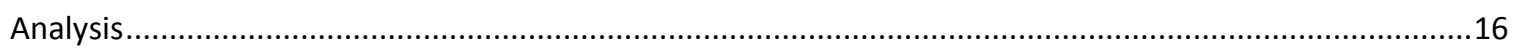

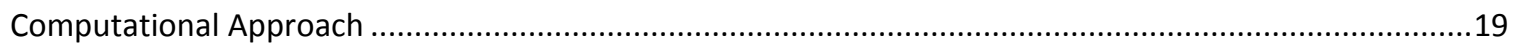

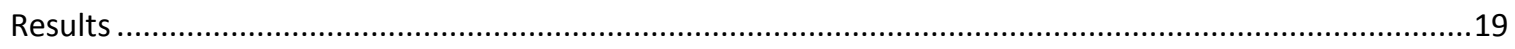

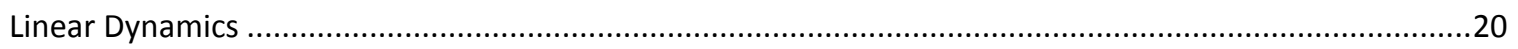

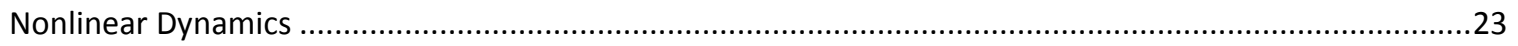

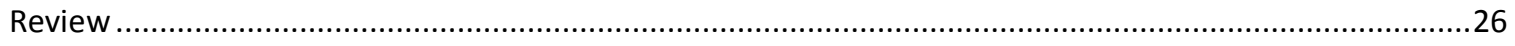

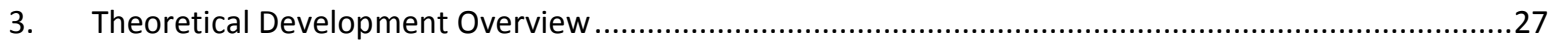

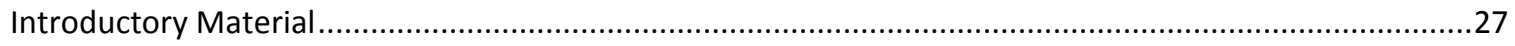

4. Modeling Flame Response to Velocity Oscillations ............................................................................

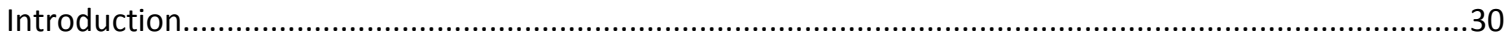

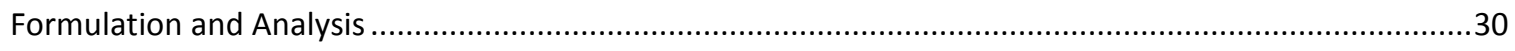


5. Modeling of Turbulent Flame Response to Flow Oscillations

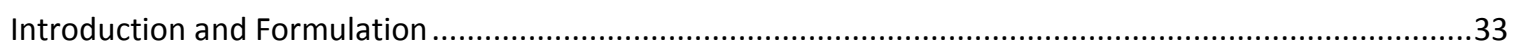

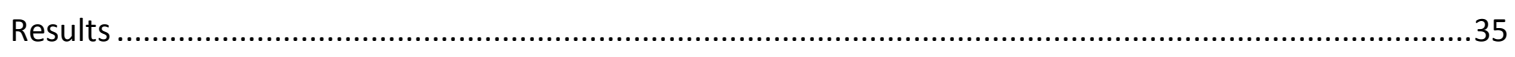

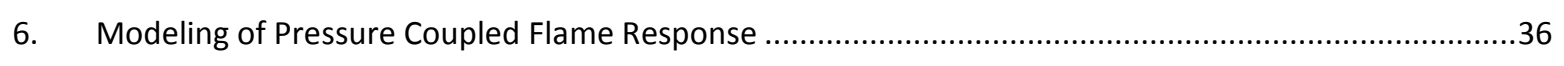

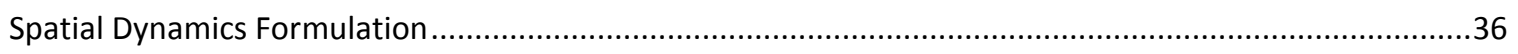

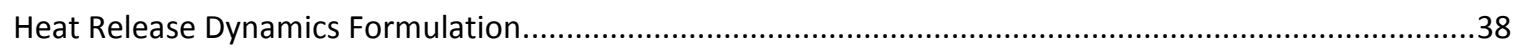

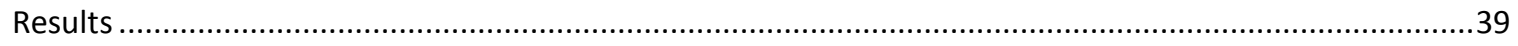

7. Response of Harmonically Forced Turbulent Flames ...........................................................41

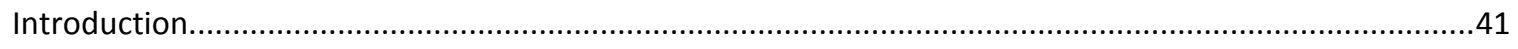

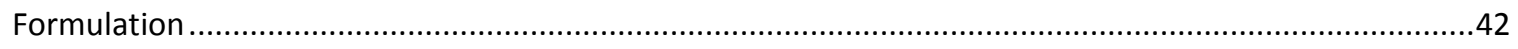

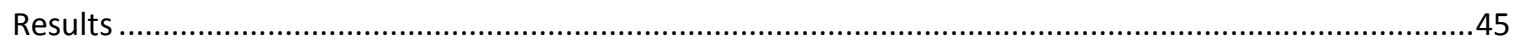

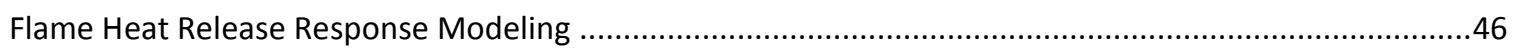

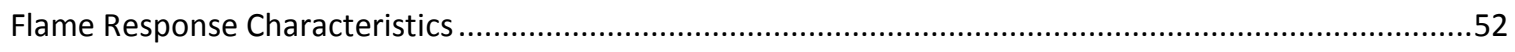

Transfer Function Predictions for Various Turbulent Flame Speed Models ...........................................57

8. Response of Premixed Flames to Equivalence Ratio Fluctuations - Comparison Between Global Heat

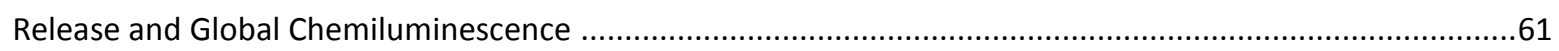

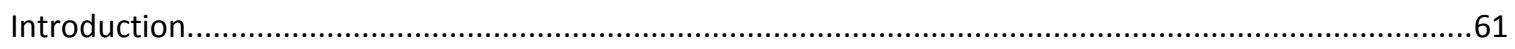

Analytical Formulation of Global Chemiluminescence Response Modeling ........................................64

Analytical Formulation of Global Heat Release Response Modeling ................................................65

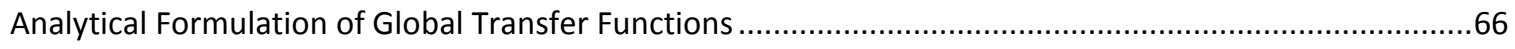

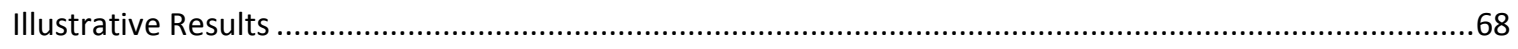

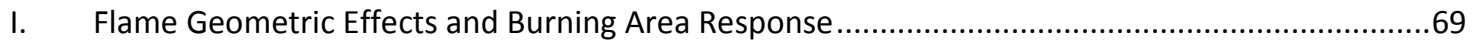

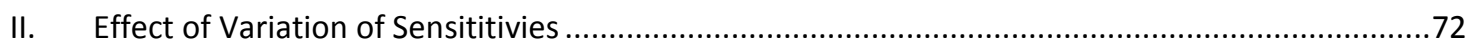

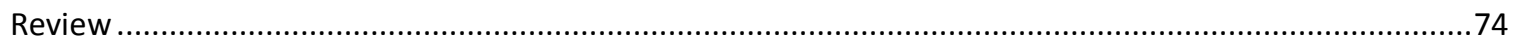

9. Response of Premixed Flames to Equivalence Ratio Perturbations - Flame Stretch and Non-Quasi-

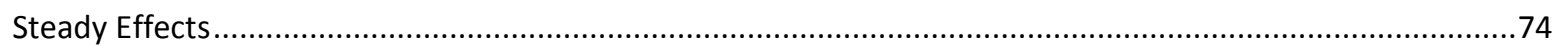

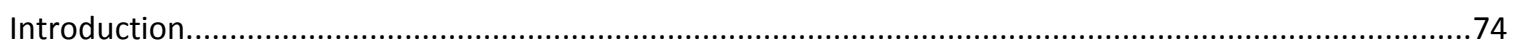




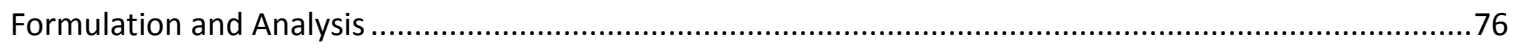

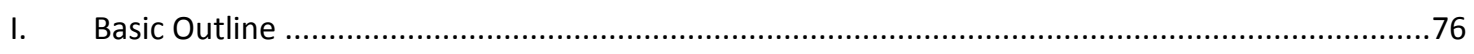

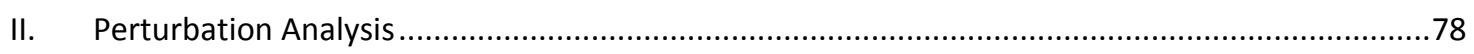

III. Quasi-steady Analysis of an Unstretched Flame ..................................................................79

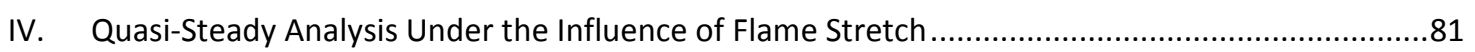

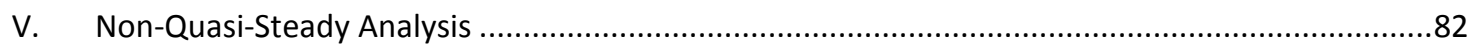

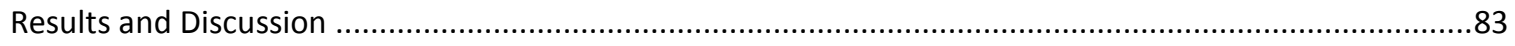

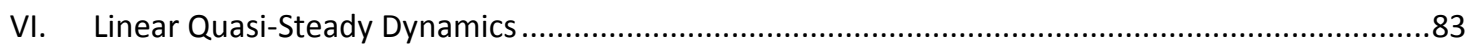

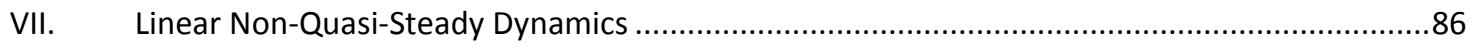

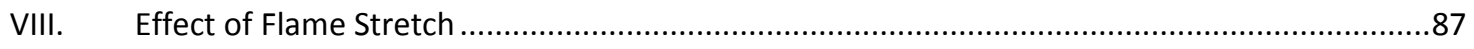

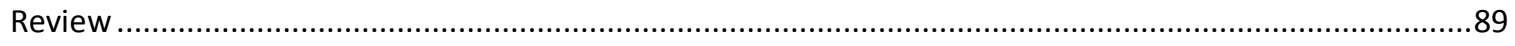

10. Response of Premixed Flames to High Frequency Acoustic Perturbations .........................................89

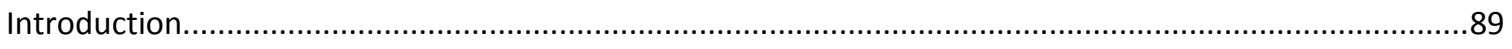

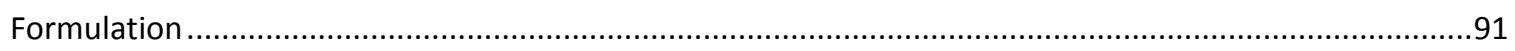

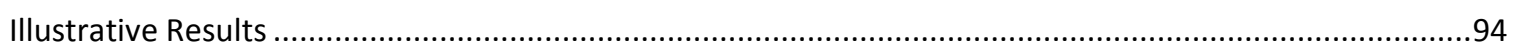

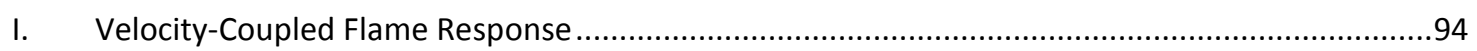

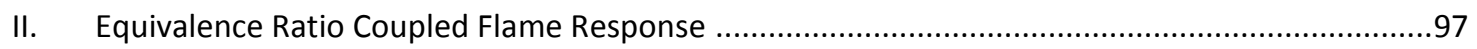

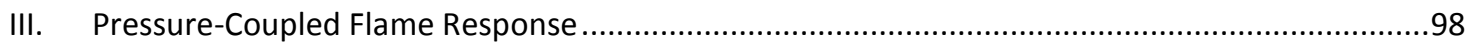

Relative Roles of Different Coupling Mechanisms ……....................................................................99

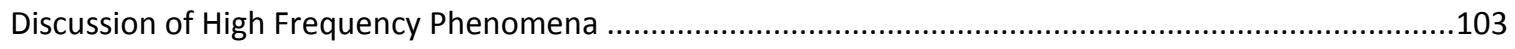

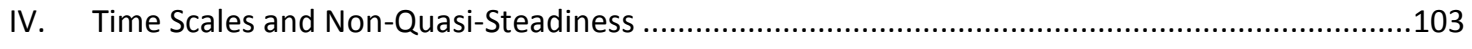

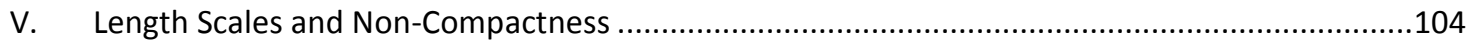

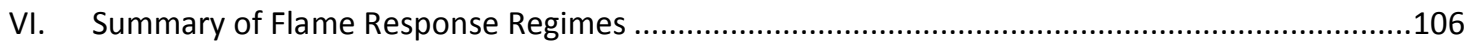

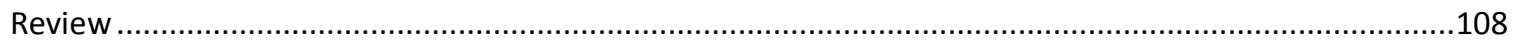

11. Swirl Effects on Transversely Forced Flame Dynamics .................................................................109

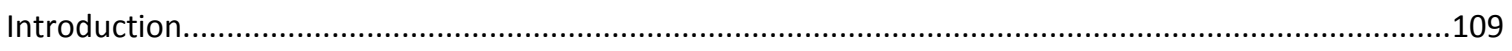

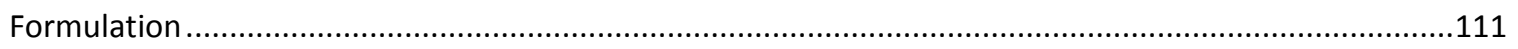


Discussion.

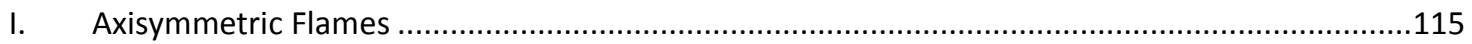

i. Uniform Transverse Velocity Results (Compact Flame) ..................................................119

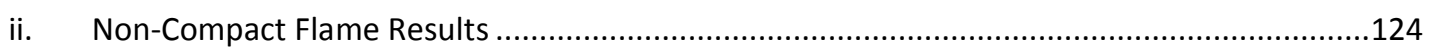

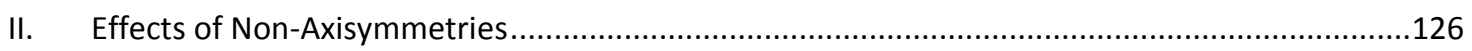

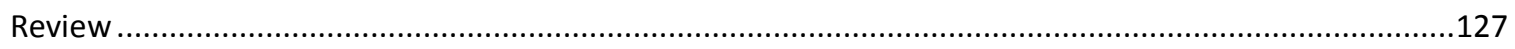

12. Swirl Effects on Transversely Forced Premixed Flame Dynamics - Extended Analysis .......................128

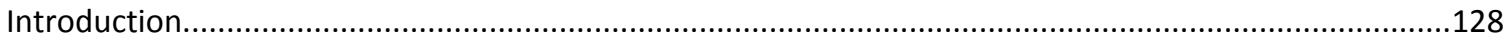

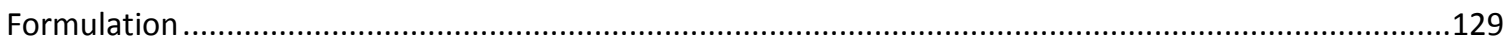

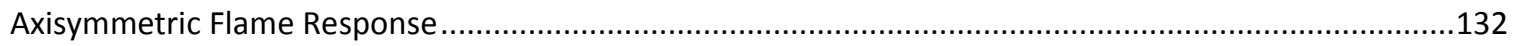

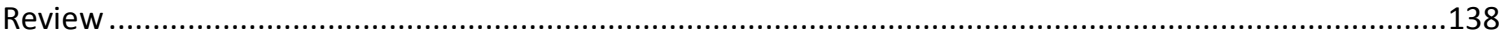

13. Effect and Contribution of Swirl Fluctuations on the Flame Response of Swirl-Stabilized Premixed

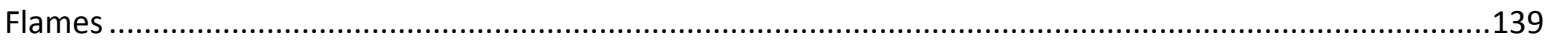

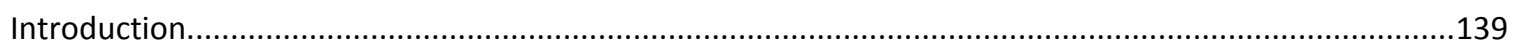

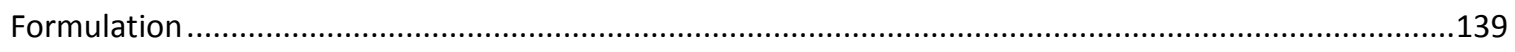

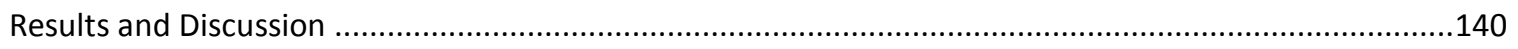

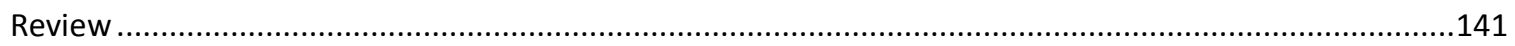

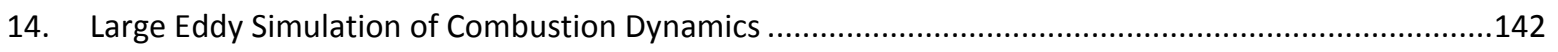

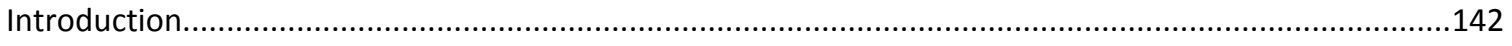

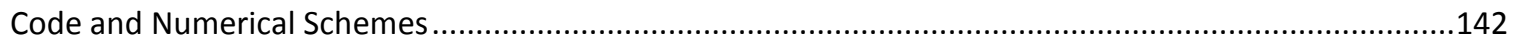

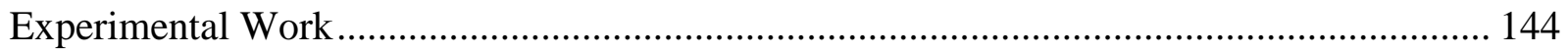

15. Heat Release Rate Fluctuation Measurements Under Partially Premixed Conditions .........................144

Chemiluminescence Measurements in Steady Flames ...............................................................144

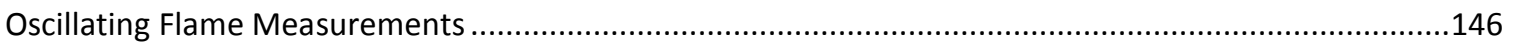

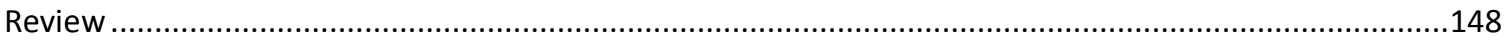

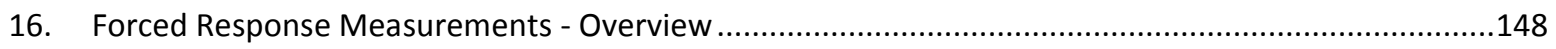


Introduction.

Velocity Fluctuation Measurements ........................................................................................148

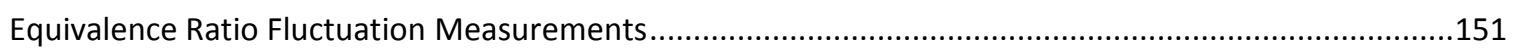

17. Forced Response Measurements - Velocity Fluctuations .......................................................151

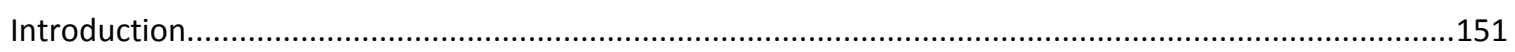

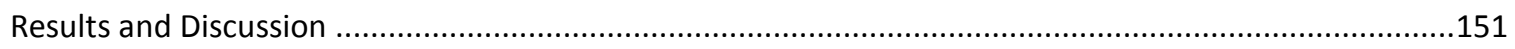

18. Forced Response Measurements - Simultaneous Velocity and Equivalence Ratio Perturbations ........162

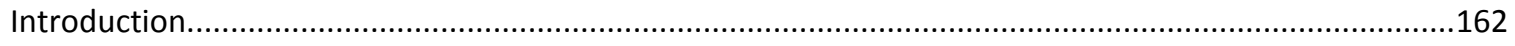

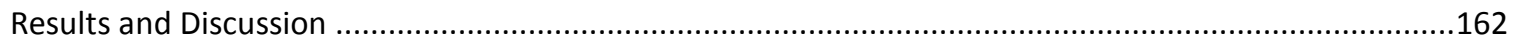

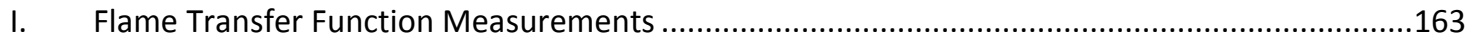

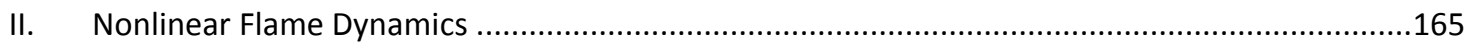

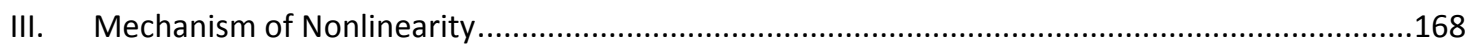

IV. Reconstruction of the Response of a Partially Premixed Flame ...........................................173

19. Measurement of the Acoustic Impedance of the Injection Swirler...............................................179

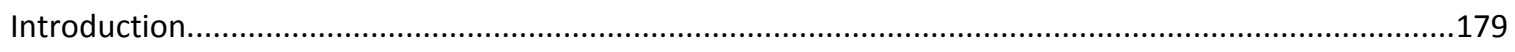

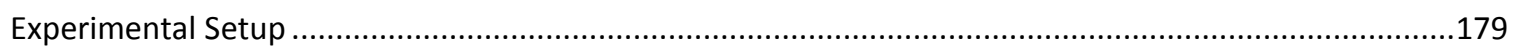

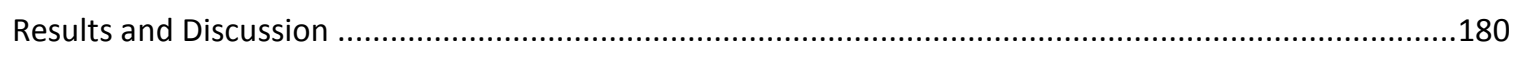

Flame transfer function measurements of lean turbulent premixed flames ......................................181

20. Flame Brush Dynamics in a Harmonically Oscillating Turbulent Jet Flame ....................................188

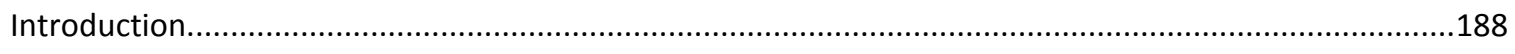

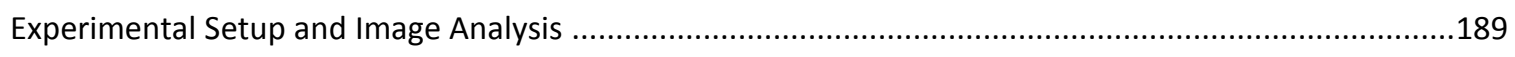

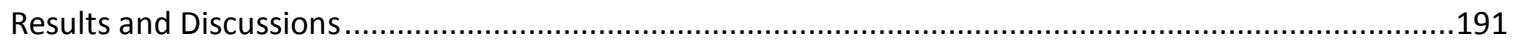

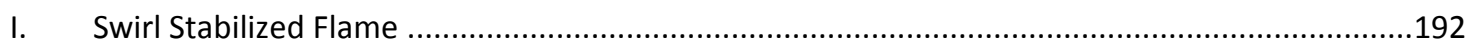

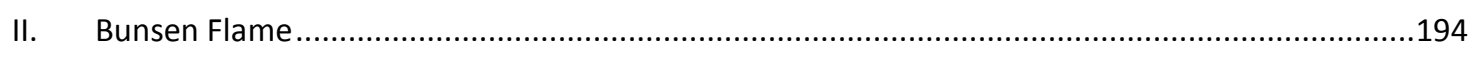

21. Acoustic Forcing of Lean-Premixed, Swirl-Stabilized, Turbulent Flames .........................................200

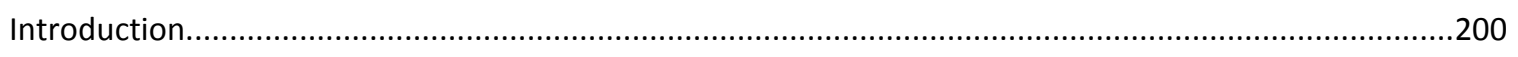

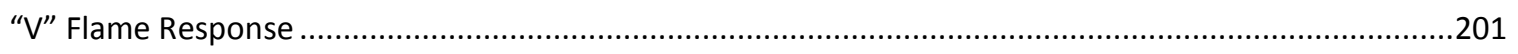




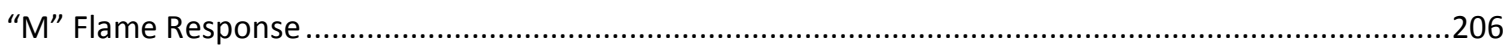

Self-Excited Instability Measurements ......................................................................................210

Comparison between Forced and Self-Excited Flame Dynamics .....................................................212

22. Nonlinear Response of a Swirl Stabilized Flame to Equivalence Ratio Oscillations............................213

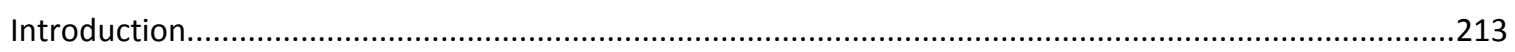

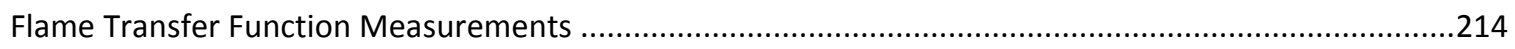

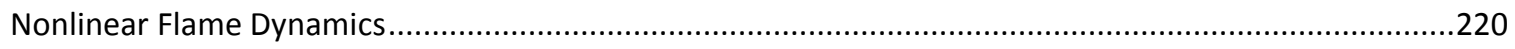

23. Inlet Velocity and Equivalence Ratio Oscillations of Swirl-Stabilized Partially Premixed Flames ...........223

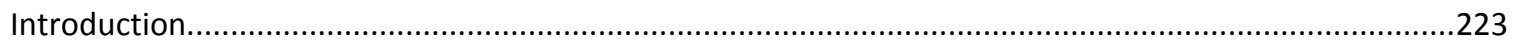

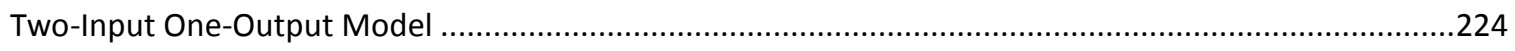

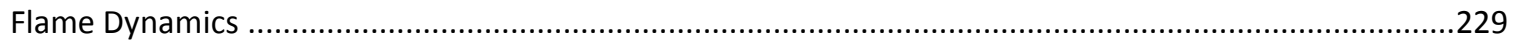

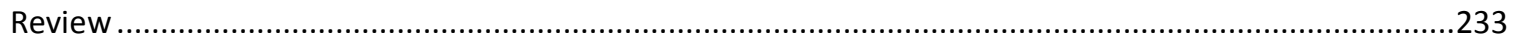

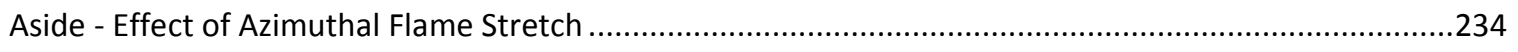

24. Response of Lean Premixed Flames to Acoustic Velocity Oscillations ...............................................235

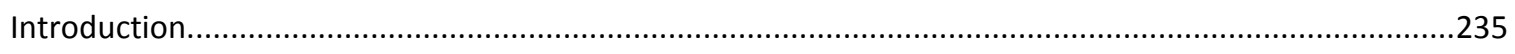

Effects of Swirl Angle on Steady-State Flame Structure .....................................................................235

Effect of Swirl Angle on the Forced Flame Response ..................................................................239

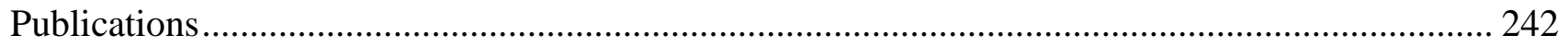

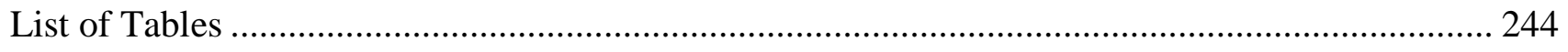

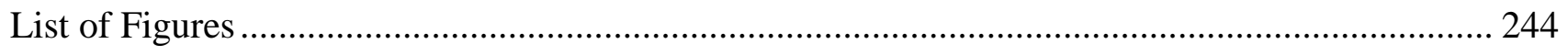




\section{$\underline{\text { Executive Summary }}$}

During the duration of this research effort, we broadened our understanding of combustion instabilities through both analytical and experimental work. "Combustion instabilities" refers to damaging pressure oscillations excited by the combustion process. If significant, the amplitude of these oscillations causes significant damage in low emissions turbines every year, through forced outages and broken parts. Predictive models were developed for flame response to transverse acoustic instabilities and for quantifying how a turbulent flame responds to velocity and fuel/air ratio forcing. A variety of physical processes were accounted for in studying the combustion dynamics of a system excited by these oscillations. Some of these are associated with the relative length scales and time scales of these disturbances in comparison to inherent length and time scales that exist in the fluid dynamics and combustion phenomena related to the problem. Results show that while at low frequencies, velocity and fuel/air ratio responses are the dominant flame response coupling mechanisms, at very high frequencies, pressure coupling tends to dominate the flame response.

Analysis was performed on the key instability mechanisms controlling heat release response for flames over a wide range of instability frequencies. Currently, there is no direct measurement approach for determining the flame's instantaneous heat release, an absolutely critical parameter for understanding combustion instabilities. As such, chemiluminescence emissions from the flame are used as an indicator. While it is known that such an approach works reasonably well for quantifying the flame's response to velocity oscillations, there have been a number of questions for flame's with varying fuel-air ratio. Our analysis of this problem show that there are differences in the flames heat release and chemiluminescence emissions for both $\mathrm{OH}^{*}, \mathrm{CH}^{*}$, and $\mathrm{CO} 2 *$ bands, but that they all give qualitatively correct results. Quantitative differences can be as large as $50 \%$, however. Importantly, work was done closely with industrial partners to transition existing models into internal instability prediction codes. Results show a significant increase in computational speed and accuracy.

In order to validate the modeling work, the flame's response was measured experimentally for various types of forcing conditions. The forced response of hydrogen-enriched natural gas/air premixed and partially premixed flames were measured. The flame's response to velocity fluctuations as large as $60 \%$ has been measured over a range of frequencies from $100 \mathrm{~Hz}$ to $450 \mathrm{~Hz}$. The velocity fluctuation is measured using a two-microphone technique near the exit of the nozzle and the flame's response is defined by the fluctuation in the flame's rate of heat release, which is determined from $\mathrm{CH}^{*}$ chemiluminescence measurements. The data shows both the linear and nonlinear flame response regimes that the models must account for to provide accurate predictions of instability frequency and amplitude. In addition to the forced response measurements, the self-excited instability characteristics of the combustor are determined in a variable length combustor. The results show that the addition of hydrogen to the fuel greatly changes the combustion dynamics. With the addition of $45 \%$ hydrogen in the fuel, the instability region shifts to combustor lengths near 42" with a frequency of approximately $380 \mathrm{~Hz}$. Other measurements show that as the hydrogen percentage increases from $0 \%$ to $45 \%$ the $\sim 210 \mathrm{~Hz}$ instability weakens and the $\sim 380 \mathrm{~Hz}$ instability strengthens, but no instabilities are observed at intermediate frequencies. 
The response of a lean premixed flame was investigated, subjected to velocity, equivalence ratio, and both forcing mechanisms simultaneously. Referring to our specific geometry, it is evident that the flame angle change over the course of modulation cycle is substantial. In the case of natural gas flames on the other hand, the flame oscillates in the flow direction, which means that the flame length changes over a period of perturbations. These oscillations in the flame position (angle) result from fluctuations in the outer shear layer. It has been reported that if the shear layers in the swirl-stabilized gas turbine combustor are acoustically excited to form a coherent vortex ring structure, the inner shear layer rolls up inward and the outer shear layer rolls up outward. This result suggests that the dynamics of $\mathrm{H}_{2}$-enriched " $\mathrm{M}$ " flames is totally different from that of "V" flames. It was found that even though periodic formation and convection of vortex structures occur at $\varphi=210 \sim 270^{\circ}$, the flame response remains in the linear regime.

In addition, important physical mechanisms controlling the response of partially premixed flames to inlet velocity and equivalence ratio oscillations were studied. This was done using a vector summation method. The decomposition of the partially premixed flame response into two subcomponent vectors provides useful insight into the underlying phenomena, and consequently suggests potential passive control methodologies to suppress combustion instabilities. The analysis demonstrates that the heat release response of a partially premixed flame can be effectively reconstructed using independent measurements of the flame's response to velocity and equivalence ratio fluctuations. In addition, the contribution of equivalence ratio oscillation to the global heat release rate oscillation of the partially premixed flame exceeds that of the velocity fluctuation by a factor of 2 . Also, the phase difference between the heat release and equivalence ratio oscillations is larger than the phase difference between heat release and inlet velocity oscillations, by a factor of approximately two. This result indicates that the heat release response of a partially premixed flame is chemical kinetics-controlled in terms of the response time scales. This final technical document summarizes our findings and major publications stemming from this research.

\section{Main Objectives}

- Remove critical barriers to usage of high hydrogen fuels in low emissions turbines by developing predictive capabilities for combustion instabilities

- Develop computational simulation capabilities to predict the conditions under which combustion instabilities occur

- Perform validating experiments of conditions under which instabilities occur

- Distill knowledge gained from simulations and experiments into reduced order models which can be used as a design tool 


\section{Accomplishments}

$\checkmark$ Developed predictive model quantifying how a turbulent flame responds to velocity and fuel/air ratio forcing

$\checkmark$ Developed model predicting flame response to transverse acoustic instabilities, such as are encountered in annular combustors

$\checkmark$ Developed a technique for measuring heat release fluctuations in unsteady flames with equivalence ratio fluctuations

$\checkmark$ Performed detailed analysis of key instability mechanism controlling heat release response for flames during low, mid and high frequency instabilities

$\checkmark$ Developed a large-eddy-simulation (LES) based numerical code for studying the flow and flame dynamics

$\checkmark$ Development of a pressure coupled flame response model

$\checkmark$ Measured the forced response of hydrogen-enriched natural gas/air premixed and partially premixed flames

$\checkmark$ Investigated the responses of a lean premixed flame to three different forcing mechanisms: velocity oscillations, equivalence ratio oscillations, and the combination of velocity and equivalence ratio oscillations

$\checkmark$ Analyzed important physical mechanisms controlling the response of a partially premixed flame to inlet velocity and equivalence ratio oscillations

$\checkmark$ Performed flame transfer function measurements on representative swirling flames

$\checkmark$ Worked closely with industrial partners to facilitate transition of models and understanding into design process for next generation gas turbines

$\checkmark$ Transitioned models into internal instability prediction codes at industrial partners who are using them as part of process to design the next generation of gas turbines

$\checkmark$ Documented results and made them available through the publication of 27 conference and journal papers.

\section{Introduction}

The objective of this research was to improve the state of the art in understanding and modeling combustion instabilities, one of the most critical problems associated with burning high hydrogen fuels in low emissions turbines. "Combustion instabilities" refers to damaging pressure oscillations excited by the combustion process. The damage due to these oscillations causes significant damage in low emissions turbines every year, through forced outages and broken parts.

\section{$\underline{\text { Approach }}$}

This work investigated these problems with a three pronged approach: experiments, computational kinetic calculations, and analytic modeling. It consisted of a collaborative effort involving two universities, both with strong expertise in dynamical combustion phenomenon, and gas turbine manufacturers. In addition, the research conducted was structured to provide significant student involvement (including high school teachers, undergraduate students, and graduate students) and therefore has and will make an important contribution to the future science and technology base of our nation. Its focus upon high fidelity simulations, coupled with validating measurements, and development of reduced order models has and will significantly improve understanding of these phenomenon and capabilities for predicting their occurrence. 


\section{$\underline{\text { Results }}$}

\section{Introductory Material and Overview}

Modern land based gas turbine manufacturers are increasingly moving towards a premixed mode of combustor operation. This operational shift and the subsequent design changes have been primarily driven by the strict emission guidelines on pollutants like $\mathrm{NO}_{\mathrm{x}}$ and $\mathrm{CO}$. One of the principal strategies adopted to satisfy these emission norms is to burn the fuel-air mixture at lean equivalence ratios. At lean conditions, the production of thermal $\mathrm{NO}_{\mathrm{x}}$ is greatly reduced due to the lowered temperatures. However, one major drawback of lean, premixed operation is that these combustors are especially prone to self excited oscillations. This dynamic phenomenon generally occurs when the unsteady combustion process couples with the natural acoustic modes of the combustor. These instabilities are destructive to hardware and adversely affect performance and emissions.

The condition under which acoustic oscillations are amplified by a fluctuating source of heat release was first postulated by Lord Rayleigh. Rayleigh's criterion essentially states that the instabilities in a combustor are driven when the unsteady combustion processes add energy in phase to the acoustic oscillations. In general, this instability is sustained if the rate at which the energy is added to the acoustic oscillations is greater than the rate at which the acoustic energy is absorbed. Essentially, a perturbation in a flow variable causes a change in the flame heat release rate. Subsequently, this unsteadiness in the flame heat release generates acoustic waves. The feedback loop is completed when these acoustic waves cause further changes in the flow variables. In a typical combustor, this feedback mechanism can be completed by a variety of driving processes like equivalence ratio oscillations, velocity oscillations, flame extinction, vortex dynamics, flame wall interaction etc. Depending on the operating conditions, one or more of these mechanisms may be driving the oscillations.

Lastly, it is important to note the organization of data being reported in this technical report. We will be following a semi-chronological approach because this way the readers get a good feel for the mindset and thinking processes endured. During some later sections, questions and additional issues that arose in earlier sections are solved. This is a great way to track the process and progress achieved. At the end of long sections we will provide a comprehensive "review" of important results, conclusions, and findings.

\section{Analytical Work}

During this sponsorship, analytical work was conducted on the development of engineering prediction models that can be used to predict flame characteristics. The basic modeling framework developed by the authors utilizes a level-set approach to quantitatively describe the flame dynamics. This approach eliminates phenomenological or heuristic descriptions of the flame dynamics. This facilitates in breaking down the much larger problem of combustion instability into a more fundamental problem at the flame. 


\section{Modeling Flame Response to Fuel/Air Ratio Oscillations}

\section{Introduction and Motivation}

Fuel/air ratio oscillations play a key role in exciting combustion instabilities. While basic models are in the literature and have been incorporated into internal codes at several OEM's, a number of questions and issues remain. Work is still needed in two areas - (1) capturing nonlinear effects (needed for predicting limit cycle amplitudes) and (2) non quasi-steady effects.

It is helpful to consider an expression for the instantaneous rate of heat release from a premixed flame sheet may be expressed as

$$
q(t)=\int_{A(t)} \rho_{u} s_{L}(t) h_{R}(t) d A(t)
$$

where, $s_{L}$ and $h_{R}$ are the flame speed and heat of reaction respectively. The subscript $u$ refers to the value in the unburned reactant mixture and the integral is performed over the entire instantaneous burning area of the flame, $A(t)$. Clearly, from Eq.(1), it may be seen that oscillations in heat release are caused by the superposition of oscillations in the flame surface area, mass burning rate, and heat of reaction. Heat release oscillations occur either as a direct or an indirect effect of equivalence ratio perturbations. Equivalence ratio perturbations directly lead to perturbations in $s_{L}$ and $h_{R}$. As may be seen from Eq.(1), this leads to oscillations in the heat release of flame. In addition, oscillations in the burning velocity cause fluctuations in flame position, leading to excitation of waves that propagate along and wrinkle the flame front. This leads to oscillations in the flame surface area, which in turn causes heat release oscillations. This is an indirect effect, as the flame area perturbations are directly related to flame speed perturbations.

Prior analyses have studied this problem in the linear regime and shown that heat release dynamics are controlled by the superposition of three processes: flame speed, heat of reaction, and flame surface area oscillations. Each contribution has somewhat different dynamics, leading to complex frequency and fuel/air ratio dependencies. The work presented in this section extends these analyses to account for two complexities.

Firstly, flame dynamics in the non-linear regime has been extensively investigated, since an understanding of the nonlinear dynamics is very essential in understanding the limit cycle characteristics. This has been achieved utilizing a third order perturbation analysis and full computations. Because of the fact that the flame response is controlled by a superposition of three processes, each with a highly nonlinear dependence upon fuel/air ratio, the results are quite rich and the key nonlinearity mechanism varies with mean fuel/air ratio, frequency, and amplitude of excitation. Two key mechanisms leading to heat-release saturation have been identified. The first mechanism of these is due to non-linearities in the flame speed and heat of reaction dependence upon equivalence ratio. The second is due to the intrinsic nonlinear property of premixed flames in that they propagate normally to themselves at each point, the "kinematic restoration mechanism". The first mechanism manifests itself in two different ways, most prominently through the so called "cross-over" mechanism. This can occur in two ways, 
depending on the mean equivalence ratio of the mixture. If the mean equivalence ratio is close to stoichiometry, then, based on the excitation amplitude, the instantaneous stoichiometry could oscillate between lean and rich stoichiometries, thereby causing non-monotonic variation of local flame speed and heat of reaction with equivalence ratio. However, for systems running very lean, as is relevant in the operation of low $\mathrm{NO}_{\mathrm{x}}$ gas turbines, it is more likely that they will encounter local extinction due to deviations of the fuel/air ratio below the flammability limits, well before they begin crossing over into the rich side. The excitation amplitude, $\varepsilon$, when this mechanism becomes significant and leads to "cross-over" into regimes of mixture composition that cannot sustain combustion, is simply the difference in value between the mean equivalence ratio and the flammability limit on the lean side. $\varepsilon=\frac{\phi_{o}-\phi_{s, \text { lean }}}{\phi_{o}}$. It can be anticipated that the perturbation amplitude where the instantaneous equivalence ratio passes through the flammability limits will also be associated with significant changes in flame dynamics, including saturation in the heat release of the flame.

Secondly, the non quasi-steady response of a premixed flame submitted to upstream equivalence ratio oscillations has been investigated. Inherent dynamics are introduced due to the time delay associated with this flame disturbance to propagate from its origin to the end of the flame. Even when the internal flame structure is neglected, the response of the flame exhibits dynamics associated with time delays proportional to $L_{f} / U_{o}$. However, incorporating flame structure effects introduces additional dynamics associated with those of the convectivediffusive-reactive balance within the flame itself. For example, the flame speed does not respond in a quasi-steady manner to fuel/air ratio fluctuations with time scales faster than those of the flame.

The objective of this second study is to extend the first study which allowed for dynamical effects in the response of the flame sheet itself, but neglected those associated with the internal flame structure. We generalize here our earlier quasi-steady results to allow for the propagation time within the diffusion zone of a premixed flame, such that the flame itself responds in a non quasi-steady manner. Two important Strouhal numbers parametrizing the flame response were identified $-S t_{L}$, based on the excitation angular frequency, mean flow velocity and mean flame length, and $S t_{\delta}$, based on the excitation angular frequency, mean flame speed and flame thickness. These will be talked about in detail later, however the former describes the flame dynamics associated with purely kinematic processes as it responds in position to fluctuations in flame speed, while the latter describes that of the internal flame structure. Interestingly, the analysis suggests that the relative roles of the three contributing processes $\left(s_{L}, h_{R}, A\right)$ do not change in the non-quasi steady case, as each respective term is scaled by the same correction term. As such, the qualitative characteristics of the flame response determined from prior quasi steady analyses remain valid, being simply scaled by a term that decays with increase in $S t_{\delta}$. This conclusion is of great significance, since if the three contributing processes scaled differently, the non quasi steady flame dynamics would have been significantly altered and the extension from the quasi steady analyses would have been more complex. While the non-quasi steady correction was determined to be quite significant for flames at atmospheric pressure, detailed chemical computations at gas turbine pressures/temperatures revealed that the effects are probably small relative to other inherent modeling uncertainties (e.g., flame position). 


\section{Formulation}

A key objective of this work is to determine the transfer function relating the heat release fluctuation of the flame to a small amplitude fuel/air perturbation; i.e.

$$
F=\frac{q^{\prime}(\omega) / q_{o}}{\hat{\phi}^{\prime} / \phi_{o}}
$$

where $q$ denotes the heat release, given by Eq.(1) and the symbols ( )' and ( )o denote the oscillatory and mean quantities respectively. The heat release can be derived from Eq. (1) and written as

$$
\frac{q(t)}{q_{o}}=\frac{A(t)}{A_{o}}+\int_{\text {flame }} \frac{s_{L}{ }^{\prime}}{s_{L_{o}}} \frac{d A}{A_{o}}+\int_{\text {flame }} \frac{h_{R}{ }^{\prime}}{h_{R_{o}}} \frac{d A}{A_{o}}+\int_{\text {flame }} \frac{s_{L}{ }^{\prime} h_{R}{ }^{\prime}}{s_{L_{o}} h_{R_{o}}} \frac{d A}{A_{o}}
$$

where it is assumed that the fuel/air ratio perturbation occurs at constant density. Referring to Eq.(3), note that modeling the heat release requires expressions for the flame speed, heat of reaction and the flame surface area. The mean values of $s_{L}$ and $h_{R}$ are presumed to be prescribed functions of the quiescent equivalence ratio, i.e., $s_{L o}=s_{L o}\left(\phi_{o}\right)$ and $h_{R o}=h_{R o}\left(\phi_{o}\right)$ respectively. We may define the flame speed and heat of reaction sensitivities, $s_{L 1}$ and $h_{R 1}$ as

$$
s_{L 1}=\left.\frac{\partial\left(s_{L} / s_{L_{o}}\right)}{\partial\left(\phi^{\prime} / \phi_{o}\right)}\right|_{\left(\phi^{\prime} / \phi_{o}\right)=0} \quad \text { and } \quad h_{R 1}=\left.\frac{\partial\left(h_{R} / h_{R_{o}}\right)}{\partial\left(\phi^{\prime} / \phi_{o}\right)}\right|_{\left(\phi^{\prime} / \phi_{o}\right)=0}
$$

respectively. In the quasi-steady case, the flame speed and heat of reaction term are functions of fuel/air ratio, fuel type, and operating condition. In the general unsteady case, however, these quantities, particularly the flame speed, introduce additional dynamics related to the flame structure so that the instantaneous flame speed is also a function of frequency.

Next, we consider the model for the flame area dynamics. The flame is assumed to consist of a thin sheet whose surface can be represented implicitly by the zero contour of a two dimensional function $G(r, \mathrm{y})$. The evolution of this contour can then be tracked using the $G$-equation

$$
\frac{\partial G}{\partial t}+\vec{U} \cdot \nabla G=s_{L}|\nabla G|
$$

where, $U$ is the local flow velocity. To make analytical progress, Eq.(5) is then solved in the explicit form using a small perturbation method, using $\phi^{\prime} / \phi_{o}$ at the flame base as the small parameter, to obtain the flame front location with respect to the chosen coordinate system. The heat release by the flame is then evaluated following Eq.(3); thereafter the heat releaseequivalence ratio transfer function may be evaluated using Eq.(2). However, in the general nonlinear case, Eq.(5) is solved as is, using robust numerical schemes that can capture highly nonlinear topological characteristics without excessive smearing. 


\section{Analysis}

A sample of the investigated geometries utilized throughout this technical report are illustrated schematically in Figure 1. For each schematic shown in Figure 1, a unique form of the flamefront tracking equation was utilized to solve for the instantaneous location of the flame surface, $\xi(x, t)$. Each form had its own simplifications and coordinate system choice for simplification. As a good example, the bluff body stabilized flame and conical flame configurations are considered. The instantaneous location of the flame surface $\xi(r, t)$ is given by the flame-front tracking equation,

$$
\frac{\partial \xi}{\partial t}+s_{L}\left[\left(\frac{\partial \xi}{\partial r}\right)^{2}+1\right\rceil^{1 / 2}=u
$$

As another example, the form used for the top-left schematic in Figure 1a is shown here:

$$
\frac{\partial \zeta}{\partial t}+u \frac{\partial \zeta}{\partial x}+v \frac{\partial \zeta}{\partial y}-w=-s_{L} \sqrt{1+\left(\frac{\partial \zeta}{\partial x}\right)^{2}+\left(\frac{\partial \zeta}{\partial y}\right)^{2}}
$$

Lastly, the form of the flame-front tracking equation used for axi-symmetric swirling flame configurations is given by the following equation,

$$
\frac{\partial \xi}{\partial t}+u_{r} \frac{\partial \xi}{\partial r}+\frac{u_{\theta}}{r} \frac{\partial \xi}{\partial \theta}+s_{L}\left[\left(\frac{\partial \xi}{\partial r}\right)^{2}+\frac{1}{r^{2}}\left(\frac{\partial \xi}{\partial \theta}\right)^{2}+1 \prod^{1 / 2}=u_{z}\right.
$$
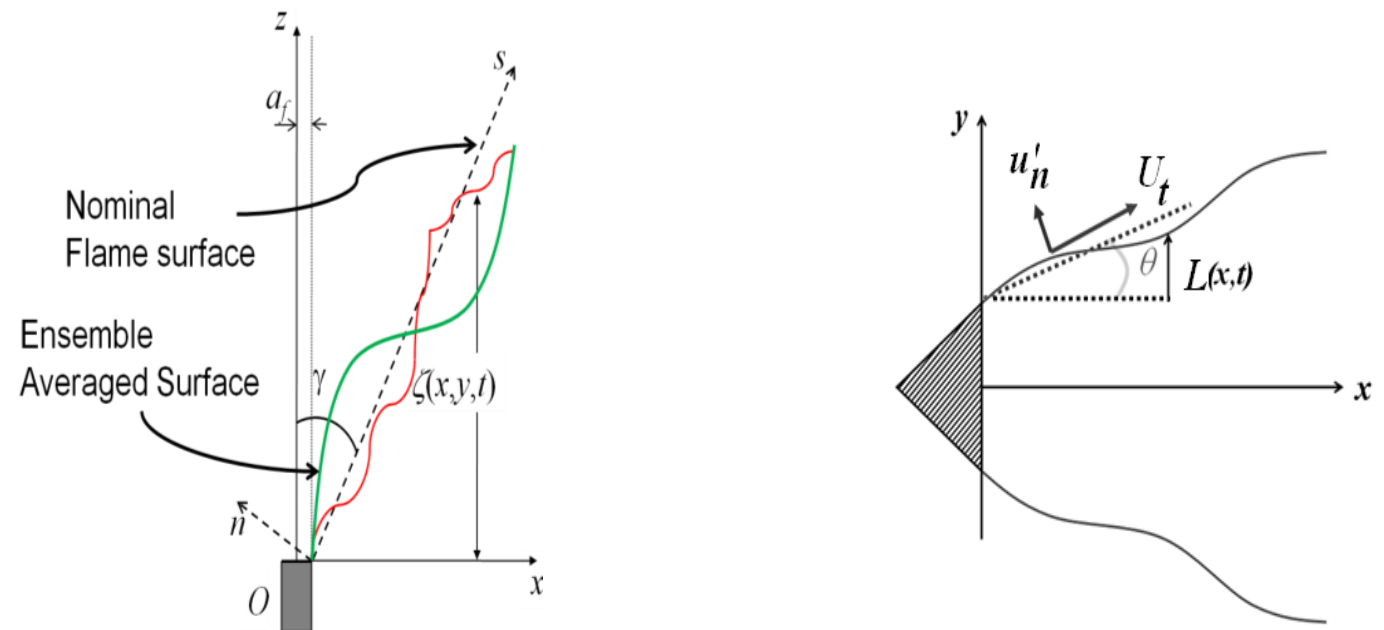

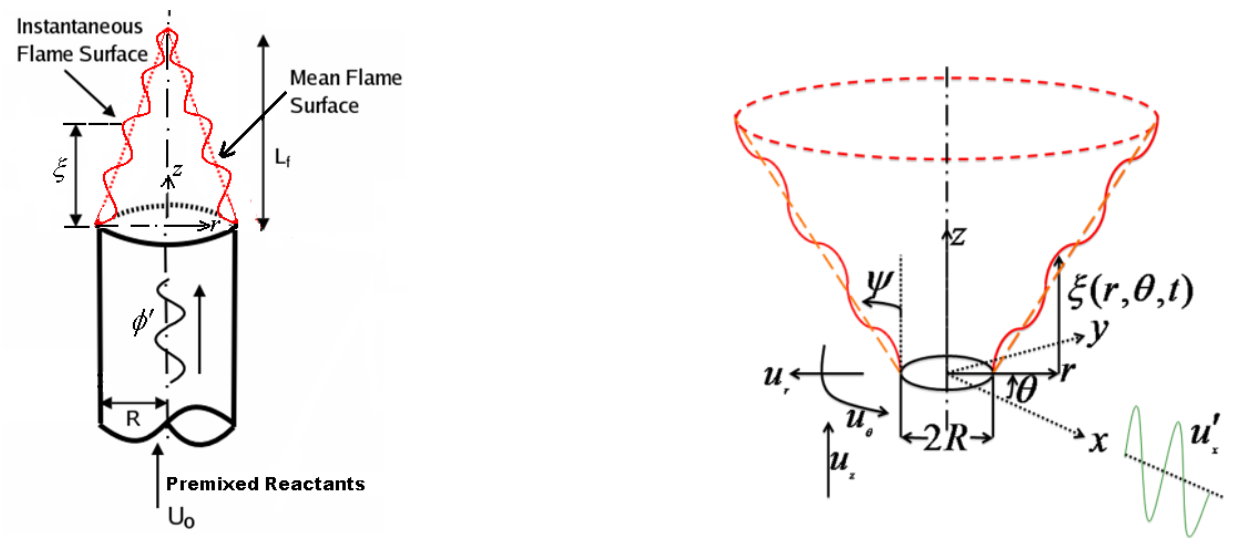

Figure 1: Schematic representations of investigated geometries. (top-left) Schematic showing one half of the instantaneous flame surface (solid red curve), the instantaneous coherent flame surface (solid green curve), and the nominal flame surface (dashed black line). (top-right) Schematic of geometry for bluff-body stabilized flame. (bottom-left) Schematic of geometry for the fuel/air ratio and pressure forcing investigation of a conical flame. (bottom-right) Schematic of geometry for effect of swirl on a velocity-forced centerbody stabilized flame.

Our focus has been the understanding of quantitative and qualitative characteristics of solutions of this nonlinear, partial differential equation. These solutions are the first of their kind. We have solved this equation in a framework where the pressure, velocity, and fuel/air ratio oscillations have frequencies ranging from low to very high values, in order to understand whether (and how) the controlling mechanisms change with operating conditions and frequency. We intended to examine the importance of each of these different mechanisms with respect to one another. This is an important piece of information that will help in bettering the models that we have developed in the last few years. Accounting for more physics will enable the model to handle a wider range of frequencies and also provide better capability for predicting real-life combustion dynamics.

A variety of physical processes were accounted for in studying the combustion dynamics of a system excited by oscillations in velocity, fuel/air ratio and pressure. Some of these are associated with the relative length scales and time scales of these disturbances in comparison to inherent length and time scales that exist in the fluid dynamics and combustion phenomena related to the problem. To this end, we define a flame to globally quasi-steady when the time scale over which these disturbances vary is much larger than the time required for the passage of these disturbances across the extent of the flame. In such a situation, the entire flame responds instantaneously to any imposed disturbances. We quantify this ratio of timescales by means of a 'global' Strouhal number,

$$
S t_{f}=\frac{f L_{f}}{u_{o}}
$$


Where $L_{f}$ is the length of the nominal flame and $u_{o}$ is the mean velocity of the reactants. This definition of the 'global' Strouhal number implies that when it is much smaller than 1, the flame responds instantaneously to disturbances.

For the purpose of illustration, consider an axi-symmetric burner stabilized conical flame, as shown in the bottom left image of Figure 1. We assume that the flame remains attached to the burner lip at all points of time. For the investigated geometry, this translates to

$$
\xi(r=R, t)=0
$$

The non quasi-steady transfer function is obtained to be the product of the quasi-steady transfer function, which is parameterized by $S t_{L}$, and a correction term that accounts for non quasisteady effects, which is a function of $s t_{\delta}$, where these Strouhal numbers are defined as $S t_{L}=\omega L_{f} / U_{o}$ and $S t_{\delta}=\omega \delta / 2 s_{L}$, where $\delta$ is the flame thickness and $\omega$ is the excitation angular frequency. Table 1 provides the analytical results for the quasi-steady linear response of a premixed flame to equivalence ratio perturbations to each of the three terms shown.

Table 1 : Analytical results for quasi-steady linear transfer function

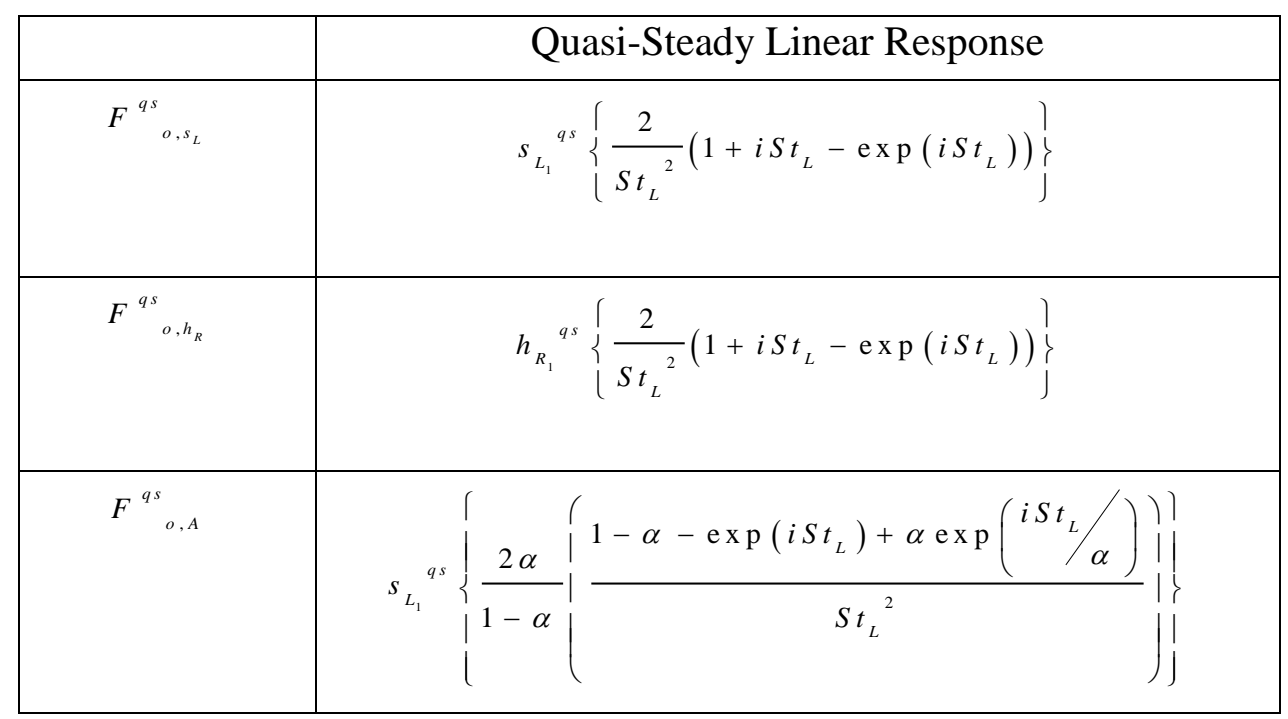

Here, the quasi-steady flame speed and heat of reaction sensitivities, $s_{L 1}{ }^{q s}$ and $h_{R 1}^{q s}$ are defined to be the quasi-steady limits of $s_{L 1}$ and $h_{R 1}$, i.e., $s_{L 1}^{q s}=\lim _{s_{t_{\delta}} \rightarrow 0} s_{L 1}$ and $h_{R 1}^{q s}=\lim _{s_{t_{\delta}} \rightarrow 0} h_{R 1}$. The total quasisteady transfer function is obtained as a linear sum of the three transfer functions in Table 1, i.e,

$$
F^{q s}=F_{o, s_{L}}^{q s}+F_{o, h_{R}}^{q s}+F_{o, A}^{q s}
$$

Finally, the non quasi-steady transfer function is the relatively simple modification: 


$$
F^{n q s}=F^{q s}\left(S t_{L}\right)\left(\sin c\left(S t_{\delta}\right)\right)^{2} \exp \left(i S t_{\delta}\right)
$$

The simplicity of this result relative to the quasi-steady case is remarkable. This is due to the reason that $s_{L 1}$ and $h_{R 1}$ have the same frequency dependence. The area contribution term is directly proportional to the $s_{L 1}$ term and necessarily has its identical form. As such, the relative magnitudes of the three contributing processes shown in Table 1 do not change in the non quasi-steady case; rather all are scaled equally so that the overall result is affected, without altering the relative balance between the contributing terms.

\section{Computational Approach}

Formally, Eq.(5) is a Hamilton-Jacobi equation. This equation is non-conservative and has the property that the non-linear term, due to flame propagation normal to itself, results in cusps, or discontinuities in derivative, and possible topological changes (i.e. pocket formation) in the solution. Hence robust numerical schemes that can capture these effects without excessive smearing are required. The solution domain is discretized using a uniform grid. The initial value for the $G$-field was constructed from the assumed quiescent flame shape. This was done by defining the value of $G$ at each grid location to be the signed distance of that location from the quiescent flame surface. The solution at later times was obtained using a low diffusion CourantIsaacson-Rees scheme with back and forth error compensation and correction (BFECC). The $G$ field was reset to a distance function after each time step. A considerable reduction in computation time can be obtained by solving Eq.(5) in only a narrow band around the actual flame location, rather than in the entire two-dimensional domain. The instantaneous heat release of the flame is given by Eq.(1). This can be written using $G$ as,

$$
q(t)=\int_{\Omega} 2 \pi r \rho s_{L} h_{R} \delta(G)|\nabla G| d \Omega
$$

where the integration is performed over the whole narrow band computational domain described earlier and $\delta(G)$ is the Dirac-delta function. The computational approach was utilized to study only the quasi steady flame dynamics.

\section{Results}

Here, we provide representative results for a conical flame with aspect ratio, $\beta=4.0$. The investigated geometry is shown schematically in Figure 1. The experimentally determined correlations for the burning velocity magnitude and heat of reaction with equivalence ratio for a methane-air flame, as given by Eq.(14) and Eq.(15) were assumed.

$$
\begin{aligned}
s_{L}(\phi)=A \phi^{B} e^{-C(\phi-D)^{2}} ; \quad A & =0.6079, B=-2.554, C=7.31, D=1.230 \\
h_{R}(\phi) & =\frac{2.9125 \times 10^{6} \mathrm{~min}(1, \phi)}{1+0.05825 \phi}
\end{aligned}
$$




\section{Linear Dynamics}

We begin with a brief discussion of the characteristics of the linear transfer function. Figure 2 shows the variation of the phase and magnitude of the linear transfer function for a representative lean mean equivalence ratio. Also shown are the phase and magnitude of the individual contributions to the total transfer function. Firstly, note that the variation of both the phase and the magnitude do not monotonically vary with $S t_{2}$. This is due to the fact that the linear flame response is determined by the net superposition of a boundary generated "wave" and a local disturbance, as discussed in the previous section. Therefore the net flame response depends on exactly how these waves superpose at different Strouhal numbers. As such, the phase of the flame speed and the heat of reaction contributions will be identical in the linear limit. Also, total gain starts from a value of nearly unity. This is due to the fact that the flame response is entirely controlled by the heat of reaction sensitivity in the quasi-steady case. The total gain then initially grows with increasing Strouhal number, because the burning area and the fluctuating flame speed terms progressively come into phase with each other. As they go out of phase, there is a decrease in the total gain and the minimum is encountered when they are 180 degrees out of phase.

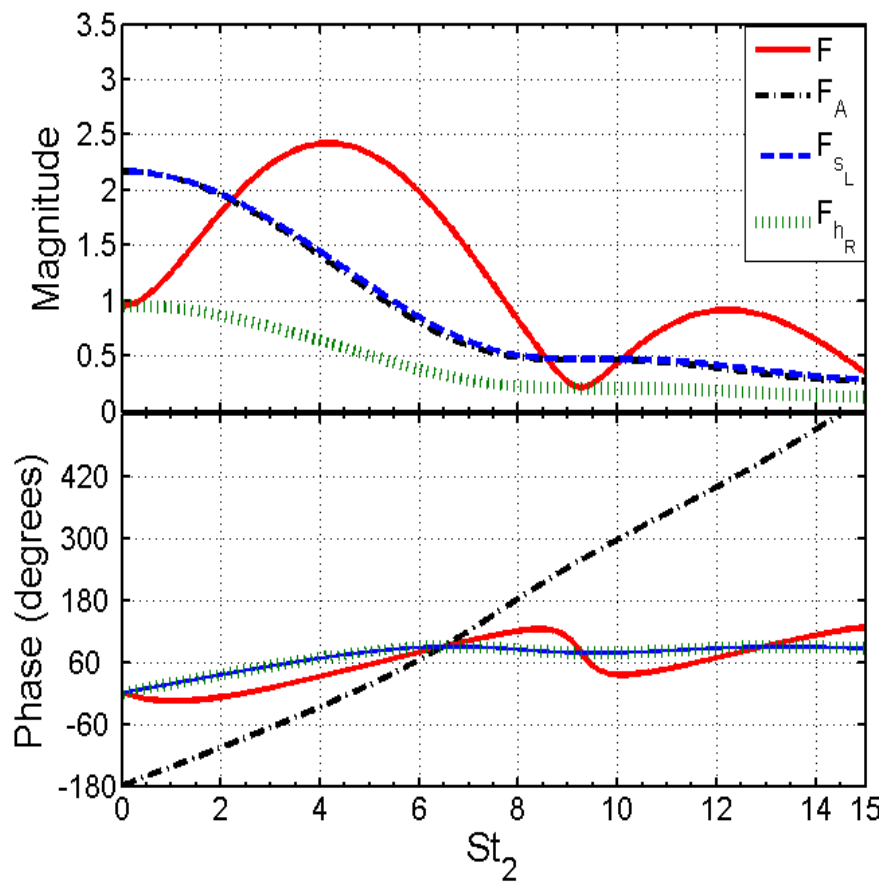

Figure 2: Linear transfer function for $\phi_{o}=0.85$ (lean), $\beta=4$ •

We next consider the effect of non quasi-steady processes on the flame dynamics and estimate the deviation of the non quasi steady transfer function from the quasi steady counterpart. This is shown in Figure 3. These results will be discussed in further detail later in the report. 


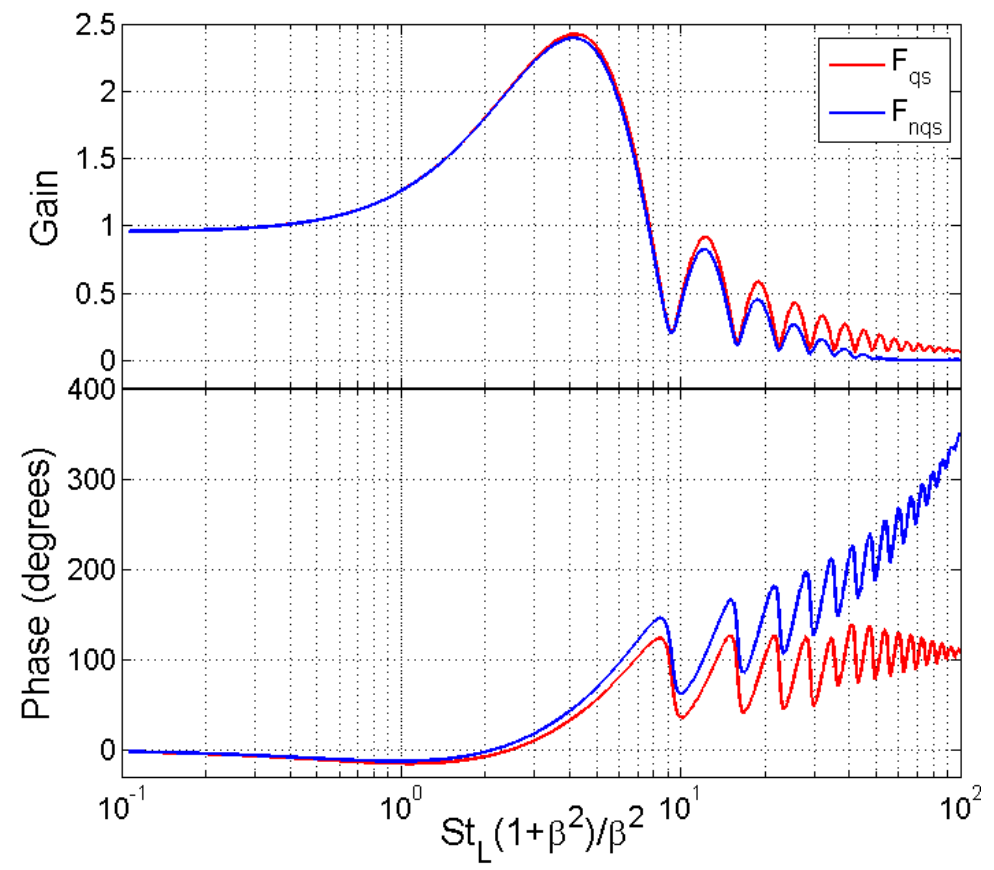

Figure 3: Quasi-steady and non quasi-steady response characteristics of a premixed flame with $\beta=4, \phi_{o}=0.85$ and $\delta / d=0.05$

The following may immediately be observed from Figure 3. In the low Strouhal number limit, both the non quasi-steady and the quasi-steady gains and the phases approach one another, as expected. In this limit, the gain attains the value of the linear heat of reaction sensitivity, as discussed in detail in our earlier work. However, as the Strouhal number increases, the gain of the non-quasi steady response begins to drop quite rapidly beyond $s t_{L}=10$; in fact, at $S t_{L}=40$, the flame is almost passive for the non quasi-steady case, while the gain of the quasi-steady response is still appreciable. It can also be seen from the phase characteristics that as the Strouhal number increases, the phase difference between the non-quasi steady and quasi-steady response increases. At very high Strouhal numbers, once the gain is almost zero, the disparity in phase becomes immaterial. However, from detailed chemical calculations, assuming an isentropic compression starting from atmospheric conditions, it may be shown that for low frequencies and pressures $\sim 10 \mathrm{~atm}$, non quasi steady effects affects the gain moderately; the effects on phase may be almost disregarded. This is shown in Figure 4, which plots the variation in the non quasi steady correction factor over a wide range of pressures, for a mean equivalence ratio of 0.6 . 


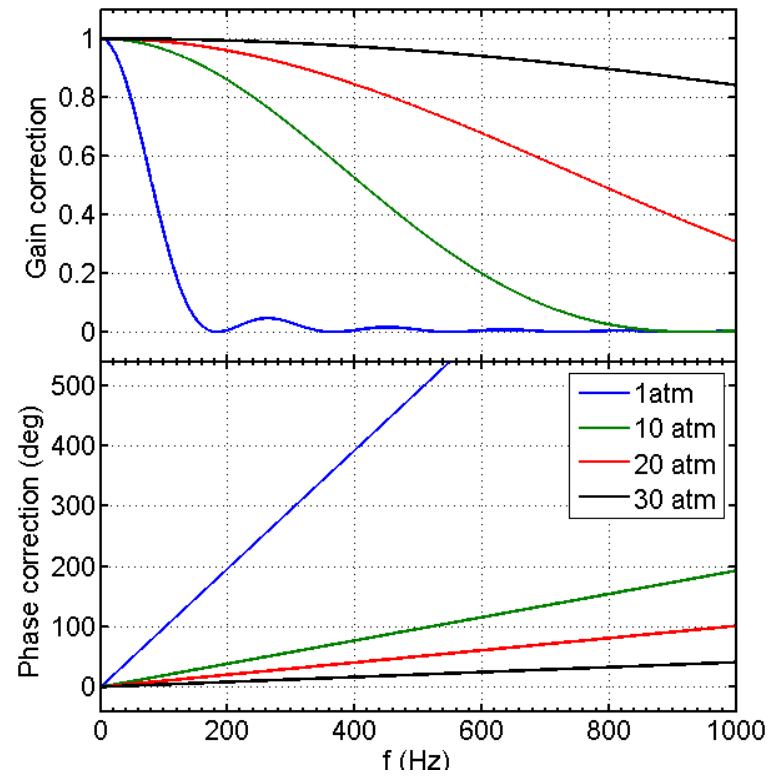

Figure 4: Variation of the non quasi steady transfer function correction for gas turbine conditions

It may be seen from Figure 4 that at frequencies of about $200 \mathrm{~Hz}$ at pressures $\sim 10 \mathrm{~atm}$, while there is about a $10 \%$ drop in the gain, there is about a 30 deg variation in the phase. As such, as the pressure increases, there seems to be hardly any appreciable effect of non quasi steady processes on the flame dynamics. Hence, it suffices to consider the quasi steady response of the flame in domains that interest gas turbine applications. Additional typical results illustrating the flame response to fuel/air ratio oscillations are shown in the top set of images in Figure 5. In addition, the bottom sets of images of Figure 5 illustrates the effect of including high frequency corrections to the flame response, important for capturing screeching flame instability characteristics. 

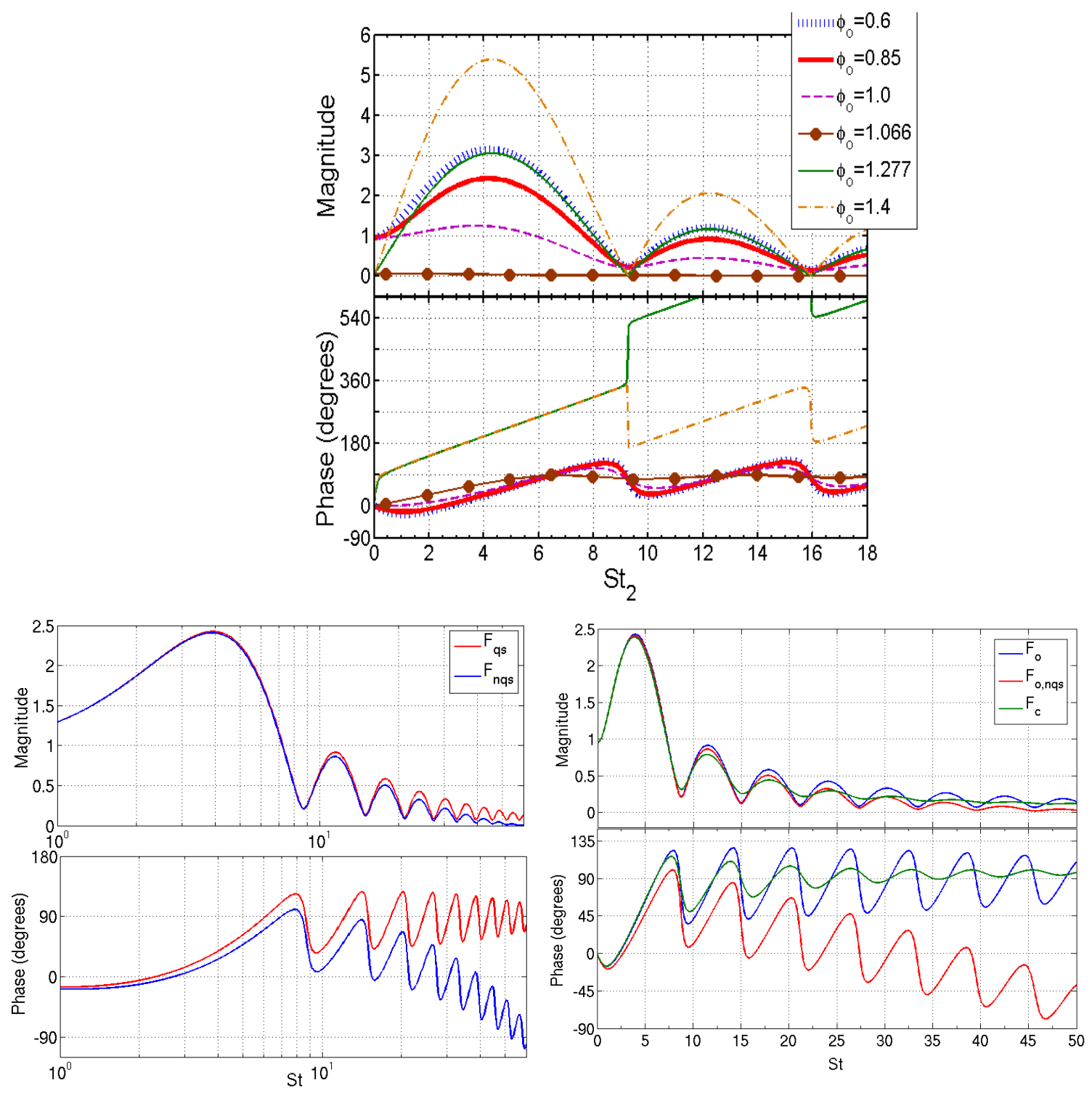

Figure 5: (left) Variation of the linear transfer function with $S t_{2}$ for different values of equivalence ratio, $\beta=4$ (center) Differences between Quasi-steady and Non quasi-steady effects. (right) Differences between stretched and un-stretched flame response.

\section{Nonlinear Dynamics}

As the excitation amplitude/frequency is increased, the higher order contributions to the transfer function become significant. Figure 6 shows typical boundaries for the regions in the parametric space of the current study where the various non-linearity mechanisms are operative. These regions are shown for the lean flame corresponding to $\phi_{o}=0.85$. Note that the heat release response in the region labeled ' 1 ' in Figure 6 is essentially linear and has the characteristics described in the previous subsection. 


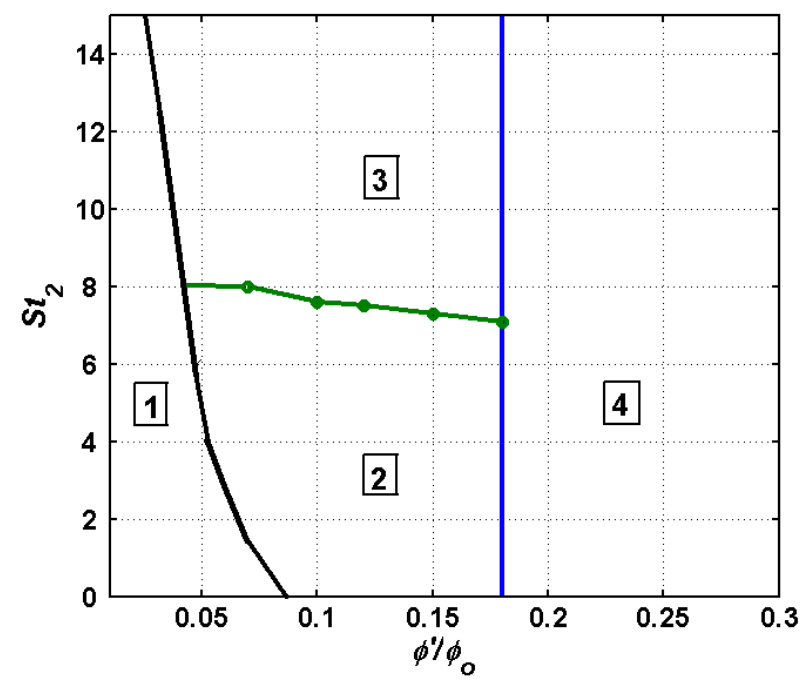

Figure 6: Qualitative map illustrating regimes of dominance of various nonlinearity mechanisms at $\phi_{o}=0.85$. (1) Linear, (2) $s_{L^{-}} \phi$ non-linearity, (3) kinematic restoration and (4) "cross-over" across stoichiometric.

There are two basic processes causing nonlinearity in the flame response:

1) Non-linearities in burning area oscillation, due to the nonlinearities in flame kinematics (term 1 in Eq.(3)) and

2) Quasi-steady nonlinearities in the $s_{L^{-}} \phi$ and $h_{R^{-}} \phi$ relationships.

There is an additional complication, however, in the fact that the $s_{L^{-}} \phi$ nonlinearity has both a direct and indirect influence on the heat release response through term 2 and term 1 in Eq.(3) respectively. It is this latter, indirect mechanism which dominates the heat release nonlinearities in Region ' 2 ' in Figure 6. The flame kinematic nonlinearities dominate the heat release nonlinearities in Region ' 3 ' in Figure 6. Larger amplitude fluctuations in the flame position slope cause kinematic nonlinearities to correspondingly grow in significance. At high frequencies, propagation of the flame surface normal to itself results in the rapid destruction of these wrinkles causing the net flame burning area to saturate. This phenomenon, known as kinematic restoration, is an additional source of flame kinematic nonlinearity and dominates the flame response at $S t_{2}>8.0$ in Region 3 in Figure 6.

We finally consider Regime 4, labeled the "crossover" mechanism. This nonlinearity is related to that of Region 2 in that, it is completely due to the second source of nonlinearity noted above, i.e. the $s_{L^{-}} \phi$ and $h_{R^{-}} \phi$ nonlinearities. However, in this region, this mechanism dominates for all Strouhal numbers and is due to the drastic change in $s_{L}$ and $h_{R}$ characteristics on the lean and rich side of stoichiometric. The trend in the variation of $s_{L}$ and $h_{R}$ qualitatively changes when this cross over occurs. This occurs at an amplitude given by $\varepsilon_{s i}=\frac{1}{\phi_{o}} \min \left\{\left|1-\phi_{o}\right|,\left|\phi_{s_{L} \max }-\phi_{o}\right|\right\}$. Furthermore, if the excitation amplitude is increased beyond $\varepsilon_{s, \text { sean }}=\frac{\phi_{o}-\phi_{s, \text { lacan }}}{\phi_{o}}$, the mixture composition can instantaneously assume values which are below the lean static flammability equivalence ratio, and local extinction can occur. This can cause heat release to saturate. In fact, 
this is the non-linearity cross-over mechanism, which is perhaps encountered at gas turbine conditions, more frequently that saturation due to crossover across the stoichiometric.

Consider the following, for instance. For methane-air, for a mean equivalence ratio of 0.85 , the crossover across the stoichiometric occurs at an excitation amplitude of about 0.18 , while crossover across the static flammability limit occurs at a critical excitation amplitude of about 0.41. Hence, saturation in heat release encountered due to the former crossover mechanism is more dominant. On the other hand, for a mean equivalence ratio of 0.6 , crossover across the lean flammability limit occurs at an amplitude of about 0.18 . As the excitation amplitude is increased, during an excitation cycle, the mixture composition attains values beneath this flammability limit for longer instances of time. Consequently, crossover into the rich side is accompanied by crossover across the lean flammability boundary, hence rendering the sub-flammability limit crossover as the dominant mechanism for saturation. Hence, if for some instantaneous oscillation amplitude $\phi$ around some lean mean equivalence ratio $\phi_{o}$, the amplitude at which the crossover mechanism causes heat release saturation may be expressed as $\varepsilon_{\text {crossover }}=\frac{1}{\phi_{o}} \min \left\{\left(\phi_{o}-\phi_{s f, \text { lean }}\right),\left(1-\phi_{o}\right),\left(\phi_{s_{L} \max }-\phi_{o}\right)\right\}$. The variation of the transfer function with excitation amplitude and frequency is shown in Figure 7.
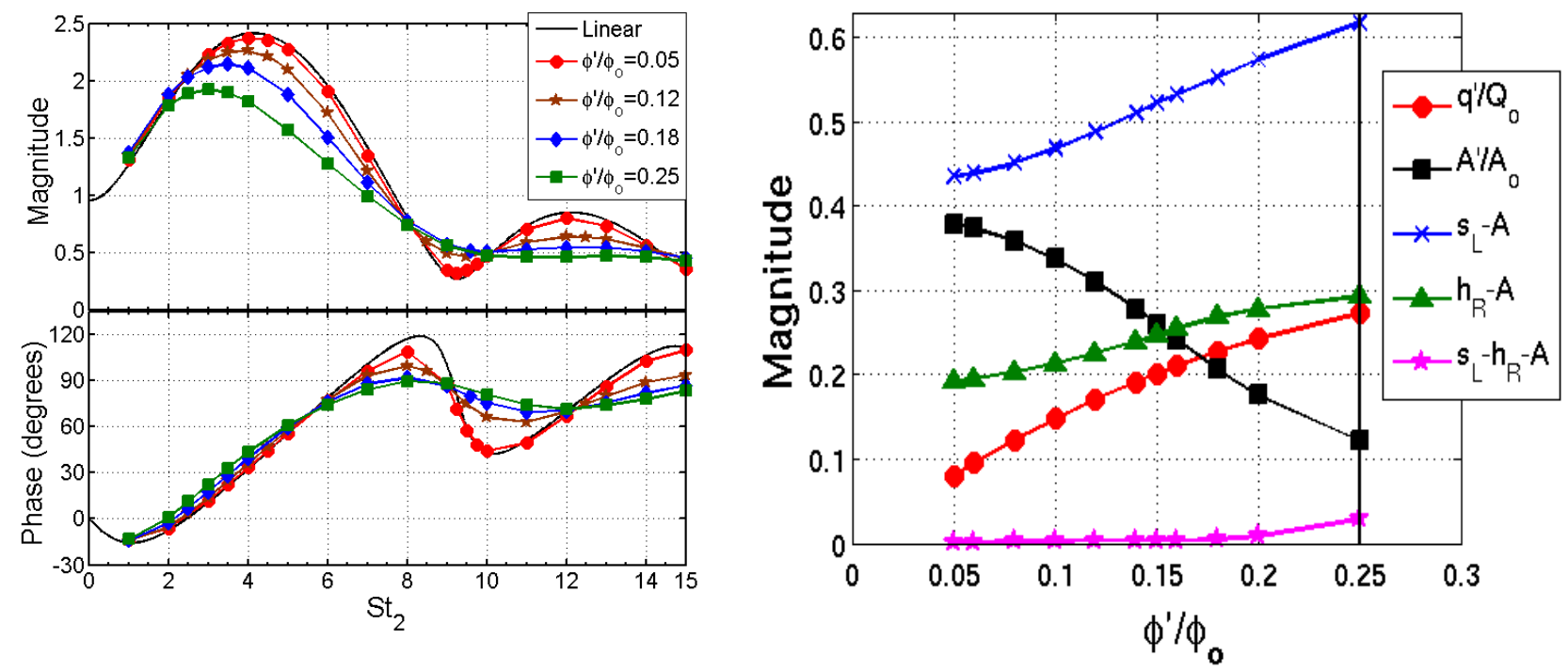

Figure 7: Variation of the transfer function with (a) Strouhal number for various excitation amplitudes (b) excitation amplitude for $S t_{2}=6.28 ; \beta=4.0, \phi_{0}=0.85$

Figure 7(a) plots the variation of the magnitude and phase of the total heat release transfer function with increasing excitation amplitudes for a lean flame. Notice first that the transfer function response for all excitation amplitudes tends toward the linear value as $S t_{2} \rightarrow 0$. With increasing $S t_{2}$, the transfer function begins to deviate significantly from the linear value. For $S t_{2}<$ 8.0 in either case, kinematic restoration effects are not important, as explained earlier. As such the slight deviation from the linear value at low amplitudes with increasing $S t_{2}$ can be ascribed to the manifestation of $s_{L^{-}} \phi$ non-linearities in both cases. Beyond $S t_{2}=8.0$, kinematic restoration 
effects contribute to slight deviation from the linear response observed at low excitation amplitudes. As the excitation amplitude is increased beyond, the crossover mechanism becomes dominant.

We now examine the converse scenario, i.e. the variation of heat release response with excitation amplitude at a fixed value of $S t_{2}$, in Figure 7(b), which plots the variation of the magnitude of the heat release response with increasing excitation amplitude for a lean flame. The solid vertical line shows the amplitude where the cross-over mechanism is initiated. Overlaid are the magnitudes of the individual constituent components of the heat release response in each case. These data have been obtained at $S t_{2} \sim 2 \pi$. From Figure 7(a), this corresponds to regions where kinematic restoration is not significant. Notice, therefore, that in either case, the amplitude of the burning area oscillation varies non-linearly with excitation. The burning rate term, labeled ' $s_{L}-A$ ', increases up to the crossover boundary in both cases. Figure 7(b) shows that the magnitude of this term saturates beyond the crossover boundary along with the net heat release amplitude. This nonlinear variation of the burning area oscillation amplitude with excitation amplitude shows that heat-release saturation is controlled completely by the cross-over mechanism at this value of $S t_{2}(\sim 2 \pi)$ and not by the kinematic restoration mechanism. This is in contrast with the results for flames perturbed by velocity oscillations, which show kinematic restoration to be the dominant mechanism leading to heat release saturation.

\section{Review}

A major aim of this section has been to better understand the response of premixed flames to fuel/air ratio perturbations. The key contribution of this work has been towards understanding the mechanisms for generation of non-linearities in the heat-release response of premixed flames. The first mechanism of these is due to non-linearities in the flame speed and heat of reaction dependence upon equivalence ratio. The second is due to the intrinsic nonlinear property of premixed flames in that they propagate normally to themselves at each point, the "kinematic restoration mechanism". The first mechanism manifests itself in two different ways, most prominently through the so called "cross-over" mechanism. Further study is needed in several areas: First, the current analysis does not consider the combined effect of flow and equivalence ratio perturbations. In most practical systems, equivalence ratio perturbations are caused by flow perturbations. It has been shown by prior analyses that the flame response to flow perturbations is highly non-linear. However, it is not clear as to when the effect of one mechanism would dominate over the other. This is however an extremely important issue which must be addressed to ensure reliability and accuracy in the prediction of limit cycle amplitudes of combustion instability and will be the subject of further research. Second, this work has emphasized the role of the "cross-over" mechanism. For systems running very lean, it is more likely that they will encounter local extinction due to deviations of the fuel/air ratio below the flammability limits, well before they begin crossing over into the rich side. It is not clear what will occur in these cases - i.e., whether the flame will locally extinguish or be sustained by the correspondingly strong regions on either side of the ultra-lean regime. In any case, it can be anticipated that the perturbation amplitude where the instantaneous equivalence ratio passes through the flammability limits will also be associated with significant changes in flame dynamics. Understanding these effects would require perhaps modeling reaction chemistry. Consideration of these effects will be a key focus for later discussions in this report. 


\section{Theoretical Development Overview}

\section{Introductory Material}

Extensive theoretical development and calculations have also been performed to understand the flame response to transverse oscillations. Figure 8 shows typical instantaneous flame shapes for swirling flames responding to transverse oscillations. This figure clearly shows that the amount of swirl in the flow has a very important influence on the flame characteristics in the transverse forced case.

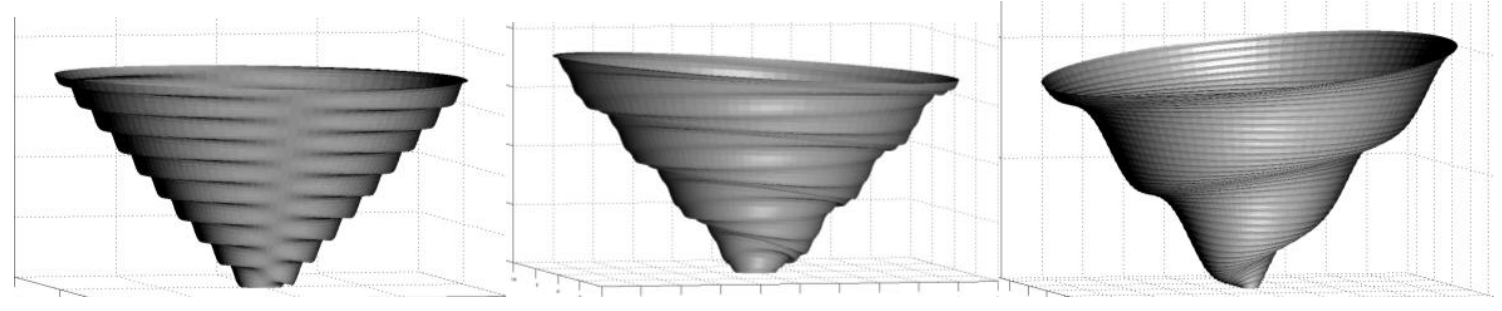

Figure 8: Typical flame shapes for characteristic swirl numbers of $0.0,0.6$ and 1.1 respectively.

A variety of physical processes were accounted for in studying the combustion dynamics of a system excited by oscillations in velocity, fuel/air ratio and pressure. Some of these are associated with the relative length scales and time scales of these disturbances in comparison to inherent length and time scales that exist in the fluid dynamics and combustion phenomena related to the problem. In addition we have been performing two parameter asymptotic expansions of this equation, where the harmonic and random turbulent excitation amplitudes can be varied. Detailed numeric solutions using level set solvers have been used for analytically intractable cases. These solutions have been worked out for flame responses to vortices excited by the oscillating flow and fuel/air ratio oscillations. A typical result showing asymptotic model results for flame response to broadband turbulence is illustrated in the figure below. 


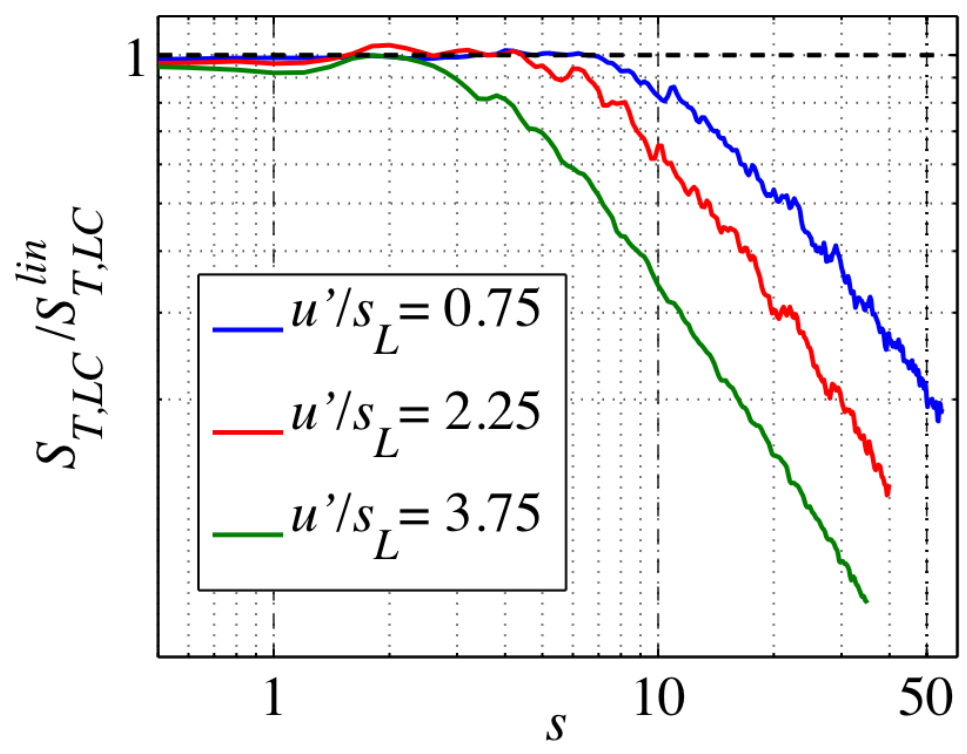

Figure 9: (left) Schematic of investigated geometry showing one half of the instantaneous flame surface (solid red curve), the instantaneous coherent flame surface (solid green curve) and the nominal flame surface (broken black line). The flow is from bottom to top. (right) Spatial variation of local consumption speed normalized by the linearized value at three different turbulence intensities (solid curves) given by the value of $u^{\prime} / s_{L}$. Flame angle, $\gamma \sim \mathbf{4}^{\mathbf{0}}$.

In addition, we have used these models to understand the relationships between unsteady flame heat release and chemiluminescence from the flame. Currently, there is no direct measurement approach for determining the flame's instantaneous heat release, an absolutely critical parameter for understanding combustion instabilities. As such, chemiluminescence emissions from the flame are used as an indicator. While it is known that such an approach works reasonably well for quantifying the flame's response to velocity oscillations, there have been a number of questions for flame's with varying fuel-air ratio. Our analysis of this problem show that there are differences in the flames heat release and chemiluminescence emissions for both $\mathrm{OH}^{*}, \mathrm{CH}^{*}$, and $\mathrm{CO}_{2} *$ bands, but that they all give qualitatively correct results. Quantitative differences can be as large as 50\%, however. Typical results are shown below. All these studies and results introduced here will be discussed in much further detail later in the report. 


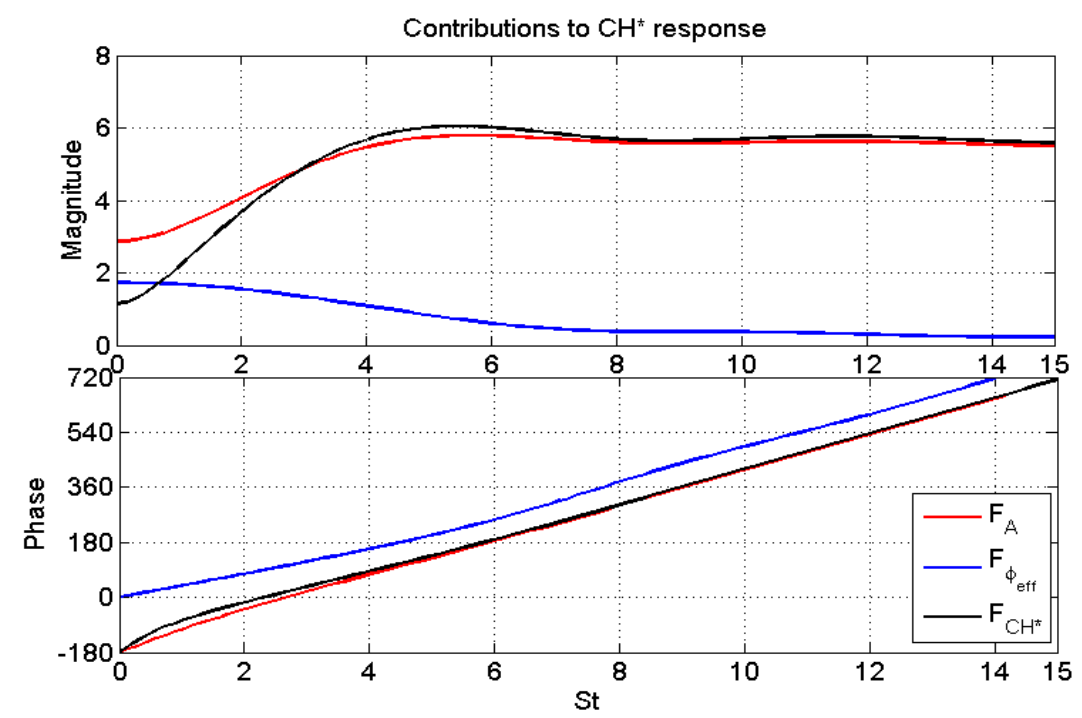

Figure 10: Chemiluminescence intensity response characteristics for $\phi_{o}=0.7, \beta=4$

A variety of physical processes were accounted for in studying the combustion dynamics of a system excited by oscillations in velocity, fuel/air ratio and pressure. Some of these are associated with the relative length scales and time scales of these disturbances in comparison to inherent length and time scales that exist in the fluid dynamics and combustion phenomena related to the problem. Along similar lines of the 'global' Strouhal number, we define 'internal' quasi-steadiness of the flame to denote the case when the flame structure responds instantaneously to disturbances imposed ahead of it. We define an 'internal' Strouhal number to quantify this process, as the ratio of the time taken for diffusive processes in the flame to that of the timescale over which the excitation evolves. This may be quantified as follows,

$$
S t_{\delta, f}=\frac{f \delta_{f}}{s_{L o}}
$$

Here $\delta_{f}$ is the flame thickness and $s_{L_{o}}$ is the nominal burning rate of the flame. These two Strouhal numbers discussed can be used to delineate the conditions at which various flame physics occur. These are shown in Figure 11. One of our accomplishments on the modeling side has been to develop a comprehensive reduced order linear model that accounts for all the physics indicated in the lower shaded region of the graph in Figure 11(a) . It can also be seen from Figure 11(b), which plots a representative graph of the flame responses to velocity, fuel/air ratio and pressure disturbances, that while at low frequencies, velocity and fuel/air ratio responses are the dominant flame response coupling mechanisms, at very high frequencies, pressure coupling tends to dominate the flame response. 


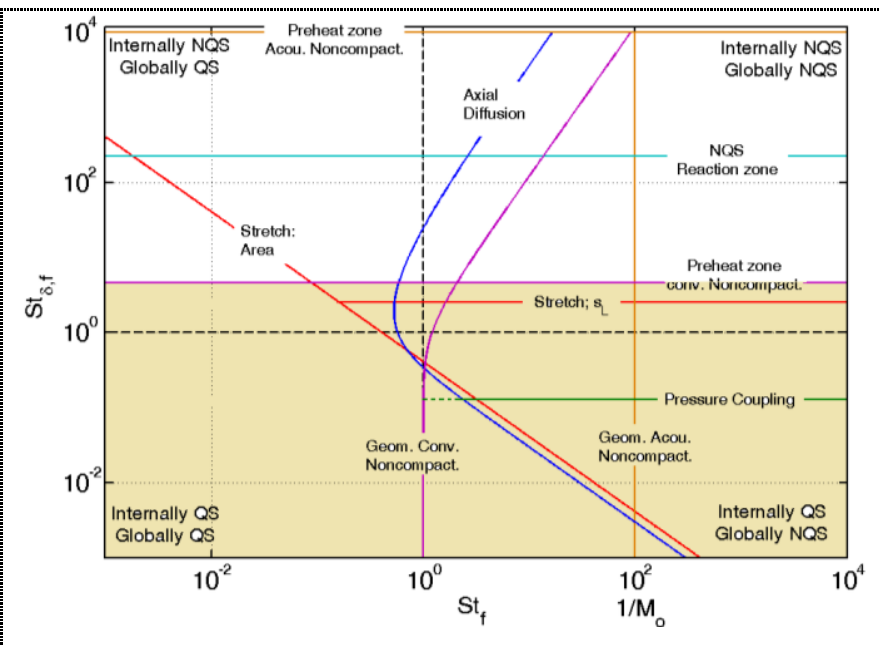

(a)

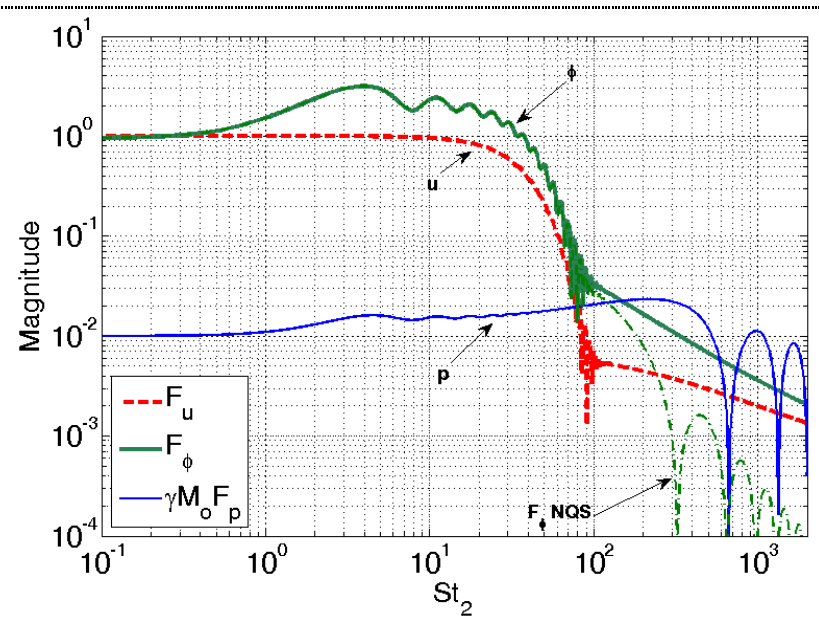

(b)

Figure 11: (a) Summary of heat release response of premixed flames to harmonic disturbances for a premixed methane/air flame, (b) Flame transfer function comparisons of premixed flame response to velocity fluctuations (red), equivalence ratio fluctuations (green), pressure perturbations (blue). These figures are for nominally two dimensional Vshaped flames with $\phi_{o}=0.85$ and aspect ratio (i.e., nominal flame length/flame duct radius) of 4.

\section{Modeling Flame Response to Velocity Oscillations}

\section{Introduction}

A model for premixed flame response in the low excitation amplitude and low turbulence intensity regimes is derived using the technique of asymptotic expansions. The results obtained from these analyses will be used to understand the fundamental issues that must be considered while developing models for turbulent premixed flame response to acoustic forcing. The investigated geometry is a V-flame stabilized on a centerbody, as illustrated schematically in Figure 12. The flame's nominal axial and radial dimensions (i.e. if the flame were to be laminar) are given by the flame length, $L_{f}$, and radius, $R$.

\section{Formulation and Analysis}

The instantaneous location of the flame surface $\zeta(r, t)$ is given by the flame-front tracking equation,

$$
\frac{\partial \zeta}{\partial t}+\frac{u_{\theta}}{r} \frac{\partial \zeta}{\partial \theta}+u \frac{\partial \zeta}{\partial r}-v=-s_{L} \sqrt{1+\frac{1}{r^{2}}\left(\frac{\partial \zeta}{\partial \theta}\right)^{2}+\left(\frac{\partial \zeta}{\partial r}\right)^{2}}
$$


The above is non-dimensionalized using the following scheme: $r^{*}=r / R, z^{*}=z / L_{f},\left(u^{*}, v^{*}, s_{L}^{*}\right)=\left(u, v, s_{L}\right) / U_{o}, t^{*}=t U_{o} / L_{f}, \beta=L_{f} / R$. The asterisks are dropped henceforth for notational convenience. It is assumed that the upstream approach flow is comprised of a uniform mean flow with a purely axial component (i.e. along $\mathrm{z}$ ), a harmonically oscillating coherent component travelling with a specified convective speed of amplitude $\varepsilon_{a}$ and a fluctuating turbulence component of with an rms value of $\varepsilon_{T}$. The following two parameter asymptotic expansion in the acoustic excitation amplitude $\varepsilon_{a}$ and turbulence intensity $\varepsilon_{T}$ is introduced into the above.

$$
\begin{aligned}
\zeta(r, \theta, t)=\zeta_{o} & (r, \theta)+\varepsilon_{a} \zeta_{1 c}(r, \theta, t)+\varepsilon_{T} \zeta_{1 T}(r, \theta, t)+ \\
& \varepsilon_{a}^{2} \zeta_{2 c}(r, \theta, t)+\varepsilon_{T}^{2} \zeta_{2 T}(r, \theta, t)+\varepsilon_{a} \varepsilon_{T} \zeta_{2 c T}(r, \theta, t)+ \\
& \varepsilon_{a}^{3} \zeta_{3 c}(r, \theta, t)+\varepsilon_{T}^{3} \zeta_{3 T}(r, \theta, t)+\varepsilon_{a}^{2} \varepsilon_{T} \zeta_{3 c c T}(r, \theta, t)+\varepsilon_{a} \varepsilon_{T}^{2} \zeta_{3 c T T}(r, \theta, t)
\end{aligned}
$$

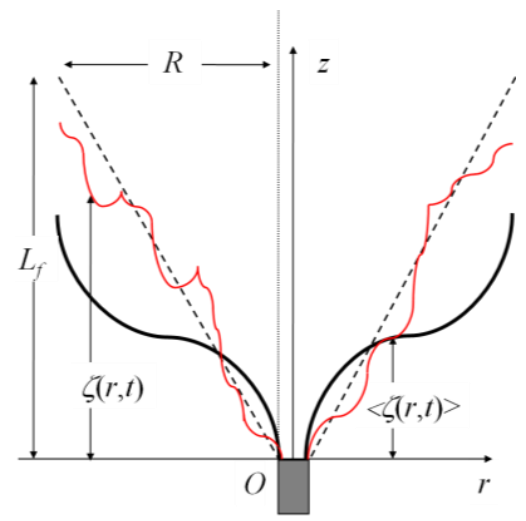

Figure 12: Schematic of investigated geometry showing the instantaneous flame surface (solid red curve), the instantaneous coherent flame surface (solid black curve) and the nominal flame surface (broken black line). The flow is from bottom to top.

Assuming further that flame speed is constant, comparing terms of similar order in $\varepsilon_{a}$ and $\varepsilon_{T}$ on both sides yields a system of linear partial differential equations for each of the coefficients in Eq.(18). Typical members of this system are presented below:

$$
\begin{gathered}
\zeta_{o}(r, \theta, t)=r-r_{f} \\
\frac{\partial \zeta_{1 t}}{\partial t}+a \frac{\partial \zeta_{1 t}}{\partial r}=f_{v}\left(r, \theta, r-r_{f}, t\right)-\sqrt{\frac{a}{1-a}} f_{u}\left(r, \theta, r-r_{f}, t\right) \\
\frac{\partial \zeta_{1 c}}{\partial t}+a \frac{\partial \zeta_{1 c}}{\partial r}=\cos \left\{S t\left(t-K\left(r-r_{f}\right)\right)\right\}+K r S t \sin \left\{S t\left(t-K\left(r-r_{f}\right)\right)\right\}
\end{gathered}
$$

Where, $a=\beta^{2} /\left(1+\beta^{2}\right)$ and $r_{f}$ is the centerbody radius. Equation (19) is just the nominal shape of the flame based on the kinematic balance between the upstream mean flow and the flame. Hence Eq.(18) essentially represents the instantaneous flame shape expressed as a perturbation 
around this nominal flame shape. The ensemble averaged heat release of the flame is given by (dimensional form),

$$
\langle q(t)\rangle=\int_{r_{f}}^{R} h_{R} \rho\left\langle s_{L} \sqrt{1+\left(\frac{\partial \zeta}{\partial r}\right)^{2}}\right\rangle 2 \pi r d r
$$

Reducing the above to non-dimensional form and using Eq. (18) yields,

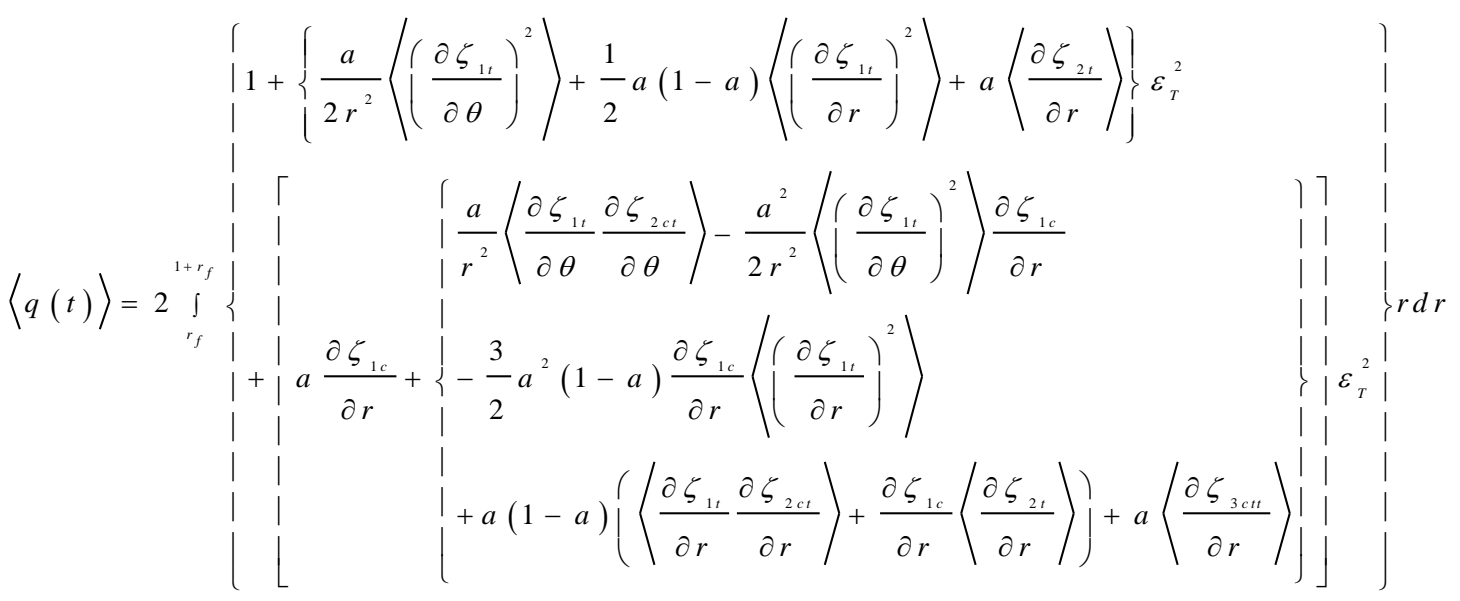

Where, ' \langle\rangle ' denotes an ensemble average. Terms of higher order in $\varepsilon_{a}$ and $\varepsilon_{T}$ have been dropped. The various averages needed to evaluate the above may be determined from the system of linear p.d.es represented by Eqs. (19) - (21). However, closed form expressions for general turbulence fields cannot be readily derived. As such, current analytical efforts are aimed at deriving expressions for the case of isotropic turbulence in order to understand fundamental physics that governs flame surface dynamics in turbulent fields. A typical result is presented below:

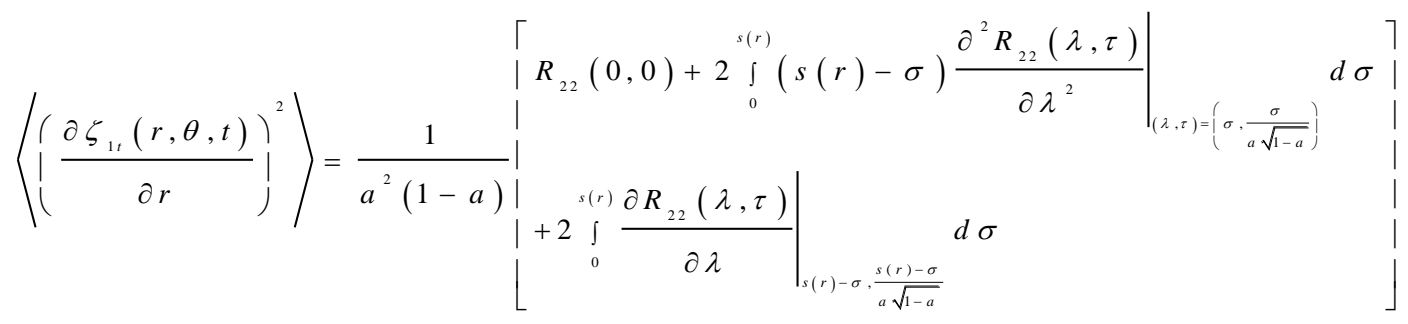

Where, $R_{22}(\lambda, \tau)$ is the space time transverse correlation function for isotropic turbulence and the integrals are performed along the nominal flame surface. The complete expressions in terms of correlation functions for Eq.(23) will be developed. This analysis will yield insight into the influence of space-time turbulent velocity correlations on the heat release response of turbulent premixed flames to acoustic forcing. 


\section{Modeling of Turbulent Flame Response to Flow Oscillations}

\section{Introduction and Formulation}

A similar analysis can be done in cartesian coordinates, following the top left image in Figure 1 and utilizing the second flame-front tracking equation provided previously. The equation is nondimensionalized using a similar scheme as before:

$\left(x^{*}, y^{*}\right)=(x, y) / L_{1 l}, z^{*}=z / L_{11},\left(u^{*}, v^{*}, s_{L}^{*}\right)=\left(u, v, s_{L}\right) / U_{0}, t^{*}=t U_{0} / L_{1 l}$. The asterisks are dropped henceforth for notational convenience. It is assumed that the upstream approach flow is comprised of a uniform mean flow with a purely axial component (i.e. along z), a harmonically oscillating coherent component travelling with a specified convective speed of amplitude $\varepsilon_{a}$ and a fluctuating turbulence component of with an rms value of $\varepsilon_{T}$. The following two parameter asymptotic expansion in the acoustic excitation amplitude $\varepsilon_{a}$ and turbulence intensity $\varepsilon_{T}$ is introduced into the above.

$$
\begin{aligned}
\zeta(x, y, t)=\zeta_{o} & (x, y)+\varepsilon_{a} \zeta_{1 c}(x, y, t)+\varepsilon_{T} \zeta_{1 T}(x, y, t)+ \\
& \varepsilon_{a}^{2} \zeta_{2 c}(x, y, t)+\varepsilon_{T}^{2} \zeta_{2 T}(x, y, t)+\varepsilon_{a} \varepsilon_{T} \zeta_{2 c T}(x, y, t)+ \\
& \varepsilon_{a}^{3} \zeta_{3 c}(x, y, t)+\varepsilon_{T}^{3} \zeta_{3 T}(x, y, t)+\varepsilon_{a}^{2} \varepsilon_{T} \zeta_{3 c c T}(x, y, t)+\varepsilon_{a} \varepsilon_{T}^{2} \zeta_{3 c T T}(x, y, t)
\end{aligned}
$$

Assuming further that flame speed is constant (i.e., $s_{L}=U_{o} \sin \gamma$ ), comparing terms of similar order in $\varepsilon_{a}$ and $\varepsilon_{T}$ on both sides yields a system of linear partial differential equations for each of the coefficients in Eq.(25). Typical members of this system are presented below.

$$
\begin{gathered}
\zeta_{o}(x, y, t)=\left(x-a_{f}\right) \cot \gamma \\
\frac{\partial \zeta_{1 t}}{\partial t}+(\cos \gamma \sin \gamma) \frac{\partial \zeta_{1 t}}{\partial x}=f_{w}\left(x, y, \zeta_{o}(x), t\right)-f_{u}\left(x, y, \zeta_{o}(x), t\right) \cot \gamma \\
\frac{\partial \zeta_{2 t}}{\partial t}+\cos \gamma \sin \gamma \frac{\partial \zeta_{2 t}}{\partial x}=-f_{v}\left(x, y, \zeta_{o}(x), t\right) \frac{\partial \zeta_{1 t}}{\partial y}-\frac{\sin ^{2} \gamma}{2}\left(\frac{\partial \zeta_{1 t}}{\partial y}\right)^{2} \\
-f_{u}\left(x, y, \zeta_{o}(x), t\right) \frac{\partial \zeta_{1 t}}{\partial x}-\frac{\sin ^{4} \gamma}{2}\left(\frac{\partial \zeta_{1 t}}{\partial x}\right)^{2} \\
+\zeta_{1 t}(x, y, t)\left\{f_{w, z}\left(x, y, \zeta_{o}(x), t\right)-\cot \gamma f_{u, z}\left(x, y, \zeta_{o}(x), t\right)\right\}
\end{gathered}
$$

$O\left(\varepsilon_{a}\right):$

$$
\begin{aligned}
& \frac{\partial \zeta_{1 c}}{\partial t}+\cos \gamma \sin \gamma \frac{\partial \zeta_{1 c}}{\partial r}=\cos \{\left.S t\left(t-K\left(x-a_{f}\right) \cot \gamma\right)\right\} \\
&+K S t x \cot \gamma \sin \left\{S t\left(t-K\left(x-x_{f}\right) \cot \gamma\right)\right\}
\end{aligned}
$$


Equation (26) is just the nominal shape of the flame based on the kinematic balance between the upstream mean flow and the flame. Hence, Eq.(25) essentially represents the instantaneous flame shape expressed as a perturbation around this nominal flame shape.

The ensemble averaged heat release density on a per unit area basis of the flame is given by (non-dimensional form):

$$
\langle q(x, y, t)\rangle=\left\langle\sin \gamma \sqrt{1+\left(\frac{\partial \zeta}{\partial x}\right)^{2}+\left(\frac{\partial \zeta}{\partial y}\right)^{2}}\right\rangle
$$

Assuming that the shape of the ensemble averaged flame surface is given by $\tilde{\zeta}(x, t)$ the RHS of the above may be rewritten as:

$$
\langle q(x, y, t)\rangle=f_{c}(x, t) \sin \gamma \sqrt{1+\left(\frac{\partial \tilde{\zeta}}{\partial x}\right)^{2}}
$$

Where, $f_{c}(x, t)$ is the local consumption speed of the flame surface. $\tilde{\zeta}(x, t)$ and $f_{c}(x, t)$ can both be expanded in terms of a series in $\varepsilon_{a}$ as:

$$
f_{c}(x, t)=f_{c o}(x)+\varepsilon_{a} f_{c 1}(x, t)+\varepsilon_{a}^{2} f_{c 2}(x, t)+\cdots
$$

and,

$$
\tilde{\zeta}(x, t)=\tilde{\zeta}_{o}(x)+\varepsilon_{a} \tilde{\zeta}_{1}(x, t)+\varepsilon_{a}^{2} \tilde{\zeta}_{2}(x, t)+O\left(\varepsilon_{a}^{3}\right)
$$

Using the above expansion in Eq.(31) and Eq.(25) in Eq. (30), and comparing terms of similar order in $\varepsilon_{a}$ yields relations for the coefficients in the series for $f_{c}(x, t)$. Thus, the first of the coefficients is given by:

$$
f_{c o}(x)=\frac{1+\frac{1}{2}\left\{\sin ^{4} \gamma\left\langle\left(\left(\frac{\partial \zeta_{1 t}}{\partial x}\right)^{2}\right\rangle+\sin ^{2} \gamma\left\langle\left(\frac{\left.\partial \zeta_{1 t}\right)^{2}}{\partial y}\right)^{2}\right\rangle+2 \cos \gamma \sin \gamma\left\langle\frac{\partial \zeta_{2 t}}{\partial x}\right\rangle\right\} \varepsilon_{T}^{2}\right.}{\sin \gamma \sqrt{1+\left(\frac{\partial \zeta_{0}}{\partial x}\right)^{2}}}
$$


where, ' \langle\rangle ' denotes an ensemble average. Further, consistency between the two series representations for the flame surface (Eqs.(32) and (25)) requires that $\tilde{\zeta}_{o}(r)=\zeta_{o}(r, \theta)+\varepsilon_{T}^{2}\left\langle\zeta_{2 t}(r, \theta, t)\right\rangle+O\left(\varepsilon_{T}^{3}\right)$. The ensemble averages in Eq.(34) can be determined from the solution of the Eqs.(26) - (28). Thus for the case of stationary isotropic turbulence we have:

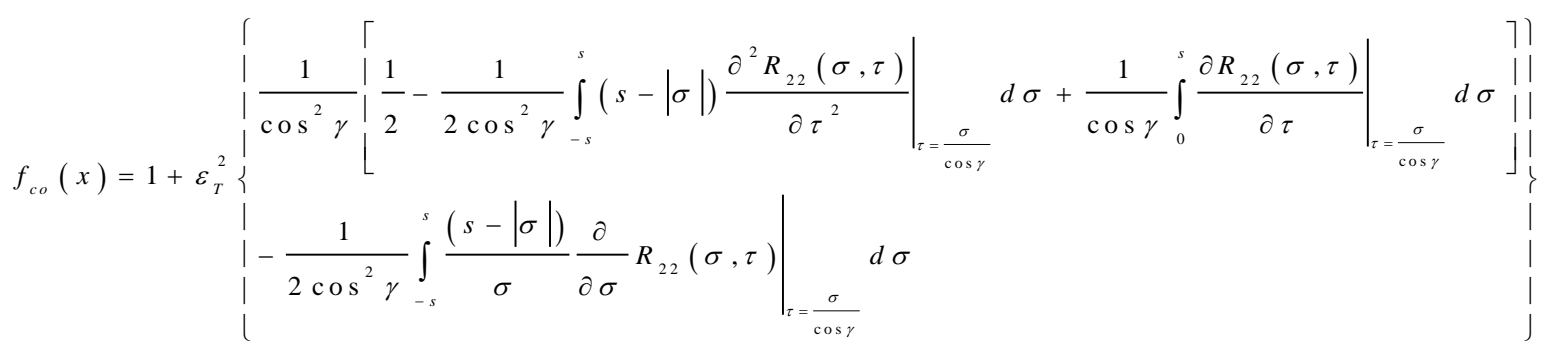

Where, $s=\left(x-a_{f}\right) / \sin \gamma$ and $R_{22}$ is the normalized transverse two-point two-time correlation of the velocity fluctuations. The results presented next assume a form for this correlation function valid at low-turbulence intensities given by:

$$
R_{22}\left(s_{12}, \tau\right)=e^{-\frac{\pi}{4}\left(s_{12}^{2}+\tau^{2}\right)\left(1-\frac{\pi s_{12}^{2}}{4}\right)}
$$

\section{Results}

Figure 13 shows the spatial variation of the correction term to the local consumption speed from Eq.(35) along the nominal flame surface co-ordinate 's' (see Figure 1). Notice that the consumption speed, which is a measure of the local mass-burning rate, increases monotonically along the flame surface. This is because of the phenomenon of flame wrinkles advecting along the flame surface due to presence of a tangential mean flow. Thus, the net flame surface wrinkling at any location is depends not only on the local turbulence properties but on the wrinkling introduced by turbulence fluctuations at upstream locations at previous times. As such the response of the flame to turbulent fluctuations is non-local in nature. Notice further that as the flame angle $\gamma$ decreases (i.e. the flame is nominally more vertical) the net normal mass flux through the flame decreases resulting in a corresponding decrease in local consumption speed.

At locations far away from the flame-holder, the influence of higher-order non-linear kinematic restoration processes increases undermining the validity of the analysis presented above. Notice that the local consumption speed in Figure 13 appears to be unbounded at large distances from the flame holder. Hence, a numerical approach must be adopted to compute the flame surface dynamics in the far-field. This will be the focus of ongoing investigation. 


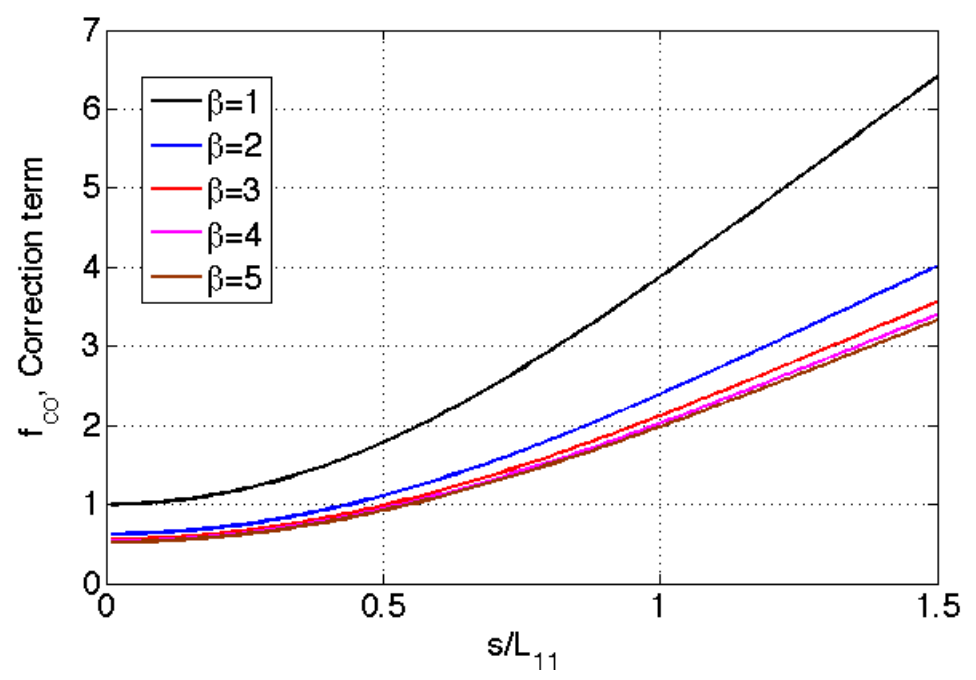

Figure 13: Variation of local consumption speed along the flame surface. $u^{\prime} / s_{L}=0.4 . \beta=\cot \gamma$ . Notice that the consumption speed is spatially varying along the flame surface due to the influence of non-locality.

\section{Modeling of Pressure Coupled Flame Response}

\section{Spatial Dynamics Formulation}

For completeness sake, an additional analysis will be performed, following the top left image in Figure 1 and utilizing a new form of the flame-front tracking equation as follows:

$$
\frac{\partial \xi}{\partial t}+s_{L}\left(1+\left(\frac{\partial \xi}{\partial r}\right)^{2}\right)^{1 / 2}=u-v \frac{\partial \xi}{\partial r}
$$

The above is non-dimensionalized using the following familiar scheme:

$z^{*}=z / L_{f},\left(u^{*}, v^{*}, s_{L}^{*}\right)=\left(u, v, s_{L}\right) / U_{o}, t^{*}=t U_{o} / L_{f}$. The asterisks are dropped henceforth for notational convenience. The pressure perturbations are assumed to harmonically oscillate in time and are advected at phase speed $u_{c}$. The following form can be assumed for the spatio-temporally varying pressure field:

$$
p=p_{o}+p^{\prime} \cos \left(\omega\left(t-\frac{z}{u_{c}}\right)\right)
$$

This form for the pressure yields a zero radial velocity and an axial velocity given by: 


$$
u=u_{o}+\left(\frac{p_{o}}{\rho_{o} c_{o}}\right) \cdot \frac{p^{\prime}}{p_{o}} \cos \left(\omega\left(t-\frac{z}{u_{c}}\right)\right)
$$

In non-dimensional form:

$$
\hat{p}=\frac{p}{p_{o}}=1+\varepsilon_{p} \cos (S t(t-K z))
$$

Here $\varepsilon_{p}=p_{b a s e}^{\prime} / p_{o}$ denotes the amplitude of the pressure disturbances, $K=u_{o} / u_{c}$, the ratio of flow velocity to phase speed and $S t$ is the Strouhal number given by:

$$
S t=\frac{\omega L_{f}}{u_{o}}
$$

Analogously, the axial velocity takes the form:

$$
\hat{u}=\frac{u}{u_{o}}=1+\left(\frac{p_{o}}{\rho_{o} c_{o} u_{o}}\right) \varepsilon_{p} \cos (S t(t-K z))
$$

For the sake of notational convenience, we may write:

$$
\begin{aligned}
& \hat{u}=1+\varepsilon_{u} \cos (S t(\hat{t}-K \hat{z})) \\
& \varepsilon_{u}=\left(\frac{p_{o}}{\rho_{o} c_{o} u_{o}}\right) \varepsilon_{p}=\zeta \varepsilon_{p}
\end{aligned}
$$

The flame front position function $\xi(r, t)$ may be expanded in terms of the parameter $\varepsilon_{p}$ as:

$$
\xi(r, t)=\xi_{o}(r)+\varepsilon_{p} \xi_{1}(r, t)+O\left(\varepsilon^{2}\right)
$$

Substituting this into Eq.(37) yields the following evolution equations:

$$
\begin{gathered}
\xi_{o}(r)=1-r \\
\frac{\partial \xi_{1}}{\partial t}-\alpha \frac{\partial \xi_{1}}{\partial r}=\left(\zeta+\gamma^{-1}\right) \cos (S t(t-K(1-r)))-s_{L 1} \cos \left(S t(t-K(1-r))+\frac{\pi}{4}\right)
\end{gathered}
$$

Together with the anchor-fixed BC, we can solve (46) to get: 


$$
\xi_{1}(r, t)=\frac{1}{(K \alpha-1) S t}\left\{\begin{array}{l}
\left\lceil s_{L 1}\{\cos (S t(K(1-r)-t)+\pi / 4)-\cos ((S t / \alpha)(1-r-\alpha t)+\pi / 4)\}+\right\} \\
\left\{\zeta+\gamma^{-1}\right)\{\sin (S t(K(1-r)-t))-\sin ((S t / \alpha)(1-r-\alpha t))\}
\end{array}\right]
$$

\section{Heat Release Dynamics Formulation}

It has to be noted in the above, that the exact form of $s_{L 1}$ is yet to be ascertained. As stated briefly before, the instantaneous heat release of the flame is then given by:

$$
q(t)=\int_{\text {flame }} \rho_{u} s_{L} h_{R} d A
$$

Assuming the heat of reaction to be unaffected by acoustic disturbances, Eq. (48) can be rewritten in terms of burning velocity and reactant density perturbations as:

$$
\frac{q(t)}{Q_{o}}=\int_{\text {flame }} \frac{d A}{A_{o}}+\int_{\text {flame }} \frac{s_{L}^{\prime}}{s_{L o}} \cdot \frac{d A}{A_{o}}+\int_{\text {flame }} \frac{\rho_{u}^{\prime}}{\rho_{o}} \cdot \frac{d A}{A_{o}}+\int_{\text {flame }} \frac{\rho_{u}^{\prime}}{\rho_{o}} \frac{s_{L}^{\prime}}{s_{L o}} \frac{d A}{A_{o}}
$$

The first term on the RHS denotes the contribution to heat release fluctuation due to oscillations in the net burning area of the flame. This in itself would include contributions due to velocity perturbations (direct mechanism) and due to flame speed perturbations (indirect mechanism). The second term represents the 'direct' contributions to heat release due to burning velocity fluctuations. The third and fourth terms represent the contributions due to reactant density oscillations and nonlinear coupling between flame speed and density respectively. These terms can be evaluated in terms of the flame surface geometry, e.g., for a conical flame geometry, the first term may be evaluated as:

$$
\frac{A(t)}{A_{o}}=2 \int_{0}^{1} r \sqrt{\frac{1+\beta^{2}\left(\frac{\partial \xi}{\partial r}\right)^{2}}{1+\beta^{2}}} d r
$$

The heat-release transfer function of the flame due to acoustic pressure fluctuations may then be defined as:

$$
F=\frac{q^{\prime}(\omega) / Q_{o}}{\hat{p}^{\prime} / p_{o}}
$$

where the numerator and denominator are the heat release and pressure perturbations, evaluated at the excitation frequency. The contributions to the transfer function from each of the physical processes (as represented by Eq.(49)) can be determined by taking a Fourier transform of Eq.(49) . This simply yields:

$$
F=\frac{q^{\prime}(\omega) / Q_{o}}{\hat{p}^{\prime} / p_{o}}=F_{A, u}+F_{A, s_{L}}+F_{s_{L}, d i r}+F_{\rho}
$$




\section{Results}

The first two terms on the RHS represent the contribution to the transfer function, which arises due to burning area oscillations, which in turn arise due to flow velocity perturbations and flame speed perturbations respectively. The last two terms represent 'direct' contributions due to flame speed and density oscillations.

The flame surface location derived earlier is used to evaluate the following expressions for the flame transfer function. Denoting $\eta=K \alpha$, explicit expressions may be written for the four terms on the RHS of Eq.(52) as follows:

$$
\begin{gathered}
F_{A, u}=\frac{2\left(1-e^{i \eta t_{2}}-\eta\left(1-e^{i S t_{2}}\right)\right)}{\eta(\eta-1) S t_{2}{ }^{2}} \zeta \\
F_{\rho}=\frac{2\left(1-e^{i \eta t_{2}}-\eta\left(1-e^{i S t_{2}}\right)\right)}{\eta(\eta-1) S t_{2}{ }^{2}} \gamma^{-1} \\
F_{s_{L}, \text { total }}=\frac{2}{\eta^{2}(\eta-1) S t_{2}{ }^{2}}\left(\eta^{2}\left(1-e^{i S t_{2}}\right)-\left(1-e^{i \eta S t_{2}}\right)+i \eta(\eta-1) S t_{2}\right) e^{-i \pi / 4} \frac{\left(1-\gamma^{-1}\right) \theta}{2} \sqrt{\frac{1}{D a_{m i x}}} \sqrt{S t} \\
\left.F_{A, s_{L}}=\frac{2\left(1-e^{i \eta S t_{2}}-\eta\left(1-e^{i S t_{2}}\right)\right)}{\eta(\eta-1) S t_{2}{ }^{2}} \cdot \mid \gamma^{-1}-e^{-i \pi / 4} \frac{\left(1-\gamma^{-1}\right) \theta}{2} \sqrt{\frac{1}{D a_{m i x}}} \sqrt{S t}\right\rceil \\
\left.F_{s_{L}, d i r}=F_{s_{L}, \text { total }}-F_{A, s_{L}}\right]
\end{gathered}
$$

A representative graph of the transfer function with its contributions is shown in Figure 14 and Figure 15. The following values are chosen: $D a=1, \theta=5, \gamma=1.3, K=1, \beta=4$. It may be observed that at very high frequencies, the flame response is mostly dominated by the contributions due to flame speed perturbations. This is due to the dependence of $F_{p}$ on the square root of the Strouhal number, as opposed to velocity contribution to the transfer function, which rolls of as $S t^{-2}$. 


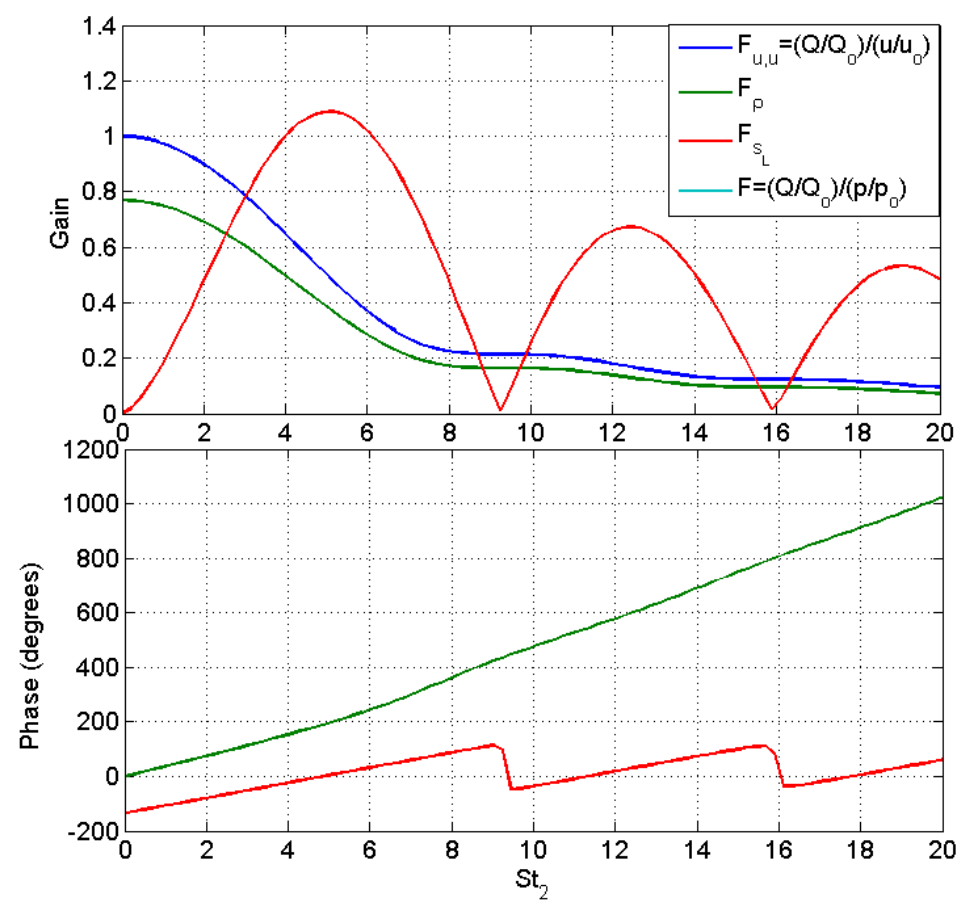

Figure 14: Comparison of heat release-pressure oscillation and heat release-velocity perturbation transfer function (and some contributions)
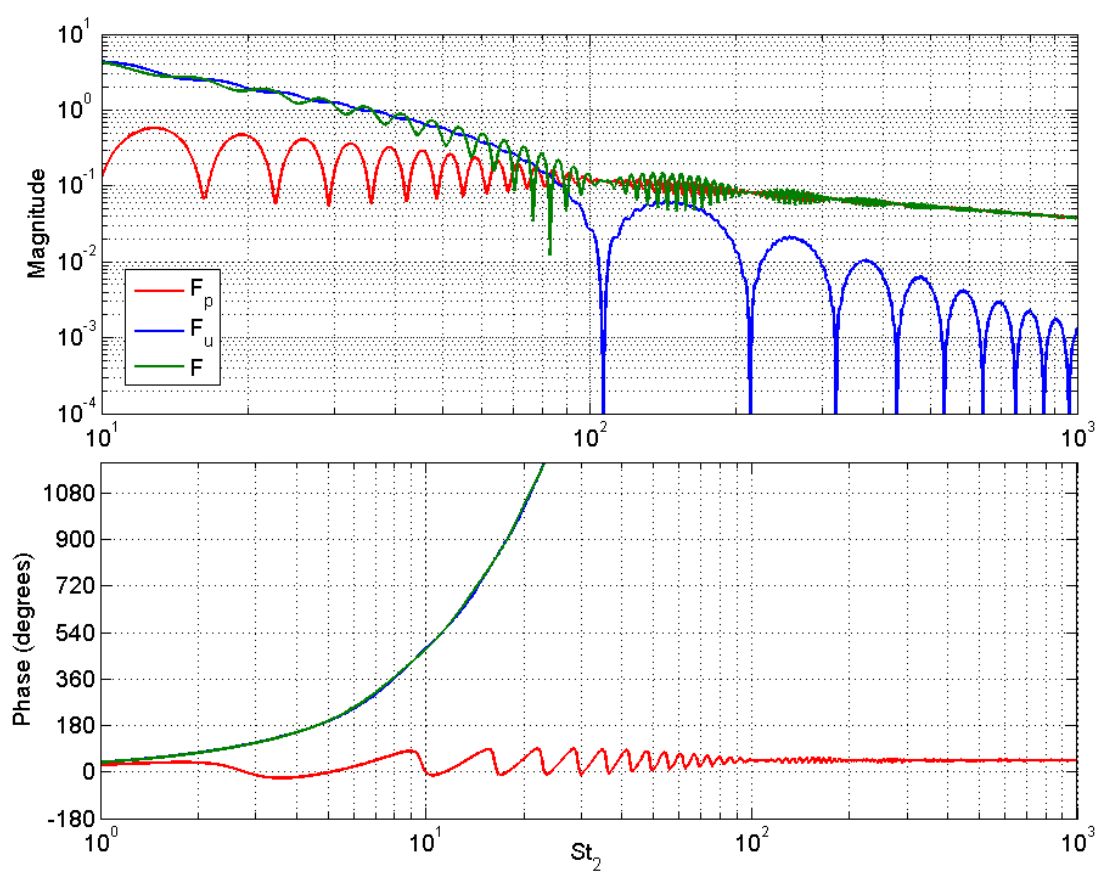

Figure 15: High frequency response of the contributions to heat release due to velocity perturbations $\left(F_{u}\right)$ and that due to flame speed perturbations $\left(F_{p}=F-F_{u}\right)$. The total transfer function $(F)$ is also plotted. 


\section{Response of Harmonically Forced Turbulent Flames}

\section{Introduction}

Similar to the top left image in Figure 1, in this section we develop a theoretical formulation for the ensemble averaged heat release response of an axis-symmetric center-body stabilized turbulent flame to coherent acoustic forcing. Prior numerical studies of Santosh et al showed that the ensemble averaged heat release response at large amplitudes of acoustic forcing is different from the equivalent value determined from existing models for laminar flame response with the laminar flame speed replaced by a modified turbulent displacement speed. Thus, it was inferred in that work that the presence of turbulence changes the ensemble averaged heat release response and that the effect of turbulence is not just that of additive noise. The theoretical formulation presented in this section formally shows this result by developing an exact expression for the leading order turbulence correction to the linear laminar transfer functions developed in Schuller et al and Preetham et al, using the technique of asymptotic expansions. The principal simplifying assumptions made in this study are as follows:

Flame:

1. Corrugated flamelet regime - the flame is a thin boundary between products and reactants.

2. Constant laminar flame speed, $s_{L}$.

Fluid Mechanics

3. Flow field upstream of flame is prescribed; as such, gas expansion effects across flame neglected.

4. Isotropic, stationary turbulence and Taylor's hypothesis. Spatial correlation: $R_{11}(s)=e^{-\pi s^{2} / 4 L_{11}^{2}}$ assumed. $L_{11}$ is the integral length scale.

The first of the above assumptions allows the flame surface motions to be tracked using a front tracking equation also known as the $G$-equation. This assumption also implies that all turbulent eddies are much larger than the laminar flame thickness. The second assumption implies a spatio/temporally constant fuel/air ratio and that the flame is insensitive to stretch. Assumption 3 essentially ignores the effect of the temperature jump on the upstream flow. This is a common assumption made in these types of analyses. This assumption allows for the flow field to be specified without having to solve the Navier-Stokes equations up and downstream of the moving flame sheet. Assumption 4 is not necessary for the posing and formulation of the problem, but invoked to derive explicit, closed form solutions. 


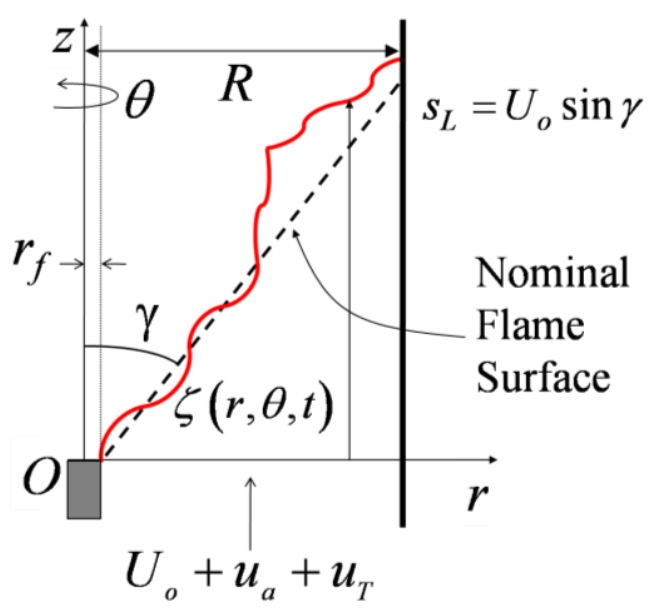

Figure 16: Schematic of investigated geometry - axis-symmetric bluff-body stabilized flame.

\section{Formulation}

The investigated geometry is shown schematically in Figure 16. It differs from Figure 1 in coordinate system and flow parameters so we thought it beneficial to include it. The analysis in this section will be performed in the burner fixed $r-z-\theta$ co-ordinate system as shown. The $G$-equation in the present burner fixed co-ordinates may be written in dimensional form as:

$$
\frac{\partial G}{\partial t}+u \frac{\partial G}{\partial r}+\frac{u_{\theta}}{r} \frac{\partial G}{\partial \theta}+v \frac{\partial G}{\partial z}=s_{L} \sqrt{\left(\frac{\partial G}{\partial r}\right)^{2}+\frac{1}{r^{2}}\left(\frac{\partial G}{\partial \theta}\right)^{2}+\left(\frac{\partial G}{\partial z}\right)^{2}}
$$

where, $u, u_{\theta}$ and $v$ are the instantaneous flow velocity components in the $r, \theta$ and $z$ directions respectively. Choosing, $G(r, \theta, z, t)=z-\zeta(r, \theta, t)$ in the above yields:

$$
\frac{\partial \zeta}{\partial t}+u \frac{\partial \zeta}{\partial r}+\frac{u_{\theta}}{r} \frac{\partial \zeta}{\partial \theta}-v=-s_{L} \sqrt{1+\left(\frac{\partial \zeta}{\partial r}\right)^{2}+\frac{1}{r^{2}}\left(\frac{\partial \zeta}{\partial \theta}\right)^{2}}
$$

The above can be written in non-dimensional form using: $\left(r^{*}, z^{*}\right)=(r, z) / L_{11}, \quad \zeta^{*}=\zeta / L_{11}$, $\left(u_{s}^{*}, v^{*}, u_{\theta}^{*}\right)=\left(u_{s}, v, u_{\theta}\right) / U_{o}$ as:

$$
\frac{\partial \zeta^{*}}{\partial t^{*}}+u^{*} \frac{\partial \zeta^{*}}{\partial r^{*}}+\frac{u_{\theta}^{*}}{r^{*}} \frac{\partial \zeta^{*}}{\partial \theta^{*}}-v^{*}=-\sin \gamma \sqrt{1+\left(\frac{\partial \zeta^{*}}{\partial r^{*}}\right)^{2}+\frac{1}{r^{*}}\left(\frac{\partial \zeta^{*}}{\partial \theta^{*}}\right)^{2}}
$$

Where, the asterisks represent non-dimensional quantities. As was done previously, these asterisks will be dropped in what follows for notational convenience with the understanding that all expressions henceforth are presented in non-dimensional form unless otherwise specified. 
It has been shown in the case of acoustically forced flames that the primary source of coherent velocity perturbations comes from the shedding of vortices caused by shear layer rollup downstream of the flame holding location due to acoustic forcing. Therefore, the net magnitude and phase of the global heat release fluctuations relative to the imposed excitation are characterized by first, the wavelength of the flame surface wrinkles relative to the characteristic dimension of the flame and second, by the length scale associated with the imposed perturbation. In practical situations, the flame is confined within the combustor liner of a finite radius. Thus, for a specified mean flow velocity and laminar flame speed $s_{L}$, the flame has a fixed nominal height denoted by $L_{f}$ for a given combustor radius, $R$ (see Figure 16). Hence, the velocity field in Eq. (59) is now specified as a superposition of a coherent harmonic excitation with a convected characteristic and a spatially isotropic and stationary turbulence field as follows:

$$
\begin{aligned}
& u(r, \theta, \zeta, t)=\frac{1}{2} \frac{K S t}{\Lambda} \varepsilon_{a} r \sin \left\lceil\frac{S t}{\Lambda}(K \zeta-t)\right\rfloor+\varepsilon_{T} f_{v}(r, \theta, \zeta, t) \\
& u_{\theta}(r, \theta, \zeta, t)=\varepsilon_{T} f_{\theta}(r, \theta, \zeta, t) \\
& v(r, \theta, \zeta, t)=1+\varepsilon_{a} \cos \left\lceil\frac{S t}{\Lambda}(K \zeta-t)\right\rfloor+\varepsilon_{T} f_{u}(r, \theta, \zeta, t)
\end{aligned}
$$

Where, the various parameters in the above are defined as follows:

- $\varepsilon_{a}=u_{a}^{\prime} / U_{o}$ - the amplitude of the coherent excitation component normalized by the mean flow velocity $U_{o}$.

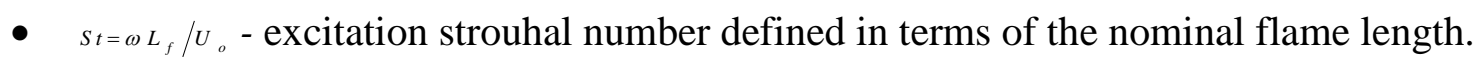

- $\Lambda=L_{f} / L_{11}$ - the normalized nominal flame length.

- $K=U_{o} / u_{c}-u_{c}$ is the convective velocity of the imposed vortical structures.

- $\varepsilon_{T}=u_{R M S}^{\prime} / U_{\circ}$, RMS velocity of the background turbulence normalized by mean velocity.

- $f_{u}, f_{v}, f_{\theta}$ zero-mean unity variance random functions of their arguments representing the underlying structure of the turbulence velocity fluctuations.

The above specification is a generalization of the velocity field specification used in the laminar flame response analysis of Preetham et al. Next, the flame surface shape can be written as a two parameter expansion in $\varepsilon_{a}$ and $\varepsilon_{T}$ as follows:

$$
\begin{aligned}
\zeta(r, \theta, t) & =\zeta_{o}(r, \theta)+\varepsilon_{T} \zeta_{1 t}(r, \theta, t)+\varepsilon_{T}^{2} \zeta_{2 t}(r, \theta, t)+\cdots \\
& +\varepsilon_{a}\left\{\zeta_{1 c}(r, \theta, t)+\varepsilon_{T} \zeta_{2 c t}(r, \theta, t)+\varepsilon_{T}^{2} \zeta_{3 c t t}(r, \theta, t)+\cdots\right\}+\varepsilon_{a}^{2}\{\cdots\}+\cdots
\end{aligned}
$$

where the contribution to the flame surface shape at each order of acoustic forcing amplitude, $\varepsilon_{a}$ has been further expanded into contributions in terms of $\varepsilon_{r}$. Physically, these contributions capture the 
effect of coupling between the response due to coherent forcing and the random flame surface wrinkling due to turbulence fluctuations. Henceforth, this coupling with be referred to as "kinematic coupling". Using the above together with the velocity field specification in Eq.(60) yields the following system of equations:

$O\left(\varepsilon_{a}^{0}\right):$

$$
\begin{gathered}
\zeta_{o}(r, \theta)=\left(r-r_{f}\right) \cot \gamma \\
\frac{\partial \zeta_{1 t}}{\partial t}+\cos \gamma \sin \gamma \frac{\partial \zeta_{1 t}}{\partial r}=\frac{f_{n}\left(r, \theta, z=\left(r-r_{f}\right) \cot \gamma, t\right)}{\sin \gamma} \\
\frac{\partial \zeta_{2 t}}{\partial t}+\cos \gamma \sin \gamma \frac{\partial \zeta_{2 t}}{\partial r}=-\frac{1}{r} f_{\theta}\left(r, \theta,\left(r-r_{f}\right) \cot \gamma, t\right) \frac{\partial \zeta_{1 t}}{\partial \theta}-f_{u}\left(r, \theta,\left(r-r_{f}\right) \cot \gamma, t\right) \frac{\partial \zeta_{1 t}}{\partial r} \\
-\frac{\sin ^{2} \gamma}{2 r^{2}}\left(\frac{\partial \zeta_{1 t}}{\partial \theta}\right)^{2}-\sin ^{4} \gamma\left(\frac{\partial \zeta_{1 t}}{\partial r}\right)^{2}+\frac{\zeta_{1 t}(r, \theta, t)}{\sin \gamma}\left\{\left.\frac{\partial f_{n}}{\partial z}\right|_{\left(r, \theta, z=\left(r-r_{f}\right) \cot \gamma, t\right)}\right\}
\end{gathered}
$$

$O\left(\varepsilon_{a}\right):$

$$
\begin{aligned}
& \frac{\partial \zeta_{1 c}}{\partial t}+\cos \gamma \sin \gamma \frac{\partial \zeta_{1 c}}{\partial r}=\cos \left\{\frac{S t}{\Lambda}\left(t-K\left(r-r_{f}\right) \cot \gamma\right)\right\}+\frac{1}{2} r \frac{K S t}{\Lambda} \cot \gamma \sin \left\{\frac{S t}{\Lambda}\left(t-K\left(r-r_{f}\right) \cot \gamma\right)\right\} \\
& \frac{\partial \zeta_{2 c t}}{\partial t}+\sin \gamma \cos \gamma \frac{\partial \zeta_{2 c t}}{\partial r}=-\zeta_{1 t}(r, \theta, t)\left(\begin{array}{l}
\frac{1}{2} r \frac{K^{2} S t^{2}}{\Lambda^{2}} \cos \left[\frac{S t}{\Lambda}\left(t-K\left(r-r_{f}\right) \cot \gamma\right)\right] \cot \gamma \\
-\frac{K S t}{\Lambda} \sin \left[\frac{S t}{\Lambda}\left(t-K\left(r-r_{f}\right) \cot \gamma\right)\right\rfloor
\end{array}\right) \\
& -f_{u}\left(r, \theta,\left(r-r_{f}\right) \cot \gamma, t\right) \frac{\partial \zeta_{1 c}}{\partial r}+\frac{1}{2} \frac{\partial \zeta_{1 t}}{\partial r} r \frac{K S t}{\Lambda} \sin \left[\frac{S t}{\Lambda}\left(t-K\left(r-r_{f}\right) \cot \gamma\right)\right\rfloor \\
& -\sin ^{4} \gamma \frac{\partial \zeta_{1 c}}{\partial r} \frac{\partial \zeta_{1 t}}{\partial r}-\frac{\zeta_{1 c}(r, \theta, t)}{\sin \gamma}\left\{\left.\frac{\partial f_{n}}{\partial z}\right|_{\left(r, \theta, z=\left(r-r_{f}\right) \cot \gamma, t\right)}\right\}
\end{aligned}
$$

where,

$$
\frac{f_{n}\left(r, \theta, z=\left(r-r_{f}\right) \cot \gamma, t\right)}{\sin \gamma}=f_{v}\left(r, \theta, z=\left(r-r_{f}\right) \cot \gamma, t\right)-f_{u}\left(r, \theta, z=\left(r-r_{f}\right) \cot \gamma, t\right) \cot \gamma
$$

The flame attachment assumption (assumption 5) implies the following boundary condition for each of the Eqs. (62) - (66):

$$
\zeta_{i}\left(r_{f}, \theta, t\right)=0
$$

The system of equations presented above must be solved to determine the heat release response of the turbulent flame to leading order in $\varepsilon_{a}$ and $\varepsilon_{T}$. Equations (62) and (65) describe laminar flame response. The remaining equations are needed because to capture the effect of kinematic coupling (to leading order). Also, from Eq.(65), it is clear that the leading order flame response contribution at 
$O\left(\varepsilon_{a}\right)$ i.e., the laminar response, is axis-symmetric. Thus, terms involving derivatives of this contribution w. r. t. ' $\theta$ 'vanish and have been omitted from the above equations. Note that the LHS of Eqs.(63) - (66) are linear advection operators in terms of the fluctuating components of the flame surface wrinkling. This means that the net flame surface wrinkling amplitude at point at any given time instant is a function of the wrinkling produced at the given point as well as the wrinkling produced at upstream points at earlier times. This property of flame surface motions is known as non-locality.

The instantaneous heat release rate of the flame normalized by the heat release rate of the nominal flame surface is given by the following:

$$
q(t)=\frac{\sin \gamma}{\pi\left(R^{2}-r_{f}^{2}\right)} \int_{r_{f}}^{R} \int_{0}^{2 \pi} d r d \theta r \sqrt{1+\frac{1}{r^{2}}\left(\frac{\partial \zeta}{\partial \theta}\right)^{2}+\left(\frac{\partial \zeta}{\partial r}\right)^{2}}
$$

Where, $R$ is the radius of the combustor normalized by $L_{11}$. Thus, using the expansion in Eq.(61) in Eq.(69) upons ensemble averaging yields:

$$
\left.\begin{array}{rl}
\left.\langle q(t)\rangle=1+\frac{2 \varepsilon_{T}^{2}}{\left(R^{2}-r_{f}^{2}\right)} \int_{r_{f}}^{R} d r r \mid \frac{\sin ^{2} \gamma}{2 r^{2}}\left\langle\left(\frac{\partial \zeta_{1 t}}{\partial \theta}\right)^{2}\right\rangle+\frac{1}{2} \sin ^{4} \gamma\left\langle\left(\frac{\partial \zeta_{1 t}}{\partial r}\right)^{2}\right\rangle\right\} \\
+\cos \gamma \sin \gamma\left\langle\frac{\partial \zeta_{2 t}}{\partial r}\right\rangle
\end{array}\right\}
$$

\section{Results}

The first two terms on the RHS of the above represent the nominal heat release rate and the leading order turbulence correction to the nominal heat release rate. The third term represents the linear laminar heat release response of the flame and the last term represents the leading order turbulence correction at linear order in acoustic forcing. The ensemble averaged heat-release rate transfer function is defined as follows:

$$
F(S t)=\frac{\langle\hat{q}(S t)\rangle / Q_{o}}{u_{a}^{\prime} / U_{o}}
$$


Where, the numerator now represents the heat release rate at the forcing frequency, expressed as the Strouhal number $(\mathrm{St})$. The denominator represents the amplitude of the coherent forcing component at the driving frequency, $\varepsilon_{a}$. From Eq.(70), the total transfer function can be written as two separate contributions as below:

$$
F(S t)=F_{L}(S t)+F_{T}(S t) \varepsilon_{T}^{2}
$$

The first term in the above represents the linear heat release transfer function for the laminar flame similar to the results derived by Preetham et al. The second term is the correction to the linear transfer function due to the presence of background turbulence. From Eq.(70), $F_{T}$ can be written as follows:

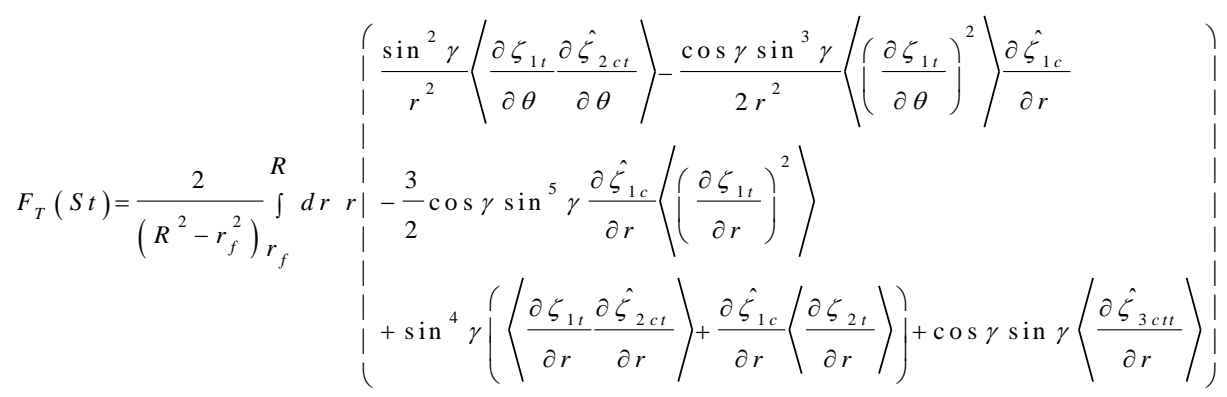

where, the caret, '^', represents the Fourier transform. These can be evaluated in terms of spatiotemporal correlation functions of isotropic turbulence. Thus, from Eq.(73) it is clear that turbulence does not behave as additive noise i.e. it changes the ensemble averaged heat release response amplitude, due to the presence of kinematic coupling. Physically, this kinematic coupling occurs because of non-linearities in flame surface kinematics as follows. First, the flame surface at each point is advected to new locations by the net local velocity field (coherent + turbulent) any instant of time. Thus, the velocities on the LHS of Eq.(58) are themselves functions of the instantaneous flame position, $\zeta(r, \theta, t)$. Second, kinematic restoration causes eventual destruction of wrinkles induced on the flame causing the net global area fluctuation amplitude to change. These processes clearly result in the coupling between the response of the flame to coherent forcing and turbulence fluctuations. Products between ensemble averaged turbulent contribution terms and laminar forcing terms in Eq.(73) control the influence of kinematic coupling.

\section{Flame Heat Release Response Modeling}

The analysis presented in this section thus far is exact in the sense that no ad-hoc assumptions of equivalent propagation speeds have been made. However, the drawback of the above approach is that it requires knowledge of the nominal flame surface shape in the absence of acoustic forcing in addition to the statistical properties of the turbulent velocity field, in order to determine the turbulence correction to the heat release transfer function. While it is possible to ascertain the latter in practical situations, defining a "nominal" flame surface becomes problematic because the definition of the equivalent "laminar" flow is itself is problematic. The presence of a temperature jump across the flame sheet further exacerbates this difficulty as the mean flow velocity upstream of the flame is modified. Hence, a modeling approach must be introduced in order to determine the heat 
release transfer function in practical situations. The utility of the present formulation, apart from providing insight into the fundamental characteristics of heat release response, is to provide a baseline against which the fidelity of different modeling approaches to determining the heat release response for the present canonical configuration can be determined. This will be discussed next.

The mean flame surface shape, i.e. the average shape of the flame in the presence of turbulence, is readily accessible from either experiments or computational approaches based on the Reynolds Averaged Navier-Stokes equations (RANS). A local "turbulent" propagation (displacement) speed and local consumption speed can be defined at all points on this mean surface. Thus, it is possible to use the G-equation approach to derive a kinematic equation for the mean flame surface in terms of the ensemble averaged velocity field and the local displacement speed. The heat release response can then be evaluated using the flame surface shape obtained from the above equation and the local consumption speed. This modeling approach is a generalization past modeling approaches for the linear heat release response transfer function of an axis-symmetric center-body stabilized turbulent flame.

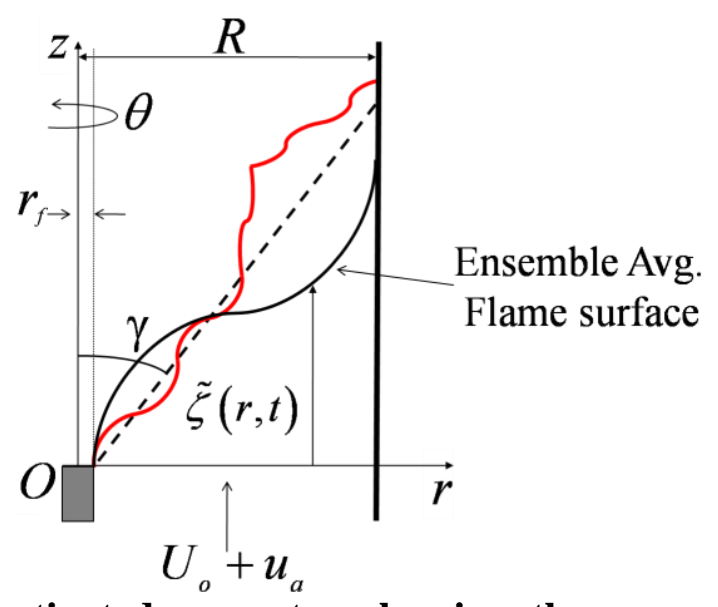

Figure 17: Schematic of the investigated geometry showing the ensemble averaged flame surface.

Let the ensemble averaged flame surface shape at any time relative to the start of the acoustic forcing cycle be denoted by $\tilde{\zeta}(r, t)$ as shown in Figure 17. This geometry differs from that shown in Figure 16 by the forcing parameters. A kinematic equation governing its dynamics in the mean flow field may now be written analogously to Eq.(59) as follows:

$$
\frac{\partial \tilde{\zeta}}{\partial t}+u \frac{\partial \dot{\zeta}}{\partial r}-\tilde{v}=-f_{T}(r) \sin \gamma \sqrt{1+\left(\frac{\partial \tilde{\zeta}}{\partial r}\right)^{2}}
$$

Where, the non-dimensionalization scheme used in Eq.(59) has been retained. The ' ' represents ensemble averaging. The function $f_{T}$ on the RHS represents the local propagation velocity of the ensemble averaged flame surface relative to the ensemble averaged upstream flow of reactants, normalized by the laminar flame speed $s_{L}$. Thus, this now is a "local" displacement speed. Due to the spatially isotropic nature of the turbulence field in this analysis, the ensemble averaged azimuthal 
velocity component is identically zero. Further, all mean and ensemble averaged quantities are independent of $\theta$ due to axis-symmetry.

The ensemble averaged velocity field can be determined from Eq.(60) as follows,

$$
\begin{aligned}
& \tilde{u}(r, \tilde{\zeta}, t)=\frac{1}{2} \frac{K S t}{\Lambda} \varepsilon_{a} r \sin \left\lceil\frac{S t}{\Lambda}(K \tilde{\zeta}-t)\right\rfloor \\
& \tilde{v}(r, \tilde{\zeta}, t)=1+\varepsilon_{a} \cos \left\lceil\frac{S t}{\Lambda}(K \tilde{\zeta}-t)\right\rfloor
\end{aligned}
$$

Next, an expansion for $\tilde{\zeta}(r, t)$ can be introduced as follows:

$$
\tilde{\zeta}(r, t)=\tilde{\zeta}_{o}(r)+\varepsilon_{a} \tilde{\zeta}_{1}(r, t)+O\left(\varepsilon_{a}^{2}\right)
$$

Using the above expansion in Eq. (74) yields the following system:

$$
-1+f_{T}(r) \sin \gamma \sqrt{1+\left(\frac{d \tilde{\zeta}_{o}}{d r}\right)^{2}}=0
$$

and,

$$
\frac{\partial \tilde{\zeta}_{1}}{\partial t}+\frac{f_{T}(r)\left(d \tilde{\zeta}_{o} / d r\right) \sin \gamma}{\sqrt{1+\left(d \tilde{\zeta}_{o} / d r\right)^{2}}} \frac{\partial \tilde{\zeta}_{1}}{\partial r}=\cos \left\lfloor\frac{S t}{\Lambda}\left\{t-K \tilde{\zeta}_{o}(r)\right\}\right\rfloor+\left(\frac{K S t}{2 \Lambda} \frac{d \tilde{\zeta}_{o}}{d r}\right) r \sin \left\lfloor\frac{S t}{\Lambda}\left\{t-K \tilde{\zeta}_{o}(r)\right\}\right\rfloor
$$

Also, the flame attachment boundary condition yields:

$$
\tilde{\zeta}_{1}(0, t)=0
$$

Next, Eq.(78) can be solved as follows. Writing Eq.(78) in terms in Fourier space at the Strouhal number $S t / \Lambda$ yields:

$$
\frac{d \tilde{\zeta}_{1}}{d r}-\frac{i(S t / \Lambda) \sqrt{1+\left(d \tilde{\zeta}_{o} / d r\right)^{2}}}{f_{T}(r)\left(d \tilde{\zeta}_{o} / d r\right) \sin \gamma} \tilde{\zeta}_{1}=e \frac{i K S t \tilde{\zeta}_{o}(r)}{\Lambda}\left(1+i\left(\frac{\left.K S t d \frac{d \tilde{\zeta}_{o}}{2 \Lambda}\right)}{d r}\right)\right) \frac{\sqrt{1+\left(d \tilde{\zeta}_{o} / d r\right)^{2}}}{f_{T}(r)\left(d \tilde{\zeta}_{o} / d r\right) \sin \gamma}
$$

Solving the above using the method of integrating factors yields:

$$
\stackrel{\varkappa}{\zeta}_{1}(r, S t)=e^{H_{1}(r)} \int_{r_{f}}^{r} d \eta_{1} e^{-H_{1}\left(\eta_{1}\right)} P\left(\eta_{1}, S t\right)
$$


where,

$$
\begin{aligned}
H(r, S t) & =\frac{i(S t / \Lambda) \sqrt{1+\left(d \tilde{\zeta}_{o} / d r\right)^{2}}}{f_{T}(r)\left(d \zeta_{o} / d r\right) \sin \gamma} \\
H_{1}(r, S t)= & \int_{r_{f}}^{r} d \eta H(\eta, S t) \\
P(r, S t) & =e^{\left.\frac{i K S t \tilde{\zeta}_{o}(r)}{\Lambda}\left(1+i \mid \frac{K S t}{2 \Lambda} \frac{d \tilde{\zeta}_{o}}{d r}\right) r\right) \frac{\sqrt{1+\left(d \tilde{\zeta}_{o} / d r\right)^{2}}}{f_{T}(r)\left(d \zeta_{o} / d r\right) \sin \gamma}}
\end{aligned}
$$

The above can be rewritten using Eq.(77) as follows:

$$
\begin{aligned}
& H(r, S t)=\frac{i(S t / \Lambda)}{f_{T}(r) \sin \gamma \sqrt{1-\left(f_{T}(r) \sin \gamma\right)^{2}}} \\
& H_{1}(r, S t)=\int^{r} d \eta H(\eta, S t) \\
& r_{f}
\end{aligned}
$$

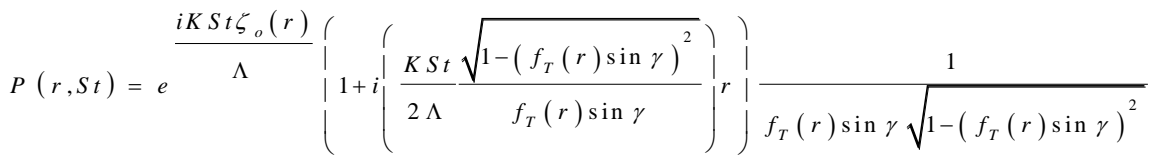

Next, the net heat release rate from the flame (normalized by the mean heat release rate of the unforced flame) can be written in terms of a local consumption speed, $f_{c}(r)$ (normalized by laminar flame speed $s_{L}$ ) as:

$$
\tilde{q}(t)=\frac{\int_{f} d r r f_{c}(r) \sqrt{1+\left(\frac{\partial \tilde{\zeta}}{\partial r}\right)^{2}}}{\int_{r_{f}}^{R} d r r f_{c}(r) \sqrt{1+\left(d \tilde{\zeta}_{o} / d r\right)^{2}}}
$$

The above represents the significant difference between the present modeling approach and past approaches that assume either $f_{c}(r)=f_{T}(r)$ or $f_{c}(r)=$ constant (You et al). It will be shown shortly that in general both of these assumptions are not true. Expanding the above as series in $\varepsilon_{a}$ yields:

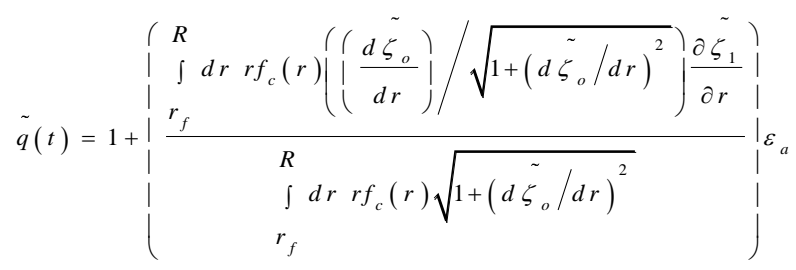


Hence the heat release transfer function can be written in Strouhal number space as:

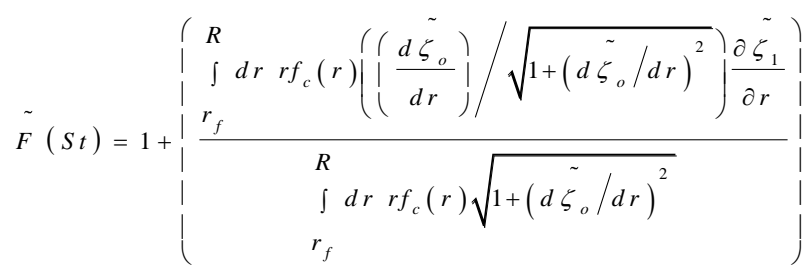

where, '^' represents the value of the Fourier transform of the corresponding quantity. Again, using Eq.(77) yields:

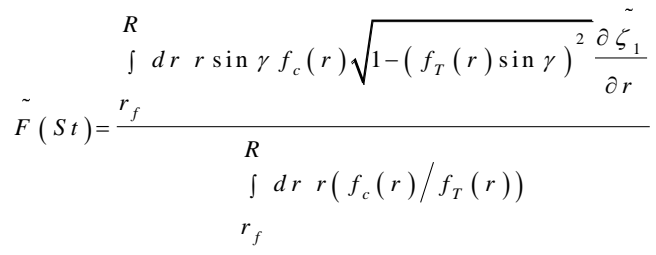

Finally, the derivative of the unsteady flame shape in the above can be evaluated by differentiating Eq.(81) w. r. t. $r$ to yield the following:

$$
\frac{d \check{\zeta}_{1}}{d r}=P(r, S t)+H(r, S t) e^{H_{1}(r, S t)} \stackrel{r}{\int_{r_{f}} d \eta_{1} e^{-H_{1}\left(\eta_{1}, S t\right)}} P\left(\eta_{1}, S t\right)
$$

Thus, for specified local displacement speed and local consumption speed, the heat release transfer function can be evaluated from Eqs. (87) and (88). Next, a model for $f_{c}(r)$ and $f_{T}(r)$ can be derived from the exact asymptotic analysis presented in the previous section as follows. The mean unforced flame geometry can be specified using the zeroth order (in forcing amplitude, $\varepsilon_{a}$ ) terms from the exact asymptotic analysis developed in the previous section as follows (see Eq.(61)),

$$
\tilde{\zeta}_{o}(r)=\left(r-r_{f}\right) \cot \gamma+\varepsilon_{T}^{2}\left\langle\zeta_{2 t}(r, \theta, t)\right\rangle+O\left(\varepsilon_{T}^{3}\right)
$$

Next, Eq.(77) represents the kinematic equation that determines the rate of propagation of the above mean flame surface into the upstream reactants, $f_{T}(r)$ i.e. the local displacement speed. Thus, using Eq.(89) in Eq.(77) and retaining term upto $o\left(\varepsilon_{T}^{2}\right)$ yields:

$$
f_{T}(r)=1-\cos \gamma \sin \gamma\left(\frac{\partial \zeta_{2 t}}{\partial r}\right) \varepsilon_{T}^{2}
$$


Equation (66) together with Eq.(90) yields:

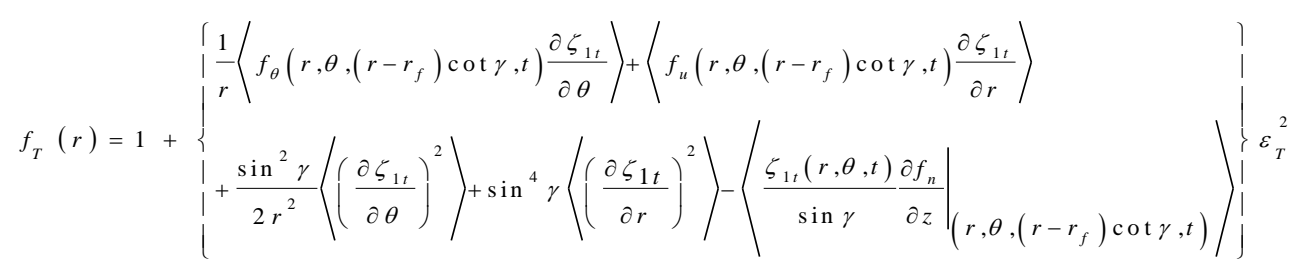

Next, the instantaneous heat release per unit area of the flame is given by:

$$
\frac{q(r, \theta, t)}{\rho s_{L} h_{R}}=\sqrt{1+\frac{1}{r^{2}}\left(\frac{\partial \zeta}{\partial \theta}\right)^{2}+\left(\frac{\partial \zeta}{\partial r}\right)^{2}}
$$

Using the expansion, Eq.(61) in the above and ensemble averaging yields:

$$
\frac{\tilde{q}_{o}(r)}{\rho s_{L} h_{R}}=\frac{1}{\sin \gamma}+\left\{\frac{\sin \gamma}{2 r^{2}}\left\langle\left(\frac{\partial \zeta_{1 L}}{\partial \theta}\right)^{2}\right)+\frac{1}{2} \sin ^{3} \gamma\left(\left(\frac{\partial \zeta_{1 L}}{\partial r}\right)^{2}\right)+\cos \gamma\left(\frac{\partial \zeta_{2 L}}{\partial r}\right)\right\} \varepsilon_{T}^{2}
$$

Where, $\tilde{q}_{o}(r)$ is the mean heat release rate per unit area for the mean flame surface shape given by Eq.(89). The mean heat release rate can be written in terms of local consumption speed $f_{c}(r)$ as follows:

$$
\frac{\tilde{q}_{o}(r)}{\rho h_{R} s_{L}}=f_{c}(r) \sqrt{1+\left(\frac{d \tilde{\zeta}_{o}}{d r}\right)^{2}}
$$

Thus, using Eq.(89) together with Eq.(93) in the above and retaining terms upto $o\left(\varepsilon_{r}^{2}\right)$ yields:

$$
f_{c}(r)=1+\left\{\frac{\sin ^{2} \gamma}{2 r^{2}}\left(\left\langle\left(\frac{\partial \zeta_{1 t}}{\partial \theta}\right)^{2}\right)+\frac{1}{2} \sin ^{4} \gamma\left\langle\left(\frac{\partial \zeta_{1 t}}{\partial r}\right)^{2}\right)+\cos \gamma \sin \gamma\left\langle\frac{\partial \zeta_{2 t}}{\partial r}\right)\right\} \varepsilon_{T}^{2}\right.
$$

and using Eq.(66) in the above yields the following result:

$$
f_{c}(r)=1+\left\{\begin{array}{l}
-\frac{1}{r}\left\langle f_{\theta}\left(r, \theta,\left(r-r_{f}\right) \cot \gamma, t\right) \frac{\partial \zeta_{1 t}}{\partial \theta}\right)-\left\langle f_{u}\left(r, \theta,\left(r-r_{f}\right) \cot \gamma, t\right) \frac{\partial \zeta_{1 t}}{\partial r}\right\rangle \\
\vdots
\end{array}\right\}
$$




\section{Flame Response Characteristics}

Explicit results for the present turbulent flame speed models have been obtained using closed form expressions for spatio-temporal correlations for stationary isotropic turbulence. Also, explicit results for the total transfer function have been obtained for the local consumption/displacement speed models given in Eqs. (91) and (96). Therefore, a comparison between that result and the flame response model developed in the present section will serve to ascertain the relative importance of the coherent variation in turbulent displacement and consumption speeds due to non-locality in determining the overall flame response. The following section discusses this and other results obtained from the analyses presented thus far.

The analysis in the previous sections showed that the presence of a spatio-temporally random velocity field results in a non-zero leading order correction to the linear transfer function. This is because of non-linear coupling between coherent flame surface perturbations induced by the acoustic forcing and random wrinkling induced by the turbulence. The leading order turbulence correction term can be determined from the expression in Eq.(73). For the spatio-temporal correlation functions assumed in this work, it was not possible to analytically determine a closed form solution for the above turbulence correction. Hence, a numerical integration technique using a 30 point GaussLegendre quadrature was adopted. The change in the value of the magnitude and phase of the leading order transfer function correction at the highest value of Strouhal number, St, between further successive refinements was less than $10^{-5}$. All lengths in this section will be presented as nondimensional numbers normalized by the integral length scale, $L_{11}$.

The value of $r_{f}$ was chosen to be 1.0. The upper limit of the outer most integral in Eq.(73) is the radius of the combustor (assumed circular in cross-section) which was assumed as $R=2.0$. Thus for a specified flame angle of $\gamma=45^{\circ}$ the nominal height of the flame is given by $\Lambda=1.0$. Figure 18 shows the variation of the total transfer function, i.e. $F_{L}\left(S t_{2}\right)+F_{T}\left(S t_{2}\right) \varepsilon_{T}^{2}$ (see Eq.(72)) with $S t_{2}$ for typical values of convection speed ratio, $K$. Also shown is the corresponding variation of the laminar transfer function for reference in each case. The turbulence intensity $\varepsilon_{T}=u_{T}^{\prime} / U_{o}=0.05$. Note that the variation of the transfer function magnitude and phase of the turbulent case are qualitatively similar to the corresponding laminar variations at low to moderate $S t_{2}$ values. This qualitative behaviour is due to the same reasons for non-monotonic behavior of laminar flame response magnitude that were discussed in Preetham et al; the net flame response is a superposition of responses from the boundary condition term and a spatial flow non-uniformity term as a result of non-locality.

The quantitative difference in the magnitude between laminar and turbulent cases (e.g. $0<\mathrm{St}_{2}<20$ for $K=1.0$ ) is because of kinematic coupling between the response of the flame surface to coherent and random velocity fluctuations and non-locality that now cause the burning rate variation along the flame to change from its unforced value. 


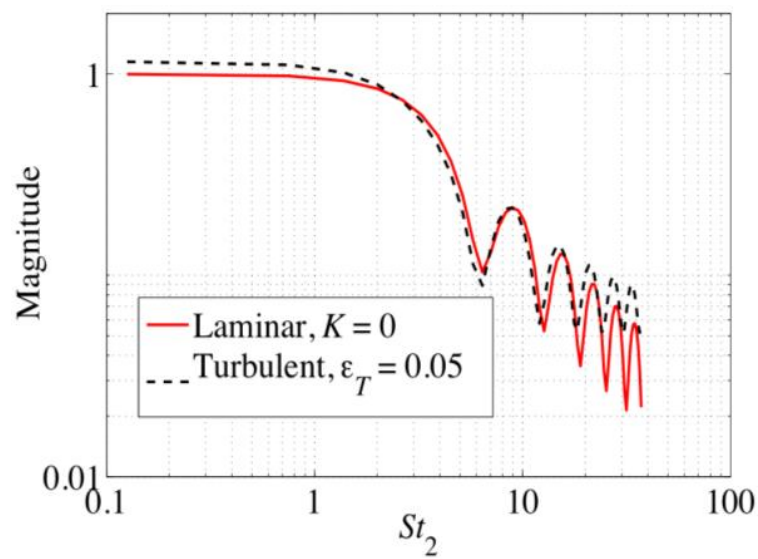

(a)

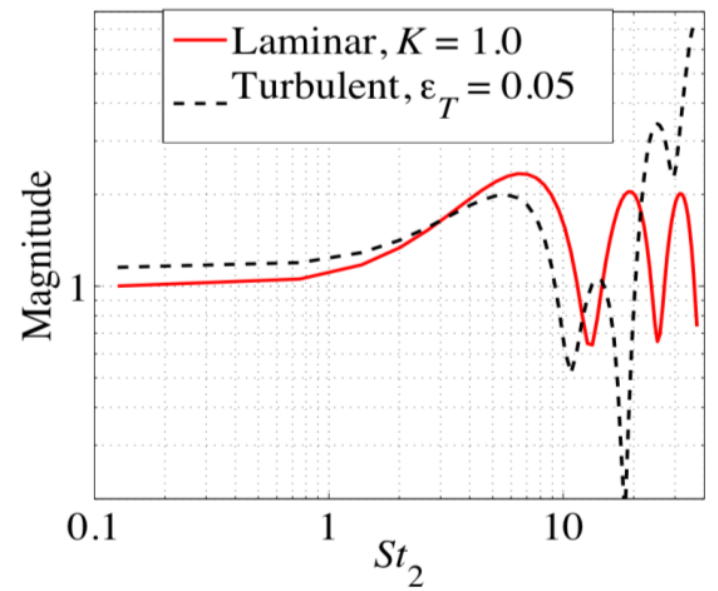

(c)

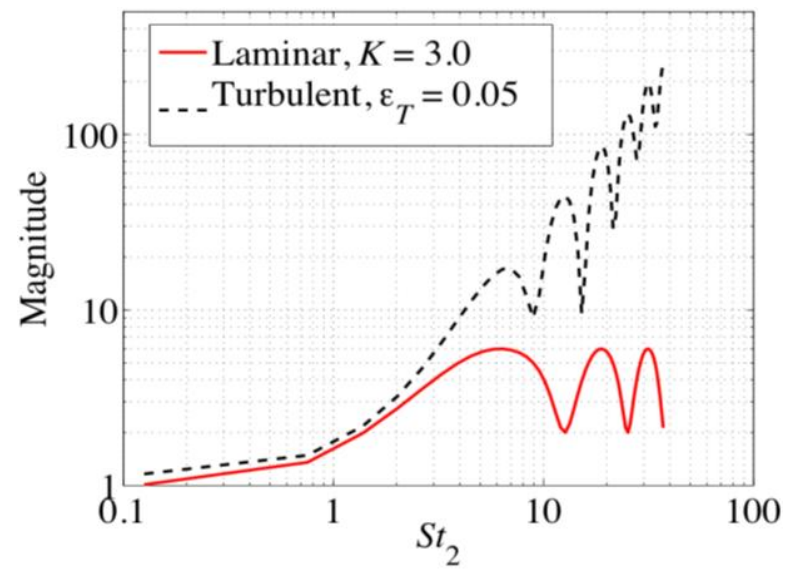

(e)

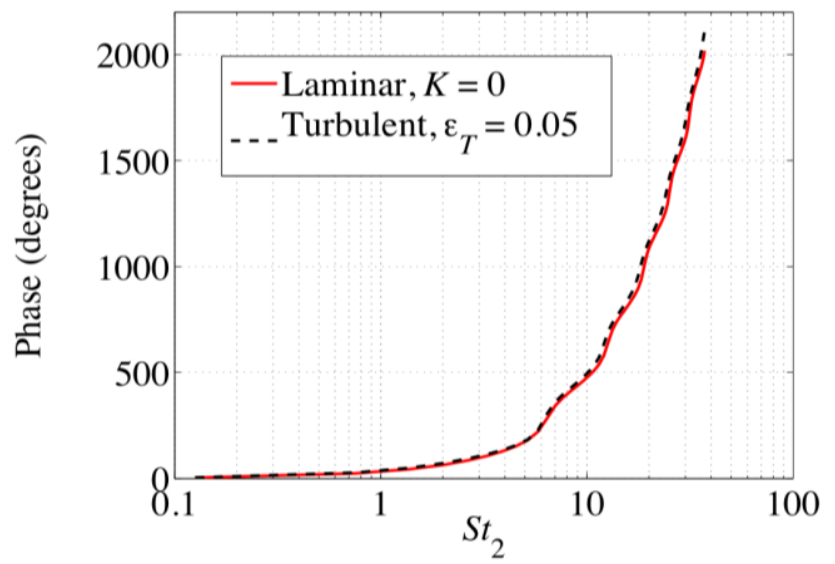

(b)

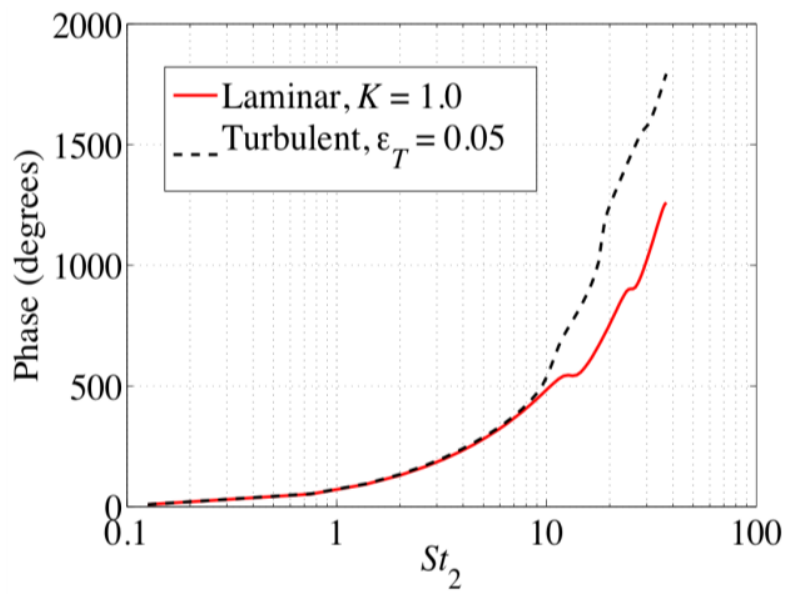

(d)

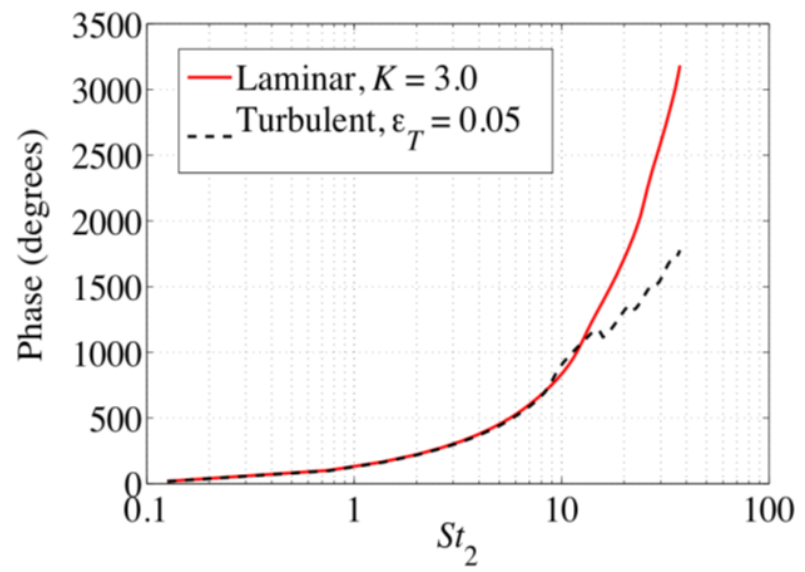

(f)

Figure 18: Variation of total transfer function magnitude and phase with Strouhal number for typical values of $K=(a)-(b) 0$, (c)-(d) 1.0 and (e)-(f) 3.0. Also shown for reference in each case is the corresponding laminar transfer function. Flame angle $\gamma=\mathbf{4 5}^{\circ}$. 
Next, note that as $S t_{2} \rightarrow 0$, each of the magnitude curves in Figure 18 start from a small non-unity value. With increasing $S t_{2}$ the magnitude and phase vary in a qualitatively similar manner as that of the corresponding laminar transfer function. This is at variance with the analysis of Polifke and Lawn who suggest that the heat release transfer function magnitude should reach unity as $S t_{2} \rightarrow 0$, for perfectly premixed flames. They give the following relation (dimensional form):

$$
\frac{\delta Q}{Q}=\frac{\delta \dot{m}_{f}}{\dot{m}_{f}}=\frac{\delta u_{B}}{u_{B}}
$$

where, $\dot{m}_{f}$ is the global mass burning rate of the flame and $u_{B}$ is a reference velocity that describes the forcing velocity field. Thus, they conclude that the transfer function magnitude must approach unity as $S t_{2} \rightarrow 0$ because, $\lim _{S t_{2} \rightarrow 0}\left\{(\delta \dot{Q} / \dot{Q}) /\left(\delta u_{B} / u_{B}\right)\right\}=1$. However, the second equality in Eq.(97) implicitly assumes that the global consumption speed of the flame is unaffected by the presence of coherent forcing. Since the net response of the flame due to the coherent forcing and turbulence fluctuations are kinematically coupled, the coherent response affects the net flame surface wrinkling due to turbulence fluctuations. Hence, global/local consumption speeds will vary in a coherent unsteady manner when the flame is acoustically forced. Therefore, writing (in dimensional form) $\dot{m}_{f}=\rho_{u} S_{T, G C}(t) A_{r e f}(t)$, where, $A_{r e f}$ is the unsteady ensemble averaged reference area, $S_{T, G C}$ is the global consumption speed of the flame in the presence of acoustic forcing and $\rho_{u}$ is the unburnt gas density, Eq.(97) can be rewritten as:

$$
\frac{\delta \dot{Q}}{Q}=\frac{\delta \dot{m}_{f}}{\dot{m}_{f}}=\frac{\delta A_{r e f}}{A_{r e f}}+\frac{\delta S_{T, G C}}{S_{T, G C}}
$$

Dividing the above expression by $\delta u_{B} / u_{B}$ yields:

$$
\frac{\delta \dot{Q} / \dot{Q}}{\delta u_{B} / u_{B}}=\frac{\delta A_{r e f} / A_{r e f}}{\delta u_{B} / u_{B}}+\frac{\delta S_{T, G C} / S_{T, G C}}{\delta u_{B} / u_{B}}
$$

The first term on the RHS approaches unity in the limit of $S t_{2} \rightarrow 0$ (i.e., low-frequency limit). However, the second term does not do so in general because of the presence of kinematic coupling between the coherent wrinkling and the random wrinkling as will be shown later. This point can be seen from Figure 19a-b that shows the variation of the magnitude of the turbulence correction and its phase relative to the laminar response with $S t_{2}$. The various $\left|F_{T}\right|$ curves tend to a constant non-zero value as $S t_{2} \rightarrow 0$. Further, Figure $19 \mathrm{~b}$ shows that the phase of this correction term relative to the laminar forcing is $<90^{\circ}$ for all values of $K$ as $S t_{2} \rightarrow 0$. This means that the contributions to the total transfer function due to the laminar response and due to the leading order turbulence correction add constructively, resulting in the magnitude of the total transfer function being $>1$ as $S t_{2} \rightarrow 0$. 


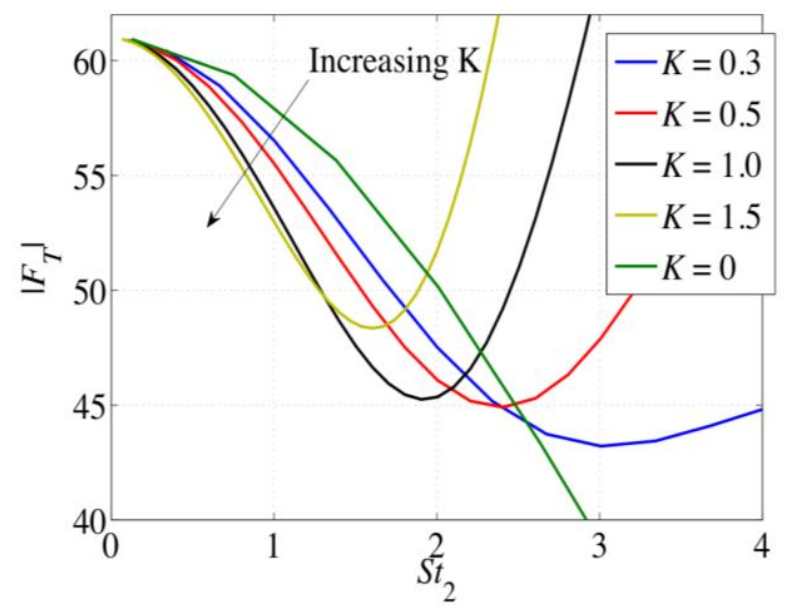

(a)

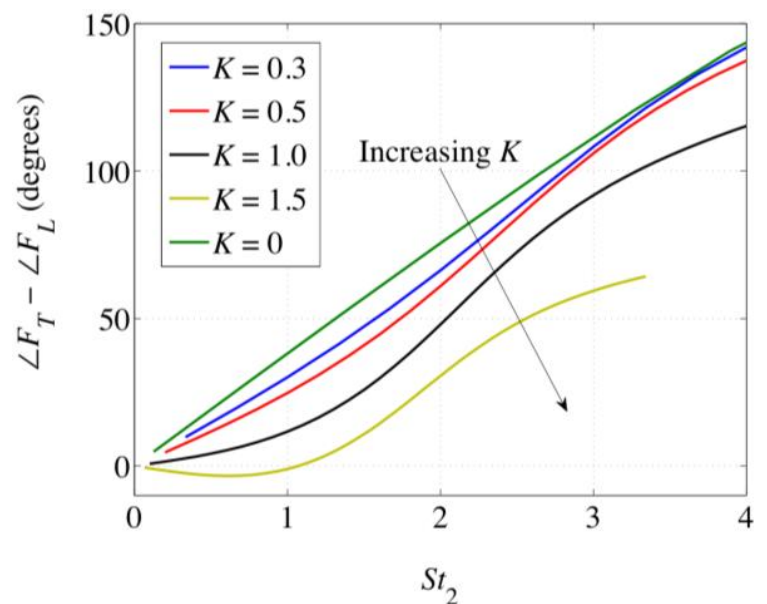

(b)

Figure 19: Variation of the leading order turbulence correction term with Strouhal number (a) magnitude and (b) phase. Flame angle, $\gamma=45^{\circ}$ and flame length, $\Lambda=1$.

Next, Figure 19 shows that $\left|F_{T}\right|$ decreases with increasing $S t_{2}$ for all values of $K$. This can be explained by consideration of kinematic restoration effects that cause destruction of small length scale wrinkles induced on the coherent flame surface wrinkling component due to turbulence fluctuations as shown schematically in Figure 20. Note first that the laminar analysis of Preetham et al shows that when $K>0$, the wavelength of flame surface wrinkling induced on the flame by coherent velocity fluctuations is controlled by $u_{c} / f$. This is shown schematically by the broken lines on both sides of the large arrows in Figure 20. Due to the presence of turbulence, additional small length scale wrinkling is induced on this coherent baseline which, in the absence of kinematic restoration would increase flame wrinkling amplitudes as shown by the corresponding solid lines to the left of the large arrows. For a given turbulence intensity, a shorter coherent wrinkling wavelength will result in wrinkles that are very quickly destroyed by kinematic restoration over a fixed time interval. The resulting flame shapes are shown by the red curves to the right of the large arrows. Hence, it is clear that with increasing frequency or decreasing $u_{\mathrm{c}}$ with all other parameters held constant, $\left|F_{T}\right|$ decreases as shown by Figure 19. The increase in $\left|F_{T}\right|$ after a certain cut-off frequency is because the higher order contributions become significant at these values of $S t_{2}$. These contributions cannot be determined in closed form. 
Large wavelength

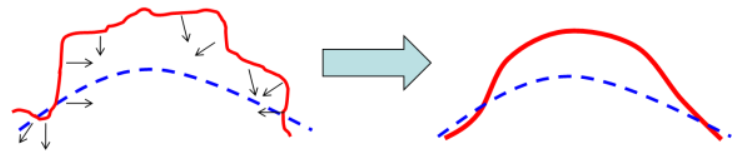

Small wavelength

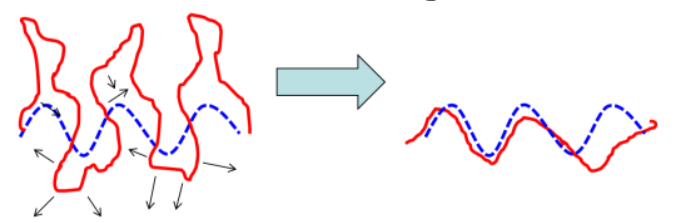

Figure 20: Schematic showing the influence of the wavelength of coherent forcing relative to the turbulent scales on burning area destruction. The solid curves on the left show schematically the resultant wrinkling if there were no kinematic restoration. The broken curves show schematically, the base-line coherent wrinkling. The small arrows show the direction of flame surface propagation.

Next, the above reasoning suggests that the turbulence correction magnitude must reduce as the laminar flame speed increases as a higher laminar flame speed causes wrinkles to be destroyed more efficiently. Note that within the framework of the present analysis, a change in flame angle $\gamma$ for a given value of $K$ implies a change in the value of $s_{L}$ because the value of $K$ fixes the mean flow velocity $U_{\mathrm{o}}$. Figure 21 shows the variation of the turbulence correction magnitude and phase (relative to the laminar correction) for three different values of flame angle. By definition, an increase $\gamma$ implies an increase in laminar flame speed. Thus, it is clear from Figure 21 that $\left|F_{T}\right|$ reduces with increasing $s_{L}$. Note also that the phase relative to the laminar correction is $<90^{\circ}$ over a larger range of $S t_{2}$ at smaller $s_{L}$ values. The implication of this result is that the change in the ensemble averaged value of heat release response due to turbulence would be more pronounced in lean premixed systems.

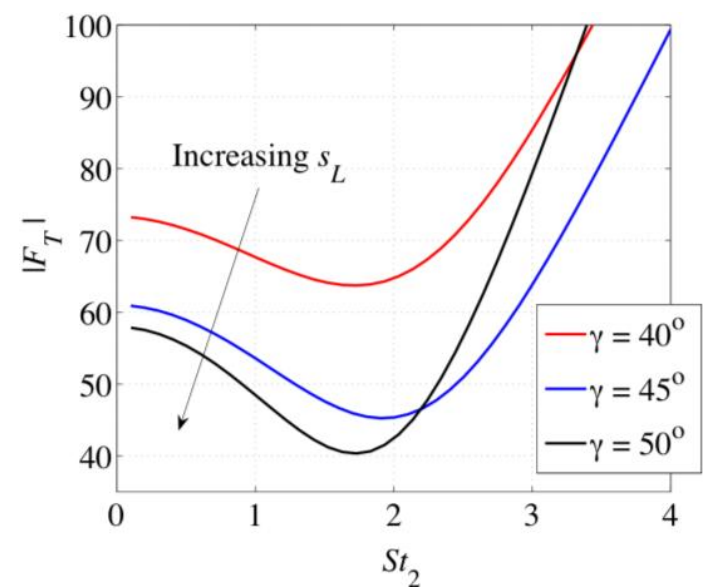

(a)

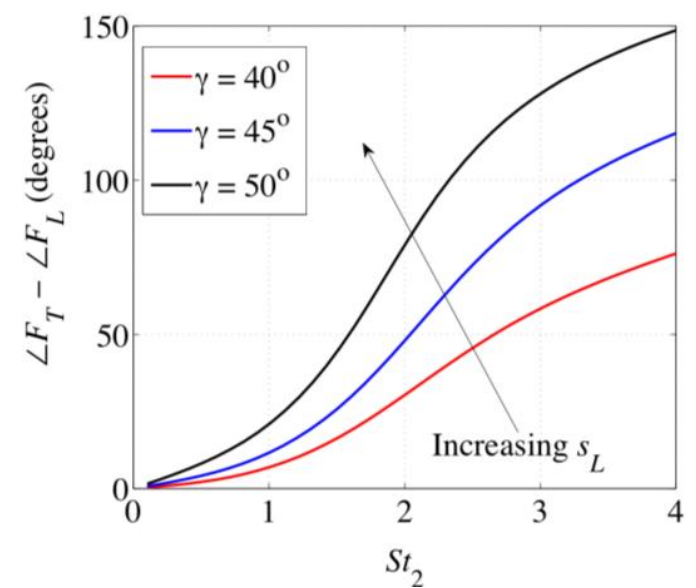

(b)

Figure 21: Variation of the (a) Magnitude and (b) phase relative to laminar response of the leading order turbulence correction for different flame angles $\gamma, K=1.0, \Lambda=1$. 
In summary, the results presented in this section show that the total ensemble averaged heat release response transfer function to leading order in turbulence intensity is qualitatively similar, but quantitatively different, from the corresponding baseline laminar transfer function at low to medium $S t_{2}$. The flame response does not go to unity in the low $S t_{2}$ limit because of the coherent response of the local/global consumption speeds to acoustic forcing. This is caused by kinematic coupling between coherent flame motions from acoustic forcing and random wrinkling due to turbulence fluctuations in addition to non-locality. This kinematic coupling causes the magnitude and phase of the total turbulent transfer function to differ quantitatively from its laminar baseline value at low to moderate Strouhal numbers. With increasing $S t_{2}$ and $s_{L}$, the decreased destruction time of short wavelength wrinkles causes the magnitude of the correlation function to drop. Beyond a certain critical Strouhal number (dependent on $K$ ) the length scale of coherent fluctuations becomes the same as the length scales of the turbulence fluctuations in the energy containing range. This results in large amplitude wrinkling of the flame surface. Thus, higher order kinematic restoration terms become significant and must be included in order to correctly predict the leading order turbulence correction to the flame transfer function.

\section{Transfer Function Predictions for Various Turbulent Flame Speed Models}

The above results provide useful insight into the fundamental physical processes that characterize the heat release response of turbulent premixed flames. However, in practical situations, it may not always be possible to determine the nominal flame surface shape except for simple configurations as analyzed in this work for the reasons discussed before. Heat release transfer function predictions have been obtained for two different turbulent flame speed models. The first of these is based on the specification of local displacement and consumption speeds $f_{T}(r)$ and $f_{c}(r)$ from Eqs. (91) and (96) respectively. Thus, this approach captures the non-local nature of unforced turbulent flame surface motions. The effect of kinematic coupling is not captured.

The second model is that of Lipatnikov and Chomiak (referred to as the LC model). The details of the derivation of this model are given in their review paper. The final expression for the local consumption speed is repeated here for convenience:

$$
f_{c}^{L i p}(r)=\frac{S_{o}}{s_{L}}\left\{1+\frac{T}{t^{\prime}(r)}\left(e^{-t^{\prime}(r) / T}-1\right)\right\}
$$

Where, $t^{\prime}(r)$ is a flame development time i.e., the time taken for a fluid particle to reach the flame surface from the burner inlet and $T$ is a turbulent mixing time. The flame development time was determined following the procedure in by first numerically evaluating the following integral (30point Gauss-Legendre quadrature) to obtain at parametrization $r=r\left(t^{\prime}\right)$ (non-dimensional form):

$$
r\left(t^{\prime}\right)=r_{f}+\frac{S_{o}}{s_{L}} \int d \tau\left\{1+\frac{T}{\tau}\left(e^{-\tau / T}-1\right)\right\}
$$


The value of $s_{o} / s_{L}$ was specified as $0.09 / \sin \gamma=0.13$. The mixing time $T$ was specified as $l_{E, \max } / \varepsilon_{T}=\sqrt{2 \pi} / 0.05 \sim 50$. Thus, the radial variation of $f_{c}^{\text {Lip }}(r)$ can be specified in parametric form using eq. (101) in terms of the parameter $t^{\prime}$. Finally, in keeping with the assumption made in, it was assumed that $f_{T}(r)=f_{c}^{\text {Lip }}(r)$. This consumption/displacement speed model amounts to assuming that the flame surface responds locally to turbulent velocity fluctuations. Again, the kinematic coupling effects are not captured in this model as well.

Figure 22(a) shows the radial variation of local consumption and displacement speeds from the nonlocal model (Eqs. (91) and (96)). Note first from Figure 22(a) that the predicted $f_{T}(r)$ and $f_{c}(r)$ variations with $r$ are not the same. The spatial variation in each of the above is induced by flame surface response non-locality. The difference between the two quantities is due to the fact that $f_{T}(r)$ is the propagation speed of the mean flame surface in the burner fixed co-ordinates, while $f_{c}(r)$ is a measure of the net mass burning rate change due to turbulence of an area element on the mean flame surface. This reference area is shown schematically in Figure 22(b). The mean surface shown in this figure is not the same as the nominal flame surface because of the presence of turbulence fluctuations. Notice that for the low value of turbulence intensity assumed in the present analysis $\left(\varepsilon_{T}=0.05\right)$, the area change due to flame wrinkling will be relatively small when compared the reference area shown in Figure 22(b) resulting in the value of $f_{c}(r) \sim 1$, suggesting that the assumption of You et al., i.e. $f_{c}(r)=$ constant is a good assumption in this case. However, the same cannot be assured at higher turbulence intensities. Thus, in the results presented next, the predictions of the present non-local model and those of You et al. will not be very different from each other. Therefore, only results from the former model will be presented in the interest of clarity.

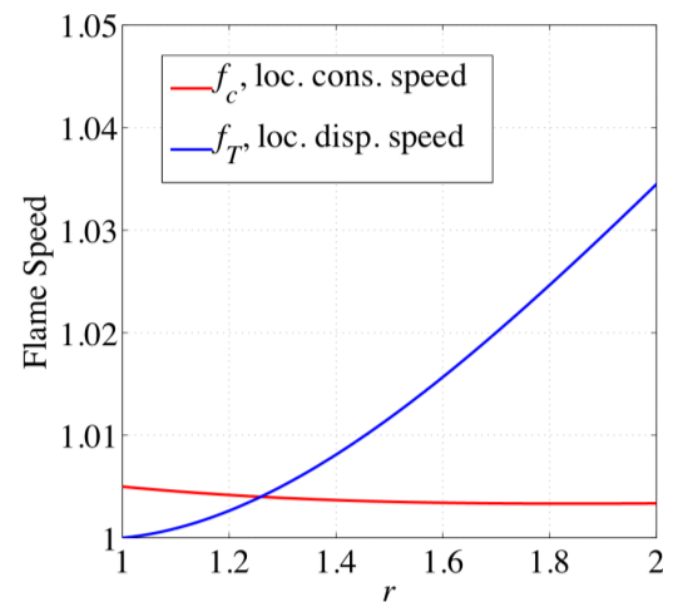

(a)

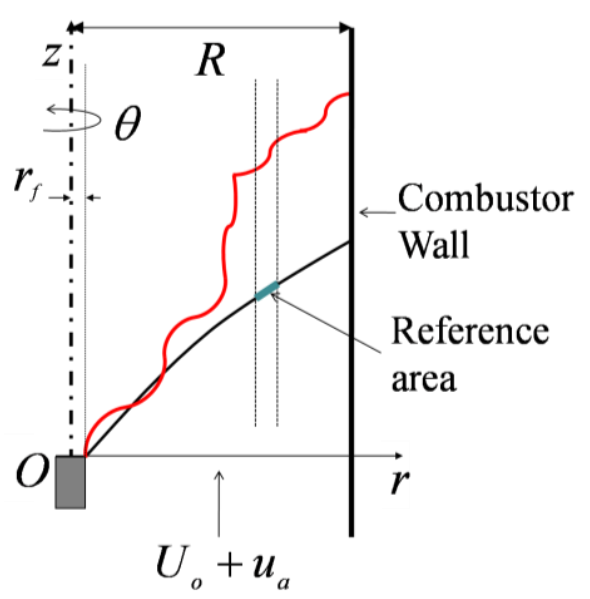

(b)

Figure 22: (a) Variation of local consumption and displacement speeds from the non-local model and (b) schematic showing the reference area on the unforced mean surface used to define $f_{c}(r), \varepsilon_{r}=0.05, \gamma=45^{\circ}$ and $\Lambda=1$. 
The corresponding variation of $f_{\mathrm{c}}$, (or $\left.f_{T}\right)$ from the $\mathrm{LC}$ model is shown in Figure 23. Note that this model predicts a consumption speed that is lower than the laminar flame speed. Figure 24 compares the predicted variation of the magnitude and phase of the heat-release transfer function from the modeling approach using the different models for $f_{T}(r)$ and $f_{c}(r)$ as described above and the exact asymptotic analysis presented in the previous section. Consider first the non-local model. Note that in every case, the agreement between the predicted magnitude and phase from the non-local model is in good agreement with the exact asymptotic result. However, the model cannot capture the nonunity magnitude of the exact result as $S t_{2} \rightarrow 0$ because the assumed flame speed model does not predict the modulation of flame speed with coherent forcing as no description of kinematic coupling effects on the local consumption and displacement speeds has been included. This result provides further evidence in support of the argument advanced earlier in this context.

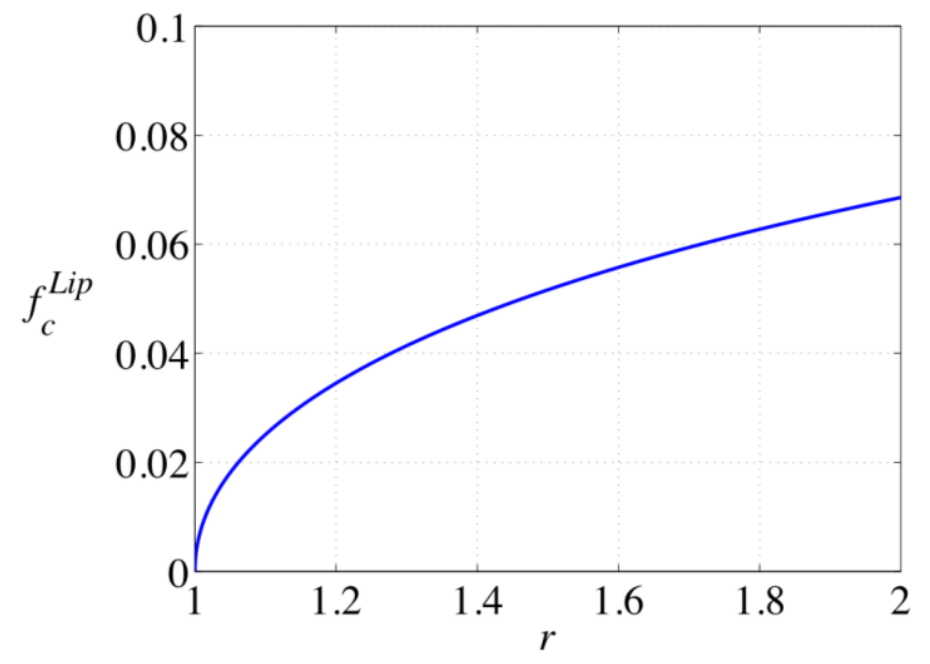

Figure 23: Variation of normalized local consumption/displacement speeds from the theory of Lipatnikov and Chomiak. Turbulent mixing time $T=50, s_{o} / s_{L}=0.13, \gamma=\mathbf{4 5}^{\circ}$ and flame length, $\Lambda=1$.

The LC model prediction is in poor agreement with the exact asymptotic result. The possible reasons for this result are as follows. First, the consumption and displacement speeds have been assumed to be identical. This is at variance with the exact kinematic result that the non-local model shows. Second, the model does not account for the non-local nature of flame surface response within its framework. Further, the model requires the specification of a fully developed flame speed value $\left(s_{o} / s_{L}\right)$.This requirement represents the general issue with, most heuristic models for displacement/consumption speed as they are based on adjustable parameters that may be hard to determine in general. The LC model is an example of such a model. However, the results from the non-local model presented here are encouraging and suggest that models that incorporate the physics of non-local response may be more successful in predicting turbulent premixed flame heat-release response for low values of $S t_{2}$ because they capture more realistically the underlying features of flame surface perturbations. 


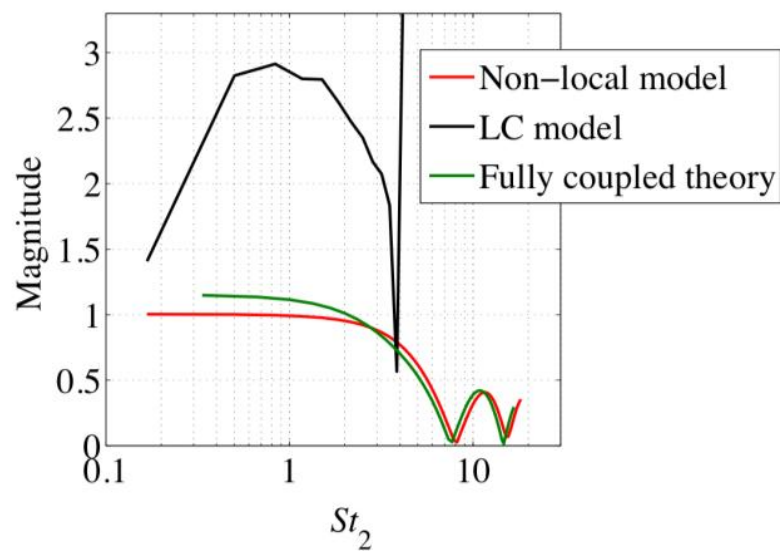

(a)

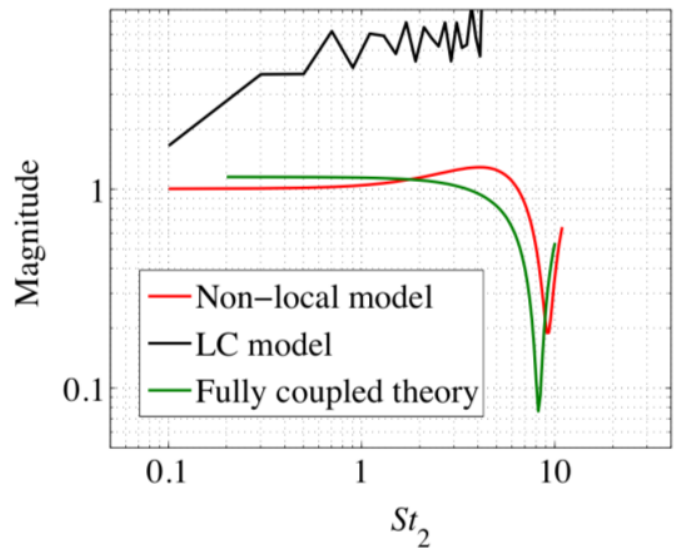

(c)

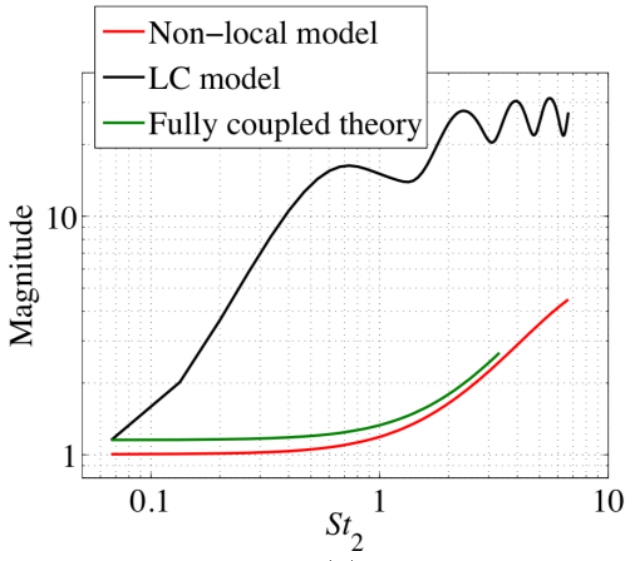

(e)

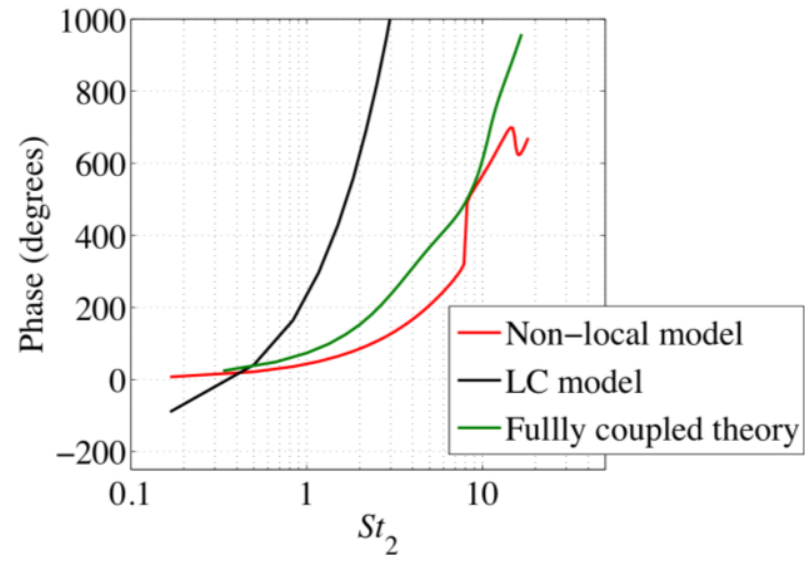

(b)

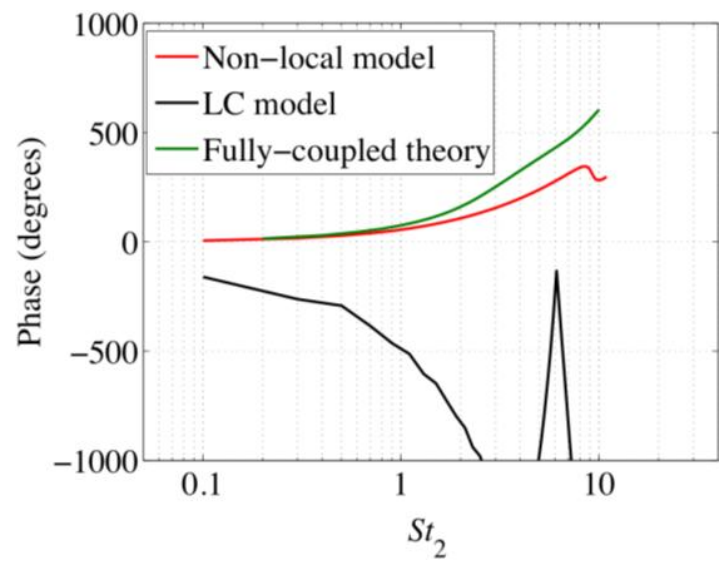

(d)

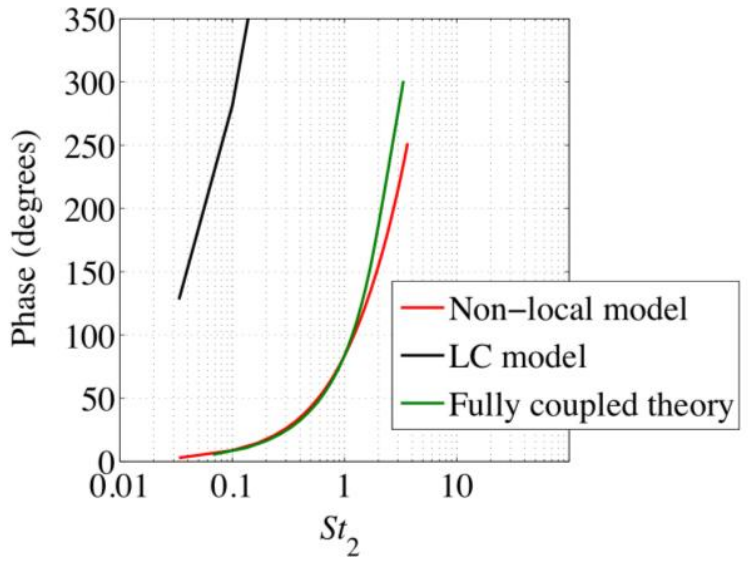

(f)

Figure 24: Comparison of transfer function magnitude and phase from the two modeling approaches and the theoretical analysis (a)-(b) $K=0.3$, (c)-(d) $K=0.5$, (e)-(f) $K=1.5$, $\varepsilon_{T}=0.05$ and $\gamma=45^{\circ}$ 


\section{Response of Premixed Flames to Equivalence Ratio Fluctuations - Comparison Between Global Heat Release and Global Chemiluminescence}

\section{Introduction}

As mentioned earlier, a major focus this technical report was to investigate the relationship between global heat release and chemiluminescence oscillations of flames excited by fuel/air ratio oscillations. This comparison is important because chemiluminescence response measurements may not directly reflect the heat release response of flames when the fuel composition is oscillating. The manner in which these oscillations disturb the heat release can be understood by considering the instantaneous global heat release rate, given by the integral over the flame surface area shown in Eq.(1). This equation shows four fundamentally different mechanisms generating heat-release disturbances in a premixed flame, viz., fluctuations in reactant density, flame speed, heat of reaction, or flame surface area. To understand how equivalence ratio oscillations lead to heat release oscillations, consider Figure 25, which provides a pictorial representation of the different possible "routes" by which the heat release oscillates. First, spatio-temporal fluctuations in equivalence ratio directly perturb the heat release by perturbing flame speed (route $2 \mathrm{a}$ ) and the heat of reaction (route 1). Second, these perturbations in the flame speed induce fluctuations in flame position, hence causing fluctuations in burning area (route $2 \mathrm{~b}$ ). This in turn causes perturbations in the heat release. This is an indirect route.

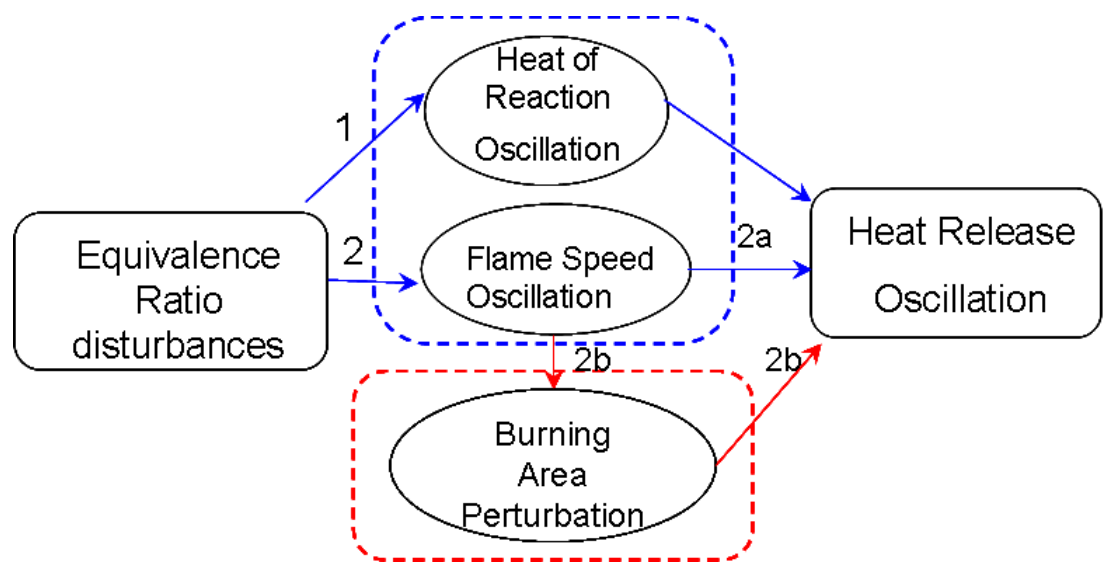

Figure 25: Physical mechanisms causing heat release oscillations due to fluctuations in reactant equivalence ratio

Hemchandra et al. showed that route 1, i.e., heat of reaction oscillations, is dominant for lean flames at very frequencies when $S t<<1$. At higher values of the Strouhal number, all three routes are seen to be of comparable importance.

Having overviewed the mechanisms by which fuel/air ratio oscillations lead to heat release oscillations, we next discuss how equivalence ratio oscillations are manifested through oscillations in flame chemiluminescence. Measurements of naturally occurring flame chemiluminescence emissions from premixed flames have been used in numerous studies as an indicator of the local and global heat release rates. Detailed chemical kinetic calculations of lean 
premixed laminar $\mathrm{CH}_{4}$-air flames show that $\mathrm{CH}^{*}, \mathrm{OH}^{*}$ and $\mathrm{CO}_{2} *$ radicals occur within the reaction zone, indicating that the reaction zone is the source of these chemiluminescence emissions. While certainly not a perfect approach, as discussed further below, chemiluminescence is really the only practical method for inferring heat release rates at present.

There is significant experimental data showing that at a fixed equivalence ratio, the quasi-steady chemiluminescence emission intensity from the flame exhibits a linear dependence on the reactant flow rate. For these reasons, unsteady chemiluminescence appears to be a good marker of unsteady heat release for flames responding to low frequency flow velocity oscillations. For example, the data below illustrate gain curves for global $\mathrm{CH}^{*}$ and $\mathrm{OH}^{*}$ chemiluminescence showing similar frequency sensitivities. Given the different chemical pathways by which these species are formed, it appears unlikely that their gains would be so similar if they were not both primarily functions of the heat release rate.
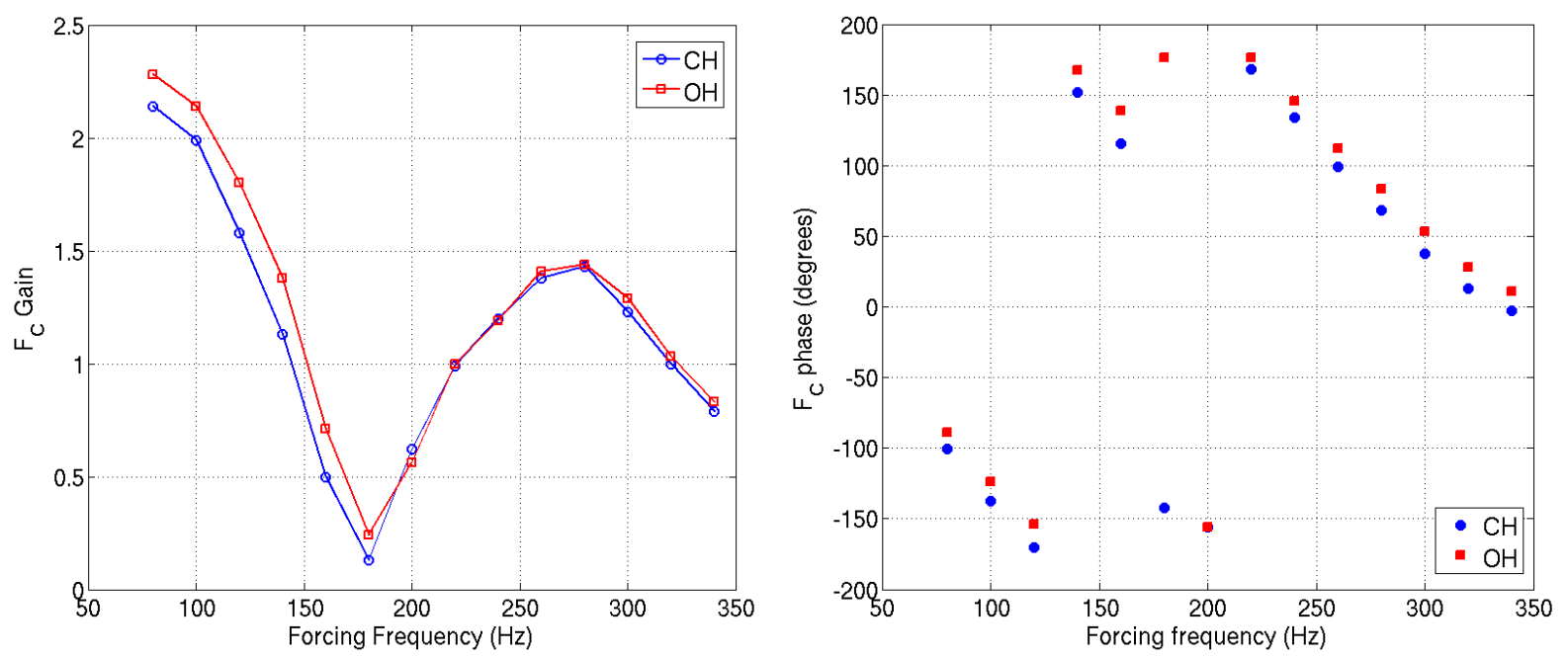

Figure 26: Chemiluminescence transfer function gain (left) and phase (right, with respect to velocity perturbations) for velocity-coupled flame response, for $\square_{o}=0.75, u_{o}=25 \mathrm{~m} / \mathrm{s}, 5 \%$ perturbation amplitude

However, chemiluminescence emissions are not only a function of the instantaneous heat release rate, but other parameters as well, including fuel/air ratio, fuel type, strain rate, and unsteady effects. We start first with unsteady effects. Due to finite rate kinetics, one can expect there to be a certain phase lag between heat release and chemiluminescence in unsteady flames, even if they track each other perfectly in the quasi-steady limit. While we are not aware of studies explicitly considering this effect, data suggests that it may be negligible for a variety of frequencies of interest. This may be seen from the difference in phases of $\mathrm{CH}^{*}$ and $\mathrm{OH}^{*}$ chemiluminescence signals with respect to those of velocity perturbations at the flame base, as shown above. Given the different chemical pathways (and presumably time scales) by which these species are formed, the fact that they give essentially the same phase strongly suggests that they are tracking the heat release in a quasi-steady manner. More data and analyses are needed, however, to further understand these effects. 
We next consider other effects such as turbulence and strain rate and flame curvature. John and Summerfield, Hurle et al. and, more recently, Lauer and Sattelmayer have shown that turbulence reduces the global chemiluminescence emission intensity. Additionally, other studies have systematically characterized the relationship between chemiluminescence and heat release fluctuations, showing that these are correlated as long as the strain rate and flame curvature are not "too large" (e.g., within a flame cusp). For highly strained flames, these studies indicate that the local chemiluminescence emission can go to zero, even without local extinction. The sensitivity to strain rate has also been discussed extensively by Nori. It appears that the strain rate sensitivity of chemiluminescence is much less than its sensitivity to equivalence ratio. Fuel sensitivities have been studied by, e.g., Clark et al. and Nori, who reported measurements using methane, propane, ethylene, $\mathrm{H}_{2} / \mathrm{CO}$ blends, and Jet A. Given the caveats already noted in this section, these studies show that chemiluminescence emissions tracked the unsteady heat release, although the specific sensitivities varied with the fuel and radical species.

Finally, we consider the fuel/air ratio sensitivity of chemiluminescence emissions, which forms the main focus of this study. The sensitivity of chemiluminescence to fuel/air ratio is well known from a number of experiments, showing that the variation of global chemiluminescence intensity with fuel-flow rate itself is an exponential function of the equivalence ratio. This is demonstrated by global $\mathrm{OH}^{*}$ and $\mathrm{CH}^{*}$ measurements and occurs due to the exponential dependence of the reaction rate upon the temperature. Due to this exponential sensitivity of chemiluminescence to fuel/air ratio, there are problems associated with its interpretation if both the fuel/air ratio and heat release rate are oscillating. In particular, it can be anticipated that the relationship between the global chemiluminescence intensity and the global heat release is not one-to-one when there is a spatial variation in equivalence ratio along the flame surface area. This occurs because the chemiluminescence intensities of the local area elements are functions of the local equivalence ratio, which is now varying along the flame surface. Hence, the overall chemiluminescence emission intensity is a non-equally weighted sum of individual elements over the flame.

Thus, an important question must be addressed: How does the global chemiluminescence response of the flame compare with the global heat release response? The current work aims to address this question by analytically calculating and comparing transfer functions for the chemiluminescence and heat release responses, which are respectively defined as follows:

$$
\begin{gathered}
F_{C}=\frac{C H^{, G} / C H_{o}^{G}}{\phi_{\text {base }}{ }^{\prime} / \phi_{o}} \\
F_{Q}=\frac{Q^{\prime} / Q_{o}}{\phi_{\text {base }} \cdot / \phi_{o}}
\end{gathered}
$$

Here, $\phi^{\prime}{ }_{\text {base }}$ denotes the fluctuations in equivalence ratio at the flame base and the hats $\left({ }^{\wedge}\right)$ denote Fourier-transformed time domain variables. We will show that the comparison between $F_{C}$ and $F_{Q}$ depends upon the chemiluminescing species considered, area-averaged fuel/air ratio, and flame geometry. 


\section{Analytical Formulation of Global Chemiluminescence Response Modeling}

The global chemiluminescence intensity can be calculated as an integral of the local chemiluminescence intensity per unit area. We write this local chemiluminescence intensity as a function of the local instantaneous heat release rate per unit flamelet surface area and local equivalence ratio. Mathematically, this may be expressed as follows:

$$
C H^{G}(t)=\int_{\text {flame }} \sigma^{L}(q(\phi), \phi(z, t)) d A
$$

Here, $q$ is the heat release per unit area of the flame. As shown previously, by definition:

$$
Q(t)=\int_{\text {flame }} q(t) d A=\int_{\text {flame }} \rho s_{L}(\phi) h_{R}(\phi) d A
$$

Further, note that the local heat release density may be estimated as the product of the local mass burning rate of the reactants and the heat of reaction of the reactants, both of which depend on the local equivalence ratio. Mathematically:

$$
q(z, t)=\rho s_{L}(\phi(z, t)) h_{R}(\phi(z, t))
$$

We shall assume here that the fuel/air ratio oscillations occur at constant reactant density. From Eq.(106), it follows that $q=q(\phi)$, so that we may write:

$$
\sigma^{L}(q, \phi)=\sigma^{L}(q(\phi), \phi)=\sigma^{L}(\phi(z, t))
$$

With these considerations, the global chemiluminescence intensity $\mathrm{CH}^{G}$, as expressed in Eq.(104), may be rewritten as follows:

$$
C H^{G}(t)=\int_{\text {flame }} \sigma^{L}(\phi(z, t)) d A
$$

For the sake of illustration, we will now assume that the mean equivalence ratio, $\phi_{0}$, is spatially uniform, so that $\phi(z, t)=\phi_{o}+\phi^{\prime}(z, t)$. Expanding $\sigma^{L}(\phi(z, t))$ about $\phi_{o}$ yields:

$$
C H^{G}(t)=\int_{\text {flame }}\left\{\left|\sigma^{L}\left(\phi_{o}\right)+\frac{\partial \sigma^{L}}{\partial \phi}\right|_{\left(q_{o}, \phi_{0}\right)} \phi^{\prime}(z, t)\right) d A
$$

Note that $\sigma^{L}\left(\phi_{o}\right)=C H_{o}^{\sigma} / A_{o}$ is the chemiluminescence intensity per unit flame surface area of the undisturbed flame, which is a constant. Further, define a flame surface area averaged "effective" equivalence ratio fluctuation as:

$$
\phi_{e f f}^{\prime}(t)=\int_{\text {flame }} \phi^{\prime}(z, t) d A
$$


The instantaneous global chemiluminescence intensity may be written in terms of $\phi_{\text {eff }}^{\prime}(t)$ as:

$$
\frac{C H^{G}(t)}{C H_{o}^{G}}=\frac{A(t)}{A_{o}}+\left.\frac{\partial\left(\sigma^{L}(\phi) / \sigma^{L}\left(\phi_{o}\right)\right)}{\partial\left(\phi / \phi_{o}\right)}\right|_{\phi_{o}} \frac{\phi_{e f f}^{\prime}(t)}{\phi_{o}}
$$

On subtracting the mean quantities, this can be written as:

where

$$
\frac{C H^{H^{G}}(t)}{C H_{o}^{G}}=\frac{A^{\prime}(t)}{A_{o}}+m\left(\phi_{o}\right) \frac{\phi_{e f f}^{\prime}(t)}{\phi_{o}}
$$

$$
m\left(\phi_{o}\right)=\left.\frac{\partial\left(\sigma^{L}(\phi) / \sigma^{L}\left(\phi_{o}\right)\right)}{\partial\left(\phi / \phi_{o}\right)}\right|_{\phi_{o}}
$$

The constant $m$ is the sensitivity of the chemiluminescence intensity of the flame per unit area to fluctuations in equivalence ratio. This will be obtained from experimental data relating chemiluminescence intensity/flow rate to equivalence ratio.

\section{Analytical Formulation of Global Heat Release Response Modeling}

Let us next consider the expression for the instantaneous heat release by the flame. Assuming constant density we may linearly perturb Eq.(105) to obtain

$$
\frac{Q^{\prime}(t)}{Q_{o}}=\frac{A^{\prime}(t)}{A_{o}}+\left(\int_{\text {flame }} \frac{s_{L}{ }^{\prime}(\phi)}{s_{L o}} \frac{d A}{A_{o}}+\int_{\text {flame }} \frac{h_{R}{ }^{\prime}(\phi)}{h_{R o}} \frac{d A}{A_{o}}\right)
$$

The first term on the RHS of the above isolates the contribution of flame surface wrinkling and consequent changes in the flame surface area, to the heat release (indirect route, 2b, Figure 25). The terms in the parentheses denote the contribution to the heat release because of the dependence of flame speed and heat of reaction on equivalence ratio (direct route, 1 and 2a, Figure 25). The variation of equivalence ratio leads to different parts of the flame responding to different local equivalence ratios.

Next, define flame speed and heat of reaction sensitivities to equivalence ratio respectively, as:

$$
\begin{aligned}
& s_{L 1}=\left.\frac{\partial\left(s_{L} / s_{L o}\right)}{\partial\left(\phi / \phi_{o}\right)}\right|_{\phi=\phi_{o}} \\
& h_{R 1}=\left.\frac{\partial\left(h_{R} / h_{R o}\right)}{\partial\left(\phi / \phi_{o}\right)}\right|_{\phi=\phi_{\sigma}}
\end{aligned}
$$


Note that, for fixed operating conditions, these sensitivities are functions of the mean equivalence ratio alone. Eq.(114) may be written in terms of these sensitivities as:

$$
\frac{Q^{\prime}(t)}{Q_{o}}=\frac{A^{\prime}(t)}{A_{o}}+\left[s_{L 1}\left(\phi_{o}\right)+h_{R 1}\left(\phi_{o}\right)\right] \frac{\phi_{e f f}^{\prime}(t)}{\phi_{o}}
$$

Note from Eq.(112) and Eq.(117) that evaluation of the instantaneous global chemiluminescence intensity and global heat release of the flame requires us to estimate the fluctuations in burning surface area and the effective equivalence ratio.

Expressions for the flame surface area may be estimated using the knowledge of the instantaneous flame surface location. For this purpose, a two-dimensional front tracking formulation is employed (Markstein, Marble and Candel, Yang and Culick, Fleifil et al., Kerstein et al., Preetham and Lieuwen). The flame is assumed to consist of a thin sheet whose surface can be represented implicitly by the zero contour of a two dimensional function $G(r, z)$. For purposes of illustration, a spatio-temporally harmonically varying equivalence ratio fluctuation advecting at the mean velocity of the reactants is assumed in this work. Mathematically:

$$
\phi=\phi_{o}\left(1+\varepsilon \cos \left(\omega\left(t-\frac{z}{u_{o}}\right)\right)\right)
$$

We non-dimensionalize the axial coordinate $z$ by the length of the quiescent flame, $L_{f}$ and $t$ by the characteristic convection time for the disturbances from the flame base to the flame tip, $L_{f} / u_{o}$. Retaining the same notations for the non-dimensional variables as their original dimensional counterparts for the sake of notational convenience, we may rewrite Eq.(118) in non-dimensional form as:

$$
\phi=\phi_{o}(1+\varepsilon \cos (S t(z-t)))
$$

\section{Analytical Formulation of Global Transfer Functions}

Here, the Strouhal number is a non-dimensional frequency defined previously in Eq.(41). With the framework presented in the previous subsections, we may evaluate transfer functions for the global chemiluminescence response, $F_{C}$ and global heat release response, $F_{Q}$, by taking the Fourier transforms of Eq.(112) and Eq.(117) at the forcing Strouhal number, to yield:

$$
\begin{gathered}
F_{C}=F_{A}+m F_{\phi_{\text {eff }}} \\
F_{Q}=F_{A}+\left(s_{L 1}+h_{R 1}\right) F_{\phi_{e f f}}
\end{gathered}
$$


Here, $F_{A}$ and $F_{\text {peff }}$ denote the transfer functions for the burning area and effective equivalence ratio fluctuation responses whose definitions are similar to those of $F_{C}$ and $F_{Q}$, see Eq.(102) and Eq.(103)

$$
\begin{gathered}
F_{A}=\frac{A^{\prime} / A_{o}}{\phi_{\text {base }}^{\prime} / \phi_{o}} \\
F_{\phi_{\text {eff }}}=\frac{\phi_{\text {eff }}^{\prime} / \phi_{o}}{\phi_{\text {base }}^{\prime} / \phi_{o}}
\end{gathered}
$$

Equations (120) and (121) are very revealing for understanding the comparison between $F_{C}$ and $F_{Q}$. First, both transfer functions have similar structures and the same two principal contributing terms - fluctuating flame surface area and fluctuating effective equivalence ratio. Furthermore, Eq.(120) and Eq.(121) differ only in the coefficient which precedes the latter contribution, $F_{\phi e f f}$. The former contribution, $F_{A}$ affects both $F_{C}$ and $F_{Q}$ in an identical manner. This has important implications. Specifically, it means that $F_{C}$ and $F_{Q}$ are the same only if either of the following two conditions is satisfied:

$$
\begin{aligned}
& \text { (1) } m \sim s_{L 1}+h_{R 1} \\
& \text { (2) }\left|F_{A}\right| \gg\left|F_{\phi, e f f}\right|
\end{aligned}
$$

The first condition reflects the fact that $F_{C}$ and $F_{Q}$ are identical if the sum of the heat of reaction and flame speed sensitivities of the heat release identically equal the fuel/air ratio sensitivity of the local chemiluminescence intensity per unit area. The second condition states that even if there is a disparity between the two coefficients, their net effect on the total transfer function would be swamped out because of the heavy domination of the area response. Equation (124) also isolates the two ways in which either response can change - viz., (a) by altering the burning area and effective equivalence ratio responses, or (b) by altering the chemiluminescence intensity sensitivity, flame speed sensitivity and heat of reaction sensitivity. The former can be achieved if the flame geometry and/or the mean flame shape changes. The latter occurs if the mean equivalence ratio, reactant pressure or reactant temperature change. Hence, for fixed operating and geometric conditions, a change in fuel composition would affect flame response through affecting the sensitivities alone.

To understand these effects, we present results from illustrative calculations for these transfer functions in the next section that account for each of these effects. For these calculations we estimate the constant $m$ from the slope of global chemiluminescence intensity/fuel flow rate versus equivalence ratio data from Lee and Santavicca and Nori. For example, a typical curve using $\mathrm{CO}_{2}{ }^{*}$ as the chemiluminescing species is presented below in Figure 27. Similarly, the flame speed and heat of reaction sensitivities were calculated from the Premix module of Chemkin (using GRIMech 3.1) and GasEq, respectively. 


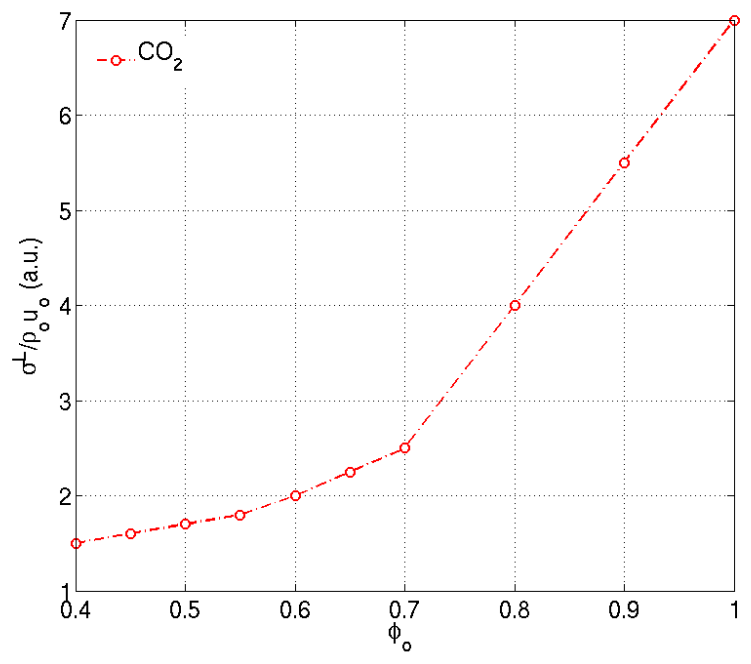

Figure 27: Variation of $\mathrm{CO}_{2}^{*}$ chemiluminescence intensity/fuel flow rate with mean equivalence ratio

\section{Illustrative Results}

Now we present illustrative results comparing $F_{C}$ and $F_{Q}$. We intend to understand how factors such as different flame geometries, mean equivalence ratios, and thermodynamic conditions of the reactants affect $F_{C}$ and $F_{Q}$. Additionally, different chemiluminescing species are considered to estimate $F_{C}$, in order to determine which of those compare best with $F_{Q}$. Two farmiliar flame geometries are considered, axisymmetric conical and V-shaped flame, as were seen in the top left and bottom left images of Figure 1. They are shown again here in Figure 28 utilizing more convenient parameters and coordinates. We assume that in either case, the flame base remains attached to the burner lip at all times.

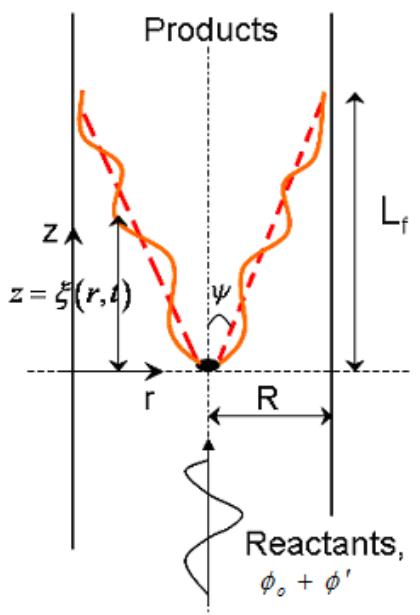

(a)

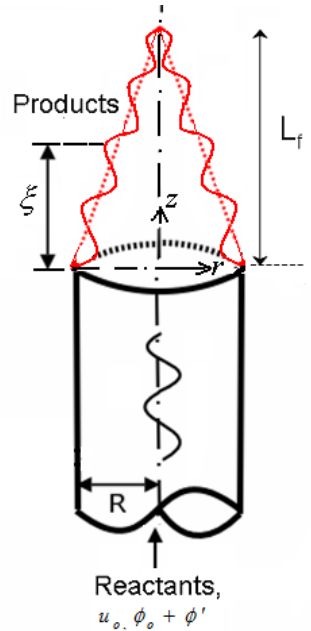

(b)

Figure 28: Schematic of the flame geometry - (a) axisymmetric V-flame and (b) axisymmetric conical flame 


\section{Flame Geometric Effects and Burning Area Response}

As noted in the earlier section, the relationship between global responses is influenced by the flame geometry or in the three sensitivity parameters. These two possibilities are discussed under subsections ' $\mathrm{A}$ ' and ' $\mathrm{B}$ ' below. We start by presenting expressions for the $\mathrm{V}$-flame and conical flame transfer functions. We will consider flame geometric effects and burning area response. For an axisymmetric V-flame, expressions for $F_{A}$ and $F_{\phi e f f}$ are:

$$
\begin{gathered}
\left.F_{A}=-s_{L_{1}}\left\{\frac{2 \alpha}{1-\alpha}\left(\frac{1-\alpha+(i S t-1) \exp (i S t)-\alpha(i S t / \alpha-1) \exp (i S t / \alpha)}{S t^{2}}\right)\right\}\right\} \\
F_{\phi_{\text {eff }}}=-\frac{2\left(1+i S t e^{i S t}-e^{i S t}\right)}{S t^{2}}
\end{gathered}
$$

Here $\alpha=\cos ^{2} \psi$ is a flame geometric parameter. For a premixed conical flame, transfer function expressions may be obtained as follows:

$$
\begin{gathered}
F_{A}=s_{L_{1}}\left\{\frac{2 \alpha}{1-\alpha}\left(\frac{1-\alpha-\exp (i S t)+\alpha \exp (i S t / \alpha)}{S t^{2}}\right)\right\} \\
F_{\phi_{e f f}}=\frac{2\left(1+i S t-e^{i S t}\right)}{S t^{2}}
\end{gathered}
$$

Using the above, $F_{C}$ and $F_{Q}$ may be evaluated using Eq.(120) and Eq.(121). Further, the low Strouhal number limits of the above may be evaluated for either geometry as:

$$
\begin{aligned}
& \lim _{s_{t \rightarrow 0}} F_{A}=-s_{L_{1}} \\
& \lim _{s_{t \rightarrow 0}} F_{\phi_{\text {eff }}}=1
\end{aligned}
$$

For the total responses, these limits hence become:

$$
\begin{aligned}
& \lim _{S_{t \rightarrow 0}} F_{C}=m-s_{L 1} \\
& \lim _{S t \rightarrow 0} F_{Q}=h_{R 1}
\end{aligned}
$$




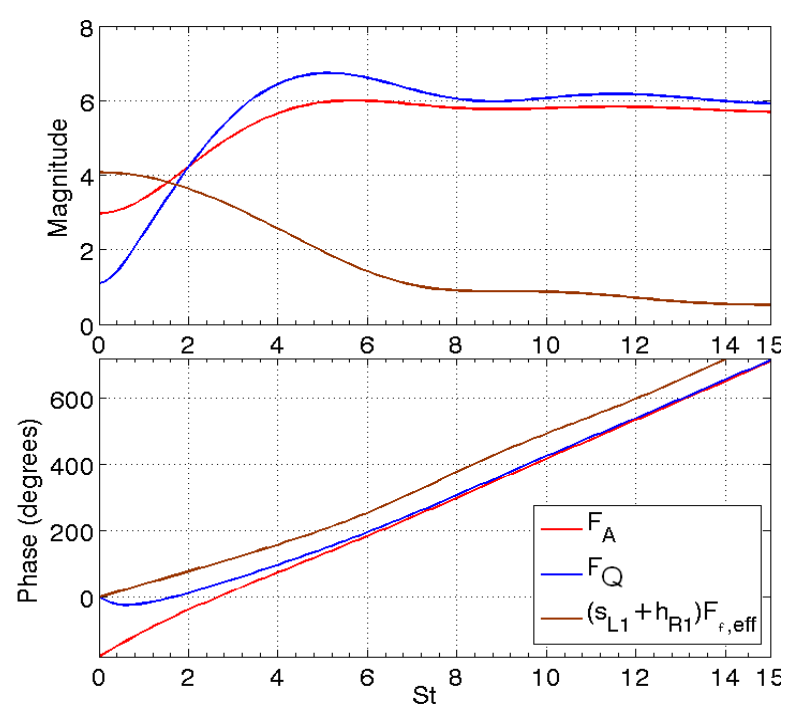

(a)
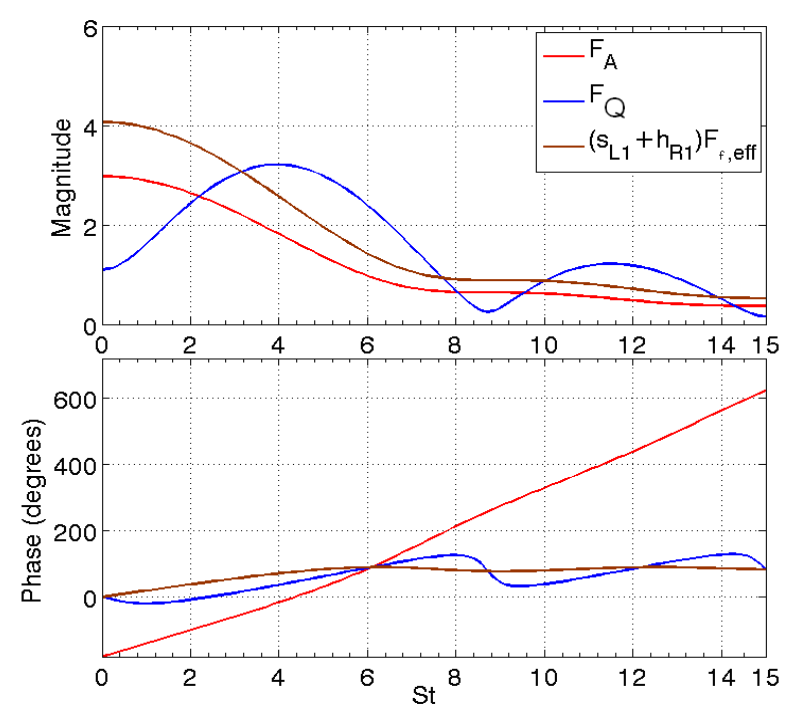

(b)

Figure 29: Contributions to $F_{Q}$ by area and effective equivalence ratio fluctuations for (a) $\mathrm{V}$-flame (b) conical flame at $1 \mathrm{~atm}, 300 \mathrm{~K}, \phi_{o}=0.6, \beta=4$.

Consider next the contribution of the area fluctuations and effective equivalence ratio fluctuations to the total transfer functions. Figure 30 plots the contributions of response of flame surface area fluctuations and effective equivalence ratio fluctuations to $F_{C}$ for a V-flame and conical flame. It can be clearly seen that the two processes contribute very differently to conical flames as compared to V-flames. In the case of V-flames, $F_{A}$ dominates over $F_{\square \text { eff }}$ over most of the Strouhal number range. Hence, the heat release response of the flame is primarily due to $F$. However, at lower Strouhal numbers, these effects are comparable. This is seen in the phase too, where the heat release response phase and burning area response phase are noticeably different in the quasi-steady limit and are identical at larger Strouhal numbers.

However, in the case of conical flames, these two effects contribute comparably over the entire Strouhal number range. This noticeable difference in the response characteristics is merely because of the fact that a V-flame has maximum surface area at the flame tip, while the conical flame has maximum surface area at the flame base. Hence, the maximum area in the V-flame is free to move and leads to higher response, while it is constrained at the flame attachment point for conical flames. 


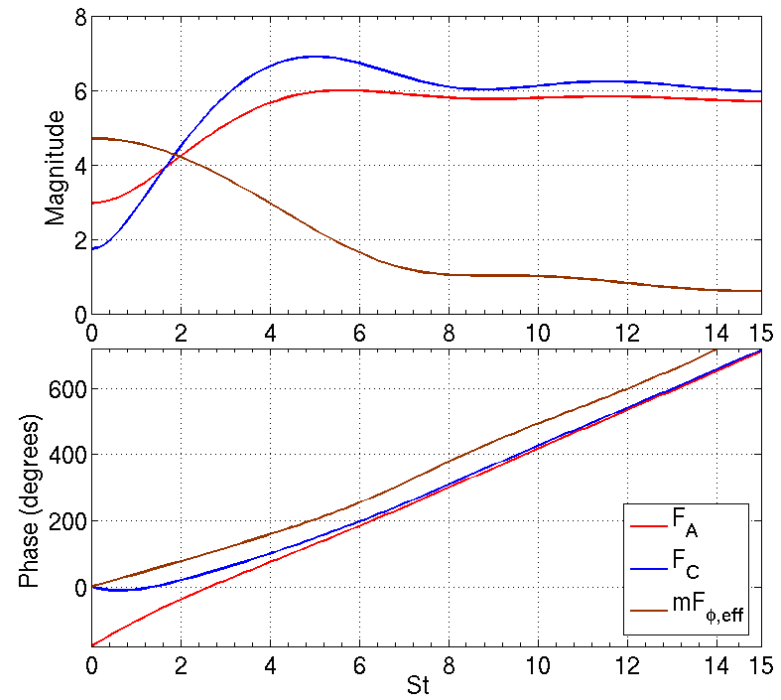

(a)
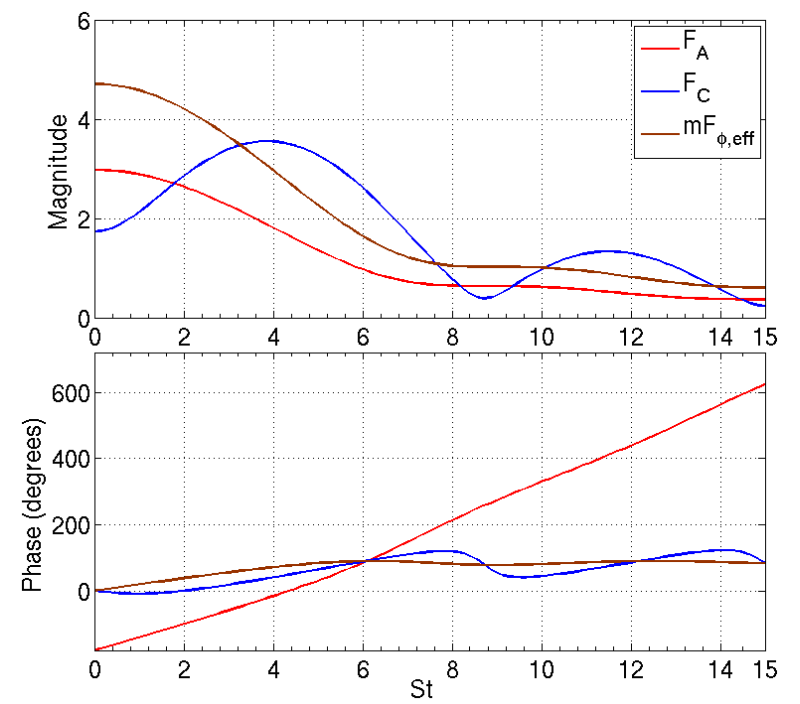

(b)

Figure 30: Contributions to $F_{C}$ by area and effective equivalence ratio fluctuations for (a) $\mathrm{V}$-flame (b) conical flame at $1 \mathrm{~atm}, 300 \mathrm{~K}, \phi_{0}=0.6, \beta=4$

The points raised in the above discussion can be directly applied to anticipate the relationship between $F_{C}$ and $F_{Q}$. The $\mathrm{V}$-flame is largely dominated by the area response at higher Strouhal numbers, while there is comparable contribution from the effective equivalence ratio response at lower Strouhal numbers. For the conical flame, these contributions are comparable over the entire range of Strouhal numbers considered. Based on the two conditions stated in Eq.(124), it can be seen that the $\mathrm{V}$-flame $F_{C}$ and $F_{Q}$ reasonably agree over the range of Strouhal numbers where area response dominates. However, in the case of conical flames, there may be some differences to the extent that the sensitivity coefficients differ. To investigate further into this issue, we next consider the comparison between $F_{C}$ and $F_{Q}$ for reactants at $1 \mathrm{~atm}, 300 \mathrm{~K}$, which is plotted in Figure 31.

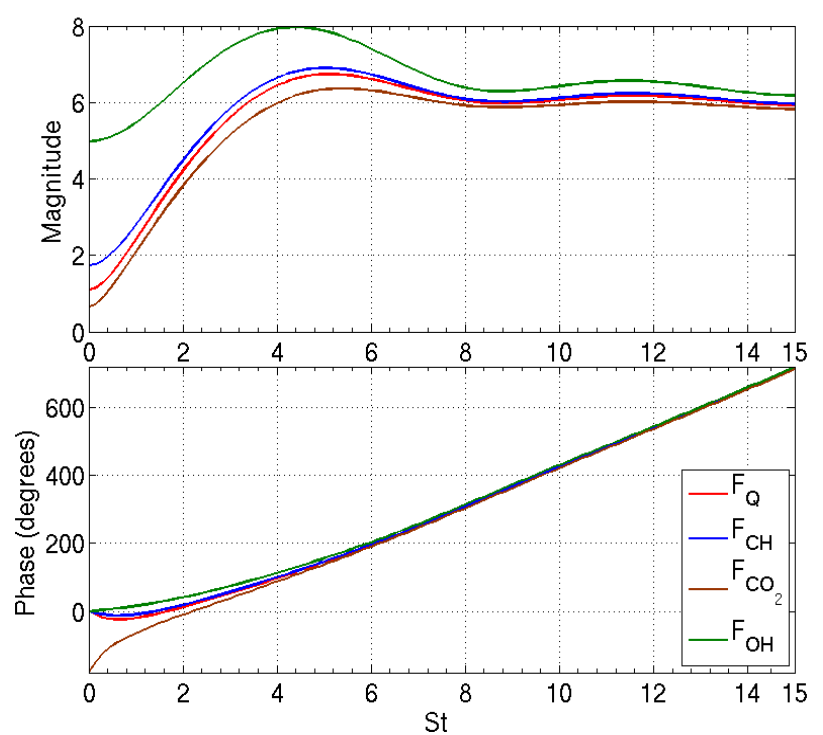

(a)

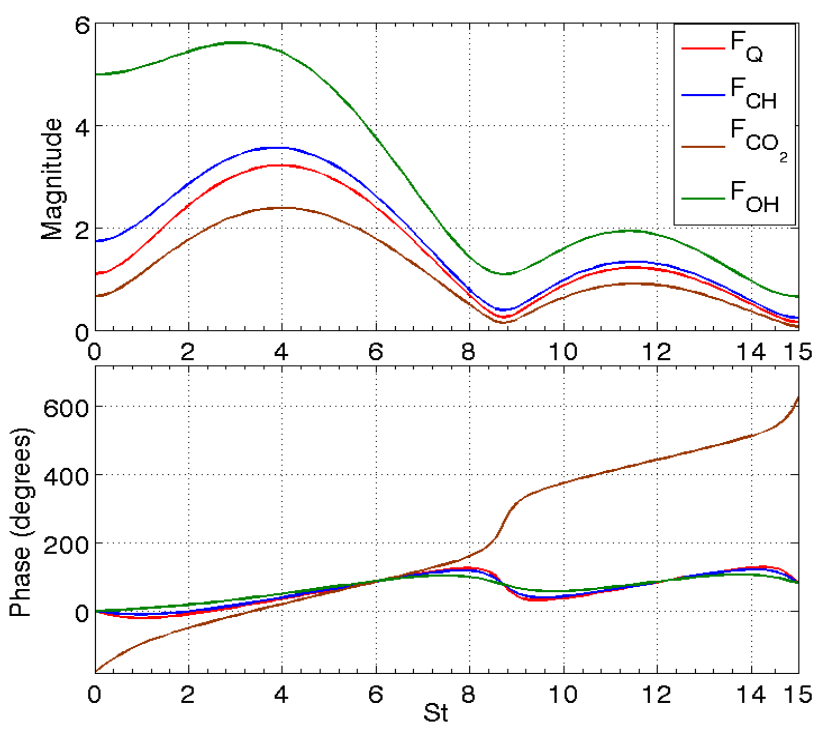

(b) 
Figure 31: Comparison between $F_{C}$ and $F_{Q}$ for (a) V-flame (b) Conical flame at $1 \mathrm{~atm}, 300$ K. $\phi_{o}=0.6, \beta=4$

The following observations may be made from Figure 31. The $F_{C}$ gain and phase for each of the three chemiluminescing species are qualitatively similar to $F_{Q}$ at all but very low Strouhal numbers. At very low Strouhal numbers, there is a disparity in gain as well as in phase. At these low Strouhal numbers, since $F_{A}$ and $F_{\text {peff }}$ are comparable; condition (1) of Eq.(124) needs to be satisfied, which is not the case. For conical flames, there is a noticeable disparity in phase if $\mathrm{CO}_{2} *$ is used, while there is a disparity in gain if $\mathrm{OH}^{*}$ is used as the chemiluminescing species. These disparities are directly related to the degree that the different sensitivities satisfy condition (1) of Eq.(124).

\section{Effect of Variation of Sensititivies}

We next consider the effect of chemiluminescence and local heat release sensitivity to fuel/air ratio. We shall start by considering the variation of the three sensitivities for reactants at 1 atm, $300 \mathrm{~K}$, for different chemiluminescing species. This is plotted in Figure 32(a). At STP, $\mathrm{CO}_{2} *$ and $\mathrm{OH}^{*}$ sensitivities, $m_{\mathrm{CO} 2}$ and $m_{\mathrm{OH}}$, are reasonably close to the sum of the flame speed and heat of reaction sensitivities, $s_{L 1}+h_{R 1}$, for mean equivalence ratios between 0.7 and 0.9 . Hence, these can be expected to yield $F_{C}$ 's that are close to $F_{Q}$. $\mathrm{CH}^{*}$ might be preferable at lower equivalence ratios, while $\mathrm{OH}^{*}$ seems to be preferable at near-stoichiometric fuel composition. Recall also that the value of $m$ relative to $s_{L 1}$ is important in controlling the response at low Strouhal numbers; specifically, there is a 180 degrees phase difference between the quasi-steady $F_{C}$ phase and $F_{Q}$ phase if $m>s_{L 1}$, see Eq.(131) and Eq.(132).

Next consider the effect of pressurizing the reactants to 5 atm. It merely suffices to consider the variation of sensitivities with equivalence ratio at 5 atm; conclusions regarding the comparison between $F_{C}$ and $F_{Q}$ can be drawn based upon the previous discussion and results. This is plotted in Figure 32(b), which suggests that at lower equivalence ratios, $\mathrm{OH}^{*}$ seems to be preferable; also, $m<s_{L 1}$ for all chemiluminescing species and hence, the quasi-steady phase difference between $F_{C}$ and $F_{Q}$ would be 180 degrees. However, at higher equivalence ratios, say $0.9, \mathrm{CO}_{2}{ }^{*}$ seems to yield closer agreement between $F_{C}$ and $F_{Q}$, owing to its sensitivity, $m_{C O 2}$ being closest to $s_{L 1}+h_{R 1}$. Also, it may be observed that at these equivalence ratios, because $m>s_{L 1}$, irrespective of the chemiluminescing species, the phase predictions would be more accurate, even in the quasi-steady limit. Finally, it may be noted that, in general, one can expect very good agreement between $F_{C}$ and $F_{Q}$ if the mean equivalence ratio lies approximately between 0.7 and 0.85 . 


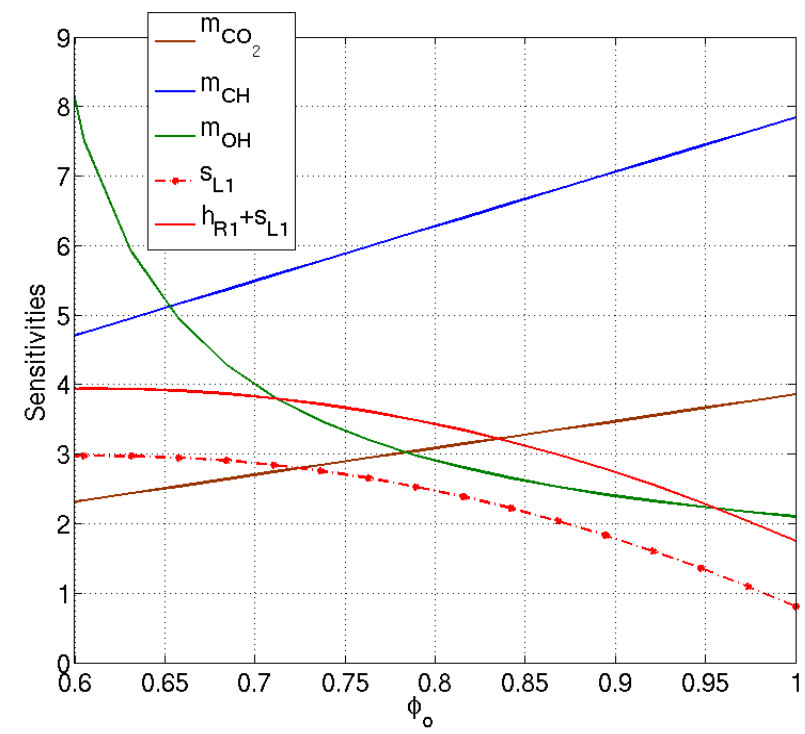

(a)

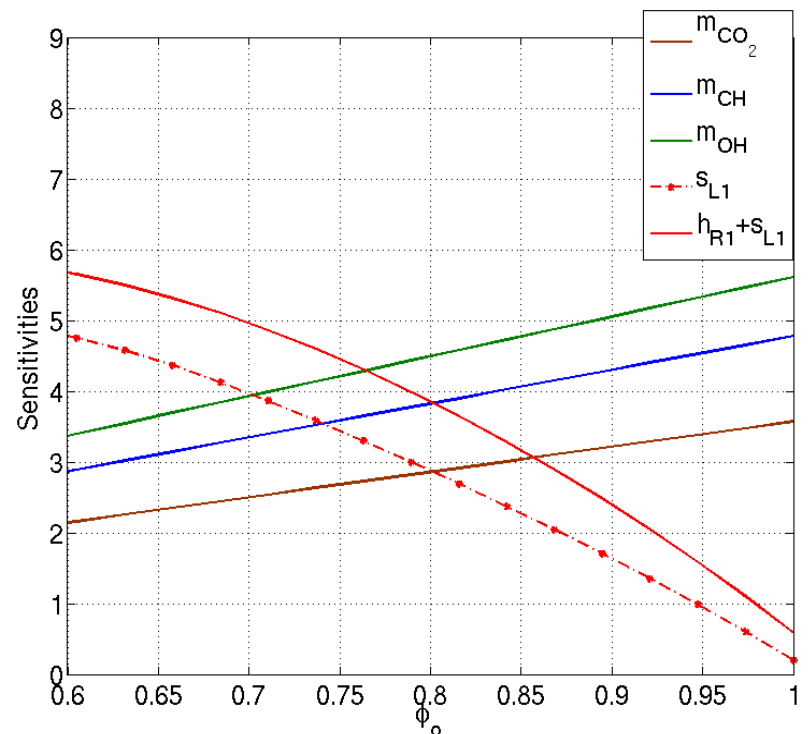

(b)

Figure 32 : Variation of various sensitivities with mean equivalence ratio at (a) 1 atm, 300 K (b) 5 atm, $300 \mathrm{~K}$

Finally, we consider the effect of preheating the reactants to a higher temperature. The variation of various sensitivities versus equivalence ratio at $5 \mathrm{~atm}, 600 \mathrm{~K}$ is plotted in Figure 33.

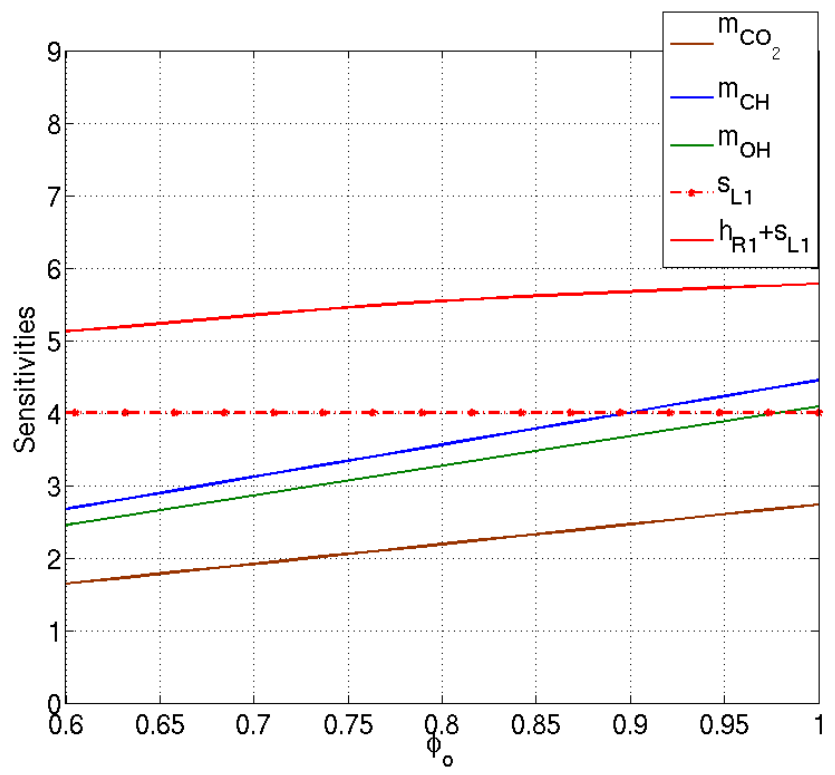

Figure 33 : Variation of various sensitivities with mean equivalence ratio at $5 \mathrm{~atm}, 600 \mathrm{~K}$.

Using similar arguments as before, it may be seen from Figure 33 that at all equivalence ratios it may be expected that $\mathrm{CH}^{*}$ and $\mathrm{OH}^{*} F_{C}$ 's would compare better with $F_{Q}$, while the low frequency phases would be off by 180 degrees for all of the chemiluminescing species, since $m<s_{L I}$ for all of them. At near-stoichiometric equivalence ratios (larger than 0.9, say), $\mathrm{CH}^{*}$ appears to be capable of also yielding good comparison between the quasi-steady phase difference between $F_{C}$ and $F_{Q}$. 


\section{Review}

The work presented in this section focused on studying the global chemiluminescence intensity response and global heat release response of premixed flame submitted to a spatio-temporally oscillating equivalence ratio field. The two responses were compared by comparing the respective transfer functions. A theoretical derivation of these linear transfer functions showed that both $F_{C}$ and $F_{Q}$ had similar forms, with the two constituent contributions coming from flame surface area response and response arising due to a spatio-temporally varying equivalence ratio oscillation. While the area response was seen to contribute identically to either transfer function, the contribution due to response of the fluctuating effective equivalence ratio differed in magnitude by a factor equal to the respective sensitivities, i.e., chemiluminescence intensity sensitivity for $F_{C}$ and the sum of flame speed and heat of reaction sensitivities of $F_{Q}$. This similarity of form yielded two criteria, at least one of which had to be satisfied for $F_{C}$ and $F_{Q}$ to compare reasonably well with each other.

To develop an actual feel for how these two transfer functions compare, calculations were performed for $\mathrm{CH}_{4}$-air mixtures at different conditions. In spite of quantitative differences, the qualitative relationship between $F_{C}$ and $F_{Q}$ seemed quite similar. The major difference seemed to be due to mean equivalence ratio and flame geometry. For the V-flame geometry, $F_{C}$ and $F_{Q}$ almost always compared very well with each other owing to the dominating contribution of the area response over the effective equivalence ratio response. At lower equivalence ratios, the quasi-steady phases differed by a factor of 180 degrees, owing to the difference in signs of the values of the respective quasi-steady transfer functions. This disparity was eliminated at higher equivalence ratios, where the agreement between $F_{C}$ and $F_{Q}$ was excellent. For the conical flame geometry, both the area response and the effective equivalence ratio response contributed comparably over the entire frequency range. While the actual quantitative values between the $F_{C}$ and $F_{Q}$ were not as close as that of the V-flame, they did follow similar trends over most of the frequency range, especially in magnitude.

The overall comparison between $F_{C}$ and $F_{Q}$ were quite encouraging for both V-flames and conical flames, hinting that, to a reasonable degree of accuracy, chemiluminescence response can be used to understand heat release response even when the equivalence ratio is oscillating spatiotemporally. However, some caution seems to be required wherever direct quantitative comparisons are sought, as well as in studying the low Strouhal number responses. Finally, it should be noted that this analysis is strictly valid for laminar flames. Clearly, the situation of most practical interest for performing these comparisons is in turbulent flames. As such, we are not aware of any factors which would lead to fundamentally different qualitative conclusions than drawn here.

\section{Response of Premixed Flames to Equivalence Ratio Perturbations - Flame Stretch and Non-Quasi-Steady Effects}

\section{Introduction}

Studying this latter equivalence ratio mechanism is the focus of this section of the final technical report. Several studies have shown strong evidence for its significance in exciting instabilities either by direct measurement of equivalence ratio oscillations during instabilities or by 
comparing the dependence of instability characteristics on geometry and operating conditions with correlations developed from theoretical analyses. From an analytical viewpoint, much insight into the phase response of the flame to such perturbations can be obtained from a simple time delay analysis that treats the flame as a concentrated source of heat release. In general, however, flames are distributed axially over a length scale where the mixture equivalence ratio can significantly vary. In other words, they are convectively non-compact, although perhaps being acoustically compact. The flame Strouhal number, $S t\left(=\omega L_{f} / U_{o}\right)$, which equals the length of the flame to the length scale of the imposed fuel/air ratio excitation determines whether the flame can be regarded as being a convectively compact or distributed source, and whether the flame response is geometrically quasi-steady or non-quasi steady. This quasi-steady limit has been discussed extensively by Polifke and Lawn, who presented a detailed analysis of the general flame response characteristics in the low forcing frequencies $(S t \rightarrow 0)$ limit.

As was stated previously, it is known that the basic phenomenology of the flame response is controlled by a superposition of three processes, shown schematically in Figure 34. This figure is an extension of Figure 25. This can be seen by noting that the instantaneous global heat release rate of a premixed flame may be expressed as the product of the local mass burning rate times the heat of reaction of the reactant mixture, integrated over the entire flame surface area, as shown in Eq.(1).

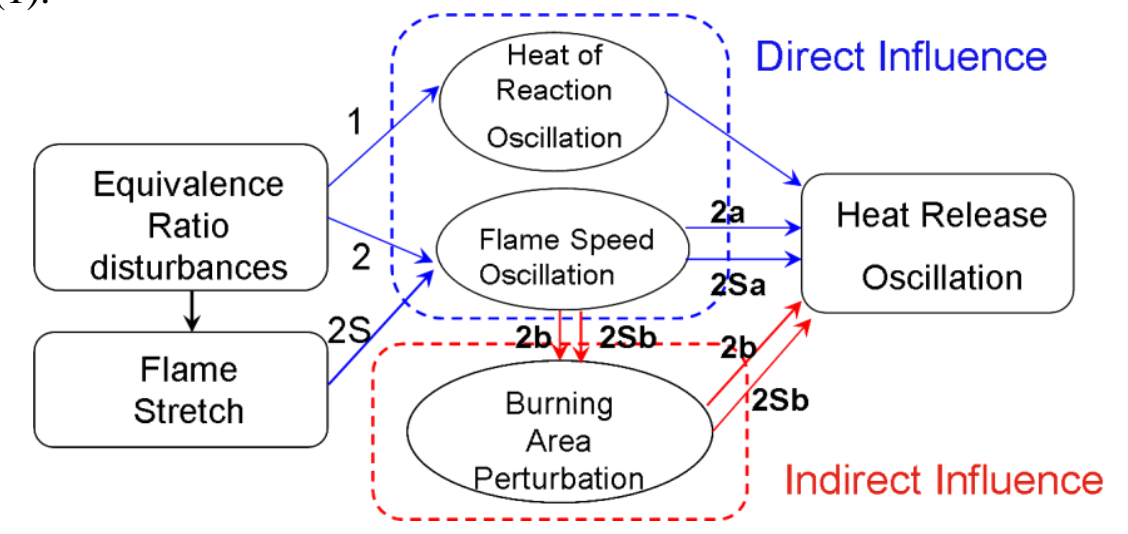

Figure 34: Fundamental processes controlling the heat release response of premixed flames to equivalence ratio oscillations

Equivalence ratio perturbations cause fluctuations in local flame speed (route 2a) and heat of reaction (route 1) along the flame surface. These fluctuations in mixture flame speed and heat of reaction then cause the local heat release rate to oscillate. This is a direct route of influence. Alternatively, flame speed variations also excite flame wrinkles that propagate along the flame. This leads to an oscillation in the burning area of the flame (route 2b), also causing the net heat release rate to oscillate. This is an indirect route of influence. It is also be noted that the indirect route of influence is also non-local; i.e., the flame area fluctuations at a given time and position are a convolution of the fuel/air ratio oscillations at all upstream locations at earlier times. Due to oscillations caused in the flame shape due to equivalence ratio perturbations, oscillations arise in the curvature of the flame front, which can now alter the flame speed, thereby establishing another route by which equivalence ratio fluctuations can cause flame speed fluctuations (route $2 \mathrm{~S}$ ). These fluctuations in flame speed can now disturb the heat release directly (route 2Sa) or indirectly by altering the burning area fluctuations (route $2 \mathrm{Sb}$ ). It is very important to note here 
that stretch rate oscillations are indirectly caused by equivalence ratio oscillations; if the latter did not exist, there would be no perturbation of the flame front location, and hence no oscillations in the stretch rate of the flame. This hence leads to a delicate coupling between equivalence ratio perturbations and flame stretch effects.

This section generalizes prior work by addressing high frequency characteristics of the flame response. We show that two processes grow in importance at higher frequency. The first is non quasi-steady response of the internal flame structure. Non quasi-steadiness here refers to "flame structural" non quasi-steadiness, i.e., a scenario when the period of oscillations in the mixture composition are comparable to the characteristic diffusion time in the preheat zone of the flame, characterized by a Strouhal number $S t_{\delta}$ This is different from "geometric" non quasi-steadiness, which occurs when $S t \sim O(1)$, where the period of oscillations in the mixture composition are comparable to the characteristic response time of the entire flame. Secondly, flame stretch effects also grow in significance with increasing frequency. The oscillating flame speed creates flame wrinkles, whose radius of curvature scales roughly as $1 / S t^{2}$. This causes the flame speed to be modulated not only directly by the fuel/air ratio oscillation, but also indirectly by the stretch effects associated with flame wrinkling due to the flame speed oscillations, analogous to the two additional routes it provides for velocity-coupled flame response.

\section{Formulation and Analysis}

\section{Basic Outline}

Once again the $G$-equation will be utilized along with a unique form of the flame front tracking equation (assuming axisymmetry):

$$
\frac{\partial \xi}{\partial t}+s_{L}\left(1+\left(\frac{\partial \xi}{\partial r}\right)^{2}\right)^{1 / 2}=u-v\left(\frac{\partial \xi}{\partial r}\right)
$$

Introducing the non-dimensionalization scheme: $r^{*}=r / R, z^{*}=z / L_{f}$ and $t^{*}=t U_{o} / L_{f}$ with $R$ being chosen to be an appropriate flame holder length scale, equation (133) is written as:

$$
\frac{\partial \xi}{\partial t}+\left(\frac{s_{L}}{s_{L_{o}}}\right)\left(\frac{s_{L_{o}}}{u_{o}}\right)\left(1+\beta^{2}\left(\frac{\partial \xi}{\partial r}\right)^{2}\right)^{1 / 2}=\frac{u}{u_{o}}-\frac{v}{u_{o}} \beta\left(\frac{\partial \xi}{\partial r}\right)
$$

where the asterisks have been dropped for convenience of notation. The subscript ' $o$ ' denotes the value of the respective quantity evaluated at the mean equivalence ratio, $\phi_{o}$, or the mean value as in the case of flow velocity. Here, the ratio $s_{L o} / u_{o}$ may be expressed in terms of the slope of the mean flame as:

$$
\frac{s_{L o}}{u_{o}}=\left(1+\beta^{2}\left(\frac{\partial \xi_{o}}{\partial r}\right)^{2}\right)^{1 / 2}
$$


The ratio of the instantaneous flame speed to mean flame speed, $s_{L} / s_{L o}$ is influenced by equivalence ratio perturbations and stretch rate of the flame. In the linear regime, we may write $s_{L} / s_{L o}$ mathematically as:

$$
\frac{s_{L}}{s_{L o}}=1+\left.\frac{\partial\left(s_{L} / s_{L o}\right)}{\partial\left(\phi / \phi_{o}\right)}\right|_{o} \frac{\phi^{\prime}}{\phi_{o}}+\left.\frac{\partial\left(s_{L} / s_{L o}\right)}{\partial \kappa}\right|_{o} \kappa^{\prime}
$$

where $\kappa^{\prime}$ is the stretch rate non-dimensionalized by the inverse of the burner radius, $(1 / R)$. The coefficients of the perturbation terms on the RHS of Eq.(136) are the sensitivities of flame speed to perturbations in equivalence ratio and stretch rate respectively. The latter sensitivity may also be expressed as:

$$
\left.\frac{\partial\left(s_{L} / s_{L o}\right)}{\partial \kappa^{\prime}}\right|_{o}=-M a \frac{\delta}{R}
$$

Where $M a$ is the Markstein number as it was previously. Equation (137) is solved by prescribing the characteristics of the disturbance field. The analysis neglects the density jump across the flame, which changes the character of the approach flow field. This assumption is well understood as a necessary approximation to making analytic progress in velocity-coupled analyses, but is also required here. Even if there are no velocity perturbations imposed upon the flame, the oscillating fuel/air ratio disturbance generates wrinkles. These wrinkles will necessarily excite velocity disturbances, which are not accounted for.

The instantaneous heat release of the flame is given by Eq.(1). For fuel/air ratio perturbation occurring at constant density, Eq.(1) can be rewritten in terms of burning velocity magnitude and heat of reaction perturbations as:

$$
\frac{q(t)}{q_{o}}=\frac{A(t)}{A_{o}}+\int_{\text {flame }} \frac{s_{L}{ }^{\prime}}{s_{L_{o}}} \frac{d A}{A_{o}}+\int_{\text {flame }} \frac{h_{R}{ }^{\prime}}{h_{R_{o}}} \frac{d A}{A_{o}}+\int_{\text {flame }} \frac{s_{L}{ }^{\prime} h_{R}{ }^{\prime}}{s_{L_{o}} h_{R_{o}}} \frac{d A}{A_{o}}
$$

The first term on the RHS denotes the contribution to heat release fluctuation due to oscillations in the net burning area of the flame. The second and third terms represent the contribution to heat release fluctuation due to burning velocity and heat of reaction oscillations, respectively. The fourth term represents the nonlinear coupling between flame speed and heat of reaction oscillations. This term is disregard in linear analyses, but has to be retained in nonlinear analyses. These terms can in fact be evaluated in terms of the flame surface geometry, e.g., the first term may be evaluated for an axi-symmetric conical flame as:

$$
\frac{A(t)}{A_{o}}=\frac{2}{\left(1+\beta^{2}\right)^{1 / 2}} \int_{0}^{1} r\left(1+\beta^{2}\left(\frac{\partial \xi}{\partial r}\right)^{2}\right)^{1 / 2} d r
$$


The heat-release transfer function of the flame due to equivalence ratio fluctuations can then be defined as:

$$
F=\frac{q^{\prime}(\omega) / q_{o}}{\hat{\phi}^{\prime} / \phi_{o}}
$$

where the numerator and denominator are the heat release and equivalence ratio perturbations, evaluated at the excitation frequency. In the quasi-steady case, the flame speed and heat of reaction terms are functions of fuel/air ratio, stretch rate, fuel type, and operating condition. In the general unsteady case, however, these quantities, particularly the flame speed, introduce additional dynamics related to the flame structure so that the instantaneous flame speed is also a function of frequency. The former study presents a model for a flat flame, describing these unsteady effects, and shows that the quasi-steady assumption is valid when $f \ll \delta / s_{L}$. They show that the non quasi-steady flame response is equal to the quasi-steady response of a flame that is excited by an equivalent mixture fraction oscillation that is obtained by spatially averaging the mixture fraction over the preheat zone thickness at each instant in time. This "effective equivalence ratio oscillation" is decreased in magnitude and shifted in phase from that of instantaneous fuel/air ratio oscillation just upstream of the preheat zone. Thereafter, modeling the non quasi-steady flame dynamics closely follows the quasi-steady modeling approach.

\section{Perturbation Analysis}

Now we present the development of the third order perturbation solution for the evolution of the flame surface. For the sake of illustration, we consider an axi-symmetric conical flame stabilized on a burner tube as shown schematically in the bottom left image of Figure 1. The velocity field was prescribed to be uniform in the axial direction; continuity equation then yields a zero radial velocity. With this assumption, equation (135) becomes:

$$
\frac{\partial \xi}{\partial t}+\frac{s_{L}}{s_{L o}} \frac{1}{\left(1+\beta^{2}\right)^{1 / 2}}\left(1+\beta^{2}\left(\frac{\partial \xi}{\partial r}\right)^{2}\right)^{1 / 2}=1
$$

Furthermore, the upstream equivalence ratio perturbations are assumed to be radially uniform, harmonically oscillating, and are advected by the mean flow:

$$
\left.\left.\phi=\phi_{o} \mid 1+\varepsilon \cos \left(\omega\left(\frac{z}{U_{0}}-t\right)\right)\right]\right\rfloor
$$

Employing the non-dimensional scheme described previously in the report, Eq.(142) may be written in dimensionless form as:

$$
\phi=\phi_{o}[1+\varepsilon \cos (S t(z-t))]
$$


Attenuation of the fuel/air ratio perturbation along the flame in the axial direction by diffusion is assumed negligible, an approximation that is reasonable if $s_{t} \cdot \frac{\omega D}{U_{0}^{2}} \ll 1$. The origin of co-ordinates is chosen to be the center of the tube exit plane. The flame is assumed to be attached at the burner. This yields the following boundary condition for $\xi$ :

$$
\left.\xi(t)\right|_{\text {flame-holder }}=0
$$

In non-dimensional form, Eq.(144) becomes:

$$
\xi(1, t)=0
$$

where the flame holder length scale parameter, $R$, is chosen to be the burner tube radius.

\section{Quasi-steady Analysis of an Unstretched Flame}

For the case of a quasi-steady flame that is not influenced by stretch, the sensitivity of flame speed to curvature is zero. We expand the flame front position function $\xi(r, t)$ linearly in terms of the parameter $\varepsilon$ as:

$$
\xi(r, t)=\xi_{0}(r)+\varepsilon \xi_{1}(r, t)+O\left(\varepsilon^{2}\right)
$$

Using the above in Eq.(135) and Eq.(145) and collecting terms of the same order in $\varepsilon$ yields the following:

$$
\begin{gathered}
\xi_{o}(r)=1-r \\
\frac{\partial \xi_{1}}{\partial t}-\alpha \frac{\partial \xi_{1}}{\partial r}+s_{L 1} \cos (S t(1-r-t))=0
\end{gathered}
$$

The parameter $\alpha$ is given by the expression, $\alpha=\beta^{2} /\left(1+\beta^{2}\right)$ and,

$$
s_{L 1}=\left.\frac{\partial\left(s_{L} / s_{L o}\right)}{\partial\left(\phi / \phi_{0}\right)}\right|_{\left(\phi^{\prime} / \phi_{0}\right)=0} \quad h_{R 1}=\left.\frac{\partial\left(h_{R} / h_{R 0}\right)}{\partial\left(\phi / \phi_{0}\right)}\right|_{\left(\phi^{\prime} / \phi_{0}\right)=0}
$$

are the linear sensitivities of the burning velocity magnitude and heat of reaction of the reactant mixture respectively, to fluctuations in equivalence ratio. For cases where the flame does not respond in a quasi-steady manner, these sensitivity derivatives are also functions of frequency. Within the linear approximation, the character of the solution does not change since the $s_{L 1}$ and $h_{R l}$ terms are now functions of mean fuel/air ratio and frequency. In the non-linear case, things are more complex and new terms arise which are not present in the above expressions. As such, the nonlinear corrections in the perturbation analysis implicitly assume quasi-steady sensitivities of flame speed and heat of reaction. 
Equation (148) can be solved to yield expressions for $\xi_{1}(r, t)$. We present below the solution for $\xi_{1}(r, t)$ :

$$
\xi_{1}(r, t)=\frac{s_{L 1}}{(1-\alpha) S t}[\sin \{S t(1-r-t)\}-\sin \{(S t / \alpha)(1-r-\alpha t)\}]
$$

This solution explicitly contains two contributions to the linear dynamics of the flame surface evolution. The first term within the brackets represents the effect of local non-uniformities in the burning velocity due to the spatial and temporal oscillations in equivalence ratio. The second term arises because of the boundary condition, i.e., Eq.(145), that the flame does not move at the burner lip, even though its flame speed is oscillating. In physical terms, Eq.(150) shows that the flame front position is controlled by two sets of waves that travel along the front - (i) waves generated at each point along the flame due to spatial variations in flame speed and (ii) waves generated at the flame attachment point due to the boundary condition, Eq.(145). Notice that the propagation velocities of these two waves along the flame surface are different. The former travels with the mean flow velocity (unity in the nondimensional case) and the latter with a nondimensional velocity $1 / \alpha$ along the axis of the flame. Thus, these two waves interfere constructively at some flame surface locations and destructively at others. This has a significant influence on the characteristics of the heat release transfer function of the flame.

This is similar to the results obtained in the previous analysis which emphasized these superposition effects upon the dynamics of flames subjected to excitation in flow velocity. The corresponding expressions for the nonlinear corrections to the flame position, $\xi_{2}$ and $\xi_{3}$ are presented later.

We next consider the transfer function. The transfer function in Eq.(140) can be decomposed in a manner similar to that of heat release in Eq.(138). In the linear limit, i.e., for very small excitation amplitudes, the transfer function $F_{\text {o }}$ can be written as a sum of three contributions, due to flame area oscillation, burning velocity oscillations and heat of reaction oscillations, which may be written as follows:

$$
\begin{gathered}
F_{o, s_{L}}=s_{L_{1}}\left\{\frac{2}{S t^{2}}(1+i S t-\exp (i S t))\right\} \\
F_{o, h_{R}}=h_{R_{1}}\left\{\frac{2}{S t^{2}}(1+i S t-\exp (i S t))\right\} \\
F_{o, A}=s_{L_{1}}\left\{\frac{2 \alpha}{1-\alpha}\left(\frac{1-\alpha-\exp (i S t)+\alpha \exp (i S t / \alpha)}{S t^{2}}\right)\right\}
\end{gathered}
$$




\section{Quasi-Steady Analysis Under the Influence of Flame Stretch}

We next account for the effects of flame stretch, which provides an additional kinematic route towards flame relaxation to high frequency disturbances. As described in Figure 34, flame stretch affects flame response by altering the flame speed directly, and therefore altering the flame burning area. A linear perturbation analysis in $\varepsilon$ is performed to evaluate the effect of flame stretch on the linear transfer function. Nonlinear effects due to stretch are neglected owing to analytical tediousness; no additional physical insight is expected. An expression can be written for the non-dimensional stretch rate of the flame due to curvature, in terms of the flame front location as follows:

$$
\kappa^{\prime}=\beta \frac{\partial^{2} \xi / \partial r^{2}}{\left(1+\beta^{2}(\partial \xi / \partial r)^{2}\right)^{3 / 2}}+\beta \frac{1}{r} \frac{\partial \xi / \partial r}{\left(1+\beta^{2}(\partial \xi / \partial r)^{2}\right)^{1 / 2}}
$$

The two terms on the RHS of Eq.(154) account for axial and azimuthal curvature respectively. The absence of an imposed velocity perturbation leads to the absence of flame stretch due to hydrodynamic strain. Equations (154) and (136) are used in Eq.(141) along with the anchor-fixed boundary condition of Eq.(145) and an additional boundary condition preserving the symmetry of the slope of the conical flame at the flame tip, i.e.:

$$
\frac{\partial \xi}{\partial r}(0, t)=0
$$

to solve for the flame location. Thereafter the transfer function may be obtained as discussed in the previous section. While it is not possible to obtain closed form solutions for this case due to the nature of the azimuthal stretch term, it may be shown by numerical calculations of the flame position and from a simple scaling analysis that the influence of azimuthal stretch is insignificant compared to axial stretch at everywhere along the flame but at the tip, and that the relative influence reduces as Strouhal number increases. Furthermore, since flame stretch effects are inherently high frequency phenomena, azimuthal stretch may be altogether neglected, to achieve analytical progress.

Accounting for axial stretch alone, for very weak curvature $(M a \rightarrow 0)$, the effect of curvature may be expressed as a correction to the linear transfer function contributions due to flame speed perturbations and area perturbations as follows:

$$
\begin{gathered}
F_{s L, c}=F_{o, s L}+\sigma_{c} S t\left\lceil\frac{2 s_{L 1}}{\left\lfloor(1-\alpha) S t^{2}\right.}\left(i \alpha\left(e^{i S t}-e^{i S t / \alpha}\right)-(1-\alpha) S t\right)\right\rfloor+O\left(\sigma_{c}^{2}\right) \\
F_{A, c}=F_{o, A}+\sigma_{c} S t\left\lceil\frac{2 i s_{L 1}}{(1-\alpha)^{2} S t^{2}}\left((1-\alpha)^{2}+\alpha e^{i S t}-\left(1-\alpha+\alpha^{2}\right) e^{i S t / \alpha}\right)\right\rfloor+O\left(\sigma_{c}^{2}\right)
\end{gathered}
$$


Here, $\sigma_{c}$ is a scaled Markstein number defined by:

$$
\sigma_{c}=M a \frac{\delta}{R} \frac{\beta}{\left(1+\beta^{2}\right)^{3 / 2}}
$$

As such, curvature effects can be seen to become important when

$$
\sigma_{c} S t \sim 1
$$

Finally, it is important to note that, in the linear regime (linear in excitation amplitude), flame stretch does not affect the heat of reaction route to heat release oscillations. Hence, the total response of the flame under the influence of stretch may be expressed as:

$$
F=F_{o, h_{R}}+F_{s L, c}+F_{A, c}
$$

\section{Non-Quasi-Steady Analysis}

We next account for non quasi-steady effects related to the time lagged response of the flame structure to equivalence ratio disturbances imposed ahead of the preheat zone. It can be shown that in the linear approximation, for an instantaneous equivalence ratio oscillation given by Eq.(142), the flame heat of reaction $\left(h_{R}\right)$ and burning rate $\left(s_{L}\right)$ responds to an equivalent equivalence ratio whose instantaneous value in dimensionless form is:

$$
\phi^{*}(z, t)=\phi_{o}\left[1+\varepsilon \sin \mathrm{c}\left(\frac{S t_{\delta}}{2}\right) \cos \left(\frac{S t_{\delta}}{2}+S t(z-t)\right)\right\rfloor
$$

where $\operatorname{sinc}(x)=\sin (x) / x$. Here, a second Strouhal number, $s_{\delta}$ is defined based on the flame thickness and flame speed as $s t_{\delta}=\omega \delta / s_{L}$. This is simply the ratio of a characteristic diffusion time in the preheat zone of the flame $\left(\tau_{\text {diff }}=\delta / s_{L}\right)$ to the characteristic time associated with mixture composition fluctuations $\left(2 \pi / \omega \sim_{\tau_{e q}}\right)$. As such, we may relate $s t_{\delta}$ and $s t$ as:

$$
\frac{S t}{S t_{\delta}}=\frac{\alpha^{1 / 2}}{(\delta / R)}
$$

On accounting for non quasi-steady effects in such a manner, the flame speed and heat of reaction sensitivities are diminished and phase shifted by a non quasi-steady scaling factor and may be expressed as:

$$
\begin{aligned}
& s_{L 1}{ }^{n q s}=s_{L 1} \operatorname{sinc}\left(S t_{\delta} / 2\right) \exp \left(-i S t_{\delta} / 2\right) \\
& h_{R 1}{ }^{n q s}=h_{R 1} \operatorname{sinc}\left(S t_{\delta} / 2\right) \exp \left(-i S t_{\delta} / 2\right)
\end{aligned}
$$


It may also be seen from Eq.(163) and (164), that, in the quasi-steady limit of $s t_{\delta} \rightarrow 0$, the quasisteady sensitivities are retrieved. Finally, the non quasi-steady transfer function, $F^{n q s}$, is a relatively simple modification of the quasi-steady transfer function, which may be expressed as:

$$
F^{n q s}=g\left(S t_{\delta}\right) F^{q s}(S t)
$$

where the correction factor accounting for non quasi-steady phenomena is given by:

$$
g\left(S t_{\delta}\right)=\operatorname{sinc}\left(S t_{\delta} / 2\right) \exp \left(-i S t_{\delta} / 2\right)
$$

By definition, non quasi-steady effects become important when $\tau_{d i f f} \tau_{e q}$, which may be written in terms of $S t_{\delta}$ as:

$$
S t_{\delta} \sim 1
$$

Finally, it is important to note that, though non quasi-steady effects and flame stretch effects are both phenomena that become important at higher frequencies, their influence on the global heat release of the flame is very different. While non quasi-steadiness seems to affect all the three routes, viz., heat of reaction oscillations, flame speed oscillations and burning area oscillations identically, flame stretch affects only the latter two routes; in fact, these two routes are affected differently due to flame stretch.

\section{Results and Discussion}

Here we present explicit results for a conical flame with aspect ratio, $\beta=4.0$. The investigated geometry is shown schematically in Figure 1. The following correlations for the burning velocity magnitude and heat of reaction for a methane-air flame at STP were assumed:

$$
\begin{gathered}
s_{L}(\phi)=A \phi^{B} \exp \left(-C(\phi-D)^{2}\right) ; \quad A=0.6079, B=-2.554, C=7.31, D=1.230 \\
h_{R}(\phi)=\frac{2.9125 \times 10^{6} \mathrm{~min}(1, \phi)}{1+0.05825 \phi}
\end{gathered}
$$

\section{Linear Quasi-Steady Dynamics}

We begin with a brief discussion of the characteristics of the linear transfer function. We focus the discussion here primarily on the manner in which the rich flame results are different from those of lean flames. In order to make this comparison, results are presented for two mean equivalence ratios, $\phi_{o}=0.85$ and $\phi_{o}=1.28$, which correspond to conditions where the flame speeds are identical, $s_{L} \sim 33 \mathrm{~cm} / \mathrm{s}$. 


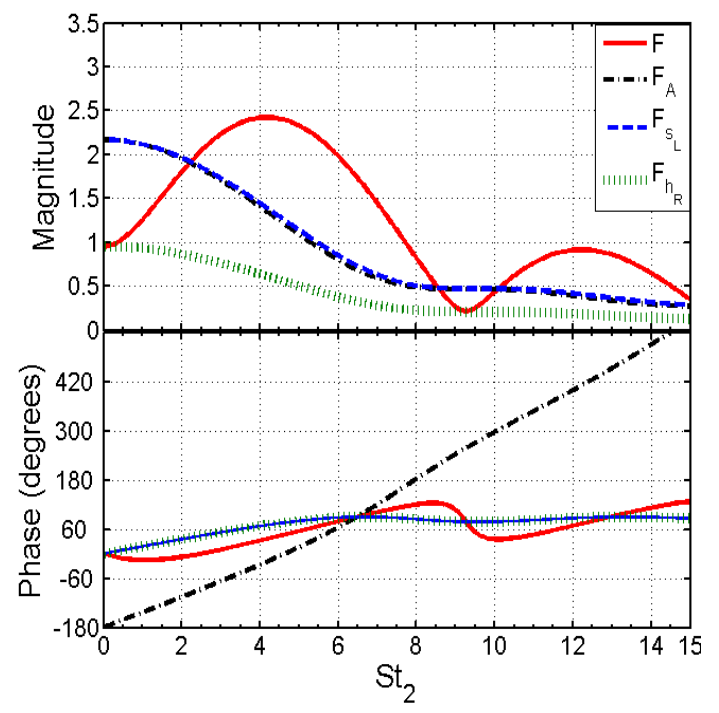

(a)

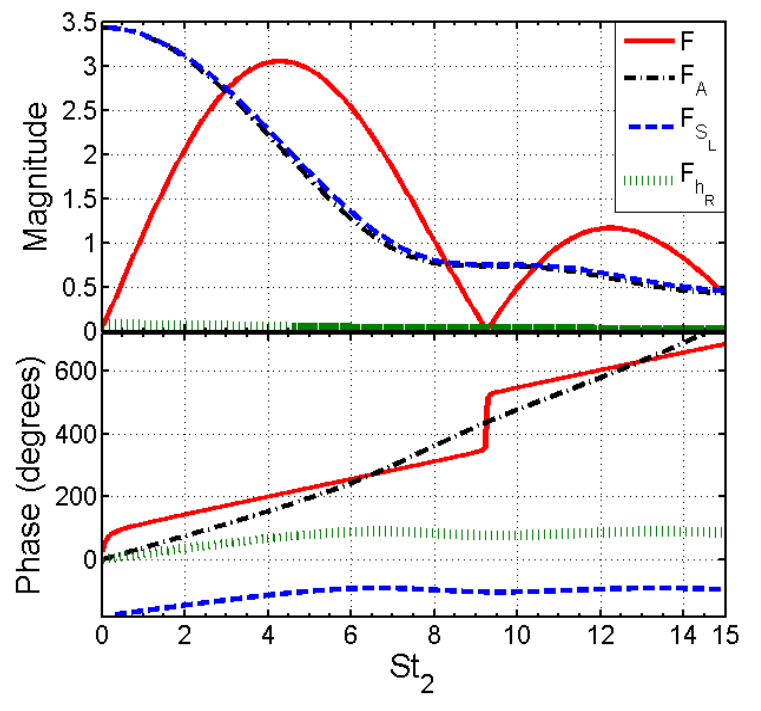

(b)

Figure 35: Linear transfer function for (a) $\phi_{o}=0.85$ (lean) (b) $\phi_{o}=1.28$ (rich), $\beta=4$.

Figure 35 shows the variation of the phase and magnitude of the linear transfer function with $S t_{2}=S t / \alpha$ for the two equivalence ratios. Also shown are the phase and magnitude of the individual contributions to the total transfer function. First, note that both the phase and the magnitude do not monotonically vary with $S t_{2}$. This is due to the fact that the linear flame response is determined by the net superposition of a boundary generated "wave" and a local disturbance, as discussed in the previous section. Therefore the net flame response depends on exactly how these waves superpose at different Strouhal numbers. It must also be noted from equation (151) and equation (152) that the phase of the flame speed and the heat of reaction contributions are identical in the linear limit. The linear transfer function for the rich case is shown in Figure 35(b). Notice that the transfer function goes to a near zero value, given by the heat of reaction sensitivity $\left(h_{R 1}\right)$, at low values of $S t_{2}$. This is in striking contrast to the corresponding lean case and is due to the fact that the heat of reaction is a nearly constant function of equivalence ratio in the rich regime. This means that in the linear regime, the heat release of a rich flame is relatively insensitive to perturbations in equivalence ratio at low values of $\mathrm{St}_{2}$.

Another difference between the two transfer functions is the presence of a zero response in the rich case, e.g. at $S t_{2} \sim 8.7$. At this point, the oscillating flame speed and area oscillation response exactly cancel each other. In the lean case, however, the node is not present. This is due to the fact that the lean case has an additional contribution to the total transfer function, viz., the heat of reaction oscillations. Therefore, in general, a zero response will not occur in the lean flame response because it consists of a superposition of three terms, whereas the rich flame has only two major contributors. However, it must be noted that these characteristics are strong functions of the sensitivities of flame speed and heat of reaction to equivalence ratio. To understand this, consider flame responses at different equivalence ratio as plotted in Figure 36. 


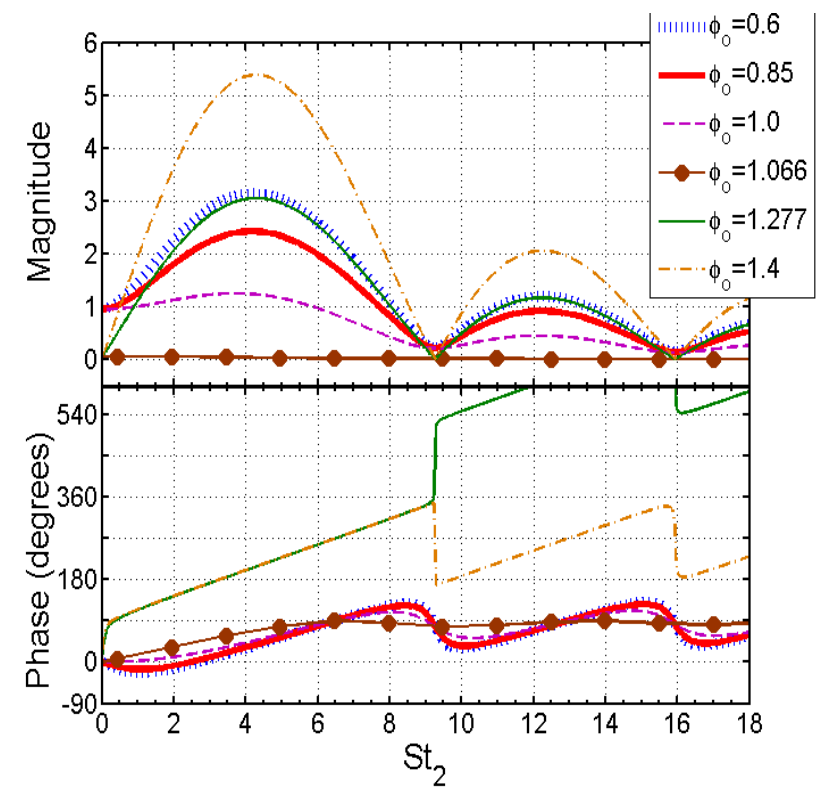

Figure 36: Variation of the linear transfer function with $S t_{2}$ for different values of equivalence ratio. $\beta=4$.

Figure 36 overlays the flame response over a range of fuel/air ratios. Note, first, that the lean cases all start with a gain of nearly unity and the rich cases with a gain of nearly zero at low Strouhal numbers. This is due to the fact that the flame response is entirely controlled by the heat of reaction sensitivity $h_{R I}$ in the quasi-steady case. All transfer functions then initially grow with increasing Strouhal number, because the burning area and the fluctuating flame speed terms progressively come into phase with each other.

As the mean equivalence ratio is increased from an initial lean value, e.g. $\phi=0.6$, the $s_{L}$ and $h_{R}$ sensitivities progressively decrease and stay nearly constant, respectively, until $\phi \sim 1.06$ where the $s_{L}$ and $h_{R}$ sensitivities vanish. Hence, the magnitude of the flame response drops to a nearly zero response at $\phi \sim 1.06$. This is due to the occurrence of the flame speed maximum at this equivalence ratio. The heat of reaction is a very weak function of equivalence ratio for $\phi_{0}>1.0$. Hence, we see, as before, that the magnitude now increases from a nearly zero value with increasing $S t_{2}$ for rich mean equivalence ratios.

Even though the flame speeds at $\phi_{o}=0.85$ and $\phi_{0}=1.28$ are identical, the magnitude of the maximum gain is higher in the rich case due to the higher $s_{L}$ sensitivity at $\phi_{o}=1.28$. Also to be noted is that the heat release response lags the excitation in the lean case, and leads it in the rich case. This again is due to the fact that the linear $s_{L}$ sensitivity, $s_{L 1}$, changes sign from positive to negative when $\phi_{o}>1.066$. This may be understood physically from the fact that the burning area response is due to $s_{L}$ fluctuations that, in turn, are induced by equivalence ratio oscillations. The change in sign of $s_{L 1}$ implies that an instantaneous increase in equivalence ratio results in an increase and decrease in the instantaneous value of $s_{L}$ on the lean and rich side, respectively. Therefore, given the same instantaneous equivalence ratio perturbation, the corresponding instantaneous burning area decreases for a lean mean equivalence ratio and increases for a rich mean equivalence ratio. 


\section{Linear Non-Quasi-Steady Dynamics}

As the frequency increases, the characteristic time associated with the fluctuations in equivalence ratio, $\tau_{c q}$, decreases. As $\tau_{c q}$ approaches the characteristic time associated with diffusion processes in the preheat zone of the flame, $\tau_{d i f f}$, the response of the flame reaction zone to upstream fluctuations in equivalence ratio fails to remain quasi-steady, though remaining linear. Non quasi-steady effects can be modeled by considering the flame response to an effective equivalence ratio given by Eq.(161). It may be recalled that the excitation amplitude of the effective equivalence ratio is smaller than the actual excitation amplitude. This implies that the linear analyses would be valid for a broader range of amplitudes than the quasi-steady case. The effective equivalence ratio also possesses a phase lag that accounts for finite time taken for the passage of the mixture composition oscillations through the flame preheat zone. This manifests itself in the phase of the flame response, Eq.(165) and Eq.(166).

We next consider actual results of the non quasi-steady response of the flame. Figure 37(a) plots the magnitude and phase of the linear total transfer function for quasi-steady and non quasisteady flame response. It may be seen that, as Strouhal number increases, there is a marked departure of the non quasi-steady response from the quasi-steady result. At moderate frequencies, corresponding to $s_{t} \sim 60$, the response is significantly attenuated, while the quasisteady response is non-zero. In fact, it may be observed from Figure 37(b), that at $s t_{s} \sim \pi$, there is already about $40 \%$ attenuation in the gain, and 90 degrees difference in phase with respect to the quasi-steady response.

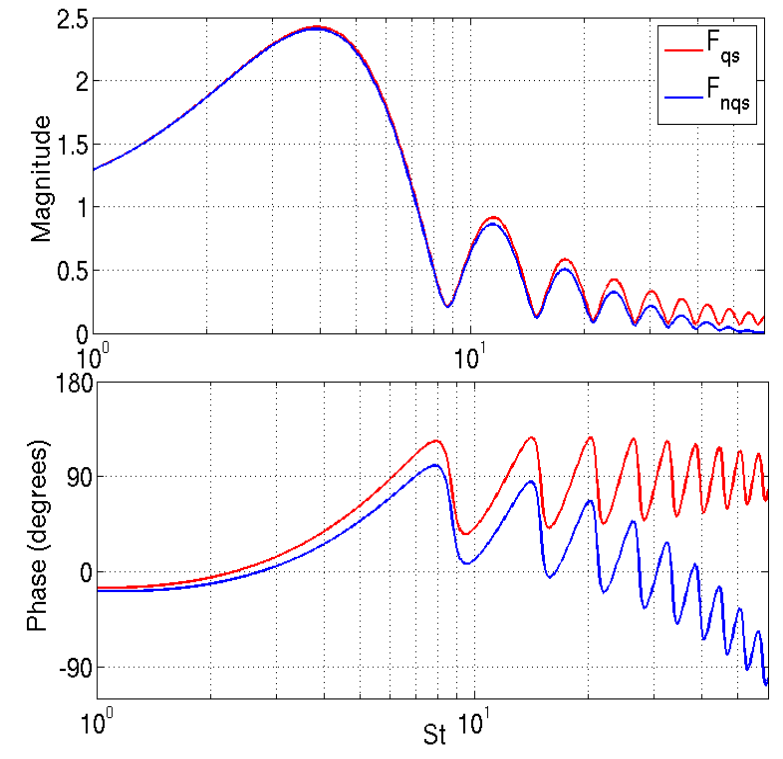

(a)

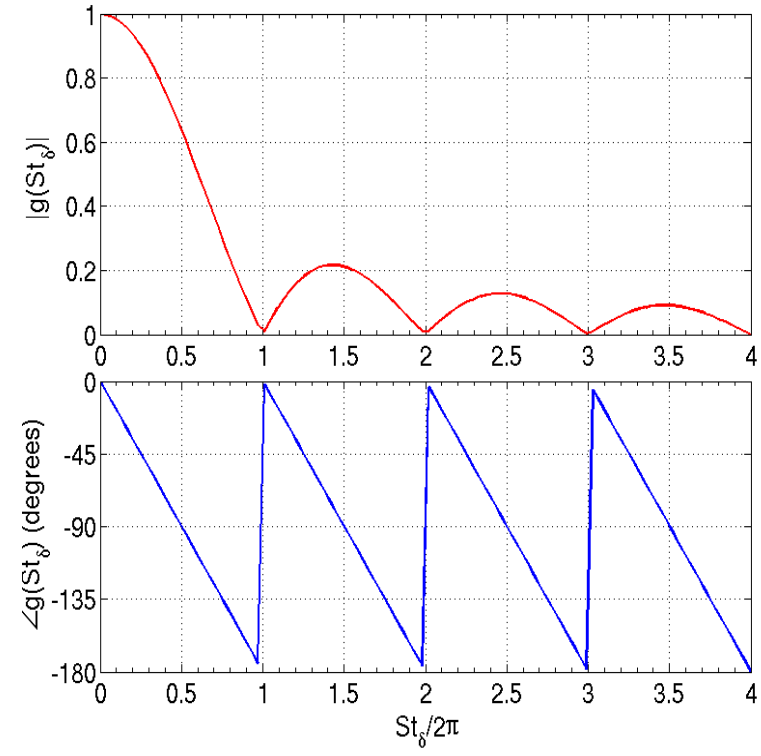

(b)

Figure 37: (a) Non quasi-steady versus quasi-steady flame response for $\phi_{o}=0.85, \beta=4, \delta=$ $0.1 R$ (b) Non quasi-steady correction factor 
To give a feel for typical numbers, let us consider a methane-air reactant mixture at $30 \mathrm{~m} / \mathrm{s}$, establishing a flame that is $10 \mathrm{~cm}$ tall. At 1atm, a typical estimate of the preheat zone thickness would be $1 \mathrm{~mm}$, while the reaction zone thickness would be about $0.1 \mathrm{~mm}$. Thus, at $1 \mathrm{~atm}$, the flame preheat zone diffusive processes become non quasi-steady at a frequency $f \sim 400 \mathrm{~Hz}$. At 10 atm, the preheat zone becomes non quasi-steady at $f \sim 4 \mathrm{kHz}$. Thus, we see that for typical combustion dynamics applications, we are most concerned with this locally non quasi-steady response of the flame, which, for typical flames would occur at frequencies of about $f \sim 400 \mathrm{~Hz}$.

\section{Effect of Flame Stretch}

We next present results that quantify the effects of flame stretch on the quasi-steady response of the flame. The effects of flame stretch in the case of premixed flames perturbed by velocity fluctuations have been studied in considerable detail in recent literature. We begin by investigating the effect of flame stretch on the contributions due to flame speed and burning area perturbations to the overall heat release for a lean flame $\left(\phi_{o}=0.85\right)$. Similar trends are seen in rich flames.

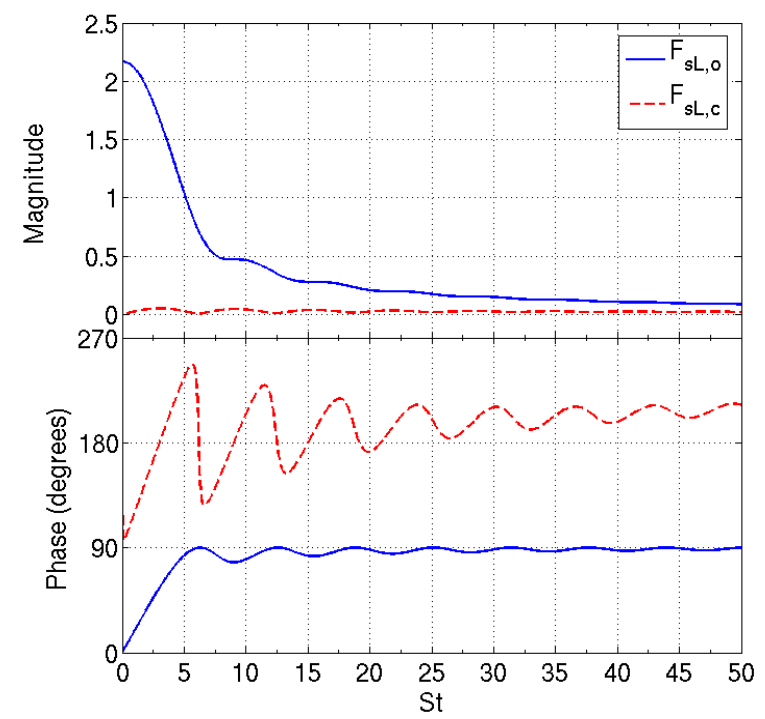

(a)

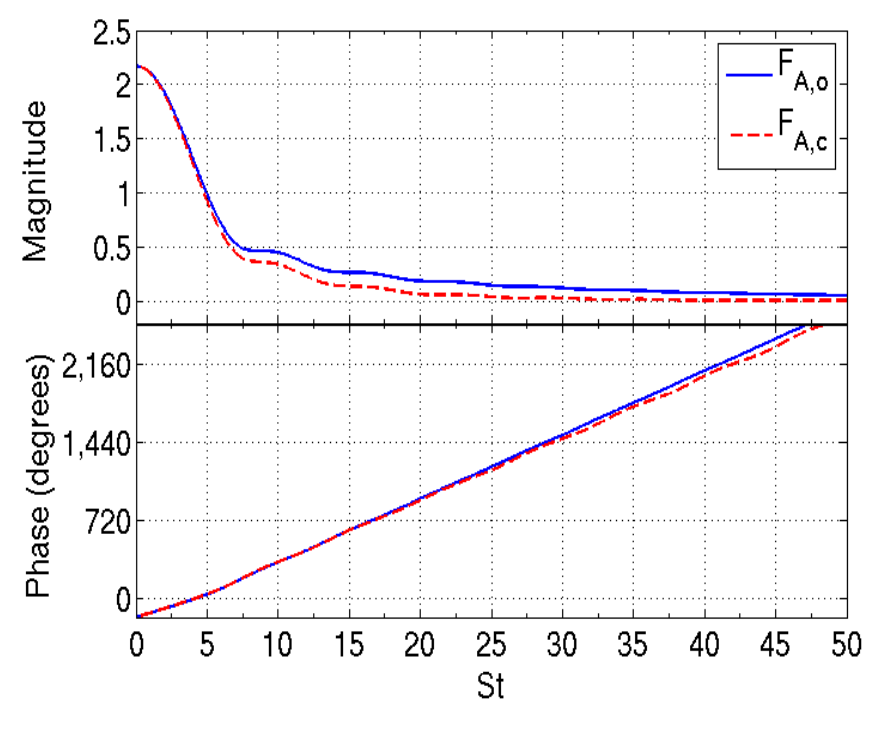

(b)

Figure 38: Effect of Flame Stretch on a lean $\mathrm{CH}_{4} /$ air flame, $\phi_{0}=0.85, \beta=4, \delta=0.1 R, M a=1-(\mathrm{a})$ Effect on Flame speed contribution to heat release. (b) Effect on burning area contribution to heat release

Figure 38(a) plots the flame speed contribution of the overall flame response for an unstretched flame (blue curve) and the stretch correction to this contribution (red curve). Hence, the total stretch corrected transfer function would be the sum of the two. As is clearly seen, this stretch correction seems to be almost negligible in comparison to the linear transfer function for the unstretched flame. A similar conclusion may be reached from Figure 38(b), which plots the contribution of burning area response with and without stretch (solid blue and dashed red curves 
respectively). From these trends, it seems reasonable to assume that the effect of flame stretch on the overall response of the flame would be more subtle, than non quasi-steady effects.

To understand the impact of flame stretch on the linear transfer function and its relative significance with respect to non quasi-steady effects, we next plot the total linear response of the flame with and without stretch and with non quasi-steadiness.

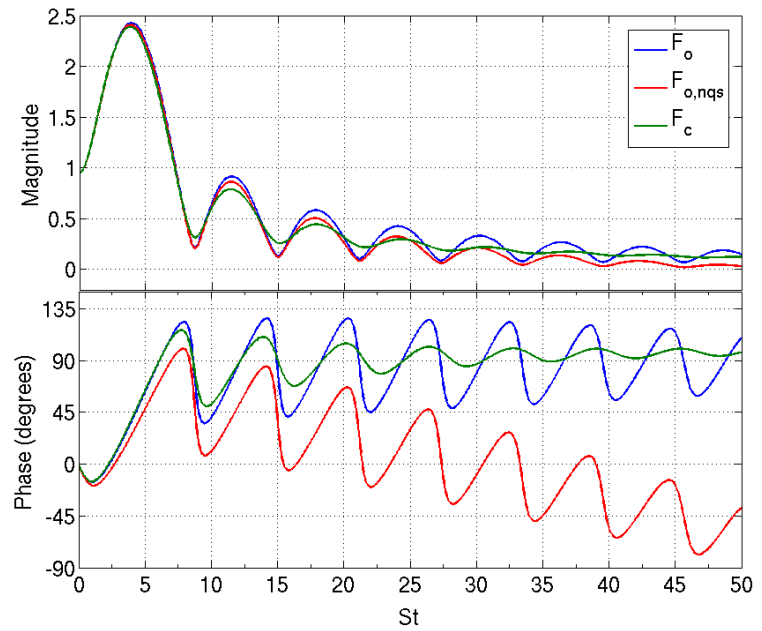

(a)

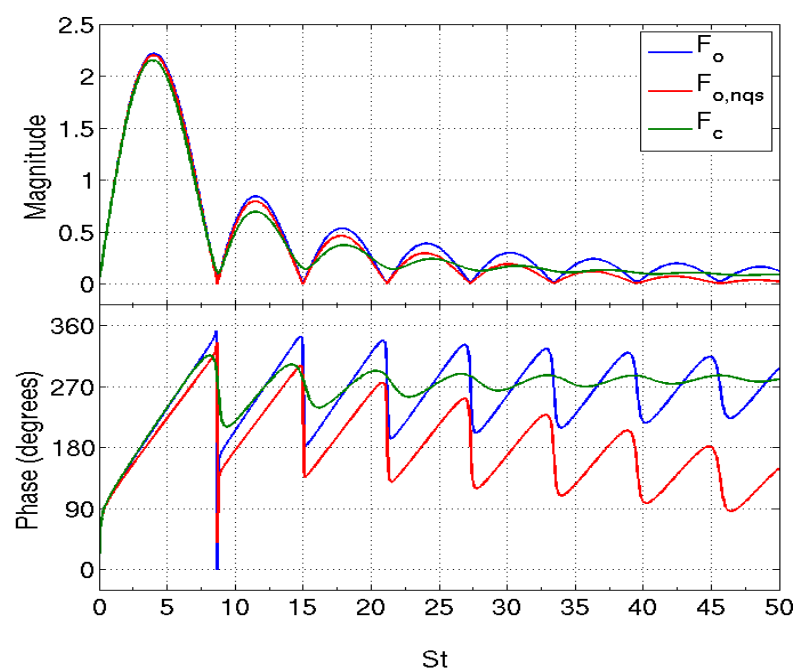

(b)

Figure 39: Comparison between unstretched, quasi-steady (solid blue), stretched, quasisteady (solid green) and unstretched, non quasi-steady (solid red) global heat release responses of a conical $\mathrm{CH}_{4} /$ air flame., $\square=4.0, \square / R=0.1, \mathrm{Ma}=1:$ (a) $\square_{0}=0.85$, (b) $\square_{0}=1.28$

Figure 39 plots the global heat release response for stretched and unstretched quasi-steady and non quasi-steady flames for lean and rich stoichiometries. It may be seen from the Figure 39 that though flame stretch and non quasi-steady effects become independently important at similar frequencies, flame stretch only smoothens out the linear unstretched transfer function, while retaining its qualitative character. However, non quasi-steady effects seem to have a larger influence than flame stretch effects, in that they scale the transfer function very differently. More specifically, while the effect of flame stretch can be envisaged in terms of a stretch correction to the unstretched flame, non quasi-steady effects "scale" the excitation amplitude that the flame responds to. The phase characteristics of these responses may be understood based on similar arguments. While flame stretch perturbs the phase about the phase of the unstretched flame, non quasi-steady effects alter the phase characteristics completely, due to incorporating the finite diffusion time in the preheat zone in the expression for the effective equivalence ratio as a phase term.

It hence appears that the non quasi-steadiness of the flame structure has far outreaching effects on the overall response of the flame, while flame stretch seems to merely add an extra layer of physics to obtain better fidelity. It is very important to recall that these transfer functions are linear transfer functions, and hence, effects arising from flame kinematic restoration and other nonlinearities have not been accounted for. 


\section{Review}

The key conclusions of the work presented in this section are the following. First, the response of rich flames to fuel/air ratio oscillations fundamentally differs from that of lean flames. This is due to the difference between the heat of reaction and flame speed sensitivities on the rich and lean sides. Second, the non quasi-steady response of premixed flames to fuel/air ratio oscillations introduces a gain scaling factor and phase shift. Third, it appears that flame stretch effects are also present at higher Strouhal numbers, and need to be accounted for. However, there are no major qualitative differences between the response of stretched flame and that of an unstretched flame, and stretch serves only to smoothen out flame wrinkles without any major contribution to the transfer function gain.

\section{Response of Premixed Flames to High Frequency Acoustic Perturbations}

\section{Introduction}

Recall Eq.(138) which shows four fundamentally different mechanisms generating heat-release disturbances in a premixed flame, viz., fluctuations in reactant density, flame speed, heat of reaction, or flame surface area. Rather than considering these four processes directly, our discussion below will focus on the perturbation that excites them - pressure, velocity, or fuel/air ratio. In general, all three perturbations are related and co-exist, but in the discussion that follows, we consider how the flame responds to each in isolation. In particular, we will focus on how these mechanisms are altered as the frequency of perturbation grows.

Having completed the equivalence ratio perturbations we now start by considering the velocity coupled mechanism, where the flame is perturbed by acoustic and/or vortical perturbations. The figure below illustrates this mechanism, showing that velocity perturbations wrinkle the flame, causing oscillations in its surface area.

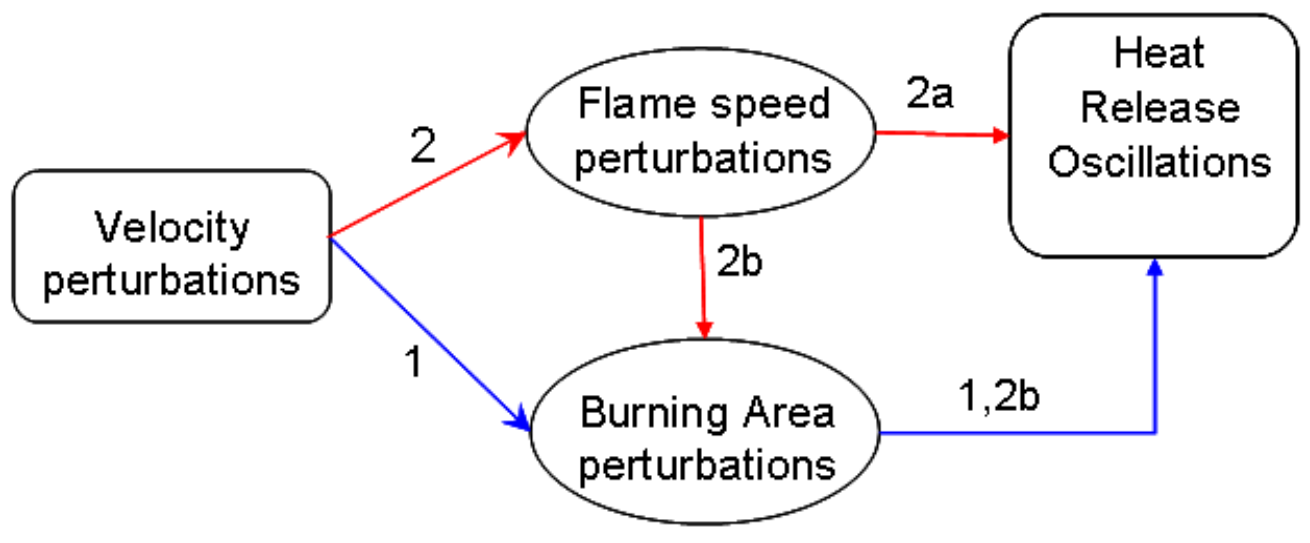

Figure 40: Physical mechanisms causing heat release oscillations due to velocity fluctuations 
At low frequencies, the flame speed remains essentially constant, rendering the heat release oscillations directly proportional to the fluctuating flame area. Route 1 is, hence, the dominating route for heat release oscillations at low frequencies. However, the oscillating stretch along the flame, due to both hydrodynamic straining and curvature, grows in importance with frequency, causing oscillations in flame speed. These flame speed oscillations perturb the heat release both directly (route 2a) and indirectly (route 2b), by affecting the burning area. Route $2 \mathrm{a}$ becomes important when $\left|\hat{\sigma}_{c}\right| S t_{2}{ }^{2} \sim 1$, while route $2 \mathrm{~b}$ becomes important at higher frequencies when $\left|\hat{\sigma}_{c}\right| S t_{2} \sim 1$.

We next recall the fuel/air ratio mechanism. As was discussed previously, the physical processes that lead to heat release oscillations are pictorially represented in Figure 25. Heat release oscillations due to equivalence ratio oscillations can occur due to oscillations in flame speed, heat of reaction or burning area. Equivalence ratio oscillations directly perturb flame speed (route 2) and the heat of reaction (route 1), while perturbations in the flame speed bring about changes in the flame wrinkling characteristics (route $2 b$ ), hence causing fluctuations in burning area. This is an indirect route. Cho and Lieuwen and Hemchandra et al. previously showed that route 1 , i.e., heat of reaction oscillations, is dominant for lean flames when $\mathrm{Stf}<<1$. At higher values of the Strouhal number, all three routes are of comparable importance. Shreekrishna and Lieuwen demonstrate that non quasi-steady effects associated with the response of the internal flame structure cause additional frequency dependence, but appear to influence all three processes in the same way so that there is no fundamental change in the key factors controlling this relationship.

We finally consider the pressure coupled mechanism or, more precisely, pressure-temperaturedensity coupling, as we assume that the three are isentropically related in the acoustic perturbation field. This is shown schematically in Figure 41. This mechanism has generally been neglected in prior studies of low frequency flame response, due to the fact that simple scaling arguments show that it is of $\mathrm{O}(\mathrm{Mf})$ lower than the velocity coupled mechanism. However, due to the increasing sensitivity of the flame speed to pressure perturbations with frequency, there is some possibility that this mechanism becomes significant at higher frequencies.

Pressure disturbances cause disturbances in the heat of reaction (route 1), unburned reactant density (route 2) and flame speed (route 3), which directly cause the heat release to oscillate. Additionally, flame speed oscillations cause the burning area to oscillate, similar to the velocity and equivalence ratio mechanism (route $3 b$ ) causing heat release oscillations indirectly. 


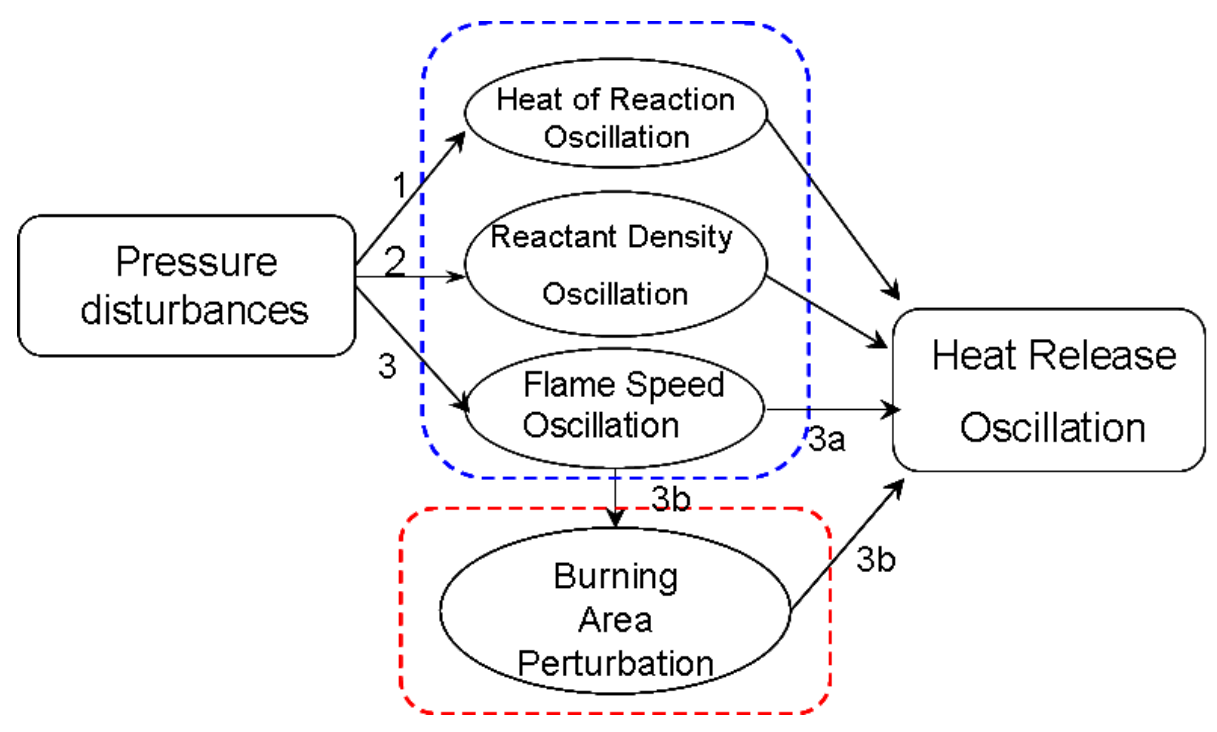

Figure 41: Physical mechanisms causing heat release oscillations due to fluctuations in acoustic pressure

Route 3 (flame speed perturbations) has received considerable theoretical treatment, through analyses of the local mass burning rate response of a flat, freely propagating premixed flame to acoustic pressure perturbations. These studies used high activation energy asymptotics with single step chemistry to analyze these interactions, and all of these show that the flame exhibits a high pass filter character, with a local response that increases as the square root of frequency at high frequencies, up to the point where the reaction zone becomes non quasi-steady. Given the fact that the velocity and fuel-air ratio coupled flame responses exhibit a low pass response character, these analyses suggest that pressure coupled flame response could grow in significance, or possibly even be dominant at high frequencies. There does not appear to be prior treatment of the global pressure coupled flame response (i.e., that includes the global flame response and incorporates all routes shown in Figure 41) to assess this point or the relative roles of the different routes shown in the figure - a key objective of this portion of the technical report.

\section{Formulation}

With this background, the rest of this discussion works out several problems related to high frequency flame response within the level set formulations. The key goal of this section is to determine the primary mechanisms of flame excitation at higher frequencies and to estimate the conditions under which pressure-coupling potentially becomes as important as velocity and fuel/air ratio coupling.

Once again, another form of the two-dimensional front tracking formulation is employed to capture the flame front location. The flame is assumed to consist of a thin sheet whose surface can be represented implicitly by the zero contour of a two dimensional function $G(r, z)$. Consequently, this analysis is most useful as long as the flame structure is thin relative to all disturbance length scales. The evolution of this contour can then be tracked using the $G$-equation as was mentioned extensively before. To achieve analytical progress, the axial location of the 
flame is given by a function $\xi(r, t)$. Thus, we may express $G$ in an explicit form as $G=z-\xi(r, t)$. Hence we obtain the following flame front tracking equation:

$$
\frac{\partial \xi}{\partial t}+s_{L}\left(1+\left(\frac{\partial \xi}{\partial r}\right)^{2}\right)^{1 / 2}=u-v \frac{\partial \xi}{\partial r}
$$

Introducing the non-dimensionalization scheme $: \hat{r}=r / R, \hat{z}=z / L_{f}$ and $\hat{t}=t U_{o} / L_{f}$ with $R$ being chosen to be the duct half-width yields:

$$
\frac{\partial \hat{\xi}}{\partial \hat{t}}+\left(\frac{s_{L}}{s_{L o}}\right) \mid\left\{\frac{1+\beta^{2}(\partial \hat{\xi} / \partial \hat{r})^{2}}{1+\beta^{2}}\right\}^{1 / 2}=\hat{u}-\hat{v} \frac{\partial \hat{\xi}}{\partial \hat{r}}
$$

Henceforth, the "hats" $\left({ }^{\wedge}\right)$ will be dropped for the sake of convenience of notation. The flame speed perturbation may be expressed in terms of its dependence on flame stretch, pressure and fuel/air ratio disturbances at the flame front location as follows:

$$
\frac{s_{L}^{\prime}}{s_{L o}}=s_{L 1, \kappa} \frac{\kappa^{\prime}}{s_{L o} / \delta_{f}}+\left.s_{L 1} \frac{p^{\prime}}{p_{o}}\right|_{z=\xi(r, t)}+\left.s_{L 1, \phi} \frac{\phi^{\prime}}{\phi_{o}}\right|_{z=\xi(r, t)}
$$

Here, $s_{L 1, k}, s_{L 1 p}$ and $s_{L 1 \phi}$ are the frequency dependent sensitivities of flame speed to flame stretch, pressure and fuel/air ratio perturbations, respectively, given by:

$$
\begin{gathered}
s_{L 1, \kappa}=M a \\
s_{L 1, p}=\left.\frac{\partial\left(s_{L} / s_{L o}\right)}{\partial\left(p / p_{o}\right)}\right|_{p=p_{o}} \\
s_{L 1, \phi}=\left.\frac{\partial\left(s_{L} / s_{L o}\right)}{\partial\left(\phi / \phi_{o}\right)}\right|_{\phi=\phi_{o}}
\end{gathered}
$$

$M a$ is the frequency dependent Markstein number and $\kappa^{\prime}$ is the flame stretch rate given by:

$$
\kappa^{\prime}=-\mathbf{n} \cdot \nabla \times\left(\left.\vec{U}\right|_{z=\xi(r, t)} \times \mathbf{n}\right)+\left(\overrightarrow{V_{f}} \cdot \mathbf{n}\right)(\nabla \cdot \mathbf{n})
$$

where $V_{f}$ is the local velocity of the flame front. Equations (171)-(176), together with a suitable model for the flame speed sensitivities to fluctuations in pressure and equivalence ratio, can be used to derive an expression for the unsteady position of the flame. 
We next calculate the instantaneous global heat release of the flame, given by Eq.(1). This equation can be linearized and written in terms of the perturbations as was done before. For convenience, the equation is repeated here:

$$
\frac{q(t)}{Q_{o}}=\int_{\text {flame }} \frac{d A}{A_{o}}+\int_{\text {flame }} \frac{s_{L}{ }^{\prime}}{s_{L o}} \cdot \frac{d A_{o}}{A_{o}}+\int_{\text {flame }} \frac{\rho_{u}^{\prime}}{\rho_{o}} \cdot \frac{d A_{o}}{A_{o}}+\int_{\text {flame }} \frac{h_{R}{ }^{\prime}}{h_{R o}} \cdot \frac{d A_{o}}{A_{o}}
$$

The first term on the RHS denotes the contribution to heat release fluctuation due to oscillations in the area of the flame. These burning area perturbations are associated with fluctuations in flame position, $\xi$, due to velocity perturbations and flame speed perturbations. For a 2D flame, the global instantaneous flame area is

$$
\frac{A(t)}{A_{o}}=\int_{0}^{1} \sqrt{\frac{1}{1+\beta^{2}}+\frac{\beta^{2}}{1+\beta^{2}}\left(\frac{\partial \xi}{\partial r}\right)^{2}} d r
$$

The normalized heat-release response of the flame is then written as:

$$
\frac{q^{\prime}(\omega)}{Q_{o}}=F_{u} \frac{u^{\prime}(\omega)}{u_{o}}+F_{p} \frac{p^{\prime}(\omega)}{p_{o}}+F_{\phi} \frac{\phi^{\prime}(\omega)}{\phi_{o}}
$$

where these transfer functions $F_{u}, F_{p}, F_{\phi}$ are defined as :

$$
\begin{aligned}
& F_{p}=\frac{q^{\prime}(\omega) / Q_{o}}{\hat{p}^{\prime} / p_{o}} \\
& F_{u}=\frac{q^{\prime}(\omega) / Q_{o}}{\hat{u}^{\prime} / u_{o}} \\
& F_{\phi}=\frac{q^{\prime}(\omega) / Q_{o}}{\hat{\phi}^{\prime} / \phi_{o}}
\end{aligned}
$$

This analytical formulation is utilized to study the flame response of a $2 \mathrm{D}$ wedge flame, see Figure 28. The flame is stabilized on a center-body, which provides the following boundary condition for $\xi$

$$
\left.\xi(t)\right|_{\text {flame-holder }}=0
$$

The duct in which the flame resides is of width $2 R$, and the undisturbed, stationary flame has a flame length $L_{f}$. The boundary condition at the wall is:

$$
\frac{\partial^{2} \xi}{\partial r^{2}}(r=0, t)=0
$$




\section{Illustrative Results}

\section{Velocity-Coupled Flame Response}

With this formulation we perform illustrative calculations to determine the relative roles of different processes. The results of these calculations are presented here. We next summarize key results on the flame response to harmonic velocity perturbations in the limit of high Strouhal numbers. This is accomplished by solving the equations presented in the prior section with $s_{L 1 p}$ and $s_{L 1 \phi}$ set to zero. We consider a harmonic velocity disturbance (in non-dimensional form) given by:

$$
\frac{u}{u_{o}}=1+\varepsilon_{u} \exp \left(2 \pi i S t_{f}(t-K z)\right)
$$

where $\varepsilon_{u}=u_{b a s e}^{\prime} / u_{o}$ denotes the amplitude of the velocity disturbances at the base of the flame, and $K=u_{d} / u_{c}$, the ratio of flow velocity to phase speed of the disturbances. Here, $S t_{f}$, is the "global" Strouhal number, defined as the ratio of the time taken by the disturbances to advect across the length of the flame at the mean flow velocity to the timescale of the imposed disturbances. Mathematically,

$$
S t_{f}=\frac{\tau_{c o n v}}{\tau_{a c}}=\frac{f L_{f}}{u_{o}}
$$

Analogous to the global Strouhal number, we define the "local" Strouhal number, $S t_{\delta, f}$, as the ratio of the time taken by disturbances to propagate through the thickness of the flame preheat zone at the laminar flame speed, to the timescale of the imposed disturbances. Mathematically,

$$
S t_{\delta, f}=\frac{\tau_{D}}{\tau_{a c}}=\frac{\delta_{f} / s_{L o}}{1 / f}=\frac{f \delta_{f}}{s_{L o}}
$$

These definitions for Strouhal number further enhance the ones previously given in the report. These two Strouhal numbers may be related as:

$$
\frac{S t_{f}}{S t_{\delta, f}}=\frac{\alpha^{1 / 2}}{\delta}=\frac{\cos \psi}{\delta}
$$

where, $\psi$ is the flame half angle. We also define a reduced Strouhal number $S_{2}$ as:

$$
S t_{2}=2 \pi \frac{S t_{f}}{\alpha}
$$


The flame speed perturbation purely due to stretch effects can be isolated from Eq.(176), and expressed as:

$$
\frac{s_{L}^{\prime}}{s_{L o}}=-L_{c}\left(\frac{\partial^{2} \xi^{\prime}}{\partial r^{2}}\right)+L_{s}\left(\left.\frac{\partial u^{\prime}}{\partial z}\right|_{z=\xi(r, t)}\right)
$$

where $L_{c}($.$) and L_{s}($.$) are the frequency dependent flame speed sensitivities to curvature and$ hydrodynamic strain, respectively. Taking a Fourier transform of Eq.(188) and linearizing yields (in dimensional form)

$$
\frac{\hat{s}_{L}{ }^{\prime}}{s_{L o}}=-\hat{L}_{c} \frac{\partial^{2} \xi_{1}}{\partial r^{2}}+\left.\hat{L} \frac{\partial u}{\partial z}\right|_{z=\xi}+O\left(\varepsilon_{u}{ }^{2}\right)
$$

Note that $\hat{L}_{c}, \hat{L}_{s}$ denote the Markstein transfer functions for curvature and hydrodynamic strain respectively. These can be non-dimensionalized by the burner duct half-width. We define:

$$
\left(\hat{\sigma}_{c}, \hat{\sigma}_{s}\right)=\left(\frac{\hat{L}_{c}}{R}, \frac{\hat{L}_{s}}{R}\right)
$$

We now present results for the velocity-coupled response of a 2D wedge flame. They show that at moderately high frequencies, flame stretch effects materially influence the velocity-coupled flame response and provide additional pathways which influence heat release fluctuations, as shown in Figure 40. The flame transfer function consists of contributions from fluctuations of both the flame surface area and flame speed. Unsteady stretch has an $O(1)$ contribution to the flame surface area fluctuations (route $2 \mathrm{~b}$ in Figure 40) when

$$
\left|\sigma_{c}^{*}\right| S t_{2}^{2}=\frac{\left|\hat{\sigma}_{c}\right|}{\beta\left(1+\beta^{2}\right)^{1 / 2}} S t_{2}^{2} \sim 1
$$

Fluctuations in flame speed (route 2a in Figure 40) become important at higher Strouhal numbers, when

$$
\left|\sigma_{c}^{*}\right| S t_{2}=\frac{\left|\hat{\sigma}_{c}\right|}{\beta\left(1+\beta^{2}\right)^{1 / 2}} S t_{2} \sim 1
$$

The effects of hydrodynamic strain become important at much larger Strouhal numbers, when

$$
\left|\sigma_{s}^{*}\right| S t_{2}=\alpha^{1 / 2}\left|\hat{\sigma}_{s}\right| S t_{2} \sim 1
$$


The dimensionless stretch sensitivities are themselves frequency dependent. An asymptotic evaluation of the high Strouhal number limits of $\hat{\sigma}_{c}, \hat{\sigma}_{s}$ yield:

$$
\begin{aligned}
& \hat{\sigma}_{c} \sim \delta \\
& \hat{\sigma}_{s} \approx \frac{\delta^{1 / 2}}{\alpha^{1 / 4}} \frac{1}{\sqrt{S t_{2}}} e^{-i \pi / 4}
\end{aligned}
$$

The above equation reduces Eq.(191), (192) and (193) respectively to:

$$
\begin{aligned}
& S t_{2} \sim \beta \delta^{-1 / 2} \\
& S t_{2} \sim \beta^{2} \delta^{-1} \\
& S t_{2} \sim \alpha^{1 / 2} \delta^{-1}
\end{aligned}
$$

We may also express Eq.(195), (196) and (197) in terms of $S t_{f}$ and $S t_{\delta, f}$ as:

$$
\begin{gathered}
S t_{f} \cdot S t_{\delta, f} \sim \frac{\beta^{4}}{4 \pi^{2}} \\
S t_{\delta, f} \sim \frac{\beta^{2}}{2 \pi} \\
S t_{\delta, f} \sim \frac{\alpha}{2 \pi}
\end{gathered}
$$

We next consider explicit results for the transfer functions. The high $S t_{2}$ limit for these transfer functions can be written as.

$$
\begin{gathered}
F_{s L, s t r} \sim-\frac{1}{\gamma M_{0}} \cdot \frac{\beta}{\eta S t_{2}} \\
F_{A, s t r} \approx \frac{1}{\gamma M_{0}} \frac{\sigma_{s}{ }^{*}{ }_{c}{ }^{*}}{e^{i \eta t_{2}}} \approx \frac{e^{-3 i \pi / 4}}{\gamma S t_{2}} \cdot \frac{\beta}{\alpha^{1 / 4} \delta^{1 / 2}} \cdot \frac{e^{i \eta S t_{2}}}{\eta S t_{2}^{3 / 2}}
\end{gathered}
$$

These transfer functions are valid when the flame thickness is small relative to the length scale of the velocity disturbance, i.e., when

$$
S t_{f}<<\frac{\beta}{K \delta}
$$


Figure 42 plots the transfer function characteristics for the baseline (unstretched, $F_{u, o}$ ) velocitycoupled response, the stretch-corrected velocity-coupled response and contributions to the stretch-corrected response due to area fluctuations and flame speed fluctuations. In order to make the graph more intelligible, the oscillations have been suppressed by only plotting the envelopes of the curves. The vertical dashed lines represent the maximum frequency of validity of these terms, as given by Eq.(203).

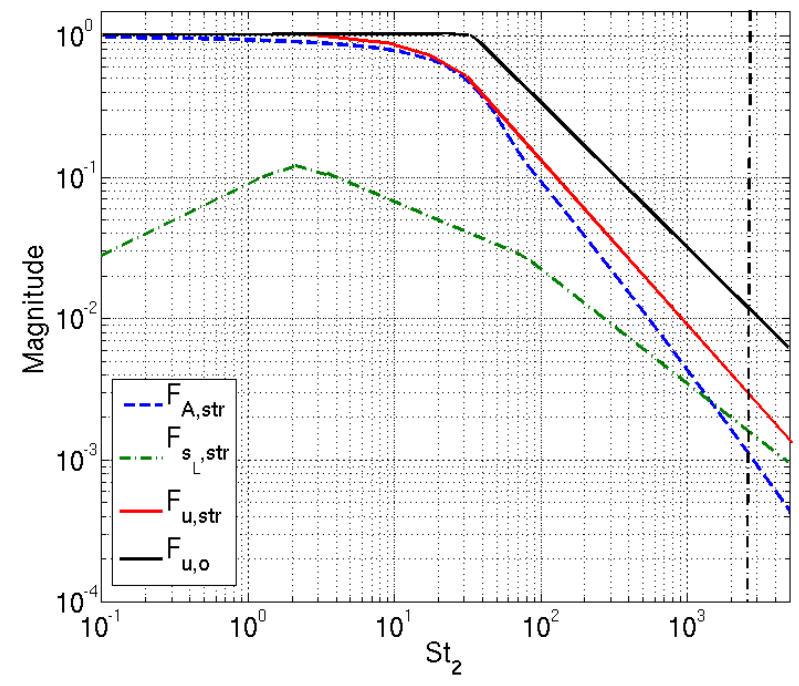

(a)

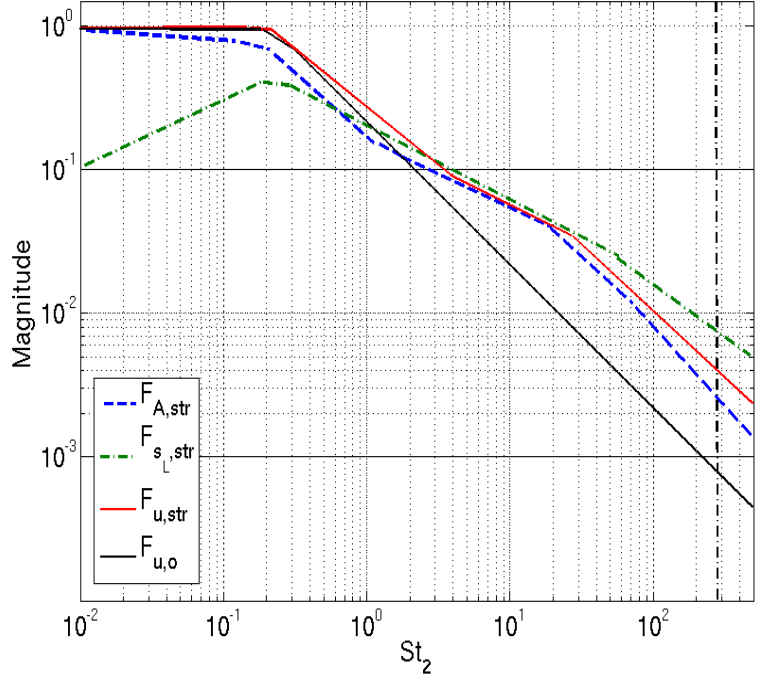

(b)

Figure 42: Strouhal number dependence of transfer function for (a) $K=1$ (b) $K=M_{o} . M_{o}=0.1$, $\beta=4, \delta=0.01$. The $S_{t_{f}}=\beta / K \delta$ limit (see Eq.(203)) is denoted by vertical dash-dot lines.

It may be seen from Figure 42 that at low Strouhal numbers, the velocity-coupled response occurs mainly through area fluctuations. Flame speed fluctuations provide a growing contribution with increasing Strouhal numbers.

\section{Equivalence Ratio Coupled Flame Response}

We next present analyses for the effect of fuel/air ratio coupling, done by setting $\sigma_{c}, \sigma_{s}$ and $s_{L, p}$ to zero. The different contributions may be evaluated as follows:

$$
\begin{gathered}
F_{A, \phi}=-s_{L 1, \phi} \sin c\left(\pi S t_{f} \frac{1-\alpha}{\alpha}\right) \sin c\left(\pi S t_{\delta, f}\right) e^{i \pi S t_{f}(1+\alpha) / \alpha} e^{-i \pi t_{\delta, f}} \\
F_{s L, \phi}=s_{L 1, \phi} \frac{i\left(1-e^{2 \pi i S t_{f}}\right)}{2 \pi S t_{f}} \sin c^{2}\left(\pi S t_{\delta, f}\right) e^{-i \pi S t_{\delta, f}} \\
F_{h_{R}, \phi}=h_{R 1, \phi} \frac{i\left(1-e^{2 \pi i S t_{f}}\right)}{2 \pi S t_{f}} \sin c^{2}\left(\pi S t_{\delta, f}\right) e^{-i \pi t_{t_{\delta, f}}}
\end{gathered}
$$


Here, $h_{R 1, \phi}$ is the quasi-steady sensitivity of heat of reaction to equivalence ratio disturbances defined as:

$$
h_{R 1, \phi}=\left.\frac{\partial\left(h_{R} / h_{R o}\right)}{\partial\left(\phi / \phi_{o}\right)}\right|_{\phi=\phi_{0}}
$$

The sinc function is defined as $\sin c(x)=\sin x / x$. We may write the quasi-steady limits for Eq.(204)-(206) as:

$$
\begin{gathered}
\lim _{S t_{f} \rightarrow 0} F_{A, \phi}=-\lim _{S t_{f} \rightarrow 0} F_{s L, \phi}=s_{L 1, \phi} \\
\lim _{S t_{f} \rightarrow 0} F_{h R, \phi}=h_{R 1, \phi}
\end{gathered}
$$

Hence, in the quasi-steady limit, the area and flame speed contributions are exactly out of phase and the total equivalence ratio coupled flame response is just $h_{R 1, \phi}$. This shows that heat of reaction fluctuations dominate the heat release response at very low Strouhal numbers. As the Strouhal number is increased, each of these processes contribute significantly to the heat release response.

\section{Pressure-Coupled Flame Response}

We next present analyses for the effect of pressure coupling on the heat release response of a $2 \mathrm{D}$ wedge flame, done by setting $\sigma_{c}, \sigma_{s}$ and $s_{L, \phi}$ to zero. This is again understood by evaluating transfer functions and their constituent contributions, that arise primarily through density and flame speed perturbations as shown in Figure 41. For this purpose, consider a harmonic pressure disturbance in non-dimensional form given by:

$$
\frac{p}{p_{o}}=1+\varepsilon_{p} \exp \left(2 \pi i S t_{f}\left(t-\frac{z}{M_{o}}\right)\right)
$$

For such disturbances, using the transfer function for the local mass burning rate fluctuations from McIntosh et al., the high frequency limit of flame speed sensitivity to pressure disturbances may written as:

$$
s_{L 1}=\frac{\left(1-\gamma^{-1}\right) \theta \delta^{1 / 2}}{2 \alpha^{1 / 4}} \sqrt{2 \pi S t_{f}} e^{i \pi / 4}-\gamma^{-1}
$$

For typical values of the parameters, such as those used in Figure 44, this dimensionless sensitivity is about 2.5 at $S t_{f} \sim 100$. Also, define the heat of reaction sensitivity to pressure perturbations as:

$$
h_{R 1, p}=\left.\frac{\partial\left(h_{R} / h_{R o}\right)}{\partial\left(p / p_{o}\right)}\right|_{p=p_{o}}+\left.\frac{\partial\left(h_{R} / h_{R o}\right)}{\partial\left(T / T_{o}\right)}\right|_{T=T_{o}} \frac{\gamma-1}{\gamma}
$$


The heat of reaction sensitivity to pressure perturbations is small in comparison to the other terms contributing to the heat release and is neglected. For example, quasi-steady equilibrium calculations for a methane-air flame with reactants at $300 \mathrm{~K}$ and $\phi=0.7$ indicate $h_{R l p} \sim 3.3 \times 10^{-2}$ and $3.2 \times 10^{-2}$ at 1 and $10 \mathrm{~atm}$, respectively. The pressure coupled flame response may be expressed as:

$$
F_{p}=F_{s L, p}+F_{\rho, p}
$$

Here, $F_{s L}$ and $F_{\rho}$ denote the contributions of flame speed and reactant density fluctuations caused due to pressure perturbations, to the transfer function. Denoting $\eta_{1}=\alpha / M$ 。

$$
\begin{gathered}
F_{\rho}=\frac{i}{\gamma} \frac{e^{i S t_{2}}-e^{i \eta_{1} S t_{2}}}{\left(\eta_{1}-1\right) S t_{2}} \\
F_{s_{L}}=\frac{e^{i \pi / 4}\left(1-\gamma^{-1}\right) \theta}{2} \delta^{1 / 2} \alpha^{1 / 4} \sqrt{S t_{2}}\left\lfloor\frac{e^{i \eta_{1} S t_{2}}-1+\eta_{1}-\eta_{1} e^{i S t_{2}}}{\eta_{1}\left(\eta_{1}-1\right) S t_{2}}\right\rfloor
\end{gathered}
$$

From the above expressions, it may be seen that

$$
\left|F_{\rho}\right| \sim \frac{1}{S t_{2}} ;\left|F_{s L}\right| \sim \frac{1}{S t_{2}{ }^{1 / 2}}
$$

This implies that the pressure coupled flame response is dominated by reactant density fluctuations at low frequencies and flame speed fluctuations at high frequencies.

\section{Relative Roles of Different Coupling Mechanisms}

We next consider the relative importance of the different coupling mechanisms as a function of frequency. As such, the perturbations in acoustic pressure and velocity are related by

$$
\frac{p^{\prime}}{p_{o}}=\frac{1}{\gamma M_{0}} \frac{u^{\prime}}{u_{0}}
$$

The perturbations in fuel/air ratio and velocity are related by:

$$
\frac{\phi^{\prime}}{\phi_{o}}=-\frac{u^{\prime}}{u_{o}}
$$

Since the ratio of the amplitudes of pressure perturbations and velocity perturbations is $1 / \gamma M_{o}$, we will multiply the velocity-coupled transfer function and equivalence ratio coupled transfer function by $1 / \gamma M_{o}$ to compare them with pressure-coupled response. Consider first the flame 
response at very low $S_{2}$. We may evaluate the very low frequency limit of the velocity-coupled, equivalence ratio coupled and pressure-coupled transfer functions as follows:

$$
\begin{gathered}
\lim _{s_{t_{2} \rightarrow 0}}\left(\frac{1}{\gamma M_{o}} F_{u, s t r}\right)=\frac{1}{\gamma M_{o}} \\
\lim _{S t_{2} \rightarrow 0} F_{p}=\frac{1}{\gamma} \\
\lim _{s_{t_{2} \rightarrow 0}}\left(\frac{1}{\gamma M_{o}} F_{\phi}\right)=\frac{h_{R 1, \phi}}{\gamma M_{o}}
\end{gathered}
$$

From Eq.(219) and Eq.(220), it may be seen that in this limit, the pressure-coupled response is smaller than the velocity-coupled response by $O\left(M_{o}\right)$ and is hence negligible. The equivalence ratio and velocity coupled responses are similar, differing only by factor $h_{R 1, \phi}$ which is of $O(1)$ for lean flames. For example, for methane-air flames at $1 \mathrm{~atm}, 300 \mathrm{~K}$ and $\phi=0.85$, this value is 0.96. In the high Strouhal number limit, we have:

$$
\begin{gathered}
\left|F_{u, s t r}\right| \sim \frac{1}{S t_{2}{ }^{3 / 2}} \\
\left|F_{p}\right| \sim \frac{1}{S t_{2}{ }^{1 / 2}} \\
\left|F_{\phi}\right| \sim \frac{1}{S t_{2}{ }^{3}}
\end{gathered}
$$

This implies that pressure coupling effects may become more significant than velocity coupling and equivalence ratio coupling at very high Strouhal numbers. 


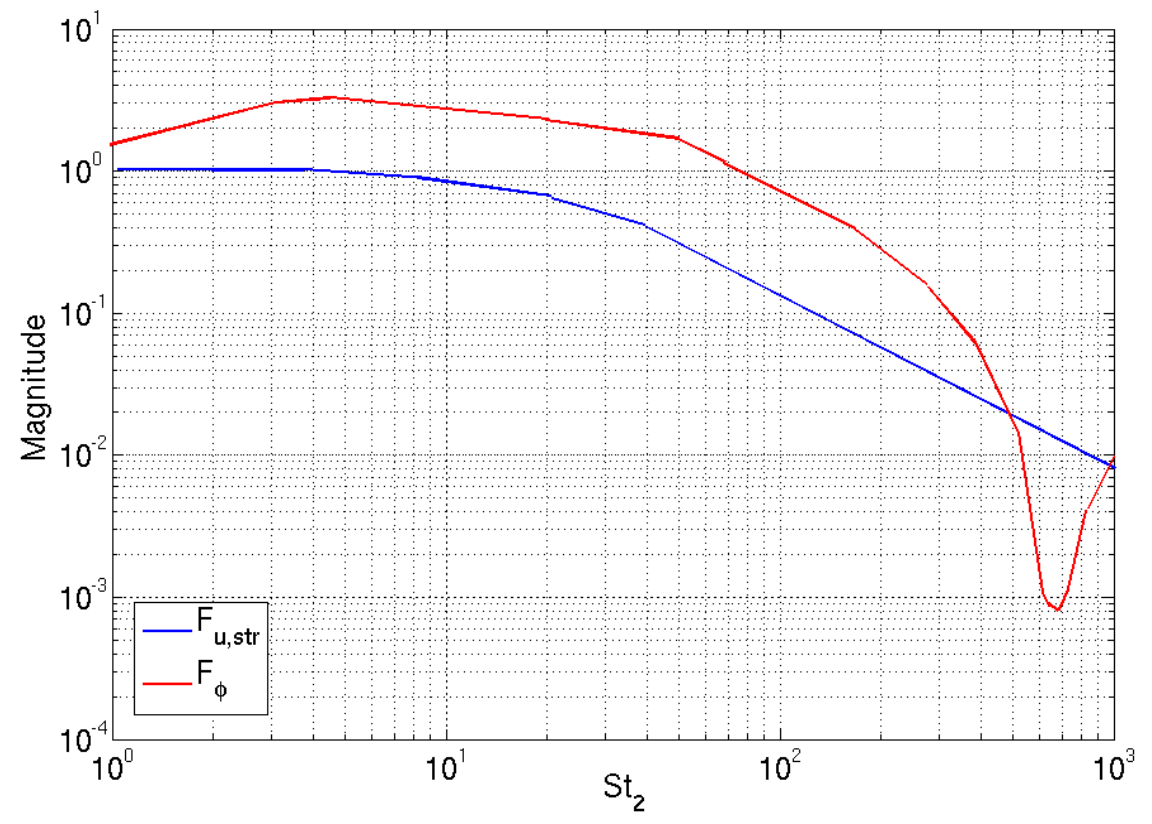

Figure 43: Gain of equivalence ratio coupled response and velocity-coupled response. $\phi_{o}=0.85, \delta=0.01, \beta=4, K=1$.

We next compare velocity coupling and equivalence ratio coupling mechanisms for the case where both disturbances are propagating at the mean flow speed $(K=1)$. Figure 43 plots the envelopes of the magnitudes of the equivalence ratio coupled and velocity-coupled response transfer functions for a methane-air flame with reactants at $1 \mathrm{~atm}, 300 \mathrm{~K}$. It can be seen that the two mechanisms have similar magnitudes, but that the fuel/air ratio response is slightly higher. This is because of the fact that, in addition to the $1 / S t_{f}$ dependence, $F_{\phi}$ decreases with frequency as the square of the sinc function (See Eqs.(204) - (206)), while $F_{u}$ reduces faster, exponentially.

Figure 44 compares the velocity and pressure coupling terms, for the case where the pressure and velocity disturbances both propagate at the sound speed. It can be seen from Figure 44 that pressure coupling grows in importance relative to velocity coupling with increasing frequency, but remains negligible over the entire frequency range indicated. For the parameters used in the figure, the Strouhal number at which pressure-coupled response becomes as significant as the velocity-coupled response is $S t_{2} \sim 6000$, corresponding to a frequency of about $100 \mathrm{kHz}$, a frequency well outside the regime of validity of this theory. Although not shown, similar calculations were performed for cases where the velocity disturbance propagated at the mean flow speed, while the pressure disturbance propagated at the sound speed. Eq.(217) was used to relate their magnitude, which assumes that the vortical velocity is of the same magnitude as the acoustic velocity disturbance that excited it. Similar results were observed here as well - i.e., that pressure coupling grows in importance but remains negligible over the frequency range over which these analyses are valid. 
In general, we may determine an approximate value for the Strouhal number at which pressure coupling and velocity coupling exert comparable effects. This occurs when:

$$
\left|F_{p}\right| \sim\left|\frac{1}{\gamma M_{o}} F_{u, s t r}\right|
$$

This may be rewritten as:

$$
\left|\sigma_{c}{ }^{*}\right| S t_{2} \sim \beta\left|\frac{\eta_{1}-1}{3 \eta_{1}{ }^{2}+1}\right| \cdot\left(\frac{2}{(\gamma-1) \theta M_{0}}\right)
$$

Equation (226) can be alternatively cast in terms of $S t_{\delta, f}$ to yield:

$$
S t_{\delta, f} \sim \frac{\beta^{3}}{2 \pi}\left|\frac{\eta_{1}-1}{3 \eta_{1}{ }^{2}+1}\right| \cdot\left(\frac{2}{(\gamma-1) \theta M_{0}}\right)
$$

However, beyond $s_{f}=\beta / K \delta$, the length scale of convective disturbances becomes of the order of the flame thickness, and the flame can no longer be treated as a gas dynamic interface. This renders the $G$-equation approach, which essentially treats the flame as a discontinuity separating reactants and products, unusable.

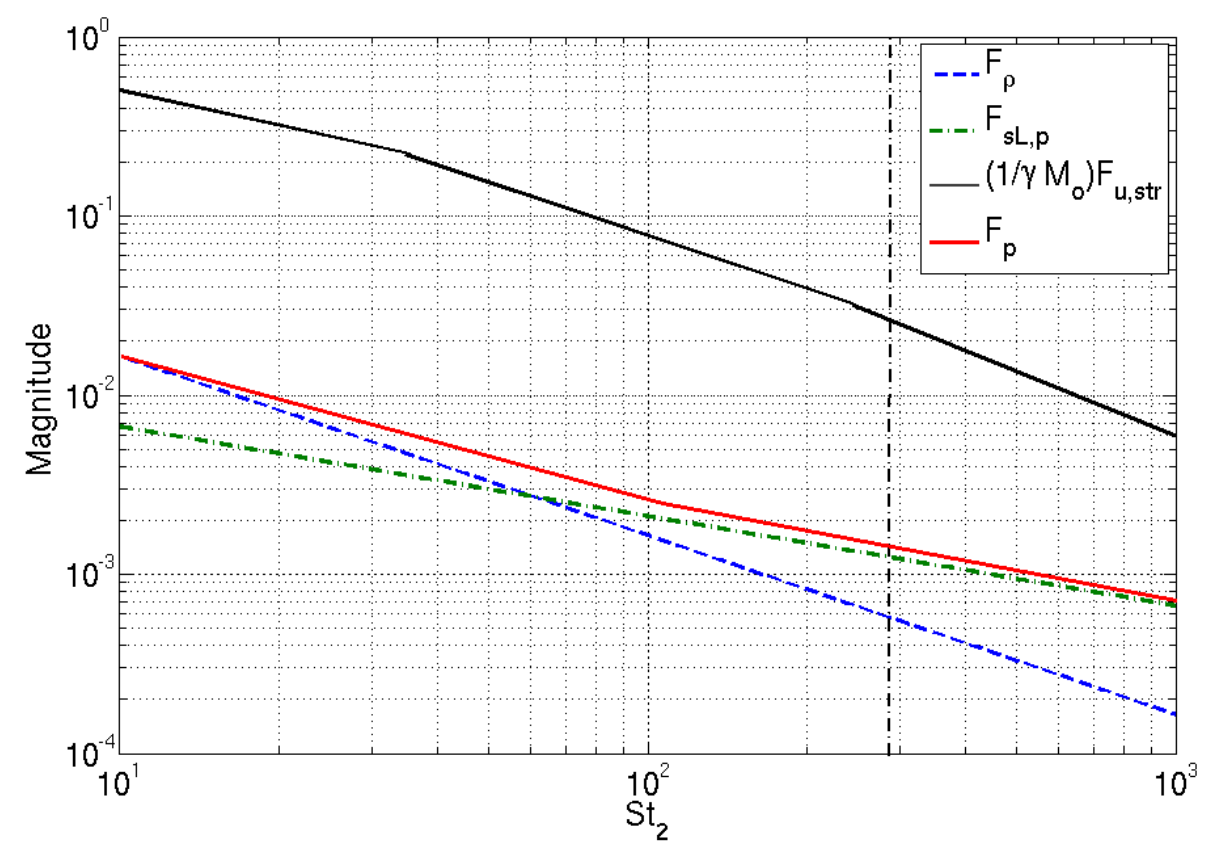

Figure 44: Magnitude of the heat release transfer function of a 2D wedge flame and its constituent contributions. $M_{0}=0.1,(\gamma-1) \theta=3, \delta=0.01, \beta=4, K=M_{0}$. The $S_{f}=\beta / K \delta$ limit (see Eq.(203)) is denoted by vertical dash-dot lines. 
Hence, within the framework of assumptions of our analysis in this section of the report, the flame response is dominated completely by equivalence ratio coupling and velocity coupling, even at higher frequencies. It is possible that pressure coupling becomes dominant at higher frequencies, but such frequencies are outside of the range of interest for any practical problem that we are aware of (100's of kHz).

\section{Discussion of High Frequency Phenomena}

\section{Time Scales and Non-Quasi-Steadiness}

Now we will present a more qualitative discussion of the various high frequency processes that occur during these interactions, without constraining ourselves to the inherent disturbance length scale restrictions of the level set approach. This can be done by considering the multitude of length and timescales present in the problem. The table below summarizes four time scales that influence the nature of flame-acoustic interactions, either through its "global" response or its internal structure.

\section{Table 2: Timescales for flame-acoustic interaction}

\begin{tabular}{|c|c|}
\hline Time scale & Approximate Scaling \\
\hline Acoustic disturbances & $\tau_{a c} \sim 1 / f$ \\
\hline Convective time along flame front & $\tau_{c o n v} \sim L_{f} / u_{o}$ \\
\hline Relaxation time associated with flame preheat zone & $\tau_{D} \sim \delta_{f} / s_{L o}$ \\
\hline Relaxation time associated with reaction zone & $\tau_{R} \sim \delta_{R} / s_{L o}$ \\
\hline
\end{tabular}

We can distinguish between flame disturbance processes that influence the local internal flame structure (such as the local burning rate) or its global geometry (such as the flame area). At very low frequencies, the acoustic timescales are much larger than the other timescales. Hence, the convective, diffusive and reactive processes respond essentially instantaneously to harmonic disturbances. However, as frequency increases, the acoustic timescale decreases and leads to non quasi-steadiness in flame response. The "global" flame position is quasi-steady when $\tau_{a c} \gg_{\tau_{c o n v}}$, or equivalently when

$$
S t_{f}=\frac{\tau_{c o n v}}{\tau_{a c}}=\frac{f L_{f}}{u_{o}} \ll 1
$$

This global quasi-steadiness implies that the overall flame shape, length, and position at each instant of time is the same as its steady state position for the same conditions. Non quasisteadiness in the global position of the flame occurs when $S t_{f} \sim O(1)$ ). Flame response modeling studies accounting for global non quasi-steadiness, but internal quasi-steadiness have been carried out in detail by various authors, and is fairly well-understood. 
We next consider the relative magnitudes of the acoustic time scale and time scale associated with internal flame processes. The ratio of the diffusive and acoustic time scales determine whether the response of the flame can be regarded as locally quasi-steady $\left(S t_{\delta, f}<<1\right)$ or non quasi-steady $\left(S t_{\delta, f} \sim O(1)\right)$. This time scale is primarily related to the relaxation of the preheat zone of the flame to imposed disturbances. McIntosh notes that the reaction zone can remain essentially quasi-steady, even under conditions where the preheat zone relaxation time is much slower than that of the harmonic disturbance. However, at even higher frequencies, the acoustic time scale can become of the order of the much faster reaction zone relaxation time. This would occur when $\tau_{a c} \sim \tau_{R}$ and can be expressed in terms of $s t_{\delta, f}$ as

$$
S t_{\delta, f} \sim \theta^{2}
$$

This scaling occurs because the reaction and preheat zone thicknesses are related as

$$
\frac{\delta_{R}}{\delta_{f}} \sim \frac{1}{\theta^{2}}
$$

Extensive research has been carried out on response of freely propagating flames to pressure disturbances in the internally non quasi-steady limit in the context of acoustic coupling of flames and in the context of non quasi-steady flame response to equivalence ratio fluctuations. To give a feel for typical numbers, let us consider a methane-air reactant mixture at $30 \mathrm{~m} / \mathrm{s}$, establishing a flame that is $10 \mathrm{~cm}$ tall. At 1atm, a typical estimate of the preheat zone thickness would be 1 $\mathrm{mm}$, while the reaction zone thickness would be about $0.1 \mathrm{~mm}$. Thus, at $1 \mathrm{~atm}$, the flame response becomes globally non quasi-steady at $f \sim 300 \mathrm{~Hz}$. Preheat zone diffusive processes become non quasi-steady at $f \sim 400 \mathrm{~Hz}$. The reaction zone becomes non quasi-steady at $f \sim 4 \mathrm{kHz}$. At $10 \mathrm{~atm}$, the preheat zone becomes non quasi-steady at $f \sim 4 \mathrm{kHz}$, while the reaction zone becomes non quasi-steady at $f \sim 40 \mathrm{kHz}$. Thus, we see that for typical combustion dynamics applications, we are most concerned with the locally non quasi-steady response of the flame, which, for typical flames would occur at frequencies of about $f \sim 400 \mathrm{~Hz}$. The analysis presented in the previous section is used to understand stretch-corrected and pressure-coupled flame response in this regime.

\section{Length Scales and Non-Compactness}

Table 3 : Relevant length scales for flame-acoustic interaction

\begin{tabular}{|c|c|}
\hline Length Scales & Approximate Scaling \\
\hline Acoustic wavelength & $\lambda=c_{o} / f$ \\
\hline Convective wavelength & $\lambda_{c}=u_{c} l f$ \\
\hline Flame length & $L_{f}$ \\
\hline Flame preheat zone & $\delta_{f}$ \\
\hline Flame reaction zone & $\delta_{R}$ \\
\hline
\end{tabular}


This section considers the ratios of important length scales, summarized in Table 3. We define two types of compactness. A region of characteristic length scale $l$ is acoustically compact if $l<<$, i.e., if it is small with respect to the acoustic wavelength. Similarly, it is convectively compact if $l<<\lambda_{c}$, where $\lambda_{c}$ denotes the distance a disturbance travels over one acoustic period at a speed of $u_{c}$. Note that the ratio of the acoustic and convective length scales is given by

$$
\frac{\lambda_{c}}{\lambda} \sim \frac{M_{o}}{K}
$$

indicating that $\lambda_{c}<<\lambda$ at low Mach numbers. With this background, we now discuss various domains of non-compactness. At very low frequencies, the acoustic and convective wavelengths are much larger than the other length scales, and hence, the flame as well as the flame structure is acoustically and convectively compact. Consider first the response of the flame to convecting disturbances. The flame length becomes on the order of the convective wavelength (assuming $K \sim 1$ ) when $S t_{f} \sim O(1)$ (or, more precisely, when $s t_{f} \sim 1 / K$ ). Thus, convective compactness implies global quasi-steadiness, and global non quasi-steadiness implies convective non-compactness of the global flame.

Similarly, the internal flame structure for flat flames $(\beta=0)$ becomes non-compact to convecting disturbances when $s_{\delta, f} \sim O(1)$. Again, non-compactness and non quasi-steadiness are directly related, but only for flat flames. More generally, the preheat zone is convectively non-compact when:

$$
S t_{\delta, f} \sim \frac{\left(1+\beta^{2}\right)^{1 / 2}}{K}
$$

This may be expressed in terms of $S t_{f}$ as:

$$
S t_{f} \sim \frac{\beta}{K \delta}
$$

From a modeling perspective, convective non-compactness implies that one has to account for the variation of properties in the preheat zone - it also implies that certain foundational assumptions of the G-equation modeling approach are suspect and one must carry out a more careful matched asymptotic expansion to couple the flow fields up and downstream of the flame. However, for $\beta>>1$ the flame structure is convectively non-compact at much higher frequencies, than when the flame response is locally non quasi-steady. This means that the analysis outlined in Section II is useful for studying locally non quasi-steady flame response. Finally, the reaction zone is convectively non-compact when $\delta_{R} \sim \lambda_{c}$, or

$$
S t_{\delta, f} \sim \theta^{2}\left(1+\beta^{2}\right)^{1 / 2}
$$


We next consider the flame response to acoustic wave disturbances. In these cases, the criteria for non quasi-steadiness and non-compactness are not the same. In fact, for low Mach number flows, the flame response becomes non quasi-steady at much lower frequencies than when the flame becomes non-compact. For example, the overall flame becomes acoustically compact when

$$
S t_{f} \sim 1 / M_{o}
$$

The acoustically non-compact limit is an important one because, global heat release transfer functions, such as considered earlier in this report become less relevant for the combustion instability problem. Rather, as stated by the Rayleigh criterion, one is interested in the spatial integral of the product of the pressure and heat release. Mathematically, the Rayleigh integral

may be written as $\int_{f a m e} p^{\prime}(x, t) q^{\prime}(x, t) d A$. For acoustically compact flames, this may be written as $p^{\prime}(x, t) Q^{\prime}(t)$. However, non-compact flames with the same global transfer functions can have very different Rayleigh products. Further, the preheat zone becomes acoustically non-compact when $\delta_{f} \sim \lambda$, i.e., when

$$
S t_{\delta, f} \sim 1 / M_{f}
$$

Hence, at these frequencies, the flame structure has to be resolved necessarily to be able to understand the physics of flame-acoustic interactions. Finally, the reaction zone loses acoustic compactness when $\delta_{R} \sim \lambda$, i.e., when

$$
S t_{\delta, f} \sim \theta^{2} / M_{f}
$$

The expression for $s_{L 1, p}$ used in this report is valid in reaction zone acoustic compactness regime, while the G-equation formulation used for obtaining flame surface location is valid only when the preheat zone is convectively compact.

\section{Summary of Flame Response Regimes}

It is useful to summarize the above discussion by means of a flame response regime diagram. These various physical processes are parameterized in terms of the two Strouhal numbers $s t_{\delta, f}$ and $s t_{f}$ which characterize local and global non quasi-steadiness respectively. The various physical phenomena occurring at different frequencies are summarized in Table 4. 
Table 4: Summary of physical processes influencing flame response at different regimes in the $S t_{f}, S t_{\delta, f}$ space

\begin{tabular}{|l|l|}
\hline Physical process & Frequency regime \\
\hline Globally quasi-steady & $S t_{f} \ll_{1}$ \\
\hline Locally quasi-steady & $S t_{\delta, f} \ll_{1}$ \\
\hline Globally non quasi-steady & $S t_{f} \sim 1$ \\
\hline Locally non quasi-steady & $S t_{\delta, f} \sim 1$ \\
\hline Geometric Convective non-compactness & $S t_{f} \sim 1 / K$ \\
\hline Geometric Acoustic non-compactness & $S t_{f} \sim 1 / M_{o}$ \\
\hline Preheat zone convective non-compactness & $S t_{f} \sim \beta / K$ \\
\hline Preheat zone acoustic non-compactness & $S t_{\delta, f} \sim 1 / M_{f}$ \\
\hline Reaction zone convective non-compactness & $S t_{\delta, f} \sim \beta \theta^{2}$ \\
\hline Reaction zone acoustic non-compactness & $S t_{\delta, f} \sim \theta^{2} / M$ \\
\hline Flame curvature affects area fluctuations & $S t_{f} \cdot S t_{\delta, f} \sim \beta{ }^{4} / 4 \pi$ \\
\hline Flame curvature alters flame speed & $S t_{\delta, f} \sim \beta^{2} / 2 \pi$ \\
\hline Hydrodynamic strain affects area fluctuations & $S t_{\delta, f} \sim \alpha / 2 \pi$ \\
\hline $\begin{array}{l}\text { Pressure coupled response } \sim \\
\text { Stretch-corrected velocity response }\end{array}$ & $S t_{\delta, f} \sim \frac{\beta^{3}}{2 \pi}(\overline{(\gamma-1) \theta M})$ \\
\hline Reaction zone non quasi-steadiness & $S t_{\delta, f} \sim \theta^{2}$ \\
\hline
\end{tabular}

Figure 45 presents a regime diagram representation and marks the different areas for the physical processes listed in Table 4. The regimes corresponding to acoustic non-compactness of the preheat and reaction zones have been excluded, since they are ultra-high frequency phenomena, and occur at couple orders of magnitude more than those of interest here viz., pressure coupling, stretch effects and convective non-compactness. 


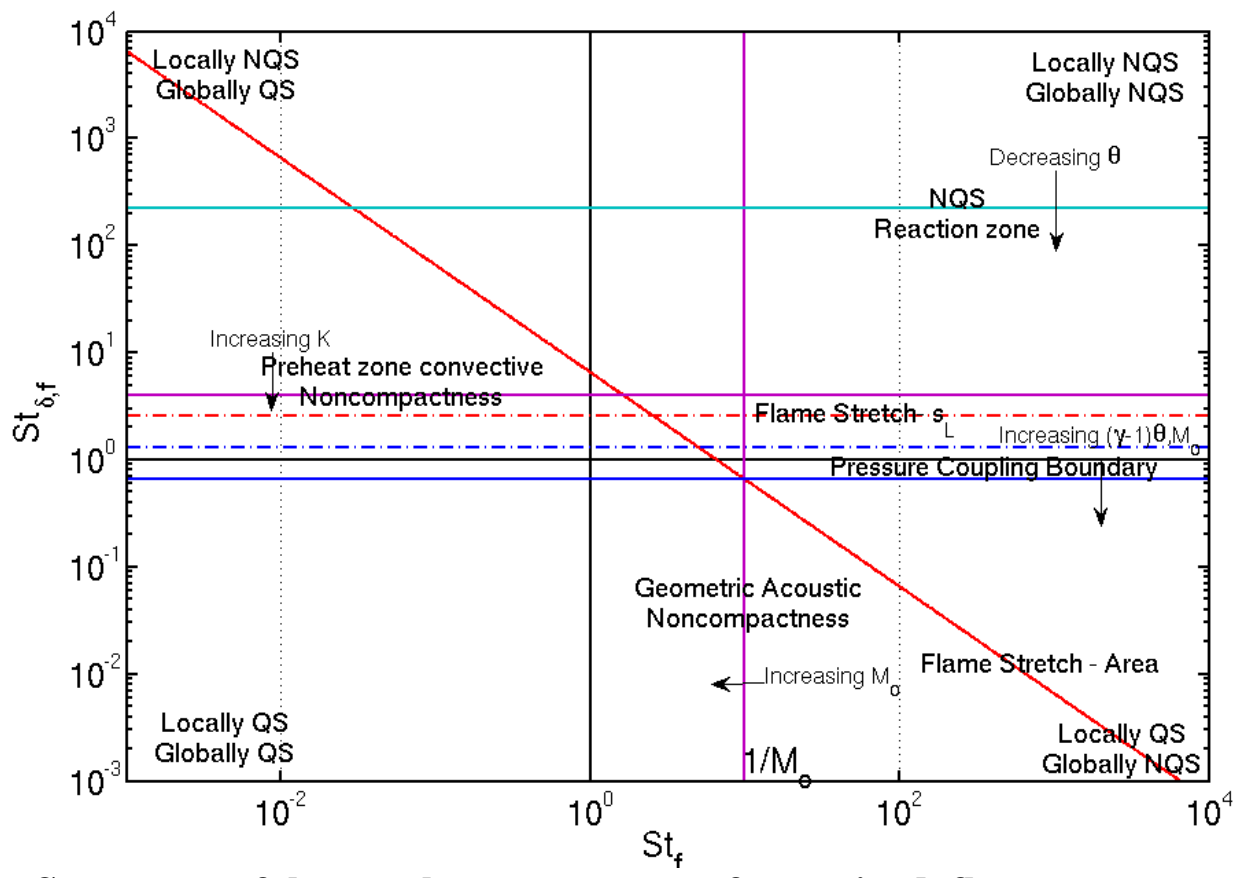

Figure 45: Summary of heat release response of premixed flames to pressure/velocity disturbances

The four corners of the graph in Figure 45 show four limits of flame response with respect to the global and local non quasi-steadiness. Our region of interest in this work has largely been the upper right quadrant, where the flame response is both locally and globally non quasi-steady. However, the transfer functions presented in this section of the report are valid only when the flame preheat zone is convectively compact. It can be seen that in this quadrant, both flame stretch and pressure coupling may become significant. However, as discussed earlier, the effect of flame stretch through area response becomes significant at far lesser Strouhal numbers than that of pressure coupling effects and dominates the high frequency response along with equivalence ratio coupling effects.

\section{Review}

As has been examined, geometric acoustic non-compactness of the flame is a critical consideration for high frequency response. The global heat release of the flame would overlook this effect, and hence it is necessary to redefine the transfer function to be able to account for flame acoustic non-compactness. The ultra-high frequency limits of flame response, which include reaction zone non quasi-steadiness, diffusion zone non-compactness and reaction zone non-compactness cannot be understood using a flame front tracking approach, and the analysis employed in this work would fail to hold. It is necessary to solve the governing equations of fluid dynamics with suitable chemistry models to be able to understand response of flames with complex geometries. 


\section{Swirl Effects on Transversely Forced Flame Dynamics}

\section{Introduction}

In following the analysis done in the previous section, this section describes the response of a swirling premixed flame to transverse acoustic excitation. This work extends prior studies of axisymmetric forcing, which have shown that wrinkles are excited on the flame that propagate axially downstream at a speed given by $U_{o} \cos \psi$, where $U_{o}$ is the mean flow velocity and $\psi$ is the flame angle. The swirl component in the flow field introduces an azimuthal transport mechanism for disturbances on the flame. As such, the flame response at any given position is a superposition of flame wrinkles excited at earlier times, upstream axial locations, and different azimuthal positions. These swirl transport effects do not show up in problems where axisymmetric flames are subjected to axisymmetric excitation, but enter quite prominently in the presence of non-axisymmetries, such as when the flame is subjected to transverse excitation. The solution characteristics are strongly dependent upon the ratio of angular rotation rate to excitation frequency, denoted by $\sigma=\Omega / \omega$, which describes the fraction of azimuthal rotation a disturbance makes in one acoustic period. When $\sigma<<1$ and $\sigma>>1$, the axial wavelength of flame wrinkles scales with the convective wavelength, $\lambda_{c}$, but becomes much longer for $\sigma \sim \mathrm{O}(1)$. For a compact flame in particular, a unique frequency matching behavior occurs at $\sigma=1$, where flame wrinkles disappear and the flame exhibits off-axis solid body rotation. The spatial variation in phase of flame wrinkling is also strongly dependent upon $\sigma$. Regardless of swirl number, flame wrinkles propagate in helical spirals along the solution characteristics at a phase speed equal to the local tangential velocity. The axial phase characteristics of flame wrinkling at a fixed azimuthal location, such as would be measured in by laser sheet imaging, are much more complex, however. For $\sigma<1$, the wrinkles exhibit the familiar negative phase-axial distance character, indicative of an axially convecting disturbance. The slope of this phase roll-off decreases with increasing $\sigma$, however, and becomes zero at $\sigma=1$ for a compact flame. For $\sigma>1$, a phenomenon analogous to aliasing occurs, where the wrinkles actually have a positive phaseaxial distance dependence, indicating a flame wrinkle with a negative trace velocity, but whose actual group velocity is positive. Finally, while the effects of swirl are quite prominent upon the local flame characteristics, their global influence upon the spatially integrated, linearized unsteady heat release depends upon an acoustic compactness parameter, $R / \lambda_{a}$, where $R$ denotes the flame width at the base. For example, in a linear acoustic framework, for $R / \lambda_{\mathrm{a}} \sim 0$ and constant flame speed limit, swirl redistributes flame area, but causes no global change.

Swirling flows possess complex dynamics associated with vortex breakdown and other flow instabilities leading to a variety of unsteady flow features, such as the precessing vortex core and helical disturbances. In particular, the presence of vortex breakdown, is one of the most prominent flow features differentiating swirling and non-swirling flow fields. Swirling flow dynamics control flow recirculation, flame stabilization, flame shape, dynamic stability limits, as well as a number of other critical combustion parameters. Because of the profound influences of the swirling flow field upon the flame shape, such as flame angle, swirl has important influences upon flame dynamics. For example, it is known that the Strouhal number, 'St', is an important parameter describing flame transfer functions. As such, changes in flame length because of modifications in swirl number exercise very significant influences upon the flame response to harmonic perturbations. This effect is implicit, however, in that swirl does not explicitly enter the 
dynamical equations for the flame dynamics, but rather through its influence upon parameters such as flame angle, $\psi$, or length, $\mathrm{L}_{\mathrm{f}}$.

Swirl also has explicit effects on the dynamics of the flame as well. Several recent papers have suggested that acoustic waves propagating through swirlers excite azimuthal vortical disturbances, leading to modulations in swirl number. These swirl number modulations, in turn, have been postulated to lead to oscillations in flame angle, $\psi$, a parameter with a well known influence upon flame dynamics. In order to proceed theoretically, these studies have postulated heuristic relationships between flame angle and instantaneous swirl number. Nonetheless, this may be an important mechanism, but one which requires further study.

Here we consider an additional mechanism through which swirl explicitly influences flame dynamics by its propagation of flame wrinkles in the azimuthal direction. The response of premixed flames to disturbances that are symmetric on either the two flame branches (for 2-D) or axisymmetric (for axisymmetric flames) has been extensively considered from a theoretical modeling perspective. In reality, no geometry or excitation field is perfectly symmetric or axisymmetric. These inherent non-symmetries do not introduce substantive changes to nonswirling flame dynamics, as we detail later. In a swirling flow, however, non-axisymmetric disturbances excite waves with an azimuthal dependence that propagates along a helical path. While numerous experimental studies of forced swirl flames have been published, they have all focused upon the axial distribution of flame wrinkling and have not discussed their azimuthal distribution.

An important problem motivating interest in non axisymmetric excitation is transverse excitation of the flame. Transverse excitation is fundamentally different from longitudinal excitation in two key ways. First, while longitudinal disturbances are generally axisymmetric, or at least approximately so, transverse disturbances excite the flame in an intrinsically non-axisymmetric manner, as shown in Figure 46(a). This causes significant azimuthal variations in local disturbance field at the flame sheet. Second, the net volumetric flow rate through the flame, which is directly related to the flame's spatially integrated low Strouhal number response, is of the same sign at all azimuthal locations for longitudinal forcing, as shown in Figure 46(b), but of opposite sign in the case of transverse forcing, as shown in Figure 46(c).

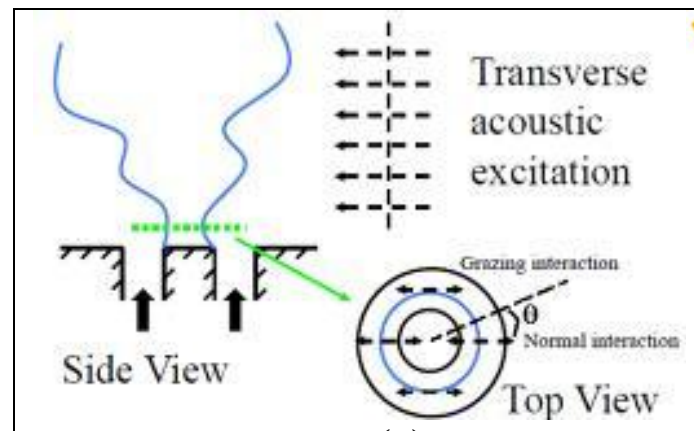

(a)

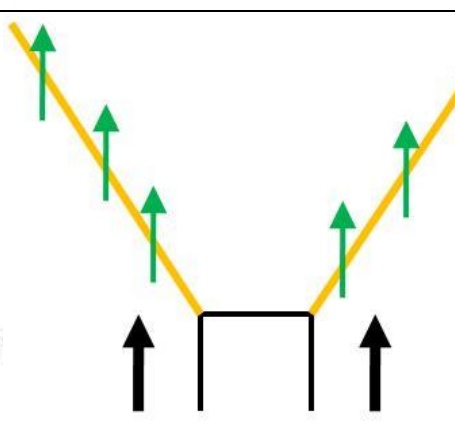

(b)

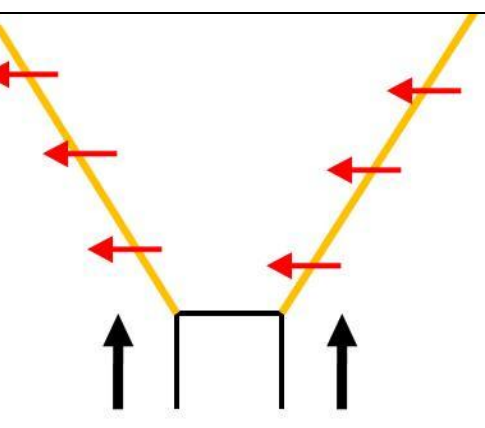

(c)

Figure 46: (a) Flame interactions with transverse excitations; (b) Longitudinal forcing; (c) Transverse forcing. 
This azimuthal degree of freedom introduces additional physics into this problem that does not appear to have been considered theoretically to date. This section of the technical report describes a theoretical analysis of this problem, identifies the key new degrees of freedom, and presents illustrative calculations showing the flame wrinkling characteristics.

\section{Formulation}

Once again, a front tracking formulation is employed to capture the flame location. Utilizing the farmiliar G-equation and assuming the flame's axial position is a single valued function of $r$, the non-dimensional form of the flame front tracking equation may be written as:

$$
\left.\frac{\partial \tilde{\xi}}{\partial \tilde{t}}+\tilde{u}_{r} \frac{\partial \tilde{\xi}}{\partial \tilde{r}}+\frac{\tilde{u}_{\theta}}{\tilde{r}} \frac{\partial \tilde{\xi}}{\partial \theta}+\tilde{s}_{L}\left[\left(\mid \frac{\partial \tilde{\xi}}{\partial \tilde{r}}\right)^{2}+\frac{1}{\tilde{r}^{2}} \mid \frac{\partial \tilde{\xi}}{\partial \theta}\right)^{2}+1\right\}^{1 / 2}=\tilde{u}_{z}
$$

The flame schematic provided in the bottom right image of Figure 1 shows a center-body stabilized premixed flame in a swirling flow. The mean flame is shown by the dashed line and the perturbed flame is shown by the solid line. The flame is stabilized on a center-body, which provides the following boundary condition for $\xi$ :

$$
\left.\xi(r=R, \theta, t)\right|_{\text {flame-holder }}=0
$$

We write $\xi$ in terms of a steady mean position and a spatio-temporally varying disturbance, as:

$$
\xi(r, \theta, t)=\bar{\xi}(r, \theta)+\varepsilon \xi^{\prime}(r, \theta, t)
$$

The evolution equations for the mean and perturbed (linear) flame positions may then be written as:

$$
\begin{gathered}
\left.\bar{U}_{r} \frac{d \bar{\xi}}{d r}+\frac{\bar{U}_{\theta}}{r} \frac{d \bar{\xi}}{d \theta}=\bar{U}_{z}-s_{L} \mid\left(\frac{d \bar{\xi}}{d r}\right)^{2}+\left(\frac{1}{r} \frac{d \bar{\xi}}{d \theta}\right)^{2}+1\right\rceil^{1 / 2} ; \frac{d \bar{\xi}}{d r}=\cot \psi(r, \theta) \\
\frac{\partial \xi^{\prime}}{\partial t}+\sin \psi\left(\vec{u}_{t} \cdot \nabla \xi^{\prime}\right)=\frac{u_{n}^{\prime}}{\sin \psi}
\end{gathered}
$$

where

$$
\bar{u}_{t}=\left(\bar{U}_{z} \cos \psi+\bar{U}_{r} \sin \psi\right) \vec{e}_{r}+\vec{U}_{\theta} \vec{e}_{\theta}
$$

denotes the net tangential velocity vector along the flame surface, due to both the azimuthal and axial velocity components. In addition: 


$$
u_{n}^{\prime}=u_{z}^{\prime} \sin \psi-u_{r}^{\prime} \cos \psi
$$

denotes the normal component of imposed velocity fluctuations to the flame surface. We assume a constant burning velocity, $s_{L}$, for analysis of unsteady stretch effects. Equation (242) shows that flame wrinkles propagate along the flame in the direction given by $\bar{u}_{t}$. The general characteristic equation for propagation of waves, in characteristic space co-ordinate $s$, is given by:

$$
d s=\frac{d t}{1}=\frac{d r}{\bar{U}_{r}+s_{L} \cos \psi}=\frac{r d \theta}{\bar{U}_{\theta}}
$$

The spatial motion of waves along the flame surface, is then given by:

$$
\frac{\bar{U}_{\theta}}{\bar{U}_{r}+s_{L} \cos \psi} \frac{d r}{r}=d \theta
$$

Equation (246) clearly shows that the spatial motion is a spiral whose nature depends on the radial and azimuthal mean velocity components. This can also be seen from the fact that the characteristic motions are along the tangential velocity vector whose components now include radial and azimuthal components. The absence of the swirling component would lead to a characteristic that is straight in the radial direction along the flame surface. Typical spiral shapes shall be presented later for specific flow-fields.

Equation (242) describes the evolution of wrinkles on the flame surface. There are two important mechanisms responsible for wrinkle evolution. The left hand side is a convection operator and describes the transport mechanism of the generated wrinkles. The right hand side shows the mechanism responsible for wrinkle generation. We can see that flame wrinkles are excited by velocity disturbances in the direction normal to mean flame front, shown in Eq.(244). In other words, the flame does not distinguish between axial, $u_{z}{ }^{\prime}$, or transverse direction, $u_{r}$, , of the disturbance field, but responds to the net scalar velocity component which is normal to itself, $u_{n}$ '. The difference lies in the inherent nature of how an imposed $u_{r}$ ' is spatially distributed as compared to an imposed $u_{z}$ ' distribution, as shown earlier in Figure 46. The effect of swirl in wrinkle evolution is not explicitly present in the velocity disturbance component, but rather seen implicitly through the wave transport mechanism. The wave operator shows that wrinkles generated by the velocity disturbances are transported by the mean tangential velocity along the flame surface. This component is shown in Eq.(243), and shows transport along the radial and azimuthal directions. As such, this creates complicated wrinkle patterns on the flame surface due to the interference of wave transport both in the radial and azimuthal direction. Hence, the presence of swirl is important both in the global flame response dynamics and in the local wrinkling behavior. In order to illustrate this, we shall first consider a general solution to the evolution equation, and then present example solutions.

The frequency domain representation of Eq.(242) is:

$$
i 2 \pi S t \hat{\xi}^{\prime}+\left(\bar{U}_{r}+s_{L} \cos \psi\right) \frac{\partial \hat{\xi}^{\prime}}{\partial r}+\frac{\bar{U}_{\theta}}{r} \frac{\partial \hat{\xi}^{\prime}}{\partial \theta}=\frac{\hat{u}_{n}^{\prime}(r, \theta, S t, \chi)}{\sin \psi}=\hat{F}(r, \theta, S t, \chi)
$$


where $F$ denotes the forcing function, which is itself a function of space $(r, \theta)$, a dimensionless frequency $(S t)$, and a non-compactness parameter, $\chi$, that compares the flame length scale and the acoustic wavelength. The function, $F$, is expanded in a Fourier series in the azimuthal direction, as below:

$$
\hat{F}(r, \theta, S t, \chi)=a_{o}(r, S t, \chi)+\sum_{n}\left(a_{n}(r, S t, \chi) \cos n \theta+b_{n}(r, S t, \chi) \sin n \theta\right)
$$

where the Fourier coefficients are:

$$
a_{o}=\frac{1}{2 \pi} \int_{0}^{2 \pi} \hat{F} d \theta, a_{n}=\frac{1}{2 \pi} \int_{0}^{2 \pi} \hat{F} \cos n \theta d \theta, b_{n}=\frac{1}{2 \pi} \int_{0}^{2 \pi} \hat{F} \sin n \theta d \theta
$$

This Fourier representation of $F$ facilitates a solution representation for the flame fluctuation, and also enables a decomposition of which features of the disturbance function influence the spatially integrated flame area response. We can write the governing partial differential equation to an ordinary differential equation, thereby enabling a generic representation for the solution, by transferring to characteristic co-ordinates. Transforming the spatial co-ordinates to the characteristic co-ordinate $s$ (from Eq.(245)), we get:

$$
\begin{aligned}
& \left.d s=\frac{d r}{\bar{U}_{r}(r, \theta)+s_{L} \cos \psi}\right\} \Rightarrow r=r\left(r_{p}, \theta_{p}, s, \sigma\right) \\
& \left.d s=\frac{r d \theta}{\bar{U}_{\theta}(r, \theta, \sigma)} \quad\right\} \quad \theta=\theta\left(r_{p}, \theta_{p}, s, \sigma\right) \\
& \therefore\left(\bar{U}_{r}+s_{L} \cos \psi\right) \frac{\partial \hat{\xi}^{\prime}}{\partial r}+\frac{\bar{U}_{\theta}}{r} \frac{\partial \hat{\xi}^{\prime}}{\partial \theta}=\frac{d \hat{\xi}^{\prime}}{d s}
\end{aligned}
$$

where $r_{p}$ and $\theta_{p}$ are the spatial co-ordinates corresponding to characteristic co-ordinate $s=s_{p}$. The suffix $p$ is used to denote position of interest on the flame surface. Note that, $s_{p}$ is a function of $r_{p}$ and $\theta_{p}$ obtained from solving the above equation for $r=r_{p}$ and $\theta=\theta_{p} . \sigma$ denotes a representative characteristic non-dimensional parameter to quantify swirl. This parameter can be explicitly specified for a given swirling flow field. Substituting in Eq.(247) and Eq.(248), we get:

$$
i 2 \pi S t \hat{\xi}^{\prime}(s)+\frac{d \hat{\xi}^{\prime}(s)}{d s}=a_{o}\left(r\left(r_{p}, \theta_{p}, s, \sigma\right), S t, \chi\right)+\sum_{n}\left\{\begin{array}{l}
\left.a_{n}\left(r\left(r_{p}, \theta_{p}, s, \sigma\right), S t, \chi\right) \cos \left(n \theta\left(r_{p}, \theta_{p}, s, \sigma\right)\right)\right) \\
\left.+b_{n}\left(r\left(r_{p}, \theta_{p}, s, \sigma\right), S t, \chi\right) \sin \left(n \theta\left(r_{p}, \theta_{p}, s, \sigma\right)\right)\right)
\end{array}\right)
$$

The solution to Eq.(251) is given by:

$$
\hat{\xi}^{\prime}\left(s_{p}\right) e^{i 2 \pi S t s_{p}}=I_{o}\left(r_{p}, \theta_{p}, \sigma, S t, \chi\right)+\sum_{n}\left(I_{n}^{c}\left(r_{p}, \theta_{p}, \sigma, S t, \chi\right)+I_{n}^{s}\left(r_{p}, \theta_{p}, \sigma, S t, \chi\right)\right)
$$


where

$$
\begin{aligned}
& I_{o}\left(r_{p}, \theta_{p}, \sigma, S t, \chi\right)=\int_{\substack{s=0 \\
s=s_{p}}} a_{o}\left(r_{p}, \theta_{p}, \sigma, S t, \chi\right) e^{i 2 \pi S t s} d s \\
& I_{n}^{c}\left(r_{p}, \theta_{p}, \sigma, S t, \chi\right)=\int_{s=0} a_{n}\left(r_{p}, \theta_{p}, \sigma, S t, \chi\right) e^{i 2 \pi S t s} \cos \left(n \theta\left(r_{p}, \theta_{p}, \sigma, S t, \chi\right)\right) d s \\
& I_{n}^{s}\left(r_{p}, \theta_{p}, \sigma, S t, \chi\right)=\int_{s=0} b_{n}\left(r_{p}, \theta_{p}, \sigma, S t, \chi\right) e^{i 2 \pi S t s} \sin \left(n \theta\left(r_{p}, \theta_{p}, \sigma, S t, \chi\right)\right) d s
\end{aligned}
$$

The suffix can be subsequently dropped, as they represent any point of interest on the flame surface where the fluctuation is being determined. The final solution can be represented as:

$$
\hat{\xi}^{\prime}(r, \theta) e^{i 2 \pi S t s_{p}(r, \theta)}=I_{o}(r, \theta, \sigma, S t, \chi)+\sum_{n}\left(I_{n}^{c}(r, \theta, \sigma, S t, \chi)+I_{n}^{s}(r, \theta, \sigma, S t, \chi)\right)
$$

The global flame surface area, proportional to its heat release for constant burning velocity flames, is given by:

$$
A=\iint_{r, \theta} r d \theta d r \sqrt{1+\left(\frac{\partial \xi}{\partial r}\right)^{2}}
$$

Linearization of this equation yields the following decomposition for the global flame area:

$$
A=\iint_{\llcorner\cdot \theta}^{\int} r d r d \theta \frac{1}{\sin \psi(r, \theta)}+\varepsilon \int \underbrace{\int}_{\bar{A}} r d \theta d r \cos \psi(r, \theta) \frac{\partial \hat{\xi}^{\prime}(r, \theta)}{\partial \theta}
$$

The first term represents the global steady mean flame area. The second term is the fluctuating flame area, which is the more relevant quantity for combustion instability dynamics. This term contains both the mean flame quantities in an explicit form, and hence the effect of swirl, and also contains the implicit effect of swirl in the slope term. From Eq.(254), we can see that this integral can be decomposed into three terms:

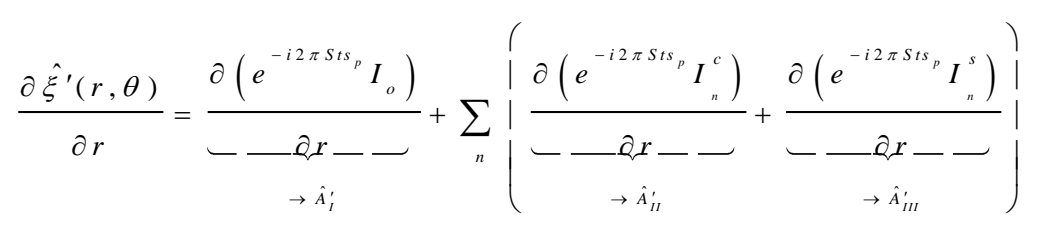

This expression will be referred to in the subsequent section, which presents explicit solution and discussions for specific flow fields. 


\section{Discussion}

Now, we shall consider different assumptions for mean flame shapes, flame compactness and disturbance field, in order to better illustrate the effect of swirl.

\section{Axisymmetric Flames}

We first illustrate the general form of the above equations for flames whose average flow field and shape is axisymmetric. Equation (241) and (242) take the form:

$$
\begin{gathered}
\bar{U}_{r} \frac{d \bar{\xi}}{d r}=\bar{U}_{z}-s_{L}\left|\left(\frac{d \bar{\xi}}{d r}\right)^{2}+1\right|^{1 / 2} ; \frac{d \bar{\xi}}{d r}=\cot \psi(r) \\
\frac{\partial \xi^{\prime}}{\partial t}+\left(\bar{U}_{r}(r)+s_{L} \cos \psi(r)\right) \frac{\partial \xi^{\prime}}{\partial r}+\frac{\bar{U}_{\theta}(r)}{r} \frac{\partial \xi^{\prime}}{\partial \theta}=\frac{u_{n}^{\prime}}{\sin \psi(r)}=u_{z}^{\prime}-u_{r}^{\prime} \cot \psi(r)-\frac{u_{\theta}^{\prime}}{r} \frac{\partial \bar{\xi}}{\partial \theta}
\end{gathered}
$$

This expression shows that fluctuations in azimuthal velocity have no influence on the flame response. The Fourier domain representation of Eq.(259) (from Eq.(247)), is:

$$
i 2 \pi S t \hat{\xi}^{\prime}+\left(\bar{U}_{r}(r)+s_{L} \cos \psi(r)\right) \frac{\partial \hat{\xi}^{\prime}}{\partial r}+\frac{\bar{U}_{\theta}(r)}{r} \frac{\partial \hat{\xi}^{\prime}}{\partial \theta}=\hat{F}(r, \theta, S t, \chi)
$$

where $F$ is as defined in Eq.(248) and (249). Following Eq.(250), we transform to characteristic co-ordinates to obtain:

$$
\begin{aligned}
& d s=\frac{d r}{\bar{U}_{r}(r)+s_{L} \cos \psi(r)} \Rightarrow r=f\left(r_{p}, s\right) \\
& d s=\frac{f\left(r_{p}, s\right) d \theta}{\bar{U}_{\theta}\left(f\left(r_{p}, s\right), \sigma\right)} \Rightarrow d \theta=\frac{\bar{U}_{\theta}\left(f\left(r_{p}, s\right), \sigma\right) d s}{f\left(r_{p}, s\right)} \Rightarrow \theta=\theta_{p}+g\left(r_{p}, s, \sigma\right)
\end{aligned}
$$

Note that the radial characteristic function $f$ is independent of the azimuthal characteristic function $g$, while the converse is not true. This also implies that the radial characteristic function is independent of swirl $\sigma$. The characteristic co-ordinate $s_{p}$ is obtained from solving for $r=r_{p}$. Also, unlike the general case, $\theta_{p}$ can be separated from the function $g$. These observations are specific to axisymmetric flow fields and play an important role in our further discussion on the effect of swirl. The spatial motion of waves on the flame surface is given by:

$$
\frac{d r}{\overline{U_{r}}(r)+s_{L} \cos \psi(r)}=\frac{r d \theta}{\bar{U}_{\theta}(r, \sigma)} \Rightarrow d \theta=\frac{\bar{U}_{\theta}(r, \sigma)}{\bar{U}_{r}(r)+s_{L} \cos \psi(r)} \frac{d r}{r}
$$

This characteristic is a general representation for a helical spiral motion. Equations (251) - (254) respectively take the form: 


$$
\begin{aligned}
& i 2 \pi S t \hat{\xi}^{\prime}(s)+\frac{d \hat{\xi}^{\prime}(s)}{d s}=a_{o}\left(f\left(r_{p}, s\right), S t, \chi\right)+\sum_{n}\left(\begin{array}{l}
\left.a_{n}\left(f\left(r_{p}, s\right), S t, \chi\right) \cos \left(n\left(\theta_{p}+g\left(r_{p}, s, \sigma\right)\right)\right)\right) \\
\left.+b_{n}\left(f\left(r_{p}, s\right), S t, \chi\right) \sin \left(n\left(\theta_{p}+g\left(r_{p}, s, \sigma\right)\right)\right)\right)
\end{array}\right) \\
& \hat{\xi}^{\prime}\left(s_{p}\right) e^{i 2 \pi S t s_{p}}=I_{o}\left(r_{p}, S t, \chi\right)+\sum_{n}\left(I_{n}^{c}\left(r_{p}, \theta_{p}, \sigma, S t, \chi\right)+I_{n}^{s}\left(r_{p}, \theta_{p}, \sigma, S t, \chi\right)\right) \\
& I_{o}\left(r_{p}, S t, \chi\right)=\int_{s=0}^{s=s_{p}\left(r_{p}\right)} a_{o}\left(f\left(r_{p}, s\right), S t, \chi\right) e^{i 2 \pi S t s} d s
\end{aligned}
$$

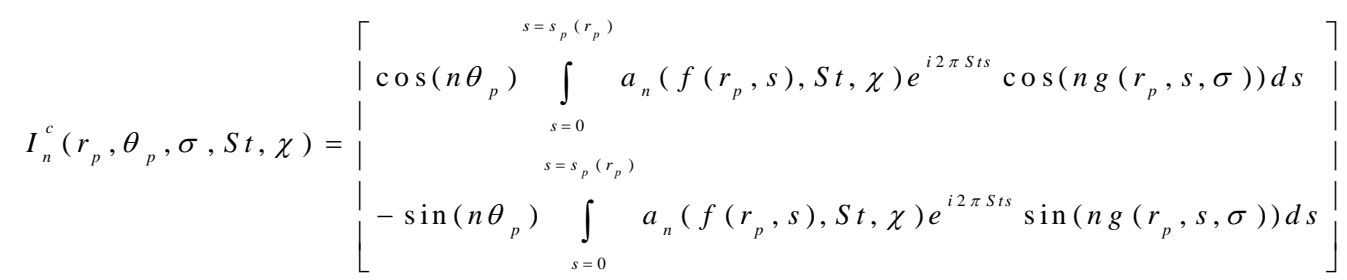

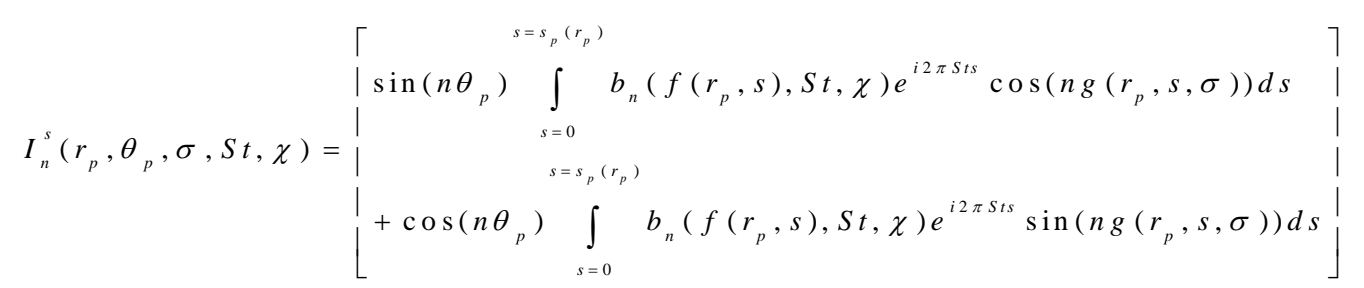

The solution can be written as:

$$
\hat{\xi}^{\prime}(r, \theta)=H_{o}(r, S t, \chi)+\sum_{n}\left(\cos (n \theta) H_{n}^{c}(r, \sigma, S t, \chi)+\sin (n \theta) H_{n}^{s}(r, \sigma, S t, \chi)\right)
$$

where

$$
\begin{aligned}
& H_{o}(r, S t, \chi)=e^{-i 2 \pi S t s_{p}(r)} \int_{s=0}^{s=s_{p}(r)} a_{o}(f(r, s), S t, \chi) e^{i 2 \pi S t s} d s \\
& H_{n}^{c}(r, \sigma, S t, \chi)=e^{-i 2 \pi S t s_{p}(r)}\left|\begin{array}{ll}
\int_{s=s_{p}(r)} a_{n}(f(r, s), S t, \chi) e^{i 2 \pi s t s} \cos (n g(r, s, \sigma)) d s \\
\vdots=0 \\
s=s_{p}(r)
\end{array}\right|
\end{aligned}
$$

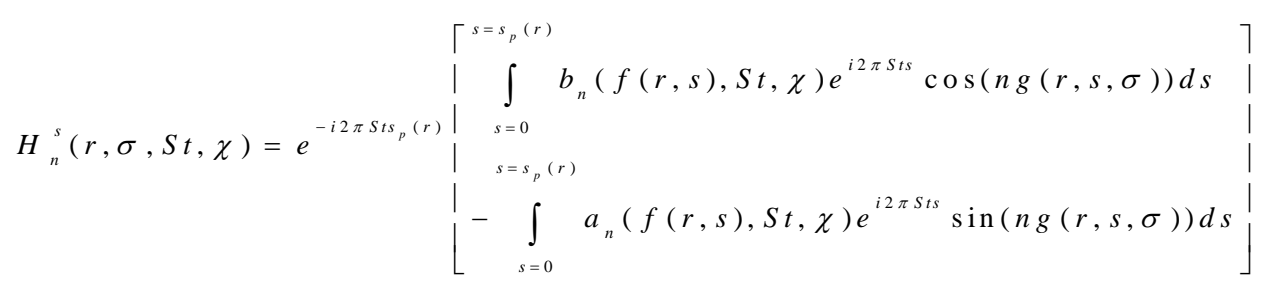


The linearized global flame area (from Eq.(256)) takes the form:

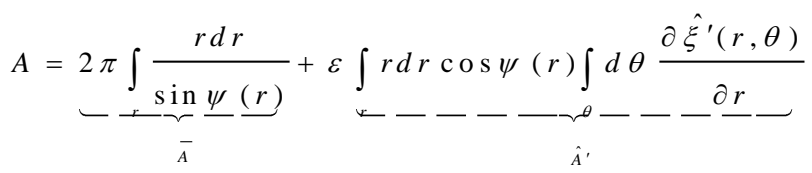

From Eq.(266) and Eq.(267), the integrand of the second term is:

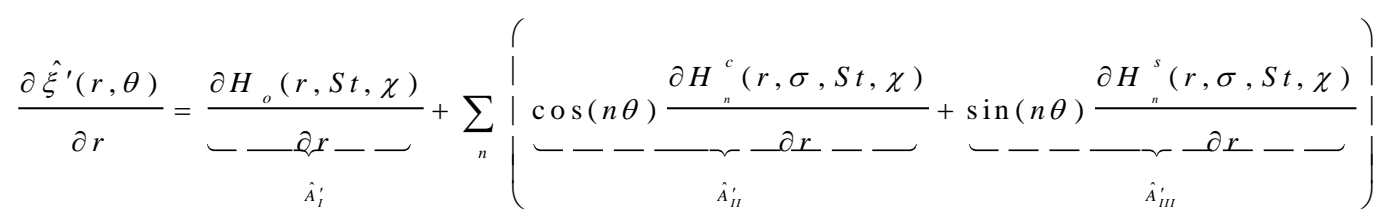

As mentioned in Eq.(257), we can split this integral into three parts as shown above:

$$
\begin{aligned}
& \hat{A}_{I}^{\prime}(S t, \chi)=\int_{r} r d r \cos \psi(r) \frac{\partial H_{o}(r, S t, \chi)}{\partial r} \int_{\theta=0}^{\theta=2 \pi} d \theta=2 \pi \int_{r} r d r \cos \psi(r) \frac{\partial H_{o}(r, S t, \chi)}{\partial r} \\
& \hat{A}_{I I}^{\prime}(\sigma, S t, \chi)=\int_{r} r d r \cos \psi(r) \frac{\partial H_{n}^{c}(r, \sigma, S t, \chi)}{\partial r} \int_{\theta=0}^{\theta=2 \pi} d \theta \cos (n \theta)=0 \\
& \hat{A}_{I I I}^{\prime}(\sigma, S t, \chi)=\int_{r} r d r \cos \psi(r) \frac{\partial H_{n}^{s}(r, \sigma, S t, \chi)}{\partial r} \int_{\theta=0}^{\theta=2 \pi} d \theta \sin (n \theta)=0
\end{aligned}
$$

Hence,

$$
\hat{A}^{\prime}=\hat{A}_{I}^{\prime}(S t, \chi)+\hat{A}_{I I}^{\prime}(\sigma, S t, \chi)+\hat{A}_{I I I}^{\prime}(\sigma, S t, \chi)=2 \pi \int_{r} r d r \cos \psi(r) \frac{\partial H_{o}(r, S t, \chi)}{\partial r}
$$

and the Flame Transfer Function (FTF) is equal to the ratio of area fluctuations and the mean flame area when the flame speed, density and heat release are constant, and is given by:

$$
F T F=\frac{\hat{A}^{\prime}}{\bar{A}}=\frac{\int_{r} r d r \cos \psi(r) \frac{\partial H_{o}(r, S t, \chi)}{\partial r}}{\int_{r} \frac{r d r}{\sin \psi(r)}}
$$

These latter two formulations for flame area are quite important, as they show that the global flame response, represented by the global fluctuating flame area, is independent of azimuthal flow velocity (i.e., swirl). In the general expression shown in Eq.(257), we can see that the effect of swirl is present in all three terms. In Eq.(270), we can see that for axisymmetric flames, the 
terms are further simplified. The first term is not a function of swirl since the radial characteristic function is independent of the azimuthal characteristics. The second and third terms contain the effect of swirl locally. However, this dependence on swirl is independent from the azimuthal functions, as shown in Eq.(266) and hence in Eq.(269). Thus, swirl effects cancel out in the azimuthal integration. Note that, this is true only for axisymmetric flames with constant burning velocity, in the linear regime.

For the purpose of illustration, consider an axially uniform mean velocity field, and a solid body azimuthal field, given by:

$$
\bar{U}_{r}=0 ; \bar{U}_{\theta}=2 \pi S t(\Omega / \omega) r=2 \pi S t \sigma r ; \bar{U}_{z}=1
$$

Note that the angular rotation rate of the mean flow field is then given by $\Omega$ and the dimensionless rotation rate by $\sigma=\Omega / \omega$. The mean flame shape from Eq.(258) is then:

$$
\bar{\xi}(r)=(r-R) \cot \psi
$$

Equation (260) takes the form:

$$
i 2 \pi S t \hat{\xi}^{\prime}+\left(s_{L} \cos \psi\right) \frac{\partial \hat{\xi}^{\prime}}{\partial r}+2 \pi S t \sigma \frac{\partial \hat{\xi}^{\prime}}{\partial \theta}=\hat{F}(r, \theta, S t)
$$

Transforming to characteristic co-ordinates as shown in Eq.(261), we have:

$$
\begin{aligned}
& d s=\frac{d r}{s_{L} \cos \psi} \Rightarrow r=\underbrace{R+s_{L^{\prime}} \cos \underline{\psi} s}_{f} \Rightarrow s_{p}(r)=\left(\frac{r_{p}-R}{s_{L} \cos \psi}\right)=\tilde{r} \\
& d s=\frac{d \theta}{2 \pi S t \sigma} \Rightarrow \theta=\theta_{p}+\underbrace{2 \pi \operatorname{St\sigma }\left(s-s_{p}\right)}_{g}
\end{aligned}
$$

These characteristic functions are used later with specific disturbance fields to extract possible flame shapes. Note, that $\tilde{r}$ is used only for convenience of notation. Using Eq.(273) in Eq.(262), we obtain the following expression for the linear spiral propagation path of disturbances from the flame base:

$$
r=\left(\frac{s_{L} \cos \psi}{2 \pi S t \sigma}\right) \theta
$$




\section{i. Uniform Transverse Velocity Results (Compact Flame)}

Now we shall detail results for disturbance fields that are compact and non-compact respectively, with respect to the flame length scale. In order to explicitly determine the flame position, the disturbance field characteristics must also be specified. An important limit is a uniformly transverse velocity field. This corresponds to the $\chi=0$ limit:

$$
u_{x}^{\prime}(t, r, \theta)=\sin (2 \pi \mathrm{St} t) \Rightarrow \hat{F}(r, \theta, S t)=i \cot \psi \cos \theta
$$

This field simulates the disturbance characteristics of a flame to a long wavelength acoustic perturbation, such as occurs in an annular combustor. This expression explicitly shows the nonaxisymmetric character of the excitation, leading to the following:

$$
\begin{aligned}
& a_{o}=0, a_{1}=i \cot \psi \\
& a_{n \geq 2}=0, b_{n \geq 1}=0
\end{aligned}
$$

Substituting into Eq.(267) we have:

$$
\begin{aligned}
& H_{o}=0 \\
& H_{1}^{c}=\frac{i \cot \psi}{2 \pi S t\left(\sigma^{2}-1\right)}\left[i-e^{-i 2 \pi \operatorname{sir}}\{i \cos (2 \pi S t \sigma \tilde{r})-\sigma \sin (2 \pi S t \sigma \tilde{r})\}\right] \\
& H_{1}^{s}=\frac{i \cot \psi}{2 \pi S t\left(\sigma^{2}-1\right)}\left[\sigma-e^{-i 2 \pi \operatorname{sir}}\{i \sin (2 \pi S t \sigma \tilde{r})+\sigma \cos (2 \pi S t \sigma \tilde{r})\}\right]
\end{aligned}
$$

Hence, the Fourier domain solution from Eq.(266) is given by:

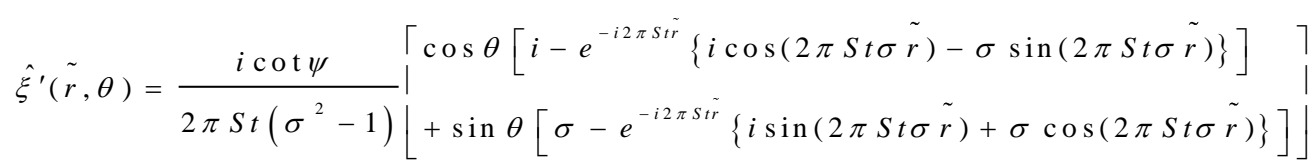

The three components of the area, from Eq.(270) and Eq.(280), are:

$$
\left.\begin{array}{l}
\hat{A}_{I}^{\prime}(S t, \chi)=2 \pi \int_{r} r d r \cos \psi(r) \frac{\partial H_{o}(r, S t, \chi)}{\partial r}=0 \\
\hat{A}_{I I}^{\prime}(\sigma, S t, \chi)=0, \hat{A}_{I I}^{\prime}(\sigma, S t, \chi)=0
\end{array}\right\} \Rightarrow \hat{A}^{\prime}=0
$$

Hence, despite a non-zero flame fluctuation (Eq.(281)), the global flame response is identically zero - regardless of frequency or swirl number. The corresponding representation of Eq.(281) in the time domain is: 


$$
\xi^{\prime}(\tilde{r}, \theta, t)=\frac{\cot \psi}{2}\left\lfloor\frac{\cos (2 \pi \mathrm{St} t+\theta)-\cos \left(2 \pi \mathrm{St} t+\theta-\tau_{+} \tilde{r}\right)}{\tau_{+}}\right]
$$

where $\tau_{ \pm}=2 \pi S t(1 \pm \sigma)$. This equation may also be written in the spatially modulated amplitude form:

$$
\xi^{\prime}(\tilde{r}, \theta, t)=W(\tilde{r}, \theta) \cos (2 \pi \mathrm{S} \mathrm{t} t+\gamma(\tilde{r}, \theta))
$$

where the amplitude and phase of flame wrinkles, $W$ and $\gamma$, are given by:

$$
\begin{gathered}
W(\tilde{r}, \theta)=\cot \psi\left[\frac{1}{\tau_{+}^{2}} \sin ^{2} \frac{\tau_{+} \tilde{r}}{2}+\frac{1}{\tau_{-}^{2}} \sin ^{2} \frac{\tau_{-} \tilde{r}}{2}+\frac{2}{\tau_{+} \tau_{-}} \sin \frac{\tau_{+} \tilde{r}}{2} \sin \frac{\tau_{-} \tilde{r}}{2} \cos (2 \theta-2 \pi \sigma \tilde{r})\right]^{1 / 2} \\
\tan [\gamma(\tilde{r}, \theta)]= \\
\frac{\frac{1}{\tau_{+}} \cos \left(\theta-\frac{\tau_{+} \tilde{r}}{2}\right) \sin \left(\frac{\tau_{+} \tilde{r}}{2}\right)+\frac{1}{\tau_{-}} \cos \left(\theta+\frac{\tau_{-} \tilde{r}}{2}\right) \sin \left(\frac{\tau_{-} \tilde{r}}{2}\right)}{\tau_{+} \sin \left(\theta-\frac{\tau_{+} \tilde{r}}{2}\right) \sin \left(\frac{\tau_{+} \tilde{r}}{2}\right)-\frac{1}{\tau_{-}} \sin \left(\theta+\frac{\tau_{-} \tilde{r}}{2}\right) \sin \left(\frac{\tau_{-} \tilde{r}}{2}\right)}
\end{gathered}
$$

The apparent convection speed of wrinkles measured at a fixed azimuthal position, $\theta$, is modified due to the interference patterns created by the swirl transport component. This apparent convection speed is obtained from the slope in a plot of the phase roll-off, shown later.

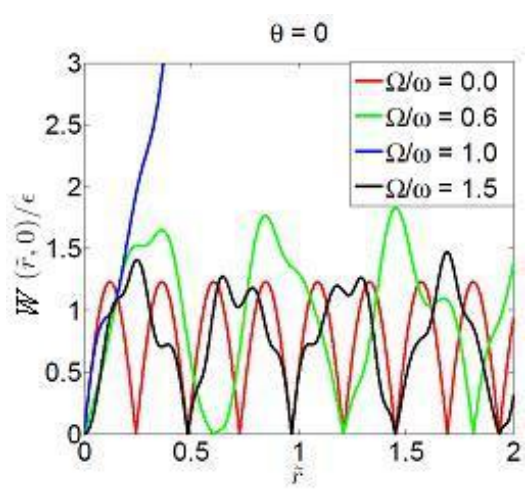

(a)

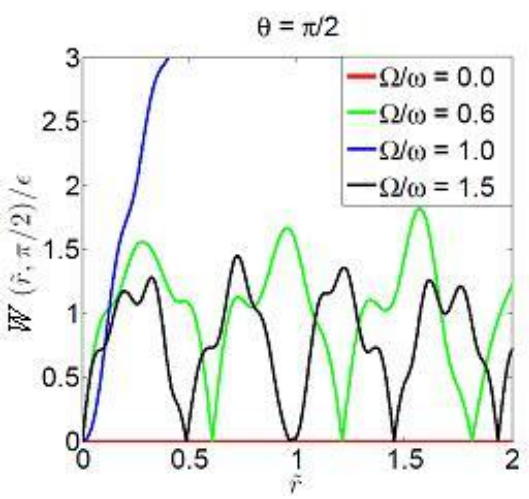

(b)

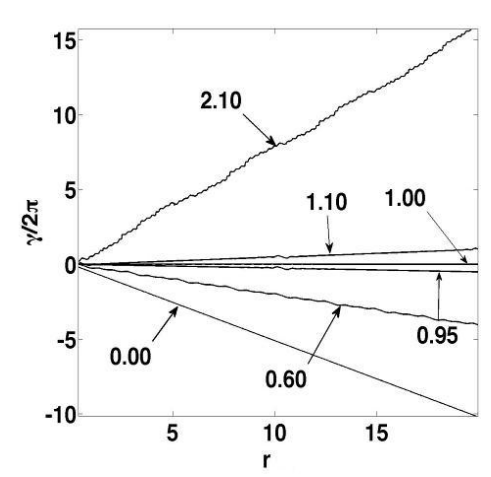

(c)

Figure 47: Amplitude variation along the flame for different values of $\sigma=\Omega / \omega$, see Eq.(285), with downstream radial distance at (a) $\theta=0$, (b) $\theta=\pi / 2$; (c) Phase variation for different values of $\sigma=\Omega / \omega$., see Eq.(286), with downstream radial distance at $\theta=0, \pi$. 
The presence of transverse excitation introduces non-axisymmetric wrinkling on the flame surface, as illustrated in Figure 46(a). In the absence of swirl, these non-axisymmetric wrinkles convect axially downstream, and the qualitative features of the flame response are essentially the same as that considered in prior analyses; they are simply not axisymmetric. The superposition of swirl onto a non-axisymmetric problem introduces fundamentally new features for the flame response. The rest of this section presents flame results for a range of dimensionless swirl numbers, $\sigma$.

We start first with the no swirl case, $\sigma=0.0$, as a baseline case. Figure 48(a) illustrates a 3-D view of the instantaneous flame surface, as well as a slice of the flame surface at $\theta=0$ and $\pi$. Notice that that even in the absence of swirl, the flame wrinkling is non-axisymmetric. There is no wrinkling at $\theta=\pi / 2$ and $3 \pi / 2$ and the wrinkles are out of phase at $\theta=0$ and $\pi$. The latter can be seen from Figure 48(b). The phase of the flame wrinkling is given by Eq.(286) for $\theta=0$ and $\pi$, is shown in Figure 47(c). Note that, the phase decreases downstream, corresponding to downstream motion of the wrinkles. The spatial dependence of the amplitude of flame wrinkling, ' $W$ ', is shown in Figure 47(a) and Figure 47(b). Note the spatially modulated character of the flame wrinkling amplitude, due to interference between wrinkles excited at the flame attachment point and by the disturbance.

We next consider a higher swirl case, $\sigma=0.6$. An image of the flame is shown in Figure 49(a), which shows that the flame is wrinkled at the grazing incidence angles, $\theta=\pi / 2$ and $3 \pi / 2$, even though the normal perturbation field is zero (see Eq.(278)). This occurs because swirl rotates wrinkles created at other azimuthal angles into these planes. This image clearly shows a more complex interference pattern along the flame sheet. This can also be seen from the flame wrinkling amplitude, $W$, shown in Figure 47(a) and Figure 47(b). Note also the change in periodicity of the nodes in flame response from this figure. The axial cut of flame wrinkling is shown in Figure 49(b). For reference, the light dashed line shows the baseline $\sigma=0.0$ case at the same instant of time. Note that there are multiple scales of wrinkling and that the effective wrinkling wavelength has increased relative to the $\sigma=0.6$ case. Furthermore, Figure $47(\mathrm{c})$ shows that the rate of axial phase roll-off is shallower than that of the baseline case, indicating a faster "effective" axial convection speed of this cut. It is important to emphasize, however, that the rate of phase roll-off corresponds to a trace velocity, not a group velocity, since it is obtained from an azimuthal cut in which wrinkles are rotating into and out of. Recall that the trace velocity does not correspond to the propagation speed of a disturbance and can be positive, negative, and have infinite values for finite propagation speed disturbances. This result has important implications on interpretations of sheet imaging of swirling flames, such as Mie scattering or planar laser induced fluorescence (PLIF), as it shows that the axial phase speed of disturbances along these cuts should not be interpreted as physical convection velocities.

We next consider $\sigma=0.95$, a value less than unity but very close, corresponding to near frequency matching. As shown in Figure 50(a), the flame surface exhibits a much larger scale helical motion. This longer wrinkling wavelength can also be seen in Figure 50(b), by comparing this case to the no swirl result. Moreover, Figure 47(c) shows that the phase roll-off rate is nearly flat, corresponding to a very high axial trace velocity of the wrinkle. 


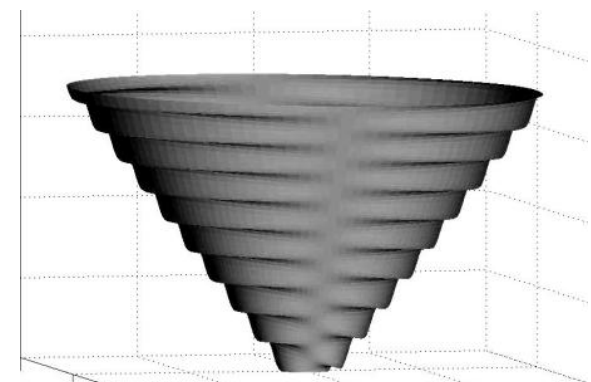

(a) 3D Visualizations

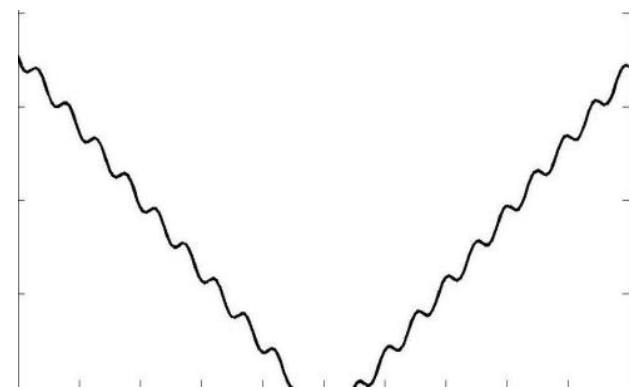

(b) Flame branches at $\theta=0, \pi$

Figure 48: Flame shape visualizations for $\psi=14^{0}, \Omega / \omega=0.00$ at $t=0.3$; (a) 3D flame surface, (b) Flame branches at $\theta=0, \pi$.

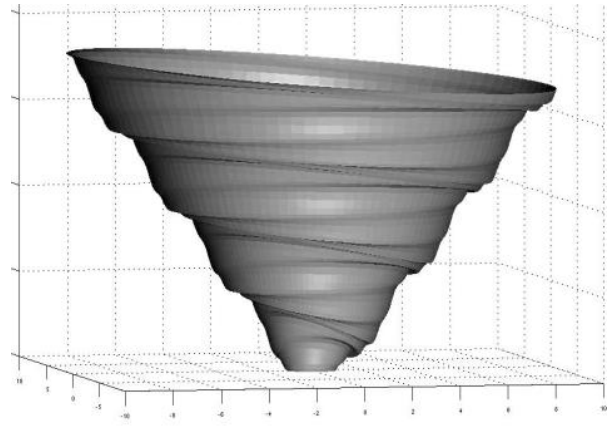

(a) 3D Visualizations

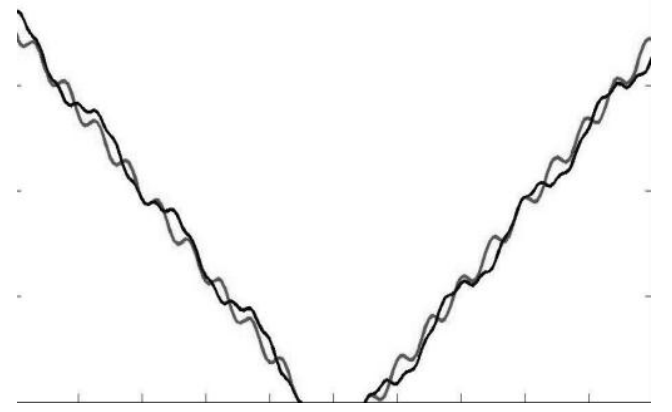

(b) Flame branches at $\theta=0, \pi$

Figure 49: Flame shape visualizations for $\psi=14^{0}, \Omega / \omega=0.60$ at $t=0.3$; (a) 3D flame surface, (b) Flame branches at $\theta=0, \pi$; black : $\Omega / \omega=0.60$, gray : $\Omega / \omega=0.00$.

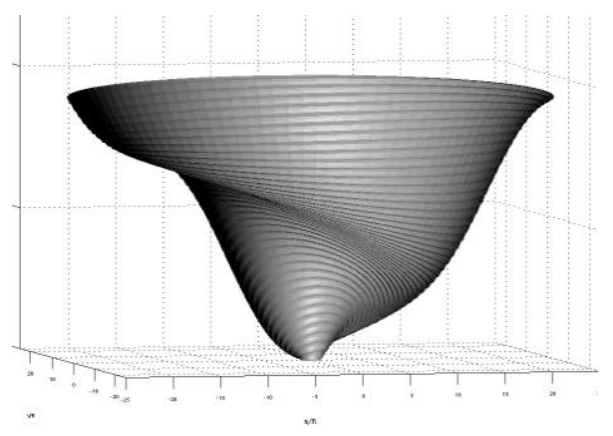

(a) 3D Visualizations

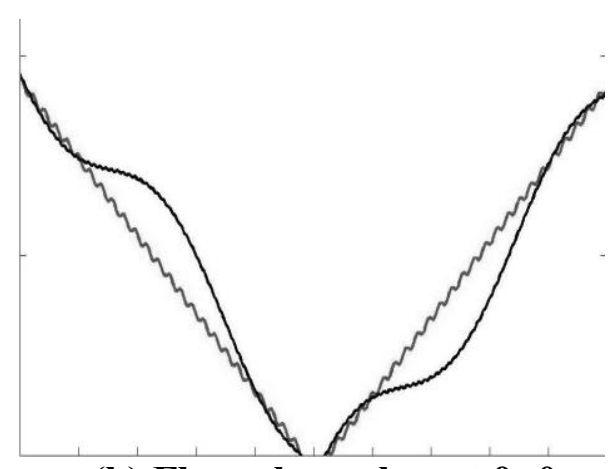

(b) Flame branches at $\theta=0, \pi$

Figure 50: Flame shape visualizations for $\psi=14^{0}, \Omega / \omega=0.95$ at $t=0.3$; (a) 3D flame surface, (b) Flame branches at $\theta=0, \pi$; black : $\Omega / \omega=0.95$, gray : $\Omega / \omega=0.00$. 
We next consider the frequency matching case, $\sigma=1.0$. As shown in Figure 51(a), there is no periodic wrinkling on the flame surface. Rather the flame exhibits a solid body rotation of the tilted conical flame surface. The degree of off-axis rotation is controlled by the excitation amplitude. As shown in Figure 47(b), the phase-axial dependence is flat. These results show that as the swirl velocity is increased, or the excitation frequency decreased, that the scale of flame wrinkling increases up to an infinite value. This is also manifested through the phase of axial cuts, which as shown in Figure 47(c), monotonically decrease toward zero. The amplitude, $A$, does not oscillate but grows monotonically with downstream distance, as illustrated in Figure 47(a) and Figure 47(b).

We next consider a dimensionless rotation rate which is greater than unity, $\sigma=1.1$, see Figure 52(a). Note that the presence of finite-scale flame wrinkling is present again. For $\sigma<1$ cases, the axial cuts of flame wrinkling propagated downstream. However, as shown in Figure 47(c), the phase now increases with downstream distance; i.e., the apparent direction of motion of wrinkles is now upstream. It is important to note that the flame wrinkles are not actually moving backward. Rather, they are moving along the characteristic curves given by Eq.(277) at a velocity of $\overrightarrow{\bar{u}}_{t}$. As noted earlier, wrinkle motion along these axial cuts of the flame should be interpreted as trace velocities, not as convection speeds of actual flame wrinkles. Returning to Figure 47(c), this positive phase-axial distance behavior is analogous to the phenomenon of aliasing in spectral analysis, where the wrinkle is rotating through the axial cut faster than the period of oscillation. A familiar example is that of the apparent backward rotation rate of a vehicles tire rims, despite the cars forward motion.

We last consider an even faster rotation rate, $\sigma=2.1$. As shown in Figure 53(a), the wrinkling wavelength has further decreased. Figure 47(c) shows that the slope of the phase continues to increase. Thus, we can see that beyond the frequency matching condition, the wrinkling wavelength decreases monotonically.

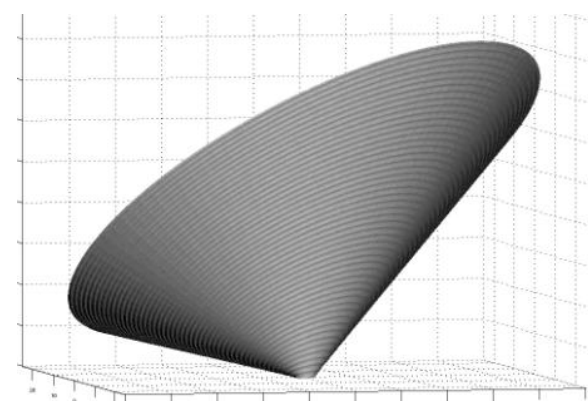

(a) 3D Visualizations

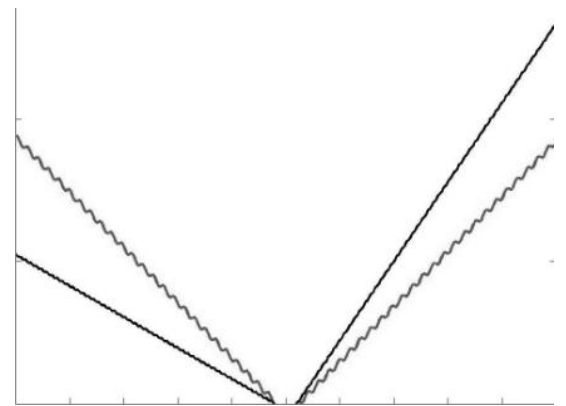

(b) Flame branches at $\theta=0, \pi$

Figure 51: Flame shape visualizations $\psi=14^{0}, \Omega / \omega=1.00$ at $t=0.3$; (a) 3D flame surface, (b) Flame branches at $\theta=0, \pi$; black : $\Omega / \omega=1.00$, gray : $\Omega / \omega=0.00$. 


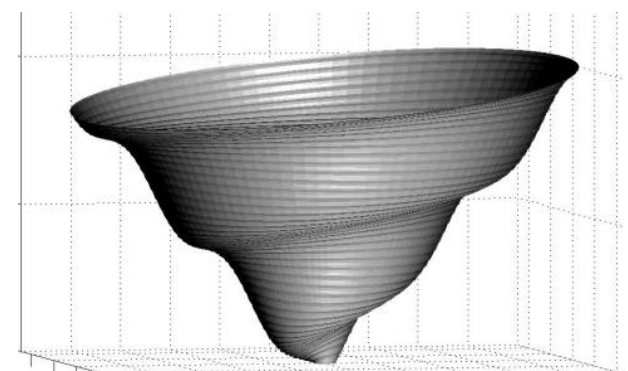

(a) 3D Visualizations

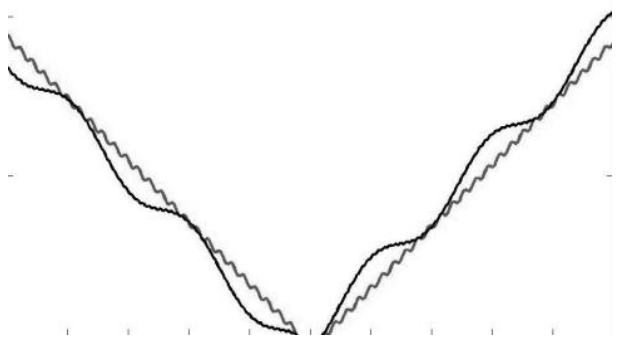

(b) Flame branches at $\theta=0, \pi$

Figure 52: Flame shape visualizations for $\psi=14^{\circ}, \Omega / \omega=1.10$ at $t=0.3$; (a) 3D flame surface, (b) Flame branches at $\theta=0, \pi$; black : $\Omega / \omega=1.10$, gray : $\Omega / \omega=0.00$.

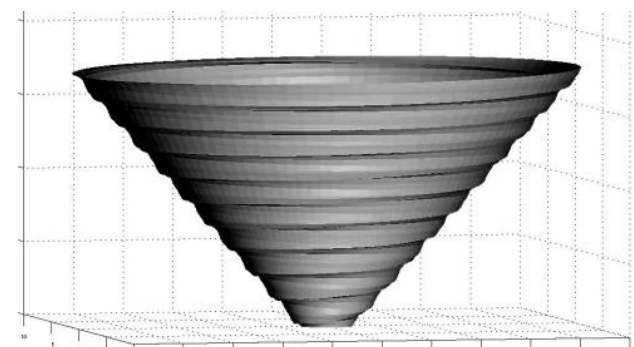

(a) 3D Visualizations

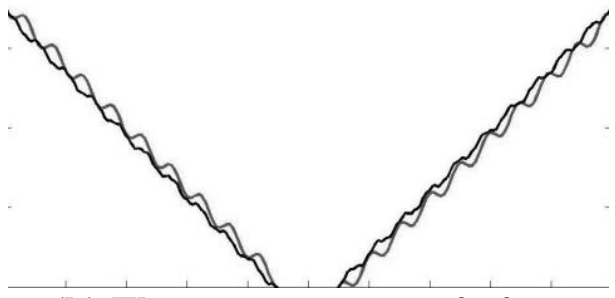

(b) Flame branches at $\theta=0, \pi$

Figure 53: Flame shape visualizations for $\psi=14^{\circ}, \Omega / \omega=2.10$ at $t=0.3$; (a) 3D flame surface, (b) Flame branches at $\theta=0, \pi$; black : $\Omega / \omega=2.10$, gray : $\Omega / \omega=0.00$.

\section{ii. $\quad$ Non-Compact Flame Results}

Having considered some basic features of the flame wrinkling field in the uniformly transverse velocity field limit, we next consider a generalization of this field corresponding to a finite disturbance length scale. The perturbation field is given by:

$$
u_{x}^{\prime}=\sin (2 \pi \mathrm{St} t-2 \pi \chi x) \Rightarrow \hat{F}(r, \theta, S t, \chi)=i \cot \psi \cos \theta e^{-i 2 \pi \chi r \cos \theta}
$$

Where $\chi$, as mentioned before is a non-compactness parameter, $\chi=L_{f} / \lambda_{a}$. Note that, $\chi=S t . M$, where $M$ is the Mach number of the incoming axial flow. The compact flame disturbance field is a special case of this disturbance field. Unlike the compact case where flame branches on either side of the axis (along the flow and perpendicular to forcing axis) would encounter the same disturbance but of opposite sign, the non-compact case introduces an additional degree of freedom to the disturbance field. The disturbance field can be decomposed into its Fourier components using the Jacobi-Anger expansion:

$$
e^{-i 2 \pi \chi r \cos \theta}=J_{0}(-2 \pi \chi r)+\sum_{n=1}^{\infty} i^{n} J_{n}(-2 \pi \chi r) \cos (n \theta)
$$


where $J_{n}(\mathrm{n} \geq 0)$ are Bessel functions of the first kind. Using, Eq.(287) - (288) in Eq.(249) and Eq.(267), we have:

$$
\begin{aligned}
& a_{0}=i \cot \psi \frac{i J_{1}(-2 \pi \chi r)}{2} \\
& a_{n \geq 1}=i \cot \psi\left\lceil\frac{i^{n-1} J_{n-1}(-2 \pi \chi r)}{2}+\frac{i^{n+1} J_{n+1}(-2 \pi \chi r)}{2}\right\rfloor \\
& b_{n \geq 1}=0 \\
& H_{o}(\tilde{r}, S t, \chi)=-\frac{1}{2} \cot \psi e^{-i 2 \pi S t s \tilde{r}} \int_{s=0}^{s=\tilde{r}} J_{1}(\tilde{s}) e^{i 2 \pi s t s} d s \\
& H_{n}^{c}(\tilde{r}, \sigma, S t, \chi)=\frac{i^{n} \cot \psi}{2} e^{-i 2 \pi \operatorname{sir}}\left[\int_{s=0}^{s=\tilde{r}}\left(J_{n-1}(\tilde{s})-J_{n+1}(\tilde{s})\right) e^{i 2 \pi s t s} \cos (2 \pi S t \sigma n(s-\tilde{r})) d s\right\rfloor \\
& H_{n}^{s}(\tilde{r}, \sigma, S t, \chi)=-\frac{i^{n} \cot \psi}{2} e^{-i 2 \pi s t r i}\left[\int_{s=0}^{s=r}\left(J_{n-1}(\tilde{s})-J_{n+1}(\tilde{s})\right) e^{i 2 \pi S t s} \sin (2 \pi S t \sigma n(s-\tilde{r})) d s\right\rfloor \\
& \tilde{s}=-2 \pi \chi f(s)
\end{aligned}
$$

Many of the solution features discussed above for the $\chi=0$ case remain similar for this case. One key difference is the spatially integrated area fluctuations. In the $\chi=0$ case, there is no net fluctuation in flame area. In contrast, area fluctuations do occur in the non-compact case. Using Eq.(272) and Eq.(290), the expression for the FTF is:

$$
\begin{aligned}
& \left.\frac{\hat{A}^{\prime}}{\bar{A}}=\frac{2 \cos \psi}{s_{L}\left(R_{\max }^{2}-R^{2}\right)} \mid s_{L} \cos \psi \hat{A}_{o}\left(R_{\text {max }}\right)\left(R_{\text {max }}+\frac{s_{L} \cos \psi}{i 2 \pi S t}\right) \exp \left(-\frac{i 2 \pi S t}{s_{L} \cos \psi}\left(R_{\text {max }}-R\right)\right)\right\} \\
& \text { w here } \\
& \hat{A}_{o}\left(R_{\max }\right)=\exp \left(-\frac{i 2 \pi S t R}{s_{L} \cos \psi}\right) \int_{r=R}^{r=R_{\max }}\left[J_{1}(2 \pi \chi r) \exp \left(-\frac{i 2 \pi S t r}{s_{L} \cos \psi}\right)\right\rfloor d r
\end{aligned}
$$

The expression in Eq.(291) indicates the presence of two different scales of interference in the Strouhal number space. One that is due to an exponential term and another that is due to a Bessel term. This is primarily due to the unique spatial distribution of the non-compact transverse forcing as seen by the conical flame surface. These aspects are depicted in Figure 54. As shown earlier in Eq.(270), there is no effect of swirl on the FTF gain, since the flame is nominally axisymmetric. Swirl has important local effects by redistributing flame surface area, but no global area effects within the assumptions of linearity, constant burning velocity, axisymmetric mean flames. 


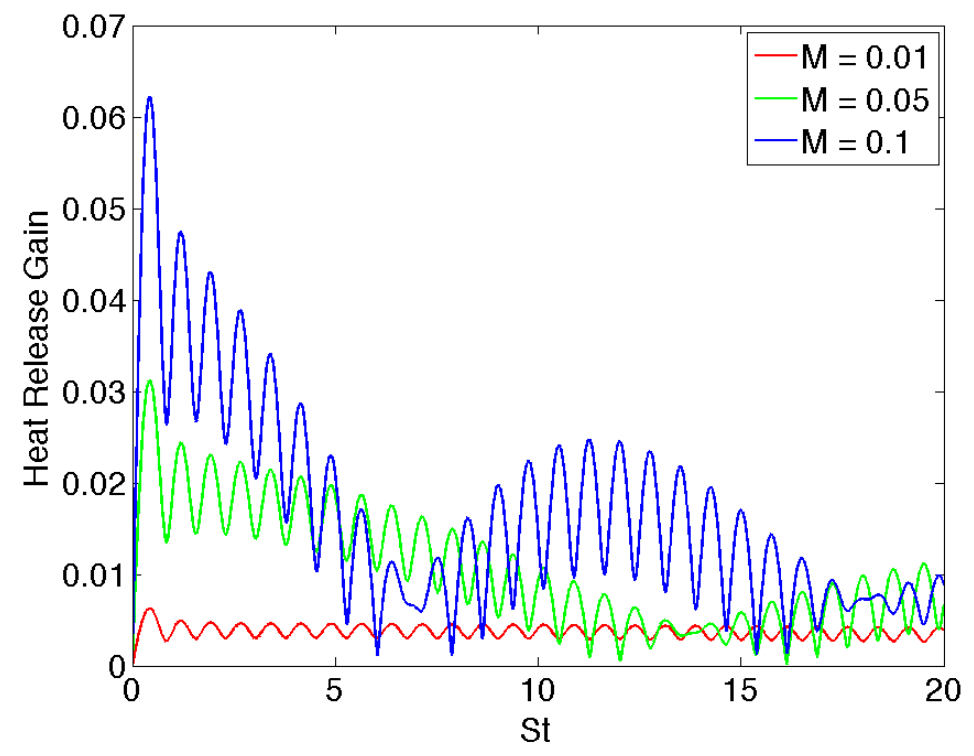

Figure 54: Flame Transfer function gain for a nominally axisymmetric flame in a noncompact transverse disturbance field. The non-compactness is controlled by the Mach Number.

\section{Effects of Non-Axisymmetries}

Having given some general results and presented illustrative calculations for axisymmetric flames, we conclude this section of the report with a brief discussion of the effect of nonaxisymmetries in mean flame shape. In this section, we shall focus on the effects of nonaxisymmetries by means of comparison with the discussion in the previous section. The Eqs.(241) - (257), essentially correspond to that of a non-axisymmetric flame. The effect of nonaxisymmetries is both implicit and explicit. The explicit effect of non-axisymmetric mean flame stems from the last term in the RHS of Eq.(259). For a given transverse velocity fluctuation, the axisymmetric flame is affected only by the radial component, whereas in case of nonaxisymmetric flames, there is an additional source of wrinkling due to the azimuthal component, resulting in a net higher wrinkling amplitude for non-axisymmetric flames. The implicit effect of swirl is through the wave terms on the LHS which contain the non-axisymmetric flow field components. Since, the local response was shown to depend on swirl irrespective of the nature of the flame; this qualitative aspect shall not be discussed here. The effect of non-axisymmetries shall be addressed in the global flame response perspective. In the discussion for axisymmetric flames, we made a critical deduction in terms of the characteristic functions, as shown in Eq. (276). Note that this is specific only to axisymmetric flames and is not possible in case of nonaxisymmetric flames. In case of axisymmetric flames, we showed that the radial characteristic function $f$ was independent of the azimuthal characteristics.

But, in case of non-axisymmetries, this does not happen because both characteristic equations are coupled and hence form an interdependent characteristic function set. In such a situation, the azimuthal characteristic function $g$ cannot be decomposed as shown in Eq.(276). This 
decomposition was essential in deducing swirl independence in Eq.(270) and Eq.(271). The global flame area calculation was decomposed into three terms, as shown in Eq.(257). The corresponding terms for the axisymmetric case are shown in Eq.(269). Note that the 'zeroth' order Fourier term is axisymmetric for the axisymmetric flame and as shown in Eq.(271), this term is the only contribution to any non-zero global flame response, as is independent of the swirl parameter $\sigma$. On the contrary, the same term is neither axisymmetric nor independent of swirl, as can be seen from Eq.(253). The primary reason for this non-axisymmetry is the coupling of the characteristic functions. This leads to an effect of swirl from the first term in the area decomposition. The second and third area terms are shown in Eq.(270), and are seen to be zero for the axisymmetric case. This occurs due to the integral of the cosine and sine azimuth functions. These separate functions stem from their decomposition in Eq.(265), which is possible only for the axisymmetric case. In case of non-axisymmetries, the characteristic function for the azimuth does not warrant an independent sine or cosine function and hence need not give a zero contribution from these terms. This leads to a non-zero dependence on swirl. However, there can be special cases where, the second and third term integrals can still be zero.

The flame wrinkling and hence its response are a resultant of the interference between radial tangential waves and the swirling azimuthal waves. In case of axisymmetric flames, the azimuthal variables and parameters are not of global importance, since there is no azimuthal bias. The lack of such bias implies any azimuthal integral results in cancellation of the effects of these waves. Hence, any circular flame surface element gives no response. However, such bias is present in non-axisymmetric flames, and can hence give a non-zero azimuthal integral, which translates to a dependence on swirl.

\section{Review}

In review, here we showed the effects of swirl on a transversely forced flame, which introduces an additional degree of freedom for wrinkle motion. The presence of swirl superposes with the upstream axial velocity, thereby creating a helical spiral motion of the wrinkles. Additionally, this transport leads to distinctive interference patters on the flame surface that changes the effective wrinkling wavelength. The flame surface wrinkling also showed a strong sensitivity to the ratio between the swirling time scale and the acoustic excitation time scale, specifically seen in the apparent direction of wrinkle motion. In case of axisymmetric flows, swirl was seen to have a local effect only. The global flame dynamics are independent of swirl regardless of the acoustic forcing mechanism, in the linear regime. The effects of non-linearity and quantitative results for non-axisymmetric nominal flame shapes are part of the ongoing research work and shall shed more light on the qualitative role of swirl in more realistic flames. 


\section{Swirl Effects on Transversely Forced Premixed Flame Dynamics - Extended Analysis}

\section{Introduction}

As a nice complement to the preceding work, here we describe the response of an axisymmetric swirling premixed flame with constant burning velocity to harmonic excitation. The wrinkles are excited on the flame that propagate both axially and azimuthally, at speeds $U_{o} \cos \psi$ and $U_{\theta}$, where $U_{o}$ is the mean flow velocity, $\psi$ is the flame angle and $U_{\theta}$ is the swirling velocity component. As such, the flame response at any given position is a superposition of flame wrinkles excited at earlier times, upstream axial locations, and different azimuthal positions. These swirl transport effects do not show up in problems where axisymmetric flames are subjected to axisymmetric excitation, but enter quite prominently in the presence of nonaxisymmetries, such as when the flame is subjected to transverse excitation. Prior work has shown that the solution characteristics are strongly dependent upon the ratio of angular rotation rate to excitation frequency, denoted by $\sigma=\Omega / \omega$, which describes the fraction of azimuthal rotation a disturbance makes in one acoustic period. The axial wavelength and phase roll-off were seen to be strong functions of $\sigma$. While the effects of swirl are quite prominent upon the local flame characteristics, their global influence upon the spatially integrated, linearized unsteady heat release depends upon an acoustic compactness parameter, $L_{f} / \lambda_{a}$, where $L_{f}$ denotes the flame length. In the $L_{f} / \lambda_{a} \sim 0$ limit (compact flames), swirl redistributes flame area, but exerts no global influence. For non-compact axisymmetric flames, the non-zero global influence while dependent on $L_{f} / \lambda_{a}$, is seen to be independent of the swirl parameter, $\sigma$. This result however, is not true for general non-axisymmetric flames.

Amongst these mechanisms, this paper focuses on perturbations in the flow velocity, specifically transverse flow oscillations and the subsequent evolution of premixed flames in a swirling upstream flow. Transverse oscillations have been a key instability concern in rockets and afterburners for decades. More recently, transverse oscillations have grown problematic in low $\mathrm{NO}_{\mathrm{x}}$ gas turbines, both in ground-based and aero-engine applications. These transverse oscillations generally occur at lower frequencies (i.e., 100's of $\mathrm{Hz}$ ) in annular combustion chambers and at higher frequencies (i.e., 1000's of Hz) in can combustion chambers. As shown in prior work, transverse excitation introduces new degrees of freedom into this problem because of its intrinsically non axisymmetric nature. While longitudinal disturbances are generally axisymmetric, or at least approximately so, transverse disturbances excite the flame in an intrinsically non-axisymmetric manner, as shown in Figure 46(a). This causes significant azimuthal variations in local disturbance field at the flame sheet. The volumetric flow rate through the flame, $\sigma^{\circ}$, which is directly related to the flame's spatially integrated low Strouhal number response, is of the same sign at all azimuthal locations for longitudinal forcing, as shown in Figure 46(b), but of opposite sign in the case of transverse forcing, as shown in Figure 46(c). 


\section{Formulation}

For this work we utilized the following form of the non-dimensional flame from tracking equation:

$$
\frac{\partial \xi}{\partial t}+u_{r} \frac{\partial \xi}{\partial r}+\frac{u_{\theta}}{r} \frac{\partial \xi}{\partial \theta}+s_{L}\left\lfloor\left(\frac{\partial \xi}{\partial r}\right)^{2}+\frac{1}{r^{2}}\left(\frac{\partial \xi}{\partial \theta}\right)^{2}+1\right\rceil^{1 / 2}=u_{z}
$$

The following analysis is similar to the previous discussion, however it is included due to the differences in coordinate system and parameters. In addition the alternative representation further enhances the clarity of the conclusions. The bottom right flame schematic provided in Figure 1 shows a center-body stabilized premixed flame in a swirling flow. The time averaged flame position is shown by the dashed line, and the perturbed flame by the solid line. The flame is stabilized on a center-body, which provides the following boundary condition for $\xi$ :

$$
\left.\xi(r=R, \theta, t)\right|_{\text {flame-holder }}=0
$$

We write quantities in terms of a steady mean and a spatio-temporally varying disturbance, as:

$$
\begin{aligned}
& \xi(r, \theta, t)=\bar{\xi}(r, \theta)+\varepsilon \xi^{\prime}(r, \theta, t) \\
& u_{i}(r, \theta, t)=\bar{U}_{i}(r, \theta)+\varepsilon u_{i}^{\prime}(r, \theta, t)
\end{aligned}
$$

where $i$ denotes the $r, \theta$, or $z$ coordinate. The evolution equations for the mean and linear perturbation in flame position may then be written as:

$$
\begin{gathered}
\bar{U}_{r} \frac{d \bar{\xi}}{d r}+\frac{\bar{U}_{\theta}}{r} \frac{d \bar{\xi}}{d \theta}=\bar{U}_{z}-s_{L}\left|\left(\frac{d \bar{\xi}}{d r}\right)^{2}+\left(\frac{1}{r} \frac{d \bar{\xi}}{d \theta}\right)^{2}+1\right|^{1 / 2} \\
\frac{\partial \xi^{\prime}}{\partial t}+\bar{u}_{t} \cdot \nabla \xi^{\prime}=u_{n}^{\prime} \sqrt{\frac{1}{\sin ^{2} \psi}+\left(\frac{1}{r} \frac{d \bar{\xi}}{d \theta}\right)^{2}}
\end{gathered}
$$

where

$$
\frac{d \bar{\xi}}{d r}=\cot \psi(r, \theta)
$$

and the net tangential velocity vector along the flame surface, due to both the azimuthal and axial velocity components is given by:

$$
\overrightarrow{\bar{u}}_{t}=\bar{U}-s_{L} \hat{n}
$$


where $\hat{n}$ is the local unit normal vector, pointing from the time averaged flame surface into the products. In addition,

$$
u_{n}^{\prime}=\overrightarrow{u^{\prime} \cdot \hat{n}}=\left(u_{z}^{\prime}-u_{r}^{\prime} \cot \psi-u_{\theta}^{\prime} \frac{1}{r} \frac{\partial \bar{\xi}}{\partial \theta}\right) / \sqrt{\frac{1}{\sin ^{2} \psi}+\left(\frac{1}{r} \frac{d \bar{\xi}}{d \theta}\right)^{2}}
$$

denotes the normal component of imposed velocity fluctuations to the time averaged flame surface. We assume a constant burning velocity, $s_{L}$; for analysis of unsteady stretch effects. Equation (242) shows that flame wrinkles propagate along the flame in the direction given by $\bar{u}_{t}$. The general characteristic equation for propagation of flame wrinkles, in characteristic space coordinate $s$, is given by:

$$
d s=\frac{d t}{1}=\frac{d r}{\left(\bar{U}_{r}-s_{L} \hat{n} \cdot \hat{e}_{r}\right)}=\frac{r d \theta}{\left(\bar{U}_{\theta}-s_{L} \hat{n} \cdot \hat{e}_{\theta}\right)}
$$

Equation (245) shows the spiral flame surface wrinkle motion, whose specific trajectory depends on the radial and azimuthal mean velocity components. There are two important processes associated with the linearized flame dynamics that are highlighted next. The left hand side of Eq.(242) is a convection operator and describes the transport of the generated wrinkles. The right hand side describes wrinkle generation, showing that flame wrinkles are excited by velocity disturbances in the direction normal to mean flame front, shown in Eq.(244). In other words, the flame does not distinguish between axial $\left(u_{z}{ }^{\prime}\right)$, or transverse $\left(u_{\theta}{ }^{\prime}, u_{r}{ }^{\prime}\right)$ disturbances, but responds to the net scalar velocity component which is normal to itself, $u_{n}$ '. We shall next consider a general solution to the evolution equation, and then present example solutions for specific mean and disturbance fields.

The frequency domain representation of Eq.(242) is

$$
i 2 \pi S t \hat{\xi}^{\prime}+\left\lceil\left(\bar{U}_{r}-s_{L} \hat{n} \cdot \hat{e}_{r}\right) \frac{\partial \hat{\xi}^{\prime}}{\partial r}+\left(\bar{U}_{\theta}-s_{L} \hat{n} \cdot \hat{e}_{\theta}\right) \frac{1}{r} \frac{\left.\partial \hat{\xi}^{\prime}\right\rceil}{\partial \theta}\right\rfloor=\hat{u}_{n}^{\prime} \sqrt{\frac{1}{\sin ^{2} \psi}+\left(\frac{1}{r} \frac{d \bar{\xi}}{d \theta}\right)^{2}}=\hat{F}(r, \theta, S t, \chi)
$$

where $F$ denotes the velocity forcing function, which is itself a function of space $(r, \theta)$, a dimensionless frequency $S t$, and a non-compactness parameter, $\chi$, that represents the ratio of a flame length scale and the acoustic wavelength. The function, $F$, is expanded in a Fourier series in the azimuthal direction as:

$$
\hat{F}(r, \theta, S t, \chi)=a_{o}(r, S t, \chi)+\sum_{n}\left(a_{n}(r, S t, \chi) \cos n \theta+b_{n}(r, S t, \chi) \sin n \theta\right)
$$

This Fourier representation of $F$ facilitates a solution representation for the flame fluctuation, and also enables a decomposition of which features of the disturbance field influence the spatially integrated flame area response. We can write the governing partial differential equation as an ordinary differential equation by transferring to characteristic co-ordinates, thereby enabling a 
generic representation for the solution. Transforming the spatial co-ordinates to the characteristic co-ordinate $s$ (from Eq.(245)), we get:

$$
\left.d s=\frac{d r}{\left(\bar{U}_{r}(r, \theta)-s_{L} \hat{n} \cdot \hat{e}_{r}\right)} ; d s=\frac{r d \theta}{\left(\bar{U}_{\theta}(r, \theta)-s_{L} \hat{n} \cdot \hat{e}_{\theta}\right)}\right\} \Rightarrow \begin{aligned}
& r=r\left(r_{p}, \theta_{p}, s, \sigma\right) \\
& \theta=\theta\left(r_{p}, \theta_{p}, s, \sigma\right)
\end{aligned}
$$

where $r_{p}$ and $\theta_{p}$ are the spatial co-ordinates corresponding to characteristic co-ordinate $s=s_{p}$. The transformed equation is now integrated along the characteristic curve on the mean flame surface, between $s=0$ and $s=s_{p}$. $\sigma$ denotes a characteristic non-dimensional parameter to quantify swirl. This parameter can be explicitly specified for a given swirling flow field. Substituting in Eq.(247) and Eq.(248), we obtain the solution as:

$$
\hat{\xi}^{\prime}\left(s_{p}\right) e^{i 2 \pi t s_{p}}=\mathscr{B}_{o}\left(r_{p}, \theta_{p}, \sigma, S t, \chi\right)+\sum_{n}\left(\mathscr{B}_{n}^{c}\left(r_{p}, \theta_{p}, \sigma, S t, \chi\right)+\mathscr{B}_{n}{ }^{s}\left(r_{p}, \theta_{p}, \sigma, S t, \chi\right)\right)
$$

where

$$
\begin{aligned}
& \mathscr{B}_{o}\left(r_{p}, \theta_{p}, \sigma, S t, \chi\right)=\int_{s=0} a_{o}\left(r_{p}, \theta_{p}, s, \sigma, S t, \chi\right) e^{i 2 \pi S t s} d s \\
& \mathscr{B}_{n=s_{p}}{ }^{c}\left(r_{p}, \theta_{p}, \sigma, S t, \chi\right)=\int_{\substack{s=0 \\
s=s_{p}}} a_{n}\left(r_{p}, \theta_{p}, s, \sigma, S t, \chi\right) e^{i 2 \pi S t s} \cos \left(n \theta\left(r_{p}, \theta_{p}, s, \sigma, S t, \chi\right)\right) d s \\
& \mathscr{B}_{n}{ }^{s}\left(r_{p}, \theta_{p}, \sigma, S t, \chi\right)=\int_{s=0} b_{n}\left(r_{p}, \theta_{p}, s, \sigma, S t, \chi\right) e^{i 2 \pi S t s} \sin \left(n \theta\left(r_{p}, \theta_{p}, s, \sigma, S t, \chi\right)\right) d s
\end{aligned}
$$

The suffix " $p$ " can be subsequently dropped, as they represent any point of interest on the flame surface where the fluctuation is being determined. The final solution can be written as:

$$
\hat{\xi}^{\prime}(r, \theta) e^{i 2 \pi t s_{p}(r, \theta)}=\mathscr{B}_{0}(r, \theta, \sigma, S t, \chi)+\sum_{n}\left(\mathscr{B}_{n}{ }^{c}(r, \theta, \sigma, S t, \chi)+\mathscr{B}_{n}{ }^{s}(r, \theta, \sigma, S t, \chi)\right)
$$

The global flame surface area, proportional to its heat release for constant burning velocity flames, is given by:

$$
A=\iint_{r, \theta} r d \theta d r \sqrt{1+\left(\frac{\partial \xi}{\partial r}\right)^{2}}
$$

Linearization of this equation yields the following decomposition for the global flame area:

$$
\bar{A}=\iint_{r, \theta} r d r d \theta \frac{1}{\sin \psi(r, \theta)} ; \hat{A}^{\prime}=\iint_{r, \theta} r d \theta d r \cos \psi(r, \theta) \frac{\partial \hat{\xi}^{\prime}(r, \theta)}{\partial r}
$$


The first and second terms describe the global mean and fluctuating flame area, respectively. In the limit of low St, this second integral can be represented using Eq.(247) as:

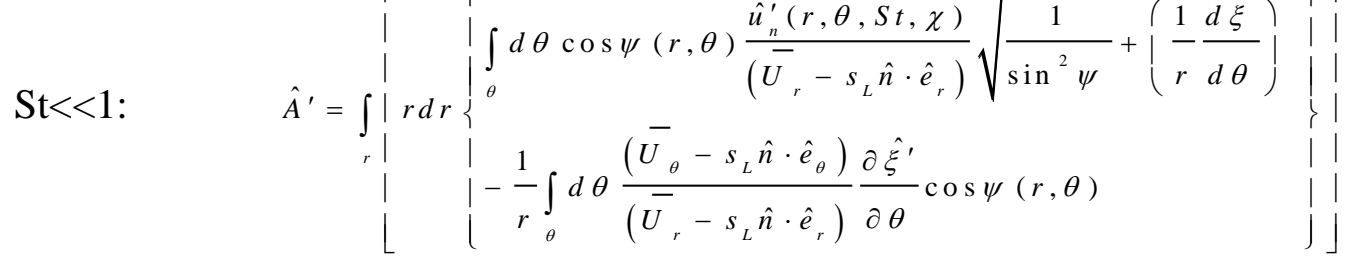

In Eq.(309), we can see two contributions to the low $S t$ flame area fluctuations. The first is from the normal component of the velocity fluctuations integrated over the flame surface, which is related to the instantaneous volume flow rate. The second contribution arises due to nonaxisymmetries in the flow field and flame shape. This term integrates to zero for an axisymmetric mean flame, as shown in the next section. For the general Strouhal number case,

the flame area fluctuation integral can be decomposed into three terms, $\hat{A}_{I}^{\prime}, \hat{A}_{I I}^{\prime}$ and $\hat{A}_{I I I}^{\prime}$ using Eq.(254),

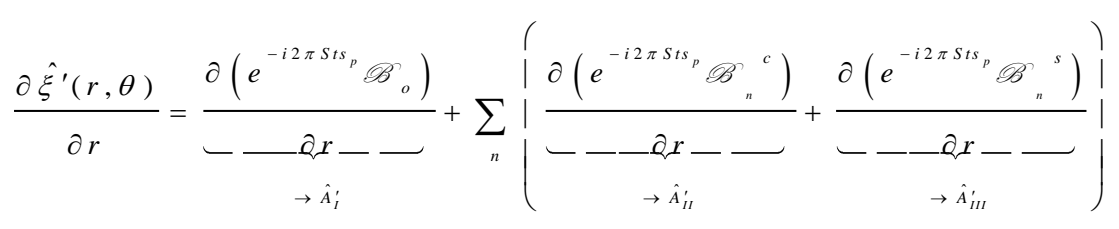

\section{Axisymmetric Flame Response}

The analysis in the above section derived general results for the flame dynamics. In this section, we consider axisymmetric mean flame shapes in a general disturbance field. Eq.(242) takes the form:

$$
\frac{\partial \xi^{\prime}}{\partial t}+\left(\bar{U}_{r}(r)+s_{L} \cos \psi(r)\right) \frac{\partial \xi^{\prime}}{\partial r}+\frac{\bar{U}_{\theta}(r)}{r} \frac{\partial \xi^{\prime}}{\partial \theta}=\frac{u_{n}^{\prime}}{\sin \psi(r)}=u_{z}^{\prime}-u_{r}^{\prime} \cot \psi(r)-\frac{u_{\theta}^{\prime}}{r} \frac{\partial \bar{\xi}}{\partial \theta}
$$

This expression shows that fluctuations in azimuthal velocity have no influence on the flame response. The Fourier domain representation of Eq.(259) (from Eq.(247)), is

$$
i 2 \pi S t \hat{\xi}^{\prime}+\left(\bar{U}_{r}(r)+s_{L} \cos \psi(r)\right) \frac{\partial \hat{\xi}^{\prime}}{\partial r}+\frac{\bar{U}_{\theta}(r)}{r} \frac{\partial \hat{\xi}^{\prime}}{\partial \theta}=\hat{F}(r, \theta, S t, \chi)
$$

where $F$ is as defined in Eq.(248). Following Eq.(250), we transform to characteristic coordinates to obtain: 


$$
\begin{aligned}
& d s=\frac{d r}{\bar{U}_{r}(r)+s_{L} \cos \psi(r)} \Rightarrow r=f\left(r_{p}, s\right) \\
& d s=\frac{f\left(r_{p}, s\right) d \theta}{\bar{U}_{\theta}\left(f\left(r_{p}, s\right), \sigma\right)} \Rightarrow d \theta=\frac{\bar{U}_{\theta}\left(f\left(r_{p}, s\right), \sigma\right) d s}{f\left(r_{p}, s\right)} \Rightarrow \theta=\theta_{p}+g\left(r_{p}, s, \sigma\right)
\end{aligned}
$$

Note that the radial characteristic function $f$ is independent of the azimuthal characteristic function $g$, while the converse is not true. This also implies that the radial characteristic function is independent of swirl $\sigma$. The characteristic co-ordinate $s_{p}$ is obtained from solving for $r=r_{p}$. Also, unlike the general case, $\theta_{p}$ can be separated from the function $g$. These observations are specific to axisymmetric flow fields and play an important role in our further discussion on the effect of swirl. The solution is as shown in Eq.(304) where the functions, $\mathscr{B}$, in Eq.(253) take the form:

$$
\begin{aligned}
& \mathscr{B}_{o}\left(r_{p}, S t, \chi\right)=\int_{s=0}^{s=s_{p}\left(r_{p}\right)} a_{o}\left(f\left(r_{p}, s\right), S t, \chi\right) e^{i 2 \pi s t s} d s \\
& \mathscr{B}_{n}^{c}{ }^{c}\left(r_{p}, \theta_{p}, \sigma, S t, \chi\right)=\left\{\begin{array}{ll}
\left\lceil\cos \left(n \theta_{p}\right)\right. & \int_{s=0}^{s=s_{p}\left(r_{p}\right)} a_{n}\left(f\left(r_{p}, s\right), S t, \chi\right) e^{i 2 \pi s t s} \cos \left(n g\left(r_{p}, s, \sigma\right)\right) d s \\
s=s_{p}\left(r_{p}\right) & \\
-\sin \left(n \theta_{p}\right) & \int_{s=0} a_{n}\left(f\left(r_{p}, s\right), S t, \chi\right) e^{i 2 \pi s t s} \sin \left(n g\left(r_{p}, s, \sigma\right)\right) d s
\end{array}\right]
\end{aligned}
$$

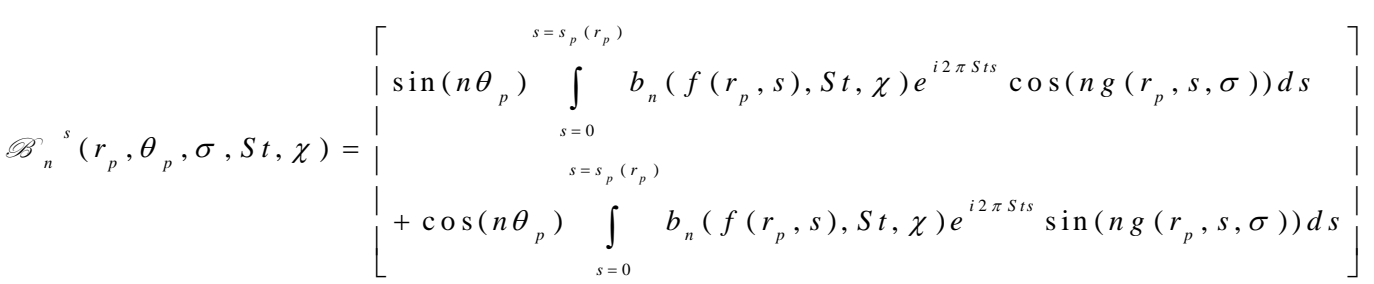

The final solution can now be written as:

$$
\hat{\xi}^{\prime}(r, \theta)=\mathscr{\sigma}_{o}(r, S t, \chi)+\sum_{n}\left(\cos (n \theta) \mathscr{C}_{n}^{c}(r, \sigma, S t, \chi)+\sin (n \theta) \mathscr{\odot}_{n}^{s}(r, \sigma, S t, \chi)\right)
$$

where 


$$
\begin{aligned}
& \mathscr{C}_{o}(r, S t, \chi)=e^{-i 2 \pi S t s_{p}(r)} \int_{s=0}^{s=s_{p}(r)} a_{o}(f(r, s), S t, \chi) e^{i 2 \pi S t s} d s \\
& \left.\mathscr{\mathscr { O }}_{n}{ }^{c}(r, \sigma, S t, \chi)=e^{-i 2 \pi S t s_{p}(r)} \mid \begin{array}{l}
\int_{s=0}^{s=s_{p}(r)} a_{n}(f(r, s), S t, \chi) e^{i 2 \pi S t s} \cos (n g(r, s, \sigma)) d s \\
s=s_{p}(r)
\end{array}\right] \\
& \left.\mathscr{G}_{n}^{s}(r, \sigma, S t, \chi)=e^{-i 2 \pi S t s_{p}(r)} \mid \begin{array}{ll}
\int_{s=0}^{s=s_{p}(r)} b_{n}(f(r, s), S t, \chi) e^{i 2 \pi s t s} \cos (n g(r, s, \sigma)) d s \\
s=s_{p}(r)
\end{array}\right]
\end{aligned}
$$

The linearized global flame area (from Eq.(256)) terms become:

$$
\begin{aligned}
& \hat{A}_{I}^{\prime}(S t, \chi)=\int_{r} r d r \cos \psi(r) \frac{\partial \mathscr{C}_{o}(r, S t, \chi)}{\partial r} \int_{\theta=0}^{\theta=2 \pi} d \theta=2 \pi \int_{r} r d r \cos \psi(r) \frac{\partial \mathscr{\mathscr { C }}_{o}(r, S t, \chi)}{\partial r} \\
& \hat{A}_{I I}^{\prime}(\sigma, S t, \chi)=\int_{r} r d r \cos \psi(r) \frac{\partial \mathscr{\mathscr { C }}_{n}{ }^{c}(r, \sigma, S t, \chi)}{\partial r} \int_{\theta=0}^{\theta=2 \pi} d \theta \cos (n \theta)=0 \\
& \hat{A}_{I I I}^{\prime}(\sigma, S t, \chi)=\int_{r} r d r \cos \psi(r) \frac{\partial \mathscr{C}_{n}{ }^{s}(r, \sigma, S t, \chi)}{\partial r} \int_{\theta=0}^{\theta=2 \pi} d \theta \sin (n \theta)=0
\end{aligned}
$$

Hence,

$$
\hat{A}^{\prime}=2 \pi \int_{r} r d r \cos \psi(r) \frac{\partial \mathscr{C}_{o}(r, S t, \chi)}{\partial r}
$$

The integrals $\hat{A}_{I I}^{\prime}$ and $\hat{A}_{I I}^{\prime}$ are identically zero, an important result for axisymmetric mean flows. This formulation for flame area in axisymmetric flows is quite important, as it shows that the fluctuating flame area is independent of azimuthal flow velocity (i.e., swirl). To restate, swirl in the flow has strong influences on the local flame characteristics, but no impact on the spatially integrated flame area. Note that, this is true only for axisymmetric flames with constant burning velocity, in the linear regime. In case of non-axisymmetric flames, the dependence on swirl is coupled into the azimuthal co-ordinate functions. Moreover, the presence of nonlinearities in the flame dynamics or, the dependence of flame speed on the local flame wrinkle curvature lead to a swirl influence on the flame area. 
Another important general result can be developed at low Strouhal numbers, St. The flame area fluctuation (from Eq.(309)) for an axisymmetric mean flame simplifies to:

$S t<<1:$

$$
\hat{A}^{\prime}=\int_{r}^{\lceil}\left\lfloor\frac{\cot \psi(r) r d r}{\left(\bar{U}_{r}(r)+s_{L} \cos \psi(r)\right)} \int_{\theta} \hat{u}_{n}^{\prime}(r, \theta, S t, \chi) d \theta\right\rfloor
$$

In order to show explicit results, consider an axially uniform mean velocity field, and a solid body azimuthal field, given by:

$$
\bar{U}_{r}=0 ; \bar{U}_{\theta}=2 \pi S t(\Omega / \omega) r=2 \pi S t \sigma r ; \bar{U}_{z}=1
$$

Note that the angular rotation rate of the mean flow field is then given by $\Omega$ and the dimensionless rotation rate by $\sigma=\Omega / \omega$. The mean flame shape is then:

$$
\bar{\xi}(r)=(r-R) \cot \psi
$$

Earlier work presented results for disturbance fields that are compact with respect to the flame length scale. We now present results for a non-compact disturbance field. The general form of the perturbation field is given by:

$$
u_{x}^{\prime}=e^{i 2 \pi \mathrm{s} t}\left(\mathscr{D}_{f} e^{-i 2 \pi x x}+\mathscr{D}_{b} e^{i 2 \pi x x}\right) \Rightarrow\left\{\begin{array}{l}
\hat{u}_{n}^{\prime}(r, \theta, S t, \chi)=i \cos \psi \cos \theta\left(\begin{array}{l}
\mathscr{D}_{f} e^{-i 2 \pi x r \cos \theta} \\
+\mathscr{D}_{b} e^{i 2 \pi x r \cos \theta}
\end{array}\right) \\
\hat{F}(r, \theta, S t, \chi)=i \cot \psi \cos \theta\left(\begin{array}{l}
\mathscr{D}_{f} e^{-i 2 \pi x r \cos \theta} \\
+\mathscr{D}_{b} e^{i 2 \pi x r \cos \theta}
\end{array}\right)
\end{array}\right.
$$

Where $\chi=L_{f} / \lambda_{a}$ is a non-compactness parameter. Note that, $\chi=S t M$, where $M$ is the Mach number of the incoming axial flow. The coefficients $\mathscr{D}_{\mathrm{f}}$ and $\mathscr{Q}_{\mathrm{b}}$ denote the weighted magnitudes of the forward and backward traveling waves, where we assume $\left|\mathscr{Q}_{\mathrm{f}}\right|+\left|\mathscr{Q}_{\mathrm{b}}\right|=1$. The compact flame disturbance field is a special case of this disturbance field where $\chi=0$. Unlike the compact case where flame branches on either side of the axis (along the flow and perpendicular to forcing axis) would encounter the same disturbance but of opposite sign, the non-compact case introduces an additional degree of freedom to the disturbance field. In case of a traveling wave, the forward wave corresponds to $\mathscr{Q}_{\mathrm{b}}=0$, and the backward wave corresponds to $\mathscr{Q}_{\mathrm{f}}=0$. A pictorial representation of the traveling wave field propagating over the flame is shown in Figure 55(a). Standing wave fields are obtained for $\mathscr{Q}_{\mathrm{f}}= \pm \mathscr{Q}_{\mathrm{b}}$. The case of $\mathscr{Q}_{\mathrm{f}}=\mathscr{Q}_{\mathrm{b}}$ corresponds to a symmetric velocity field where flame branches on either side encounter the same disturbance field, but of opposite volumetric flux. The case $\mathscr{Q}_{\mathrm{f}}=-\mathscr{Q}_{\mathrm{b}}$ corresponds to an anti-symmetric disturbance field, leading to a non-zero flame area response. This standing wave field in time is shown in Figure 55(b). 


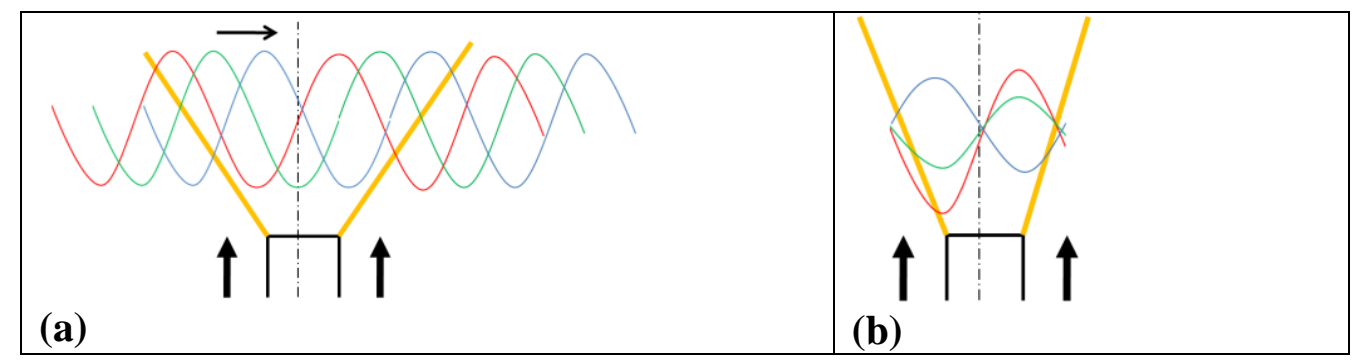

Figure 55: Time sequence evolution of velocity perturbation field for (red, green, blue in order) a (a) traveling wave, black arrow shows direction of wave and (b) standing wave

Consider first the case of a traveling wave form for the disturbance field. This is given by $\mathscr{Q}_{\mathrm{f}}=1$ and $\mathscr{Q}_{\mathrm{b}}=0$. The effects of a backward traveling wave are the same as those of a forward one, since the flame is axisymmetric. The perturbation field is given by:

$$
u_{x}^{\prime}=e^{i 2 \pi \operatorname{stt-i2\pi \chi x}} \Rightarrow\left\{\begin{array}{l}
\hat{u}_{n}^{\prime}(r, \theta, S t, \chi)=i \cos \psi \cos \theta e^{-i 2 \pi x \cos \theta} \\
\hat{F}(r, \theta, S t, \chi)=i \cot \psi \cos \theta e^{-i 2 \pi x r \cos \theta}
\end{array}\right.
$$

The disturbance field can be decomposed into its Fourier components using the Jacobi-Anger expansion:

$$
e^{-i 2 \pi \chi r \cos \theta}=J_{0}(-2 \pi \chi r)+2 \sum_{n=1}^{\infty} i^{n} J_{n}(-2 \pi \chi r) \cos (n \theta)
$$

where, $J_{n}(\mathrm{n} \geq 0)$ are Bessel functions of the first kind. Using Eq.(267), Eq.(323) and Eq.(288) we have:

$$
\begin{aligned}
a_{0} & =-\cot \psi J_{1}(2 \pi \chi r) \\
a_{n \geq 1} & =i \cot \psi\left[i^{n-1} J_{n-1}(-2 \pi \chi r)+i^{n+1} J_{n+1}(-2 \pi \chi r)\right] \\
b_{n \geq 1} & =0 \\
\mathscr{\sigma}_{o}(\tilde{r}, S t, \chi) & =-\cot \psi e^{-i 2 \pi s t r} \int_{s=0}^{s=\tilde{r}} J_{1}\left(-2 \pi \chi\left(s_{L} \cos \psi s+R\right)\right) e^{i 2 \pi s t s} d s
\end{aligned}
$$

Many of the solution features discussed above for the $\chi=0$ case remain similar for this case. A key difference, however, is the spatially integrated area fluctuations. In the $\chi=0$ case, there is no net fluctuation in flame area. In contrast, area fluctuations do occur in the non-compact case. Using Eq.(271) and Eq.(290), the expression for the normalized fluctuating flame area is:

$$
\hat{A}_{t w}^{\prime} / \bar{A}=\frac{2 \cot \psi}{\left(R_{\max }^{2}-R^{2}\right) \mid} \mid \begin{aligned}
& \mathscr{S}_{0} s_{L} \cos \psi\left(R_{\max }+\frac{s_{L} \cos \psi}{i 2 \pi S t}\right) \\
& \left.+\frac{i s_{L} \cos \psi}{4 \pi^{2} S t \chi}\left\{J_{0}(2 \pi \chi R)-J_{0}\left(2 \pi \chi R_{\max }\right)\right\}\right\rfloor
\end{aligned}
$$


where

$$
\hat{S_{0}}=\exp \left(-\frac{i 2 \pi S t R_{\max }}{s_{L} \cos \psi}\right) \int_{r=R}^{r=R_{\max }}\left\lceil J_{1}(2 \pi \chi r) \exp \left(-\frac{i 2 \pi S t r}{s_{L} \cos \psi}\right)\right\rfloor d r
$$

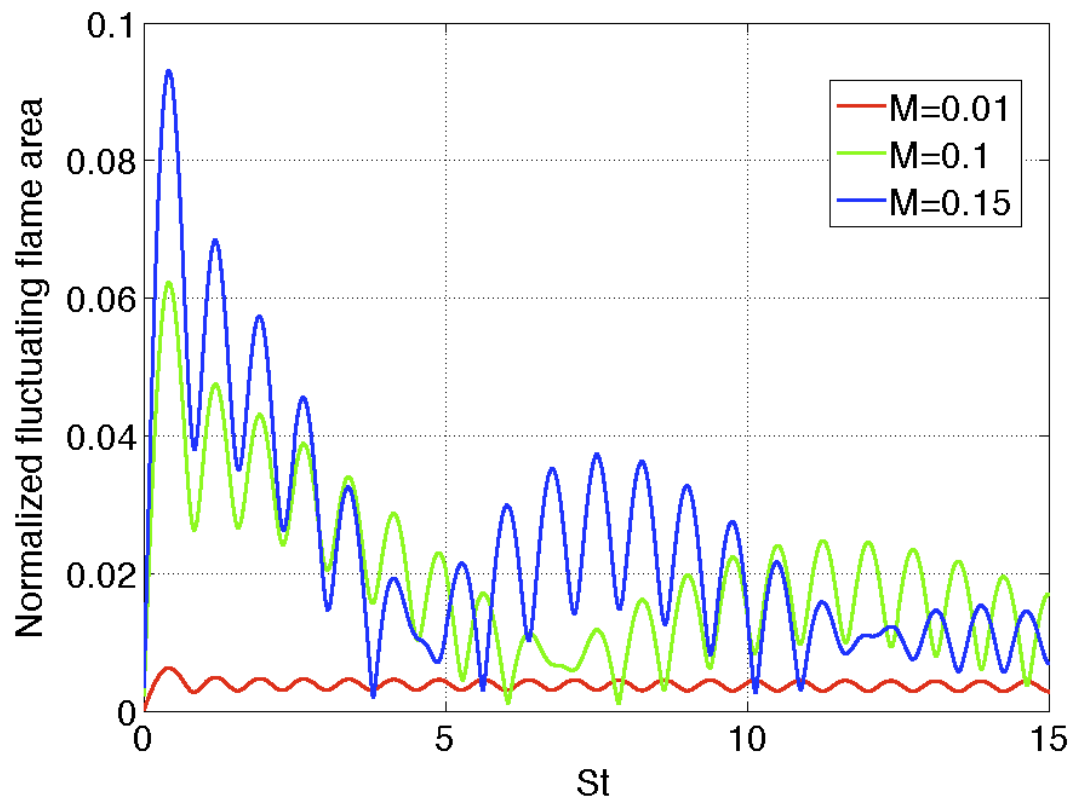

Figure 56: Normalized fluctuating flame area (Eq.(291)) for a nominally axisymmetric flame in a non-compact disturbance field (Eq.(323) and Eq.(329)). The ratio of flame size to wavelength is controlled by the Mach number, but also varies along the $x$-axis with Strouhal number.

The subscript ' $t w$ ' is used to denote traveling wave. The expression in Eq.(291) indicates the presence of two different scales of interference in the Strouhal number space, one due to the exponential term and the other to the Bessel term. This is primarily due to the spatial distribution of the non-compact transverse forcing at the conical flame surface. These aspects are depicted in Figure 56. Note how all the curves start at zero for $S t=0$, then they rise and oscillate with the two scales mentioned above. Note also that larger transfer function gains are achieved for shorter length scale disturbances parameterized here by $M$.

Next, we consider the anti-symmetric standing wave pattern. This is given by $\int_{\mathrm{f}}=-$ Ob $_{\mathrm{b}}$. The perturbation field is given by:

$$
\begin{aligned}
& u_{x}^{\prime}=e^{i 2 \pi s t} \sin (2 \pi \chi x) \\
& \Rightarrow\left\{\begin{array}{l}
\hat{u}_{n}^{\prime}(r, \theta, S t, \chi)=i \cos \psi \cos \theta \sin (2 \pi \chi r \cos \theta) \\
\hat{F}(r, \theta, S t, \chi)=i \cot \psi \cos \theta \sin (2 \pi \chi r \cos \theta)
\end{array}\right.
\end{aligned}
$$


We use the same procedure as earlier to evaluate the normalized fluctuating flame area. Consider the features of Eq.(323) and Eq.(325). The real part of $F$ in Eq.(323), corresponds to the real part of $a_{0}$ in Eq.(325), and likewise for the imaginary parts. Note that the real part of $F$ in Eq.(323) is $\sin (2 \pi \chi r \cos \theta)$ and that $a_{0}$ is purely real, corresponding to the Fourier expansion of this term. The imaginary part, $\cos (2 \pi \chi r \cos \theta)$, has a corresponding zero imaginary part in $a_{0}$. Since the fluctuating flame area depends only on $a_{0}$ it is clearly seen that a non-zero response for the traveling wave case is purely due to the $\sin (2 \pi \chi r \cos \theta)$ term, which is essentially the standing wave case we are analyzing here. This is also evident from the fact mentioned earlier that the $\cos (2 \pi \chi r \cos \theta)$ form corresponds to a symmetric perturbation distribution and, hence, leads to no net global flame response. Thus, the traveling wave and asymmetric standing wave fields lead to the same magnitude of flame area fluctuations, but a phase difference of $\pi / 2$.

However, the transfer function depends upon the choice of reference velocity. The magnitude of velocity oscillations is spatially uniform for the traveling wave. However, it varies between zero and $2 Q_{\mathrm{f}}$ for the standing wave. Moreover, the unsteady velocity along the flame centerline is identically zero. As such, the fluctuating flame area for both cases scale with $M$, but for different reasons. In the traveling wave case, $\chi=S t M$ describes the slight difference in disturbance field between the two halves of the flame, so that the disturbances do not identically cancel each other out. In the asymmetric standing wave case, $\chi$ describes the deviation of the disturbance field from non-zero at the flame. If the velocity at the flame, as opposed to the incident wave amplitude, is used as the reference velocity for the standing wave case, then the transfer function is of $\mathrm{O}(1)$. In contrast, it is of $\mathrm{O}(M)$ if the incident wave amplitude is used as the normalizing factor.

\section{Review}

As a review, this work shows the effects of swirl on a transversely forced flame, which introduces an additional degree of freedom for wrinkle motion. The presence of swirl superposes with the upstream axial velocity, thereby creating a helical spiral motion of the wrinkles. In case of axisymmetric flows, swirl was seen to have a local effect only. The global flame dynamics are independent of swirl regardless of the acoustic forcing mechanism, in the linear regime. Future work should include the additional effects of nonlinearity and stretch sensitivity. Because of the strong effects of the swirl parameter, $\sigma$, upon length scale of wrinkling, these additional processes will be strongly sensitive to these effects. For example, nonlinear terms arising from kinematic restoration will result in fast smoothing of shorter length-scale disturbances, as the flame propagates normal to itself. Similarly, smaller length-scale, small curvature wrinkles will be more strongly influenced by flame stretch. As such, inclusion of either of these effects will cause swirl to influence the global flame response as well and, also, to cause differing effects of swirl upon the flame response depending upon the disturbance amplitude and Markstein number. 


\section{Effect and Contribution of Swirl Fluctuations on the Flame Response of Swirl- Stabilized Premixed Flames}

\section{Introduction}

To bring our analysis full circle, additionally we focused on analysis of the effect and contribution of swirl fluctuations to the flame response of swirl-stabilized premixed flames. This work is motivated by the fact that acoustic waves that pass through the swirler section in the nozzle and excite swirl fluctuations that propagate at the local mean flow velocity. These swirl velocity fluctuations lead to an additional source of flame response and interference with that due to the acoustic waves.

\section{Formulation}

We employ a combination of level-set flame front tracking and numerical simulation of the flow field. The level-set equation governing the flame position dynamics is as shown below.

$$
\frac{\partial \xi^{\prime}}{\partial t}+\overrightarrow{U_{t}} \cdot \vec{\nabla} \xi^{\prime}=u_{z}^{\prime}-u_{r}^{\prime} \frac{\partial \bar{\xi}}{\partial r}-\frac{u_{\theta}^{\prime}}{r} \frac{\partial \bar{\xi}}{\partial \theta}=u_{n}^{\prime}|\nabla \bar{\xi}|
$$

Here, the overbar denotes mean (steady, time-averaged) quantities. The primes denote fluctuating quantities. The subscripts $\mathrm{t}, \mathrm{z}, \mathrm{r}, \theta$ and $\mathrm{n}$ denote the tangential, axial, radial, azimuthal and normal components respectively. Notice that the flame position fluctuation (about the steady time averaged flame) is controlled by the normal component of the fluctuating velocity. The swirl fluctuations have a direct impact on flame response for non-axisymmetric flames as can be seen from the equation, and also have an indirect impact through affecting the axial and radial velocity fluctuations. The first part of study is to focus on axisymmetric mean flames where the direct effects of swirl fluctuations are not experienced by the flame. However, the indirect effect requires extracting the nature of interactions between the swirl component of the velocity and the axial and radial components. This is obtained using CFD simulations of a typical swirl combustor in a two dimensional axisymmetric configuration as shown in Figure 57.

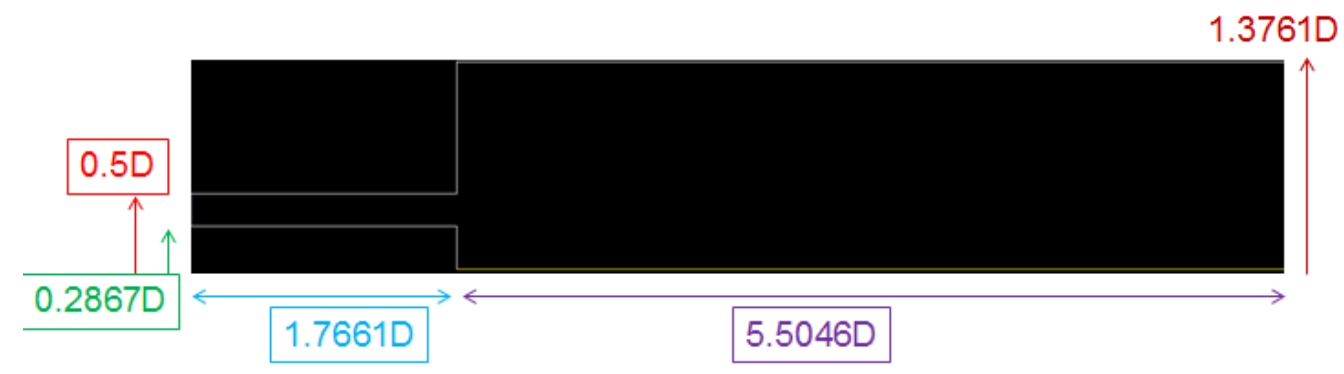

Figure 57: Schematic of the two dimensional axisymmetric swirl flow configuration used in the CFD study. The wall boundaries are indicated by white lines. The inlet is on the left section and the outlet is on the right. The axis is the base of the figure. 


\section{Results and Discussion}

The simulation is first solved for steady state using a mean flow input for the flow components based on velocity field that exits the three dimensional swirler blade passages. This steady state is then acoustically forced by adding time varying harmonic velocity to the inlet boundary. The objective of these simulations is to compare the resulting unsteady flow fields for a case with only axial forcing with that of both axial forcing and swirl velocity forcing. The differences in the resulting flow field region at the flame between these cases would illustrate the effect of swirl fluctuations on the normal component of velocity fluctuations at the flame and hence the flame response. The steady state flow fields for an inlet axial velocity of $26 \mathrm{~m} / \mathrm{s}$ and swirl velocity of $20 \mathrm{~m} / \mathrm{s}$ are shown in Figure 58. For a sample case of $10 \%$ forcing amplitude and $400 \mathrm{~Hz}$ forcing frequency, the time snapshots for the different forcing configurations are shown in Figure 59 to Figure 61.

(a)

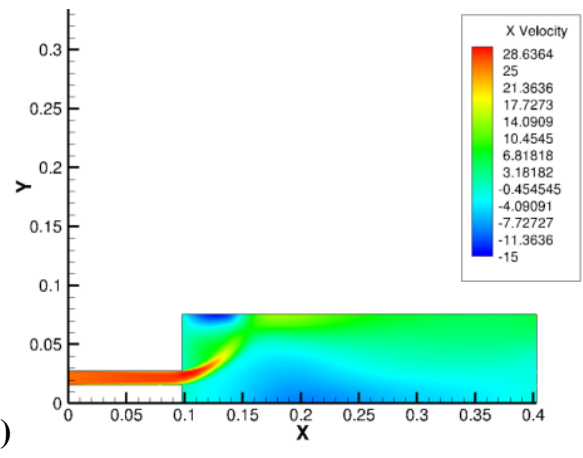

(b)

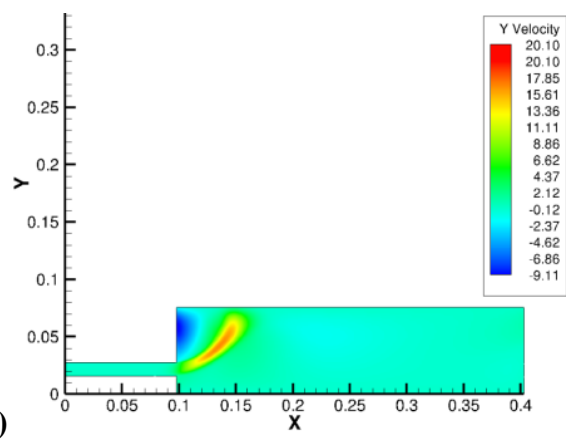

Figure 58: Steady state solution contours for unforced case, used as initial reference state for forced cases, showing (a) axial velocity $(\mathrm{X})$ and $(\mathrm{b})$ radial velocity $(\mathrm{Y})$.

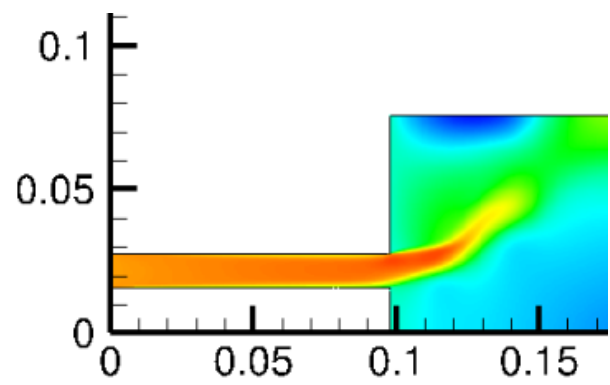

(a)

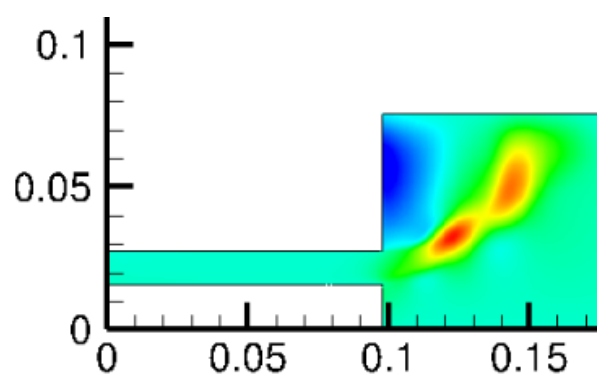

(b)

Figure 59: Snapshot of the flow field contours at a time instant during the acoustic forcing for a case with only axial forcing, showing (a) axial velocity and (b) radial velocity. Contour levels same as their respective in Figure 58. 


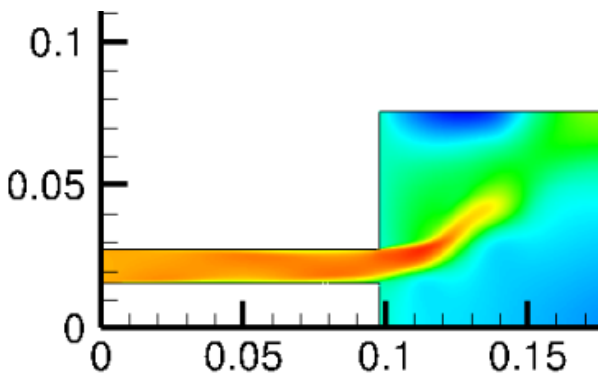

(a)

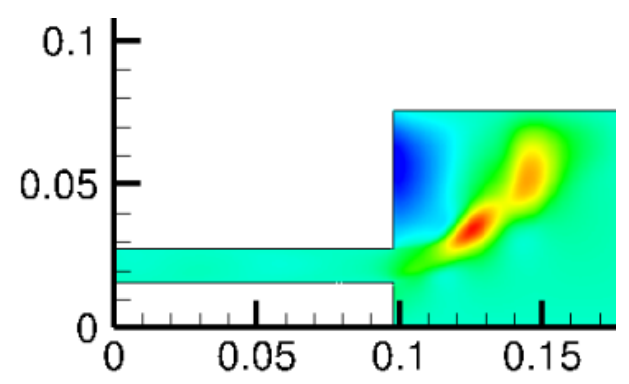

(b)

Figure 60: Snapshot of the flow field contours at a time instant during the acoustic forcing for a case with both axial and azimuthal forcing, showing (a) axial velocity and (b) radial velocity. Contour levels same as their respective in Figure 58.

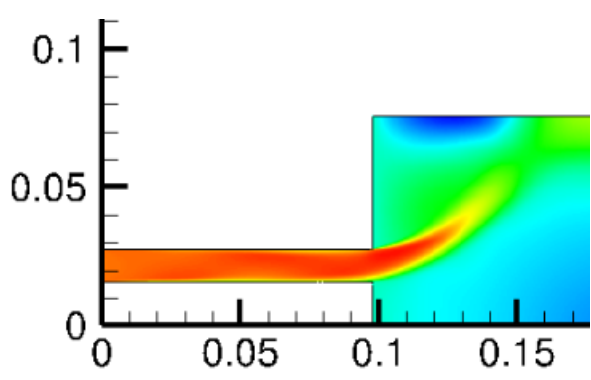

(a)

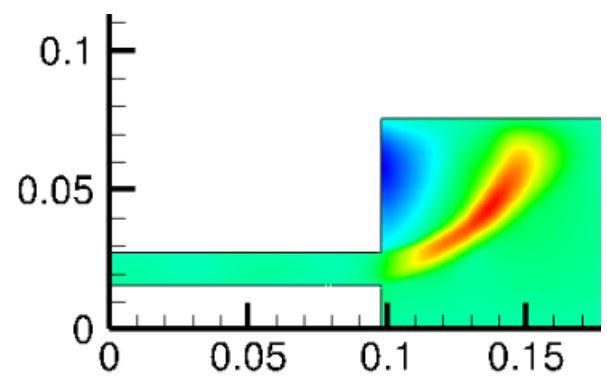

(b)

Figure 61: Snapshot of the flow field contours at a time instant during the acoustic forcing for a case with only azimuthal forcing, showing (a) axial velocity and (b) radial velocity. Contour levels same as their respective in Figure 58.

\section{Review}

For the cases with axial forcing (Figure 59) and with both axial and azimuthal forcing (Figure 60) we can clearly see that the flow components in the combustor region show appreciable fluctuations. This can be seen by comparing with the steady state in Figure 58. However, the case of only azimuthal forcing (Figure 61) shows very negligible fluctuations. This indicates that the fluctuations in the combustor are predominantly due the axial component fluctuations at the inlet. This implies that the azimuthal forcing at the inlet does not affect the flame response appreciably. However note that these conclusions are valid for low amplitude fluctuations of the velocity and axisymmetric mean flames. Further study is being conducted for other forcing amplitudes and frequencies. 


\title{
14. Large Eddy Simulation of Combustion Dynamics
}

\begin{abstract}
Introduction
Lastly, a comprehensive theoretical/numerical framework has been developed and implemented to explore the flame dynamics and its response to impressed flow fluctuations. The work presented in this section aims to perform high-fidelity simulations of the experiments described previously. Results will also be used to support the development of analytical models of flame response to fuel/air ratio oscillations. The theoretical formulation is based on the Favre-filtered conservation equations of mass, momentum, and energy in three dimensions. Turbulence closure is achieved by means of a large-eddy-simulation technique, in which unclosed subgrid-scale terms are modeled using a compressible-flow version of the dynamic Smagorinsky model (DSM). The static Smagorinsky model is also included in the code to provide a more computationally efficient way to treat subgrid-scale flow motions. Turbulence/chemistry interaction is treated by means of a flamelet model augmented with a level-set approach.
\end{abstract}

\section{Code and Numerical Schemes}

The numerical scheme employs a density-based, finite-volume approach. Spatial discretization is obtained using a fourth-order central-differencing method in generalized coordinates. A sixthorder numerical dissipation with a total-variation-diminishing (TVD) switch is included to ensure computational stability and to prevent numerical oscillations in regions with steep gradients. Temporal integration is obtained using a third-order Adam-Bashforth predictorcorrector scheme. All the calculations are performed on an in-house parallel computing facility consisting of 180 Pentium processors. A multi-block technique based on domain decomposition is employed to parallelize the code, along with the use of the Message Passing Interface (MPI) library for exchanging information among processors. The parallelization methodology is robust and scalable. The overall approached has been verified and validated against several test problems including decaying isotropic turbulence, cold-flow evolution in swirler injectors with either axial or tangential entries, premixed flames in a combustor rig with a single-element swirl injector.

The large eddy simulation (LES) code developed has been carefully calibrated and benchmarked to assess its numerical accuracy and robustness. The spatial discretization is achieved using a second-order accurate center-differencing methodology, along with the employment of fourthorder artificial dissipation. The coefficient of the dissipation term is selected to have a value of 0.01 , in order to prevent numerical oscillations at high wave numbers and to minimize numerical contaminations. The overall accuracy of the present scheme within the context of LES was first assessed based on the decay of the kinetic energy of isotropic turbulence. The experimental results of Comet-Bellot and Corrsin $(\mathrm{CBC})^{1}$ were selected as the benchmark with an initial Taylor Reynolds number of 80 . Calculations were performed on a $32 \times 32 \times 32$ grid in a cubic box of a width $2 \pi$ with periodic boundary conditions. Excellent agreement was obtained with the experimental and DNS (direct numerical simulation) results in terms of the decay rate of the turbulent kinetic energy (TKE). Figure 62 shows the temporal evolution of resolved turbulent kinetic energy. The subgrid-scale model leads to excellent agreement with experimental data, 
whereas a slower decay of TKE is observed when the sgs model is turned off. The present numerical scheme offers a reasonable predictive capability with minimal numerical dissipation.

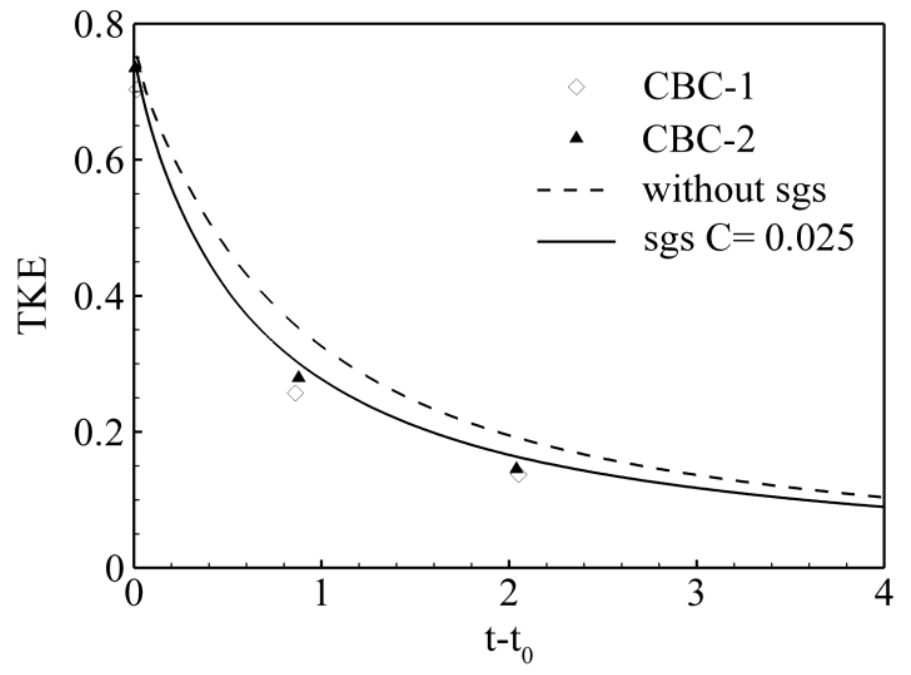

Figure 62: Decay of resolved turbulent kinetic energy.

The LES code is currently being applied to investigate the fuel/air mixing process in a jet-incrossflow configuration. The study proceeds in three steps. First, calculations are conducted to simulate the experiments of Su and Mungal. A grid of 300 million cells is employed to provide detailed information about the mean flow properties, turbulence statistics, and fuel/air mixedness. The purpose is to assess the turbulence-modeling (especially the sgs closure scheme) and numerical accuracy. In addition, detailed flow physics is explored in terms of the vorticity evolution, fuel/air interfacial dynamics, and characteristic time and length scales in various parts of the flowfield. The second step involves the simulation of the mixing process in the experimental setup of this part. Emphasis is placed on the interactions between the fuel jets from the whole array of orifices in a realistic gas-turbine mixing channel, as opposed to the situation with a single orifice in the experiment of $\mathrm{Su}$ and Mungal. The third step treats of the mixing process in an oscillatory environment. Periodic oscillations are imposed at the downstream boundary of the computational domain, simulating the acoustic fluctuations originating from the combustor. A wide range of frequencies is considered, so that a complete spectrum of the response of the fuel/air mixedness to acoustic excitation can be obtained. The result will be used directly in the reduced-order modeling of flame oscillations. 


\section{Experimental Work}

In order to validate the modeling work, the flame's response was measured experimentally for various types of forcing conditions. Accurate measurements of the time-varying rate of heat release are essential to the formulation and validation of flame response models. The technique that is used for this measurement in fuel lean premixed flames is based on the well-established linear relationship between the intensity of the flame's overall chemiluminescence emission and the fuel flow rate, i.e., the rate of heat release. The slope of this linear relationship, however, is dependent on the equivalence ratio. Therefore the equivalence ratio must also be known in order to determine the rate of heat release. In combustors where the fuel and air are mixed upstream of a choked inlet, the equivalence ratio is not affected by the acoustic fluctuations in the combustor and is therefore constant. In practical combustors, however, the flow is not choked after the fuel and air are mixed, therefore during unstable combustion it is possible, in fact likely, that the rate of heat release and the equivalence ratio will both be fluctuating. Under such conditions, the instantaneous, time-varying equivalence ratio must be measured in order to determine the corresponding time-varying rate of heat release. It has been shown by a number of researchers that the ratio of the $\mathrm{OH}^{*}$ and $\mathrm{CH}^{*}$ chemiluminescence intensities (from the entire flame) can be used to measure the overall equivalence ratio of the flame. As described in the previous section, the objective of this work was to develop and validate a flame response model that accounts for the effect of equivalence ratio fluctuations. To date, however, the capability for measuring the time varying rate of heat release when the equivalence ratio is also fluctuating has not been demonstrated. Therefore an important first step in the experimental part of this technical report is the development of this capability.

\section{Heat Release Rate Fluctuation Measurements Under Partially Premixed Conditions}

\section{Chemiluminescence Measurements in Steady Flames}

The general characteristics of the chemiluminescence spectrum from a lean premixed natural gas and air flame are presented in Figure 63. As shown, the $\mathrm{OH}^{*}$ and $\mathrm{CH}^{*}$ spectra occur over relatively narrow wavelength intervals, while the $\mathrm{CO}_{2} *$ spectra is very broad, extending over a wavelength range of several hundred nanometers. Figure 4 also shows that when measuring the $\mathrm{OH}^{*}$ chemiluminescence intensity at $307 \mathrm{~nm}$ and the $\mathrm{CH}^{*}$ chemiluminescence intensity at 430 $\mathrm{nm}$, it is necessary to subtract the background $\mathrm{CO}_{2}$ chemiluminescence. This is easily done if a spectrometer is used the measure the entire spectrum, such as shown in Figure 63. However, in order to make real-time measurements of the time-varying chemiluminescence emission it is necessary to use photomultiplier tubes with appropriate band pass filters. The methodology that has been developed as part of this work for subtracting the $\mathrm{CO}_{2}$ chemiluminescence emission background from measurements of the $\mathrm{OH}^{*}$ chemiluminescence intensity at $307 \mathrm{~nm}$ and the $\mathrm{CH}^{*}$ chemiluminescence intensity is as follows. Chemiluminescence spectra, such as that shown in Figure 63, have been made in our laboratory scale optically-accessible lean premixed combustor over a broad range of operating conditions. From these spectra, the relationship between the magnitude of the $\mathrm{CO}_{2} *$ intensity at $365 \mathrm{~nm}$ and the magnitudes at $307 \mathrm{~nm}$ and 430 $\mathrm{nm}$ are determined. These results are plotted in Figure 64a and Figure 64b. These 
measurements, which were made over a range of combustor inlet velocities $(25,27.5,30,32.5$, and $35 \mathrm{~m} / \mathrm{s})$ and a range of equivalence ratios $(0.65,0.675,0.7,0.725,0.75$, and 0.775$)$, show that there is a relatively well defined relationship between the $\mathrm{CO}_{2} *$ chemiluminescence intensities at $365 \mathrm{~nm}$ and $307 \mathrm{~nm}$ and $430 \mathrm{~nm}$. Knowing this relationship one can use a measurement of the $\mathrm{CO}_{2}$ * chemiluminescence intensity at $365 \mathrm{~nm}$ to determine the magnitude of the $\mathrm{CO}_{2} *$ chemiluminescence intensity at $307 \mathrm{~nm}$ and $430 \mathrm{~nm}$.

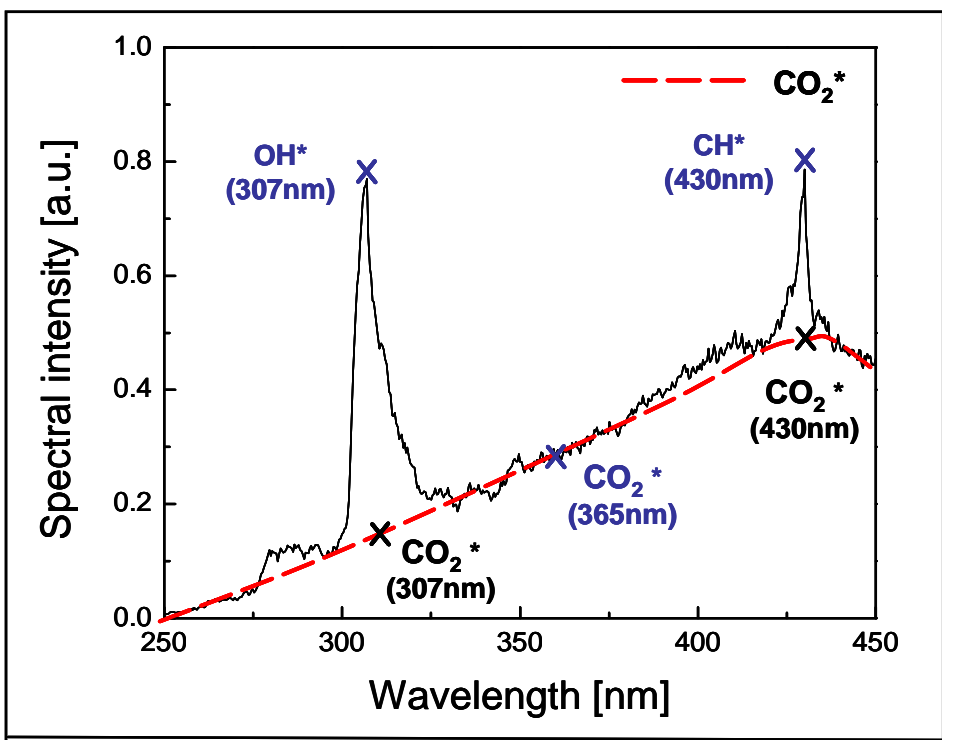

Figure 63: $\mathrm{OH}^{*}, \mathrm{CH}^{*}$, and $\mathrm{CO}^{*}$ chemiluminescence spectra showing spectra.

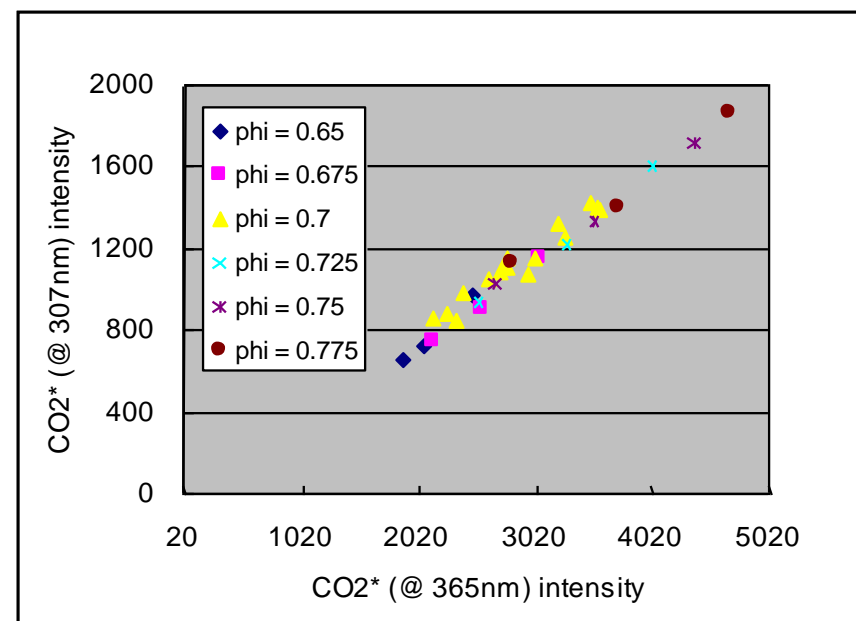

(a)

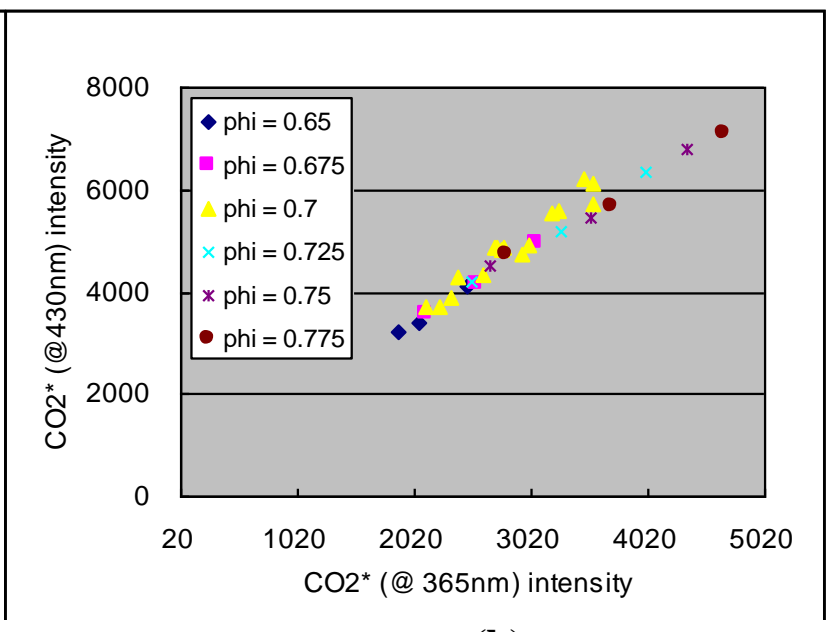

(b)

Figure 64: Relationship between the $\mathrm{CO}_{2} *$ chemiluminescence intensity at (a) $307 \mathrm{~nm}$ and $365 \mathrm{~nm}$ and (b) $430 \mathrm{~nm}$ and $365 \mathrm{~nm}$. 
As mentioned above, the relationship between the chemiluminescence intensity and the rate of heat release is dependent on the flame's equivalence ratio. It was also mentioned that the technique that can be used to measure the flame's equivalence ratio is based on the ratio of the $\mathrm{OH}^{*}$ and $\mathrm{CH}^{*}$ chemiluminescence intensities. Figure 65 illustrates this relationship and the importance of using $\mathrm{CO}_{2} *$ background subtracted $\mathrm{OH}^{*}$ and $\mathrm{CH}^{*}$ chemiluminescence measurements. The red data corresponds to the raw data and the blue data corresponds to the background subtracted data. Also shown in this figure are straight line correlations to the two sets of data. These results clearly indicate the advantages of using the background subtracted data.

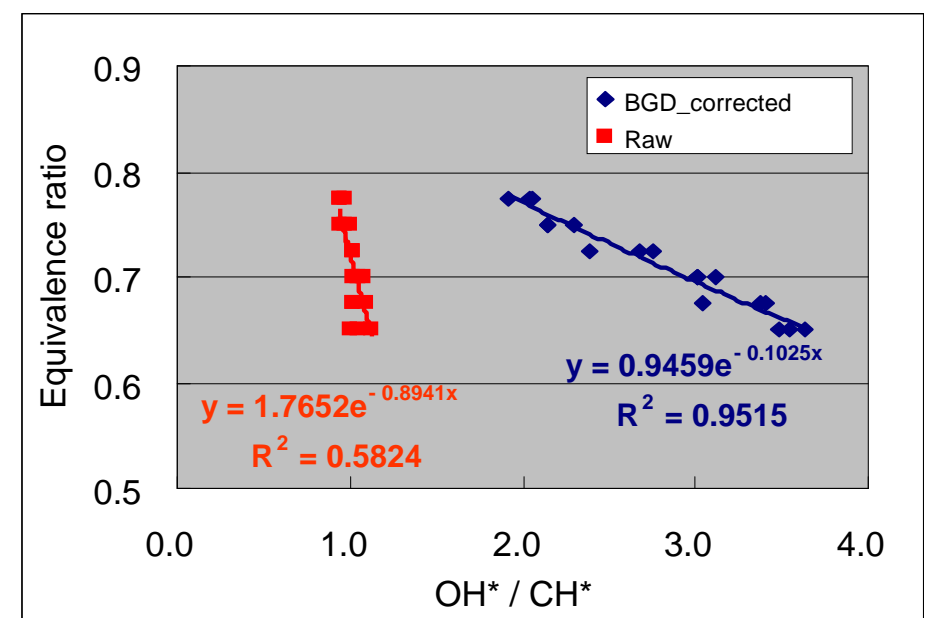

Figure 65: Actual equivalence ratio versus the measured $\mathrm{OH} * / \mathrm{CH}^{*}$ chemiluminescence intensity. Red data corresponds to the raw data; while the blue data corresponds to the background subtracted data.

\section{Oscillating Flame Measurements}

The next step was to apply and evaluate this technique in an oscillating flame. In order to evaluate the equivalence ratio measurement technique in an oscillating flame, the actual timevary equivalence ratio must be independently known. Unfortunately, measuring the time-varying equivalence ratio along the flame front is a difficult measurement. Therefore, it was decided to apply the equivalence ratio measurement to an oscillating flame with no equivalence ratio fluctuations. As noted previously, this is achieved by mixing the fuel and air upstream of a choked inlet to the combustor. If the time-varying nature of the flame introduces an error in the equivalence ratio measurement, this should also be evident in the case when the equivalence ratio is constant. In fact the uncertainty in the actual equivalence ratio will be significantly smaller, compared to the case when the fluctuating equivalence is independently measured.

The results for two operating conditions are shown in Figure 66(a) and Figure 66(b), where the velcity fluctuation in the mixing section and the chemiluminescence-based equivalence ratio measurement are plotted versus time. In each figure, two results are presented for the equivalence ratio measurement. In one case (the yellow curve) the $\mathrm{CO}_{2}{ }^{*}$ chemiluminescence background has been subtracted from the $\mathrm{OH}^{*}$ and $\mathrm{CH}^{*}$ chemiluminescence intensities. In the other case (the red curve) the $\mathrm{CO}_{2}{ }^{*}$ background is not removed. In both cases the inlet velocity is $30 \mathrm{~m} / \mathrm{s}$ and the equivalence ratio is 0.7 . The results shown in Figure $66 \mathrm{a}$ are for a $100 \mathrm{~Hz}$ forced 
oscillation with a $10 \%$ velocity fluctuation; while the results shown in Figure $66 \mathrm{~b}$ are for a 250 $\mathrm{Hz}$ forced oscillation with a velocity fluctuation of $8 \%$. Ideally there should be no fluctuation in the measured equivalence ratio, however, that is clearly not the case. The results show that subtracting the background $\mathrm{CO}_{2}{ }^{*}$ chemiluminescence and increasing the oscillation frequency both significantly reduce the magnitude of the measured equivalence ratio fluctuation, i.e., with background subtraction at $100 \mathrm{~Hz}$, the equivalence fluctuation is $5.1 \%$, while at $250 \mathrm{~Hz}$ with background subtraction the equivalence ratio fluctuation is only $1.7 \%$. This behavior was observed at all conditions tested as shown in Figure 67which summarizes the results that were obtained over a range of frequencies from $75 \mathrm{~Hz}$ to $280 \mathrm{~Hz}$ and velocity fluctuations from $2 \%$ to $15 \%$. These results provide an indication of the minimum equivalence fluctuation magnitude that can be measured with this technique.

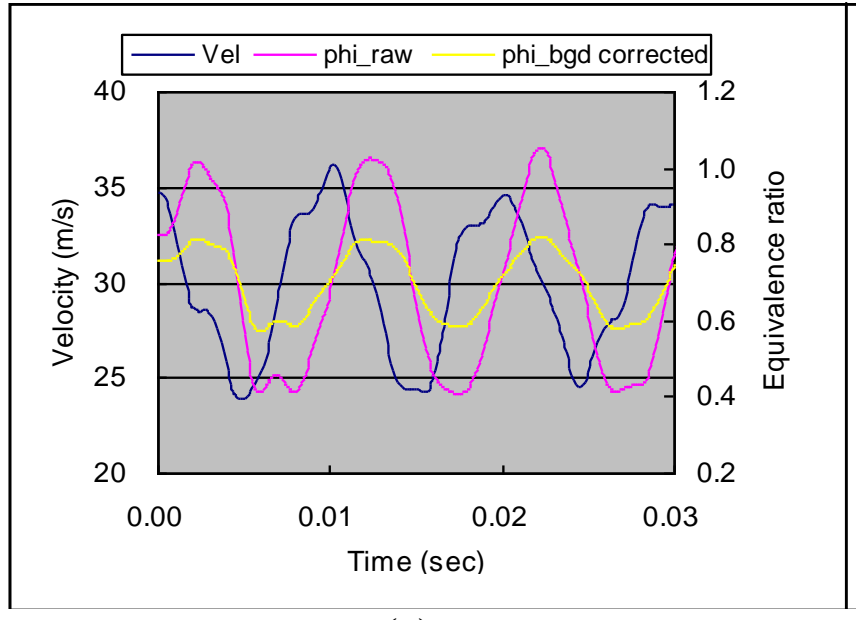

(a)

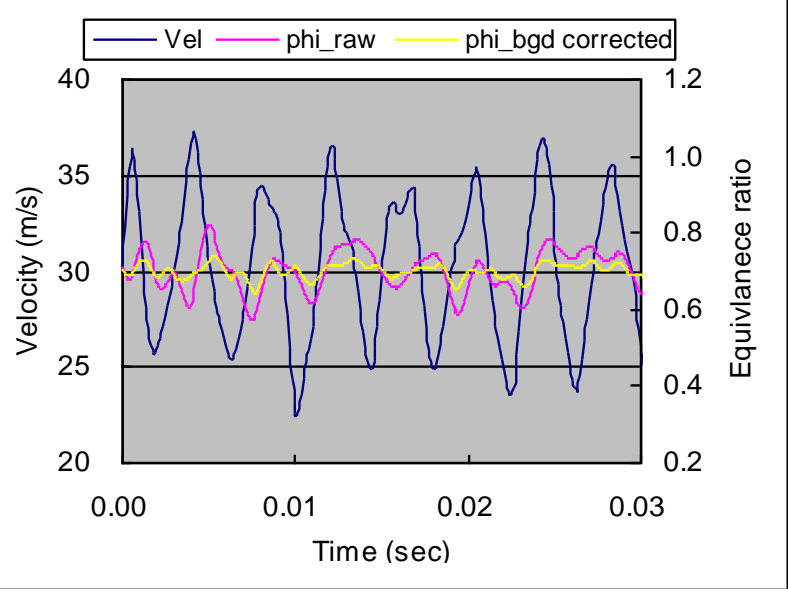

(b)

Figure 66: Equivalence ratio fluctuation measurements for a (a) $100 \mathrm{~Hz}$ forced oscillation with a $10 \%$ velocity fluctuation, (b) $250 \mathrm{~Hz}$ forcd oscillation with an $8 \%$ velocity fluctuation. $(\mathrm{V}=\mathbf{3 0} \mathrm{m} / \mathrm{s}, \phi=0.7)$

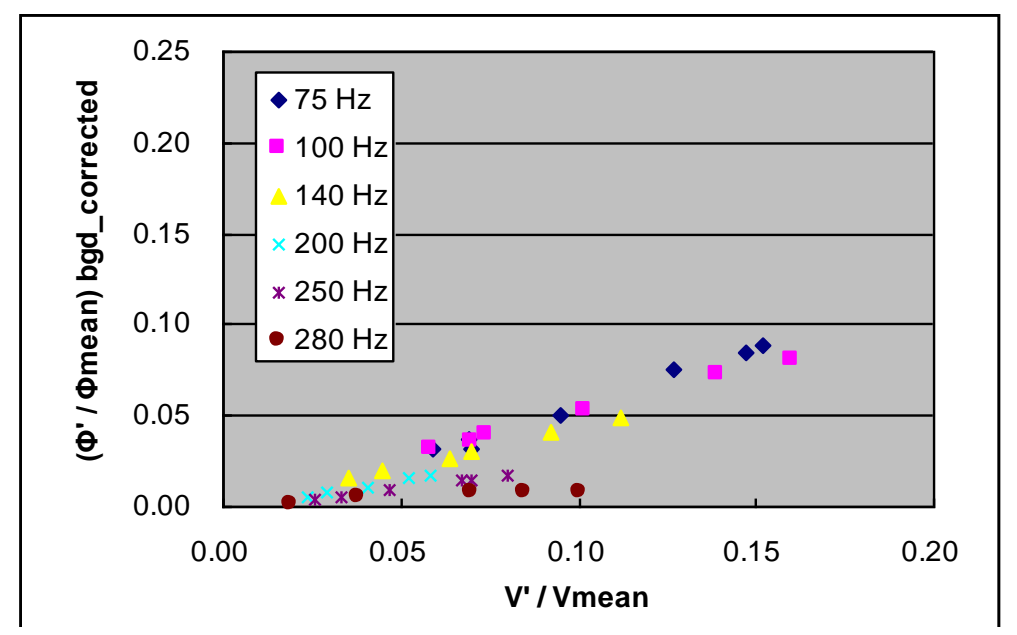

Figure 67: The error in the equivalence ratio fluctuation measurement as a function of fluctuation frequency and the magnitude of the forced velocity fluctuation. 


\section{Review}

There is a linear relationship between the overall chemiluminescence from lean premixed hydrocarbon - air flames and the flame's rate of heat release. Because this relationship is also dependent on the equivalence ratio, making chemiluminescence-based heat release measurements under conditions where the equivalence ratio is fluctuating requires an independent measurement of the time-varying equivalence ratio. It has been demonstrated in steady flames that the ratio of the $\mathrm{OH}^{*}$ and $\mathrm{CH}^{*}$ chemiluminescence intensity, after subtracting the background $\mathrm{CO}_{2} *$ chemiluminescence, is proportional to the equivalence ratio. As part of this work, a methodology for making such measurements in unsteady flames with has been developed and its limitations assessed. It has been shown that the technique's ability to measure equivalence ratio fluctuations varies with the frequency of the oscillation. At frequencies as low as $75 \mathrm{~Hz}$, there is an "artificial" fluctuation in the equivalence ratio measurement that can be as large as $5 \%$, while at a frequency of $250 \mathrm{~Hz}$ the "artificial" equivalence ratio fluctuation is reduced to approximately $2 \%$.

\section{Forced Response Measurements - Overview}

\section{Introduction}

Once again, the successful development of the flame response model discussed previously in the analytical results section will depend on the availability of experimental flame response data obtained over a broad range of flow conditions. This data is necessary to both guide the formulation of the model and to validate the model's predictions. Determination of the flame response function requires measurements of the system input and the system output. The system input includes the velocity fluctuation and the equivalence fluctuation near the exit of the mixing section, where both the amplitude and phase of the fluctuations must be measured. The system output is the fluctuation in the flame's rate of heat release.

\section{Velocity Fluctuation Measurements}

In an additional experimental setup, the velocity fluctuations are produced by a siren that has been designed for operation at inlet temperatures up to $300^{\circ} \mathrm{C}$ and modulation frequencies up to $500 \mathrm{~Hz}$. The magnitude of the velocity fluctuation is controlled by varying the fraction of the air that bypasses the siren and goes directly into the plenum upstream of the mixing section. Before installing the siren in the combustor test rig, its performance was evaluated in a simpler flow configuration which consisted of a 1" diameter pipe that was configured to have the same flow path as in the combustor test rig. In addition, the velocity fluctuation was measured at a distance downstream of the siren which is the same as in the combustor test rig. A representative result from these tests is shown in Figure 68. In this case the air was at room temperature, the air flow rate was $50 \mathrm{SCFM}$, the mean air velocity in the pipe was $20 \mathrm{~m} / \mathrm{s}$, and all of the air flowed through the siren, ie., the bypass valve was closed. 


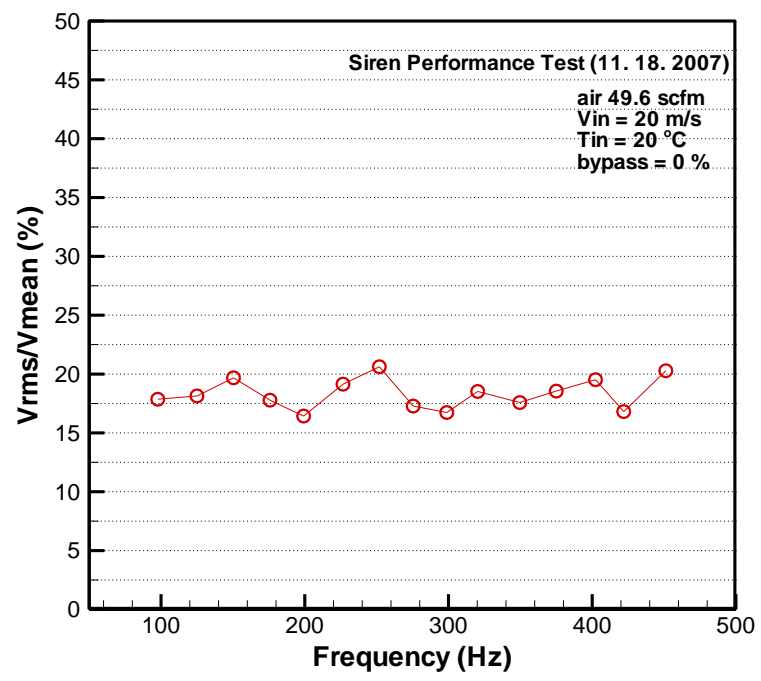

Figure 68: Normalized rms velocity fluctuation versus modulation frequency with $0 \%$ bypass measured in the performance test rig.

As shown in Figure 68, the siren is capable of producing a relatively constant velocity fluctuation over a range of modulation frequencies from $75 \mathrm{~Hz}$ to $450 \mathrm{~Hz}$. This behavior was also observed at other velocities and bypass ratios. The measurement of the velocity fluctuations was performed using both hot wire anemometry and the two microphone technique, where the velocity fluctuations measured by the two techniques was found to agree within $10 \%$.

Next, the siren was installed and its performance evaluated in the combustor test rig. The primary differences between the performance test rig and the combustor test rig are 1) the flow passage in the mixing section is annular due to the centerbody and 2) there is a partial restriction of the flow at the entrance to the mixing section due to the ring that supports the centerbody. The blockage of the support ring is $25 \%$. These features are illustrated in Figure 69, which is a schematic drawing of the mixing section in the combustor test rig. Also shown in this figure is the location of the hot wire measurement and the two-microphone measurement.

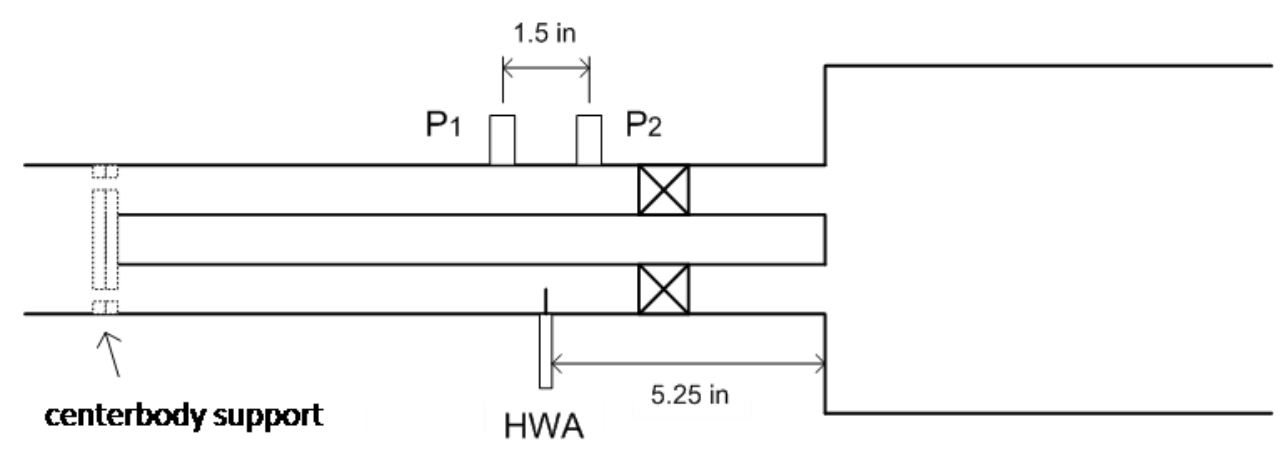

Figure 69: Schematic drawing of the mixing section in the combustor test rig. 
Normalized velocity fluctuation results obtained in the combustor test rig are presented in Figure 70, where the air flow rate is 85 SCFM and there was no bypass air. This figure shows that there is a significant attenuation of the fluctuation over a range of frequencies centered on $\sim 350 \mathrm{~Hz}$. This frequency corresponds closely with the Helmholtz resonance frequency of the mixing section, which is given by:

$$
f_{0}=\frac{c}{2 \pi} \sqrt{\frac{S}{L_{e f f} V}}
$$

where $\mathrm{c}$ is the speed of sound, $\mathrm{S}$ is the surface area of the inertance (i.e., the neck), $\mathrm{V}$ is the volume of the compliance, and $\mathrm{L}_{\text {eff }}$ is the effective length of the neck. This equation indicates that as the speed of sound increases or as the effective length of the neck decreases that the Helmholtz frequency should increase. Additional tests were performed whereby the speed of sound was increased by heating the intake air, and the effective length of the neck was decreased by removing a section of the centerbody support. Both cases showed the expected shift in the frequency at the minimum amplitude, supporting the argument that the attenuation is due to damping at the Helmholtz resonance frequency of the mixing section. The mixing section is currently being modified to relate the centerbody support so as to avoid this effect.

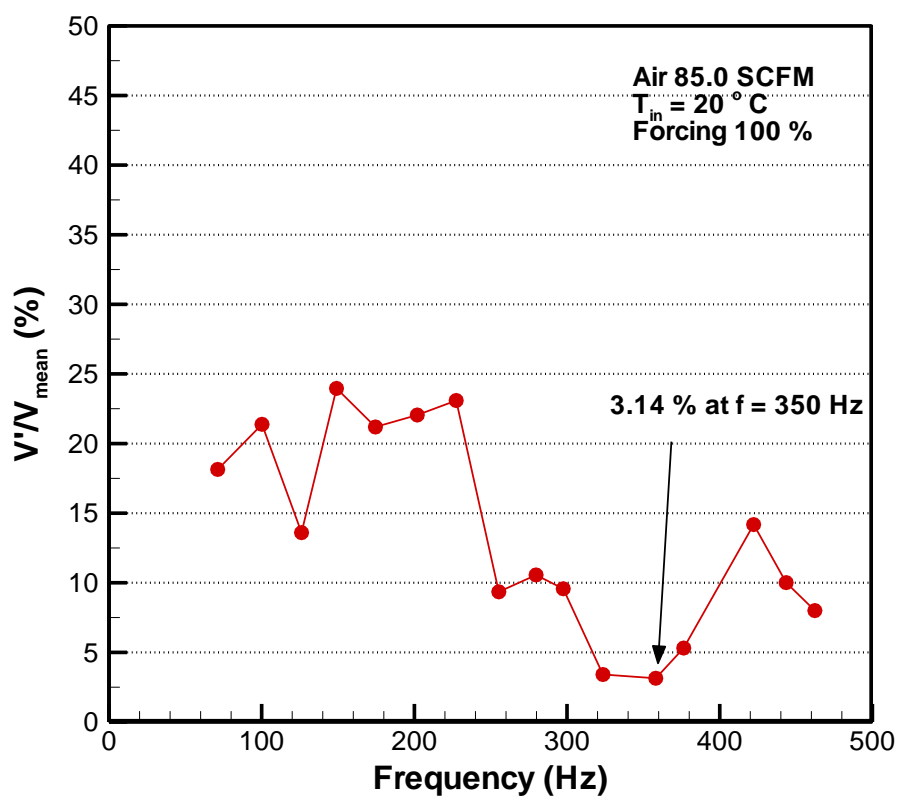

Figure 70: Normalized velocity fluctuation versus modulation frequency with $0 \%$ bypass measured in the combustor test rig. 


\section{Equivalence Ratio Fluctuation Measurements}

The fluctuation in the equivalence ratio at the exit of the mixing section will be measured using an infrared absorption technique. This involves the use of an infrared Helium Neon laser which passes through the mixing section at a location approximately $5 \mathrm{~mm}$ upstream of the dump plane. Infrared detectors are used to measure the intensity of the laser beam before and after it passes through the mixing section. The ratio of these intensities is related to the concentration of natural gas at the measurement location. This technique will be implemented on the combustor test rig in a future discussion. The fluctuation in the overall rate of heat release will be measured using chemiluminescence emission from excited state $\mathrm{CH}, \mathrm{OH}$ and $\mathrm{CO}_{2}$ molecules. The status of efforts to develop a methodology for applying this technique under partially premixed conditions was reported previously.

\section{Forced Response Measurements - Velocity Fluctuations}

\section{Introduction}

Additional forced flame response measurements were performed. These measurements were made with natural gas and under premixed conditions, where the fuel and air were premixed upstream of the choked inlet to the mixing section. Under these conditions the equivalence ratio is constant and the flame response is due to the imposed velocity fluctuations. These measurements were made at a fixed mean velocity $(60 \mathrm{~m} / \mathrm{s})$, inlet temperature $\left(200^{\circ} \mathrm{C}\right)$, and equivalence ratio (0.6), over a range of modulation frequencies from $100 \mathrm{~Hz}$ to $450 \mathrm{~Hz}$, in $25 \mathrm{~Hz}$ increments and fluctuations as large as $60 \%$. Measurements were made of the $\mathrm{OH}^{*}, \mathrm{CH}^{*}$ and $\mathrm{CO}_{2} *$ chemiluminescence emission, the combustor pressure and the velocity near the exit of the mixing section. The velocity fluctuation is measured using a two-microphone technique near the exit of the nozzle and the flame's response is defined by the fluctuation in the flame's rate of heat release, which is determined from $\mathrm{CH}^{*}$ chemiluminescence measurements. $\mathrm{H}_{2}$-blended natural gas fuels with $\mathrm{X}_{\mathrm{H} 2}=0.00 \sim 0.60$ were utilized.

\section{Results and Discussion}

Figure 71 shows a typical time trace of $\mathrm{OH}^{*}, \mathrm{CH}^{*}$, and $\mathrm{CO}_{2} *$ chemiluminescence, inlet velocity, and combustor pressure at a forcing frequency of $100 \mathrm{~Hz}$. The combustor pressure and the pressures for the two-microphone method are digitally filtered from $50 \mathrm{~Hz}$ to $150 \mathrm{~Hz}$, while the chemiluminescence intensity measurements are not filtered. Figure 71 shows that $\mathrm{OH}^{*}, \mathrm{CH}^{*}$, and $\mathrm{CO}_{2} *$ chemiluminescence intensity fluctuations are almost in phase with each other, and lead the combustor pressure fluctuation by approximately 70 degrees; while the inlet velocity fluctuation leads the combustor pressure fluctuation by approximately 110 degrees. The corresponding power spectra are shown in Figure 72, where both the fundamental frequency at $100 \mathrm{~Hz}$ and the higher harmonics are observed. 


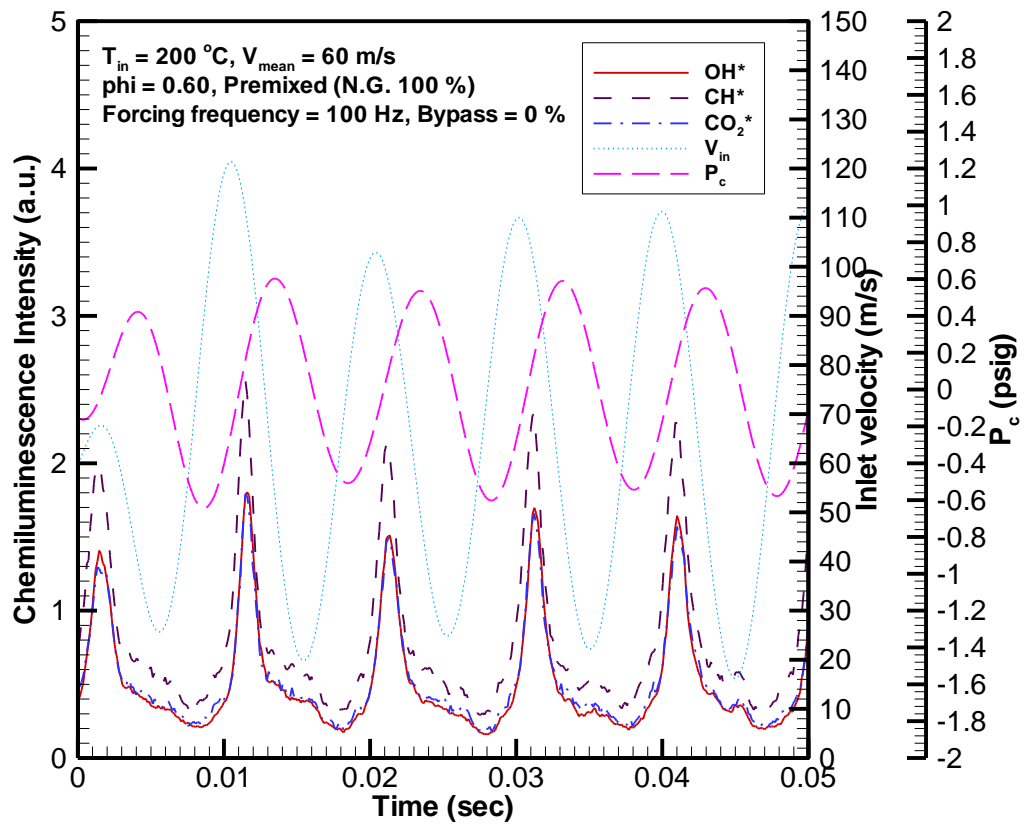

Figure 71: $\mathrm{OH}^{*}, \mathrm{CH}^{*}$, and $\mathrm{CO}_{2}^{*}$ chemiluminescence intensity, inlet velocity, and combustor pressure versus time for a $100 \mathrm{~Hz}$ forcing frequency.

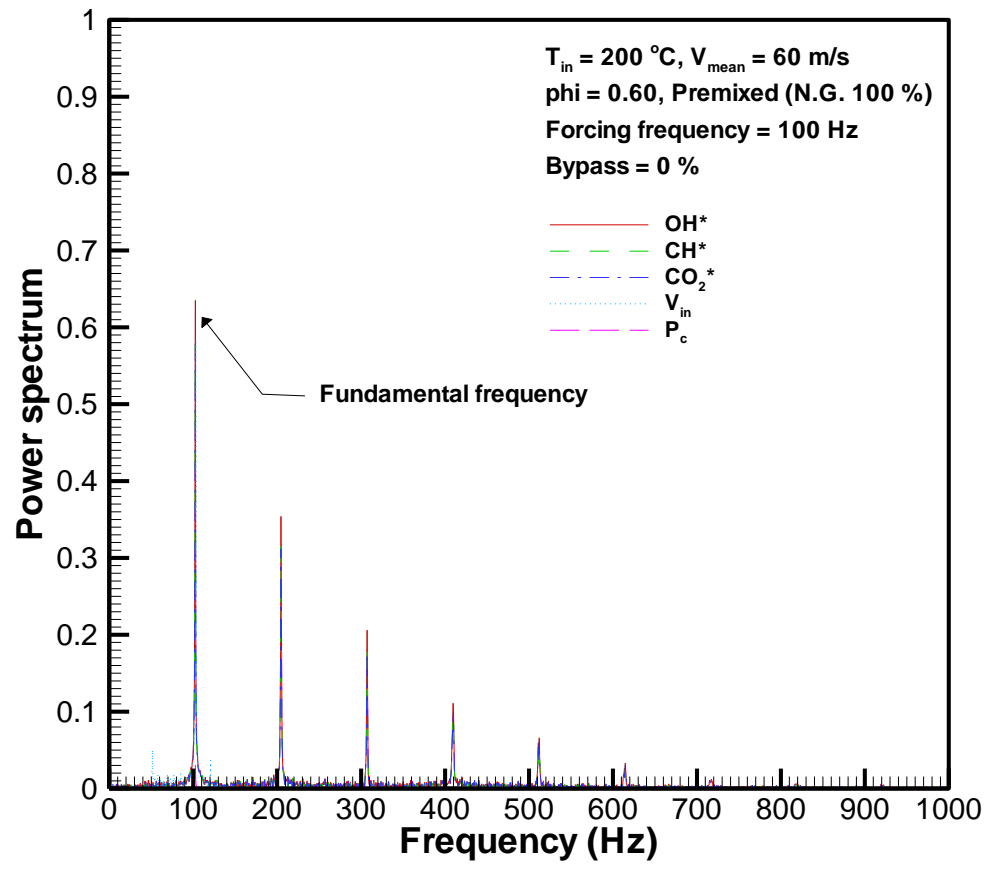

Figure 72: Power spectra of $\mathrm{OH}^{*}, \mathrm{CH}^{*}$, and $\mathrm{CO}_{2}^{*}$ chemiluminescence intensity, inlet velocity, and combustor pressure fluctuations with a forcing frequency of $100 \mathrm{~Hz}$. 
Figure 73 shows typical flame response measurements for fixed inlet temperature, mean nozzle velocity, and equivalence ratio for the case of natural gas fuel. For these tests the fuel and air are mixed upstream of the choked inlet to the nozzle to eliminate the possibility of equivalence ratio fluctuations. These data show both the linear and nonlinear flame response regimes that the model must account for to provide accurate predictions of instability frequency and amplitude. The heat release fluctuation increases linearly with the velocity fluctuation at $100 \mathrm{~Hz}$. At higher forcing frequencies the heat release response shows evidence of nonlinear behavior, which leads to saturation, most notably in the higher frequency cases presented in Figure 73. Also note that the forcing amplitude at which the heat release fluctuation becomes saturated decreases with increasing frequency. This behavior is consistent with the observations of Lieuwen et al. and Balachandran et al. and is associated with vortex rollup of the flame sheet.

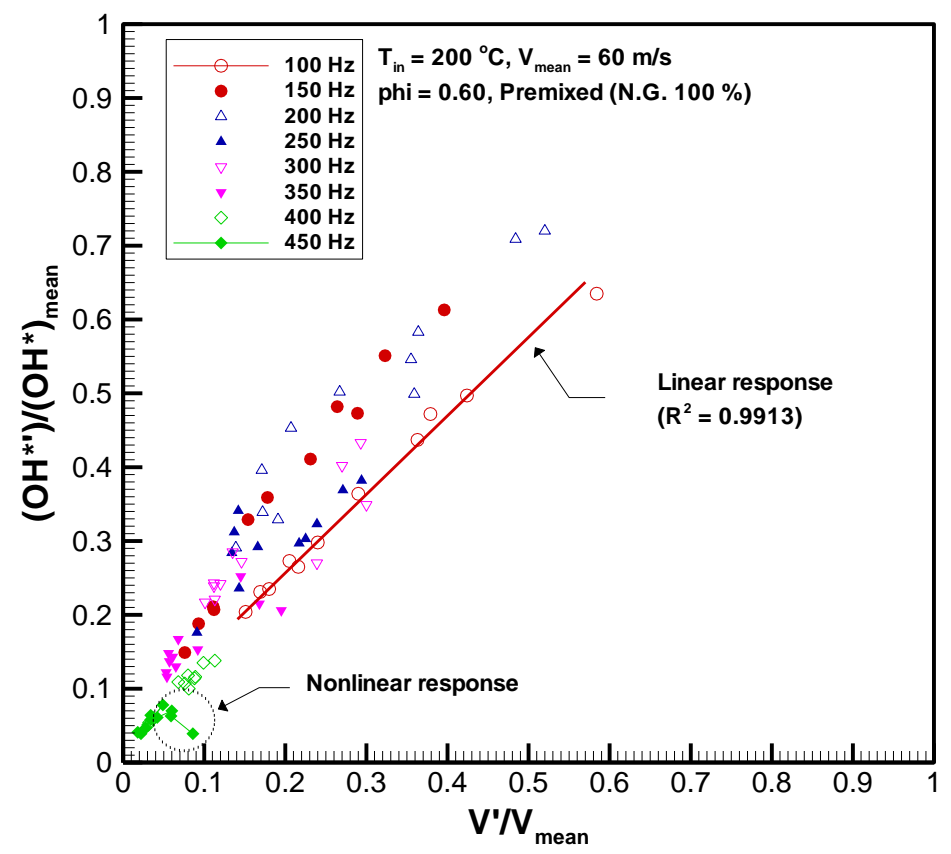

Figure 73: The dependence of normalized global heat release fluctuation (as indicated by the $\mathrm{OH}^{*}$ chemiluminescence intensity) on the forcing velocity amplitude. Normalized fluctuation in the rate of heat release, as indicated by $\mathrm{OH}^{*}$ chemiluminescence, versus the normalized velocity fluctuation for an inlet temperature of $200^{\circ} \mathrm{C}$, a mean nozzle velocity of $60 \mathrm{~m} / \mathrm{s}$ and an equivalence ratio of 0.6 over a range of forcing frequencies from 100 to 450 Hz with natural gas fuel.

Figure 74 (a) and (b) show the gain and phase of the flame transfer function as a function of inlet velocity amplitude. As the forcing frequency increases, nonlinear response of a flame becomes dominant. When the modulation frequency is less than $200 \mathrm{~Hz}$, the phase is constant with respect to the amplitude of the inlet velocity oscillations. However, at higher modulation frequency, they are not constant any more. This could be due to the fact that at these forcing frequencies the flame might not be acoustically convectively compact. Global flame image measurements are required to confirm this hypothesis. 


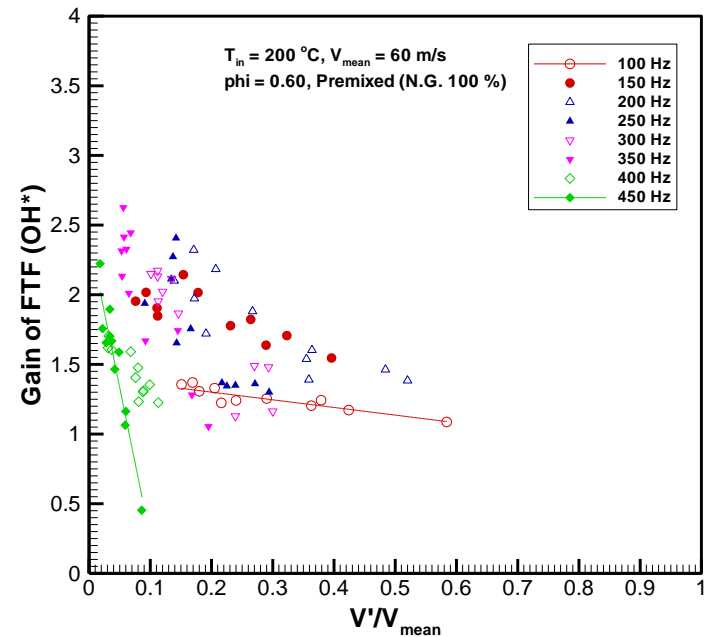

(a)

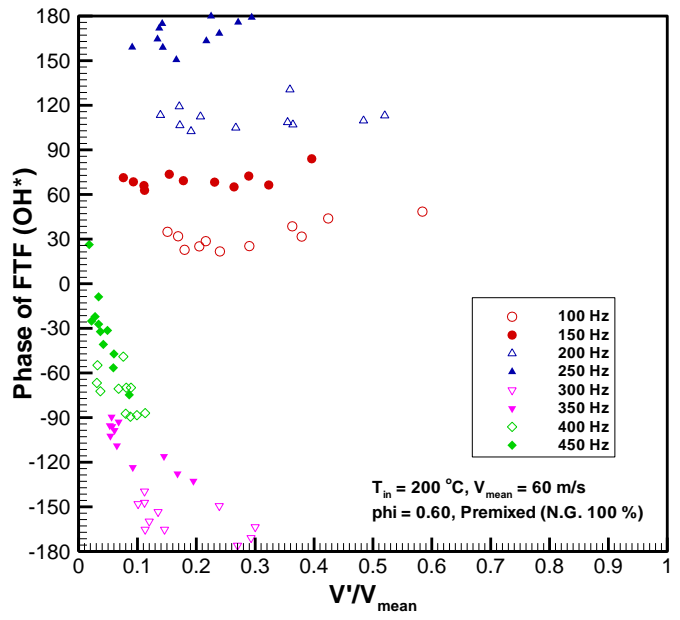

(b)

Figure 74: (a) Gain and (b) Phase of the flame transfer function based as a function of forcing velocity amplitude.

Figure 75(a) shows the gain of the flame transfer function as a function of the forcing frequency for a constant inlet velocity fluctuation magnitude of $15 \%$. Initially the gain increases with increasing forcing frequency, until it reaches a maximum, and then decreases. Although measurements were not made at frequencies less than $100 \mathrm{~Hz}$, because of an inherent limitation of the two microphone technique, the results appear to be approaching a gain of 1 in the limit of zero frequency. Similarly, although modulation amplitudes of $15 \%$ were not possible at frequencies above $400 \mathrm{~Hz}$, the results indicate that the gain is approaching zero in the high frequency limit. Figure 75(b) shows the phase of the FTF as a function of frequency for a constant inlet velocity fluctuation magnitude of $15 \%$. It can be seen that the phase evolves quasilinearly with forcing frequency. Using the phase information, the variation in convection delay time as the function of forcing frequency is plotted in Figure 75(c). As shown, the convective time decreases with increasing frequency, from $11 \mathrm{msec}$ to $5 \mathrm{msec}$. 


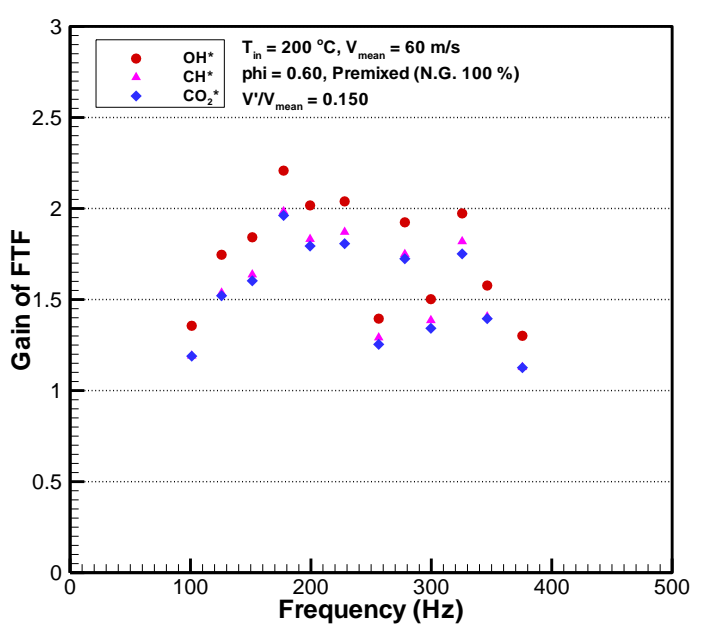

(a)

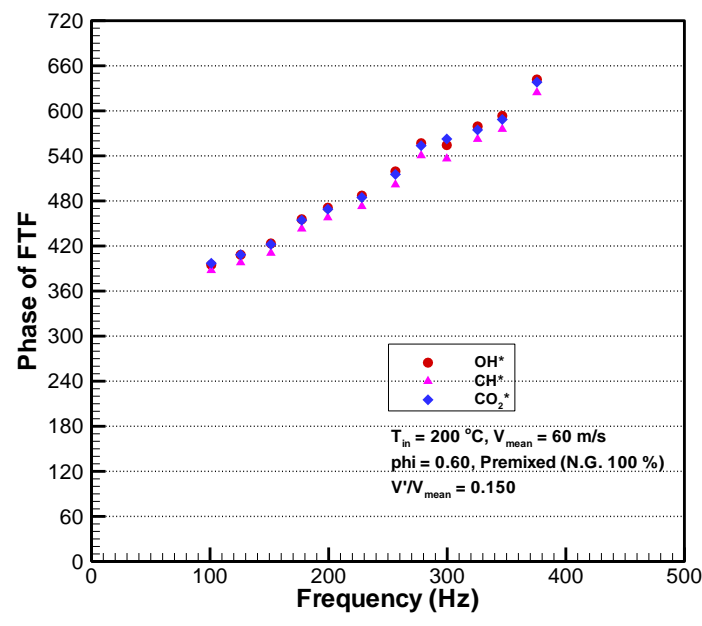

(b)

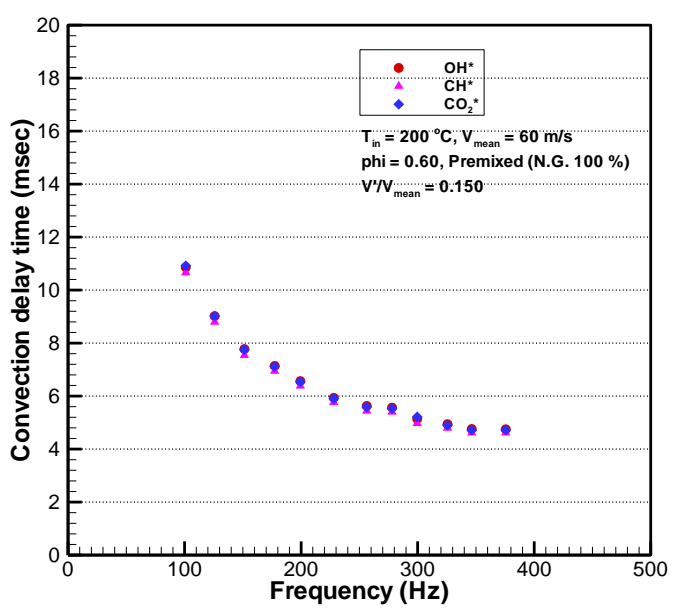

(c)

Figure 75: (a) Gain and (b) Phase of the flame transfer function, and (c) Convection time delay at a constant inlet velocity fluctuation magnitudes of $15 \%$.

Figure 76 (a) shows the gain of the FTF at $\mathrm{V}^{\prime} / \mathrm{V}_{\text {mean }}=0.11,0.15,0.20$, and 0.25 . It can be observed that with increasing forcing amplitude the gain decreases. The phase and convection delay time of the FTF at $\mathrm{V}^{\prime} / \mathrm{V}_{\text {mean }}=0.11,0.15,0.20$, and 0.25 are presented in Figure $76(\mathrm{~b})$ and (c), respectively. These results show that there is no influence of the pulsation level on the time delay and phase angle, respectively. 


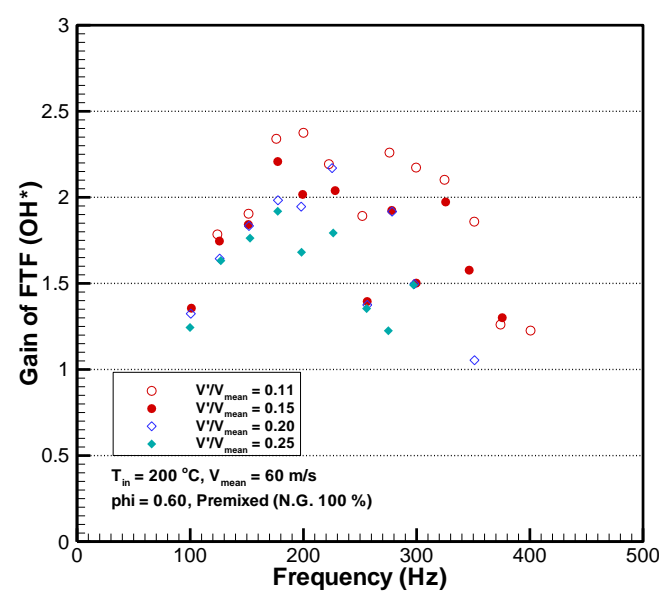

(a)

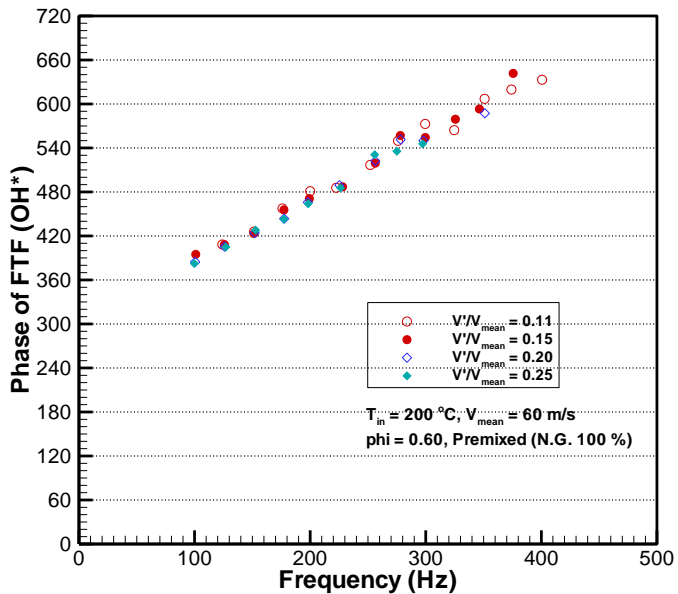

(b)

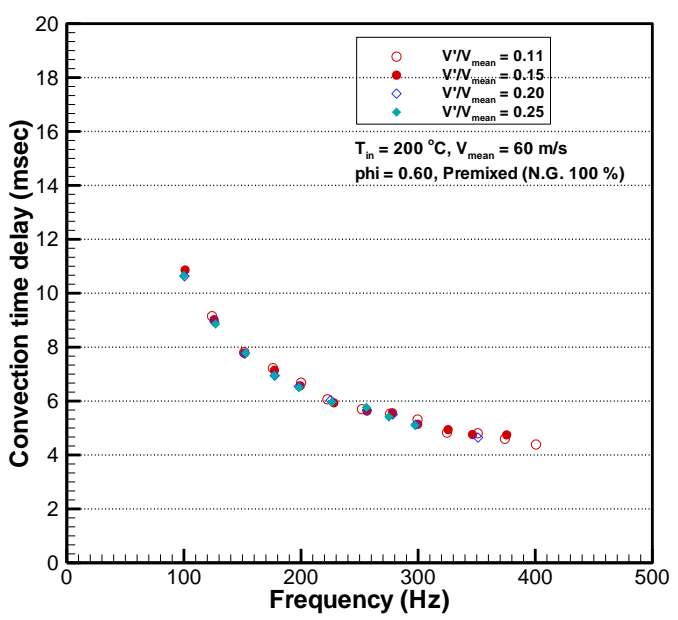

(c)

Figure 76: (a) Gain and (b) Phase of the flame transfer function, and (c) Convection time delay at a constant inlet velocity fluctuation magnitudes of $11,15,20$, and $25 \%$.

In addition to the forced response measurements, the self-excited instability characteristics of the combustor are determined in a variable length combustor. Typical results are shown in Figure 77, where the normalized combustor pressure fluctuation (upper graphs) and the instability frequency (lower graphs) are plotted versus the combustor length for the case of $100 \%$ natural gas on the left and $45 \%$ hydrogen-55\% natural gas on the right. These results show that with $100 \%$ natural gas unstable combustion occurs at combustor lengths near 58" with a frequency of approximately $210 \mathrm{~Hz}$. With the addition of $45 \%$ hydrogen in the fuel, the instability region shifts to combustor lengths near 42" with a frequency of approximately $380 \mathrm{~Hz}$. Other measurements show that as the hydrogen percentage increases from $0 \%$ to $45 \%$ the $\sim 210 \mathrm{~Hz}$ instability weakens and the $\sim 380 \mathrm{~Hz}$ instability strengthens, but no instabilities are observed at intermediate frequencies. 

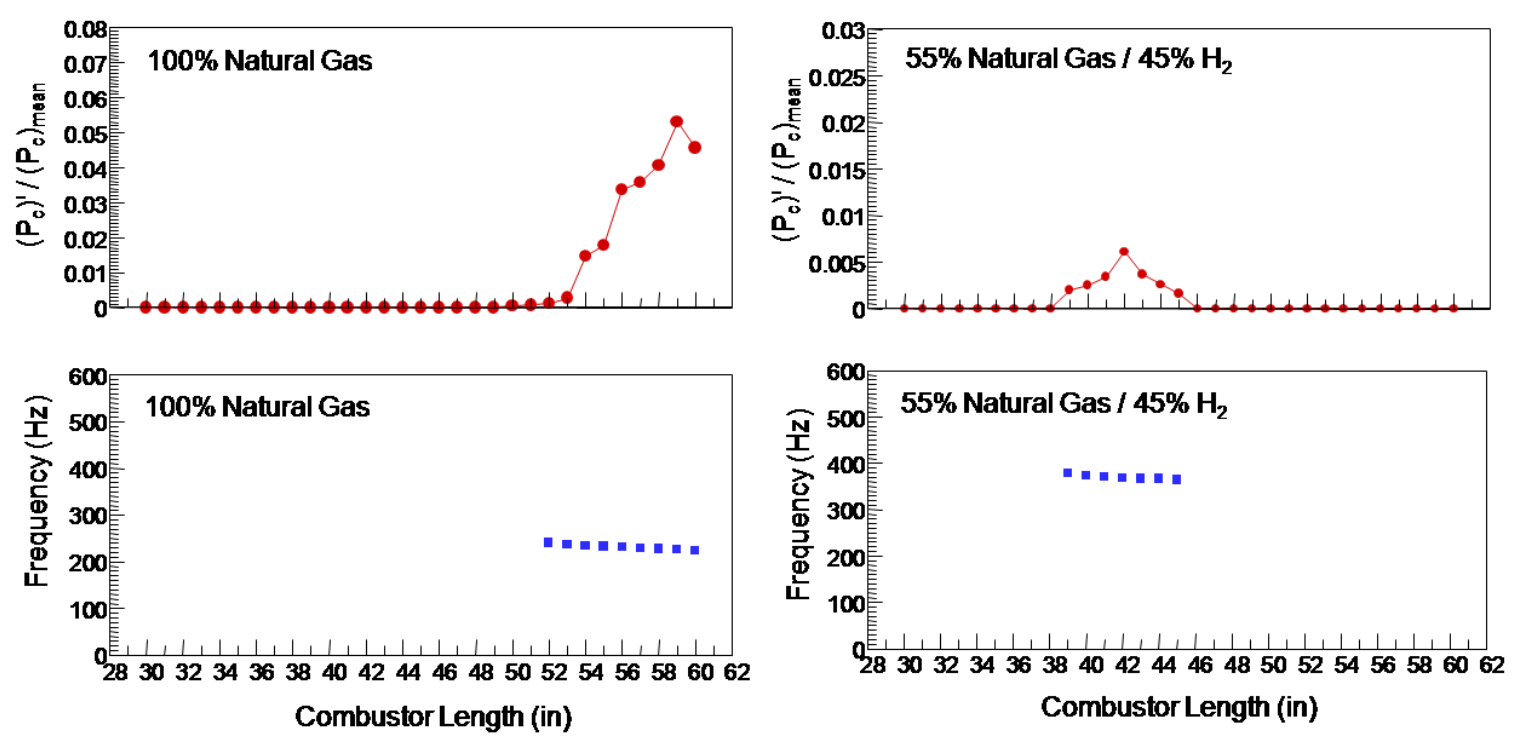

Figure 77: Normalized combustor pressure fluctuation and corresponding frequency of self-excited instabilities as a function of the combustor length at an inlet temperature of $200^{\circ} \mathrm{C}$, a mean inlet velocity of $60 \mathrm{~m} / \mathrm{s}$, and an equivalence ration of 0.6 . The graphs on the left are for $100 \%$ natural gas and the graphs on the right are for a $55 \% / 45 \%$ natural gashydrogen fuel mixture.

Figure 78 presents the flame transfer function for a constant forcing amplitude. The amplitude of the velocity perturbation imposed on the airflow is limited to $10 \%$ of the mean velocity, to ensure a linear response of the flame to the acoustic forcing. Other operating conditions are the same, except for the $\mathrm{H}_{2}$ mole fraction: $\mathrm{X}_{\mathrm{H} 2}=0 \%$ and $15 \%$. The global flame response can be described as a low-pass filter, in that the gain decreases as the frequency increases. These results also show that with increasing $\mathrm{X}_{\mathrm{H} 2}$, the maximum gain of FTF decreases by $43 \%$ and the phase decreases at a given forcing frequency, indicating that the gain depends strongly on flame length. The phase can be approximated by $\Delta \varphi=\tau(2 \pi \mathrm{f})$, since the phase linearly evolves with increasing modulation frequency.
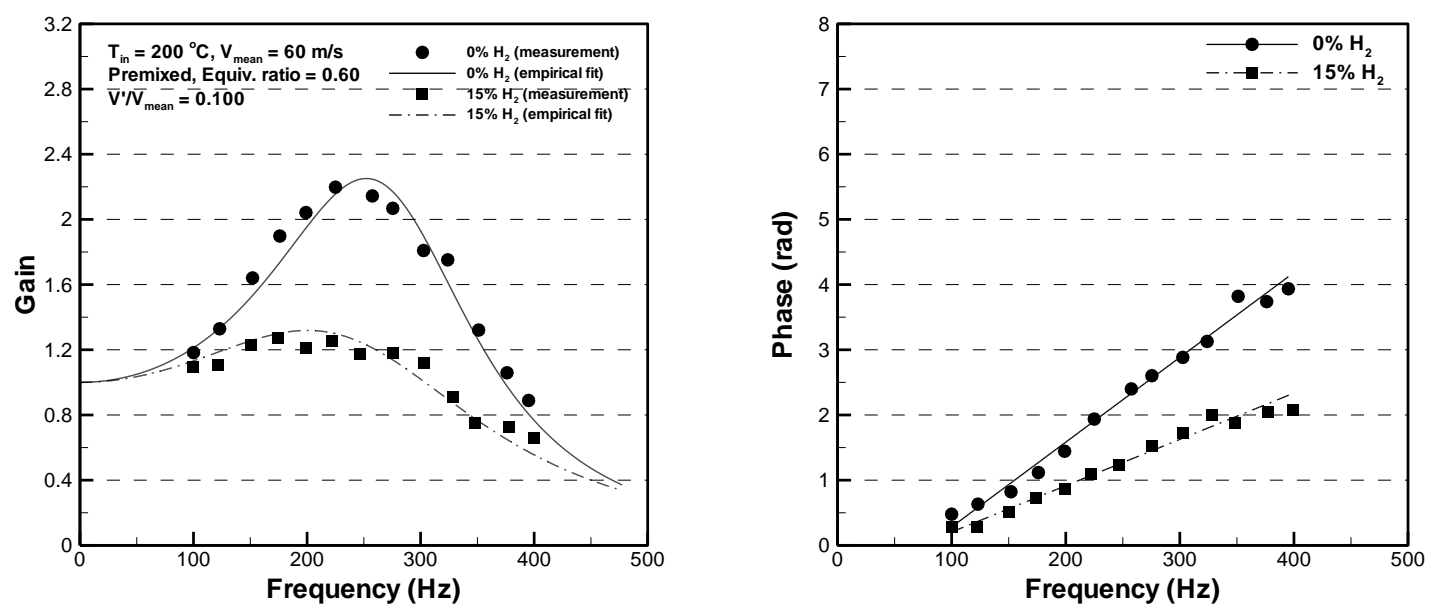
Figure 78: Gain and phase of the flame transfer function vs. forcing frequency at $V^{\prime} / V_{\text {mean }}$ $=0.100$. Inlet conditions: $T_{\text {in }}=200{ }^{\circ} \mathrm{C}, V_{\text {mean }}=60 \mathrm{~m} / \mathrm{s}, \Phi=0.60$, premixed, and $X_{\mathrm{H} 2}=0.00$, 0.15. Note that the gain of FTF is well fitted by a second-order oscillator model (lines).

The nonlinear dynamics of swirled flames were characterized by phase-resolved flame image measurements. Deconvoluted and line-of-sight integrated phase-averaged $\mathrm{CH}^{*}$ chemiluminescence images at a modulation frequency of $200 \mathrm{~Hz}$ and amplitude of $\mathrm{V}^{\prime} / \mathrm{V}_{\text {mean }}=$ $25 \%$ are shown in Figure 79. As forcing amplitude increases, the flame front bends toward the inner recirculation zone at $\varphi=270^{\circ}$. The interaction between a vortex-ring structure and the flame is significant at $\varphi=180^{\circ} \sim 300^{\circ}$. In particular, line-of-sight integrated images at $\varphi=150$ and $240^{\circ}$ clearly show rollup of the shear layer and convection in the flow direction, respectively. It has been reported that the nonlinear flame response is related to the shear layer rollup. The shear layer rollup shortens the flame length, which in turn decreases the flame area in a nonlinear manner.

(A)

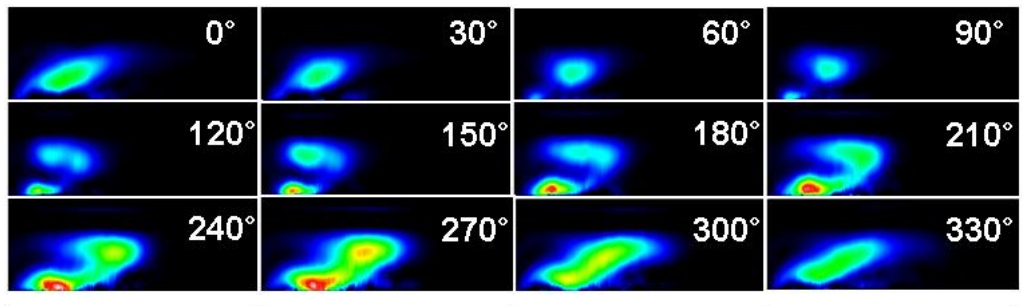

(B)

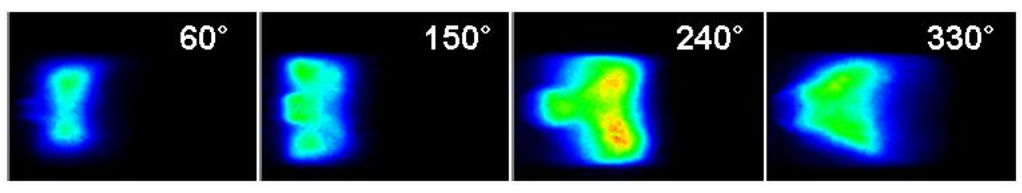

Figure 79: (A) Phase-synchronized $\mathrm{CH}^{*}$ chemiluminescence imaging at modulation frequency of $200 \mathrm{~Hz}$ and $V^{\prime} / V_{\text {mean }}=25 \%$. (B) line-of-sight integrated, backgroundcorrected $\mathrm{CH}^{*}$ images at $\varphi=60,150,240,330^{\circ}$. Inlet conditions: $T_{\text {in }}=200{ }^{\circ} \mathrm{C}, \mathrm{V}_{\text {mean }}=60$ $\mathrm{m} / \mathrm{s}, \Phi=0.60$, premixed, and $\mathrm{X}_{\mathrm{H} 2}=0.00$.

(A)

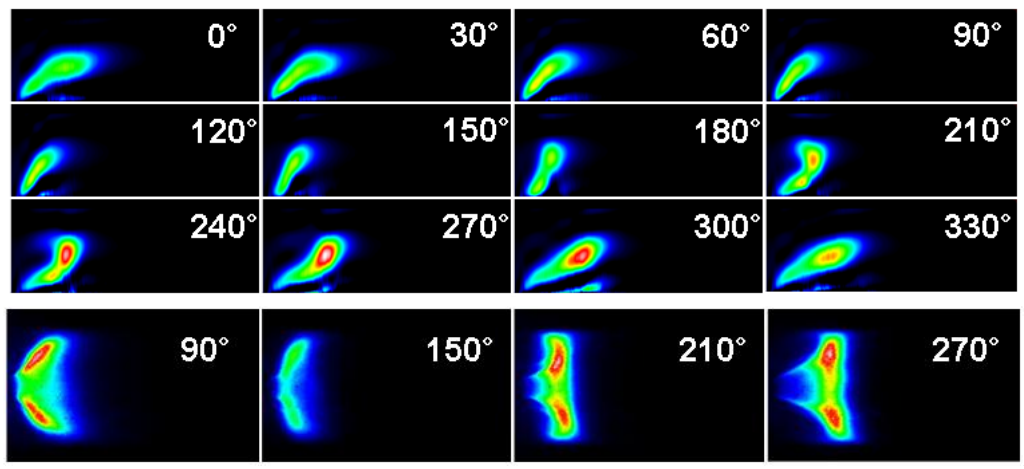

Figure 80: (A) Phase-synchronized $\mathrm{CH}^{*}$ chemiluminescence imaging at $f=200 \mathrm{~Hz}$ and $V^{\prime} / V_{\text {mean }}=25 \%$, (B) line-of-sight integrated $\mathrm{CH}^{*}$ images at $\varphi=90^{\circ}, 150^{\circ}, 210^{\circ}$, and $270^{\circ}$. Inlet conditions: $T_{\text {in }}=200{ }^{\circ} \mathrm{C}, V_{\text {mean }}=60 \mathrm{~m} / \mathrm{s}, \Phi=0.60$, premixed, and $X_{\mathrm{H} 2}=0.30$. 
The dynamics of $\mathrm{H}_{2}$-enriched natural gas/air premixed flames are shown in Figure 80. It is evident that the flame angle changes over the course of modulation cycle is substantial. In the case of natural gas flames on the other hand, the flame oscillates in the flow direction, which means that the flame length changes over a period of perturbations, as shown in Figure 79. These oscillations in the flame position (angle) result from fluctuations in the outer shear layer. It has been reported that if the shear layers in the swirl-stabilized gas turbine combustor are acoustically excited to form a coherent vortex ring structure, the inner shear layer rolls up inward and the outer shear layer rolls up outward. This result suggests that the dynamics of $\mathrm{H}_{2}$-enriched "M" flames is totally different from that of "V" flames. It was found that even though periodic formation and convection of vortex structures occur at $\varphi=210 \sim 270^{\circ}$, the flame response remains in the linear regime.

Having focused primarily on premixed flames up until now, an additional purpose of the work presented in this section of the report is to investigate a superposition/decomposition method of analyzing the heat release response of a partially premixed flame by using a vector summation method. The decomposition of the partially premixed flame response into two sub-component vectors provides useful insight into the underlying phenomena, and consequently suggests potential passive control methodologies to suppress combustion instabilities.

The heat release response of a partially premixed flame can be mathematically expressed as the following equation (two-input one-output model):

$$
\frac{Q^{\prime}}{\bar{Q}}=H_{V}(\omega) \frac{V^{\prime}}{\bar{V}}+H_{\Phi}(\omega) \frac{\Phi^{\prime}}{\bar{\Phi}}
$$

where $H_{V}$ and $H_{\phi}$ denote the flame transfer functions for independent oscillations of the inlet velocity and the equivalence ratio. Physically, the equation means that the response of a partially premixed flame can be linearly superposed from two independent responses. Each term in the equation is a complex quantity. Therefore, the response of the partially premixed flame can be reconstructed by a vector summation. In Figure 81, the two vectors, $Q_{v}$ and $Q_{\Phi}$, denote the heat release response to velocity oscillations and equivalence ratio oscillations, respectively. They were measured separately. The phase of the individual heat release response, which is represented by the angle between the vector and the x-axis and is equal to the phase of the corresponding oscillation, i.e., $V^{\prime}$ or $\Phi^{\prime}$. The resulting summation vector, $Q_{v+\Phi}$, gives the predicted heat release response of the partially premixed flame with the indicated inlet velocity and equivalence ratio oscillations. Also shown in Figure 81 is a symbol ( $\square$ ) which represents the actual flame response of a partially premixed flame at the inlet conditions. 


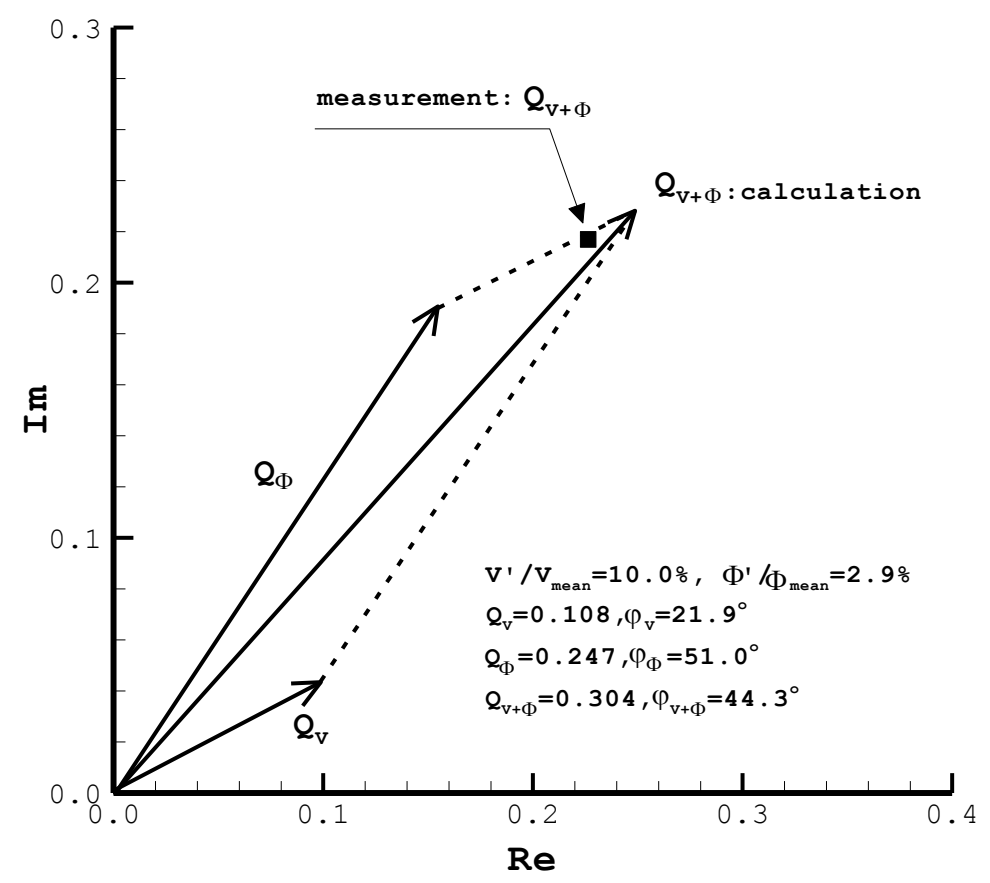

Figure 81: Prediction of the normalized heat release response of a partially premixed flame at a modulation frequency of $100 \mathrm{~Hz}$ and amplitude of $v^{\prime} / V_{\text {mean }}=10.0 \%, \Phi \Phi_{\text {mean }}=2.9 \%$. Inlet conditions: $T_{i n}=200{ }^{\circ} \mathrm{C}, v_{\text {mean }}=60 \mathrm{~m} / \mathrm{s}, \Phi_{\operatorname{mean}}=0.60$, and $x_{H_{2}}=0.00$.

First of all, this analysis demonstrates that the heat release response of a partially premixed flame can be effectively reconstructed using independent measurements of the flame's response to velocity and equivalence ratio fluctuations. Second, the contribution of equivalence ratio oscillation to the global heat release rate oscillation of the partially premixed flame exceeds that of the velocity fluctuation by a factor of 2 . Third, the phase difference between the heat release and equivalence ratio oscillations is larger than the phase difference between heat release and inlet velocity oscillations, by a factor of approximately two. This result indicates that the heat release response of a partially premixed flame is chemical kinetics-controlled in terms of the response time scales. The vector plots further suggest that depending on the phase difference between the velocity and equivalence ratio oscillations, the response of a partially premixed flame can be reconstructed constructively and destructively. 


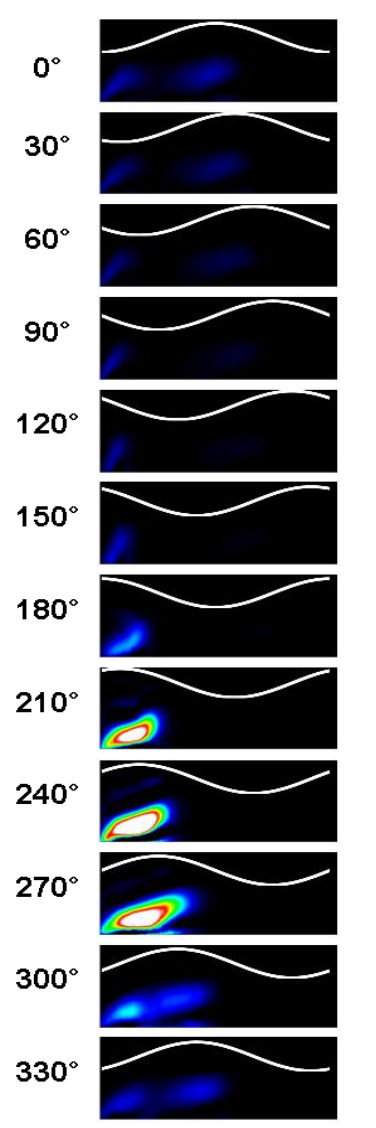

(A) In-phase interference

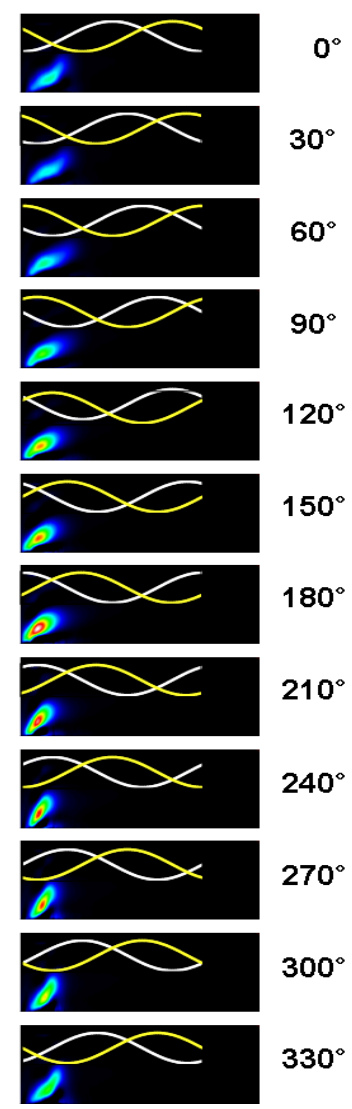

(B) Out-of-phase interference

Figure 82: Phase-synchronized $\mathrm{CH}^{*}$ chemiluminescence images at a modulation frequency of $200 \mathrm{~Hz}$. (A) in-phase interference, (B) out-of-phase interference. Inlet conditions: $T_{i n}=200$ ${ }^{\circ} \mathrm{C}, v_{\text {mean }}=60 \mathrm{~m} / \mathrm{s}, \Phi_{\text {matan }}=0.60, \mathrm{x}_{H_{2}}=0.60,{ }_{f}=200 \mathrm{~Hz}$, and $v^{\prime} /_{\text {mean }}=42 \%$.

As stated previously, we wanted to analyze the influence of multiple parametric oscillations upon the flame dynamics. Returning to the premixed regime, the effect of the in-phase and out-ofphase interference between the velocity and equivalence ratio oscillations on the flame dynamics is shown in Figure 82. It is evident at the phase angle of $330^{\circ}$ (A) that the flame starts to divide into two regions, as the upstream part of the flame moves in the direction of increasing flame angle. In the middle of the projected reaction zone, a thin local region with weak $\mathrm{CH}^{*}$ chemiluminescence intensity is observed; the reaction zone appears to be divided in two. At a phase-angle of $60^{\circ}$, a continuous reaction region is not sustained, and consequently unsteady local extinction occurs. The in-phase interaction of the two inlet disturbances results in the unsteady destruction of flame surface area, leading to the nonlinear response of the partially premixed flame. On the other hand, significant differences are observed when the phase difference is close to $120^{\circ}$, as shown in Figure 82(B). Localized flame extinction is not seen. $\mathrm{CH}^{*}$ chemiluminescence intensity does not vary significantly during an oscillation cycle, since reactants with high equivalence ratio enter the combustion chamber with low velocity and reactants with low equivalence ratio reach the flame front with high inlet velocity. As a result, the flame "meets" almost the same amount of fuel per unit time. 


\section{Forced Response Measurements - Simultaneous Velocity and Equivalence Ratio Perturbations}

\section{Introduction}

In addition to this analysis, during the duration of this work, the response of a partially premixed flame to perturbations in inlet velocity and equivalence ratio oscillations was experimentally examined in depth. The combined action of inlet velocity and equivalence ratio perturbations at the combustor inlet induces the nonlinear response of hydrogen-enriched flames, whose response to high amplitude acoustic velocity perturbations was almost linear. The nonlinearity of the flame was manifested by unsteady localized flame extinction events, resulting from flame stretching. It was shown that the response of the flame at a modulation frequency of $300 \mathrm{~Hz}$ is almost linear; the response is highly nonlinear at a lower frequency of $200 \mathrm{~Hz}$. This characteristic of the partially premixed flame is due to the phase relationship between inlet velocity and equivalence ratio oscillations at the combustor inlet. Depending on the phase difference between the two perturbations, the flame response can be linear even at high perturbation frequency and amplitude. Results also showed that in the linear regime, the response of a partially premixed flame can be calculated from each response of the flame to velocity and equivalence ratio oscillations. This is the first experimental demonstration of a two-input one-out model of a partially premixed flame.

\section{Results and Discussion}

All measurements were performed at atmospheric pressure (1 atm) and an inlet temperature of $200{ }^{\circ} \mathrm{C}$. The mean equivalence ratios were varied from 0.55 to 0.70 , and mean velocity at the nozzle was $60 \mathrm{~m} / \mathrm{s}$. Modulation frequencies were varied from 100 to $400 \mathrm{~Hz}$ in $25 \mathrm{~Hz}$ increment and the amplitude of modulation was varied from minimum to maximum. In most cases, the maximum forcing amplitude was determined by the limitations of the modulating device. Fuel (natural gas + hydrogen) is injected in the mixing section, while preheated air is modulated by a siren-type modulating device. Due to high pressure drop through the fuel injector, the acoustic field of the fuel line is isolated from the acoustic field in the mixing section. The phase of equivalence ratio oscillations at the fuel injection location is therefore $180^{\circ}$ out-of-phase with the air mass flow rate at that location. The equivalence ratio modulation generated is then convected with the mean flow and impinges on the flame. Hence, the siren provides perturbations in acoustic velocity and equivalence ratio at the combustor inlet. The full list of operating conditions and fuel compositions for forced flame response measurements is listed in Table 5. 
Table 5: Test conditions for forced flame response measurements.

\begin{tabular}{cc}
\hline Parameters & Test conditions \\
\hline pressure $(\mathrm{P})$ & $1 \mathrm{~atm}$ \\
inlet temperature $\left(\mathrm{T}_{\text {in }}\right)$ & $200{ }^{\circ} \mathrm{C}$ \\
nozzle velocity $\left(\mathrm{V}_{\text {mean }}\right)$ & $60 \mathrm{~m} / \mathrm{s}$ \\
equivalence ratio $(\Phi)$ & $0.55,0.60,0.65,0.70$ \\
forcing frequency $(f)$ & $100 \sim 400 \mathrm{~Hz}(\Delta f=25$ \\
forcing amplitude $(\mathrm{V} /$ & $\mathrm{Hz})$ \\
$\left.\mathrm{V}_{\text {mean }}\right)$ & up to $60 \%$ \\
fuel composition $\left(\mathrm{X}_{\mathrm{H} 2}\right)$ & $0.00,0.30,0.60$ \\
\hline
\end{tabular}

\section{Flame Transfer Function Measurements}

Figure 83 shows the normalized heat release response and gain of FTF at a modulation frequency of $200 \mathrm{~Hz}$. The response of totally premixed (PM) and partially premixed (PPM) flames are shown together. For a partially premixed flame, fuel $\left(\mathrm{X}_{\mathrm{H} 2}=0.30\right)$ is injected $152 \mathrm{~mm}(6$ inches $)$ upstream of the combustor dump plane. At these inlet conditions, the steady-state flame structure exhibits an enveloped " $\mathrm{M}$ " geometry. Result shows that the response of the premixed flame is totally linear, up to an amplitude of approximately 50\%; the gain is nearly constant. When the flame is modulated by two disturbances (PPM), i.e., V' and $\Phi^{\prime}$, however, it exhibits highly nonlinear behavior. The response of the partially premixed flame levels off at $\mathrm{V}^{\prime} / \mathrm{V}_{\text {mean }} \sim 0.35$ and increases again. It is expected that this nonlinear behavior could be related to equivalence ratio nonuniformities, because the only difference between the two cases considered is the number of inlet disturbances. In particular, the response $\left(\mathrm{CH}^{*} / \mathrm{CH}^{*}{ }_{\text {mean }}\right)$ of a partially premixed flame is much greater than that of a premixed flame. It is believed that the actual heat release rate of the partially premixed flame is to some extent lower than the normalized $\mathrm{CH}^{*}$ chemiluminescence intensity, due to the sensitivity of chemiluminescence to equivalence ratio. As mentioned above, care should be taken when interpreting chemiluminescence intensity data quantitatively, but the trend of variation is expected to be qualitatively correct. 

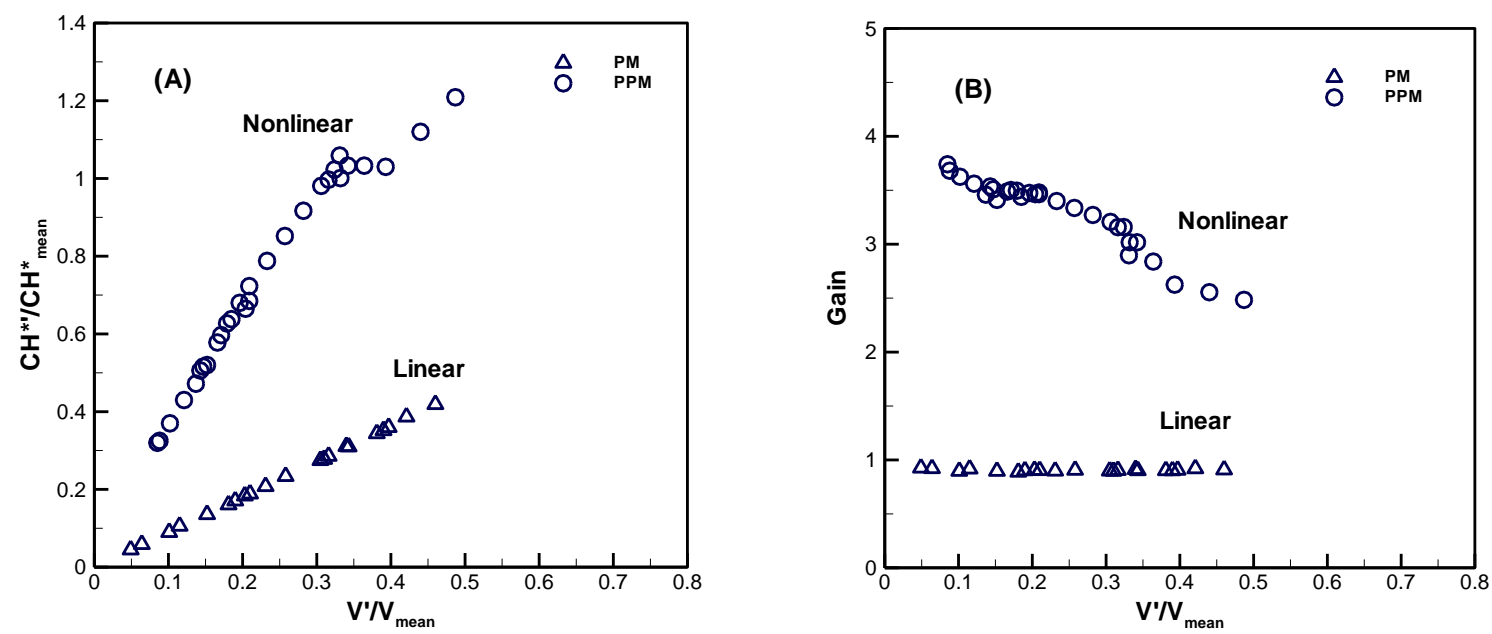

Figure 83: (A) Normalized heat release response and (B) gain of the flame transfer function plotted as a function of the forcing amplitude. Inlet conditions: $T_{\text {in }}=200{ }^{\circ} \mathrm{C}, V_{\text {mean }}=60 \mathrm{~m} / \mathrm{s}$, $\Phi_{\text {mean }}=0.60, \mathrm{X}_{\mathrm{H} 2}=0.30, \mathrm{PM}, \operatorname{PPM}\left(\mathrm{L}_{\mathrm{f}, \text { inj }}=152 \mathrm{~mm}\right)$, and $f=200 \mathrm{~Hz}$.

The response of a dihedral "V" flame to perturbations in equivalence ratio and velocity at a modulation frequency of $100 \mathrm{~Hz}$ is presented in Figure 84. As a reference, the response of a perfectly premixed flame to velocity oscillations is also shown. At this condition, the response is linear when the flame is subjected to inlet velocity oscillations only. This is due to the fact that the excitation of the shear layer into the vortex-ring structure is not achieved, insofar as the modulation frequency is relatively low. The evolution of flame surface area is influenced by the turbulent motion of the flame. For a partially premixed flame, however, the gain of FTF gradually decreases with amplitude and levels off at amplitude of approximately $20 \%$. It can be conjectured that the nonlinearity could be related to equivalence ratio oscillations or the coupled effects of $\Phi^{\prime}$ and $V^{\prime}$ perturbations. Details of relevant processes will be discussed in the following section.
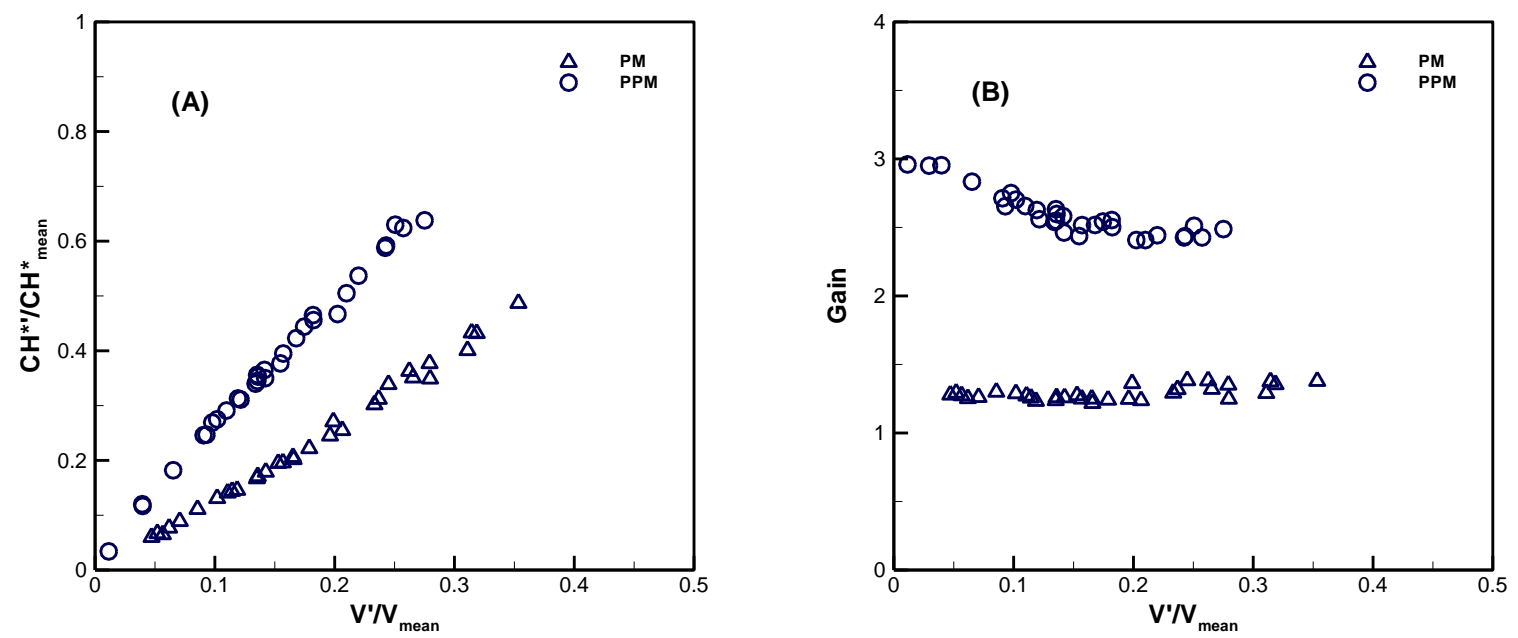

Figure 84: (A) Normalized heat release response and (B) gain of the flame transfer function plotted as a function of the forcing amplitude. Inlet conditions: $T_{\text {in }}=200{ }^{\circ} \mathrm{C}, V_{\text {mean }}=60 \mathrm{~m} / \mathrm{s}$, $\Phi_{\text {mean }}=0.60, \mathrm{X}_{\mathrm{H} 2}=0.00, \mathrm{PM}, \operatorname{PPM}\left(\mathrm{L}_{\mathrm{f}, \text { inj }}=6 "\right), f=100 \mathrm{~Hz}$. 


\section{Nonlinear Flame Dynamics}

In order to understand the nonlinear response of a partially premixed, hydrogen-enriched, " $\mathrm{M}$ " flame, phase-locked $\mathrm{CH}^{*}$ chemiluminescence images are analyzed. It provides details of the response of the flame to inlet disturbances. Figure 85 presents phase-averaged $\mathrm{CH}^{*}$ chemiluminescence images for a totally premixed (PM) flame at a modulation frequency of 200 $\mathrm{Hz}$ and amplitude of $41 \%$. Its corresponding transfer functions are shown in Figure 83. Figure 85 clearly shows that the dynamics of enveloped " $\mathrm{M}$ " flames remains in the linear region, regardless of the shedding of a vortex structure. This indicates that the dynamics of the flame is influenced only minimally by the vortex dynamics and their interaction with the flame. Therefore, the influence of the vortex dynamics on the heat release rate oscillations can be effectively ruled out for a hydrogen enriched flame. The response of the flame to velocity and equivalence ratio modulation will be studied in detail below.

(A)

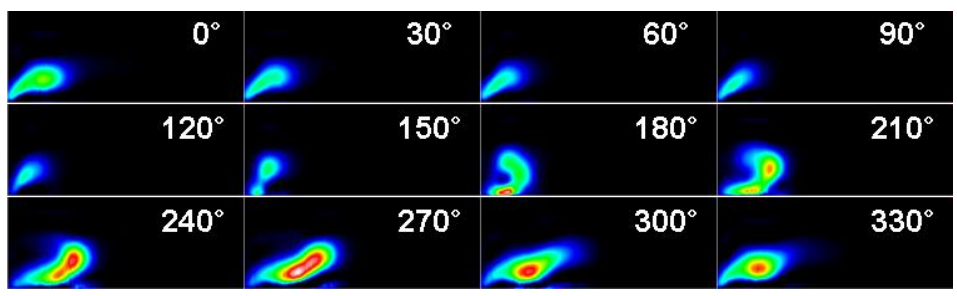

(B)

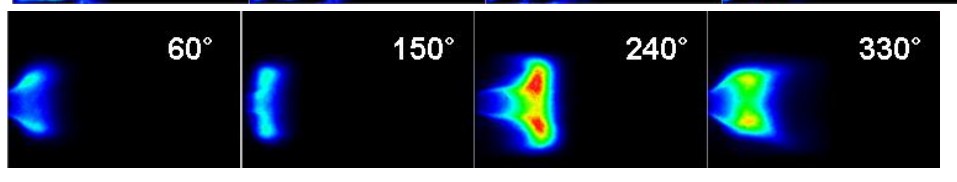

Figure 85: (A) Phase-synchronized $\mathrm{CH}^{*}$ chemiluminescence imaging at a modulation frequency of $200 \mathrm{~Hz}$ and $V^{\prime} / \mathrm{V}_{\text {mean }}=41 \%$ (PM). (B) Bottom row shows line-of-sight integrated, background-corrected $\mathrm{CH}^{*}$ chemiluminescence images at $\varphi=60,150,240$, and $330^{\circ}$. Inlet conditions: $\mathrm{T}_{\mathrm{in}}=200{ }^{\circ} \mathrm{C}, \mathrm{V}_{\text {mean }}=60 \mathrm{~m} / \mathrm{s}, \Phi_{\text {mean }}=0.60, \mathrm{X}_{\mathrm{H} 2}=0.30, \mathrm{PM}$, and $f=$ $200 \mathrm{~Hz}$.

(A)

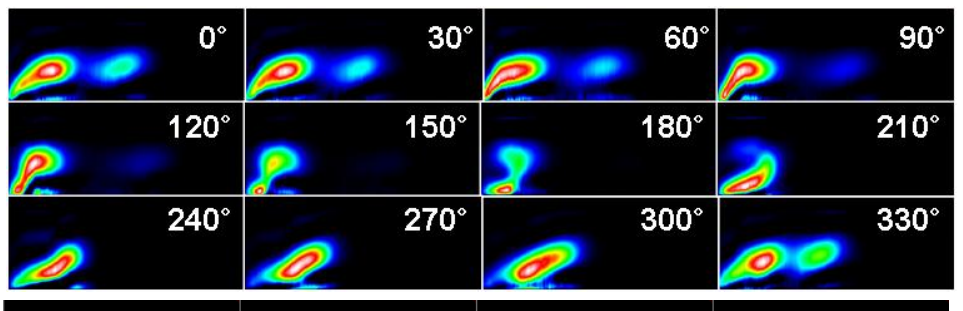

(B)

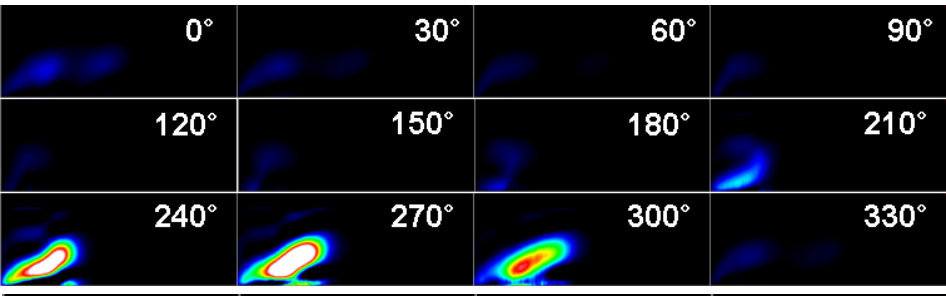

(C)

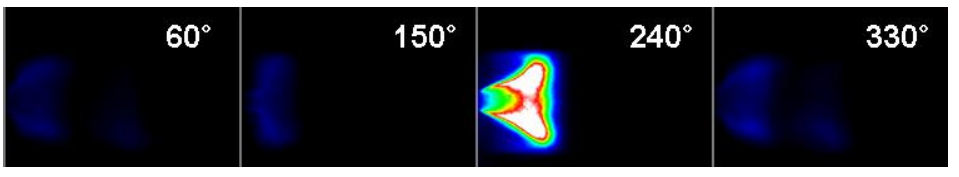


Figure 86: Phase-synchronized $\mathrm{CH}^{*}$ chemiluminescence imaging at a modulation frequency of $200 \mathrm{~Hz}$ and $V^{\prime} / V_{\text {mean }}=42 \%$ (PPM). Top row (A) shows individually-scaled images to highlight the flame structure. Middle row (B) shows images normalized by maximum intensity. Bottom row $(C)$ shows line-of-sight integrated, background-corrected $\mathrm{CH}^{*}$ chemiluminescence images at $\varphi=60,150,240$, and $330^{\circ}$. Inlet conditions: $\mathrm{T}_{\text {in }}=200$ ${ }^{\circ} \mathrm{C}, \mathrm{V}_{\text {mean }}=60 \mathrm{~m} / \mathrm{s}, \Phi_{\text {mean }}=0.60, \mathrm{X}_{\mathrm{H} 2}=0.30, \operatorname{PPM}\left(\mathrm{L}_{\mathrm{f}, \text { inj }}=6 "\right)$, and $f=200 \mathrm{~Hz}$.

The nonlinear dynamics of a partially premixed flame are characterized by phase-resolved $\mathrm{CH}^{*}$ chemiluminescence images in Figure 86. The transfer function is shown in Figure 83. The amplitude and frequency of modulation are the same as for the premixed counterpart, Figure 85. Figure 86(A) shows individually-scaled flame images highlighting the flame structure at each phase. Figure 86(B) shows the same images as Figure 86(A) but normalized by the maximum intensity at $\varphi=270^{\circ}$. It is obvious that the $\mathrm{CH}^{*}$ chemiluminescence intensity varies significantly during a cycle of oscillation. It can also be clearly seen that when the flame moves in the direction of increasing flame angle $\left(\varphi=330-60^{\circ}\right)$, the downstream part of the flame experiences localized flame extinction events. This could be due to the fact that the unsteady local stretch rate exceeds the extinction stretch rate. Destruction of the flame surface area results in a nonlinear decrease in the gain of the flame transfer function. The unsteady local extinction, which was not observed for a premixed flame, is therefore a mechanism of the nonlinear response of the partially premixed "M" flame.

Time-domain results of global $\mathrm{CH}^{*}$ chemiluminescence intensities (PMT measurements) for premixed and partially premixed flames are shown in Figure 87. The modulation frequency and amplitude are $200 \mathrm{~Hz}$ and $42 \%$, respectively. This corresponds to the flame images shown in Figure 85 and Figure 86 . The global $\mathrm{CH}^{*}$ chemiluminescence of the premixed flame exhibits nearly sinusoidal variations, responding to sinusoidal perturbations in inlet velocity. The siren produces a nearly sinusoidal velocity fluctuation at the combustor inlet. On the other hand, the partially premixed flame shows a highly nonlinear behavior, manifested by a strong peak, followed by a rapid falloff. The high peak could be related to rapid burning of fuel-rich mixture. This will be verified by a convection wave analysis. 


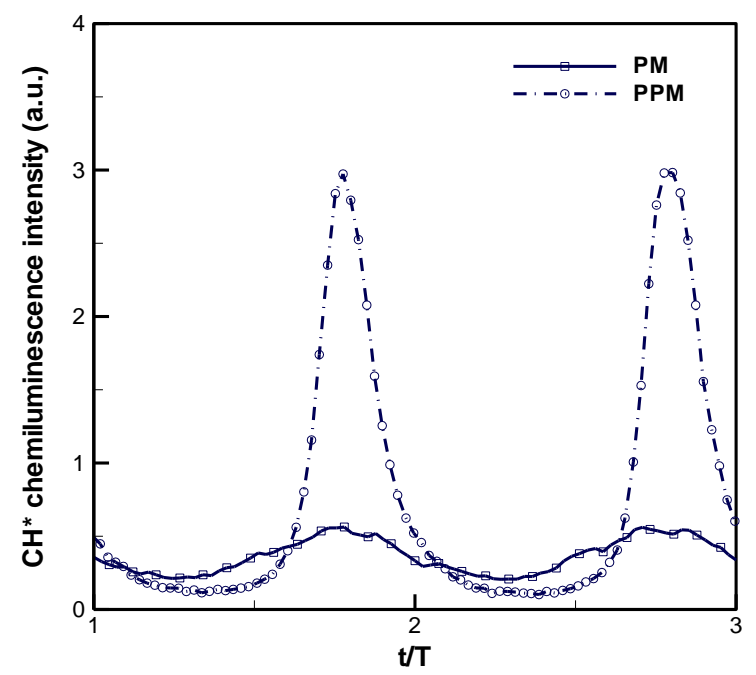

Figure 87: Global $\mathrm{CH}^{*}$ chemiluminescence signal variations over two cycles of oscillations at a modulation frequency of $200 \mathrm{~Hz}$ and $V^{\prime} / V_{\text {mean }}=42 \%$. Inlet conditions: $T_{\text {in }}=200{ }^{\circ} \mathrm{C}$, $\mathrm{V}_{\text {mean }}=60 \mathrm{~m} / \mathrm{s}, \Phi_{\text {mean }}=0.60, \mathrm{X}_{\mathrm{H} 2}=0.30,\left(\mathrm{~L}_{\mathrm{f}, \text { inj }}=6 "\right)$, and $f=200 \mathrm{~Hz}$.

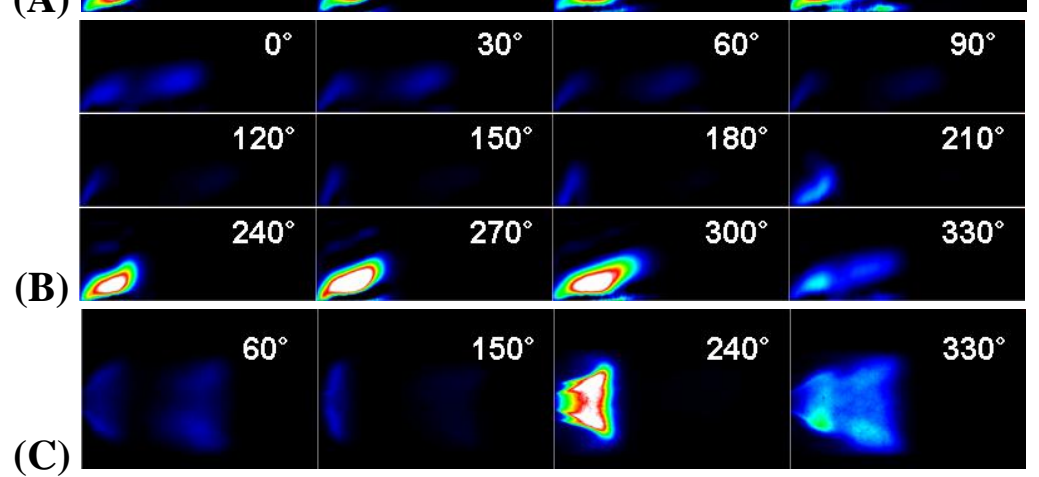

Figure 88: Phase-synchronized $\mathrm{CH}^{*}$ chemiluminescence imaging at a modulation frequency of $200 \mathrm{~Hz}$ and $V^{\prime} / V_{\text {mean }}=42 \%$ (PPM). Top row (A) shows auto-scaled intensity images. Middle row (B) shows images normalized by maximum intensity. Bottom row (C) shows line-of-sight integrated, background-corrected $\mathrm{CH}^{*}$ chemiluminescence images at $\varphi$ $=60,150,240,330^{\circ}$. Inlet conditions: $T_{\text {in }}=200{ }^{\circ} \mathrm{C}, V_{\text {mean }}=60 \mathrm{~m} / \mathrm{s}, \Phi=0.60, X_{\mathrm{H} 2}=0.60$, $\operatorname{PPM}\left(\mathrm{L}_{\mathrm{f}, \mathrm{inj}}=6\right.$ "'), and $f=200 \mathrm{~Hz}$. 
The nonlinear flame dynamics of a partially premixed flame with high hydrogen mole fraction of $\mathrm{X}_{\mathrm{H} 2}=0.60$ is shown in Figure 88. Other inlet conditions are the same as for the previous cases, including modulation frequency $(200 \mathrm{~Hz})$ and amplitude $(42 \%)$. Figure 88 clearly shows that the unsteady local flame extinction event is more distinct when the flame is more compact. At $\varphi=$ $330^{\circ}$ in Figure 88(C), the flame area starts to divide into two regions. The upstream part moves in the direction of increasing the flame angle, and thus the stretch rate is expected to be large in the middle of the flame, where the local extinction occurs. The line-of sight integrated image at $\varphi$ $=60^{\circ}$ and deconvoluted images at $\varphi=0-90^{\circ}$ clearly show that there are two distinct regions of heat release and the flame is locally extinguished due to stretching.

\section{Mechanism of Nonlinearity}

Figure 89 presents the dependence of normalized heat release response and gain of flame transfer function upon modulation frequency: 200 and $300 \mathrm{~Hz}$. Hydrogen-blended natural gas fuel is injected $152 \mathrm{~mm}$ from the combustor dump plane. It can be observed that the response at $200 \mathrm{~Hz}$ exhibits nonlinear behavior, but it becomes nearly linear at a modulation frequency of $300 \mathrm{~Hz}$. This is not consistent with the response characteristics of a totally premixed flame with respect to inlet velocity perturbations. In the perfectly premixed flame case, the nonlinearity occurs at lower amplitude $\left(\mathrm{V}^{\prime} / \mathrm{V}_{\text {mean }}\right)$, when the frequency of modulation increases. This is because the minimum level of velocity perturbation for the excitation of vortex roll-up decreases with an increase in forcing frequency. Figure 89 (A) and (B) show the opposite trend. It can be conjectured that inlet conditions with two perturbations can make the response of a partially premixed flame linear even at high modulation frequency. This would also imply that general observations about the governing parameters of the nonlinear response of a perfectly premixed flame cannot be applied to that of partially premixed flames.
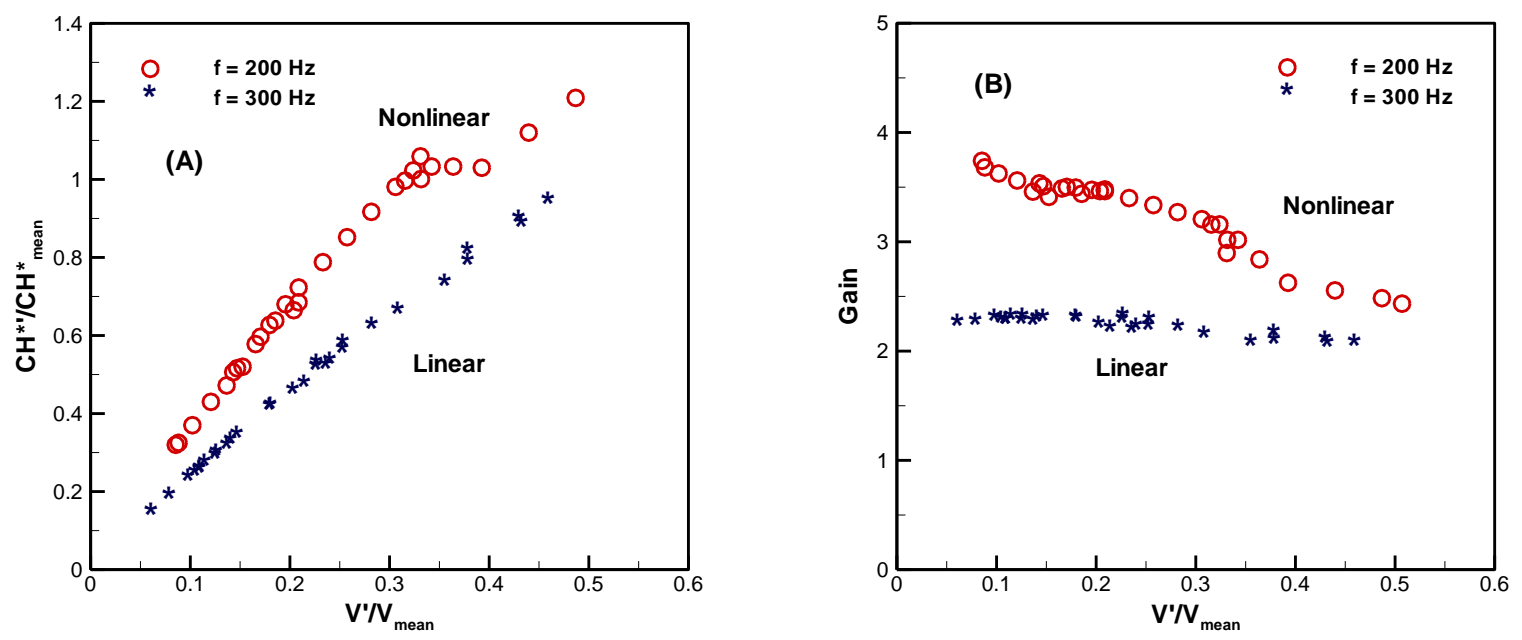

Figure 89: (A) Normalized heat release response $\left(\mathrm{CH}^{*} / \mathrm{CH}^{*}{ }_{\text {mean }}\right)$ and (B) gain of flame transfer function as a function of the magnitude of inlet velocity perturbation. Inlet conditions: $T_{\text {in }}=200{ }^{\circ} \mathrm{C}, \mathrm{V}_{\text {mean }}=60 \mathrm{~m} / \mathrm{s}, \Phi_{\text {mean }}=0.60, \mathrm{X}_{\mathrm{H} 2}=0.60, f=200,300 \mathrm{~Hz}$, and $\mathrm{L}_{\mathrm{f}}$, inj $=6$." 
Figure 90 (A) and (B) present corresponding phase-averaged flame imaging at modulation frequencies of 200 and $300 \mathrm{~Hz}$, respectively. The magnitude of perturbation is set to the same at $\mathrm{V}^{\prime} / \mathrm{V}_{\text {mean }}=42 \%$. It is evident from Figure 90 (A) that the nonlinearity at $200 \mathrm{~Hz}$ is caused by unsteady local extinction, as previously mentioned. Figure 90 (B) shows that there is no localized extinction at a frequency of $300 \mathrm{~Hz}$. The overall dynamics of the flame is similar to the dynamics of a flame subjected to pure velocity perturbations. The magnitude of heat release oscillations is reduced when the frequency is altered from 200 to $300 \mathrm{~Hz}$. This result reconfirms that the nonlinear dynamics of enveloped " $\mathrm{M}$ " flames are induced by the local extinction mechanism. This also suggests that the nonlinearity occurs only at certain inlet conditions, such as certain frequencies and amplitudes of modulations. The relationship between the combined action of two perturbations ( $V^{\prime}$ and $\Phi^{\prime}$ ) and the corresponding linear and/or nonlinear flame response requires further elucidation.

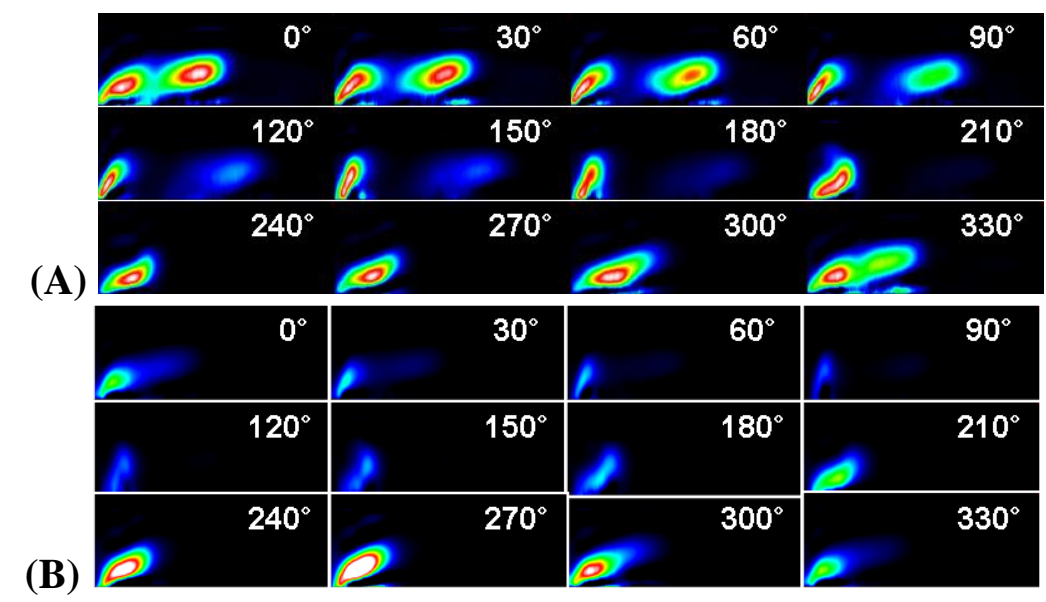

Figure 90: Phase-synchronized $\mathrm{CH}^{*}$ chemiluminescence imaging at modulation frequencies of (A) $200 \mathrm{~Hz}$ (individually scaled) and (B) $300 \mathrm{~Hz}$ (normalized by maximum intensity). Inlet conditions: $T_{\text {in }}=200{ }^{\circ} \mathrm{C}, \mathrm{V}_{\text {mean }}=60 \mathrm{~m} / \mathrm{s}, \Phi_{\text {mean }}=0.60, X_{\mathrm{H} 2}=0.60, \operatorname{PPM}\left(L_{f, \text { inj }}=6 "\right)$, and $\mathrm{V} / \mathrm{V}_{\text {mean }}=42 \%$.

Figure 91 presents time signals of inlet velocity, equivalence ratio, and $\mathrm{CH}^{*}$ chemiluminescence intensity at a modulation frequency of $200 \mathrm{~Hz}$, corresponding to the flame images shown in Figure 90 (A). In Figure 91, each parameter is normalized by its corresponding maximum values. High-frequency noise was removed from the inlet velocity and equivalence ratio signals, for the purpose of showing the fundamental component of oscillations. The magnitude of the higher harmonic components of inlet velocity and equivalence ratio oscillations is orders of magnitude lower than that of the fundamental component. It is obvious from Figure 91 that the inlet velocity and equivalence ratio at the combustor inlet are essentially in phase. Physically, this indicates that fuel-rich mixtures enter the reaction zone with high velocity. The $\mathrm{CH}^{*}$ chemiluminescence signal features a finite phase delay with respect to the modulation signal, that is, $\mathrm{V}^{\prime}$ and $\Phi$ '. 


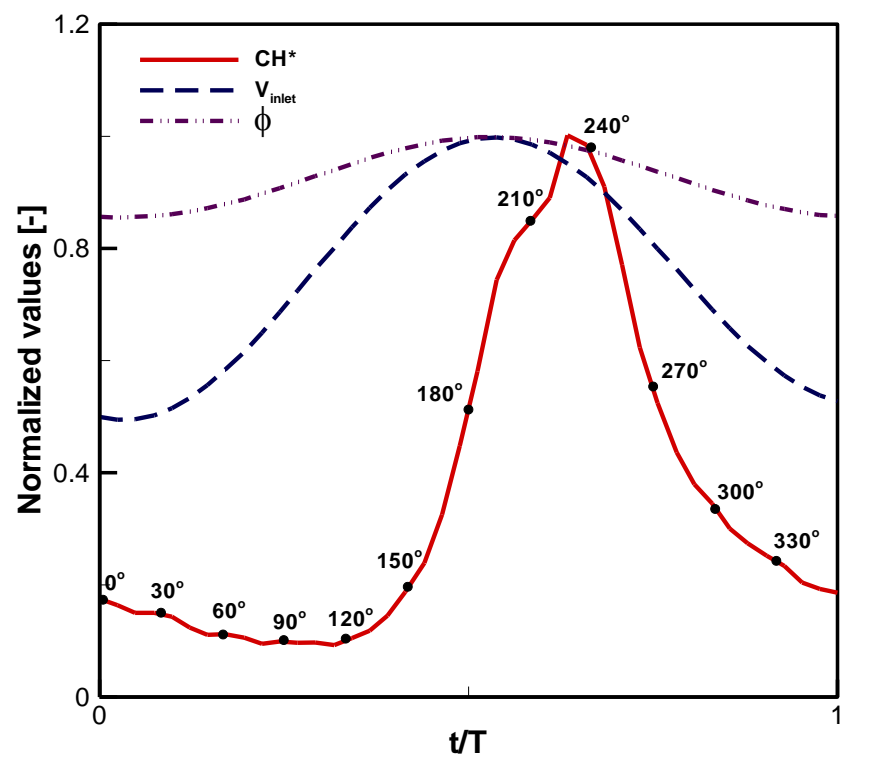

Figure 91: Time signals of inlet velocity, equivalence ratio, and $\mathrm{CH}^{*}$ chemiluminescence during a cycle of oscillation at a modulation frequency of $200 \mathrm{~Hz}$ and amplitude of $\mathrm{V}^{\prime} / \mathrm{V}_{\text {mean }}$ $=42 \%$. Inlet conditions: $T_{\text {in }}=200{ }^{\circ} \mathrm{C}, \mathrm{V}_{\text {mean }}=60 \mathrm{~m} / \mathrm{s}, \Phi_{\text {mean }}=0.60, X_{\mathrm{H} 2}=0.60$, and PPM $\left(\mathbf{L}_{\mathrm{f}, \mathrm{inj}}=\mathbf{6}^{\prime \prime}\right)$.

The nonlinear dynamics of the partially premixed flame are analyzed using the time signals of acoustic velocity and equivalence ratio oscillations measured at the combustor inlet. The convection delay time for two perturbations to reach the flame can be calculated from Figure 91. The convection waves of velocity and mixture ratio oscillations in the combustion chamber are then related to the unsteady flame dynamics, as shown in Figure 92. 


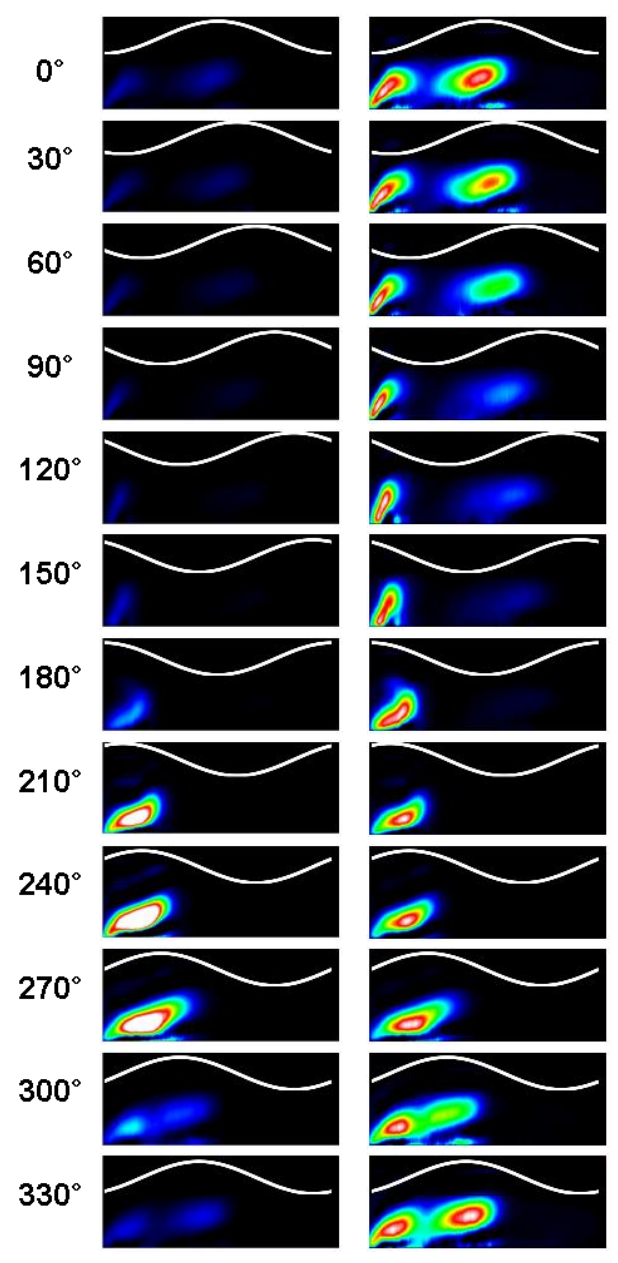

Figure 92: Phase-synchronized $\mathrm{CH}^{*}$ chemiluminescence imaging at a modulation frequency of $200 \mathrm{~Hz}$. Right column shows individually-scaled images to highlight the flame structure. Left column is images normalized by their maximum intensity. Inlet conditions: $\mathrm{T}_{\mathrm{in}}=200{ }^{\circ} \mathrm{C}, \mathrm{V}_{\text {mean }}=60 \mathrm{~m} / \mathrm{s}, \Phi_{\text {mean }}=0.60, \mathrm{X}_{\mathrm{H} 2}=0.60, \operatorname{PPM}\left(\mathrm{L}_{\mathrm{f}, \text { inj }}=6 "\right), f=200 \mathrm{~Hz}$, and $\mathrm{V}^{\prime} / \mathrm{V}_{\text {mean }}=\mathbf{4 2 \%}$.

At these inlet conditions, the convective wavelength $\left(\lambda_{\Phi \text {, conv }}=\mathrm{V}_{\text {mean }} * \mathrm{~T}\right)$ of the two disturbances is approximately $30 \mathrm{~cm}$, which is slightly smaller than the length of the quartz combustor, $\mathrm{L}_{\text {quartz }}$ $=33 \mathrm{~cm}$. The white lines denote convection waves of both acoustic velocity and equivalence ratio oscillations. It can be seen that at $\varphi=180-210^{\circ}$, fuel-rich mixtures impinge on the flame front, leading to the high heat release rate at $\varphi=240^{\circ}$ with a phase delay. At $\varphi=270-300^{\circ}$, the flame moves upstream because the amplitude of velocity oscillation starts to decrease toward the minimum value. At the same time, fuel-lean reactants start to enter the reaction zone at $\varphi=270$ $300^{\circ}$. The flame burning fuel-lean mixture is susceptible to unsteady flame stretching. Therefore, a continuous region of combustion reaction can not be sustained, owing to the combined action of the velocity field and equivalence ratio oscillations at $\varphi=330^{\circ}$. Note that the convection waves of equivalence ratio shown in Figure 92 have meaning only near the flame front. This result suggests that the phase relationship between $V^{\prime}$ and $\Phi^{\prime}$ at the combustor inlet is important to yield the nonlinear flame dynamics, that is, unsteady local extinction events. 


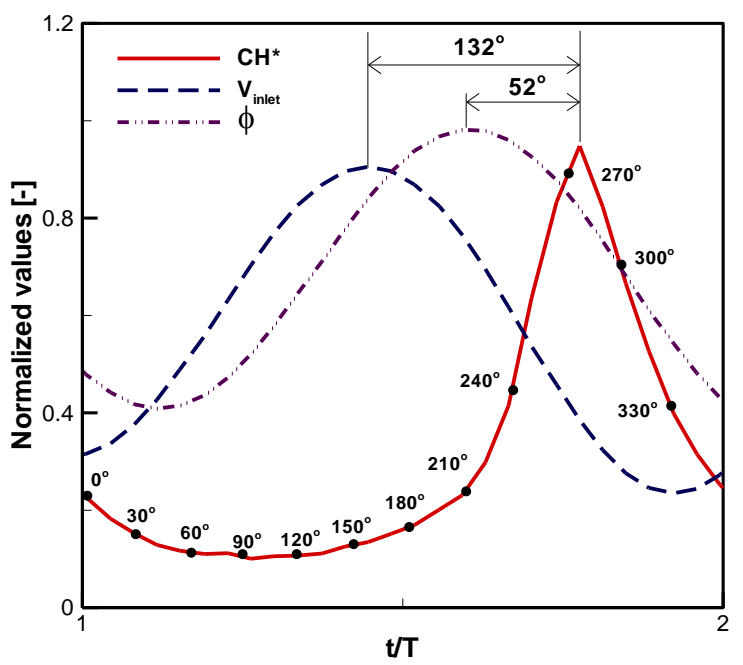

Figure 93: Time signals of inlet velocity, equivalence ratio, and $\mathrm{CH}^{*}$ chemiluminescence during a cycle of oscillation at a modulation frequency of $300 \mathrm{~Hz}$ and $V^{\prime} / V_{\text {mean }}=42 \%$. Inlet conditions: $T_{\text {in }}=200{ }^{\circ} \mathrm{C}, V_{\text {mean }}=60 \mathrm{~m} / \mathrm{s}, \Phi_{\text {mean }}=0.60, X_{\mathrm{H} 2}=0.60$, and PPM $\left(L_{f, \text { inj }}=6 "\right)$.

Figure 93 shows time traces of inlet velocity, equivalence ratio, and $\mathrm{CH}^{*}$ chemiluminescence intensity at a modulation frequency of $300 \mathrm{~Hz}$ and amplitude of $42 \%$, corresponding to Figure 90(B). The result clearly shows that there exists a phase difference between velocity and equivalence ratio oscillation. Changes in forcing frequency modify the phase difference between $V^{\prime}$ and $\Phi$ ' at the combustor inlet. This indicates that the flame may respond to the disturbances differently than it responds to $200 \mathrm{~Hz}$ forcing. Because the two disturbances, V' and $\Phi$, ' have different phases with respect to a reference signal, two lines (yellow and white) are used to represent velocity and equivalence ratio oscillations, respectively.

Corresponding phase-resolved flame imaging with convection waves of V' and $\Phi^{\prime}$ 'perturbations is presented in Figure 94. In this forcing condition, the convective wavelength of the perturbations is $20 \mathrm{~cm}$. Figure 94 shows that at $\varphi=180-210^{\circ}$, reactant mixtures with high equivalence ratio impinge on the flame front, and thus the heat release rate reaches its peak at $\varphi$ $=270^{\circ}$ with some phase delay. In fact, the fuel-rich reactants start to burn very near the centerbody; note that the white region at $\varphi=270^{\circ}$ in Figure 94 starts near the combustor inlet. This situation enables the fresh reactants to burn rapidly, and thus a sharp peak in $\mathrm{CH}^{*}$ chemiluminescence intensity is observed in Figure 93. At $\varphi=240-270^{\circ}$, the amplitude of velocity oscillation starts to decrease toward its lowest value, indicating that the flame moves upstream. At the same instant, $\varphi=240-270^{\circ}$, fuel-rich reactants are still entering the reaction region and burning, with some phase delay. Accordingly, the flame can sustain a continuous reaction region due to the high rate of heat release. The flame is "robust" with respect to flow disturbances. As a result, the unsteady local extinction events observed in Figure 92 do not occur. This analysis explains why the nonlinear flame dynamics manifested by the localized flame extinction only occur at a modulation frequency of $200 \mathrm{~Hz}$. This result also suggests that the linear and/or nonlinear dynamics of hydrogen-enriched partially premixed flames is contributed to the phase relationship between $V^{\prime}$ and $\Phi^{\prime}$ at the combustor inlet. 


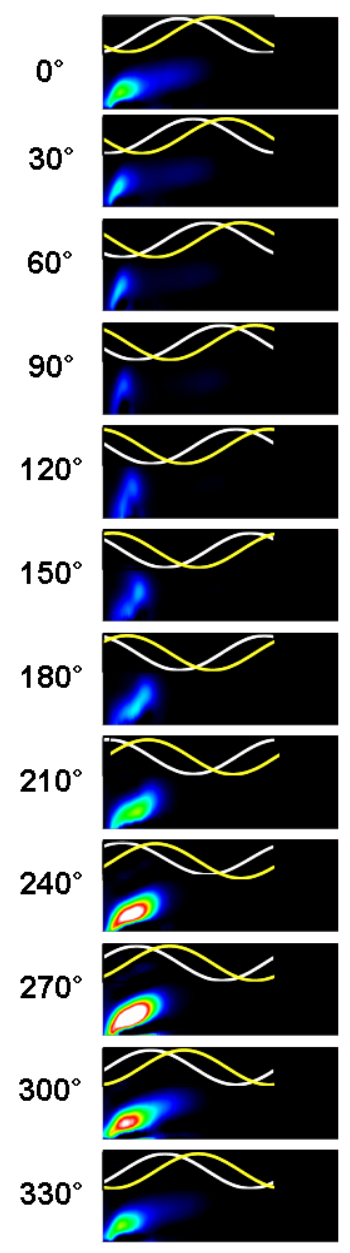

Figure 94: Phase-synchronized $\mathrm{CH}^{*}$ chemiluminescence imaging at a modulation frequency of $300 \mathrm{~Hz}$. White lines denote equivalence ratio oscillations, and yellow lines denote velocity perturbations. Inlet conditions: $T_{\text {in }}=200{ }^{\circ} \mathrm{C}, \mathrm{V}_{\text {mean }}=60 \mathrm{~m} / \mathrm{s}, \Phi_{\text {mean }}=0.60$, $\mathrm{X}_{\mathrm{H} 2}=0.60, \operatorname{PPM}\left(\mathrm{L}_{\mathrm{f}, \text { inj }}=6^{\prime}\right), f=300 \mathrm{~Hz}$, and $V^{\prime} / \mathrm{V}_{\text {mean }}=42 \%$.

\section{Reconstruction of the Response of a Partially Premixed Flame}

The heat release response of a partially premixed flame can be mathematically expressed, similar to Eq.(332), as the following equation:

$$
\frac{\mathrm{Q}^{\prime}}{\overline{\mathrm{Q}}}=\mathrm{FTF}_{\mathrm{v}}(\omega) \frac{\mathrm{V}^{\prime}}{\overline{\mathrm{V}}}+\mathrm{FTF}_{\phi}(\omega) \frac{\phi^{\prime}}{\bar{\phi}}
$$

where $\mathrm{FTF}_{\mathrm{V}}$ and $\mathrm{FTF}_{\Phi}$ are specific functions used to denote the flame transfer functions due to two independent perturbations of inlet velocity and equivalence ratio. Physically, Eq.(333) means that the response of a partially premixed flame can be linearly superposed from two independent responses. The two-input one-put system representation of the partially premixed 
flame is valid only when the response of the flame remains in the linear region with respect to both perturbations. This concept was recently introduced by Huber and Polifke. They tried to calculate the response of a partially premixed flame using two flame transfer functions; the flame transfer functions were obtained by numerical simulation of transient computational fluid dynamics of turbulent reacting flow. Experimental verification of the principle has not been explored to date. In this section, the concept will be experimentally demonstrated and its physical significance will be discussed.

Each term in Eq.(333) is complex quantity. Each has magnitude and phase with respect to a reference signal. Therefore, the response of the partially premixed flame can be reconstructed by a vector summation, as shown in Figure 95 and Figure 96. In Figure 95, $\mathrm{Q}_{\mathrm{V}}$ and $\mathrm{Q}_{\Phi}$ denote the responses $\left(\mathrm{CH}^{*} / \mathrm{CH}^{*}{ }_{\text {mean }}\right)$ of a flame to velocity perturbation and equivalence ratio oscillations, respectively. They were measured separately. The amplitudes of oscillation are low enough to ensure that the response of the flame is linear with respect to each perturbation. The phase of heat release responses, the angle between the each arrow and the $\mathrm{x}$-axis, is designated by their corresponding phases of perturbations, V' or $\Phi^{\prime}$. The arrow, $\mathrm{Q}_{\mathrm{V}+\Phi}$ (calculation) in Figure 95, indicates the predicted heat release response of a partially premixed flame by means of a vector summation, when the flame is perturbed by inlet velocity and equivalence ratio with given magnitudes and phases. The symbol ( $\mathbf{a})$ indicates the actual response of the partially premixed flame at the inlet conditions considered here. A simple arithmetic mean of $\varphi_{Q^{\prime}-\Phi^{\prime}}$ and $\varphi_{Q^{\prime}-V^{\prime}}$ was tried to calculate the phase of the heat release response, since there are two phases of heat release oscillations with respect to inlet perturbations; refer to Figure 93.

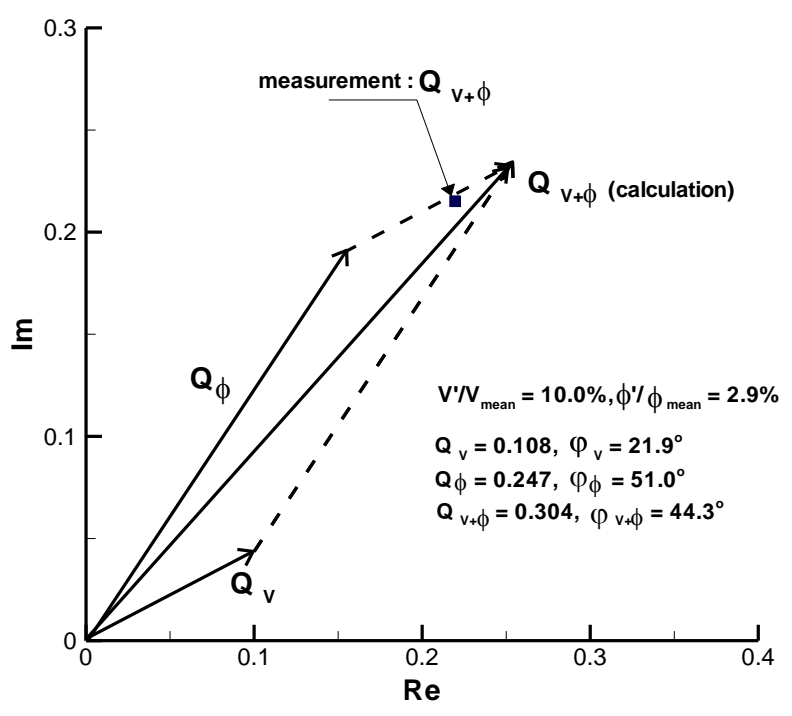

Figure 95: Calculation of the normalized $\mathrm{CH}^{*}$ chemiluminescence intensity (heat release) of a partially premixed flame at a modulation frequency of $100 \mathrm{~Hz}$ and amplitude of $V^{\prime} / V_{\text {mean }}=10.0 \%, \Phi^{\prime} / \Phi_{\text {mean }}=2.9 \%$. Inlet conditions: $T_{\text {in }}=200{ }^{\circ} \mathrm{C}, V_{\text {mean }}=60 \mathrm{~m} / \mathrm{s}, \Phi_{\text {mean }}=$ 0.60 , and $\mathrm{X}_{\mathrm{H} 2}=0.00$. 


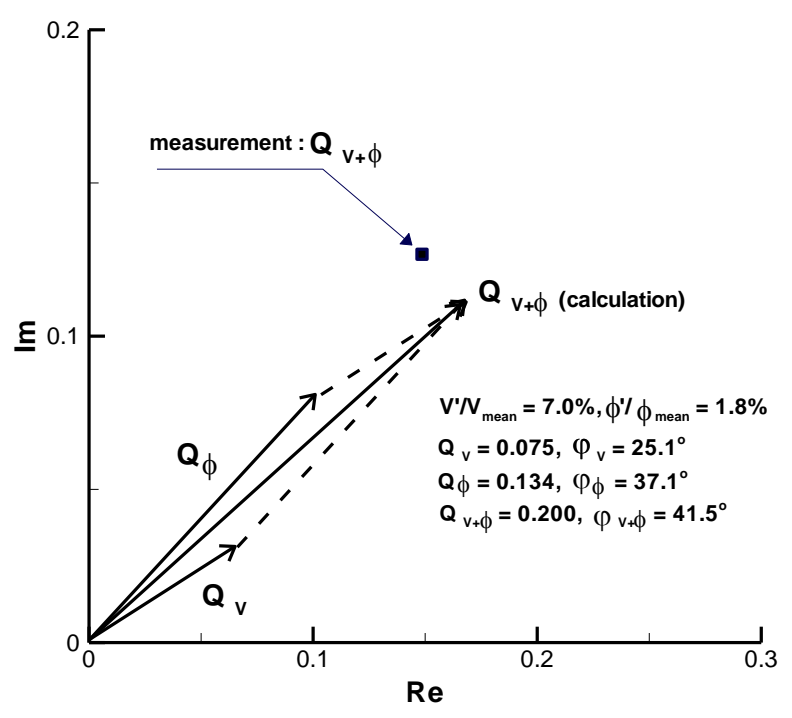

Figure 96: Calculation of the normalized $\mathrm{CH}^{*}$ chemiluminescence intensity (heat release) of a partially premixed flame at a modulation frequency of $100 \mathrm{~Hz}$ and amplitude of $V^{\prime} / V_{\text {mean }}=7.0 \%, \Phi^{\prime} / \Phi_{\text {mean }}=1.8 \%$. Inlet conditions: $T_{\text {in }}=200{ }^{\circ} \mathrm{C}, V_{\text {mean }}=60 \mathrm{~m} / \mathrm{s}, \Phi_{\text {mean }}=$ 0.60 , and $\mathrm{X}_{\mathrm{H} 2}=0.00$.

Results show that the heat release response of a partially premixed flame can be effectively reconstructed using the individual measurements of the responses to velocity and equivalence ratio. Figure 96 illustrates another example of the analysis. In this case, the phase between measurement and prediction show discrepancy, while the magnitude agrees well. This could be due to the fact that a simple average of phases, $\varphi_{Q^{\prime}-\Phi^{\prime}}$ and $\varphi_{Q^{\prime}-V^{\prime}}$, was used to obtain the phase of $\mathrm{Q}_{\mathrm{V}+\Phi}$ (measurement).

The results shown in Figure 95 and Figure 96 imply that the heat release response of a partially premixed flame in the linear region is governed by four parameters: frequency, amplitude of inlet velocity fluctuation, amplitude of inlet equivalence ratio perturbation, and phase difference between velocity and equivalence ratio at the inlet of the combustor. The amplitudes of velocity perturbation and equivalence ratio oscillation are not independent. In fact, they are linearly related, as shown in Figure 97. Figure 97 shows the dependence of the normalized amplitude of equivalence ratio upon the amplitude of velocity perturbations at a modulation frequency of 100 Hz. This relationship is due to the fact that the fuel is choked at the fuel injector and the magnitude of the equivalence ratio at the fuel injector is determined by the magnitude of the air mass flow rate at that location. The phase difference between velocity and equivalence ratio at the combustor inlet, $\Delta \varphi_{\Phi}{ }^{\prime}-V^{\prime}$, is a function of modulation frequency, fuel injection location, fuel injector impedance, and mean velocity in the nozzle. The phase difference plays a critical role in controlling the linear and nonlinear response of a partially premixed flame, as previously presented. 


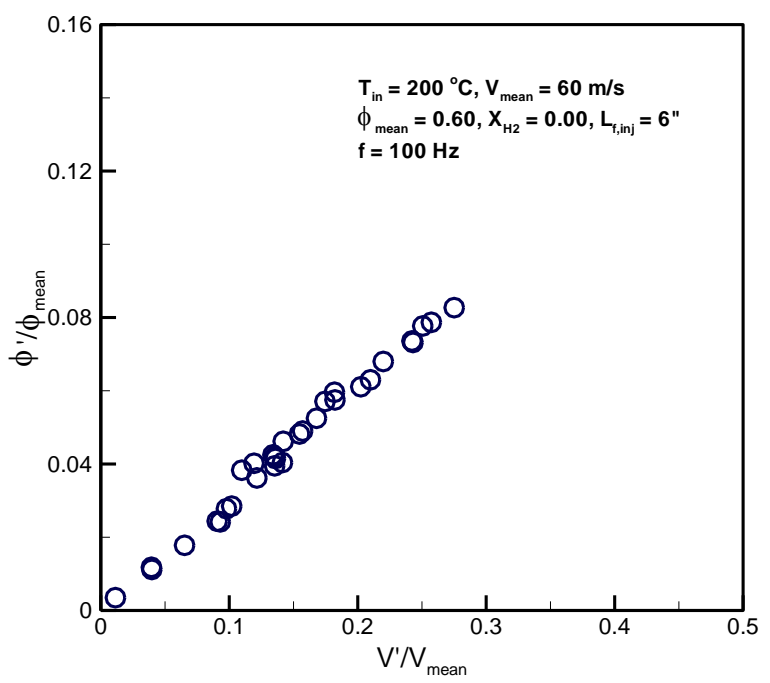

Figure 97: Dependence of the amplitude of equivalence ratio oscillations upon the amplitude of inlet velocity perturbations at a modulation frequency of $100 \mathrm{~Hz}$. Inlet conditions: $T_{\text {in }}=200{ }^{\circ} \mathrm{C}, V_{\text {mean }}=60 \mathrm{~m} / \mathrm{s}, \Phi_{\text {mean }}=0.60$, and $X_{\mathrm{H} 2}=0.00$.

An additional way to validate our modeling approach, by means of comparison with experimental data, is through use of the model equation shown below:

$$
i 2 \pi\left(\frac{L^{\prime}}{\lambda_{c}}\right)+\frac{U_{t}}{U_{0}} \cos \theta \frac{d}{d\left(x / \lambda_{c}\right)}\left(\frac{L^{\prime}}{\lambda_{c}}\right)=\frac{1}{\cos \theta}\left(\frac{u_{n}^{\prime}}{U_{0}}\right)
$$

where the flame location fluctuations, $L^{\prime}$, are measured as function of distance from bluff-body, $x$, and the other quantities such as $\lambda_{c}$ and $U_{0}$ are the characteristic length and velocity scales and $\theta$ is the mean flame angle. This is a two dimensional representation of the earlier front tracking equation, tailored for this comparison. The equation represents a relation between the flame dynamics and flow dynamics, both of which were measured experimentally.

The comparison study was performed in two ways: flow dynamics experimental data as input, to predict the flame dynamics and compare with its experimentally measured value, and vice-versa. This is a comparison of linearized flame response characteristics, so we can expect these linearized calculations to also increasingly grow in error downstream where nonlinear effects grow. This point should be recalled in the ensuing comparisons of model predictions. A typical flame response spectrum and flow field as measured by experiments is shown in Figure 98. These flame response results show an interference pattern, reflected in the ripple in the plots, due to the interaction between flame wrinkles/fluctuations, vortical disturbances, and acoustic disturbances and their different propagation speeds. The flow field plot shows the location of the mean flame and flame brush, which represents the extreme of the flame position fluctuations at each location. 

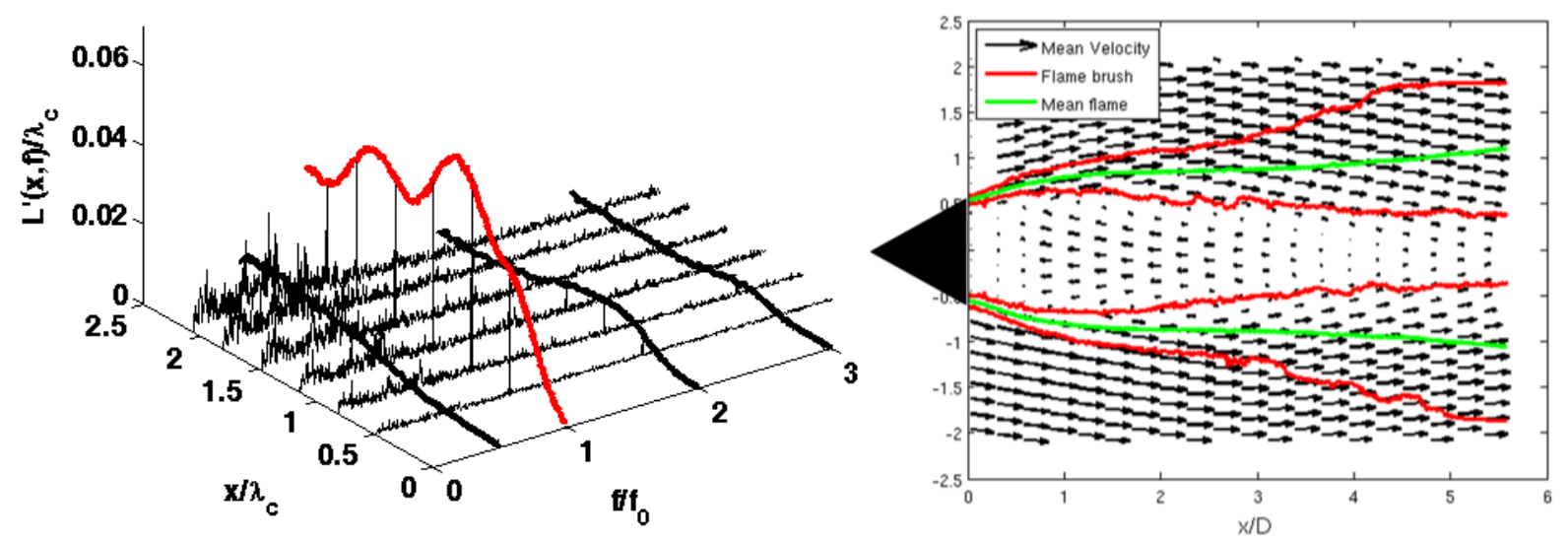

Figure 98: (left) Spectrum of flame sheet fluctuations at different downstream locations (50 $\mathrm{m} / \mathrm{s}, 644 \mathrm{~K})$. The $x$-coordinate is the downstream distance with $x=0$ located at the bluff body trailing edge and $f_{o}$ is the acoustic forcing frequency. The flame response is enveloped at the forcing frequency as well as the first sub-harmonic and the first two harmonics. (right) Sample mean velocity fields obtained from PIV with flame brush and mean flame position overlaid. $(100 \mathrm{~m} / \mathrm{s}, 755 \mathrm{~K})$

The results of the comparison study using these experimental data, are shown in Figure 99 and Figure 100 for two different flow conditions. Figure 99 (a) and (b) plot comparisons using the flame edge as input. Figure 99 (c) and (d) plot comparisons using the velocity field as input. Both validation studies show that the interference patterns, such as crests and troughs, are clearly captured spatially. The phase also shows similar spatial trends. However, the quantitative gain amplitude trends in both studies are different. For example, Figure 99(c) clearly shows that the flame edge motion is under-predicted since the velocity field magnitude is less than that required for a correct prediction, as shown in Figure 99(a). Closer examination of cases with different behaviors between theory and model emphasize the coupling of gain and phase in predicting either quantity. For example, Figure 100(a) shows that the magnitude and spatial interference trends are qualitatively similar, but shifted in space relative to each other. This shift can be seen in the phase comparison of Figure 100(b) where the phase trend is similar, but there is a uniform phase shift. Although the magnitude is well captured, the shift in phase plays a major role in the prediction of the flame edge as can be seen in Figure 100(c).

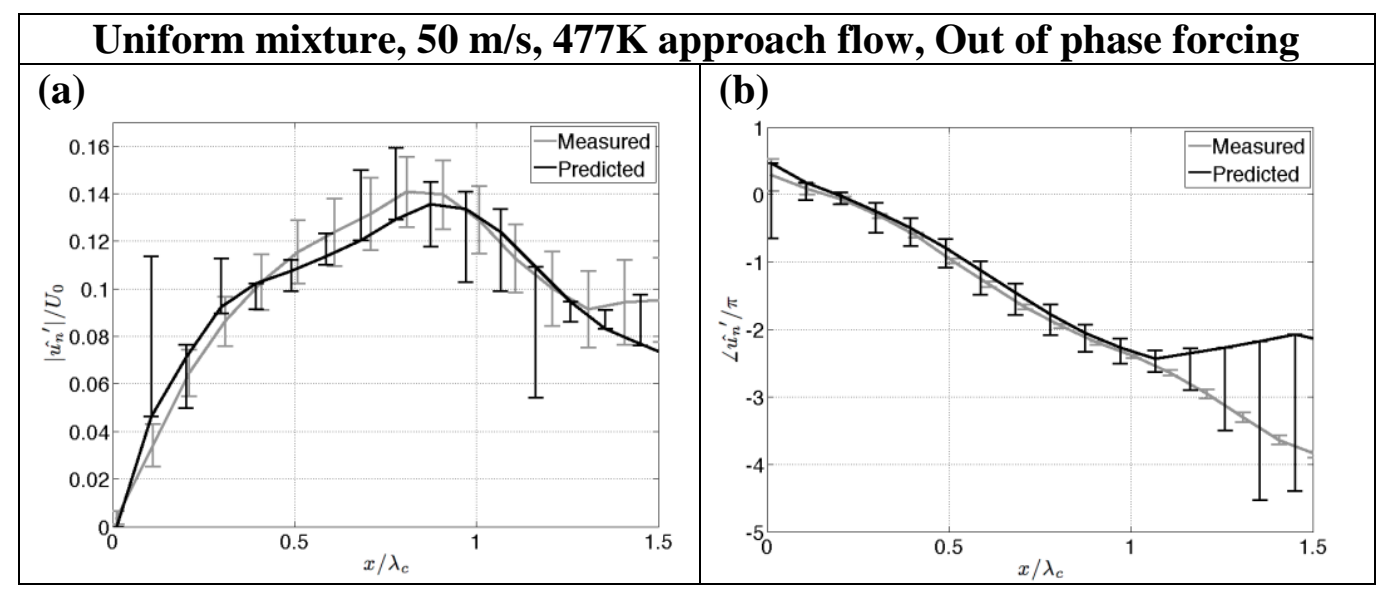




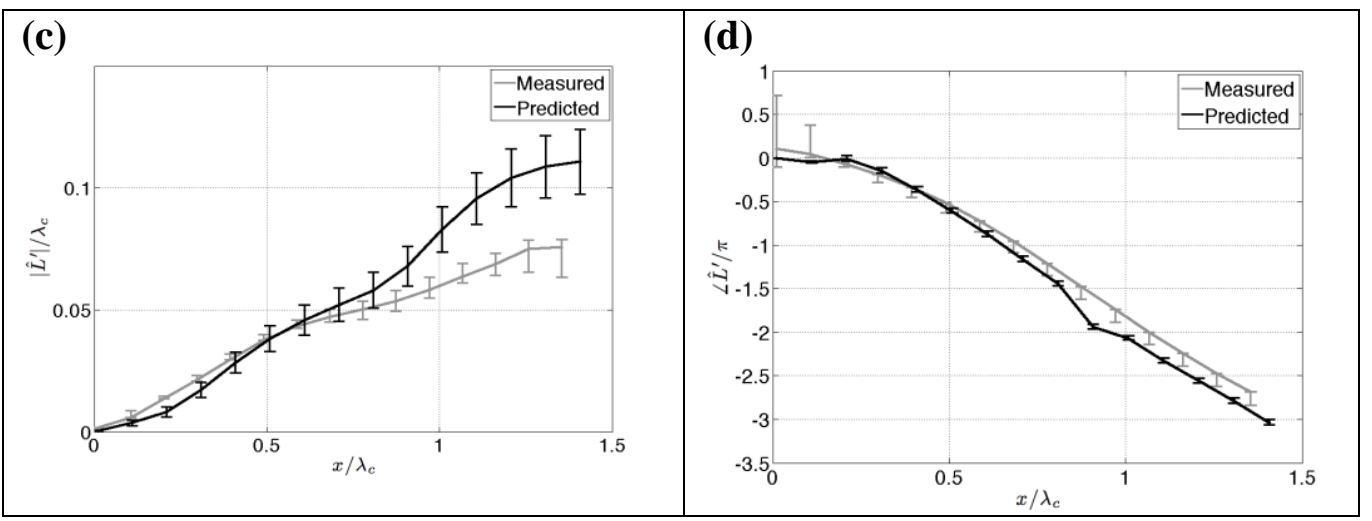

Figure 99: Velocity validation study with flame edge as input : (a) amplitude comparison, (b) corresponding phase comparison; Flame edge validation study with velocity as input : (c) amplitude comparison, (d) corresponding phase comparison.

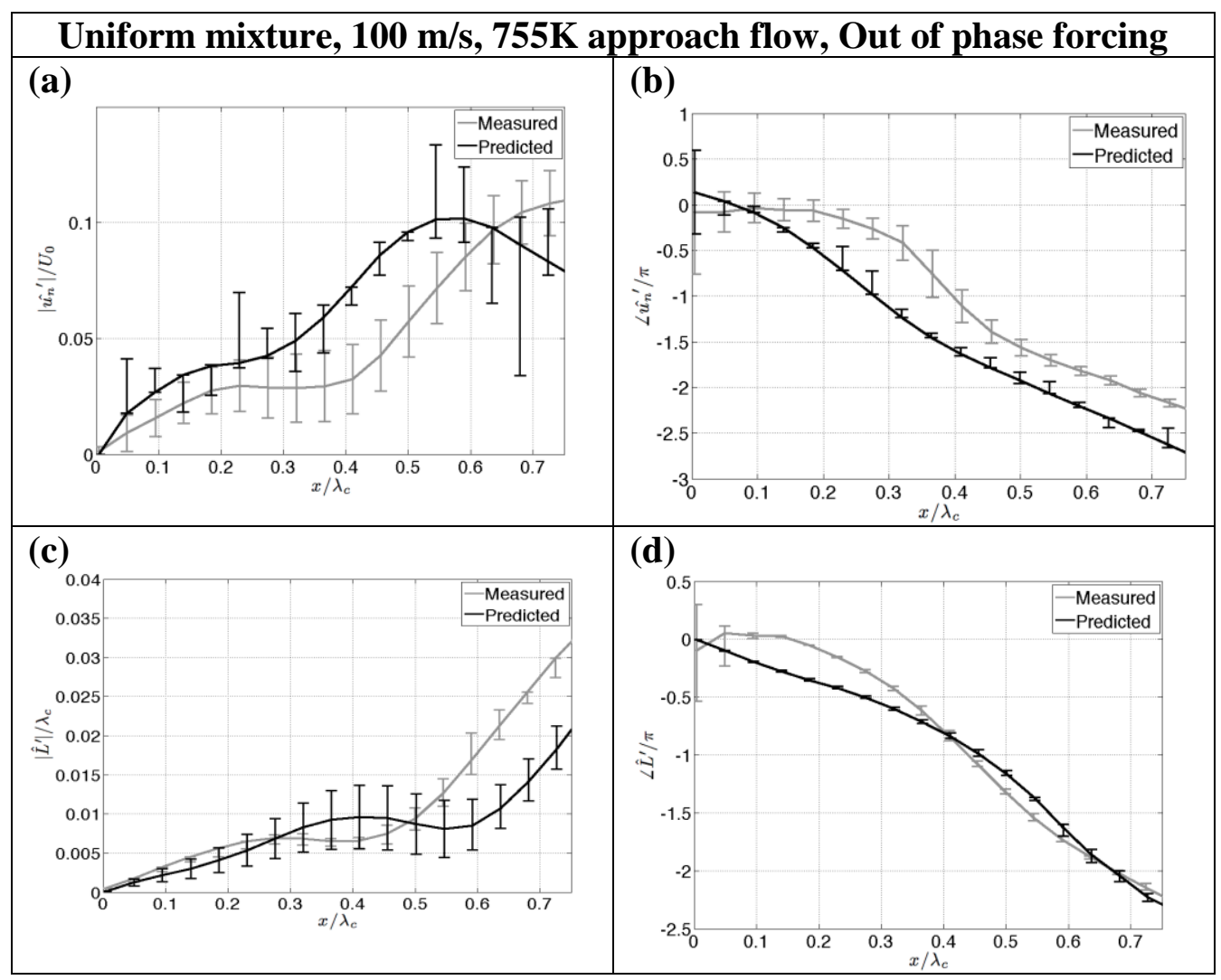

Figure 100: Velocity validation study with flame edge as input : (a) amplitude comparison, (b) corresponding phase comparison; Flame edge validation study with velocity as input : (c) amplitude comparison, (d) corresponding phase comparison. 


\section{Measurement of the Acoustic Impedance of the Injection Swirler}

\section{Introduction}

As stated previously, the successful development of a flame response model which accounts for the effects of both velocity and equivalence ratio fluctuations requires the ability to predict the acoustic flow field in the injector. Of particular importance is the ability to account for the acoustic impedance of the swirler. During this section of the technical report, measurements were made of the acoustic characteristics of the injector used in this study from which the acoustic impedance of the swirler was determined. The status of this work is summarized in the following discussion.

\section{Experimental Setup}

The mixing section of the Penn State combustor is illustrated in Figure 101. Also shown are the swirler and the various pressure transducer pressure measurement locations, designated by "PT1", "PT2", etc. From the dynamic pressure measurements at pressure transducer locations 2 and 3 and locations 4 and 5 the velocity fluctuations were calculated upstream and downstream of the swirler, respectively. These measurements were made both with and without the swirler at a fixed air flow rate of $50 \mathrm{SCFM}$ and temperature of $20^{\circ} \mathrm{C}$. Using the siren described in previous reports, the air flow rate was modulated at frequencies from 100 to $500 \mathrm{~Hz}$ in $25 \mathrm{~Hz}$ increments. The results are shown in Figure 102, which is a plot of the phase difference between the velocity fluctuation measured upstream and downstream of the swirler location. Results are presented both with and without the swirler. In the case without the swirler, the phase difference is shown to increase approximately linearly with the forcing frequency, as one would expect. In the case with the swirler, the phase difference increases markedly from $100 \mathrm{~Hz}$ to $200 \mathrm{~Hz}$, and is approximately constant at frequencies above $200 \mathrm{~Hz}$ at a value of nearly 180 degrees.
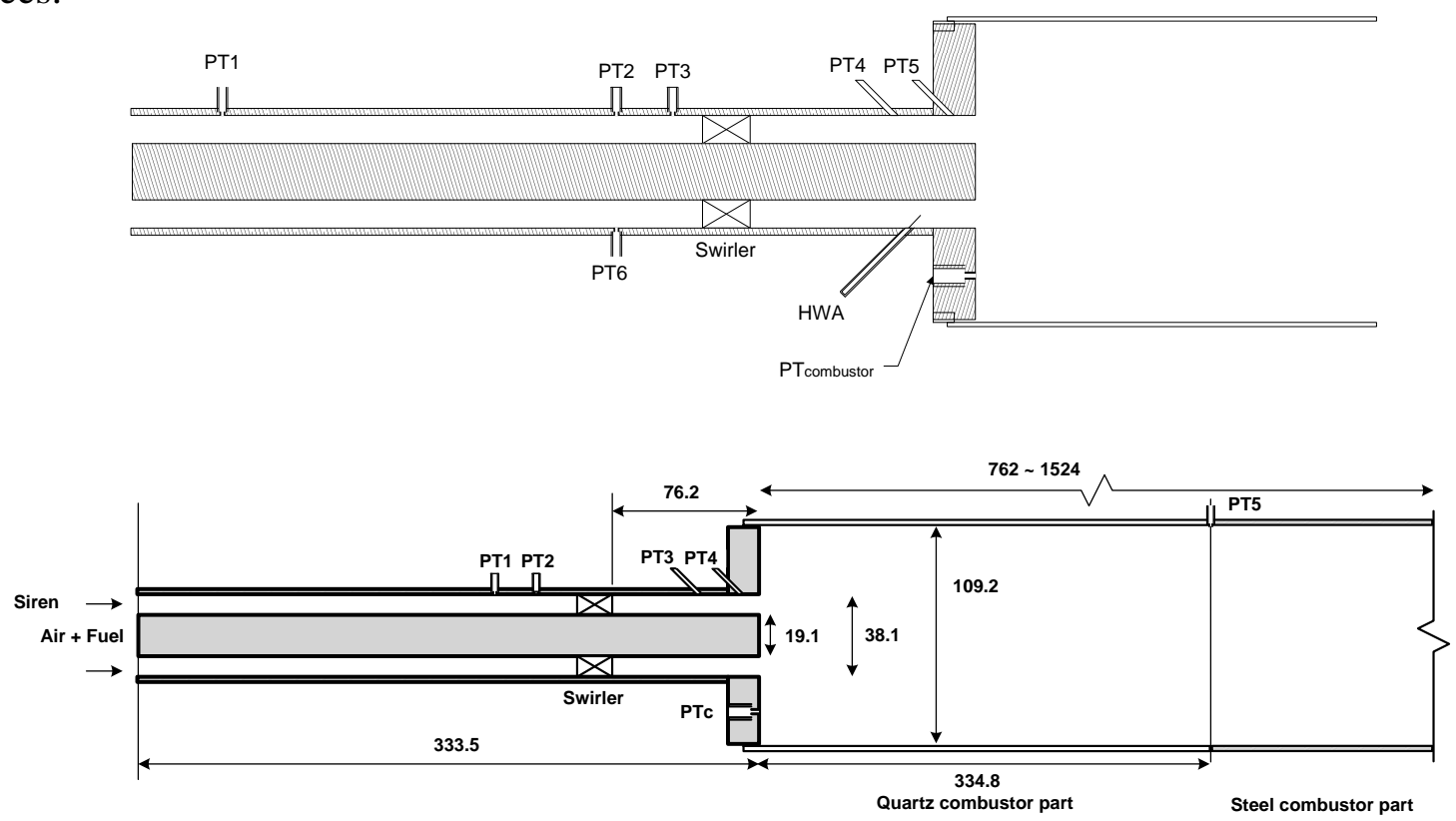

Figure 101: Experimental set-up for swirler acoustic impedance measurements. Bottom figure includes dimensions given in millimeters. 


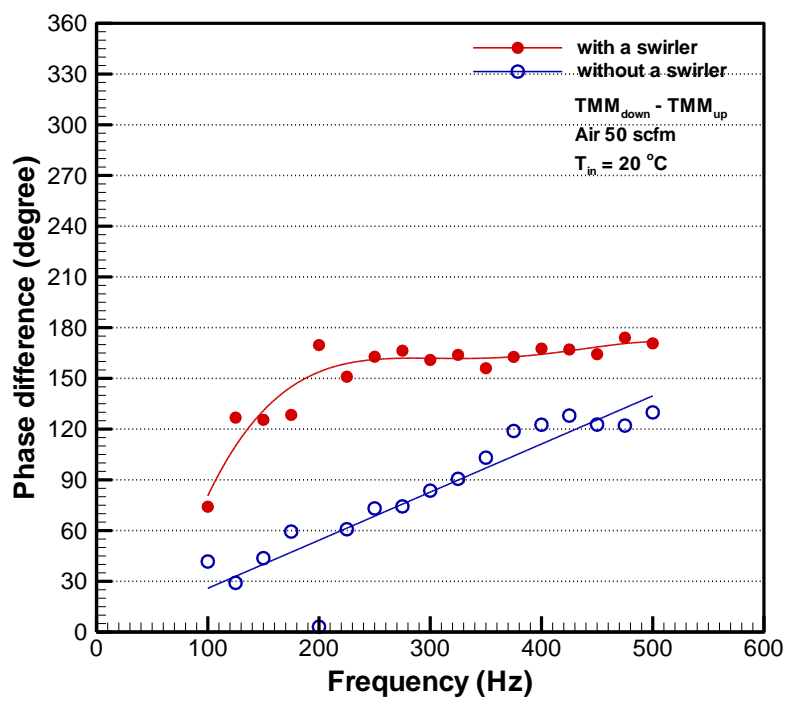

Figure 102: The phase-difference between the velocity fluctuation upstream and downstream of the swirler location, both with and without the swirler.

\section{Results and Discussion}

The effect of the swirler on the velocity fluctuation can be characterized in terms of a transfer function, which is defined by:

$$
T F_{V}(f, A)=\frac{V_{d}^{\prime}(f) / V_{d, m}}{V_{u}^{\prime}(f) / V_{u, m}}
$$

The transfer function gives the phase and gain between the upstream and downstream velocity fluctuation. The phase and gain of the swirler transfer function is plotted versus the Strouhal number for five different values of the velocity in Figure 103.

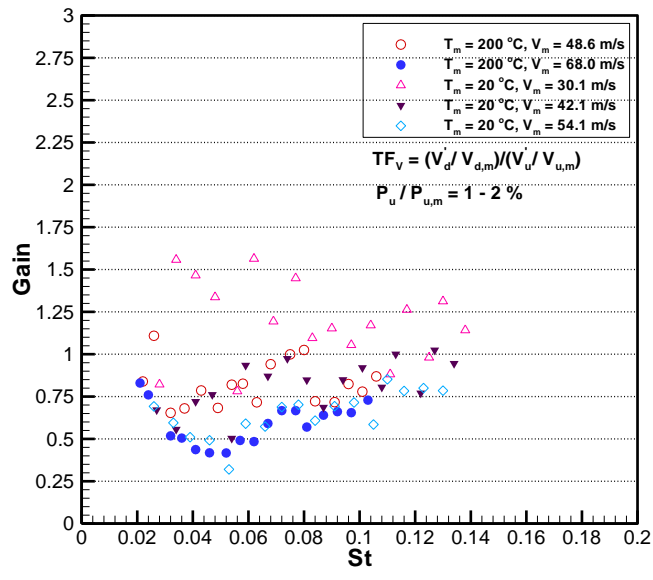

(a) Gain

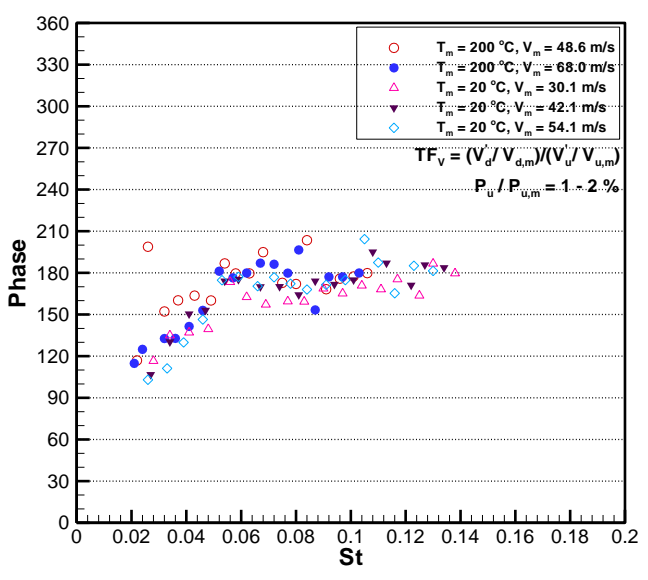

(b) Phase

Figure 103: (a) Gain and (b) phase of swirler transfer function (velocity) as a function of Strouhal number when the upstream pressure oscillation amplitude is kept constant, $1 \sim 2$ $\%$. 
And lastly, these results are used to determine the acoustic impedance of the swirler, as shown in Figure 104. The plot on the left corresponds to upstream of the swirler, while the plot on the right corresponds to downstream of the swirler. These results clearly show that the swirler acts as an acoustic damper.
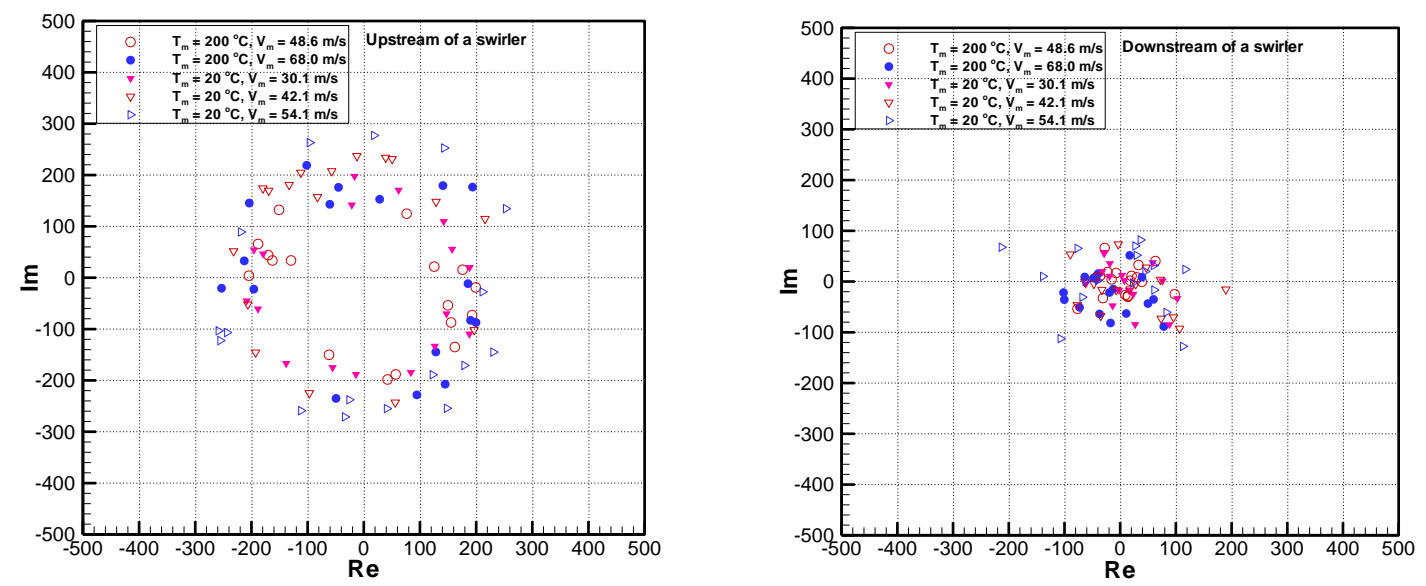

\section{Figure 104: Acoustic impedance in complex domain upstream and downstream of the swirler}

\section{Flame transfer function measurements of lean turbulent premixed flames}

During this sponsorship, extensive flame transfer function measurements of turbulent premixed flames were taken in a lean premixed, swirl-stabilized, gas turbine combustor, shown in Figure 101. $\mathrm{OH}^{*}, \mathrm{CH}^{*}$, and $\mathrm{CO}_{2} *$ chemiluminescence emissions were used to measure heat release oscillations from a whole flame, and the two-microphone technique was used to estimate inlet velocity disturbances. 2-D $\mathrm{CH}^{*}$ chemiluminescence imaging was used to characterize the flame structure: the flame length $\left(\mathrm{L}_{\mathrm{CH}^{*} \max }\right)$ and flame angle $(\alpha)$. Using $\mathrm{H}_{2}$-natural gas composite fuels, $\mathrm{X}_{\mathrm{H} 2}=0.00 \sim 0.60$, very short flame structures were obtained. All tests were performed at a mean pressure of $1 \mathrm{~atm}$ and at mean equivalence ratios ranging from 0.55 to 0.70 . Mean velocities at the nozzle were $60 \sim 100 \mathrm{~m} / \mathrm{s}$ and the inlet temperature was kept constant at $200{ }^{\circ} \mathrm{C}$, giving a Reynolds number of approximately 33,000 54,000. Forcing frequencies were varied from 100 to $400 \mathrm{~Hz}$.

The overall understanding of the flame structure is gained from time-averaged $\mathrm{CH}^{*}$ chemiluminescence images, shown in Figure 105. It presents the dependence of the spatial distribution of the flame's heat release on $\mathrm{H}_{2}$ mole fraction $\left(\mathrm{X}_{\mathrm{H} 2}\right)$ and the equivalence ratio. In these deconvoluted images, only the upper half is shown because the reconstructed images are axisymmetric. The direction of flow in these chemiluminescence images is from left to right. With increasing $\mathrm{X}_{\mathrm{H} 2}$ (from 0.00 to 0.60 ) or equivalence ratio (from 0.55 to 0.70 ) at a fixed inlet velocity $(60 \mathrm{~m} / \mathrm{s})$, the flame length decreases because of the increased flame propagation speed. The flame extends into the corner recirculation zone as well as the inner shear layer at the equivalence ratio of 0.55 with $\mathrm{X}_{\mathrm{H} 2}=0.00$. To quantitatively describe the flame structure, the distance between the edge of the centerbody and the maximum $\mathrm{CH}^{*}$ chemiluminescence intensity point $\left(\mathrm{L}_{\mathrm{CH}^{*} \max }\right)$, and the flame angle $(\alpha)$ between $\mathrm{L}_{\mathrm{CH}^{*} \text { max }}$ and the flow direction are defined from the deconvoluted flame images, shown in Figure 105. $\mathrm{L}_{\mathrm{CH}^{*} \text { max }}$ represents the flame length or the convection length scale of a given flame. 


\begin{tabular}{|c|c|c|c|c|}
\hline 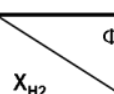 & 0.55 & 0.80 & 0.65 & 0.70 \\
\hline 0.00 & 50 & 8 & 2 & 2 \\
\hline 0.15 & 2 & 8 & 8 & 0 \\
\hline 0.30 & D & e & 0 & 0 \\
\hline 0.45 & e & e & e & $e$ \\
\hline 0.50 & e & e & e & e \\
\hline
\end{tabular}

Figure 105: Deconvoluted stable flame images $\left(\mathrm{CH}^{*}\right.$ chemiluminescence). Operating conditions: $T_{\text {in }}=200{ }^{\circ} \mathrm{C}, \mathrm{V}_{\text {mean }}=60 \mathrm{~m} / \mathrm{s}, \Phi=0.55,0.60,0.65,0.70$, premixed, $\mathrm{X}_{\mathrm{H} 2}=0.00$, $0.15,0.30,0.45,0.60$.

Coordinates of maximum $\mathrm{CH}^{*}$ chemiluminescence intensity locations and flame angle $(\alpha)$ versus the flame length, $\mathrm{L}_{\mathrm{CH}^{*} \text { max }}$, of 95 stable flames are shown in Figure $106\left(\mathrm{~T}_{\text {in }}=200{ }^{\circ} \mathrm{C}, \mathrm{V}_{\text {mean }}=60\right.$, $70,80,90,100 \mathrm{~m} / \mathrm{s}$, premixed, $\mathrm{X}_{\mathrm{H} 2}=0.00,0.15,0.30,0.45,0.60$, and $\Phi=0.55,0.60,0.65,0.70$ ). The origin of these plots corresponds to the edge of the centerbody, where the flame is stabilized. It shows that the maximum $\mathrm{CH}^{*}$ chemiluminescence intensity locations can be characterized by a well-defined curve: $\mathrm{L}_{\mathrm{CH}^{*} \text { max }}$ varies along this curve over a range of input conditions. This figure also shows that the flame angle, $\alpha$, increases up to $\alpha_{\text {crit }} \sim 36^{\circ}$, and then decreases, while $\mathrm{L}_{\mathrm{CH}^{*} \text { max }}$ keeps decreasing. The critical flame angle, $\alpha_{\text {crit }}$ corresponds to the demarcation point where " $\mathrm{V}$ " flame structures are changed to " $\mathrm{M}$ " flames. Therefore, the flame length, $\mathrm{L}_{\mathrm{CH}^{*} \text { max }}$, and the flame angle, $\alpha$, are necessary parameters to define the flame structure. These two parameters, $\mathrm{L}_{\mathrm{CH}^{*} \max }$ and $\alpha$, will be used to analyze the forced response of turbulent premixed flames.
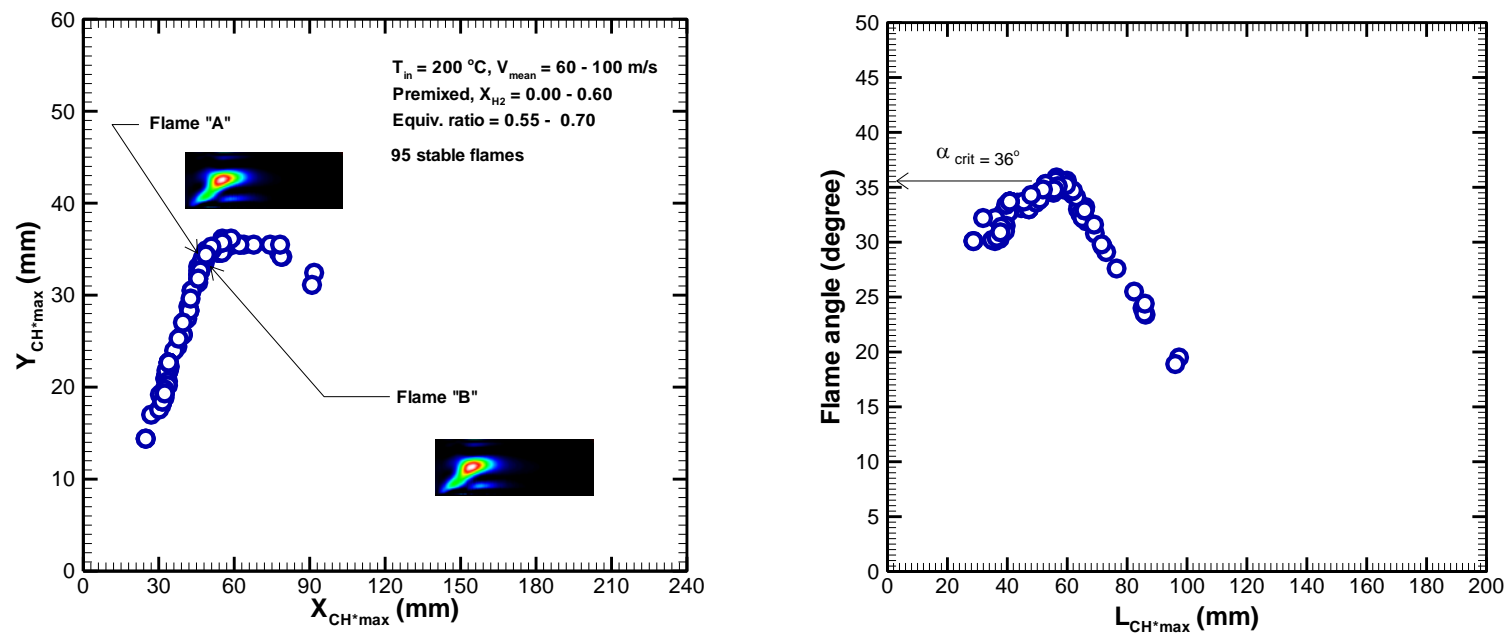

Figure 106: Coordinates of maximum $\mathrm{CH}^{*}$ chemiluminescence intensity locations and flame angle $(\alpha)$ versus the flame length $\left(\mathrm{L}_{\mathrm{CH}^{*} \text { max }}\right)$. 
To compare heat release response of two different flames, Flame " $\mathrm{A}$ " and "B" were chosen. Their input conditions and deconvoluted flame structures are presented in Table 6 and Figure 106, respectively. Flames "A" and "B" have different input parameters such as the inlet velocity, equivalence ratio, the adiabatic flame temperature, and input power, but they have the similar flame structure, i.e., $\mathrm{L}_{\mathrm{CH}^{*} \max }$ and $\alpha$. Figure 4 shows the gain of FTF of Flame "A" and "B" at the forcing frequency of $f=100 \mathrm{~Hz}$ and $250 \mathrm{~Hz}$. The amplitude dependence of flame heat release and the gain of two different flames are almost identical to each other at both forcing frequencies. The results shown in Figure 107 indicate that Flame " $\mathrm{A}$ " and "B" show saturation phenomena at the same forcing amplitude, $\mathrm{V}^{\prime} / \mathrm{V}_{\text {mean }} \sim 0.22(f=100 \mathrm{~Hz})$ and $\mathrm{V}^{\prime} / \mathrm{V}_{\text {mean }} \sim 0.13(f=$ $250 \mathrm{~Hz}$ ). The higher the forcing frequency, the lower the forcing amplitude at which the inception of nonlinearity appears. Figure 107 suggests that the flame structure is one of important parameters governing the flame transfer functions of turbulent premixed flames.

Table 6: Comparison of two different flames: Flame "A" and "B".

\begin{tabular}{cccccccccc}
\hline & $\begin{array}{c}\mathrm{T}_{\mathrm{in}} \\
\left({ }^{\circ} \mathrm{C}\right)\end{array}$ & $\begin{array}{c}\mathrm{V}_{\text {mean }} \\
(\mathrm{m} / \mathrm{s})\end{array}$ & $\Phi$ & $\mathrm{X}_{\mathrm{H} 2}$ & $\begin{array}{c}\mathrm{T}_{\mathrm{ad}} \\
(\mathrm{K})\end{array}$ & $\begin{array}{c}\mathrm{Q}_{\text {in }} \\
(\mathrm{kW})\end{array}$ & $\begin{array}{c}\mathrm{X}_{\mathrm{CH} \text { max }} \\
(\mathrm{mm})\end{array}$ & $\begin{array}{c}\mathrm{Y}_{\mathrm{CH}^{*} \max } \\
(\mathrm{mm})\end{array}$ & $\begin{array}{c}\alpha \\
(\text { degree })\end{array}$ \\
\hline $\begin{array}{c}\text { Flame } \\
\mathbf{A}\end{array}$ & 200 & 60 & 0.65 & 0.00 & 1681 & 67.91 & 55.1 & 35.0 & 32.5 \\
$\begin{array}{c}\text { Flame } \\
\text { B }\end{array}$ & 200 & 70 & 0.70 & 0.00 & 1767 & 84.90 & 53.3 & 34.6 & 33.0 \\
\hline
\end{tabular}
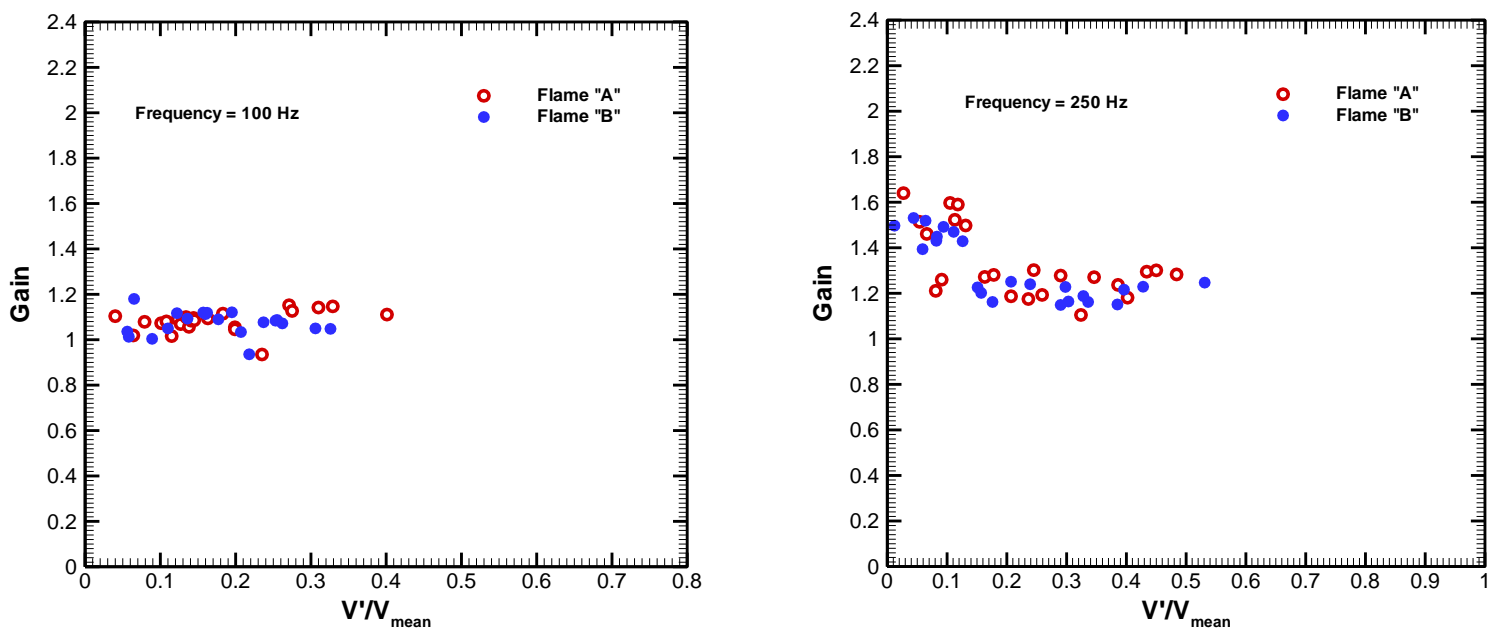

Figure 107: Comparison of normalized heat release response and gain of Flame "A" and "B": $f=100 \mathrm{~Hz}$ and $250 \mathrm{~Hz}$.

For the present study, to quantitatively describe the forced flame response, the second order model was used to theoretically fit the gain of FTF. Figure 108 shows theoretical fits (18 different operating conditions) of gain of FTF at a fixed forcing amplitude, $\mathrm{V}^{\prime} / \mathrm{V}_{\text {mean }}=0.100$, operating on $\mathrm{H}_{2}+$ natural gas mixture fuels $\left(\mathrm{X}_{\mathrm{H} 2}=0.00,0.15,0.30\right)$ at an inlet temperature of 200 ${ }^{\circ} \mathrm{C}$ over a range of inlet velocities from 60 to $80 \mathrm{~m} / \mathrm{s}$ and a range of equivalence ratios from 0.55 
to 0.70 . This result shows that the gain of FTF decreases with decreasing the flame length, $\mathrm{L}_{\mathrm{CH}^{*}}$ $\max$, due to two possible reasons. One reason is that the flame structure is changed from " $\mathrm{V}$ " flames to "M" flames with increasing flame propagation speed, and therefore the "M" flames have higher capability to dampen upstream velocity perturbations. The other possible explanation could be illustrated by using the following Strouhal number:

$$
S t=\frac{f \cdot L_{C H^{*} \mathrm{max}}}{V_{c o n v}}=\frac{L_{C H^{*} \mathrm{max}}}{L_{\text {conv }}}=\frac{\tau_{\text {conv }}}{T_{\text {acoustic }}}
$$

As was discussed previously in the analytical work section, the Strouhal number (St) represents the ratio of the flame length and convective wavelength, or equivalently, the ratio of convective time to the acoustic forcing period. Figure 109 shows the dependence of the Strouhal number upon the $\mathrm{H}_{2}$ mole fraction. All operating conditions $\left(\mathrm{T}_{\text {in }}=200{ }^{\circ} \mathrm{C}, \mathrm{V}_{\text {mean }}=60 \mathrm{~m} / \mathrm{s}\right.$, premixed, and $\Phi=0.60)$ are the same except for the $\mathrm{H}_{2}$ mole fraction. The Strouhal number decreases with increasing $\mathrm{X}_{\mathrm{H} 2}$ at a fixed forcing frequency. The Strouhal number approaches unity, suggesting that the relative ratio of convection time to acoustic time scale decreases. Therefore, the flame is less influenced by the acoustic perturbations with increasing $\mathrm{X}_{\mathrm{H} 2}$.

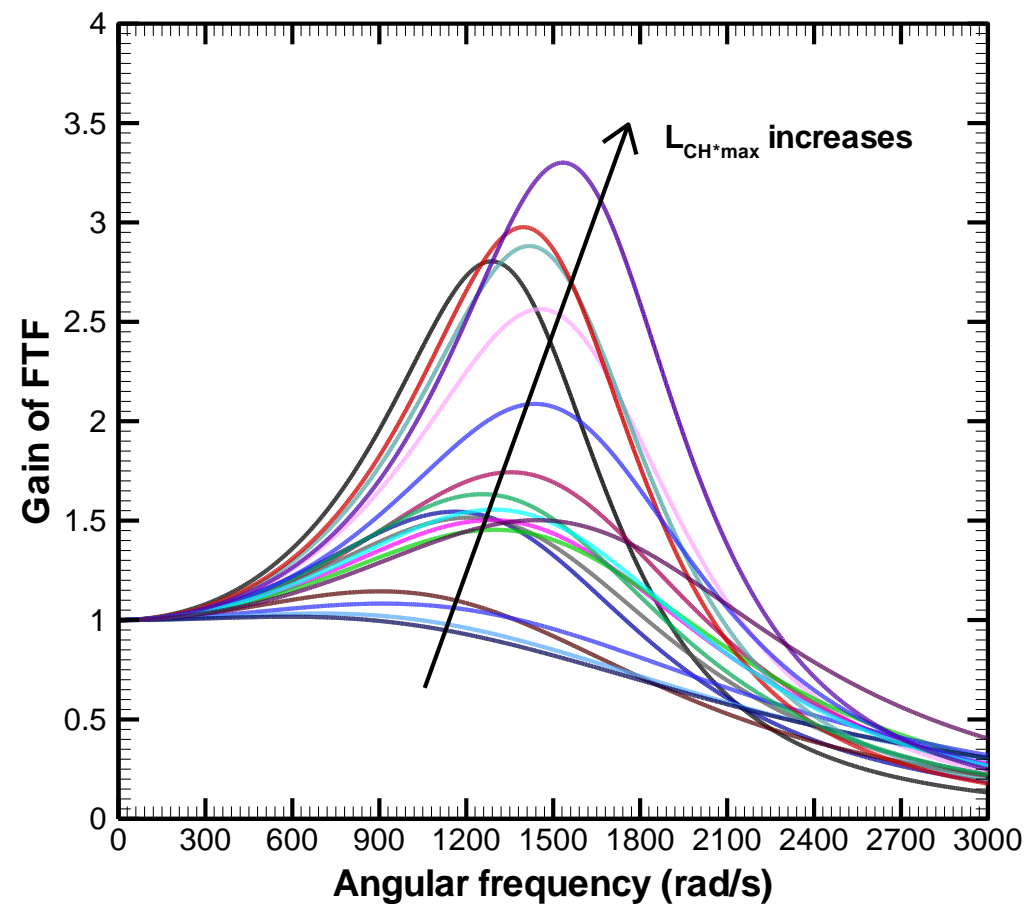

Figure 108: Theoretical fits (second order model) of gain of FTF. Operating conditions: $T_{\text {in }}$ $=200{ }^{\circ} \mathrm{C}, \mathrm{V}_{\text {mean }}=60,70,80 \mathrm{~m} / \mathrm{s}, \Phi=0.55,0.60,0.65,0.70$, premixed, $X_{\mathrm{H} 2}=0.00,0.15,0.30$. 


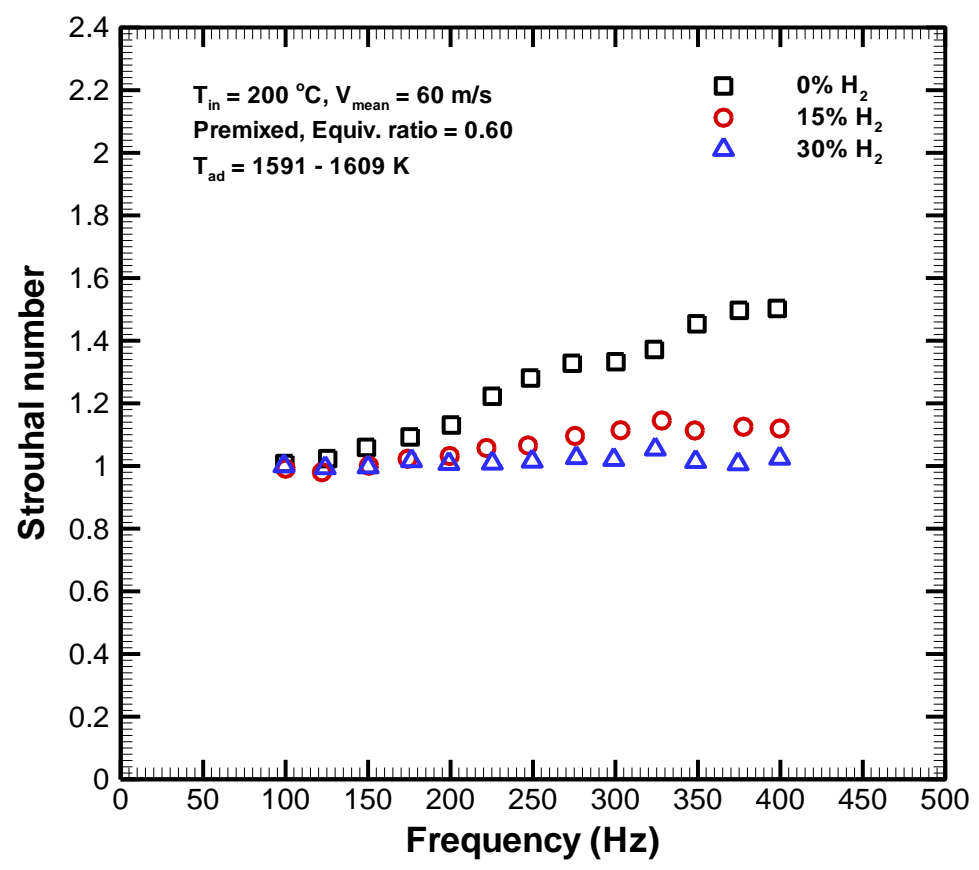

Figure 109: Dependence of the Strouhal number $(\mathrm{St})$ upon $X_{\mathrm{H} 2}$.

The modulation frequency where the flame response is largest is $150 \mathrm{~Hz} \sim 250 \mathrm{~Hz}$, irrespective of the input parameters, clearly shown in Figure 107. This indicates that the turbulent premixed flames amplify velocity disturbances in a certain range of frequencies. Also, this frequency range is consistent with one of the self-induced instability frequencies observed in this combustor facility. At high forcing frequency, $f>300 \mathrm{~Hz}$, the gain of FTF asymptotically approaches zero, showing a low-pass filter behavior. Low-frequency flow perturbations significantly affect the global evolution of flame surface area, i.e., flame heat release rate. On the other hand, highfrequency velocity perturbations have a limited effect on flame surface fluctuations. Linear fits of phase difference of FTF are shown in Figure 110. Operating conditions are the same as those in Figure 107. The phase difference of FTF increases with increasing $\mathrm{L}_{\mathrm{CH}^{*} \max }$, due to the fact that with increasing the flame length, the convection time for disturbances to convect to the flame's center-of-heat-release location also increases. In particular, at low frequency limit, the influence of flame structure on the phase difference of FTF becomes negligible. 


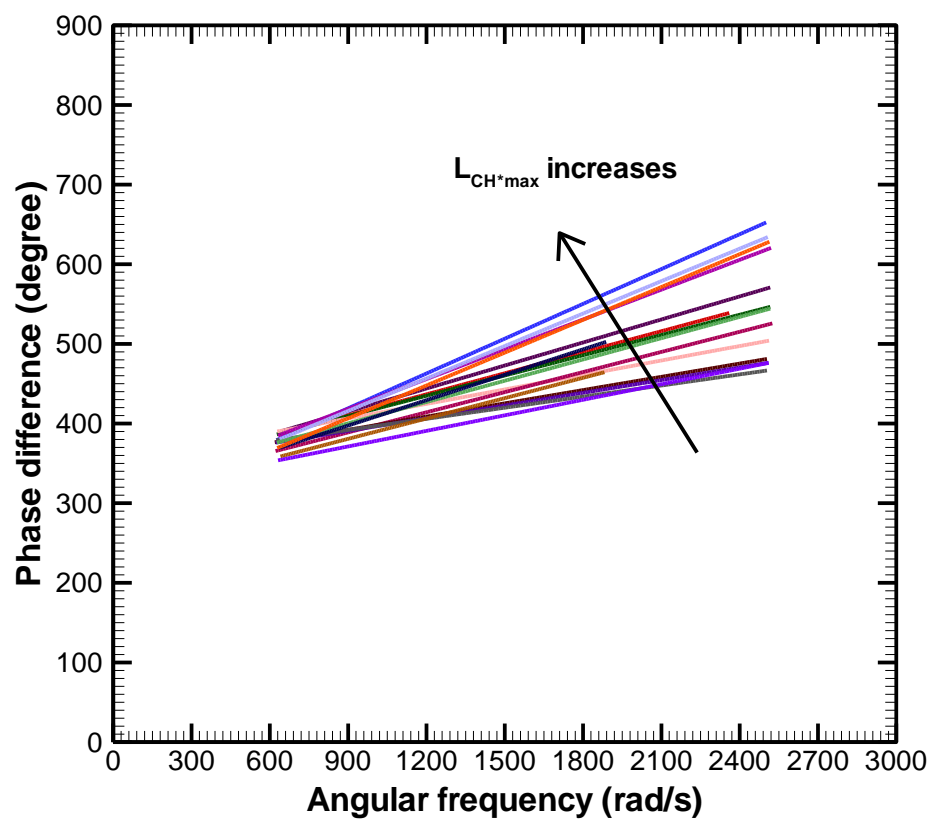

Figure 110: Linear fits of phase difference of FTF. Operating conditions: $T_{\text {in }}=200{ }^{\circ} \mathrm{C}$, $\mathrm{V}_{\text {mean }}=60,70,80 \mathrm{~m} / \mathrm{s}, \Phi=0.55,0.60,0.65,0.70$, premixed, $X_{\mathrm{H} 2}=0.00,0.15,0.30$.

In order to quantify the responses of different flames, the damping coefficient $(\xi)$ and the convection time delay $(\tau)$ were obtained from theoretical fits. Figure 111 plots the dependence of the damping coefficient and the convection time delay upon the normalized flame length, $\mathrm{L}_{\mathrm{CH}^{*}}$ $\max / R$, where $R$ denotes the inner radius of the quartz combustor. Note that the damping coefficient, $\xi$, is inversely proportional to $\mathrm{L}_{\mathrm{CH}^{*}}{ }_{\max } / \mathrm{R}$, and the convection time delay, $\tau$, is proportional to $\mathrm{L}_{\mathrm{CH}^{*}}$ max $/ \mathrm{R}$. This shows that the dynamic response of totally premixed turbulent flames can be characterized by the flame structure.
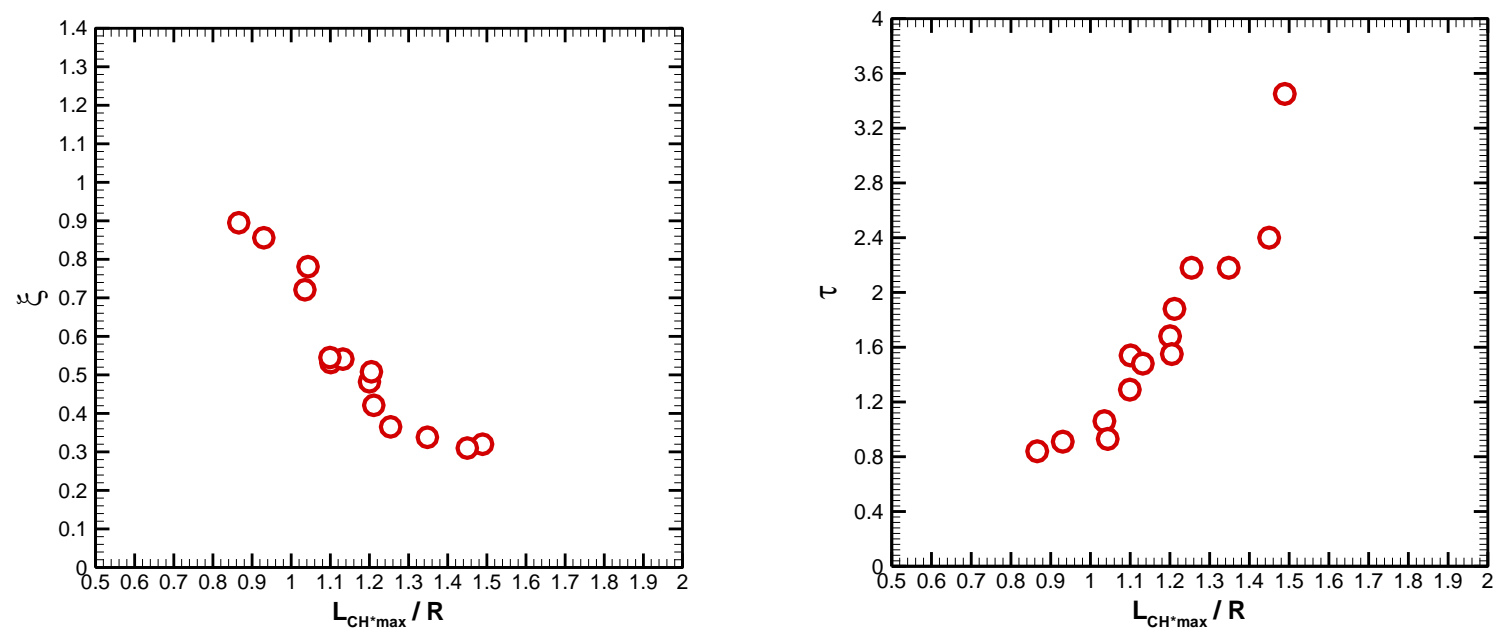

Figure 111: The dependence of damping coefficient $(\xi)$ and convection time delay $(\tau)$ upon the normalized flame length $\left(\mathrm{L}_{\mathrm{CH}^{*} \max } / \mathrm{R}\right)$. Operating conditions: $\mathbf{T}_{\mathrm{in}}=200{ }^{\circ} \mathrm{C}, \mathrm{V}_{\text {mean }}=60$, $70,80 \mathrm{~m} / \mathrm{s}, \Phi=0.55,0.60,0.65,0.70$, premixed, $X_{\mathrm{H} 2}=0.00,0.15,0.30$. 

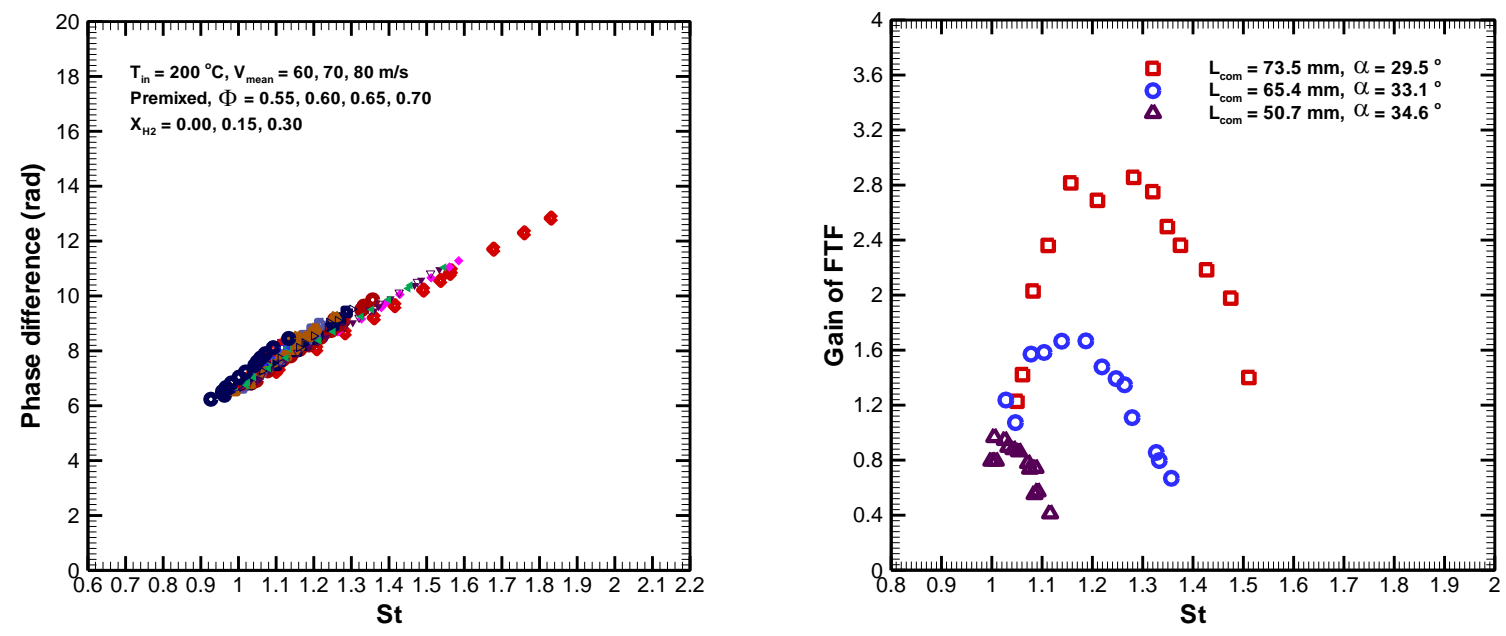

Figure 112: The dependence of phase difference and gain of FTF upon the Strouhal number $(\mathrm{St})$. Operating conditions: $T_{\text {in }}=200{ }^{\circ} \mathrm{C}, \mathrm{V}_{\text {mean }}=60,70,80 \mathrm{~m} / \mathrm{s}, \Phi=0.55,0.60,0.65$, 0.70, premixed, $\mathrm{X}_{\mathrm{H} 2}=0.00,0.15,0.30$.

In Figure 112, the gain and phase difference of FTF are plotted against the non-dimensional parameter, the Strouhal number (St). The definition of St was presented in Equation (1), and it was calculated from the phase difference of FTF at each operating condition. It is evident that the phase differences of FTF are well-characterized by the Strouhal number only in the investigated range of St. When the flame angle changes significantly, the phase difference starts to deviate from the trend. On the other hand, the gain of FTF is a strong function of the flame structure as well as the Strouhal number. The gain decreases with increasing the flame angle $(\alpha)$. These results are reminiscent of the results from analytic and experimental studies about laminar premixed flames. In an analytic study of V-flame transfer functions, Schuller et al. ${ }^{[1]}$ reported that the smaller the flame angle, the larger and the higher the overshoot. They also showed that phase difference is not significantly influenced by the flame angle, $\alpha$, compared to the gain of FTF. Buchner et al. ${ }^{[2]}$ reported that the dynamics of pulsated premixed axial jet flames are governed by the Strouhal number $\left(\mathrm{St}=\mathrm{f}^{*} \mathrm{x}_{\mathrm{OH} \text { max }} / \mathrm{U}\right)$ only, but they didn't take the flame structure into account. The present results strongly suggest that the dynamics of swirl-stabilized, turbulent premixed flames are characterized by three relevant parameters, the Strouhal number $\left(\mathrm{St}=\mathrm{L}_{\mathrm{CH}^{*}}\right.$ $\left.\max / \mathrm{L}_{\text {conv }}\right)$, the normalized flame length $\left(\mathrm{L}_{\mathrm{CH}^{*} \max } / \mathrm{R}\right)$, and the flame angle $(\alpha)$, therefore, extending previous experimental and analytical results from laminar to turbulent premixed flames. There are no other experimental data of controlling parameters of turbulent premixed flames in literature yet. Systematic investigation and generalization about the forced flame response measurements in a practical, lean-premixed, gas turbine combustor could be incorporated into an analytic model and enable the development of theoretical models to control and suppress self-sustained combustion instabilities. 


\section{Flame Brush Dynamics in a Harmonically Oscillating Turbulent Jet Flame}

\section{Introduction}

The evolution of the turbulent flame brush is an important fundamental quantity that describes the spatial distribution of heat release of a turbulent flame. One objective of this work was to investigate this spatial/temporal evolution of the flame brush in an acoustically forced case under different flow/flame configurations, and compare this response to the unforced case. Two different configurations were explored: a conical Bunsen flame and an annular swirl stabilized flame. The flame's heat release response to a perturbation generally increases linearly with perturbation amplitude over a certain range of (low) amplitudes, before saturating or exhibiting more complex nonlinear behavior at high amplitudes. These low amplitude linear dynamics generally control the balance between driving and damping processes at low amplitudes of oscillation and, thus, determine the regions under which self-excited oscillations can occur. On the other hand, the nonlinear combustor processes control the finite amplitude dynamics of the oscillations.

Although many studies have obtained measurements under turbulent conditions, little work has been done to elucidate the influence of acoustic forcing upon the actual turbulent flame parameters, such as turbulent flame speed or flame brush thickness. This present study was motivated by measurements in a non-swirling flat flame that reported coherent fluctuations in flame brush thickness of a forced flame. Such coherent oscillations would certainly influence the flame's response to such forcing; e.g. fluctuations in turbulent flame speed will exert important influences on both the linear and nonlinear flame response characteristics.

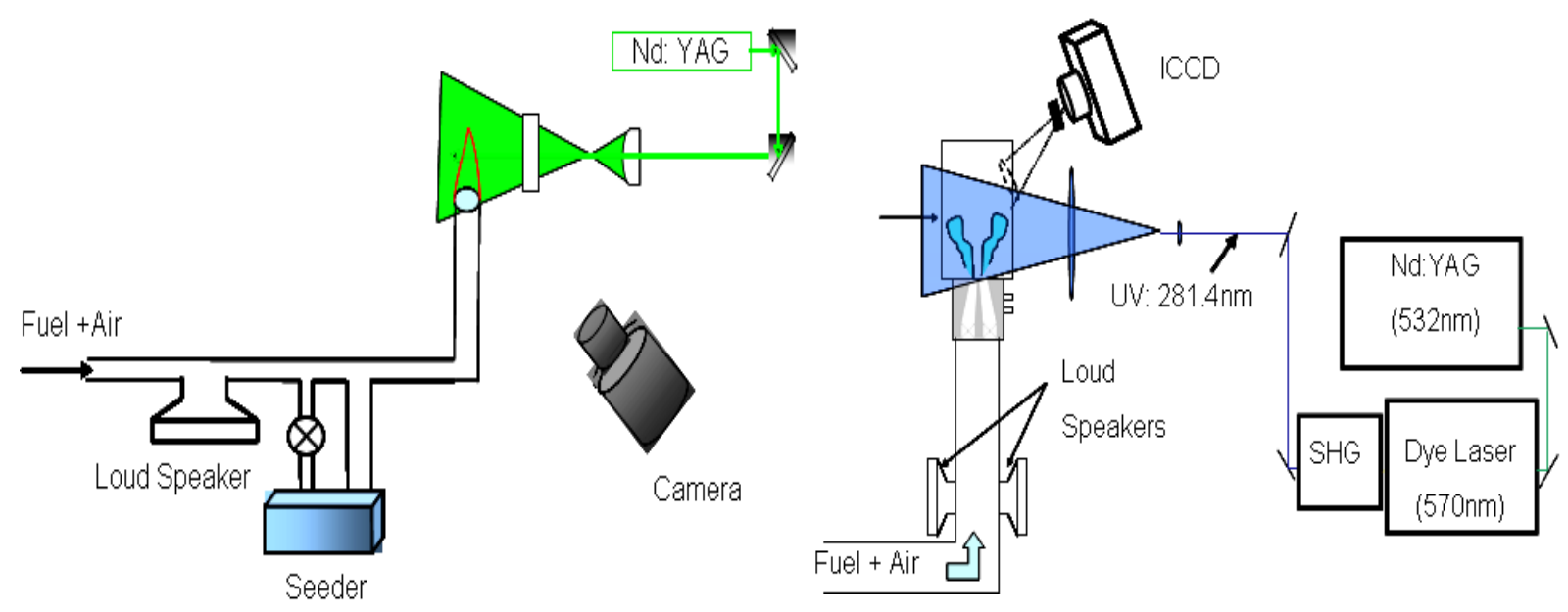

(a)

(b)

Figure 113: Schematic of the experimental setup used for studying the (a) Bunsen flame and (b) swirl flame. 
A number of prior studies have characterized the turbulent flame brush evolution in the unforced case, as discussed in the review by Lipatnikov \& Chomiak. For example, prior experimental works have reported the growth of the flame brush for conical Bunsen flames, V-shaped flames, and other confined oblique flames. These studies have shown that the flame brush thickness increases monotonically with distance from the flame holder. This monotonic growth of the flame brush thickness has been described by the Taylor theory of turbulent diffusion, as shown by Eq.(337) below:

$$
\left.\delta_{t}^{2}=4 \pi u_{t}^{\prime} L_{L}{ }^{t} f d\left\{1-\frac{{ }^{\tau} L}{{ }^{t} f d}\left[1-\exp \left(-\frac{{ }^{t} f d}{{ }{ }_{L}}\right)\right]\right\}\right\}
$$

Lipatnikov and Sathiah extended the flame speed closure model proposed by Zimont to account for turbulent flame speed variations along the flame length. The rationale behind this was that development of the turbulent flame speed and the flame brush differed qualitatively. The development of the flame brush is governed by turbulent diffusion and hence the large length scales, while the development of the turbulent flame speed is governed more by the small length scales. These effects were incorporated into the unsteady problem by using the above quasisteady relations by Lipatnikov and Sathiah on the heat release fluctuations of an acoustically forced flame. In an acoustically forced flame, the flame length changes throughout the cycle. A key question we were interested in exploring was whether the flame brush evolution tracked this quasi-steady response, such as assumed by Lipatnikov and Sathiah, or exhibited totally different dynamics. The objective of the work presented in this section of the technical report is to study the spatialltemporal evolution of the turbulent flame brush in the presence of acoustic forcing.

\section{Experimental Setup and Image Analysis}

The study was conducted for two configurations: (1) turbulent Bunsen flame and (2) annular swirl stabilized flame. Schematics of the two facilities are shown in Figure 113. Figure 113 (a) illustrates the axisymmetric Bunsen burner (ID $25.4 \mathrm{~mm}$ ). The fuel (natural gas) and air were premixed upstream at an equivalence ratio of 0.85 . Acoustic oscillations were introduced through a loudspeaker mounted upstream of the combustor. The flame was stabilized by a lean premixed pilot flame at low flow rates relative to the main burner ( $2 \%$ of the main flow). Time resolved turbulence characteristics of the flow were obtained using hot wire anemometry at a sampling frequency of $10 \mathrm{kHz}$. The spatial characteristics of the flame and the velocity field were measured using PIV. A schematic of the burner and optics is shown in Figure 113. The premixed fuel and air were seeded with 0.9-2.2 $\mu \mathrm{m}$ aluminum oxide particles. The light source was a dualcavity, frequency doubled pulsed $N d$ : $Y A G$ laser (Surelite I-10 PIV). The infrared emission $(1064 \mathrm{~nm})$ from both cavities was combined before it entered a frequency-doubling crystal that produced collinear beams of the same polarization. Each pulse had a maximum energy of $110 \mathrm{~mJ}$ (at $532 \mathrm{~nm}$ ) and duration of $\sim 9 \mathrm{~ns}$. The output from the laser was directed into the combustor section using high power $532 \mathrm{~nm}$ laser mirrors (CVI Laser). The sheet forming optics consisted of two lenses: a plano-convex cylindrical glass lens $(50 \mathrm{~mm}$ focal length, and $25.4 \mathrm{~mm}$ in diameter) and a plano-convex cylindrical lens (500 $\mathrm{mm}$ focal length and $90 \mathrm{~mm}$ in diameter), mounted with its axis perpendicular to the first lens to narrow the thickness of the sheet over the burner. The coplanar vertically-expanding laser sheets (approximately $400 \mathrm{~mm}$ thick) illuminated particles seeded along the injector center-plane from the base of the combustor. 


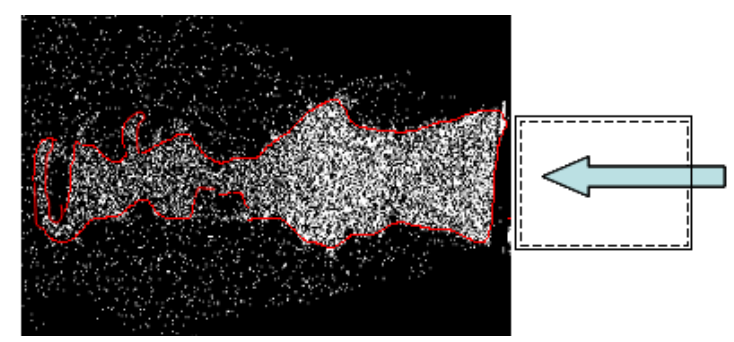

(a)

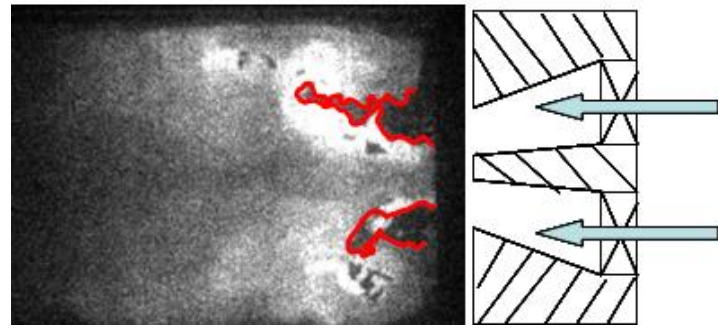

(b)

Figure 114: Sample instantaneous (a) Mie-scattering image used for the PIV analysis $\left(f_{o}=200 \mathrm{~Hz}, u^{\prime}{ }_{A} / u_{o}=0.2,0^{\circ}\right.$ phase with respect to the forcing signal) and (b) OH PLIF image of the unforced swirl flame $(R e=21,000)$.

Light scattered by the particles was collected at right angles onto an interline CCD camera (Micro Max) fitted with a $50 \mathrm{~mm} f / 1.8$ Nikon lens. The camera had a high resolution array of $1300 \square 1030$ pixels, where each pixel is a $6.7 \square \mathrm{m}$ square, with a 12-bit signal resolution. The spatial resolution achieved by using this camera was around 500 microns/pixel. The velocity field was computed using a 64 pixel square window, with an overlap of 50\%. To achieve phase locking, the timing between the signal generator, the two laser heads and the camera shutter was synchronized using a delay generator (Stanford Research Systems DG-535). Pulse delays between the PIV image pairs varied from 7 to $8 \mu \mathrm{s}$. At each excitation amplitude and at 8 phases of an acoustic cycle, 400 phase locked images were recorded. The captured images were corrected for background noise and filtered using a 3-pixel width Gaussian filter. Figure 114(a) shows a sample instantaneous Mie-scattering image used for the PIV analysis. It illustrates the high contrast between the high seeding density in reactants and the low seeding density in products. This high contrast allows for tracking of the flame edge, as indicated by the red line in the figure. The flame can be seen to be highly corrugated and multi-connected and although not shown here, the formation and burnout of pockets were also seen near the tip region of the flame.

Flame parameters were calculated in the following manner. First, each instantaneous image was converted into a binary image of reactants (progress variable $\bar{c}=0$ ) and products $(\bar{c}=1)$. Next, these binary images were averaged at each phase to yield an ensemble averaged image with the progress variable varying spatially from 0 to 1 . The flame brush thickness was then determined as the distance between the contours of $\bar{c}=0.3$ and 0.7 , along the normal drawn from the $\bar{c}=$ 0.5 contour. Also, the variations in the flame brush thickness were obtained along a flame coordinate "s". This flame coordinate was measured along the arc length of the $\bar{c}=0.5$ contour.

In these ensemble averaged images, only pixels achieving a threshold correlation value were used. Also, the averages at each pixel were computed only if a minimum number of "good" ensembles (100) were present. Hence, it should be noted that each pixel in the velocity plots is the average of a different number of ensembles but with a minimum number of at least 100 . Figure 113(b) illustrates the confined, swirl stabilized flame configuration used for the second set of experiments. The nozzle of the combustor consisted of an annular flow passage with a 4.5 $\mathrm{mm}$ ID and $15.9 \mathrm{~mm}$ OD centerbody, a $40^{\circ}$ axial swirler (swirl number, $\mathrm{S} \sim 0.65$ ), followed by rapid expansion into the dump plane of the combustor (70 $\mathrm{mm}$ diameter). Fuel (natural gas) and 
air are premixed $(\Phi=0.8)$ upstream of a choke point to prevent the occurrence of fuel/air ratio oscillations. The equivalence ratio was also maintained constant throughout the course of the experiment. Acoustic oscillations were introduced using two drivers mounted upstream of the combustor. Velocity oscillations were calculated using the two microphone method using two transducers mounted just upstream of the combustor.

Phase locked OH PLIF was used to visualize the spatial dynamics of the flame. This system consists of a cluster of an Nd: YAG laser, a dye laser, and a high-resolution ICCD camera. The frequency-doubled output from the dye laser was tuned near $281.4 \mathrm{~nm}$ to pump the $R 1$ (9) transition of the $A 1 \square-X^{2} \square \square(1,0)$ band. OH fluorescence integrated from 300-380 nm was captured by an ICCD camera through a WG-305 and UG-11 Schott glass filter. Since the frequency of imaging was limited by the $N d$ : $Y A G$ laser to $10 \mathrm{~Hz}$, successive phase-locked images were actually obtained several cycles apart from each other. Fifty flame images per phase were obtained at eight equally spaced phase angles, at a spatial resolution of about $270 \mu \mathrm{m} /$ pixel. The captured images were corrected for background noise and for beam profile inhomogeneities and filtered using a 3-pixel width Gaussian filter. Flame edges were captured manually from a combination of the PLIF images and its gradient image. Determination of the flame front location was straightforward in most cases, except in regions where the flame was close to the combustor walls and/or where there was back mixing of $\mathrm{OH}$ laden products. A sample OH PLIF image is shown in Figure 114(b), with the red line indicating the location of the flame edge. In the latter case, determination of whether the interface between regions of high and low $\mathrm{OH}$ levels corresponded to a flame or a non-reacting, product-reactant interface was determined from the $\mathrm{OH}$ gradient. Gradient values that fell substantially below typical gradient levels for regions with known flames were not marked as flame fronts.

\section{Results and Discussions}

The flame brush was computed after digitizing the images and using the same progress variable approach used for the Bunsen flame. Figure 115(b) shows a sample image with a typical spatial variation in the progress variable. Analysis of the progress variables were based on an ensemble average of 25 images for the unforced case, and 10 images at each phase for the forced case.

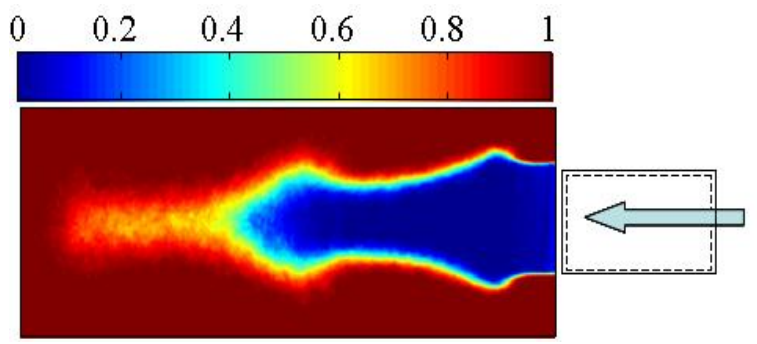

(a)

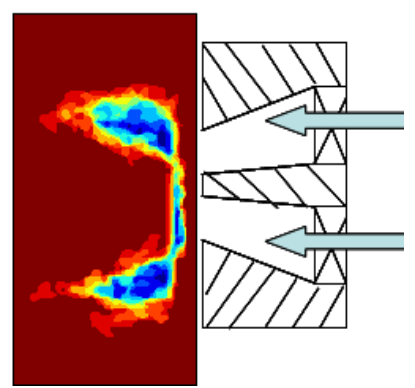

(b)

Figure 115: Sample images showing the variation of the ensemble averaged progress variable $(\bar{c}=0$ indicates reactants, while $\bar{c}=1$ indicates products) for the (a) forced Bunsen flame and (b) forced swirl flame. 


\section{Swirl Stabilized Flame}

Discussed here are the results for the swirl stabilized flame. The results presented in this subsection were based on experimental data obtained at $R e$ of 21,000 (based on the nozzle exit diameter and a mean nozzle exit flow velocity of nearly $21 \mathrm{~m} / \mathrm{s}$ ). The acoustic excitation was at a frequency of $130 \mathrm{~Hz}$ and the amplitude of excitation was maintained at $u^{\prime}{ }_{A} / u_{o}=0.6$. In the case of the swirl flames, Figure 116 shows the flame brush contours of the unforced flame corresponding to the progress variables of $\bar{c}=0.3$ and 0.7 . From, this figure it can be observed that the flame brush seems to grow, although at a very small rate with downstream distance. Also, note that the flame height in this case was only around $30 \mathrm{~mm}, \sim 3$ times the width $(11.9$ $\mathrm{mm}$ ) of the annular passage at the nozzle exit.

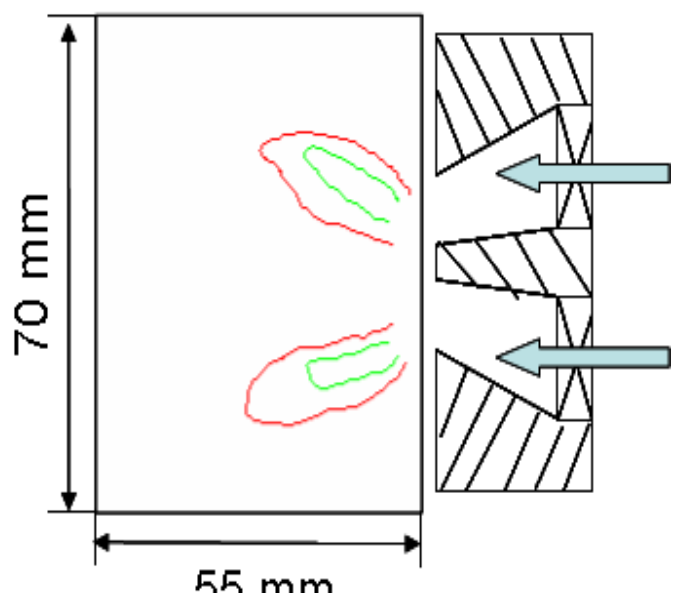

$55 \mathrm{~mm}$

Figure 116: Evolution of the flame brush as given by the progress variable contours of $\bar{c}=\mathbf{0 . 3}$ (green line) and $\bar{c}=\mathbf{0 . 7}$ (red line) of an unforced swirl flame at $\boldsymbol{R e}=\mathbf{2 1 , 0 0 0}$.

Next, in the presence of acoustic oscillations, Figure 117, describes the typical variation of the flame brush contours with phase. It can be observed that length of the flame is clearly modulated with phase through the acoustic cycle. From this figure one can clearly see that despite the clear modulation of the flame length, the flame brush does not seem to increase significantly as one moves downstream from the nozzle exit. 


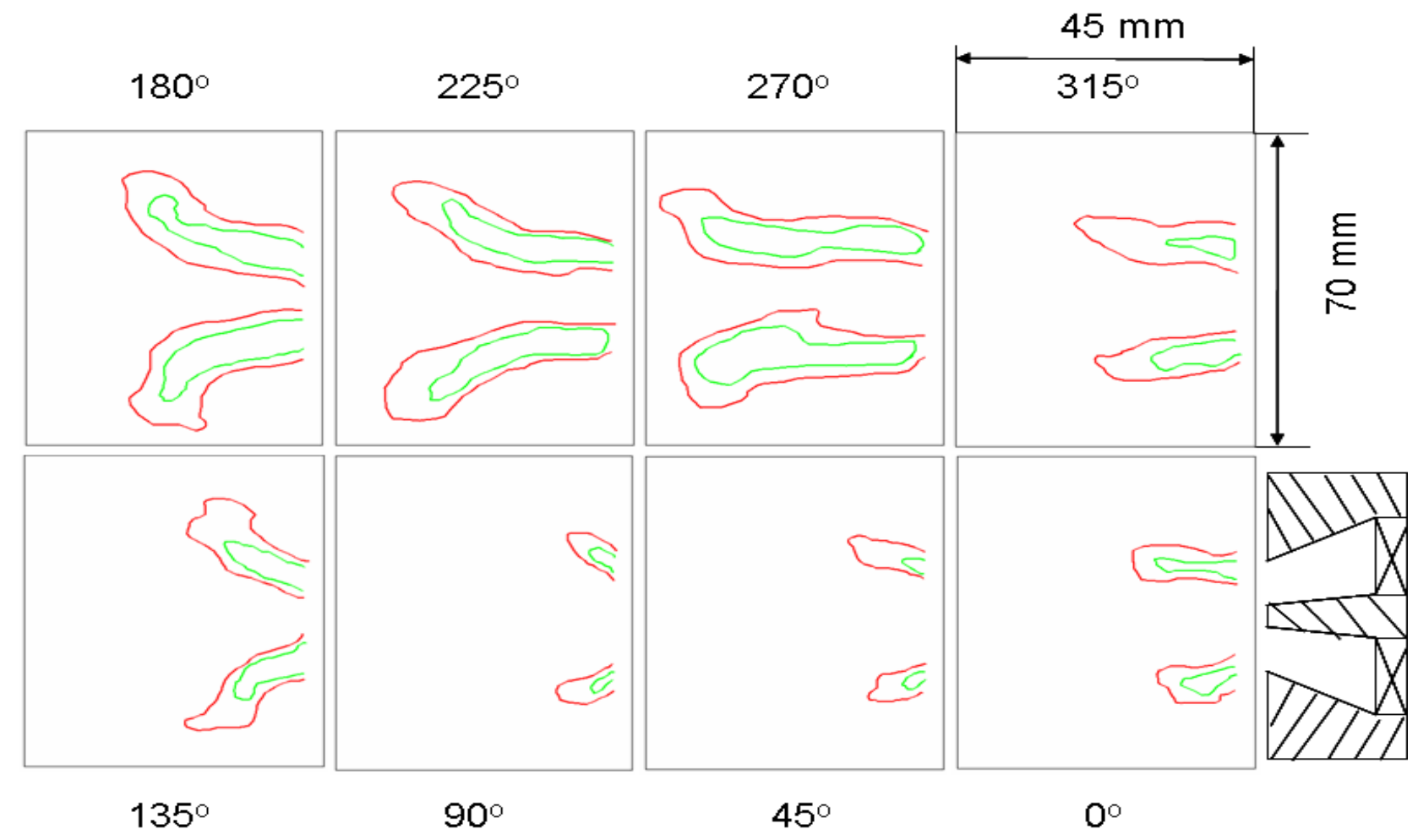

Figure 117: Contour plots of the progress variables $\bar{c}=\mathbf{0 . 3}$ (green line) and $\bar{c}=\mathbf{0 . 7}$ (red line) showing the development of the flame brush at $R e=21,000, f_{o}=130 \mathrm{~Hz}$ and $u^{\prime} / u_{o}=$ 0.6.

Quantitative plots showing the variation of $\delta_{\mathrm{t}}$ for the swirling flame are shown in Figure 118. Only the regions close to the center body prior to the merging of the inner and outer annular $\bar{c}=$ 0.3 contours are shown. From Figure 118, we observe that, the flame brush thickness for all the four phases rises very slowly relative to the forced case. Possible explanations for this could include, phase jitter, non-quasi steady effects and flame stretch effects. Since the flow field of a swirl flame is extremely complex, physical insights are drawn from detailed measurements involving a turbulent Bunsen flame. 


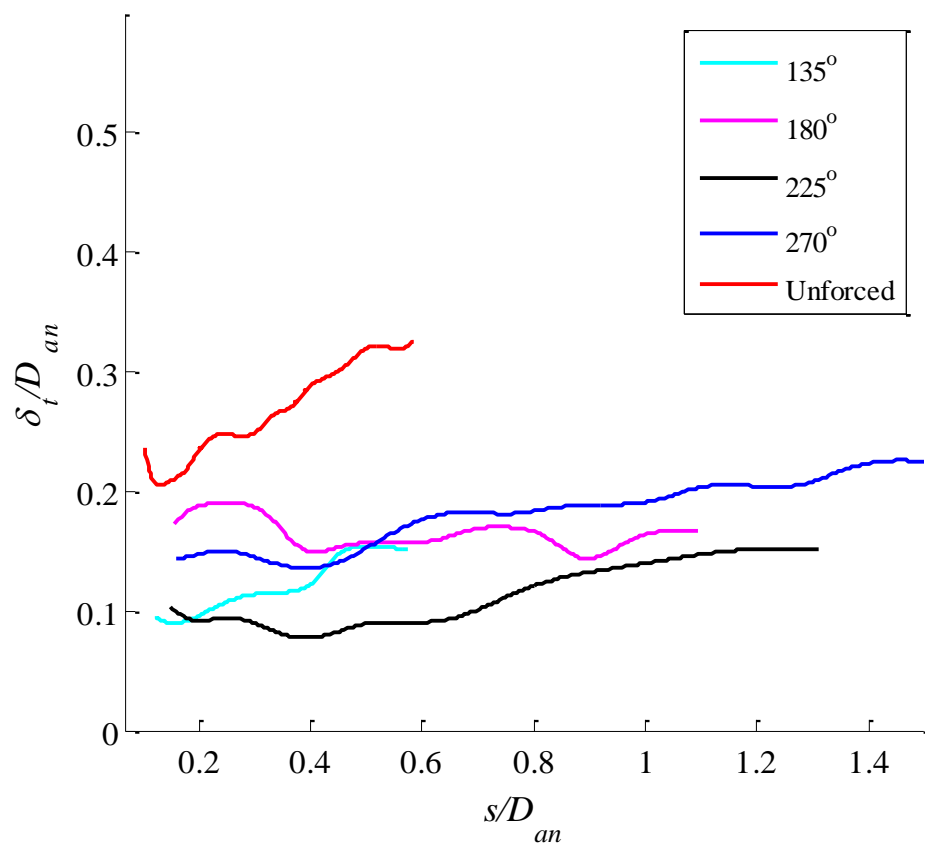

Figure 118: Variation of the normalized flame brush thickness $\left(\delta_{t} / D_{a n}\right)$ along the normalized flame coordinate $\left(s / D_{a n}\right)$ at $R e=21,000, f_{o}=130 \mathrm{~Hz}$ and $u^{\prime} / u_{o}=0.6$.

\section{Bunsen Flame}

The results for the Bunsen flame reported in this subsection were obtained at a Reynolds number of 10,200 (based on the burner exit diameter and mean flow velocity of $u_{o} \sim=10 \mathrm{~m} / \mathrm{s}$ ). The turbulent spectrum at the centerline of the jet just downstream of the burner is shown in Figure 119. A line of slope $-5 / 3$ is also shown for reference. In the unforced case, the centerline turbulent fluctuations were of the order of $u^{\prime} t / u_{o}=0.05$. Forced excitation cases were obtained at $f_{o}=200 \mathrm{~Hz}$ and at the disturbance amplitude of $u^{\prime}{ }_{A} / u_{o}=0.15$. 


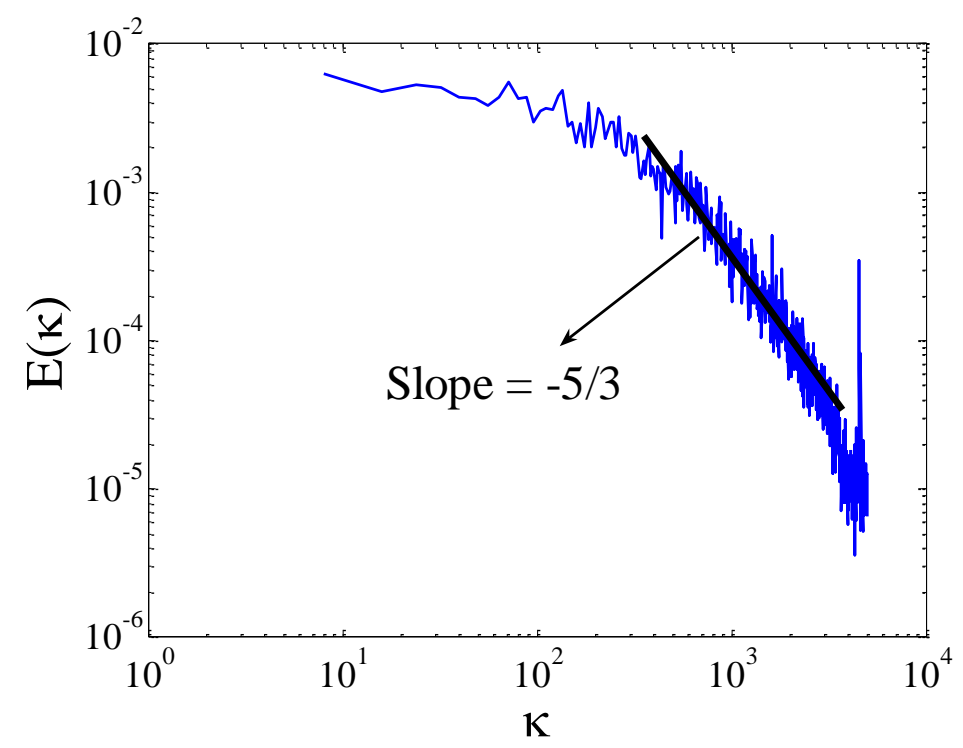

Figure 119: Centerline turbulence kinetic energy spectrum for Bunsen flame.

Figure 120 overlays a number of instantaneous images of the flame edge, showing the development of the flame brush for the unforced case. The clear growth of the flame brush can be seen with increasing distance in the downstream direction from the flame holder, an observation consistent with prior studies.

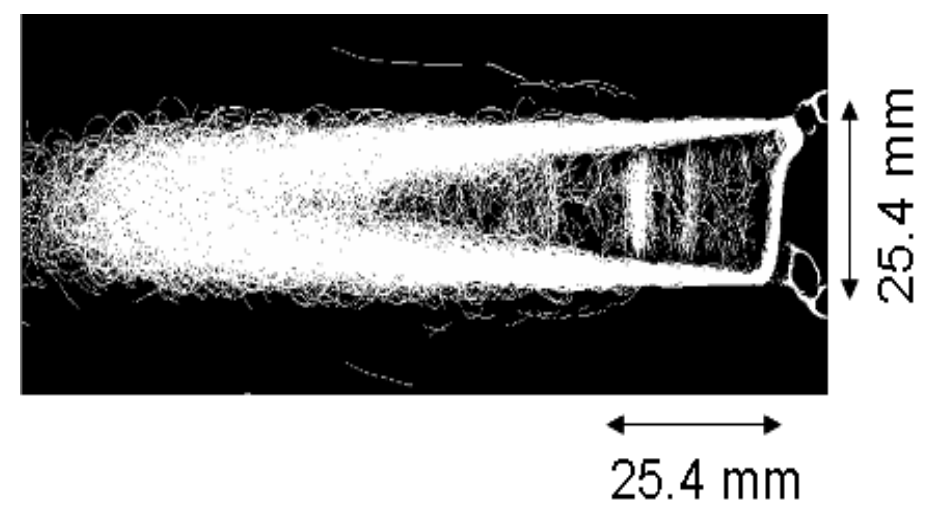

Figure 120: Overlay of several extracted flame edges showing flame brush development for the unforced case.

The mean velocity field for the unforced and forced case is shown in Figure 121 (a) and (b), respectively. The effect of acoustic forcing is evident in this latter figure. The regions of high velocity near the center of the jet, at the locations of $x=0.02 \mathrm{~m}$ and at $0.07 \mathrm{~m}$ indicates the spatial distribution of the coherent velocity field due to the acoustic forcing. 
The instantaneous flame edges obtained from the Mie-scattering images were used to visualize the flame brush. Figure 122 overlays a number of instantaneous phase locked flame edges for 8 phases of an acoustic cycle. From these images, the flame brush thickness $\left(\delta_{t}\right)$ can be seen to exhibit a step-like behavior, i.e. growing very slowly for a certain distance before increasing rapidly across the rollup region, and then increasing slowly again. For example, in Figure 122 at the phase of $0^{\circ}$ and $315^{\circ}$, it can be observed that close to the base of the burner, the flame brush thickness increases rapidly at the location of the first rollup. Downstream of this rollup region, $\delta_{\mathrm{t}}$ increases quite slowly before increasing again at the location of the next rollup. The evolution of the flame brush at the other phases is also shown in Figure 122, in which the progress variable contours indicating the edges of the flame brush are shown. These results illustrate that $\delta_{\mathrm{t}}$ grows very slowly up to the location of the convecting vortex, after which it rises rapidly and then grows slowly again. This behavior is significantly different from the unforced case, where the flame brush thickness increases monotonically, and at a much faster rate, along the flame coordinate.

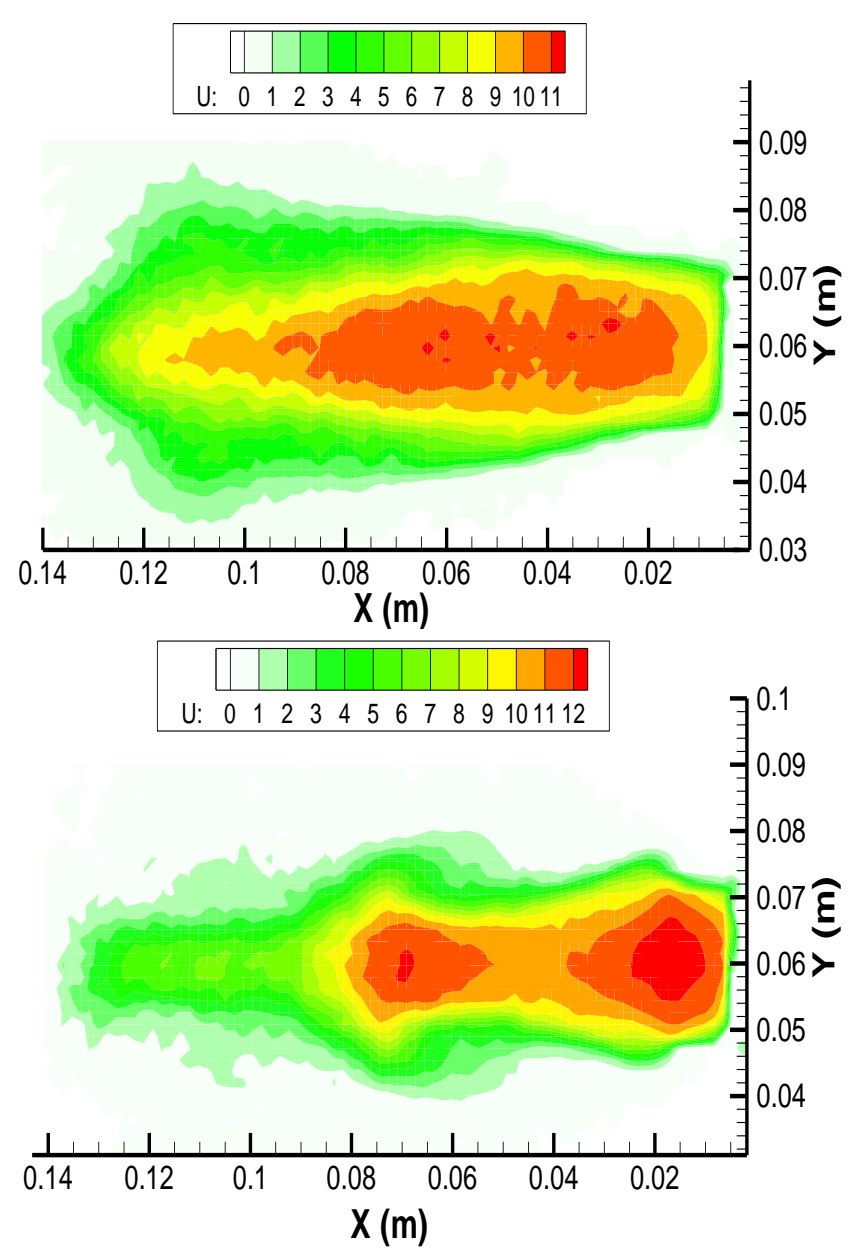

(a)

(b)

Figure 121: Mean axial velocity contours for (a) the unforced case and (b) forced case at $u^{\prime} / u_{o}=0.2$ and phase angle of $90^{\circ}$. 

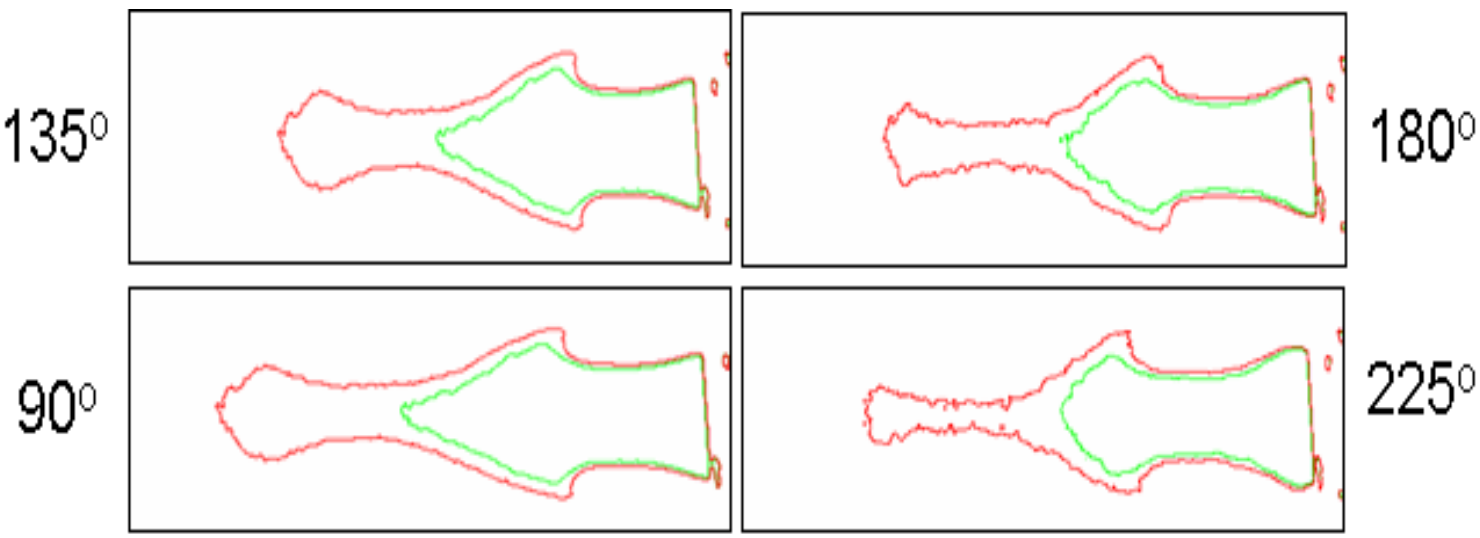

$225^{\circ}$
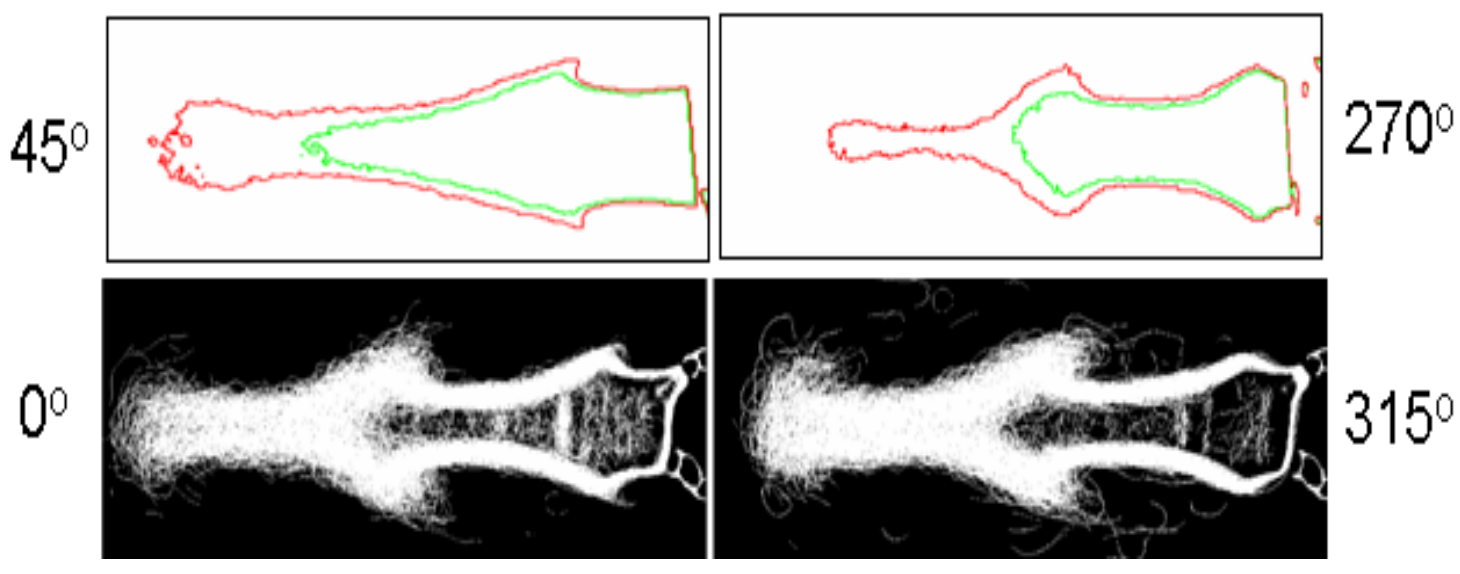

Figure 122: Sample ensemble averaged images and progress variable contours of $\bar{c}=0.3$ (green line) and $\bar{c}=\mathbf{0 . 7}$ (red line) illustrating the development of the flame brush at $R e=$ $10,200, f_{o}=200 \mathrm{~Hz}$ and $u^{\prime} / u_{o}=0.15$.

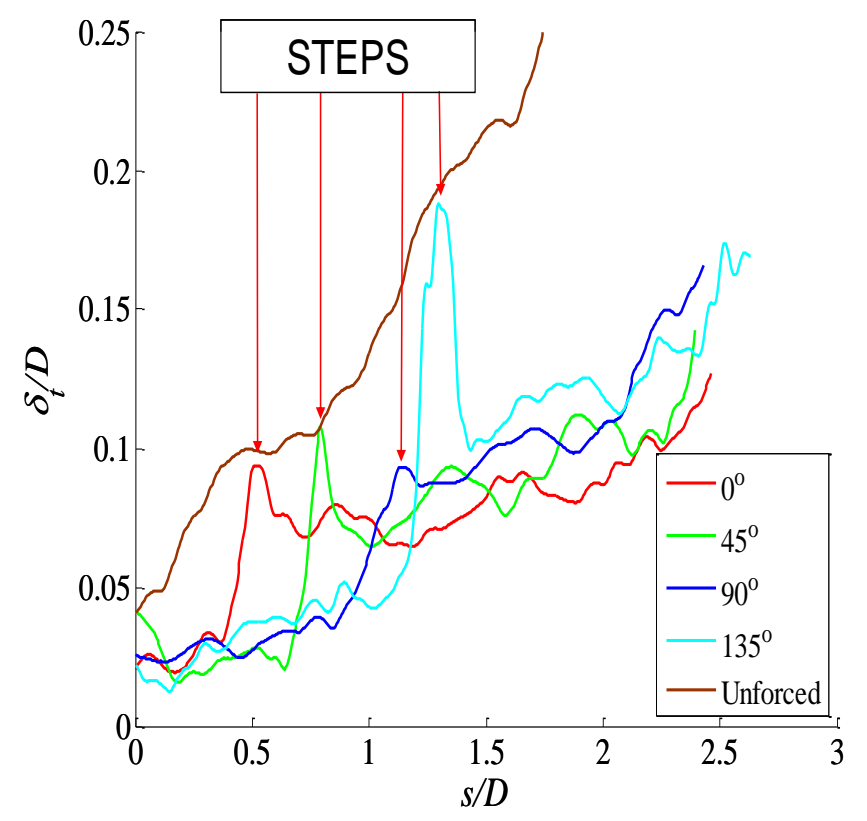

Figure 123: Variation of the normalized flame brush thickness $\left(\delta_{t} / D\right)$ along the normalized flame coordinate $(s / D)$. 
The spatial evolution of $\delta_{\mathrm{t}}$ for the unforced case and for the forced case at several phases is plotted in Figure 123. It can be seen that $\delta_{\mathrm{t}}$ increases monotonically in the downstream direction for the unforced case, as discussed earlier. However, in the presence of acoustic oscillations, $\delta_{\mathrm{t}}$ exhibits the step like behavior explained above. For example, consider the variation of the $\delta_{\mathrm{t}}$ at the phase of $45^{\circ}$ in Figure 123. The flame brush is initially a constant before rising rapidly at the flame coordinate $s / D=0.75$. After rising rapidly, $\delta_{\mathrm{t}}$ remains nearly constant. Similar behavior can also be seen at $135^{\circ}$, in which, the flame brush thickness jumps at the flame coordinate $s / D \sim 1.1$ and then again at $s / D \sim 2.5$ and remaining almost constant in between.

This shows that in the presence of acoustic excitation, $\delta_{\mathrm{t}}$ does not evolve in the same manner as in the unforced case. This indicates that $\delta_{\mathrm{t}}$ for the forced cases evolves in a non-quasi steady manner. The spatial convection of these "jumps" in $\delta_{\mathrm{t}}$ can also be visualized from Figure 123 as, for example from the phase of $0^{\circ}$ to the phase of $135^{\circ}$, the location of the occurrence of the first step increases in the increasing flame coordinate direction.

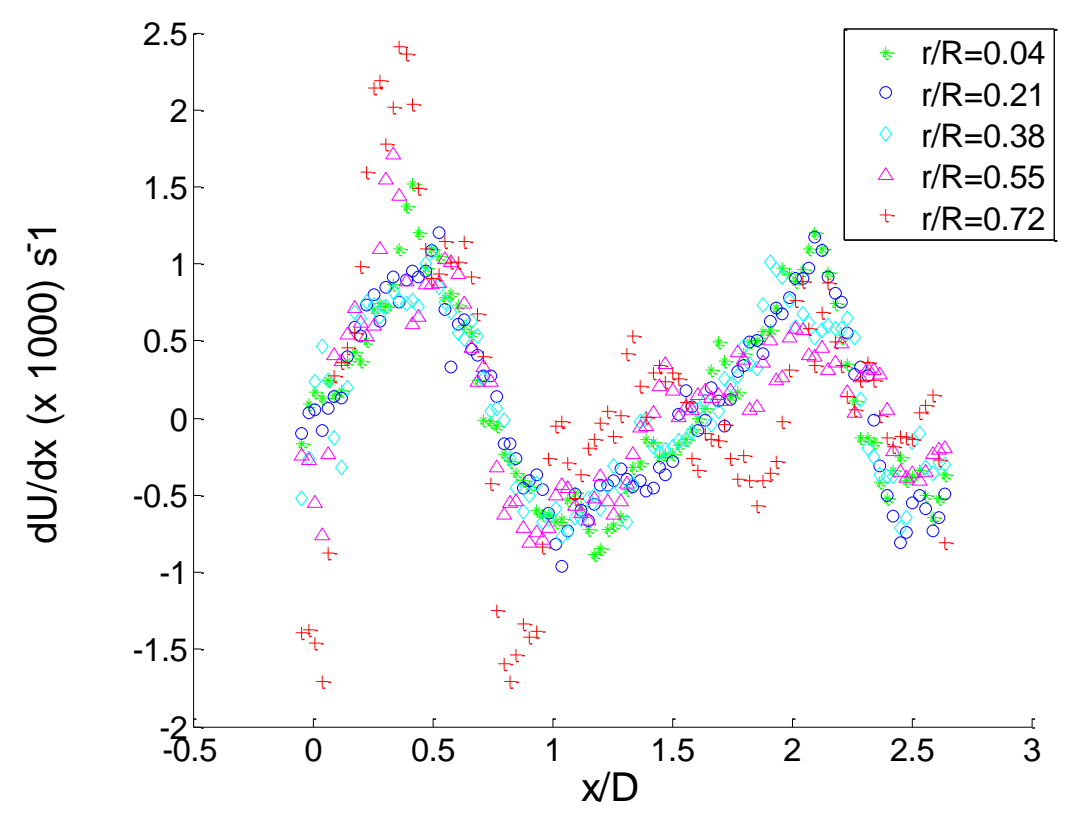

Figure 124: Variation of axial velocity gradients at different radial locations.

Next, in order to gain an insight into the physics of this behavior, high resolution PIV images were captured focusing only on a small region of the flame $(70 \mathrm{~mm})$ - close to the burner exit. The variation in the axial velocity with downstream distance is plotted in Figure 124 for different radial locations. From this Figure it may be observed that there is very little variation in the radial distance indicating that the velocities (and strain rates) at the center of the burner are similar to those close to the flame. This is an important result as it indicates that the strain rates that might influence the flame are similar in magnitude as those at the center of the burner. Since there exists a velocity jump at the location of the flame, if the velocity field is not conditioned (into reactants and products), there might exist biases close to the location of the flame. To tackle this problem, the velocities along the burner center are used instead (since they are similar in magnitude to those close to the flame) in all further analysis. 
Figure 125 plots the variation of the flame brush, the gradient in the flame brush along with the axial velocities and the gradients in the axial velocities. From this figure, it can be seen that the flame brush thickness grows in the regions of positive axial gradients, while it remains constant in the regions of negative axial gradients. The reasons behind this could be that either the effect could be because of increased kinematic restoration (in regions of positive strain rates) and hence flame speed along positive regions of the velocity gradients. Other reasons could be that it could be a non-quasi steady effect (i.e. a time delay between the growth of the flame brush thickness and the velocity gradients) or it could also be caused by phase jitter: caused by random variations in the location of the vortex which could affect the flame brush thickness in return. The first reason is examined in this technical report while the other two are currently being examined.

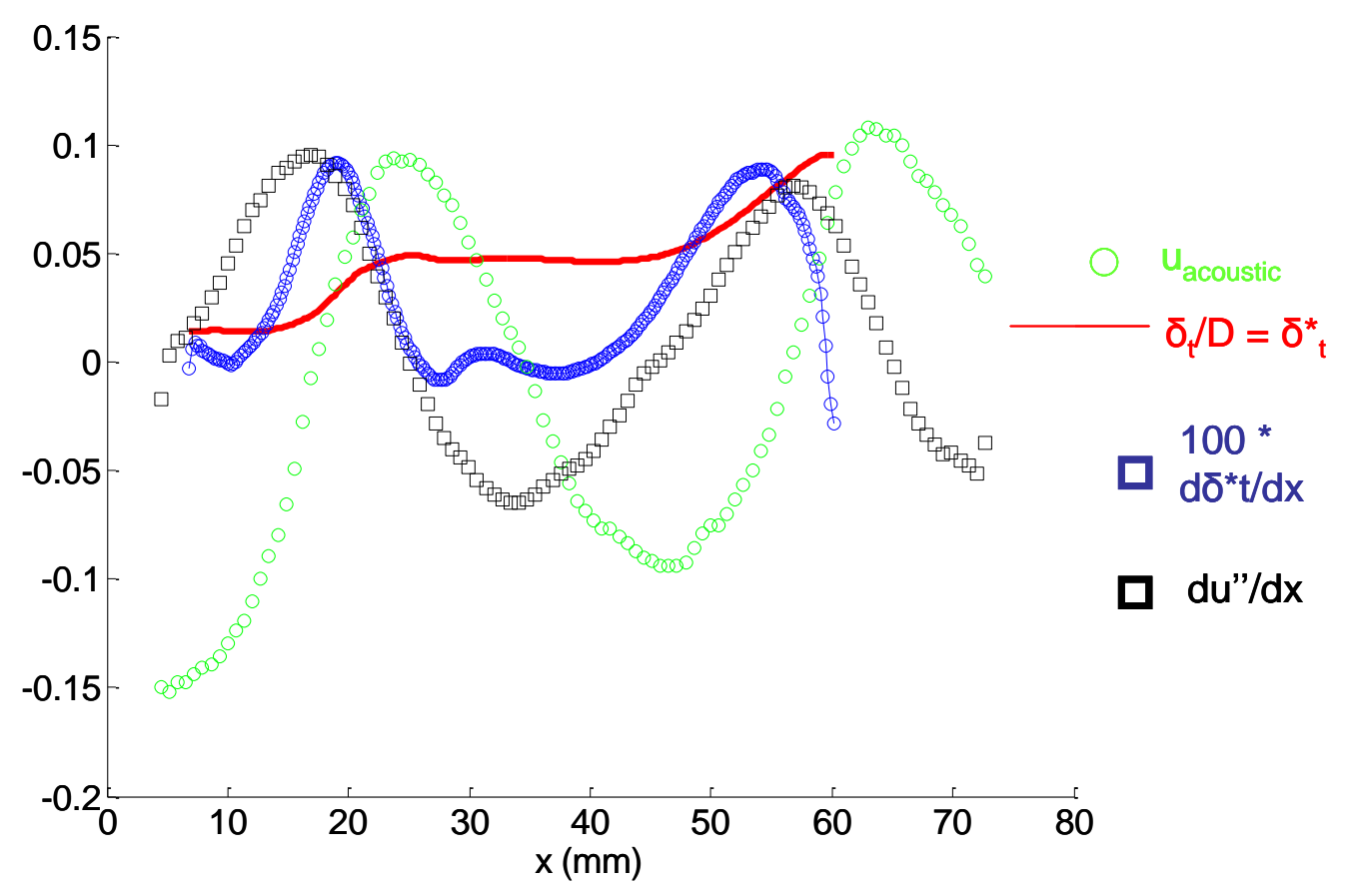

Figure 125: Variation of flame brush thickness, acoustic velocity and their gradients along axial distance for lean methane. 


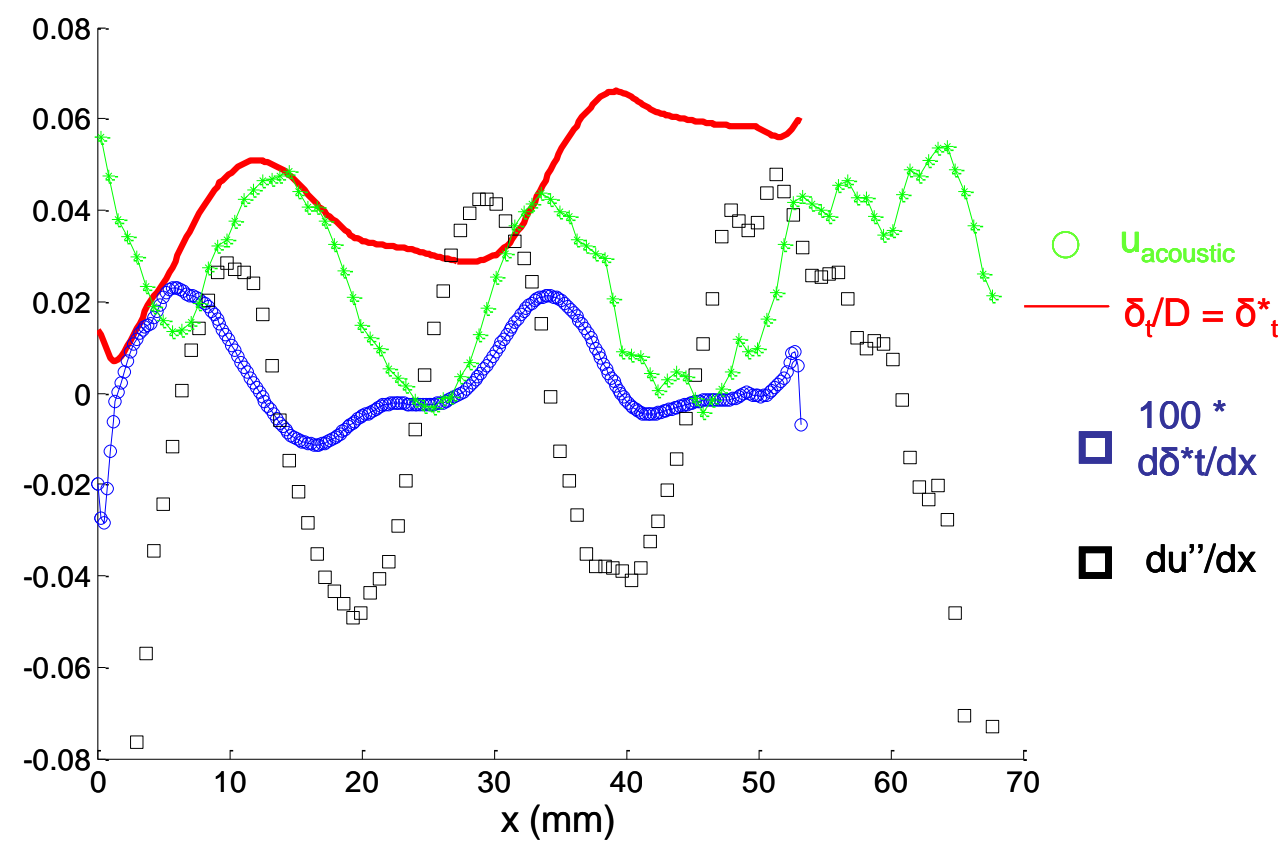

Figure 126: Variation of flame brush thickness, acoustic velocity and their gradients along axial distance for lean propane.

Figure 126 shows the variation of the flame brush thickness, axial velocity and the gradients in the flame brush thickness and velocity with axial distance for the case of lean propane. Lean propane was chosen because of the fact that it has a markstein length opposite to that of lean methane - hence the effect of stretch on the flame speed is expected to be opposite. So if strain rates were to be the dominant factor in controlling the growth of the flame brush thickness, then one would expect opposite trends to that of lean methane. Now from Figure 126, it can be seen that a similar trends as those of lean methane are seen. This seems to suggest that the strain rates and hence kinematic restoration might not be the dominating factor in curbing the growth of the flame brush thickness and that phase jitter might be the dominating effect.

\section{Acoustic Forcing of Lean-Premixed, Swirl-Stabilized, Turbulent Flames}

\section{Introduction}

During this sponsorship, the linear and nonlinear dynamics of lean-premixed, swirl-stabilized, turbulent premixed flames under acoustically forced oscillations were characterized. This analysis shows that the flame dynamics are characterized by several regimes: the linear (L), transition (TR), and first (NL1) and second (NL2) nonlinear regimes, depending upon steadystate flame configuration, modulation frequency, and amplitude of excitation. Phase-resolved $\mathrm{CH}^{*}$ chemiluminescence images of acoustically excited swirl flames show that in the linear regime the flame area modulation is determined by an oscillating flow field in the annular jet region, and the reaction zone is located in the inner shear layer. In the transition regime, a coherent large-scale vortex structure starts to interact with the flame periodically, but the flame 
structures at each phase are similar to those of the linear regime. The first and second nonlinear regimes are characterized by shear layer dynamics and unsteady flame liftoff, respectively. The present study compares these characteristics of acoustically forced flame dynamics with those of self-sustained flame dynamics. Results show that when the limit-cycle oscillation amplitude is relatively weak, i.e., $\mathrm{p}_{\mathrm{c}}{ }^{\prime} / \mathrm{p}_{\mathrm{c}}$, mean $\leq 0.05$, the Kelvin-Helmholtz instability (shear layer dynamics) controls the self-sustained instability. In the case of high instability intensity, i.e., $\mathrm{p}_{\mathrm{c}}{ }^{\prime} / \mathrm{p}_{\mathrm{c}}$, mean $\geq$ 0.10 , however, the unsteady flame liftoff governs the self-excited instability, and the reaction region penetrates into the inner recirculation zone.

\section{"V" Flame Response}

All tests were performed at a mean pressure of 1 atm and at mean equivalence ratio of 0.60 . Mean velocity at the nozzle was $60 \mathrm{~m} / \mathrm{s}$ and the inlet temperature was kept constant at $200{ }^{\circ} \mathrm{C}$, giving a Reynolds number of approximately 33,000. Forcing frequencies were varied from 100 to $400 \mathrm{~Hz}$. The range of forcing frequency was determined based on the two-microphone method calibration results. Also, the frequency range of $100 \sim 400 \mathrm{~Hz}$ includes self-induced instability frequencies observed in the investigated lean premixed gas turbine combustor. $\mathrm{H}_{2}$-blended natural gas fuels with $\mathrm{X}_{\mathrm{H} 2}=0.00$ and 0.30 were used to examine the effects of steady-state flame structure on the response of a flame. Fuel is injected and mixed upstream of the choked inlet in order to ensure that the reactant mixtures are spatially and temporally homogeneous before they enter the reaction zone. The full list of operating conditions and fuel compositions for the forced flame response measurements is listed in

Table 7.

Table 7: Test conditions for forced flame response measurements.

\begin{tabular}{cc}
\hline Parameters & Forced response tests \\
\hline pressure $(\mathrm{P})$ & $1 \mathrm{~atm}$ \\
inlet temperature $\left(\mathrm{T}_{\mathrm{in}}\right)$ & $200{ }^{\circ} \mathrm{C}$ \\
nozzle velocity $\left(\mathrm{V}_{\text {mean }}\right)$ & $60 \mathrm{~m} / \mathrm{s}$ \\
equivalence ratio $(\Phi)$ & 0.60 \\
forcing frequency $(f)$ & $100 \sim 400 \mathrm{~Hz}(\Delta f=25$ \\
forcing amplitude $\left(\mathrm{V}^{\prime} /\right.$ & $\mathrm{Hz})$ \\
$\left.\mathrm{V}_{\text {mean }}\right)$ & up to 0.60 \\
fuel composition $\left(\mathrm{X}_{\mathrm{H} 2}\right)$ & $0.00,0.30$ \\
\hline
\end{tabular}

This discussion leads us into the linear and nonlinear dynamics of inverted dihedral "V" flames. Figure 127 presents the amplitude dependence of the normalized heat release response, the gain, and phase of the flame transfer function at a modulation frequency of $200 \mathrm{~Hz}$. Inlet conditions are: $\mathrm{T}_{\text {in }}=200{ }^{\circ} \mathrm{C}, \mathrm{V}_{\text {mean }}=60 \mathrm{~m} / \mathrm{s}, \Phi=0.60$, and $\mathrm{X}_{\mathrm{H} 2}=0.00 . \mathrm{CH}^{*}$ chemiluminescence intensities are used as an indicator of heat release rate oscillations. High coherence between inlet velocity and heat release fluctuations, close to unity, was found at the forcing frequency of $200 \mathrm{~Hz}$ enabling flame transfer functions to be accurately measured. Also, a high velocity perturbation 
magnitude of up to approximately $60 \%$ can be achieved at this forcing frequency. At these inlet conditions, the flame is stabilized in the inner shear layer and it is attached to the centerbody, exhibiting an inverted dihedral "V" structure. The responses of the "V" flame to acoustic perturbations are divided into four distinct regimes: linear (L), transition (TR), and the first (NL1) and second (NL2) nonlinear regimes, as shown in Figure 127. In the linear regime, the normalized heat release response increases linearly with the forcing amplitude; thus, the gain is almost constant. When the magnitude of inlet velocity fluctuation reaches approximately $23 \%$, the normalized heat release response levels off and the gain decreases, representing typical nonlinear behavior. It is noteworthy that when the modulation amplitude is greater than $40 \%$ of the mean value, the gain is constant with respect to the forcing amplitude. Such characteristics of acoustically forced swirl-stabilized flames were also observed by Thumuluru and Lieuwen.
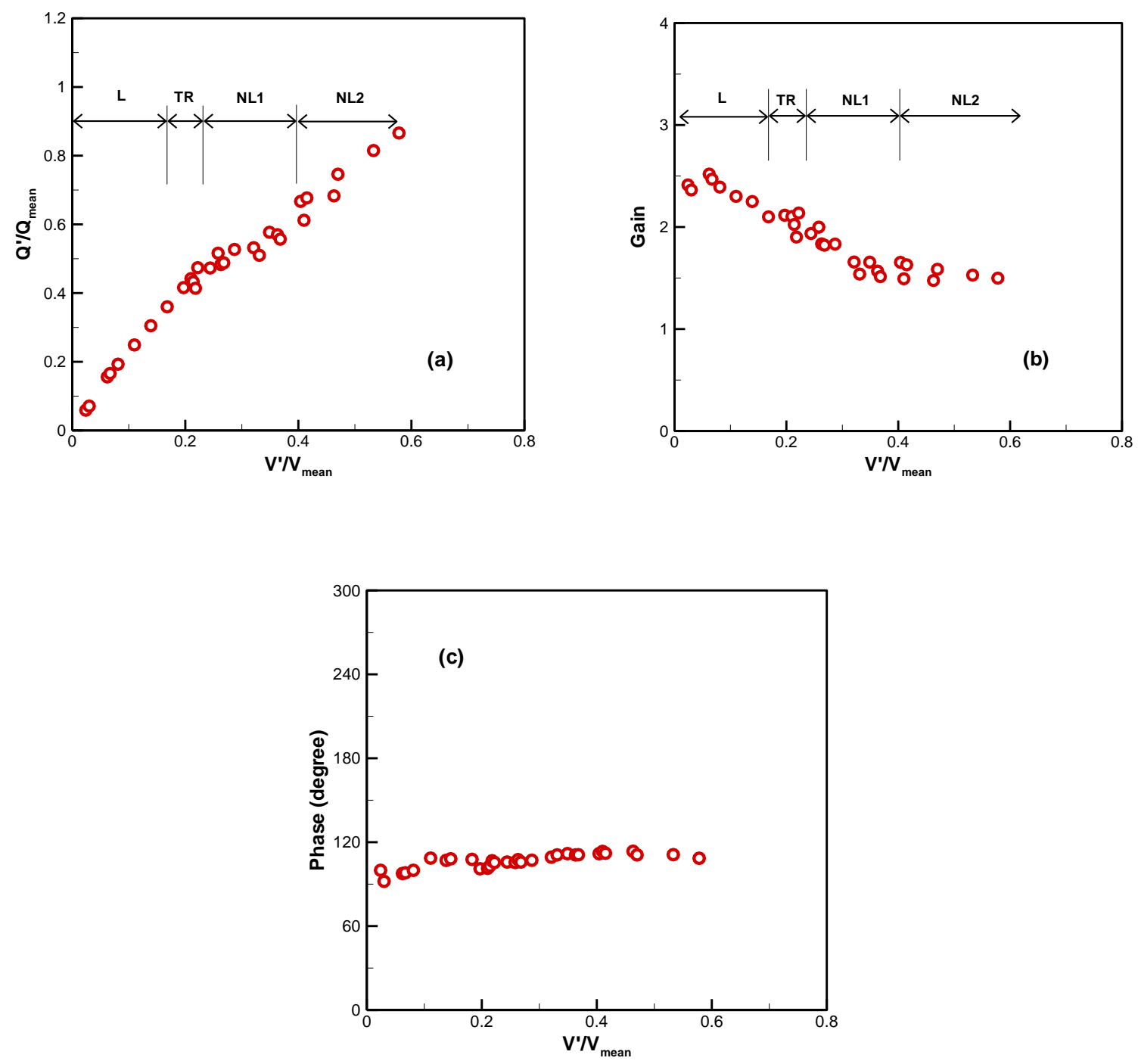

Figure 127: (a) Normalized heat release response $\left(\mathrm{CH}^{*} / \mathrm{CH}^{*}\right.$ mean), (b) gain, and (c) phase of flame transfer function as a function of the magnitude of inlet velocity perturbation. $T_{\text {in }}=$ $200{ }^{\circ} \mathrm{C}, \mathrm{V}_{\text {mean }}=60 \mathrm{~m} / \mathrm{s}, \Phi=0.60, \mathrm{X}_{\mathrm{H} 2}=0.00, f=200 \mathrm{~Hz}$. 
(a)

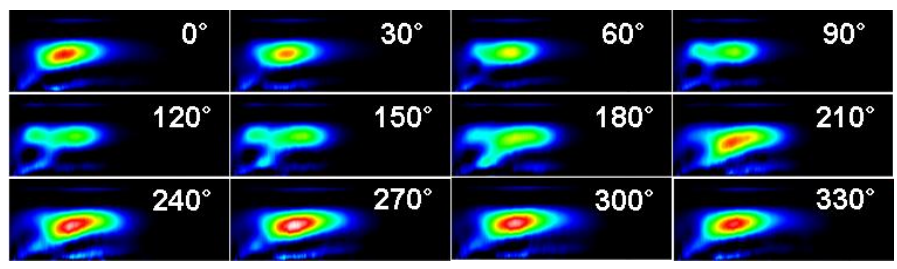

(b)

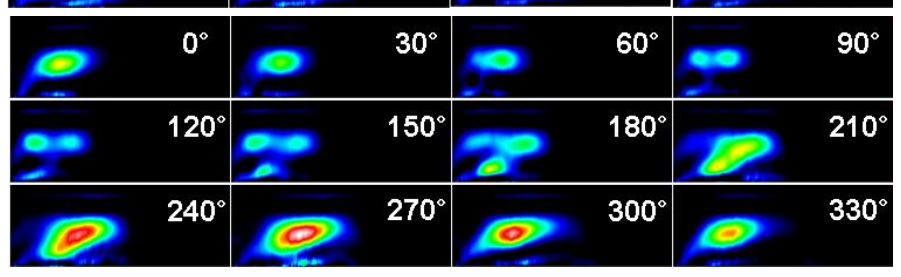

Figure 128: Phase-synchronized $\mathrm{CH}^{*}$ chemiluminescence imaging at modulation frequency of $200 \mathrm{~Hz}$ : (a) $V^{\prime} / V_{\text {mean }}=9 \%$ for linear regime (L) and (b) $V^{\prime} / V_{\text {mean }}=23 \%$ for transition regime (TR).

Phase-resolved flame imaging measurements in each regime will elucidate the amplitudedependent dynamic characteristics of acoustically forced swirl flames. Figure 127(c) shows that the phase measured between the heat release signal and the inlet velocity fluctuation is essentially independent of the modulation amplitude of up to $\mathrm{V}^{\prime} / \mathrm{V}_{\text {mean }}=0.60$. This is consistent with studies on laminar premixed flames. This indicates that the frequency-dependent convection times for a velocity perturbation to reach the flame are not a function of the modulation amplitude, irrespective of linear and nonlinear regimes.

Figure 128 presents phase-synchronized $\mathrm{CH}^{*}$ chemiluminescence images at a modulation frequency of $200 \mathrm{~Hz}$ for amplitudes of (a) $\mathrm{V}^{\prime} / \mathrm{V}_{\text {mean }}=9 \%$ and (b) $23 \%$. They represent the response of the flame in the linear (L) and transition (TR) regimes, respectively. Only the upper half of these deconvoluted images is shown, because the reconstructed images are axisymmetric. The direction of flow is from left to right. It can be observed from Figure 128(a) that the flame moves back and forth due to the oscillation in the annular jet region, and the reaction zone is located in the inner shear layer. Reaction occurs in the corner recirculation zone (CRZ) at $\varphi=$ $90 \sim 180^{\circ}$. Shear layer rollup is not seen because the minimum excitation level for the formation of a large-scale coherent structure is not achieved. Figure 128(b) shows the forced flame response in transition regime. The flame shapes at each phase are similar to those of the linear flame behavior. The formation and reaction of large-scale, coherent vortex structures are, however, observed at $\varphi=180 \sim 270^{\circ}$. It is convected by the mean flow, and the heat release reaches its maximum at $\varphi=270^{\circ}$. The vortices periodically entrain a large amount of combustible material into the reaction zone at the modulation frequency, and therefore the evolution of flame surface area is determined by the interplay between the coherent structure and the flame. 


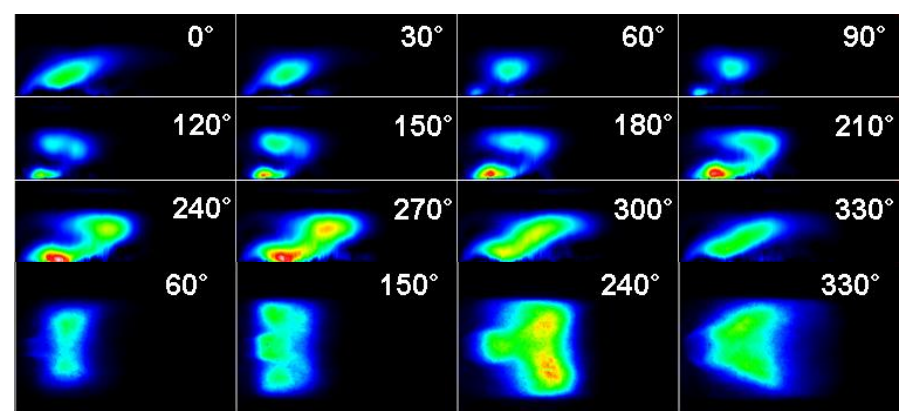

Figure 129: Phase-synchronized $\mathrm{CH}^{*}$ chemiluminescence imaging at modulation frequency of $200 \mathrm{~Hz}$ and $V^{\prime} / V_{\text {mean }}=25 \%$ for the first nonlinear regime (NL1). Bottom row shows lineof-sight integrated, background-corrected $\mathrm{CH}^{*}$ images at $\varphi=60,150,240,330^{\circ}$.

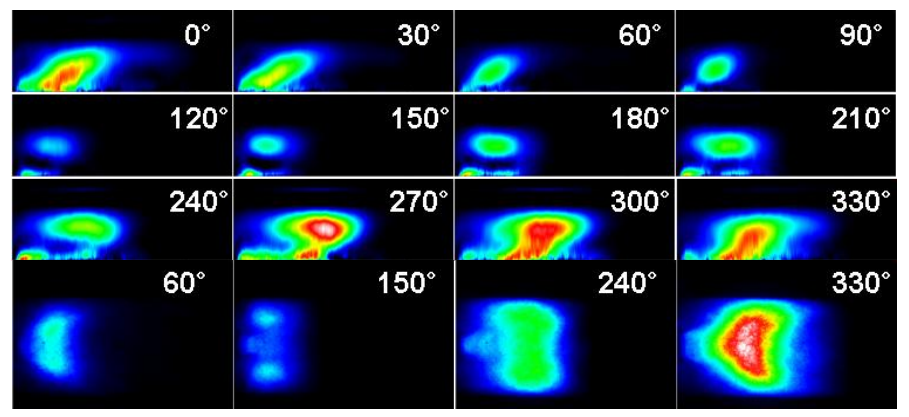

Figure 130: Phase-synchronized $\mathrm{CH}^{*}$ chemiluminescence imaging at modulation frequency of $200 \mathrm{~Hz}$ and $V^{\prime} / V_{\text {mean }}=59 \%$ for the second nonlinear regime (NL2). Bottom row shows line-of-sight integrated, background-corrected $\mathrm{CH} *$ images at $\varphi=60,150,240,330^{\circ}$.

Deconvoluted and line-of-sight integrated phase-averaged $\mathrm{CH}^{*}$ images in the first nonlinear regime (NL1) are shown in Figure 129. All input conditions are the same as for Figure 128, except for the modulation amplitude. The most distinct difference in flame behavior between the transition (TR) and the first nonlinear (NL1) regimes is that in the first nonlinear regime, the flame front bends toward the inner recirculation zone at $\varphi=270^{\circ}$, as compared to the deconvoluted flame image at $\varphi=270^{\circ}$ in Figure 128(b). The interaction between a vortex-ring structure and the flame is significant at $\varphi=180^{\circ} \sim 300^{\circ}$. It has been reported that the nonlinear flame response is related to the shear layer rollup. The shear layer rollup shortens the flame length, which in turn decreases the flame area in a nonlinear manner. Hence, the gain of FTF decreases in nonlinear regime, as shown in Figure 127.

Figure 130 shows phase-resolved flame images at a modulation amplitude of 59\%, corresponding to the second nonlinear regime (NL2). The deconvoluted and line-of-sight integrated images at $\varphi=330^{\circ}$ clearly show the unsteady flame liftoff from the attachment point, i.e., the centerbody. The high level of inlet velocity perturbation causes the flame attachment point to move off of the centerbody to the downstream inner recirculation zone. 


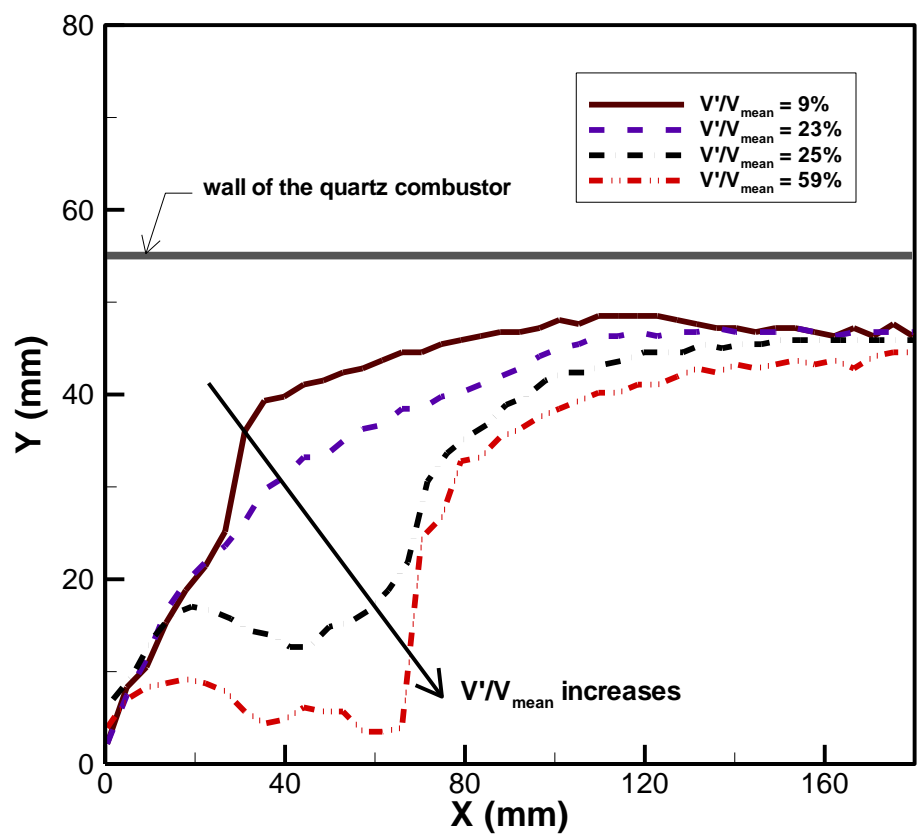

Figure 131: Coordinates of maximum $\mathrm{CH}^{*}$ intensity location at phase of $\varphi=270^{\circ}$ (maximum intensity) for inlet velocity magnitude of $V^{\prime} / V_{\text {mean }}=9,23,25$, and 59\%.

This evolution of the flame is totally different from that of the first nonlinear regime, as shown in Figure 129. This indicates that when the inlet velocity perturbation magnitude is greater than $40 \%$ of the mean value, the dynamics of swirl-stabilized premixed flames subjected to upstream acoustic forcing are not governed by the Kelvin-Helmholtz instability, but by the unsteady flame liftoff, due to high perturbation amplitude. At $\mathrm{V}^{\prime} / \mathrm{V}_{\text {mean }}>40 \%$, a vortex ring structure is shed, but its convection path is mainly determined by the annular jet. The main reaction region is located in the inner recirculation zone, however. Therefore, the forced flame response levels off when $\mathrm{V}^{\prime} / \mathrm{V}_{\text {mean }}>40 \%$, as shown in Figure 127.

Coordinates of maximum $\mathrm{CH}^{*}$ chemiluminescence intensity locations at $\varphi=270^{\circ}$ for $\mathrm{V}^{\prime} / \mathrm{V}_{\text {mean }}=$ 9, 23, 25, and 59\% are plotted in Figure 131. With increasing the modulation amplitude, the reaction region moves toward the inner recirculation zone. The amplitude-dependent dynamics of inverted "V" flames suggest that limit-cycle pressure oscillation magnitudes are governed by different mechanisms of flame area modulation under self-sustained oscillations, depending on the magnitude of inlet velocity fluctuation. The dynamics of self-sustained flames will be characterized and discussed later. 


\section{“M” Flame Response}

With regards to the forced response of "M" flames, it has been shown that the flame geometry changes from an inverted " $V$ " flame to an enveloped " $M$ " flame with increasing $\mathrm{H}_{2}$ mole fraction. The "M" flames have unique characteristics that allow them to damp flow perturbations, as compared to "V" flames. Thus, the flame transfer function gain of " $\mathrm{M}$ " flames is much smaller than that of "V" flames for a given forcing frequency and amplitude. Schuller et al. reported that a "V" flame behaves as an amplifier in a certain range of frequencies and the gain of laminar "V" flames is greater than that of conical and "M" flames.

(a)

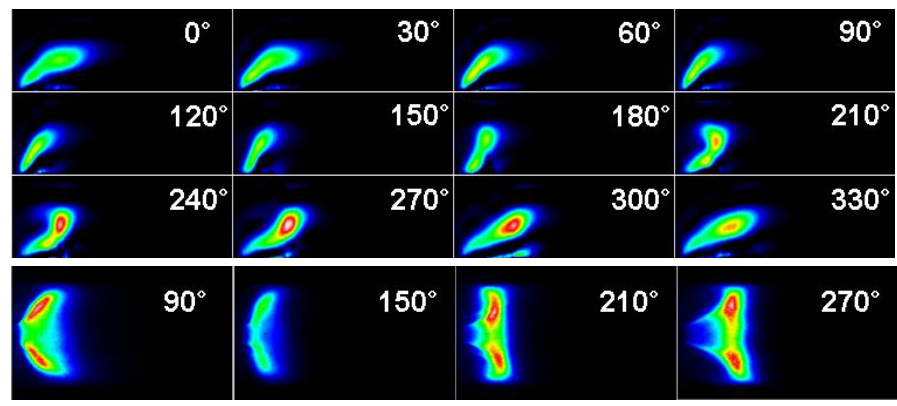

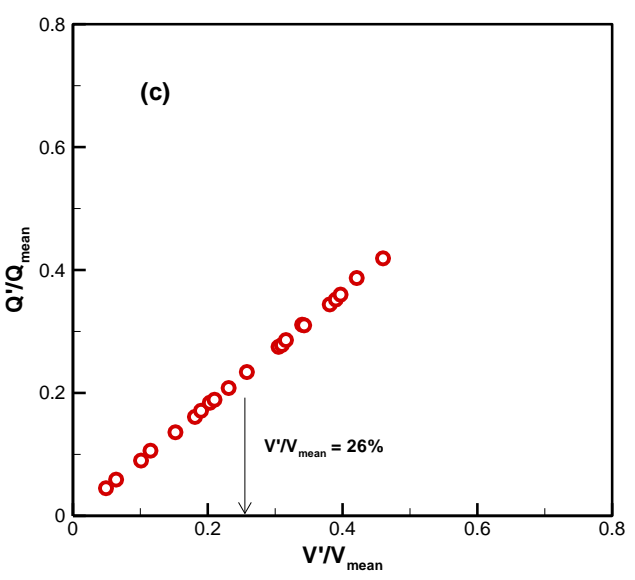

(c)

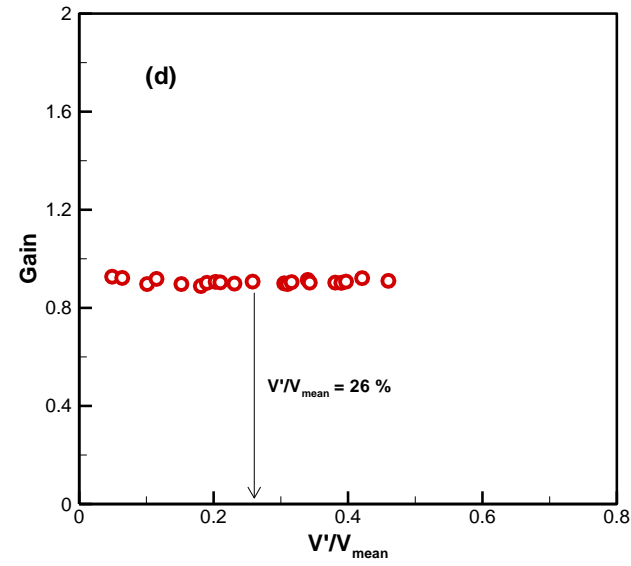

(d)

Figure 132: (a) Phase-synchronized $\mathrm{CH}^{*}$ chemiluminescence imaging at $f=200 \mathrm{~Hz}$ and $V^{\prime} / V_{\text {mean }}=26 \%$, (b) line-of-sight integrated $\mathrm{CH}^{*}$ images at $\varphi=90^{\circ}, 150^{\circ}, 210^{\circ}$, and $270^{\circ}$, (c) normalized heat release response, and (d) gain of flame transfer function as a function of modulation amplitude. Operating conditions: $T_{\text {in }}=200{ }^{\circ} \mathrm{C}, V_{\text {mean }}=60 \mathrm{~m} / \mathrm{s}, \Phi=0.60$, and $\mathrm{X}_{\mathrm{H} 2}=\mathbf{0 . 3 0}$.

Figure 132 shows twelve successive phases $\left(\mathrm{CH}^{*}\right)$ during a cycle at a modulation frequency of $200 \mathrm{~Hz}$ and amplitude of $\mathrm{V}^{\prime} / \mathrm{V}_{\text {mean }}=26 \%$. Line-of-sight integrated $\mathrm{CH}^{*}$ chemiluminescence images at $\varphi=90^{\circ}, 150^{\circ}, 210^{\circ}$, and $270^{\circ}$ are also included $\left(\mathrm{T}_{\text {in }}=200{ }^{\circ} \mathrm{C}, \mathrm{V}_{\text {mean }}=60 \mathrm{~m} / \mathrm{s}, \Phi=\right.$ $\left.0.60, \mathrm{X}_{\mathrm{H} 2}=0.30\right)$. With $\mathrm{H}_{2}$-enrichment $\left(\mathrm{X}_{\mathrm{H} 2}=0.30\right)$, the flame is very compact in both axial and radial directions and the steady-state flame structure exhibits an " $\mathrm{M}$ " geometry. The flame is 
attached to the combustor dump plane as well as the centerbody. The local flame geometry is similar to a conical flame. Figure 132 (c) and (d) plots the dependence of the normalized heat release response and the gain of flame transfer function upon the modulation amplitude at a modulation frequency of $200 \mathrm{~Hz}$. It is evident from Figure 132(a) that the flame angle change is substantial during a modulation cycle. In the case of "V" flames, however, the flame oscillates in the flow direction, which means that the flame length changes over a period of perturbations, as shown in Figure 128 and Figure 129.

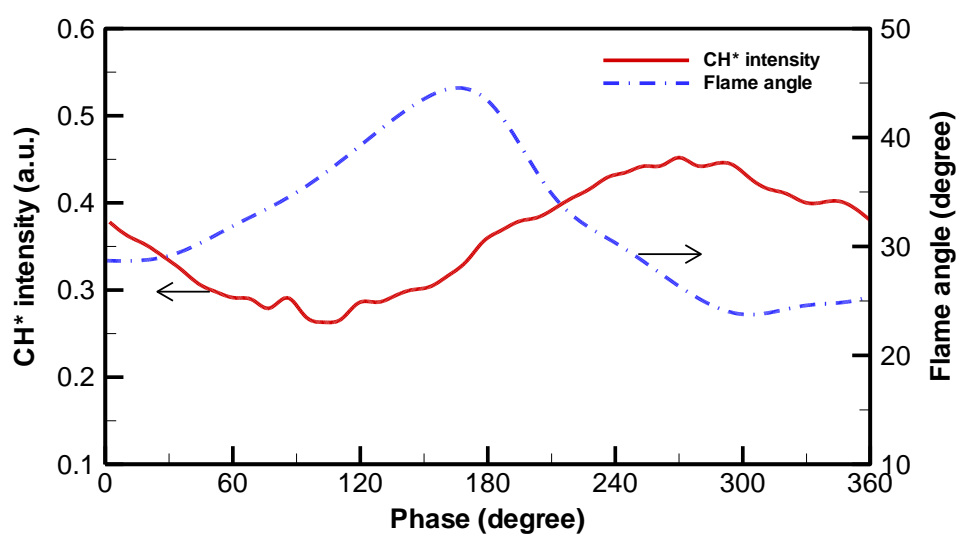

Figure 133: $\mathrm{CH}^{*}$ chemiluminescence intensity and flame angle variation over one period of oscillation. Operating conditions: $\mathrm{T}_{\text {in }}=200{ }^{\circ} \mathrm{C}, \mathrm{V}_{\text {mean }}=60 \mathrm{~m} / \mathrm{s}, \Phi=0.60, \mathrm{X}_{\mathrm{H} 2}=0.30 f=200$ $\mathrm{Hz}$, and $\mathrm{V}^{\prime} / \mathrm{V}_{\text {mean }}=26 \%$.

The flame angle and $\mathrm{CH}^{*}$ chemiluminescence intensity variations over one period of oscillation are shown in Figure 133. These oscillations in the flame position (angle) result from fluctuations in the outer shear layer. It has been reported that if the shear layers in the swirl-stabilized gas turbine combustor are acoustically excited to form a coherent vortex ring structure, the inner shear layer rolls up inward and the outer shear layer rolls up outward. This result suggests that nonlinear flame dynamics of " $\mathrm{M}$ " flames is totally different from that of "V" flames. Note that even though periodic formation and convection of vortex structures occurs at $\varphi=210 \sim 270^{\circ}$, the flame response remains in the linear regime, as shown in Figure 132(c) and (d). The formation and convection of large scale structures are determined by fluid dynamic conditions, such as inlet velocity, forcing frequency, and amplitude. As already shown in Figure 127(b) and Figure 129, at the same fluid dynamic conditions the inverted "V" flame with long flame length exhibits strong interaction with a large scale structure, leading to highly nonlinear behavior. In contrast, an " $\mathrm{M}$ " flame is less influenced by the Kelvin-Helmholtz instability, since the relative ratio of convective time to the acoustic forcing period is very small.

Figure 134 shows the normalized heat release response and phase of the flame transfer function as a function of forcing amplitude for a $\mathrm{H}_{2}$ mole fraction of $\mathrm{X}_{\mathrm{H} 2}=0.30$. Unlike the cases without $\mathrm{H}_{2}$-enrichment at the same inlet flow condition, the flame tends to remain in the linear regime even at high forcing frequency $(f=350 \mathrm{~Hz})$ and amplitude $\left(\mathrm{V}^{\prime} / \mathrm{V}_{\text {mean }}=0.211\right)$. This result is consistent with data from theoretical studies. We previously reported that the response of the 
conical flame is nearly linear over the entire velocity disturbance amplitude while the "V" flames exhibit substantial nonlinearity, which is manifested as saturation. This evidence strongly suggests that " $\mathrm{M}$ " flames are more stable than inverted "V" flames. The modification of the steady-state flame configuration from a "V" to an " $\mathrm{M}$ " geometry by $\mathrm{H}_{2}$-enrichment was found to reduce the limit-cycle pressure oscillation amplitude significantly. It is interesting to note that in contrast with " $\mathrm{V}$ " flames the normalized heat release response of " $\mathrm{M}$ " flames is insensitive to the forcing frequencies $(f=100-300 \mathrm{~Hz})$, as shown in Figure 134. The response starts to decrease when $f \geq 350 \mathrm{~Hz}$.
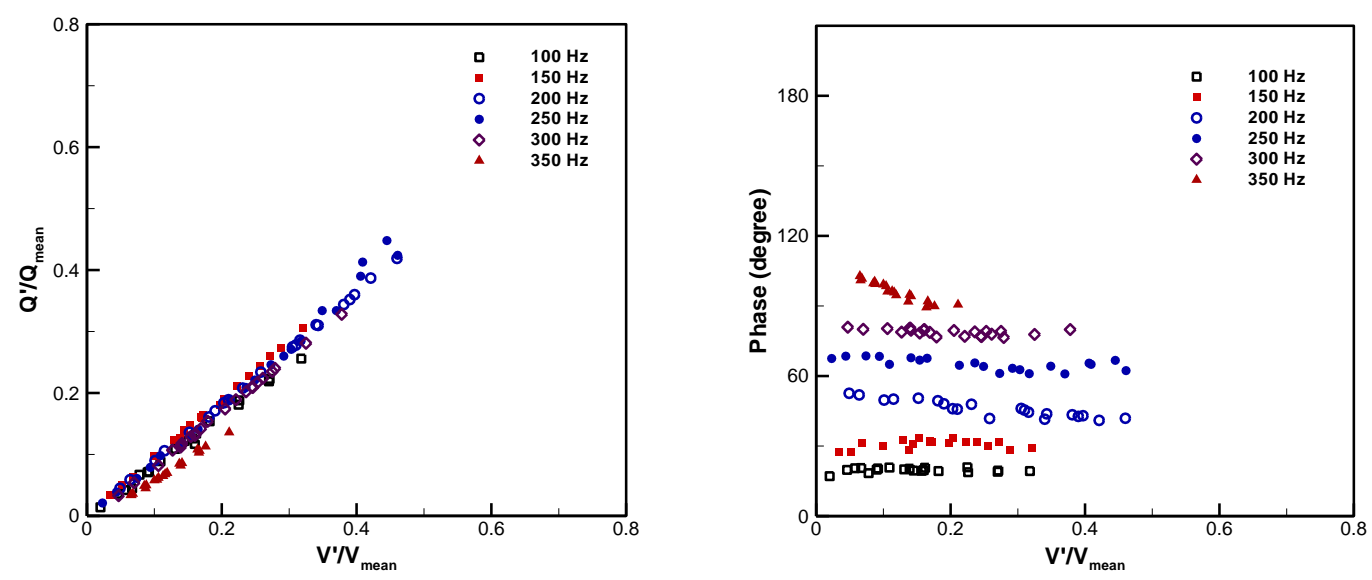

Figure 134: Normalized heat release response and phase of flame transfer function as a function of forcing amplitude. Operating conditions: $T_{\text {in }}=200{ }^{\circ} \mathrm{C}, \mathrm{V}_{\text {mean }}=60 \mathrm{~m} / \mathrm{s}, \Phi=0.60$, and $\mathrm{X}_{\mathrm{H} 2}=\mathbf{0 . 3 0}$.

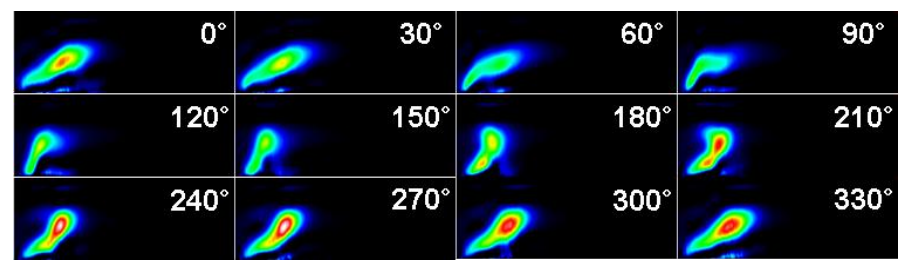

Figure 135: Phase-synchronized $\mathrm{CH}^{*}$ chemiluminescence imaging at $f=300 \mathrm{~Hz}$ and $V^{\prime} / V_{\text {mean }}=31 \%$. Operating conditions: $T_{\text {in }}=200{ }^{\circ} \mathrm{C}, V_{\text {mean }}=60 \mathrm{~m} / \mathrm{s}, \Phi=0.60$, and $X_{\mathrm{H} 2}=$ 0.30.

Figure 135 shows phase-resolved $\mathrm{CH}^{*}$ chemiluminescence images at a forcing frequency of 300 $\mathrm{Hz}$ and amplitude of $31 \%$ at the same inlet conditions as those shown in Figure 132. It can be observed from Figure 132 and Figure 135 that the dynamics of forced flames appear very similar, regardless of the forcing frequencies and amplitudes. The reason is that the response of the flames is linear in both cases and the forced response is independent of forcing frequencies. Shear layer rollup does not play a role in inducing nonlinearity. The phase is independent of the forcing amplitude and it evolves quasi-linearly with the forcing frequency, as shown in Figure 134. 
Figure 136 shows the flame transfer function gain and phase for a constant forcing amplitude of $\mathrm{V}^{\prime} / \mathrm{V}_{\text {mean }}=10 \%$. At the investigated operating conditions, the forced flame response is linear below the level of acoustic velocity perturbation of $\mathrm{V}^{\prime} / \mathrm{V}_{\text {mean }}=10 \%$. The global flame response can be described as a low-pass filter. These results also show that with increasing $\mathrm{X}_{\mathrm{H} 2}$, the maximum gain of FTF decreases significantly and the phase decreases at a given forcing frequency, indicating that the gain depends strongly on flame length. For the inverted "V" flames, the gain of the flame transfer function exceeds unity at the modulation frequency of $f \leq$ $375 \mathrm{~Hz}$ and the flame amplifies flow perturbation at certain frequencies. In the case of "M" flames, however, the overshoot behavior is not observed, and the gain is almost independent of forcing frequency when the frequency is less than $300 \mathrm{~Hz}$. Furthermore, the gain is less than unity, which suggests that " $\mathrm{M}$ " flames damp flow perturbations. At high forcing frequencies, the gain asymptotically approaches zero. The phase difference of " $\mathrm{V}$ " flames is greater than that of "M" flames, due to the fact that the convection time for velocity disturbances to reach the flame increases with increasing the flame length.
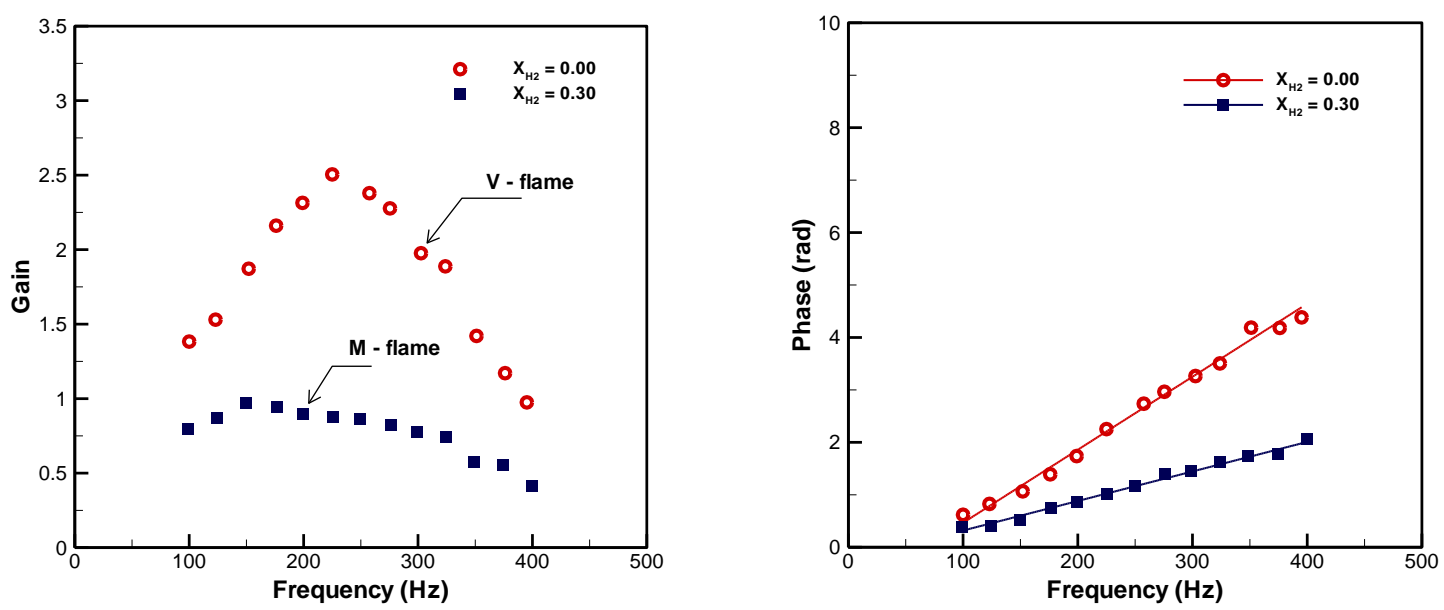

Figure 136: Gain and phase difference of FTF vs. forcing frequency at a constant forcing amplitude, $V^{\prime} / V_{\text {mean }}=0.100$. Operating conditions: $T_{i n}=200{ }^{\circ} \mathrm{C}, V_{\text {mean }}=60 \mathrm{~m} / \mathrm{s}, \Phi=0.60$, and $\mathrm{X}_{\mathrm{H} 2}=0.00,0.30$. 

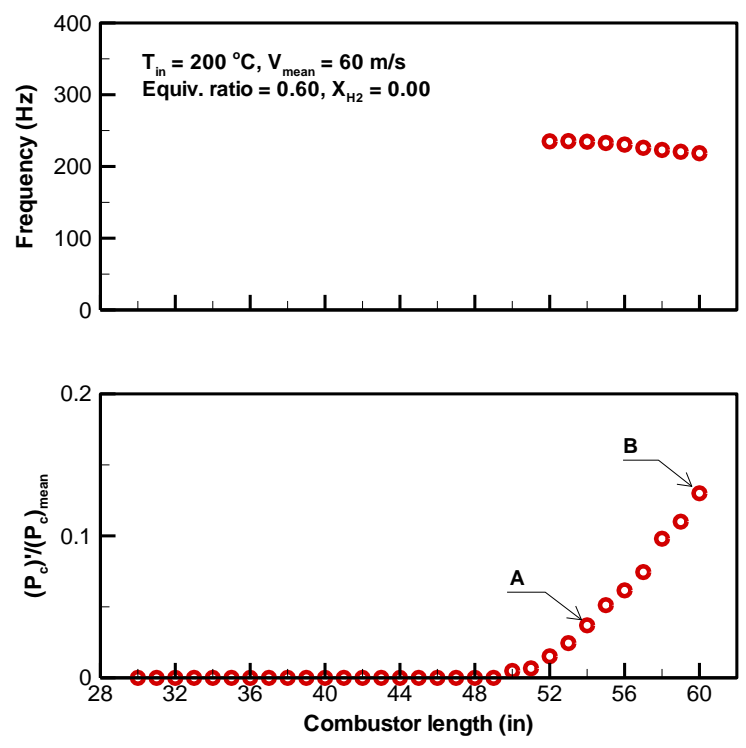

Figure 137: Self-excited instability frequencies and the normalized combustor pressure oscillation magnitude plotted as a function of the combustor length. $T_{\text {in }}=200{ }^{\circ} \mathrm{C}, \mathrm{V}_{\text {mean }}=60$ $\mathrm{m} / \mathrm{s}, \Phi=0.60, \mathrm{X}_{\mathrm{H} 2}=0.00$, and $\mathrm{L}_{\mathrm{c}}=30 " \sim 60 "$.

\section{Self-Excited Instability Measurements}

To compare the relationship between the dynamics of acoustically forced and self-excited flames, self-excited instability measurements were characterized in a lean-premixed, variablelength, gas turbine combustor facility. Figure 137 shows the dependence of self-excited instability frequencies and the normalized combustor pressure oscillation magnitude upon the combustor length $\left(\mathrm{T}_{\text {in }}=200{ }^{\circ} \mathrm{C}, \mathrm{V}_{\text {mean }}=60 \mathrm{~m} / \mathrm{s}, \Phi=0.60\right.$, and $\left.\mathrm{X}_{\mathrm{H} 2}=0.00\right)$. The combustor length was used as a bifurcation parameter; it can be continuously varied between 30 and 60 inches. The longer the combustor length, the lower the instability frequency, due to an increase in acoustic wave length. The observed instability corresponds to the first longitudinal eigenmode of the system. The strongest instability is found for the combustor length of $\mathrm{L}_{\mathrm{c}}=60$ " at $f=218$ $\mathrm{Hz}$.

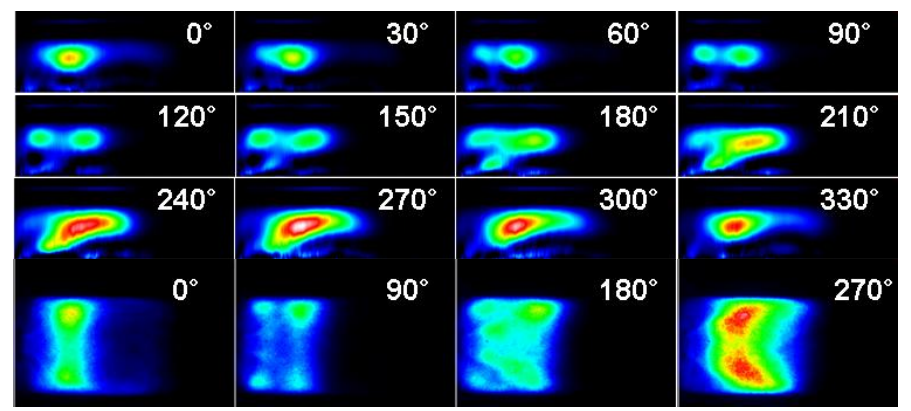

Figure 138: Phase-synchronized (self-excited) flame imaging at $\mathrm{L}_{\mathrm{c}}=54$ " and $f=234 \mathrm{~Hz}$. $\mathrm{V}^{\prime} / \mathrm{V}_{\text {mean }}=0.165, \mathbf{P}_{\mathrm{c}} / \mathrm{P}_{\mathrm{c}}$, mean $=0.037$. Bottom row shows line-of-sight integrated, background-corrected $\mathrm{CH}^{*}$ images at $\varphi=0,90,180,270^{\circ}$. 


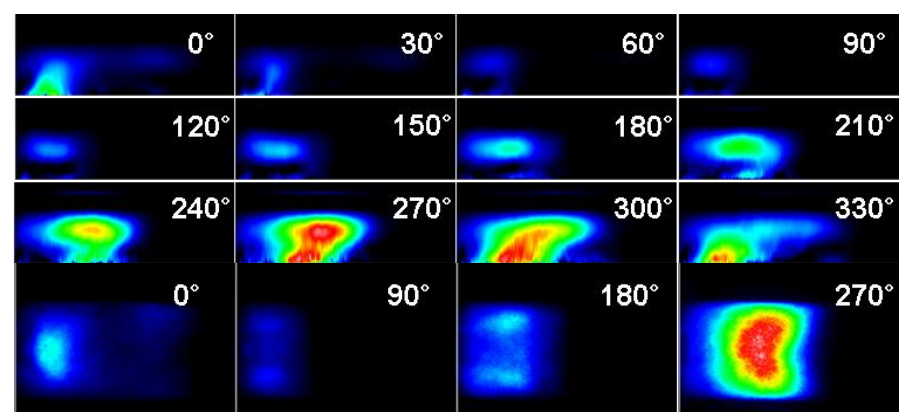

Figure 139: Phase-synchronized (self-excited) flame imaging at $\mathrm{L}_{c}=60$ " and $f=218 \mathrm{~Hz}$. $V^{\prime} / V_{\text {mean }}=0.548, P_{c} / P_{c}$, mean $=0.130$. Bottom row shows line-of-sight integrated, background-corrected $\mathrm{CH}^{*}$ images at $\varphi=0,90,180,270^{\circ}$.

Figure 138 shows phase-synchronized (self-excited) flame imaging at $\mathrm{L}_{\mathrm{c}}=54$ " and $f=234 \mathrm{~Hz}$, representing marginally unstable conditions (marked as " $\mathrm{A}$ " in Figure 137). At this combustor length, the amplitudes of inlet velocity and the combustor pressure oscillations are measured to be $16.5 \%$ and $3.7 \%$, respectively. The flame oscillates back and forth in the flow direction and reaction occurs in the corner recirculation zone at $\varphi=60^{\circ} \sim 210^{\circ}$. Periodic vortex shedding also occurs at $\varphi=180^{\circ} \sim 210^{\circ}$. The flame area variation in Figure 138 is very similar to that seen in Figure 128(b), qualitatively, although the oscillation frequencies are different. $\left(f_{\text {forced }}=200 \mathrm{~Hz}\right.$ and $f_{\text {self-excited }}=234 \mathrm{~Hz}$, respectively)

Figure 139 shows phase-averaged flame imaging at $\mathrm{L}_{\mathrm{c}}=60$ " and $f=218 \mathrm{~Hz}$, which is the point marked as "B" in Figure 137. Contrary to the case of the marginally unstable condition, the flame at $\varphi=270^{\circ}$ is almost detached from the centerbody for part of the cycle and intense reaction occurs in the inner recirculation zone. This is very similar to flame behavior under highamplitude forcing, as shown in Figure 130. The interaction between a large-scale structure and the flame is not dominant. As a consequence, this suggests that when the self-excited instability intensity is weak, the Kelvin-Helmholtz instability (shear layer dynamics) controls the selfsustained instability. In the case of strong instability intensity, however, the unsteady liftoff governs the self-excited instability. In the intermediate ranges, the flame dynamics are controlled by a superposition of shear layer dynamics and unsteady liftoff. These unsteady flame dynamics are directly related to the acoustic fields in the combustor.

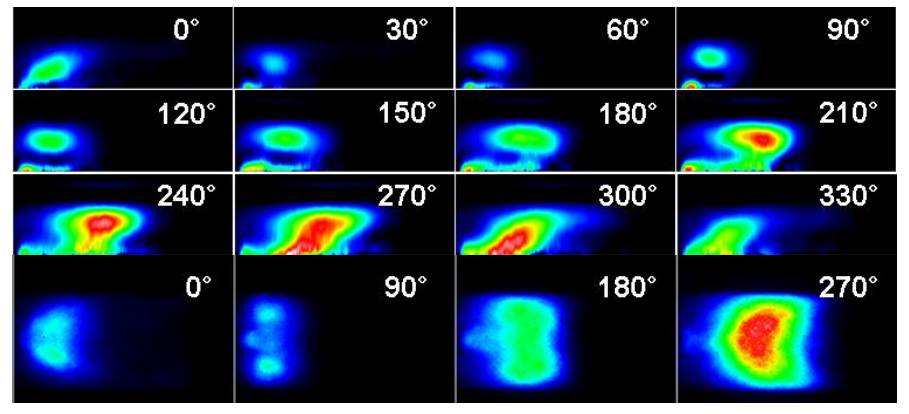

Figure 140: Phase-synchronized $\mathrm{CH}^{*}$ chemiluminescence imaging at modulation frequency of $218 \mathrm{~Hz}$ and $V^{\prime} / V_{\text {mean }}=57 \%\left(P_{c}{ }^{\prime} / P_{c}\right.$, mean $\left.=0.031\right)$. Bottom row shows line-of-sight integrated, background-corrected $\mathrm{CH}^{*}$ images at $\varphi=0,90,180,270^{\circ}$. 


\section{Comparison between Forced and Self-Excited Flame Dynamics}

The dynamics of unsteady flames under forced and self-excited oscillations are compared in an attempt to comprehend the dependence of the heat release responses of a premixed flame upon acoustic velocity and pressure perturbations. Phase-resolved (acoustically forced) $\mathrm{CH}^{*}$ chemiluminescence images at the same input conditions and modulation frequency $(218 \mathrm{~Hz})$ as the self-sustained instability (Figure 139) are presented in Figure 140. The amplitude of inlet velocity fluctuation is set to $57 \%$, which is very close to that of the self-excited instability, i.e., $\mathrm{V}^{\prime} / \mathrm{V}_{\text {mean }}=55 \%$. In the case of the forced response, the normalized combustor pressure amplitude was found to be smaller than for the self-excited instability by a factor of 4 , since the unsteady heat release is not coupled with the acoustics of the system. Flame area variations at each phase for forced and self-excited oscillations are very similar, including their corresponding $\mathrm{CH}^{*}$ chemiluminescence power spectra, although their acoustic pressure amplitudes are different by an order of magnitude, as shown in Figure 141. This result strongly suggests that the flame area modulation (unsteady heat release) under self-excited operations is not determined by pressure perturbations, but by inlet velocity perturbations, for a totally premixed flame. Under self-excited oscillations, however, the velocity disturbance in the mixing section is induced by the pressure perturbations. Pressure perturbations may affect small-scale flame structures, but it is difficult to resolve fine flame structures such as a short-scale wrinkling of the flame front from the chemiluminescence-based imaging technique. It is noted that in the investigated inlet condition the period of acoustic oscillations $(\mathrm{T}=4.587 \mathrm{msec})$ is the same order of magnitude as the convective transport time $(\tau=6.460 \mathrm{msec})$. This indicates that the flame would breathe rather than develop wrinkles in response to low frequency acoustic perturbations. In contrast, at high frequency regime the influence of an acoustic wave upon a flame is expected to be significant and the mechanism of heat release variation in the forced response may be to some extent different from the limit-cycle oscillation under strong acoustic pressure perturbations.
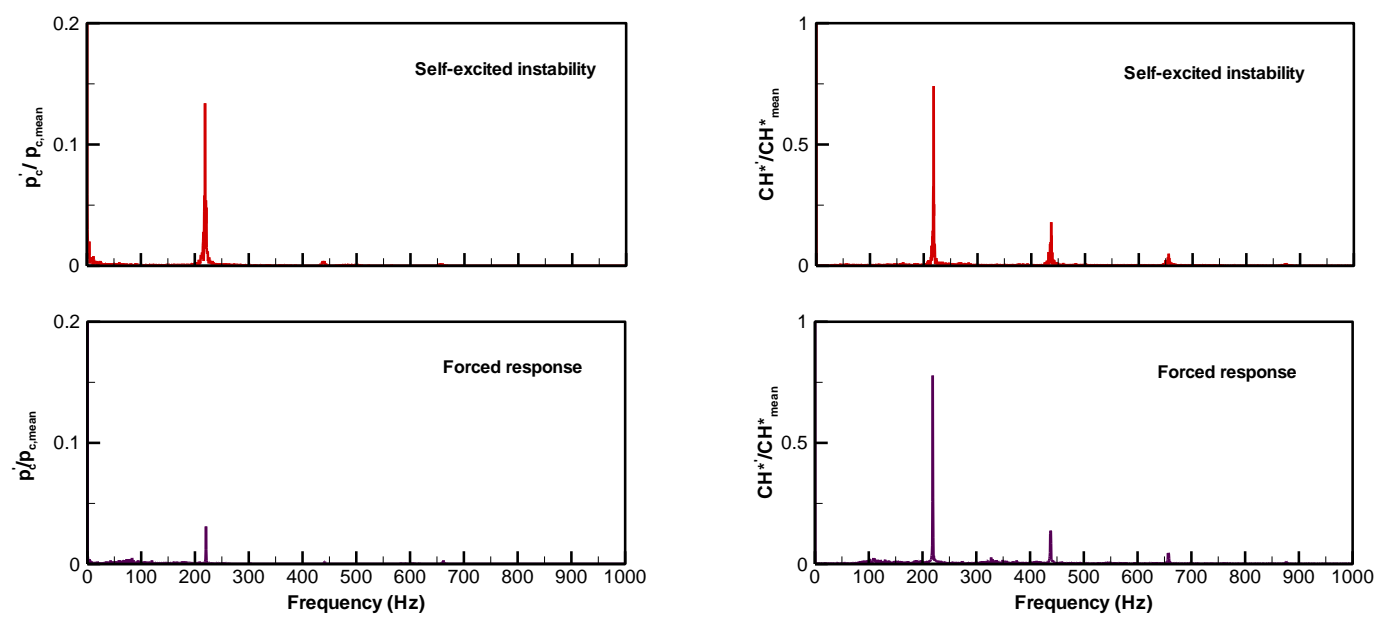

Figure 141: Normalized combustor pressure amplitude and $\mathrm{CH}^{*}$ chemiluminescence intensity for forced response and self-excited instability at $218 \mathrm{~Hz}$. Operating conditions: $T_{\text {in }}=200{ }^{\circ} \mathrm{C}, V_{\text {mean }}=60 \mathrm{~m} / \mathrm{s}, \Phi=0.60$, and $X_{\mathrm{H} 2}=0.00$. 
Nonlinear features of acoustically excited swirl-stabilized lean-premixed flames are characterized by nonlinear flame transfer function measurements and a phase-resolved analysis. Two distinct mechanisms of nonlinearity are investigated. The first mechanism is shear layer dynamics, which plays a key role in causing the nonlinearity of inverted dihedral "V" flames when the modulation amplitude is less than $40 \%$ at $f=200 \mathrm{~Hz} . \mathrm{H}_{2}$-enriched enveloped "M" flames are found to be more stable in terms of combustion instability. Their interaction with a large-scale coherent structure is very weak, which leads to a linear response even at high forcing frequency and amplitude. This implies that steady-state flame configuration is one important controlling parameter controlling linear and nonlinear dynamics. The second mechanism is unsteady flame liftoff, which causes rapid reduction in the flame surface area due to a merging of two flame branches. This mechanism is manifested when the forcing amplitude is greater than $40 \%$ and at a frequency of $200 \mathrm{~Hz}$. Phase-resolved $\mathrm{CH}^{*}$ chemiluminescence images under limitcycle oscillations reveal that when the instability intensity is weak, the Kelvin-Helmholtz instability controls the unsteady heat release process. In the case of high amplitude limit-cycle oscillations, however, the unsteady flame liftoff governs the self-sustained instability. This is consistent with observations from the forced flame dynamics. A systematic investigation of forced and self-excited flame response measurements in a practical, lean-premixed, swirlstabilized, gas turbine combustor could be incorporated into an analytic model and would enable the development of theoretical models for the control and suppression of self-sustained combustion instabilities.

\section{Nonlinear Response of a Swirl Stabilized Flame to Equivalence Ratio Oscillations}

\section{Introduction}

Additionally, the nonlinear response of a swirl-stabilized flame to equivalence ratio nonuniformities was experimentally investigated in an atmospheric-pressure, high-temperature, lean-premixed gas turbine combustor. To generate high-amplitude equivalence ratio oscillations, fuel was modulated using a siren-type modulating device. The mixture ratio oscillations at the inlet of the combustion chamber were measured by the infrared absorption technique and the flame's response to upstream $\Phi$ ' perturbations was estimated by $\mathrm{CH}^{*}$ chemiluminescence emission intensity. Phase-resolved $\mathrm{CH}^{*}$ chemiluminescence images were taken to characterize the dynamic response of the flame. Results show that amplitude and frequency dependence of the flame's response to equivalence ratio modulation is qualitatively consistent with the flame's response to acoustic excitation. However, the underlying physics of the nonlinear response of the flame to $\Phi$ ' modulation is associated with the intrinsically nonlinear dependence of heat of reaction and burning velocity upon equivalence ratio. It was found that combustion cannot be sustained at high-amplitude equivalence ratio oscillations.

All tests were performed at a mean pressure of 1 atm and at mean equivalence ratios ranging from 0.55 to 0.65 . Mean inlet velocity at the nozzle was $60 \mathrm{~m} / \mathrm{s}$ and the inlet temperature was kept constant at $200{ }^{\circ} \mathrm{C}$, giving a Reynolds number of approximately 33,000. Forcing 
frequencies were varied from 100 to $400 \mathrm{~Hz}$ in $25 \mathrm{~Hz}$ increments. The frequency range of 100 $400 \mathrm{~Hz}$ includes self-induced instability frequencies observed in the lean-premixed gas turbine combustor. A small-size siren-type modulation device driven by a DC motor was used to modulate the fuel flow rate. The device consists of a rotor ( $\#$ of holes $=12, \mathrm{~d}_{\text {hole }}=1.5 \mathrm{~mm}$ ) and a stator (\# of holes $=1, d_{\text {hole }}=1.5 \mathrm{~mm}$ ). One needle valve was used to adjust the bypass fuel flow rate, thereby changing the amplitude of modulation at a given frequency. Modulated fuel is injected in the mixing section and mixed with steady-flow, high-temperature air. The fuel injector has a ring-shaped configuration with 24 holes $\left(\mathrm{d}_{\text {hole }}=0.5 \mathrm{~mm}\right)$ on its circumference with equal spacing. The fuel flow rate is not choked here, so that modulation amplitudes are high for any given modulation frequency. The full list of operating conditions is summarized in Table 8 .

Table 8: Test conditions for forced flame response measurements.

\begin{tabular}{cc}
\hline Parameters & Test conditions \\
\hline pressure $(\mathrm{P})$ & $1 \mathrm{~atm}$ \\
inlet temperature $\left(\mathrm{T}_{\text {in }}\right)$ & $200^{\circ} \mathrm{C}$ \\
nozzle velocity $\left(\mathrm{V}_{\text {mean }}\right)$ & $60 \mathrm{~m} / \mathrm{s}$ \\
mean equivalence ratio & $0.55,0.60,0.65$ \\
$\left(\Phi_{\text {mean }}\right)$ & $100 \sim 400 \mathrm{~Hz}(\Delta f=25 \mathrm{~Hz})$ \\
forcing frequency $(f)$ & up to $\mathrm{LBO}$ \\
forcing amplitude $\left(\Phi^{\prime} / \Phi_{\text {mean }}\right)$ & 0.00 \\
fuel composition $\left(\mathrm{X}_{\mathrm{H} 2}\right)$ & \\
\hline
\end{tabular}

\section{Flame Transfer Function Measurements}

The response of a turbulent swirl-stabilized flame to equivalence ratio oscillations is experimentally examined. Fuel modulation is used to induce significant mixture ratio perturbations at the combustor inlet, while the amplitude of the velocity perturbations is kept negligible, due to the high mass flow rate ratio of air and fuel. Therefore, the effect of velocity disturbances on the flame's heat release response is not considered here. If the fuel is modulated $\left(\mathrm{m}_{\mathrm{a}}=0\right)$, the amplitude of equivalence ratio inhomogeneities, the input function of the flame transfer function, is directly related to that of the fuel mass flow rate:

$$
\left(\frac{\Phi^{\prime}}{\bar{\Phi}}\right)=\frac{\frac{\overline{\mathrm{m}}_{\mathrm{f}}}{\overline{\mathrm{m}_{\mathrm{f}}}}-\frac{\mathrm{m}_{\mathrm{a}}}{\overline{\mathrm{m}_{\mathrm{a}}}}}{1+\frac{\mathrm{m}_{\mathrm{a}}^{\prime}}{\overline{\mathrm{m}_{\mathrm{a}}}}}=\frac{\mathrm{m}_{\mathrm{f}}}{\overline{\mathrm{m}_{\mathrm{f}}}}
$$

A typical example of the flame's heat release response to equivalence ratio modulation is shown in Figure 142, which presents the normalized $\mathrm{CH}^{*}$ chemiluminescence intensity $\left(\mathrm{CH}^{*} / \mathrm{CH}^{*}{ }_{\text {mean }}\right)$ and phase of the flame transfer functions at a modulation frequency of $100 \mathrm{~Hz}$, when the flame is subjected to equivalence ratio modulations. At the condition considered here, the amplitude of inlet velocity fluctuations is less than $3 \%$ of the mean velocity. This indicates that the influence of inlet velocity fluctuations on heat release oscillations is negligible, and therefore the response of a flame is essentially determined by mixture ratio modulations. Figure 142(A) shows that the 
heat release response increases almost linearly with an increase in the amplitude of the equivalence ratio oscillation up to $\Phi^{\prime} / \Phi_{\text {mean }} \approx 3 \%$. This represents the linear flame response at relatively low forcing frequency. The gain is nearly constant with respect to modulation amplitude. Note that the maximum driving amplitude point is not caused by a limitation of the modulating device, but by flame blowoff. When the amplitude of mixture ratio perturbations is greater than 3\%, the flame becomes detached from the centerbody and lean blowoff occurs. The flame near blowoff exhibits a tornado-like vortex core structure, as shown in Figure 143. The flame is highly unsteady and it is detached from the centerbody. It is believed that incomplete combustion near the lean blowoff is a major source of pollutant emissions. Figure 142(B) shows that the phase between the heat release and equivalence ratio oscillations at the dump plane gradually increases with an increase in amplitude.
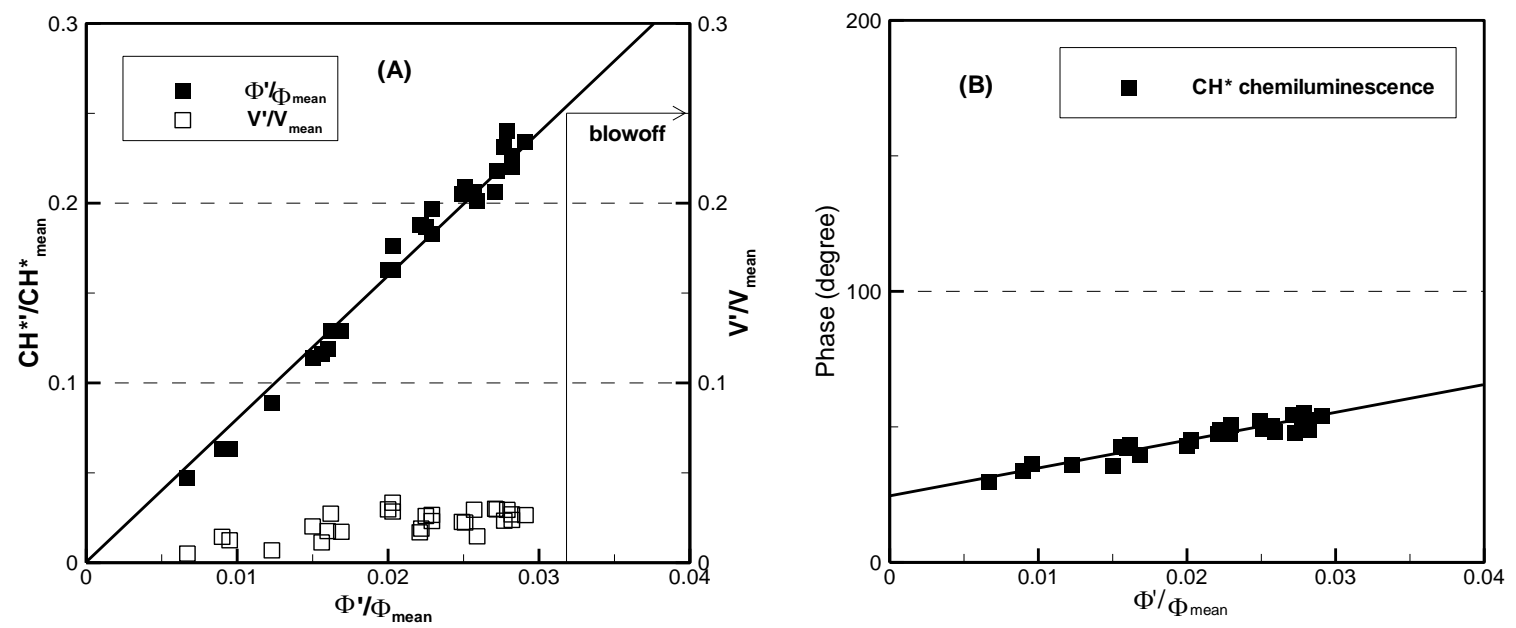

Figure 142: (A) The normalized $\mathrm{CH}^{*}$ chemiluminescence intensity and (B) the phase of the flame transfer function at a modulation frequency of $100 \mathrm{~Hz}$. Inlet conditions: $\mathrm{T}_{\text {in }}=200{ }^{\circ} \mathrm{C}$, $V_{\text {mean }}=60 \mathrm{~m} / \mathrm{s}$, and $\Phi_{\text {mean }}=0.60$.

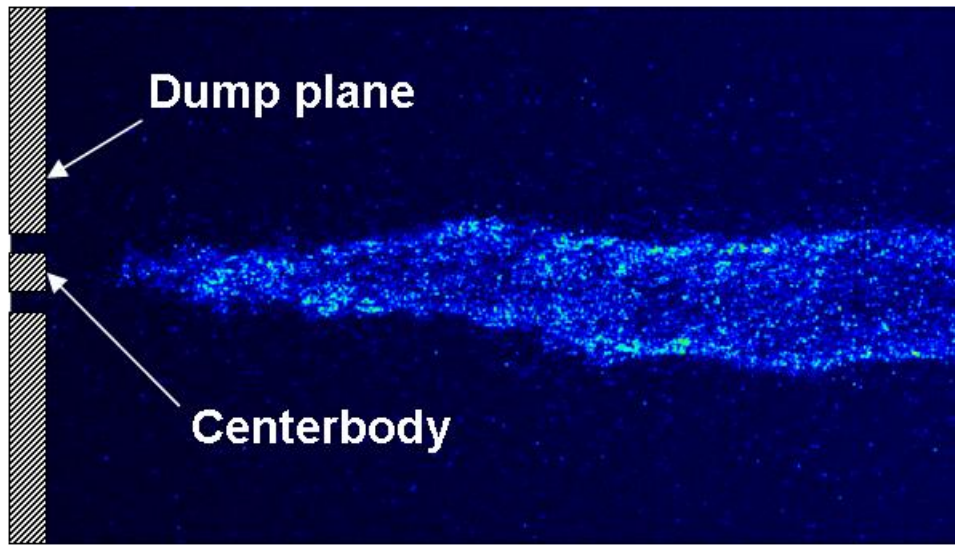

Figure 143: Instantaneous $\mathrm{CH}^{*}$ chemiluminescence image at a modulation frequency of $100 \mathrm{~Hz}$ and $\Phi^{\prime} / \bar{\Phi}=4.3 \%$. Inlet conditions: $T_{\text {in }}=200{ }^{\circ} \mathrm{C}, \mathrm{V}_{\text {mean }}=60 \mathrm{~m} / \mathrm{s}$, and $\Phi_{\text {mean }}=0.55$. 

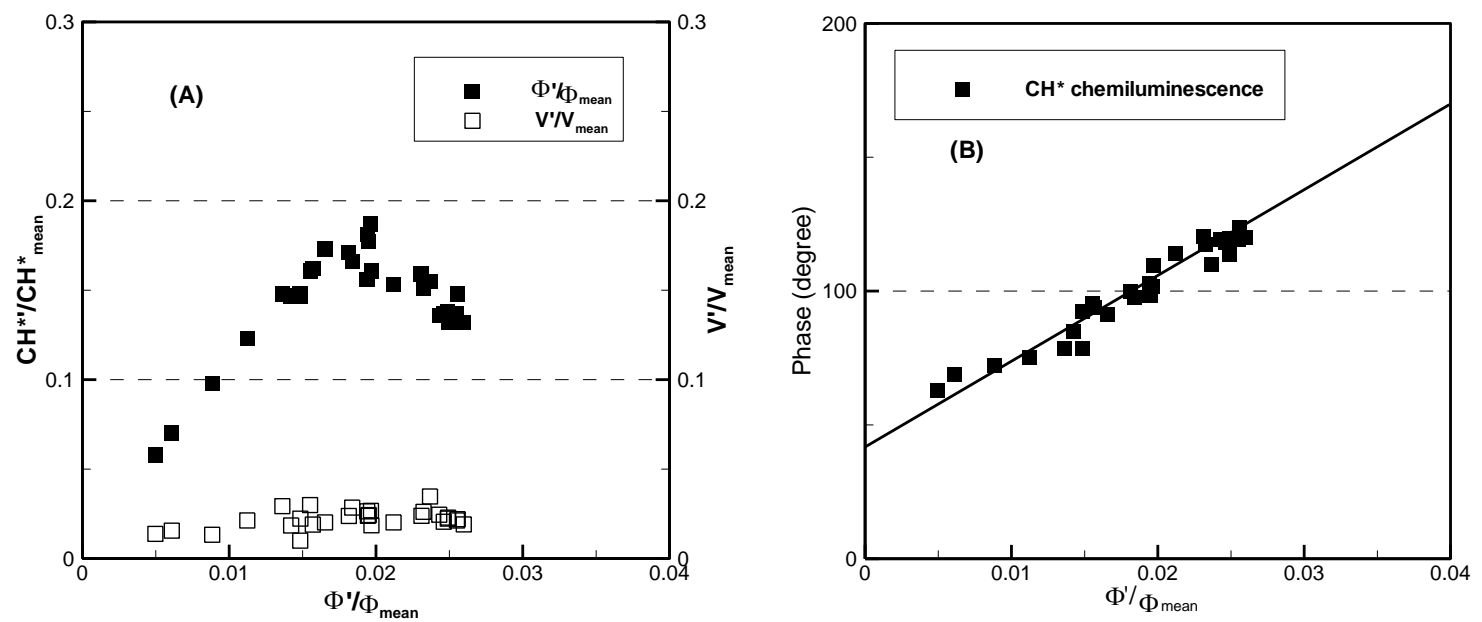

Figure 144: (A) The normalized $\mathrm{CH}^{*}$ chemiluminescence intensity and $(\mathrm{B})$ the phase of the flame transfer function at a modulation frequency of $150 \mathrm{~Hz}$. Inlet conditions: $T_{\text {in }}=200{ }^{\circ} \mathrm{C}$, $V_{\text {mean }}=60 \mathrm{~m} / \mathrm{s}$, and $\Phi_{\text {mean }}=0.60$.

Figure 144 shows the forced response at a modulation frequency of $150 \mathrm{~Hz}$. Figure 144(A) clearly shows that the response increases linearly up to $\Phi^{\prime} / \Phi_{\text {mean }} \approx 2 \%$, and it starts to decrease with increasing modulation amplitude. This represents the nonlinear behavior of a flame subjected to $\Phi$ perturbations: the gain starts to steeply decrease when $\Phi^{\prime} / \Phi_{\text {mean }}$ is greater than $2 \%$. The detailed mechanisms of nonlinearity will be discussed with reference to the phaselocked flame images. It should be noted that as in the prior case, the phase gradually increases with increasing amplitude, indicating that the effective flame length increases with modulation amplitude. This behavior was not observed when the flame is perturbed purely by acoustic velocity oscillations; it was shown that the phase is nearly constant with respect to the amplitude of inlet velocity modulations.

It can be seen from Figure 142 and Figure 144 that the nonlinearity occurs at lower $\Phi^{\prime} / \Phi_{\text {mean }}$ as the modulation frequency increases. The flame response is linear up to $\Phi^{\prime} / \Phi_{\text {mean }} \approx 3 \%$ at a frequency of $100 \mathrm{~Hz}$, but the nonlinear response starts at $\Phi^{\prime} / \Phi_{\text {mean }} \approx 2 \%$ at a frequency of 150 $\mathrm{Hz}$. Figure 145 plots the dependence of the normalized $\mathrm{CH}^{*}$ chemiluminescence intensity upon modulation frequency between 100 and $400 \mathrm{~Hz}$. It is evident from Figure 145 that the normalized $\mathrm{CH}^{*}$ chemiluminescence intensity decreases with excitation frequency at a fixed perturbation amplitude, and the flame's response at modulation frequencies of 300 and $400 \mathrm{~Hz}$ is definitely nonlinear. The dependence of the inception of the nonlinear response to $\Phi$ oscillations upon modulation frequency shows the same trend as the response of the flame to acoustic excitation. The response characteristic of a flame in terms of frequency is qualitatively similar regardless of the nature of the disturbances, i.e., acoustic velocity or equivalence ratio, but the flame response is quantitatively very different. The gain of the flame transfer function is an order of magnitude greater for equivalence ratio fluctuations than when the flame is subjected to velocity oscillations. The nonlinearity appears for very low amplitude perturbations $\left(\Phi^{\prime} / \Phi_{\text {mean, transition }} \approx\right.$ $2 \%$ ), whereas the nonlinear flame response to acoustic velocity modulation occurs at a modulation amplitude of approximately $\mathrm{V}^{\prime} / \mathrm{V}_{\text {mean, transition }} \approx 20 \%$. This result indicates that the 
flame's response to equivalence ratio nonuniformities is much more sensitive than it is to velocity perturbations. When the flame is perturbed by inlet velocity oscillations, high amplitude perturbations are required for shear layer rollup, leading to shedding of a vortex-ring structure. Flame area modulation due to flame-vortex interaction is a key mechanism, inducing heat release oscillations. On the other hand, equivalence ratio modulations directly affect the heat release rate oscillations, by means of variations in burning velocity and heat of reaction of incoming reactants. Perturbations of flame surface area are also indirectly induced by the burning velocity fluctuations.

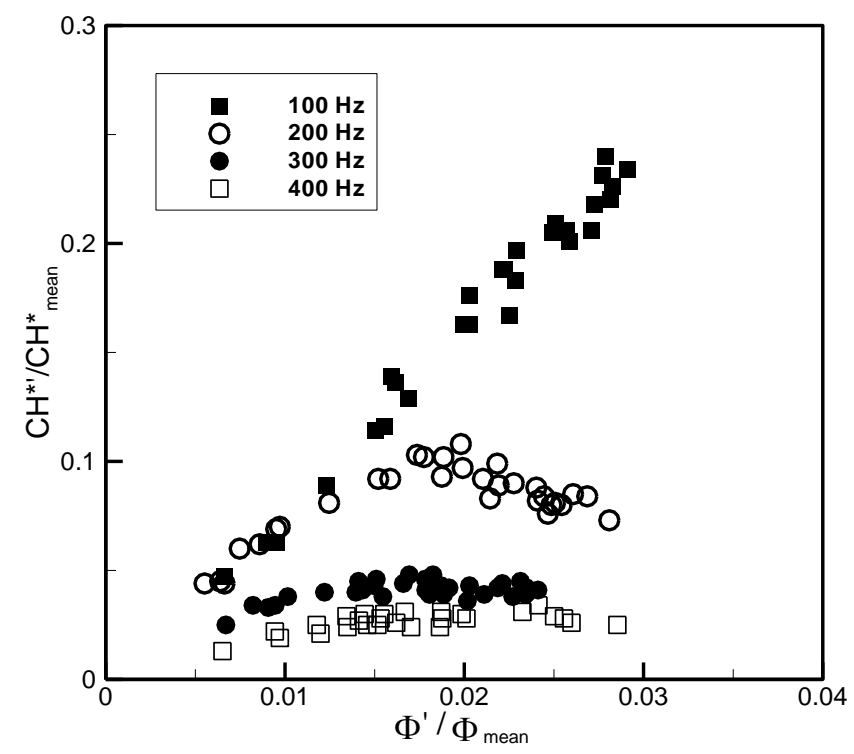

Figure 145: Dependence of the normalized $\mathrm{CH}^{*}$ chemiluminescence intensity upon modulation frequency. Inlet conditions: $T_{\text {in }}=200{ }^{\circ} \mathrm{C}, V_{\text {mean }}=60 \mathrm{~m} / \mathrm{s}$, and $\Phi_{\text {mean }}=0.60$. 


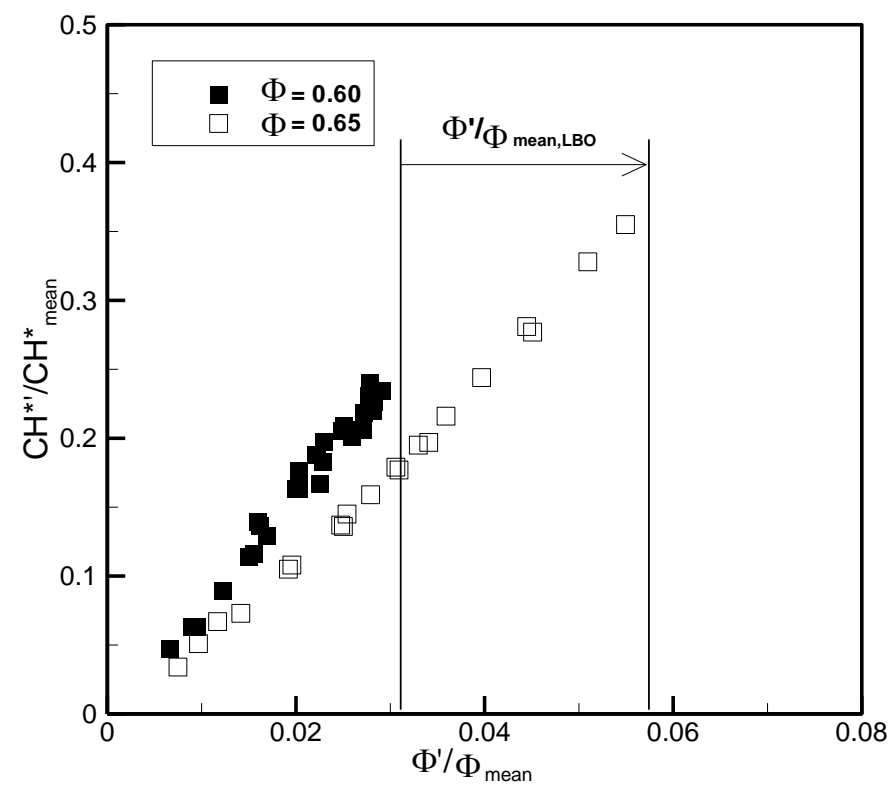

Figure 146: Dependence of the response of a flame upon mean equivalence ratio at a modulation frequency of $100 \mathrm{~Hz}$. Inlet conditions: $T_{\text {in }}=200{ }^{\circ} \mathrm{C}, \mathrm{V}_{\text {mean }}=60 \mathrm{~m} / \mathrm{s}$, and $\Phi_{\text {mean }}=$ 0.60, 0.65 .

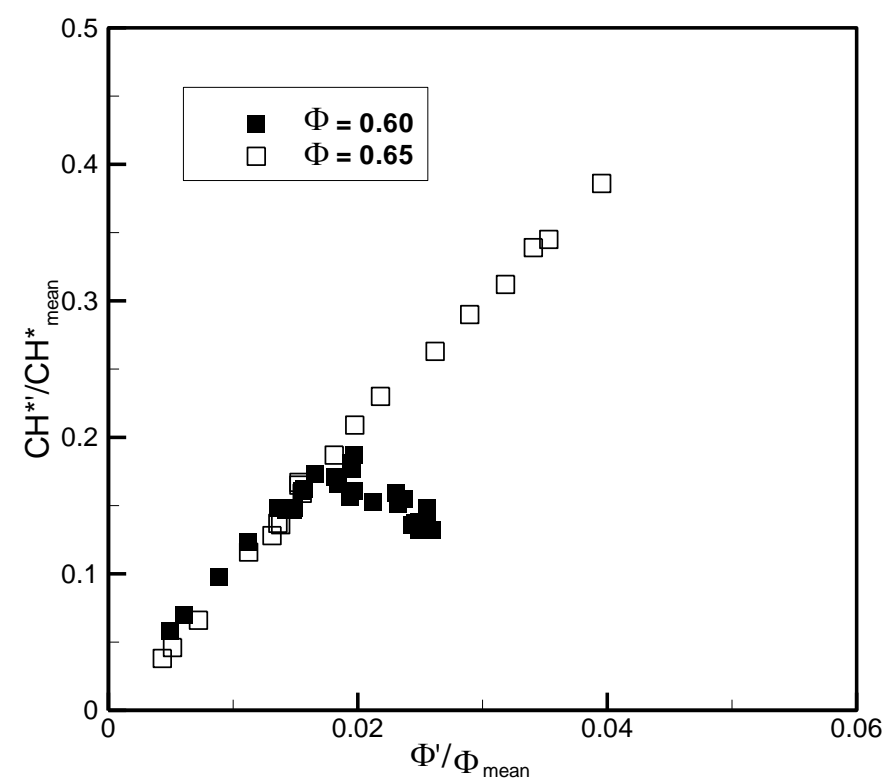

Figure 147: Dependence of the response of a flame upon mean equivalence ratio at a modulation frequency of $150 \mathrm{~Hz}$. Inlet conditions: $T_{\text {in }}=200{ }^{\circ} \mathrm{C}, V_{\text {mean }}=60 \mathrm{~m} / \mathrm{s}$, and $\Phi_{\text {mean }}=$ 0.60, 0.65 . 
Figure 146 shows the dependence of the flame's response to changes in the mean equivalence ratio at a modulation frequency of $100 \mathrm{~Hz}$. As the mean equivalence ratio increases from $\Phi_{\text {mean }}=$ 0.60 to $\Phi_{\text {mean }}=0.65$, the magnitude of the heat release response decreases for a fixed amplitude of perturbation, but the response remains in the linear region. This indicates that a flame with high mean equivalence ratio is less perturbed, because its "robustness" with respect to disturbances is enhanced. When the mean equivalence ratio is near the static lean blowoff limit, on the other hand, the flame is more easily destabilized when subjected to upstream disturbances. It is also noted that the dynamic flammability limit, defined as the minimum equivalence ratio above which the unsteady flame can sustain combustion, is extended from $\Phi^{\prime} / \Phi_{\text {mean }} \approx 3 \%$ to $6 \%$ with increasing mean equivalence ratio.

At a modulation frequency of $150 \mathrm{~Hz}$, the flame at a mean equivalence ratio of 0.60 exhibits highly nonlinear behavior, while the flame at $\Phi_{\text {mean }}=0.65$ shows linear response characteristics, as shown in Figure 147. This is associated with the improved stability characteristic of the flame as mean equivalence ratio increases. Note that the dynamic flammability limits are also improved by approximately $60 \%$, as mean equivalence ratio increases. Figure 146 and Figure 147 suggest that the linear/nonlinear dynamics of a flame submitted to $\Phi$ oscillations are strongly dependent on the mean equivalence ratio. As the mean equivalence ratio decreases, e.g., when $\Phi_{\text {mean }}$ is near static LBO limit, the nonlinear response of a flame to equivalence ratio perturbations is more prominent.
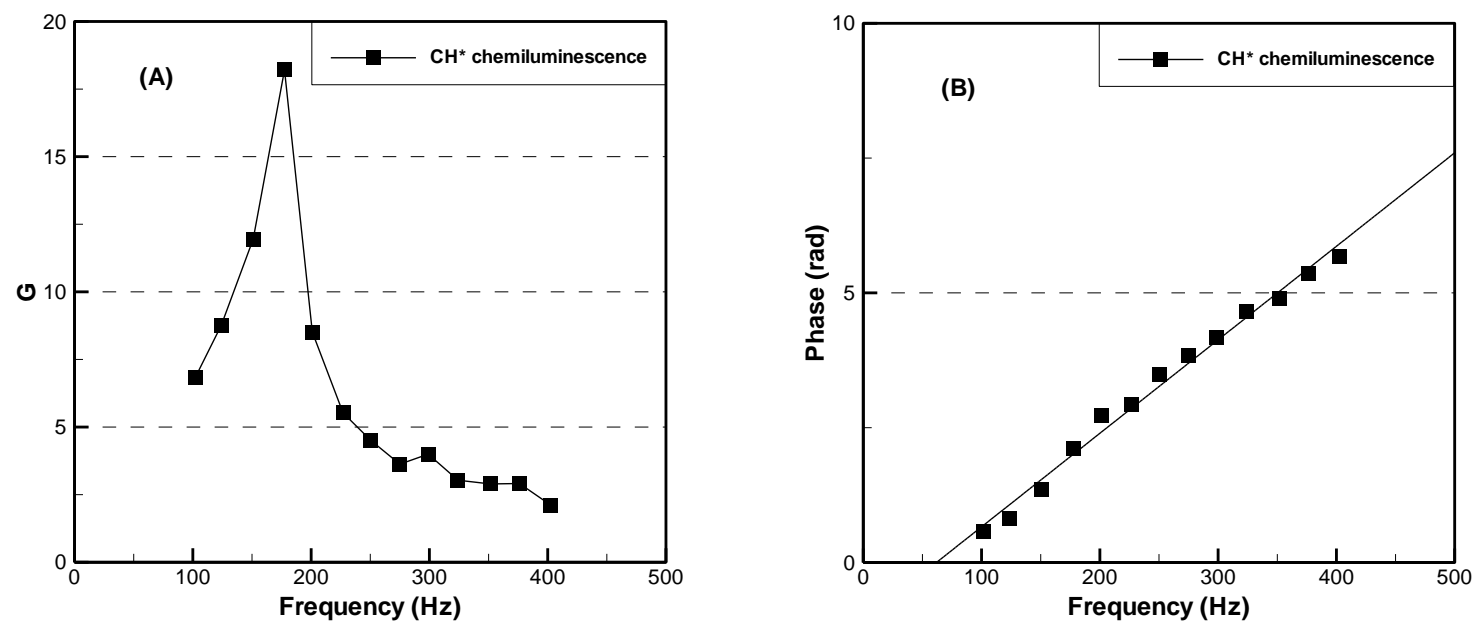

Figure 148: (A) Gain and (B) phase of the flame transfer function in frequency domain. Inlet conditions: $T_{\text {in }}=200{ }^{\circ} \mathrm{C}, V_{\text {mean }}=60 \mathrm{~m} / \mathrm{s}$, and $\Phi_{\text {mean }}=0.60$. 
The gain and phase of the flame transfer function can be expressed in the frequency domain at the same magnitude of equivalence ratio perturbations, $\Phi^{\prime} / \Phi_{\text {mean }}=1 \%$. This amplitude limits the flame response to the linear region. Figure 148 plots the flame transfer function against modulation frequencies, for a mean equivalence ratio of 0.60 and a mean inlet velocity of $60 \mathrm{~m} / \mathrm{s}$. The flame transfer function exhibits a low-pass filter behavior, as when the flame is perturbed by velocity oscillations. It can be seen from Figure 148(A) that a strong overshoot at a modulation frequency of $175 \mathrm{~Hz}$ is observed and the gain asymptotically decreases to zero with increasing modulation frequency. The physical significance of this result is that the flame selectively amplifies equivalence ratio perturbations with certain frequency ranges. The frequency where the response of the flame is amplified is consistent with one of self-excited instability frequencies observed in the test rig. A small peak at a frequency of $300 \mathrm{~Hz}$ is observed. Figure 148(B) shows that the phase evolves quasi-linearly with frequency. This phase dependence indicates a roughly constant time delay between the chemiluminescence and $\Phi$ perturbation, and it implies that their relationship is purely convective in nature.

\section{Nonlinear Flame Dynamics}

Presented here are the nonlinear features of a flame subjected to equivalence ratio modulation. Phase-averaged $\mathrm{CH}^{*}$ chemiluminescence flame images were measured using an ICCD camera with a phase interval of $30^{\circ}$. Figure 149 shows phase-averaged $\mathrm{CH}^{*}$ chemiluminescence images during a cycle of oscillation at a modulation frequency of $200 \mathrm{~Hz}$ and an amplitude of 2.2 and $3.2 \%$. The intensity of $\mathrm{CH}^{*}$ chemiluminescence emission is highest at $\varphi=270^{\circ}$. It can be seen that shear layer rollup is not observed, due to the low amplitude of velocity perturbation at the combustor inlet. This confirms that the heat release oscillations are caused by equivalence ratio modulations. The overall flame length is nearly constant over a period of oscillation, but the heat release rate varies significantly (see Figure 150). Figure 150 plots a time series of the global $\mathrm{CH}^{*}$ chemiluminescence, measured by a PMT. It clearly shows that the heat release signal is highly nonlinear, in that a sharp peak of heat release is produced in each cycle $\left(\tau_{2} \gg \tau_{1}\right)$. Similar observation was made by Birbaud et al.. They described that the flame behaves like a relaxation oscillator with a slow growth followed by a sharp drop. It is interesting to note that the overall flame length is almost constant over a cycle of oscillation. This behavior is similar to Large Eddy Simulation (LES) results of Sengissen et al. They found that the flame does not move significantly when the equivalence ratio modulation impinges on the swirl-stabilized flame. This result suggests that the influence on the heat release rate oscillation of the variation in heat of reaction is more significant than that of flame speed. If the flame speed varies substantially during a period of oscillation, the flame length change will be prominent; $L_{\text {flame }} \propto S_{\mathrm{T}}\left(\mathrm{S}_{\mathrm{T}}=\right.$ turbulent flame speed).

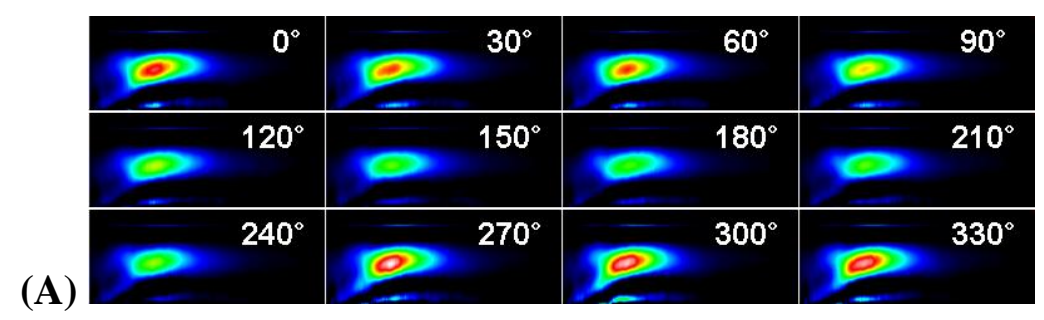




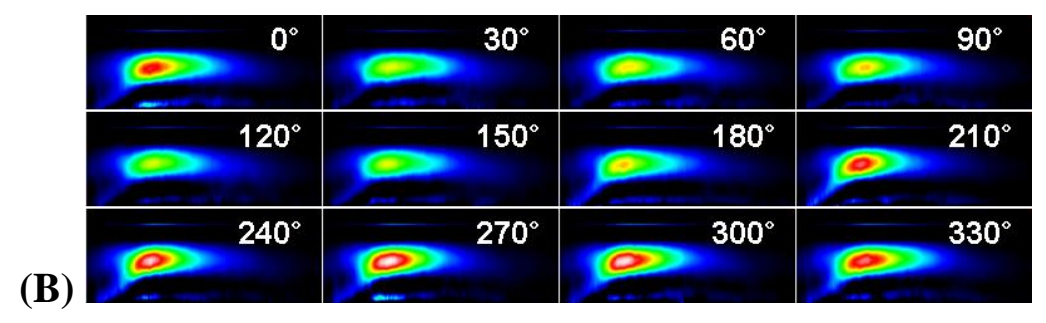

Figure 149: Phase-resolved $\mathrm{CH}^{*}$ chemiluminescence imaging during a period of oscillation at a modulation frequency of $200 \mathrm{~Hz}$ and amplitudes of $(\mathrm{A})_{\Phi} / \bar{\Phi}=\mathbf{2 . 2 \%}$ and $(\mathrm{B})_{\Phi} / \bar{\Phi}=$ $3.2 \%$. Inlet conditions: $T_{\text {in }}=200{ }^{\circ} \mathrm{C}, V_{\text {mean }}=60 \mathrm{~m} / \mathrm{s}$, and $\Phi_{\text {mean }}=0.60$.

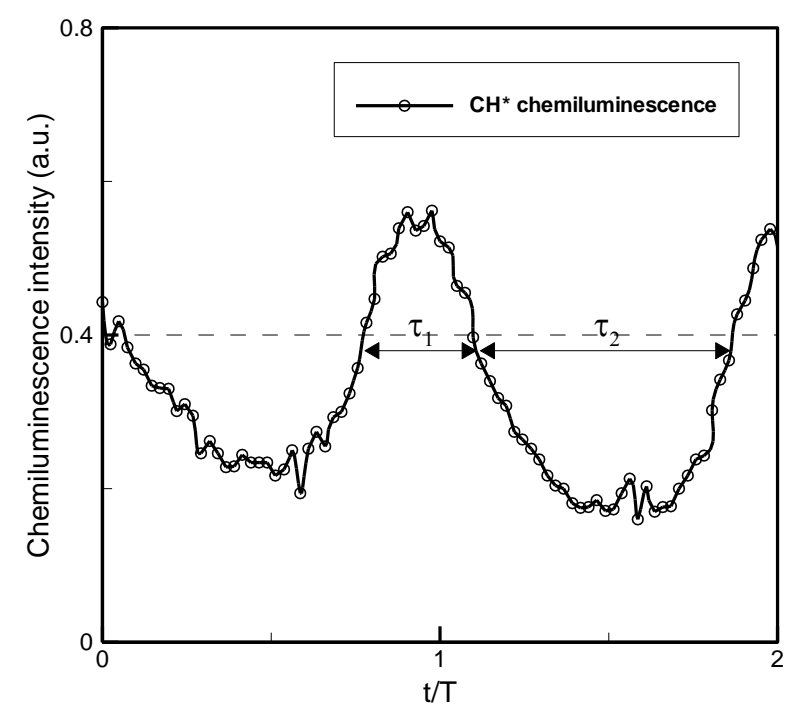

Figure 150: Time signal of $\mathrm{CH}^{*}$ chemiluminescence intensity at a modulation frequency of $200 \mathrm{~Hz}$ and amplitude of $\Phi / \bar{\Phi}=2.2 \%$. Inlet conditions: $T_{\text {in }}=200{ }^{\circ} \mathrm{C}, \mathrm{V}_{\text {mean }}=60 \mathrm{~m} / \mathrm{s}$, and $\Phi_{\text {mean }}=0.60$.

Figure 149(B) shows phase-resolved $\mathrm{CH}^{*}$ chemiluminescence images over a period of oscillation $(200 \mathrm{~Hz})$ at a modulation amplitude of $\Phi^{1 / \bar{\Phi}}=3.2 \%$. Other inlet conditions are the same as in the case shown in Figure 149(A). This forcing condition is very close to the blowoff limit. It is noteworthy that with increasing modulation amplitude, the flame length at each phase is larger than when it is modulated at $\Phi / \bar{\Phi}=2.2 \%$. The increase in the phase with respect to perturbation amplitude, as shown in Figure 144(B), is attributed to the increased flame length, meaning that effective convection time for disturbances to impinge the flame increases. The nonlinear dynamics of the flame exhibits enhanced unsteadiness near blowoff. However, unsteady local extinction events, which are known as the first stage of near-blowoff dynamics of bluff-body stabilized flames, are not observed here. This indicates that the instantaneous stretch rate does not exceed the local extinction stretch rate. 


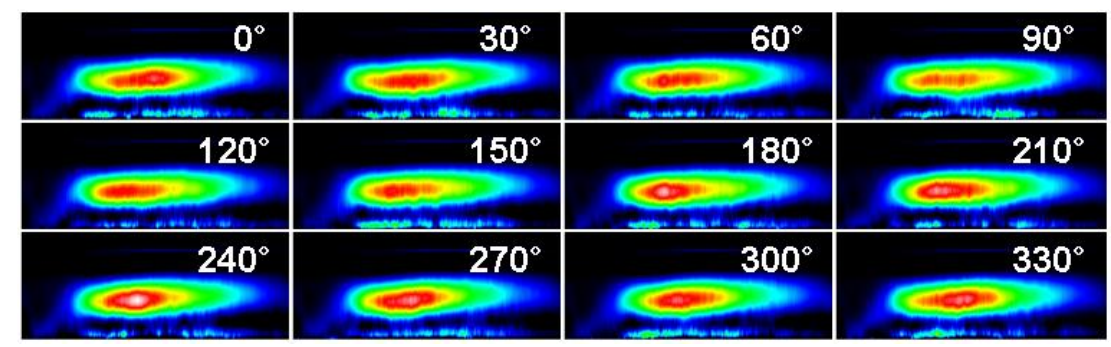

Figure 151: Phase-resolved $\mathrm{CH}^{*}$ chemiluminescence imaging during a period of oscillation at a modulation frequency of $400 \mathrm{~Hz}$ and $\Phi / \bar{\Phi}=2.3 \%$. Inlet conditions: $\mathrm{T}_{\text {in }}=200{ }^{\circ} \mathrm{C}, \mathrm{V}_{\text {mean }}$ $=60 \mathrm{~m} / \mathrm{s}$, and $\Phi_{\text {mean }}=0.55$.

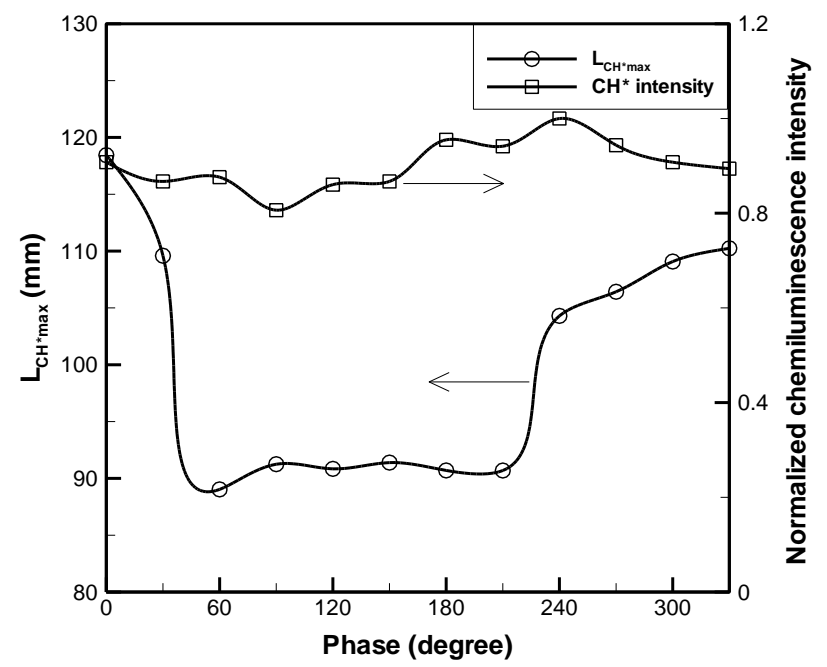

Figure 152: The dependence of the maximum $\mathrm{CH}^{*}$ chemiluminescence intensity locations $\left(\mathrm{L}_{\mathrm{CH}^{*} \text { max }}\right.$ ) and $\mathrm{CH}^{*}$ chemiluminescence intensity upon the phase during a period of oscillation at a modulation frequency of $400 \mathrm{~Hz}$ and $\Phi^{\prime} / \bar{\Phi}=2.3 \%$. Inlet conditions: $T_{\text {in }}=$ $200{ }^{\circ} \mathrm{C}$, Vmean $=60 \mathrm{~m} / \mathrm{s}$, and $\Phi_{\text {mean }}=0.55$.

Figure 151 shows phase-synchronized $\mathrm{CH}^{*}$ chemiluminescence imaging at a modulation frequency of $400 \mathrm{~Hz}$ and amplitude of $2.3 \%$. The mean equivalence ratio is 0.55 , and therefore the steady-state flame length is relatively long. At this inlet condition, the convective wavelength of the equivalence ratio perturbation $\left(\lambda_{\Phi, \text { conv }}=15 \mathrm{~cm}\right)$ is comparable with the flame length. Figure 152 shows the corresponding variations of $\mathrm{CH}^{*}$ chemiluminescence intensity and the maximum intensity location of the $\mathrm{CH}^{*}$ chemiluminescence, $\mathrm{L}_{\mathrm{CH}^{*} \text { max }}$. Figure 142 and Figure 143 show that the overall flame length is almost unchanged and $\mathrm{CH}^{*}$ chemiluminescence intensity is also nearly constant during a cycle of modulation. The maximum $\mathrm{CH}^{*}$ chemiluminescence intensity location varies, however, as displayed in Figure 152. At $\varphi=60-210^{\circ}$, the fuel-rich mixture impinges on the near field of the flame and the fuel-lean mixture impinges on the farfield of the flame. Locally, the flame is prone to stretching. Hence, when the flame is modulated by both perturbations of velocity and equivalence ratio, it is highly possible for localized flame extinction to occur, resulting in nonlinear flame dynamics; refer to the previous section of this report about the response of a partially premixed flame to acoustic velocity and equivalence ratio oscillations. 
Even though it is well understood that equivalence ratio nonuniformities play an important role in inducing self-sustained pressure oscillations in lean-premixed gas turbines, this key issue has not been well explored experimentally, primarily because of the difficulties of making simultaneous measurements of inlet equivalence ratio and heat release rate. The response of the swirl-stabilized flames to equivalence ratio oscillations was determined experimentally. Equivalence ratio perturbations were achieved by modulating fuel flow rate, giving nearly sinusoidal oscillations of fuel/air ratio at the combustor inlet. It was found that the response of the flame is divided into linear and nonlinear regimes, depending on the magnitude of perturbations. For high perturbation amplitudes, the heat release response shows nonlinear behavior with abrupt changes in the waveform. The nonlinearity is related to the nonlinear dependence of heat of reaction and burning velocity upon equivalence ratio. The nonlinear response becomes more dominant as modulation frequencies increase and mean equivalence ratio decrease. It was found that the flame acts as an amplifier of equivalence ratio perturbations at modulation frequencies of approximately $175 \mathrm{~Hz}$ and the phase measured between $\mathrm{CH}^{*}$ chemiluminescence and inlet equivalence ratio modulation evolves linearly with excitation frequency. This suggests that the overall response characteristics of the flame to equivalence ratio modulation are very similar to results obtained from the response to acoustic excitation, but the mechanisms of nonlinearity are quite different. The data presented in the present section of this technical report will provide insight into the controlling physics of the response of the flame to equivalence ratio modulation, and it can be used to validate an analytic flame response model.

\section{Inlet Velocity and Equivalence Ratio Oscillations of Swirl-Stabilized Partially Premixed Flames}

\section{Introduction}

In following the previous experiments, the forced response of a swirl-stabilized, partially premixed flame to inlet velocity and equivalence ratio oscillations was further experimentally investigated in a model lean-premixed gas turbine combustor. Three different forcing mechanisms were studied: the response of a premixed flame to velocity oscillations, the response of a partially premixed flame to equivalence ratio oscillations, and the response of a partially premixed flame to velocity and equivalence ratio oscillations. The overall heat release response of the flame was determined from measurements of the $\mathrm{CH}^{*}$ chemiluminescence emission intensity from the entire flame, while the response of the spatially distributed heat release was determined from phase-synchronized chemiluminescence images. In addition, simultaneous measurements were made of the inlet velocity and equivalence ratio oscillations using the two-microphone method and the IR absorption technique, respectively. The results show that in the linear regime, the response of a partially premixed flame to simultaneous velocity and equivalence ratio oscillations can be reconstructed from independent measurements of the flame's response to velocity oscillations and to equivalence ratio oscillations using a vector summation method. This is the first experimental demonstration of a two-input one-output model of a swirl-stabilized partially premixed flame. It suggests that the response of a partially premixed flame is governed by four physical parameters, i.e., the oscillation frequency, the amplitude of velocity oscillation, the amplitude of equivalence ratio oscillation, and the phase 
difference between the two oscillations. As a result, the heat release response of a partially premixed flame can be amplified or damped, depending on the phase difference between the velocity and equivalence ratio oscillations at the combustor inlet.

Three different forcing methods were implemented to investigate the flame response. The first method involves the use of a siren device to modulate the flow rate of premixed fuel and air. The siren is driven by a variable-speed DC motor, which allows for the variation of the forcing frequency. Two high-temperature globe valves are used to control the siren bypass flow rate, which changes the magnitude of the velocity oscillation. For these tests the fuel and air are mixed far upstream of a choked orifice to ensure that there are no spatial or temporal variations in the equivalence ratio, in which case, the flame response is solely the result of the oscillating velocity and the related shear layer dynamics. The second forcing method involves the use of a small siren device to modulate the fuel flow rate. Again, a bypass valve is used to change the modulation amplitude. Modulated fuel flows through the injector and into the air flowing through the mixing section. Since the air flow is steady, this results in a time-varying equivalence ratio which is convected by the mean flow to the flame. The fuel injector is ringshaped with 24 equally-spaced holes $\left(\mathrm{d}_{\text {hole }}=0.5 \mathrm{~mm}\right)$ around its circumference, and is located $152 \mathrm{~mm}$ upstream of the combustor dump plane, which is upstream of the swirler. The third forcing method involves the use of the siren to modulate the air flow, while the fuel is injected directly into the mixing section using the fuel injector described in the previous paragraph. The fuel injector orifices are choked, and as a result, the fuel flow rate entering the mixing section is constant. The net effect of a steady fuel flow rate and a modulated air flow rate is a time varying equivalence ratio, which is convected from the fuel injection location to the flame. The fuel injection location, i.e., the distance from the dump plane, can be continuously varied between $152 \mathrm{~mm}$ and $304 \mathrm{~mm}$, which corresponds to a change in the time required for the equivalence ratio oscillation to convect from the injector to the flame. This also changes the phase difference between the equivalence ratio oscillation and the velocity oscillation. The response of the flame is therefore determined by the combined effects of the oscillating velocity and equivalence ratio.

\section{Two-Input One-Output Model}

The heat release response of a partially premixed flame can be mathematically expressed by Eq.(332) where $\mathrm{H}_{V}$ and $\mathrm{H}_{\Phi}$ denote the flame transfer functions for independent oscillations of the inlet velocity and the equivalence ratio. This concept is illustrated schematically in Figure 153. Physically, Eq.(332) means that the response of a partially premixed flame can be linearly superposed from two independent responses, i.e., $\mathrm{Q}_{\mathrm{V}}$ and $\mathrm{Q}_{\Phi}$. The two-input one-output system representation of the partially premixed flame is valid only when the response of the flame remains in the linear region. The concept of a two-input one-output system was recently introduced by Huber and Polifke. They used it to calculate the response of a partially premixed flame using two flame transfer functions obtained by numerical simulation of the transient fluid dynamics of a turbulent reacting flow. Experimental verification of the principle has not been explored to date. The concept will be experimentally demonstrated and its physical significance will be discussed in what follows. 


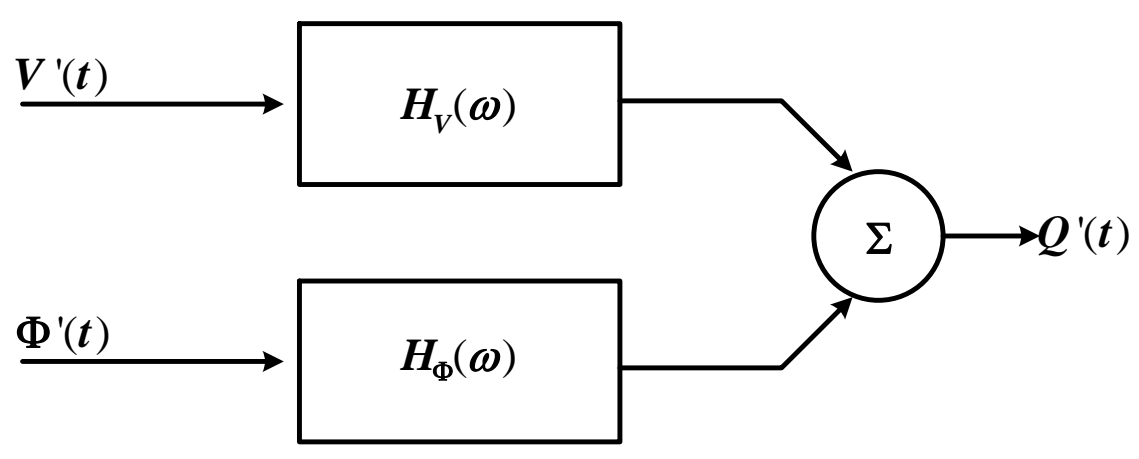

Figure 153: Two input/one output model

Each term in Eq.(332) is a complex quantity. Each has a magnitude and phase with respect to a reference signal. Therefore, the response of the partially premixed flame can be reconstructed by a vector summation, as shown in Figure 81. As was discussed previously, in Figure 81, the two vectors, $\mathrm{Q}_{\mathrm{V}}$ and $\mathrm{Q}_{\Phi}$, denote the heat release response $\left(\mathrm{CH}^{*} / \mathrm{CH}^{*}{ }_{\text {mean }}\right)$ to velocity oscillations and equivalence ratio oscillations, respectively. They were measured separately. The velocity and equivalence ratio oscillations are small enough to ensure that the response of the flame is in the linear regime. The normalized inlet velocity oscillation $\left(\mathrm{V}^{\prime} / \mathrm{V}_{\text {mean }}\right)$ and equivalence ratio oscillation $\left(\Phi^{\prime} / \Phi_{\text {mean }}\right)$ were $10 \%$ and $2.9 \%$, respectively, while the oscillation frequency was 100 $\mathrm{Hz}$. The phase of the individual heat release response, which is represented by the angle between the vector and the $\mathrm{x}$-axis and is equal to the phase of the corresponding oscillation, i.e., $\mathrm{V}^{\prime}$ or $\Phi^{\prime}$. (These were determined from independent velocity forced and equivalence ratio forced experiments.) The resulting summation vector, $\mathrm{Q}_{\mathrm{V}+\Phi}$ in Figure 81, gives the predicted heat release response of the partially premixed flame with the indicated inlet velocity and equivalence ratio oscillations (see the insert for numerical values). Also shown in Figure 81 is a symbol (घ) which represents the actual flame response of a partially premixed flame at the inlet conditions. A simple arithmetic mean of $\varphi_{\mathrm{Q}^{\prime}-\Phi^{\prime}}$ and $\varphi_{\mathrm{Q}^{\prime}-\mathrm{V}^{\prime}}$ was used to calculate the phase of the heat release response, however, since the phase difference between $\mathrm{Q}^{\prime}$ and $\mathrm{V}^{\prime}$ is not necessarily same as the phase difference between $Q^{\prime}$ and $\Phi^{\prime}$. The phase relationship has a strong dependence upon perturbation frequency, fuel injection location, mean nozzle velocity, and fuel injector impedance.

The result presented in Figure 81 provides important insight into the key physical processes related to the heat release response of a partially premixed flame. First of all, this analysis demonstrates that the heat release response of a partially premixed flame can be effectively reconstructed using independent measurements (or numerical simulation) of the flame's response to velocity and equivalence ratio fluctuations. Second, the contribution of equivalence ratio oscillation to the global heat release rate oscillation of the partially premixed flame exceeds that of the velocity fluctuation by a factor of 2 , even though the normalized magnitude of the equivalence ratio oscillation $\left(\Phi^{\prime} / \Phi_{\text {mean }}=0.029\right)$ is much smaller than that of velocity perturbation $\left(\mathrm{V}^{\prime} / \mathrm{V}_{\text {mean }}=0.100\right)$. This indicates that the heat release response of a swirl-stabilized flame is more sensitive to equivalence ratio oscillations than to velocity oscillations. The decomposition of the partially premixed flame transfer function into the transfer functions of the sub-systems demonstrates the relative significance of $\mathrm{Q}_{V}$ and $\mathrm{Q}_{\Phi}$. Third, this result also illustrates that the 
phase difference between the heat release and equivalence ratio oscillations $\left(\varphi_{\Phi}=51.0^{\circ}\right)$ is larger than the phase difference between heat release and inlet velocity oscillations $\left(\varphi_{V}=21.9^{\circ}\right)$, by a factor of approximately two. This indicates that the flame's response time $\left(\tau_{\text {conv, }}\right)$ to equivalence ratio oscillations is longer than the flame's response time $\left(\tau_{\text {conv, }} v\right)$ to velocity oscillations. The heat release response of a partially premixed flame is chemical kineticscontrolled in terms of the response time scales, while the contribution of flame front kinematics induced by oscillating velocity fields is much smaller than the former. This is consistent with CFD results of Huber and Polifke. They reported that the mean time delays for the response to equivalence ratio and velocity fluctuations are about $\tau_{\text {conv, } \Phi}=6 \mathrm{~ms}$ and $\tau_{\text {conv, }} \mathrm{v}=1.8 \mathrm{~ms}$, respectively.

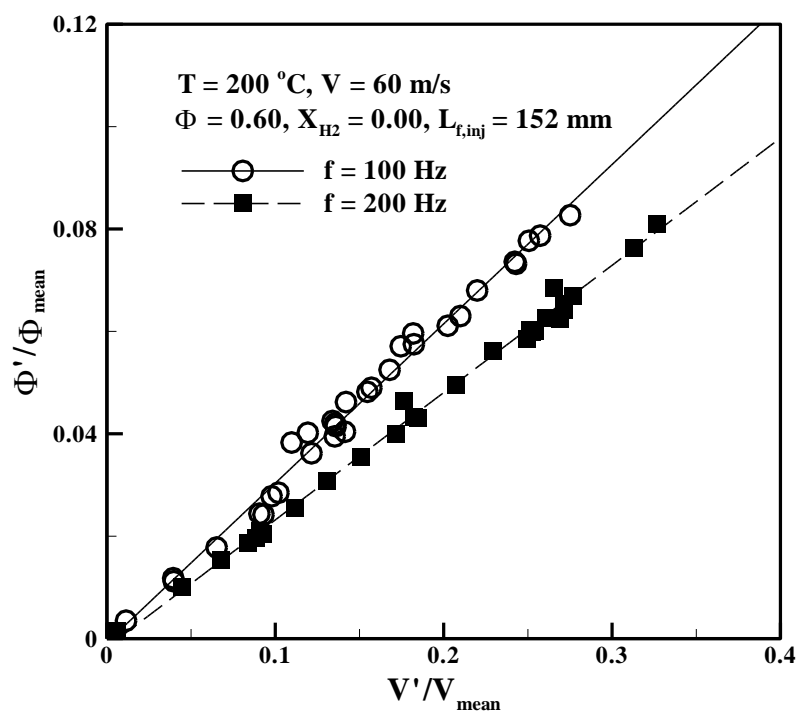

Figure 154: Dependence of the amplitude of equivalence ratio oscillations upon the amplitude of inlet velocity perturbations at a modulation frequency of 100 and $200 \mathrm{~Hz}$. Inlet conditions: $T_{\text {in }}=200{ }^{\circ} \mathrm{C}, V_{\text {mean }}=60 \mathrm{~m} / \mathrm{s}, \Phi_{\text {mean }}=0.60$, and $X_{\mathrm{H} 2}=0.00$.

The (de)composition of the partially premixed flame response by means of a vector summation also indicates that chemiluminescence emission intensity can be used as a semi-quantitative indicator of the heat release rate, when the equivalence ratio oscillation is small. Heuristically, the chemiluminescence intensity has been used to estimate the heat release rate of a premixed flame, inasmuch as at a fixed equivalence ratio, the $\mathrm{CH}^{*}, \mathrm{OH}^{*}$, or $\mathrm{CO}^{*}$ chemiluminescence intensity increases linearly with fuel flow rates. Meanwhile, in the case of fuel modulation $\left(m_{a}^{\prime}=\right.$ 0 ), the amplitude of equivalence ratio oscillation is directly related to oscillation of the fuel mass flow rate through Eq.(338).

Accordingly, measured chemiluminescence intensity represents the fluctuations of the heat release rate induced by fuel flow rate modulation. For a partially premixed case, fuel is injected in the mixing section, while preheated air is modulated. In spite of the choked fuel flow at the fuel injection nozzle, the equivalence ratio oscillates with respect to time, due to air mass flow rate oscillations at that location. Different physiochemical processes are related to the fluctuations of $\mathrm{CH}^{*}$ chemiluminescence intensity, but the analysis using the vector summation 
indicates that the heat release response of a partially premixed flame can be estimated by the chemiluminescence-based measurement technique, unless the heat release response of the partially premixed flame is nonlinearly related to the amplitude of inlet disturbances.

In addition, the results shown in Figure 81 suggest that the heat release response of a partially premixed flame in the linear regime is governed by four physical parameters: frequency, amplitude of inlet velocity oscillation, amplitude of inlet equivalence ratio oscillation, and phase difference between $\mathrm{V}^{\prime}$ and $\Phi^{\prime}$ at the inlet of the combustor. The amplitudes of the velocity and equivalence ratio oscillations are not independent. In fact, they are almost linearly related, as shown in Figure 154. Figure 154 plots the dependence of the normalized amplitude of the equivalence ratio oscillation versus the amplitude of the velocity oscillation at modulation frequencies of 100 and $200 \mathrm{~Hz}$. This relationship is due to the fact that the fuel is choked at the fuel injector and the magnitude of the equivalence ratio at the fuel injector is determined by the magnitude of the air mass flow rate at that location. It is important to note that the normalized magnitude of the equivalence ratio oscillation is one order-of-magnitude smaller than that of the inlet velocity oscillation. Theoretically, they should be of the same order of magnitude, with $180^{\circ}$ phase difference at the fuel injection location, but turbulent mixing plays a significant role in dissipating the magnitude of the equivalence oscillation at the combustor inlet. Note that fuel is injected $152 \mathrm{~mm}$ upstream of the combustor dump plane. Figure 154 also shows that the magnitude of the equivalence ratio oscillation at a frequency of $200 \mathrm{~Hz}$ is smaller than at a frequency of $100 \mathrm{~Hz}$. This can be attributed to the influence of the oscillation frequency on the fine scale mixing process.

The vector plots presented in Figure 81 further suggest that depending on the phase difference between the velocity and equivalence ratio oscillations, the response of a partially premixed flame can be reconstructed both constructively and destructively, as illustrated in Figure 155. Due to the convective-acoustic characteristics of the inlet disturbances, the phase difference between the velocity and equivalence ratio oscillations at the combustor inlet, $\Delta \varphi_{\Phi^{\prime}-\mathrm{V}^{\prime}}$, is a function of modulation frequency, fuel injection location, fuel injector impedance, and mean velocity in the nozzle. This phase difference plays a critical role in determining the linear and non-linear response of a partially premixed flame. The dynamics of a partially premixed flame subjected to in-phase and out-of-phase interactions between $V^{\prime}$ and $\Phi^{\prime}$ will be presented and discussed using flame transfer function measurements and phase-locked flame imaging measurements. 

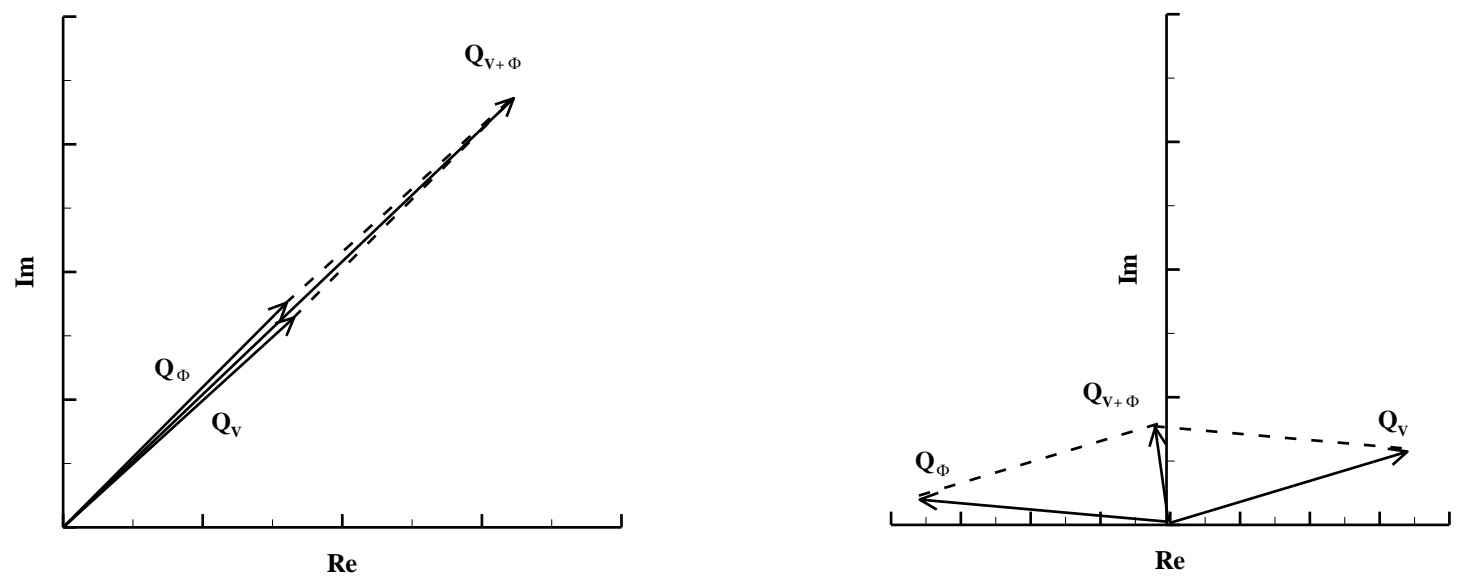

Figure 155: Constructive and destructive reconstruction of the response of a partially premixed flame.

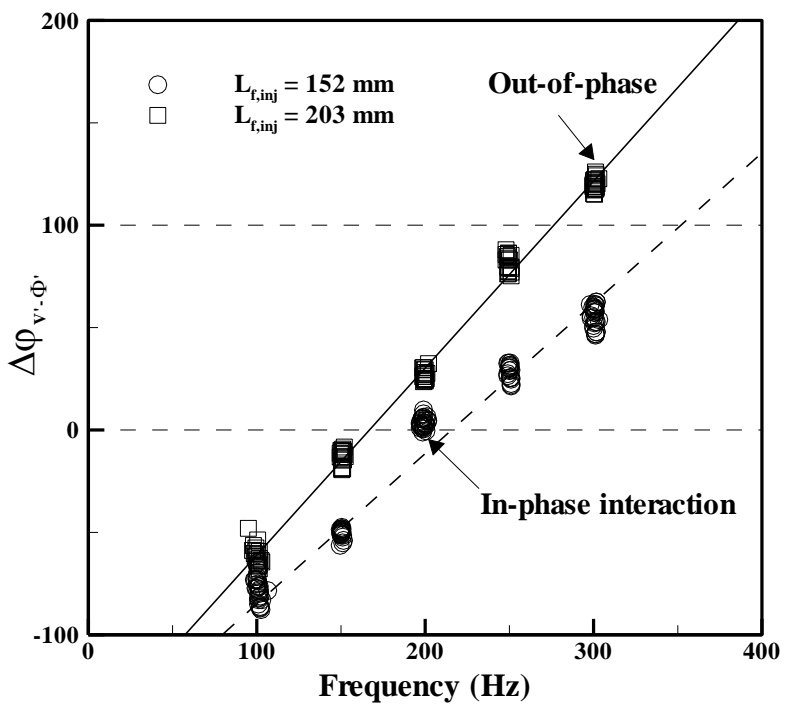

Figure 156: Phase difference between acoustic velocity and equivalence ratio at the combustor inlet as a function of modulation frequency for two fuel injection locations of $L_{\mathrm{f}, \mathrm{inj}}=152 \mathrm{~mm}$ and $203 \mathrm{~mm}$. Inlet conditions: $T_{\text {in }}=200{ }^{\circ} \mathrm{C}, \mathrm{V}_{\text {mean }}=60 \mathrm{~m} / \mathrm{s}, \Phi_{\text {mean }}=0.60$, $\mathrm{X}_{\mathrm{H} 2}=0.60$, and $\mathrm{V}^{\prime} / \mathrm{V}_{\text {mean }}=\min \sim \max$. 


\section{Flame Dynamics}

Oscillations of the equivalence ratio and velocity near the combustor inlet were measured using the IR absorption technique and the two-microphone method at modulation frequencies between $100 \sim 300 \mathrm{~Hz}$ and for normalized velocity oscillations from a minimum of $\sim 5 \%$ to a maximum of $\sim 50 \%$. Figure 156 shows the phase difference between the acoustic velocity and equivalence ratio oscillations at the combustor inlet as a function of modulation frequency for two fuel injection locations $(152 \mathrm{~mm}$ and $203 \mathrm{~mm}$ ). It can be observed that the phase difference between the velocity and equivalence ratio oscillations increases almost linearly with increasing modulation frequency, and it is also affected by the fuel injection location, as discussed in the previous section. The amplitude of the oscillation does not, however, influence the phase difference: each overlapped symbol at a fixed frequency corresponds to 30 different forcing amplitudes. Note that when fuel is injected $152 \mathrm{~mm}$ upstream of the combustor inlet and is modulated at a frequency of $200 \mathrm{~Hz}$, the phase difference between acoustic velocity and equivalence ratio oscillations is nearly zero. Physically, this condition indicates that unburned reactant mixtures with high equivalence ratio enter the combustion chamber at high inlet velocity, possibly leading to a large fluctuation of the heat release rate.

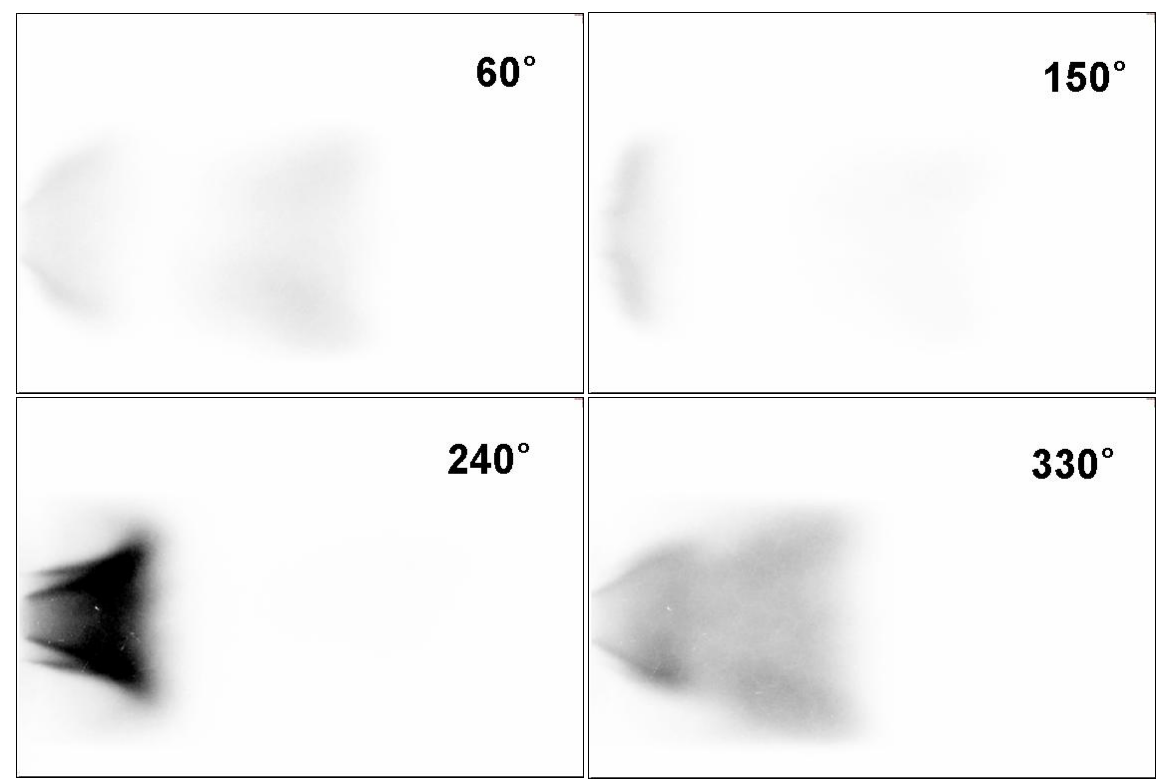

Figure 157: Phase-synchronized $\mathrm{CH}^{*}$ chemiluminescence images at a modulation frequency of $200 \mathrm{~Hz}$ and $\mathrm{V}^{\prime} / \mathrm{V}_{\text {mean }}=42 \%$. The inverted gray scale is linear from black to white, denoting the highest and the lowest chemiluminescence intensity. Inlet conditions: $T_{\text {in }}=200{ }^{\circ} \mathrm{C}, V_{\text {mean }}=60 \mathrm{~m} / \mathrm{s}, \Phi=0.60, X_{\mathrm{H} 2}=0.60$, and $L_{f, i n j}=152 \mathrm{~mm}$.

The effect of the in-phase interaction between the velocity and equivalence ratio oscillations on the flame dynamics is shown in Figure 157. This figure shows phase-synchronized $\mathrm{CH}^{*}$ chemiluminescence images at a modulation frequency of $200 \mathrm{~Hz}$ and a normalized velocity oscillation of $42 \%$ at different phase angles of $60,150,240$, and $330^{\circ}$. It is evident at the phase angle of $330^{\circ}$ that the flame starts to divide into two regions, as the upstream part of the flame moves in the direction of increasing flame angle. In the middle of the projected reaction zone, a thin local region with weak $\mathrm{CH}^{*}$ chemiluminescence intensity is observed; the reaction zone 
appears to be divided in two. At a phase-angle of $60^{\circ}$, a continuous reaction region is not sustained, and consequently unsteady local extinction occurs in the middle of the flame area. The upstream part of the flame moves further upstream, while the downstream part of the flame is convected at the mean flow and is eventually extinguished. At a phase-angle of $150^{\circ}$, the $\mathrm{CH}^{*}$ chemiluminescence intensity is very weak and the flame structure exhibits a very large flame angle. The heat release reaches its peak at the phase-angle of $240^{\circ}$ when reactants with high equivalence ratio reach the flame front. The in-phase interaction of acoustic velocity and equivalence ratio oscillations results in the unsteady destruction of flame surface area, leading to the nonlinear response of the partially premixed flame. Figure 158 plots the gain of the flame transfer function as a function of the amplitude of the inlet velocity oscillation at the same inlet conditions. This result confirms that local extinction contributes to the nonlinear behavior of the partially premixed $\mathrm{H}_{2}$-enriched "M" shaped flame.

Changing the fuel injection location influences the convection delay time needed for equivalence ratio oscillation to convect from the fuel injection location to the flame's center-of-heat-release. This in turn modifies the phase difference between velocity and equivalence ratio oscillations at the combustor inlet. As illustrated in Figure 156, when the fuel injection location is changed from $152 \mathrm{~mm}$ to $203 \mathrm{~mm}$ and air is modulated at a frequency of $300 \mathrm{~Hz}$, the phase difference between the two oscillations is approximately $120^{\circ}$. Phase-synchronized $\mathrm{CH}^{*}$ chemiluminescence images during a period of oscillation are presented in Figure 159. Significant differences are observed between in-phase and out-of-phase relationships in terms of instantaneous flame shape, chemiluminescence intensity variation, and measured flame transfer functions. Localized flame extinction is not seen, and the oscillation in angle direction is dominant for the "M" shaped flame. In the case of out-of-phase interaction, $\mathrm{CH}^{*}$ chemiluminescence intensity does not vary significantly during an oscillation cycle, since reactants with high equivalence ratio enter the combustion chamber with low velocity and reactants with low equivalence ratio reach the flame front with high inlet velocity. As a result, the flame "meets" almost the same amount of fuel per unit time.

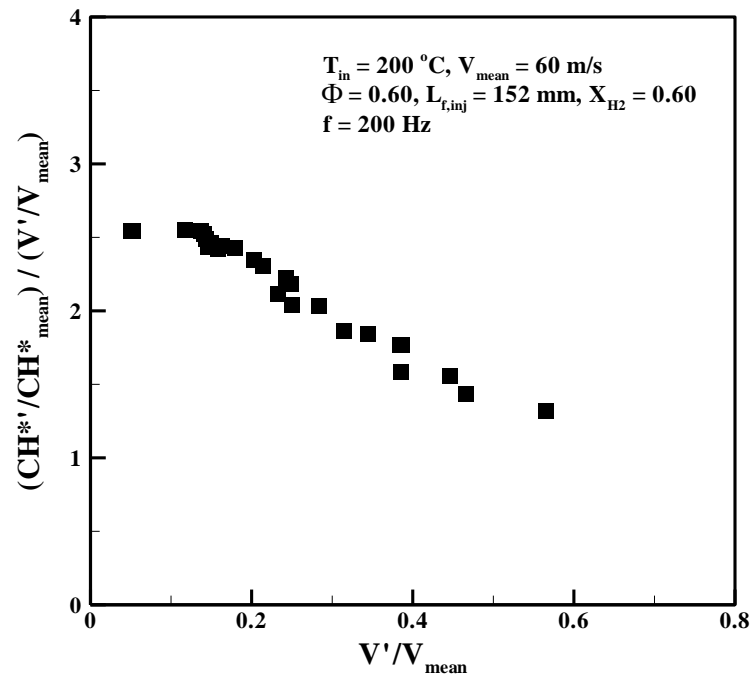

Figure 158: Gain of the flame transfer function plotted as a function of the forcing amplitude. Inlet conditions: $T_{\text {in }}=200{ }^{\circ} \mathrm{C}, V_{\text {mean }}=60 \mathrm{~m} / \mathrm{s}, \Phi_{\text {mean }}=0.60, X_{\mathrm{H} 2}=0.60, L_{f, i n j}=$ $152 \mathrm{~mm}$, and $f=200 \mathrm{~Hz}$. 


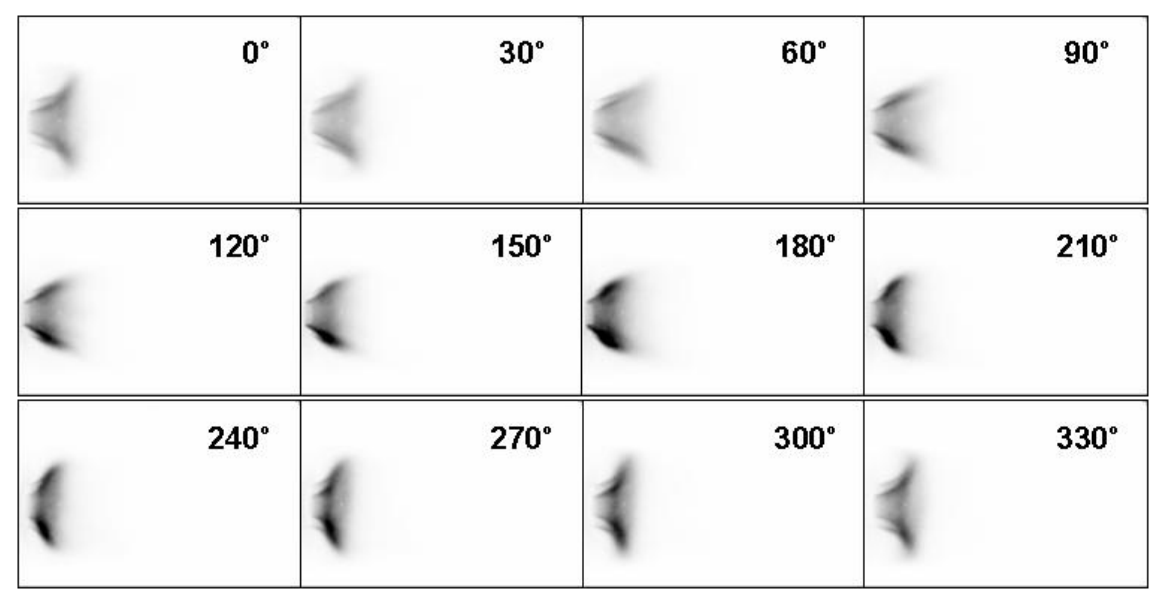

Figure 159: Phase-synchronized $\mathrm{CH}^{*}$ chemiluminescence images at a modulation frequency of $300 \mathrm{~Hz} \mathrm{~V}^{\prime} / \mathrm{V}_{\text {mean }}=27 \%$. The inverted gray scale is linear from black to white, denoting the highest and the lowest chemiluminescence intensity. Inlet conditions: $T_{\text {in }}=200$ ${ }^{\circ} \mathrm{C}, \mathrm{V}_{\text {mean }}=60 \mathrm{~m} / \mathrm{s}, \Phi_{\text {mean }}=0.60, X_{\mathrm{H} 2}=0.60$, and $L_{f, i n j}=203 \mathrm{~mm}$.

Figure 160 shows the dependence of the flame transfer function gain on modulation frequency for a range normalized velocity oscillations, from $\mathrm{V}^{\prime} / \mathrm{V}_{\text {mean }}=0.03$ to 0.50 . This result shows that the response of the flame is at its maximum at a frequency of $150 \mathrm{~Hz}$, at which point the phase difference between the velocity and equivalence ratio oscillations is almost zero. At frequencies in the range of $100 \sim 200 \mathrm{~Hz}$, the nonlinear response is apparent, in that the heat release response decreases with oscillation amplitude. The response of the partially premixed flame remains in the linear regime when $\mathrm{f}>250 \mathrm{~Hz}$. This observation is important because it is well documented that the nonlinear response of premixed flames to velocity oscillations becomes more dominant as the oscillation frequency increases; this is related to the frequency-dependent response characteristic of shear layers. As illustrated in Figure 160, the behavior of the partially premixed flame does not agree with previous observations about premixed flame dynamics. 


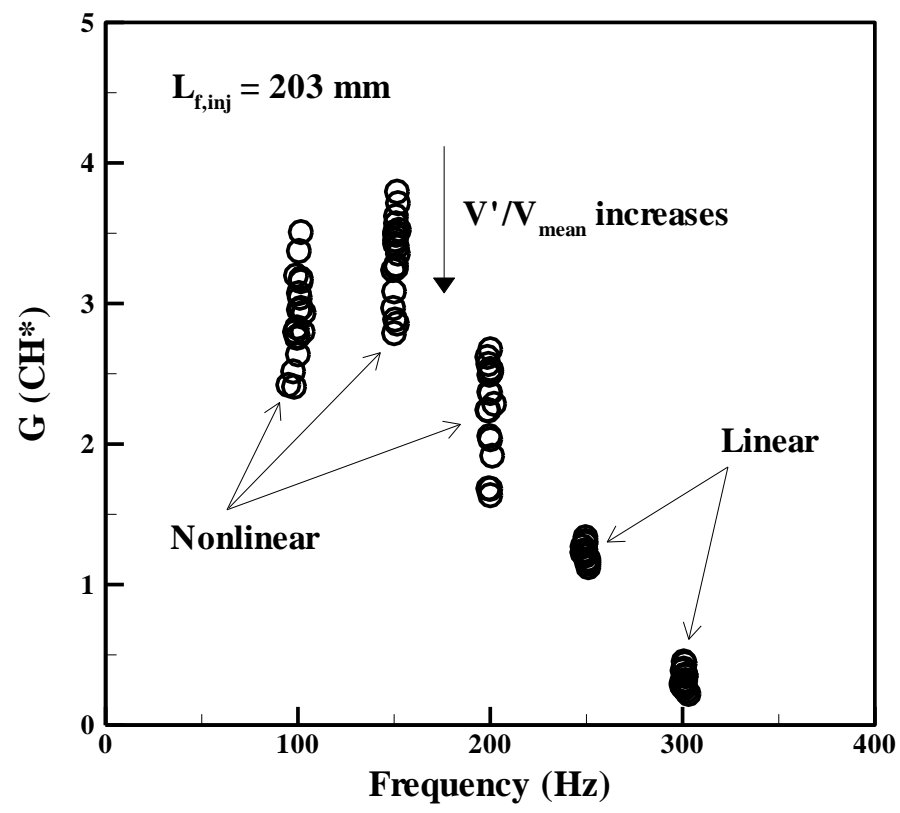

Figure 160: The dependence of the gain of the flame transfer function on modulation frequency for a range of magnitude of inlet velocity oscillations, $V^{\prime} / V_{\text {mean }}=0.03 \sim 0.50$. Inlet conditions: $T_{\text {in }}=200{ }^{\circ} \mathrm{C}, V_{\text {mean }}=60 \mathrm{~m} / \mathrm{s}, \Phi_{\text {mean }}=0.60, X_{\mathrm{H} 2}=0.60$, and $L_{\text {f,inj }}=203 \mathrm{~mm}$.

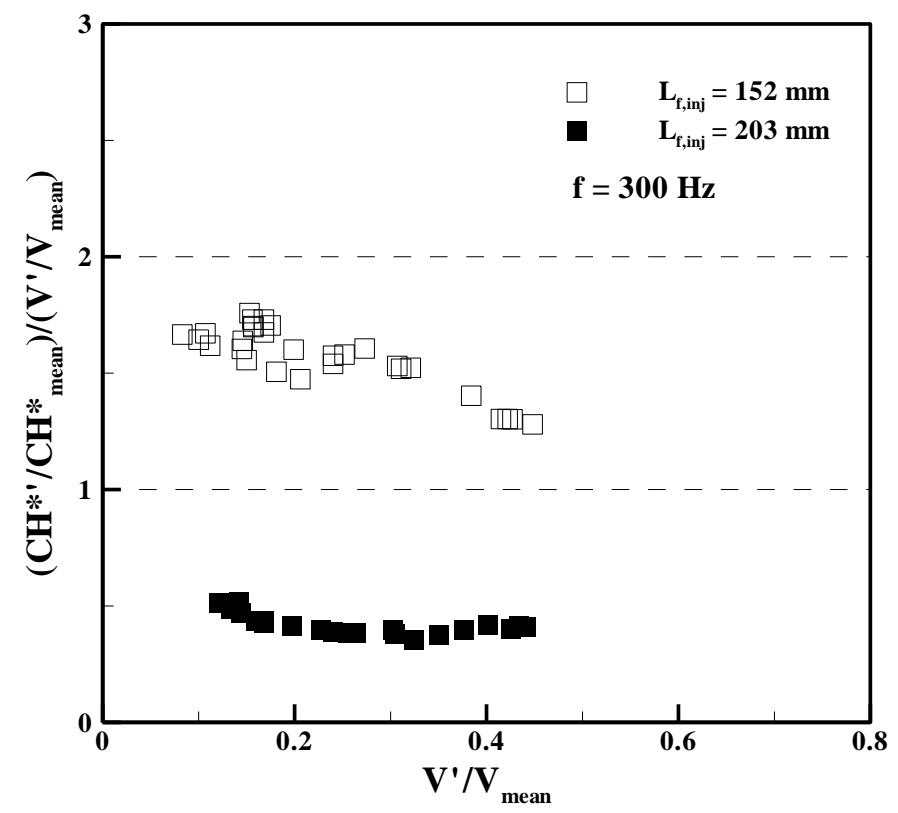

Figure 161: The dependence of the gain of the flame transfer function on magnitude of inlet velocity oscillations at a modulation frequency of $300 \mathrm{~Hz}$ and for fuel injection locations of $L_{\mathrm{f}, \mathrm{inj}}=152 \mathrm{~mm}$ and $203 \mathrm{~mm}$. Inlet conditions: $T_{\mathrm{in}}=200{ }^{\circ} \mathrm{C}, \mathrm{V}_{\text {mean }}=60 \mathrm{~m} / \mathrm{s}, \Phi_{\text {mean }}=0.60$, $\mathrm{X}_{\mathrm{H} 2}=0.60$, and $\mathrm{V}^{\prime} / \mathrm{V}_{\text {mean }}=\min \sim \max$. 
The out-of-phase relationship between the velocity and equivalence ratio oscillations yields very low gain of the flame transfer function, as illustrated in Figure 161. The ratio of the normalized heat release oscillation to the normalized inlet velocity oscillations close to 0.5 for all oscillation amplitudes investigated. The flame acts to damp the flow oscillations. The flame transfer function gain is nearly constant with respect to the oscillation amplitude, representing a linear flame response even at a normalized velocity oscillation of $45 \%$, as mentioned previously. In contrast, the response of a partially premixed flame at the fuel injection location of $152 \mathrm{~mm}$ is larger than unity and it is nonlinear at the same inlet condition. Several inlet parameters affecting the phase difference between the velocity and equivalence ratio oscillations, such as frequency, mean velocity, fuel injection location, and fuel injector impedance, were systematically investigated to study the role of the parameters on the response of the partially premixed flame. It was found that the nonlinear response of " $\mathrm{M}$ " shaped partially premixed flames manifested by unsteady local extinction takes place only when the condition, $\Delta \varphi_{\Phi^{\prime}-\mathrm{V}^{\prime}} \approx 0^{\circ}$, is met.

\section{Review}

As a review, the present investigation describes a method for (de)composing the heat release response of a partially premixed swirl flame using a vector summation method, in which each vector represents magnitude and phase of the heat release oscillation with respect to perturbations of acoustic velocity and equivalence ratio in the complex domain. The decomposition method provides an improved understanding of the behavior of partially premixed flames. In terms of both magnitude and response time, the contribution of equivalence ratio oscillations to the global heat release response of partially premixed flames is more significant relative to that of acoustic velocity fluctuations. The present results suggest that the partially premixed flame response is governed by the superposition of magnitude of velocity oscillation, magnitude of equivalence ratio fluctuation, and the phase difference between the two inlet disturbances. In particular, the importance of the phase difference between $\mathrm{V}^{\prime}$ and $\Phi^{\prime}$ was demonstrated. When fuel rich mixture pockets enter the combustion chamber with high inlet velocity, i.e., in the in-phase interaction, the peak-to-peak variations of heat release rate are substantial during a single cycle of oscillation, and are typified by unsteady local extinction of flame surface area. The rapid reduction of flame area induces the nonlinear response. In contrast, the out-of-phase interaction between $\mathrm{V}^{\prime}$ and $\Phi^{\prime}$ disturbances significantly reduces the heat release response of partially premixed flames, so that the flame transfer function gain is less than unity at all perturbation levels investigated. In the present section of the report, the spatial distribution of equivalence ratio is excluded, in an attempt to simplify the given problem, and therefore only the response of swirl-stabilized flames to temporal oscillation of the mixture ratio nonhomogeneities is considered. 


\section{Aside - Effect of Azimuthal Flame Stretch}

As an aside, here we aim to justify neglecting azimuthal flame stretch effects while calculating transfer functions in the case of equivalence ratio coupled flame response. Analogous to the case of velocity-coupled flame response for an axisymmetric wedge flame as dealt with by Preetham et. al., we shall show that azimuthal curvature does not largely alter the flame shape (and hence response).

Writing the flame front location as a perturbation about the mean flame, we have:

$$
\xi(r, t)=\xi_{o}(r)+\varepsilon \xi_{1}(r, t)
$$

An equation may be written for the Fourier transform of the perturbation at the forcing frequency, $\zeta_{1}(r, S t)=\hat{\xi}_{1}$ as follows:

$$
\sigma_{c} \frac{d^{2} \zeta_{1}}{d r^{2}}+\left(\frac{\sigma_{c}}{r}-\alpha\right) \frac{d \zeta}{d r}-i S t \zeta=e^{-i(1-r) S t}
$$

This equation is solved numerically with the fixed-anchor and symmetry boundary conditions:

$$
\begin{aligned}
& \zeta_{1}(r=1)=0 \\
& \frac{d \zeta_{1}}{d r}(r=0)=0
\end{aligned}
$$

A representative result is provided for the case of $S t=10$ in Figure 162.

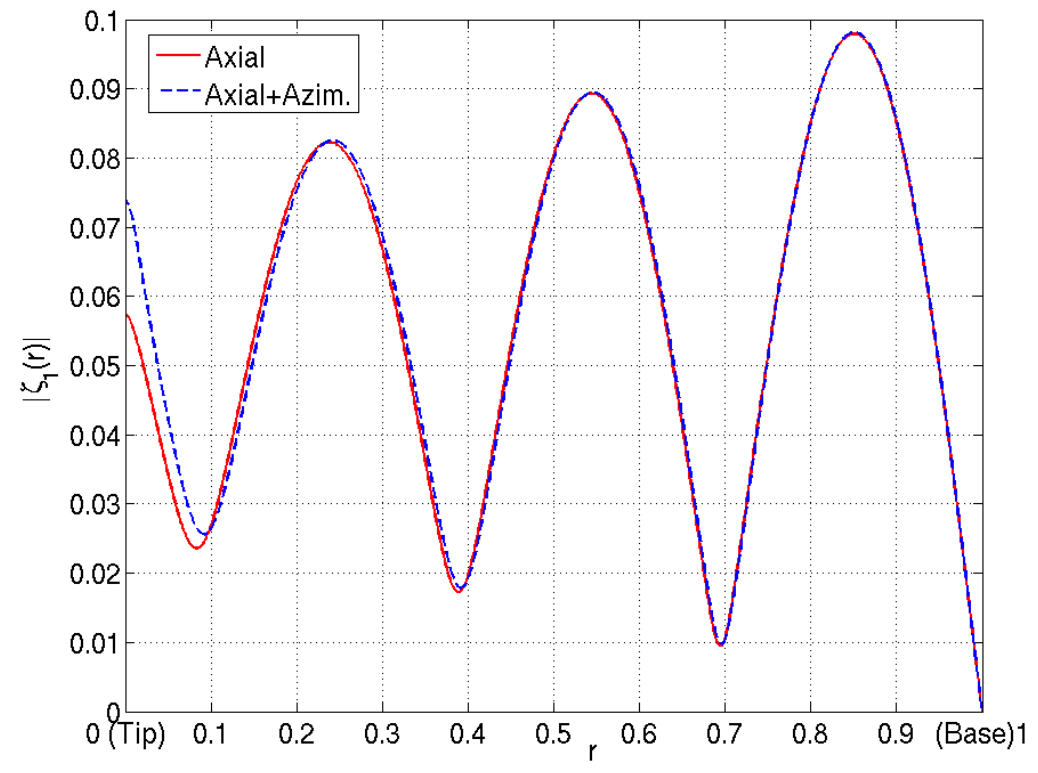

Figure 162: Variation of the response amplitude of perturbation about the mean flame for a conical flame with $\beta=4.0, M a=1, \delta R=0.1$ at a forcing Strouhal number of $S t=10$. 
It may be clearly seen that the effect of azimuthal stretch is seen only at the flame tip and that over most of the flame, there is no significant influence of azimuthal stretch. Hence, it seems reasonable to neglect azimuthal stretch effects while solving Eq.(340). To evaluate the importance of azimuthal stretch with increasing forcing Strouhal number, a comparison of orders of azimuthal stretch effects to axial stretch effects may be carried out as follows:

$$
\frac{\kappa_{a z i}}{\kappa_{a x}} \sim \frac{\sigma_{c}(1 / r)(\partial \zeta / \partial r)}{\sigma_{c}\left(\partial^{2} \zeta / \partial r^{2}\right)} \sim \frac{\lambda_{c} \sin \psi}{r}
$$

Here, $\lambda_{c}$ is the convective wavelength that reduces with increasing Strouhal number and $\psi$ is the flame half-angle, given by $\cot \psi=\alpha$. Expressing $\lambda_{c}$ in terms of the flame height, burner radius and flame half-angle, Eq.(342) can be simplified to yield:

$$
\frac{\kappa_{a z i}}{\kappa_{a x i a l}} \sim \frac{\alpha}{r} \cdot \frac{1}{S t}
$$

Hence, it can be seen from Eq.(343) that the influence of azimuthal stretch decreases as frequency increases.

\section{Response of Lean Premixed Flames to Acoustic Velocity Oscillations}

\section{Introduction}

Lastly, the effects of swirl angle on steady-state flame structure and the response of lean premixed flames to acoustic velocity perturbations were experimentally investigated. The twomicrophone technique and $\mathrm{CH}^{*}$ chemiluminescence intensity measurements were used to determine the input (inlet velocity perturbation) and the output functions (heat release oscillation) of the flame transfer function, respectively. For stable flame image measurements, an ICCD camera (Princeton Instruments model 576G) with a $\mathrm{CH}^{*}$ band pass filter centered at 430 $\mathrm{nm}\left(10 \mathrm{~nm}\right.$ FWHM) was used. It was found that an increase in swirl angle from $30^{\circ}$ to $45^{\circ}$ has a significant impact on flame structure, particularly, flame angle. The modification of steady state flame geometry consequently affects the response of the swirl stabilized flame to upstream perturbations. As swirl angle increases, the magnitude of the flame response decreases due to enhanced flame stiffness and convection time decrease at a constant effective flame length. In the next section, experimental results are presented and discussed.

\section{Effects of Swirl Angle on Steady-State Flame Structure}

Figure 163(A) plots the X-Y coordinates of the location of maximum $\mathrm{CH}^{*}$ chemiluminescence intensity for a broad range of inlet conditions: $\mathrm{T}_{\text {in }}=200,300{ }^{\circ} \mathrm{C}, \mathrm{V}_{\text {mean }}=60,70,80,90,100 \mathrm{~m} / \mathrm{s}$, $\mathrm{X}_{\mathrm{H} 2}=0.00,0.15,0.30,0.45,0.60, \Phi=0.55,0.60,0.65,0.70$, and $\alpha_{\mathrm{sw}}=30,45^{\circ}$. It is evident that as the swirl angle increases from $30^{\circ}$ to $45^{\circ}$, $\mathrm{X}-\mathrm{Y}$ coordinates of maximum $\mathrm{CH}^{*}$ 
chemiluminescence intensity locations, the center of heat release, are characterized by the different trajectories. In particular, the change in flame angle is distinct in the near field of the reaction region. The dependence of the modification of the swirl angle upon the flame angle is presented in Figure 163(B). It clearly shows that when $\mathrm{L}_{\mathrm{CH}^{*} \max }$ is less than $60 \mathrm{~mm}$, the two curves diverge. Flame angle is larger for $45^{\circ}$ swirler than when $30^{\circ}$ swirler is mounted, due to changes in the flow fields in the combustion chamber. As the effective flame length $\left(\mathrm{L}_{\mathrm{CH}^{*} \max }\right)$ decreases, the flame angle approaches to $45^{\circ}$ which is consistent with the swirl angle of the axial swirler.

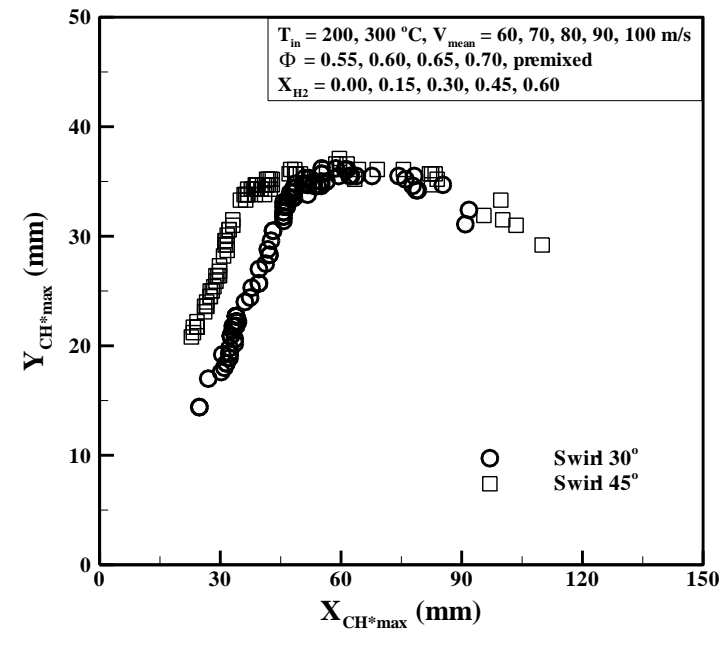

(A)

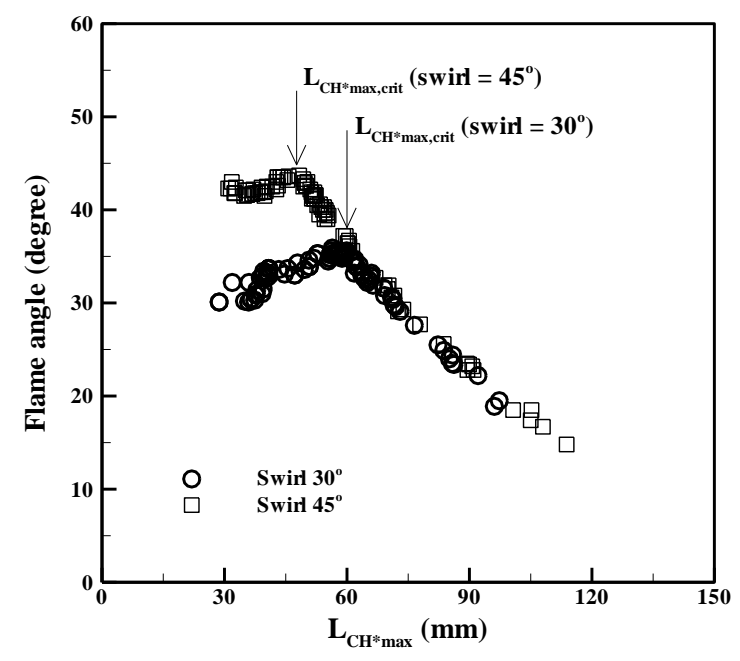

(B)

Figure 163: (A) $\mathrm{X}-\mathrm{Y}$ coordinates of maximum $\mathrm{CH}^{*}$ chemiluminescence intensity locations and (B) Flame angle as a function of effective flame length, $\mathrm{L}_{\mathbf{C H} \text { max }}$ for $30^{\circ}$ and $45^{\circ}$ swirlers. Inlet conditions: $T_{\text {in }}=200,300{ }^{\circ} \mathrm{C}, \mathrm{V}_{\text {mean }}=60,70,80,90,100 \mathrm{~m} / \mathrm{s}$, perfectly premixed, $X_{\mathrm{H} 2}=0.00,0.15,0.30,0.45,0.60$, and $\Phi=0.55,0.60,0.65,0.70$.

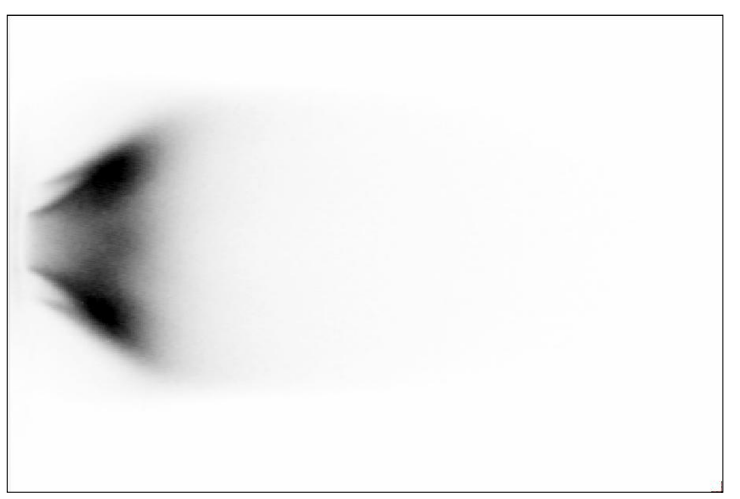

(A) $\boldsymbol{\alpha}_{\mathrm{sw}}=\mathbf{3 0}^{\circ}$

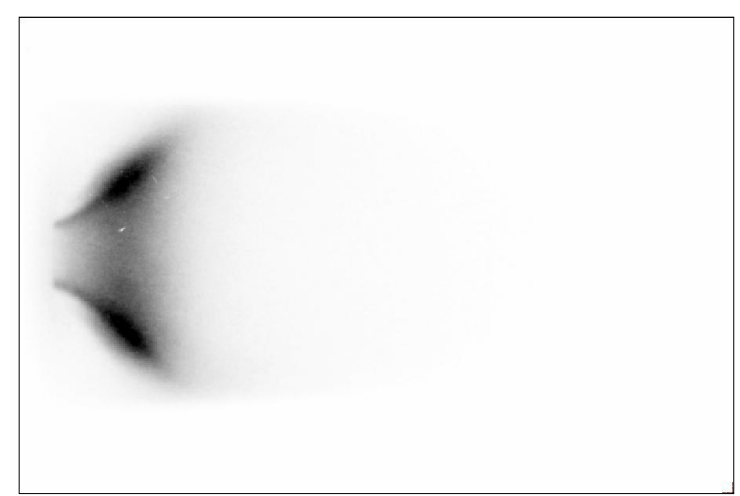

(B) $\alpha_{\mathrm{sw}}=\mathbf{4 5}^{\circ}$

Figure 164: Line-of-sight integrated $\mathrm{CH}^{*}$ chemiluminescence images for $(\mathrm{A}) \boldsymbol{\alpha}_{\mathrm{sw}}=30^{\circ}$ and (B) $\alpha_{\mathrm{sw}}=45^{\circ}$. Inlet conditions: $T_{\text {in }}=200{ }^{\circ} \mathrm{C}, V_{\text {mean }}=60 \mathrm{~m} / \mathrm{s}, X_{\mathrm{H} 2}=0.60$, and $\Phi=0.70$. 
Figure 164 shows two compact flame images $\left(\mathrm{CH}^{*}\right.$ chemiluminescence) for $\alpha_{\mathrm{sw}}=30^{\circ}$ and $45^{\circ}$ at the same inlet conditions: $\mathrm{T}_{\mathrm{in}}=200{ }^{\circ} \mathrm{C}, \mathrm{V}_{\text {mean }}=60 \mathrm{~m} / \mathrm{s}, \mathrm{X}_{\mathrm{H} 2}=0.60$, and $\Phi=0.70$. It shows that as swirl angle changes from $30^{\circ}$ to $45^{\circ}$, flame's diverging angle increases and the thickness of the flame brush (thickness of the reaction zone in the radial direction) becomes thinner. This indicates that in the case of the flame with $\alpha_{\mathrm{sw}}=45^{\circ}$, the intense reaction occurs in more compact region than its counterpart does. This is attributed to the fact that the turbulent intensity is enhanced as swirl angle increases. Quantitative interpretation about the effect of swirl angle on stable flame structure will be presented for various equivalence ratios next.

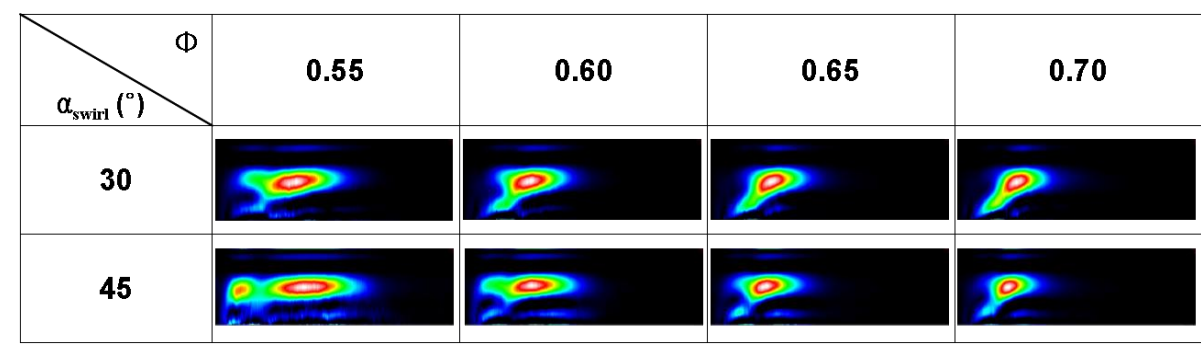

(A) $\quad V_{\text {mean }}=90 \mathrm{~m} / \mathrm{s}$

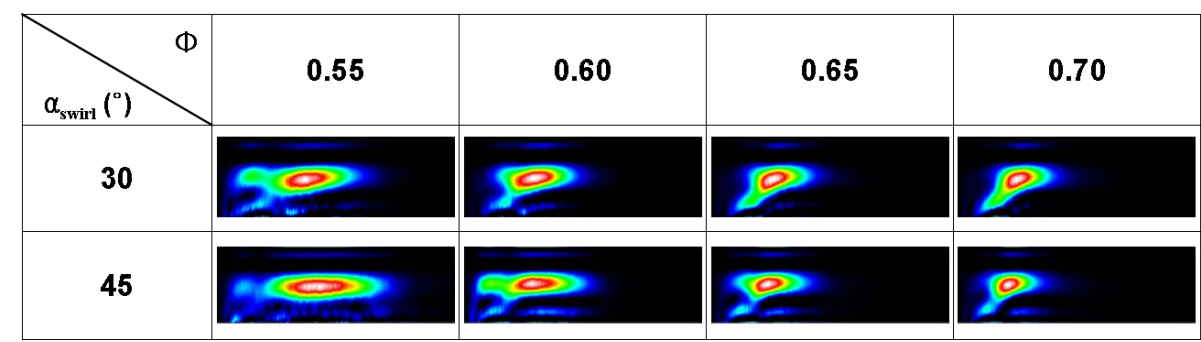

(B) $\quad V_{\text {mean }}=100 \mathrm{~m} / \mathrm{s}$

Figure 165: Dependence of steady-state $\mathrm{CH}^{*}$ chemiluminescence images upon swirl angle $\left(\alpha_{\mathrm{sw}}=30^{\circ}\right.$ and $\left.45^{\circ}\right)$ : (A) $\mathrm{V}_{\text {mean }}=90 \mathrm{~m} / \mathrm{s}$ and $(B) V_{\text {mean }}=100 \mathrm{~m} / \mathrm{s}$. Inlet conditions: $T_{\text {in }}=300$ ${ }^{\circ} \mathrm{C}, \mathrm{V}_{\text {mean }}=90,100 \mathrm{~m} / \mathrm{s}, \mathrm{X}_{\mathrm{H} 2}=0.00$, and $\Phi=0.55,0.60,0.65,0.70$. 


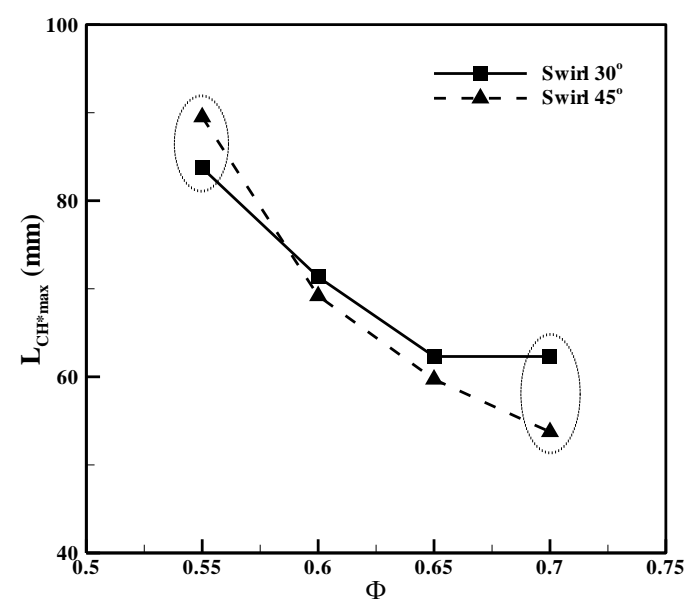

(A) $\mathrm{V}_{\text {mean }}=90 \mathrm{~m} / \mathrm{s}$

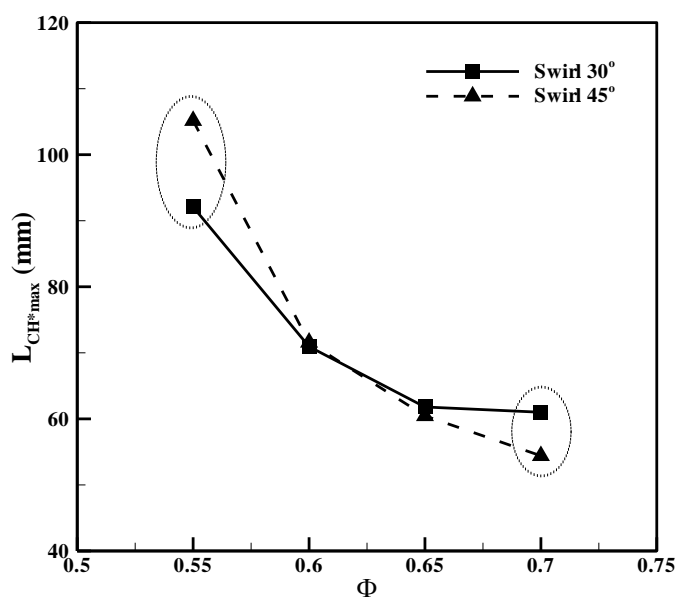

(B) $\mathrm{V}_{\text {mean }}=100 \mathrm{~m} / \mathrm{s}$

Figure 166: Dependence of effective flame length $\left(\mathrm{L}_{\mathrm{CH}^{*} \max }\right)$ upon mean equivalence ratio for two swirl angles of $\alpha_{\mathrm{sw}}=30^{\circ}$ and $45^{\circ}$ : (A) $V_{\text {mean }}=90 \mathrm{~m} / \mathrm{s}$ and (B) $V_{\text {mean }}=100 \mathrm{~m} / \mathrm{s}$. Inlet conditions: $T_{\text {in }}=300{ }^{\circ} \mathrm{C}, V_{\text {mean }}=90,100 \mathrm{~m} / \mathrm{s}, X_{\mathrm{H} 2}=0.00$, and $\Phi=0.55,0.60,0.65,0.70$.

Figure 165 shows the dependence of steady-state $\mathrm{CH}^{*}$ chemiluminescence images upon swirl angle for a range of mean equivalence ratio, $0.55 \sim 0.70$. In these deconvoluted images, only the upper half is shown because the reconstructed images are axisymmetric. The flow direction is from left to right. For a given inlet velocity, $V_{\text {mean }}=90 \mathrm{~m} / \mathrm{s}$, the flame length for $45^{\circ}$ swirler is greater than that of $30^{\circ}$ swirler when equivalence ratio is less than 0.60 , or vice versa. The effective flame length for those images is quantitatively compared in Figure 166 for mean inlet velocities of 90 and $100 \mathrm{~m} / \mathrm{s}$, which correspond to the $\mathrm{CH}^{*}$ chemiluminescence images illustrated in Figure 165. The result shows that the rate of change of the effective flame length with respect to mean equivalence ratio is larger for the swirl angle of $45^{\circ}$ in comparison with that of $30^{\circ}$ swirler. It can be mathematically expressed as the following equation:

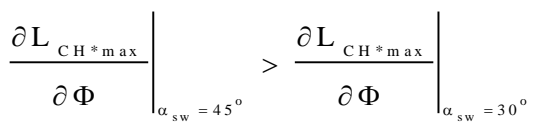

This could be due to the fact that the rate of change of velocity with respect to flow direction is greater for $45^{\circ}$ swirler, since fluid elements in the annular jet region reaches the combustor wall earlier than its counterpart does (see Figure 163(A)). It is well documented that the flame becomes more compact for the high swirler number, because of the enhanced flame speed resulting from the increased turbulent intensity. However, experimental data presented in Figure 165 and Figure 166 demonstrate that the effect of swirl angle on the flame length is also associated with mean equivalence ratio, and that the argument is only valid when the flame does not impinge on the wall of the combustion chamber. If the flame impinges on the combustor wall, the rate of heat transfer increases and also the reaction rates could be reduced by the flamewall interaction. When the equivalence ratio is less than 0.60 , therefore, a larger flame area is needed to completely burn the incoming reactants for higher swirler number cases, although the turbulent intensity increases due to the swirl effect. 

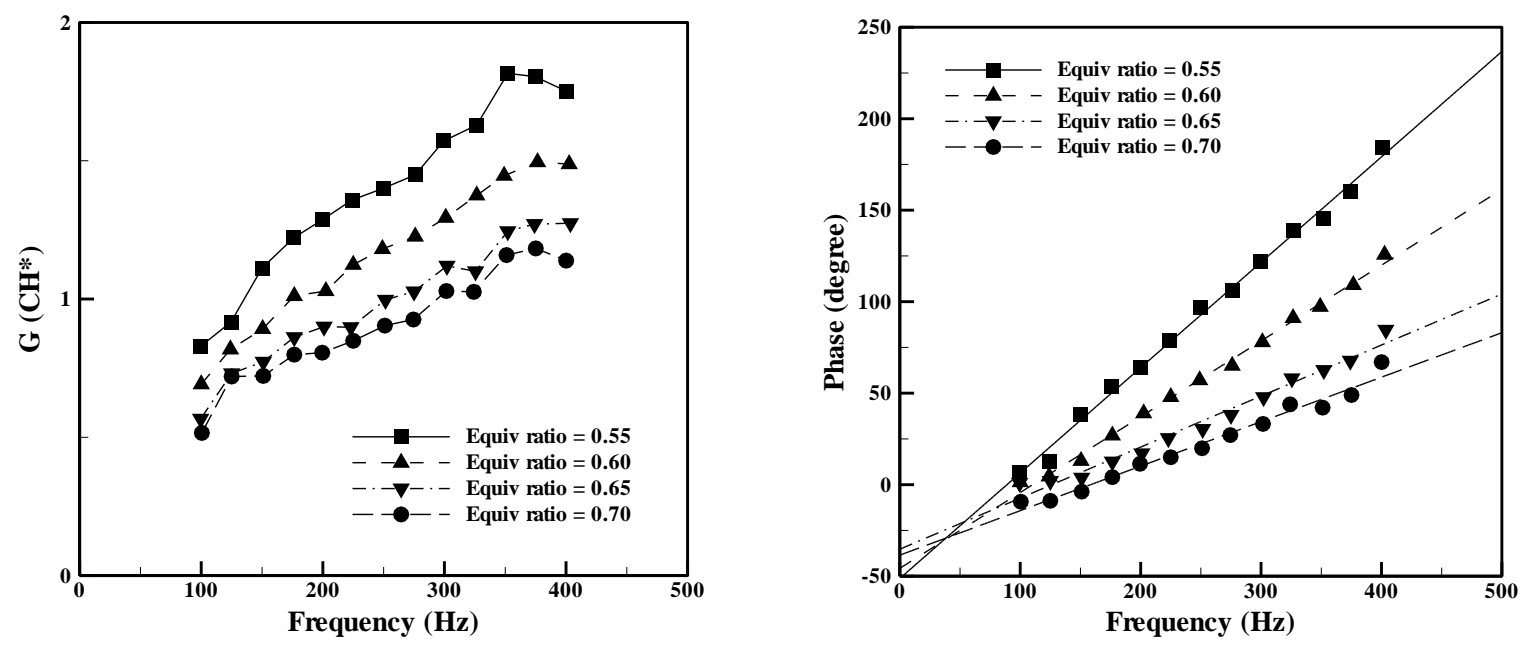

Figure 167: Flame transfer function gain and phase as a function of modulation frequency. Inlet conditions: $T_{\text {in }}=300{ }^{\circ} \mathrm{C}, V_{\text {mean }}=100 \mathrm{~m} / \mathrm{s}, X_{\mathrm{H} 2}=0.00, \Phi=0.55,0.60,0.65,0.70$, and $\alpha_{\mathrm{sw}}$ $=45^{\circ}$.

\section{Effect of Swirl Angle on the Forced Flame Response}

The dependence of the response of premixed flames to acoustic excitation upon swirl angle is investigated in this section. Figure 167 shows the gain and phase of the flame transfer function as a function of modulation frequency for mean equivalence ratios of $0.55 \sim 0.70$. It is noteworthy that the largest response of the flame is observed at a modulation frequency of approximately $400 \mathrm{~Hz}$, and the gain decreases with increasing equivalence ratio. The phase increases almost linearly with frequency and the flame's response time decreases with increasing equivalence ratio. This indicates that the response of the flame to acoustic velocity oscillations is purely convective in nature and the response time is closely associated with flame length. Comparison between the response of the flame for $30^{\circ}$ swirler and $45^{\circ}$ swirler is presented in Figure 168. At the conditions considered here, all inlet conditions are the same except for the swirl angle. The flame transfer function gain and phase decrease as the swirl angle increases from $30^{\circ}$ to $45^{\circ}$. The reduction in the flame response could be attributed to the enhanced flame stiffness with increasing swirl angle. 

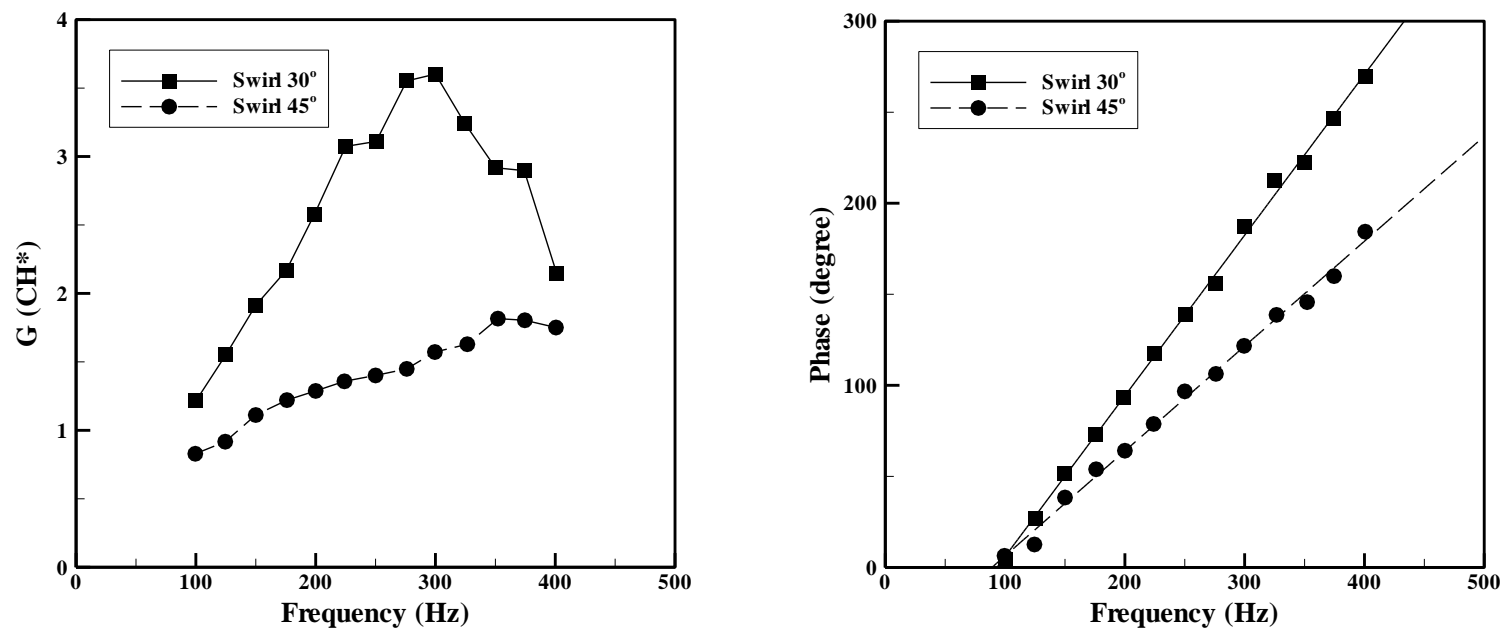

Figure 168: Dependence of flame transfer functions upon swirl angle. Inlet conditions: $T_{\text {in }}$ $=300{ }^{\circ} \mathrm{C}, \mathrm{V}_{\text {mean }}=100 \mathrm{~m} / \mathrm{s}, \mathrm{X}_{\mathrm{H} 2}=0.00, \Phi=0.55$, and $\alpha_{\mathrm{sw}}=30^{\circ}, 45^{\circ}$.

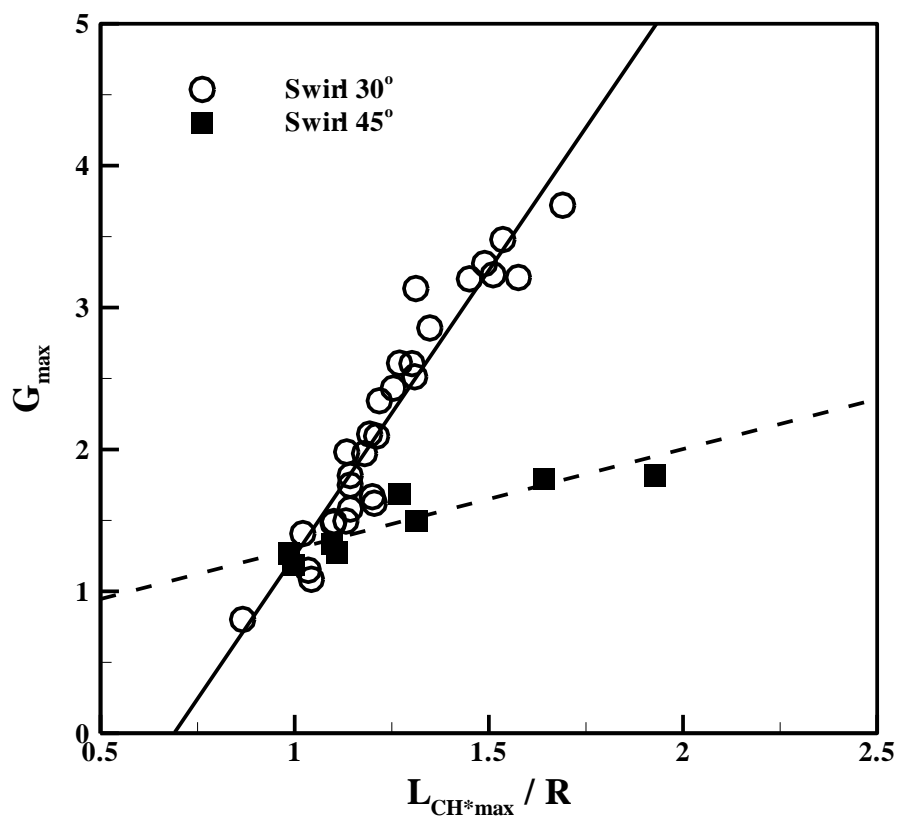

Figure 169: The maximum gain of the flame transfer functions plotted against the normalized effective flame length for swirl angles of $30^{\circ}$ and $45^{\circ}$. Inlet conditions: $T_{\text {in }}=$ $200,300{ }^{\circ} \mathrm{C}, \mathrm{V}_{\text {mean }}=60,70,80,90,100 \mathrm{~m} / \mathrm{s}, \Phi=0.55,0.60,0.65,70$, and $X_{\mathrm{H} 2}=0.00,0.15$, 0.30.

To generalize the response of swirl stabilized premixed flames to upstream perturbations, the flame transfer functions are plotted against the normalized effective flame length, as illustrated in Figure 169 and Figure 170. Figure 169 plots the maximum gain of the flame transfer functions as 
a function of the normalized effective flame length for swirl angles of $30^{\circ}$ and $45^{\circ}$. It can be concluded from Figure 169 that the maximum gain is almost linearly related to the normalized effective flame length ( flame area) for a given swirl angle, and that as swirl angle increases the response of the flame decreases at a constant normalized effective flame length. The reduction in the flame transfer function gain may be attributed to the enhanced flame stiffness, which reduces flame sensitivity to imposed disturbances.

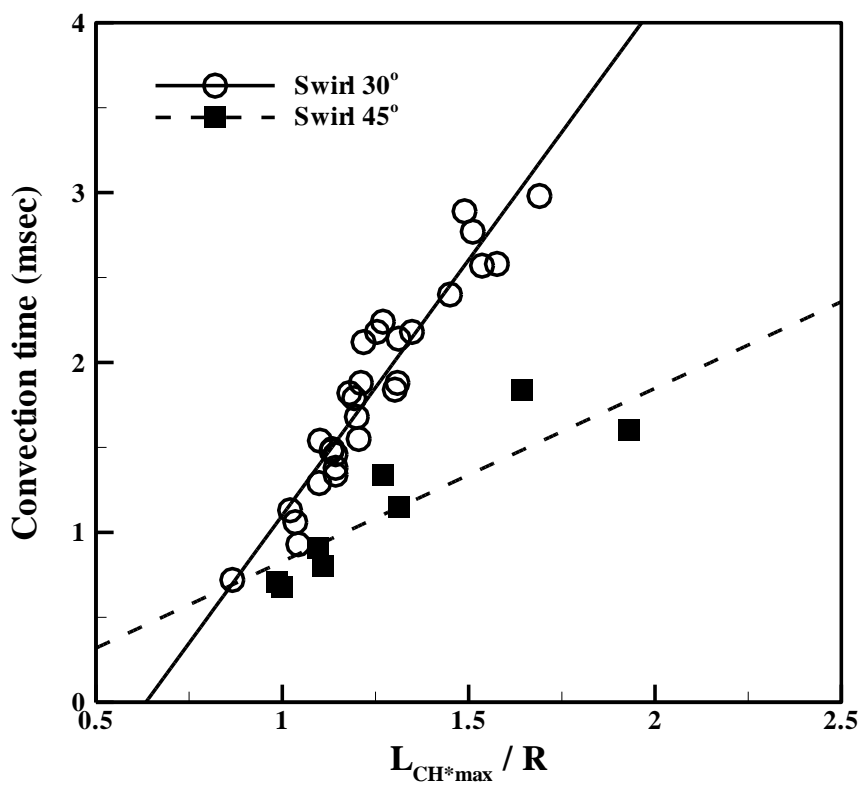

Figure 170: The convection times as a function of the normalized effective flame length for swirl angles of $30^{\circ}$ and $45^{\circ}$. Inlet conditions: $T_{\text {in }}=200,300{ }^{\circ} \mathrm{C}, V_{\text {mean }}=60,70,80,90,100$ $\mathrm{m} / \mathrm{s}, \Phi=0.55,0.60,0.65,70$, and $X_{\mathrm{H} 2}=0.00,0.15,0.30$.

Figure 170 shows the convection times as a function of the normalized effective flame length for swirl angles of $30^{\circ}$ and $45^{\circ}$. It can be clearly seen that the convection time increases almost linearly with the effective flame length for a fixed swirl angle. As the swirl angle increases, the convection time decreases as well as the slope decreases at a constant effect flame length. This indicates that the flame's response time becomes shorter as swirl angle increases, even though they have the same effective flame length scales. This result suggests that an increase in swirl angle may affect the frequency of limit-cycle pressure oscillations. The self-excited instability frequency is expected to increase for the high swirl number case. The results presented in Figure 169 and Figure 170 suggest that the dynamics of a turbulent premixed flame is strongly dependent on the flame structure, the flame length $\left(\mathrm{L}_{\mathrm{CH}^{*} \max }\right)$ and the flame angle $(\alpha)$. These results are reminiscent of the results from analytic and experimental studies about laminar premixed flames. 


\section{Publications}

$\checkmark$ Vishal S.A., Santosh H., Lieuwen, T.C., "Influence of Flame Wrinkling on the Upstream Disturbance Field of An Acoustically Excited Premixed Flame," Proc. of the Twelfth International Conference on Numerical Combustion, SIAM, 2008.

$\checkmark$ Preetham, Hemchandra S., Lieuwen, T., "Forced Response of Premixed Flames: Effect of Flow Non-Uniformity", AIAA paper \# 2009-0978, Presented at the 46th AIAA Aerospace Sciences Meeting, 2008.

$\checkmark$ Shreekrishna, Hemchandra S., Lieuwen, T., "Premixed Flame Response to Equivalence Ratio Perturbations", Combustion Theory and Modeling, Vol. 14(5), 2010.

$\checkmark$ Shanbogue, S., Shin, D.H., Hemchandra, S., Plaks, D., Lieuwen, T., "Flame Sheet Dynamics of Bluff Body Stabilized Flames During Longitudinal Acoustic Forcing", Proceedings of the Combustion Institute, Vol. 32, pp. 1787-1794, 2009.

$\checkmark$ Sai K. Thumuluru, Periagaram K., Lieuwen T., "Flame Brush Dynamics in a Harmonically Oscillating, Turbulent Jet Flame", Proceedings of the 2008 Technical Meeting of the Central States Section of the Combustion Institute, 2008.

$\checkmark$ Thumuluru Sai K., Lieuwen, T., "Characterization of Acoustically Forced Swirl Flame Dynamics", Proceedings of the Combustion Institute, Vol. 32, pp.2893-2900, 2009.

$\checkmark$ Shin, D.H., Shanabogue, S., Lieuwen, T., "Premixed Flame Kinematics in an Axially Decaying, Harmonically Oscillating Vorticity Field", AIAA Paper \#2008-5042, Presented at the $44^{\text {th }}$ AIAA/ASME/SAE/ASEE Joint Propulsion Conference, 2008.

$\checkmark$ Shreekrishna, Lieuwen, T., "High Frequency Premixed Flame Response to Acoustic Perturbations", Presented at $15^{\text {th }}$ AIAA/CEAS Aeroacoustics Conference, Miami, FL, 2009, AIAA\#2009-3261.

$\checkmark$ Preetham, Thumulur Sai K., Lieuwen, T., "Linear Response of Laminar Premixed Flames to Flow Oscillations: Unsteady Stretch Effects", Journal of Propulsion and Power, Vol. 26(3), 2010.

$\checkmark$ Wang, H., Law, C.K., Lieuwen, T., "Linear Response of Stretch-Affected Premixed Flames to Flow Oscillations", Combustion and Flame, Vol. 156(4), 2009.

$\checkmark$ Preetham, Hemchandra, S., Lieuwen, T., "Dynamics of Premixed Flames Forced by Harmonic Velocity Disturbances", Journal of Propulsion and Power, Vol. 24(6), 2008.

$\checkmark$ Vishal S. A., Shreekrishna, Shin, D.H., Lieuwen, T., "Response of Premixed Swirling Flames to Transverse Excitation", Proc. Of the 2009 Fall Technical Meeting of the Eastern States Section of the Combustion Institute, 2009.

$\checkmark$ B. Emerson, U.M. Mondragon, C.T. Brown, Vishal S. A., D-H. Shin, V.G. McDonell and T. Lieuwen, "Dynamics of a Transversely Forced Bluff Body Stabilized Flame", presented at the 49th AIAA Aerospace Sciences Meeting \& Exhibit, Orlando, FL, USA, Jan. 2011.

$\checkmark$ Vishal S. A., B. Emerson, U.M. Mondragon, D-H. Shin, C.T. Brown, V.G. McDonell and T. Lieuwen, "Comparison between Velocity and Flame data from a Transversely Forced, Bluff Body Stabilized Flame", presented at the $7^{\text {th }}$ US National Technical Meeting of the Combustion Institute, Atlanta, GA, USA, March 2011.

$\checkmark$ Vishal S. A. and T. Lieuwen, "Analytical Description of Axisymmetric Swirling Flame Dynamics in a Transverse Excitation Field", presented at the $7^{\text {th }}$ US National Technical Meeting of the Combustion Institute, Atlanta, GA, USA, March 2011.

$\checkmark$ Vishal S. A., B. Emerson, U.M. Mondragon, C.T. Brown, D-H. Shin, V.G. McDonell and T. Lieuwen, "Measurements and Analysis of Bluff Body Flame Response to Transverse Excitation", presented at the ASME Turbo Expo Turbine Technical Conference \& Exposition, Vancouver, BC, Canada, June 2011. 
$\checkmark$ Vishal S. A., Shreekrishna, D-H. Shin and T. Lieuwen, "Swirl Effects on Harmonically Excited, Premixed Flame Kinematics", Combustion and Flame, Vol. 159 (2012), pp. 11391150.

$\checkmark$ Shreekrishna, Lieuwen, T., "Response of Premixed Flames to Equivalence Ratio Oscillations: Non Quasi Steady Effects", Proc. of the 2007 Fall Technical Meeting of the Eastern States Section of the Combustion Institute, 2007.

$\checkmark$ Hemchandra, S., Shreekrishna, Lieuwen, T., "Premixed Flame Response to Equivalence Ratio Perturbations", AIAA Paper \#2007-5656, Presented at the 43rd AIAA/ASME/ SAE/ASEE Joint Propulsion Conference, 2007.

$\checkmark$ Kim, K.T., H.J. Lee, J.G. Lee, B.D. Quay, D.A. Santavicca "Flame transfer function measurement and instability frequency prediction using a thermoacoustic model," Proceedings of ASME Turbo Expo 2009, \#GT2009-60026, June 2009.

$\checkmark$ Kim, K.T., J.G. Lee, H.J. Lee, B. Quay, D. Santavicca "Characterization of forced flame response of swirl-stabilized turbulent lean-premixed flames in a gas turbine combustor," Proceedings of ASME Turbo Expo 2009, \#GT2009-60031, June 2009.

$\checkmark$ Shanbogue, S., Shin, D.H., Hemchandra, S., Plaks, D., Lieuwen, T., "Flame Sheet Dynamics of Bluff Body Stabilized Flames During Longitudinal Acoustic Forcing", Proceedings of the Combustion Institute, Vol. 32, pp. 1787-1794, 2009.

$\checkmark$ Thumuluru, S.K., Lieuwen, T., "Characterization of Acoustically Forced Swirl Flame Dynamics", Proceedings of the Combustion Institute, Vol. 32, pp.2893-2900, 2009.

$\checkmark$ Kim, K.T., Lee, J.G., Quay, B.D., Santavicca, D.A., "Spatially distributed flame transfer functions for predicting combustion dynamics in lean premixed gas turbine combustors," Combustion and Flame, 2010, doi:10.1016/j.combustflame.2010.04.016.

$\checkmark$ Kim, K.T., Lee, J.G., Quay, B.D., Santavicca, D.A., "Response of partially premixed flames to acoustic velocity and equivalence ratio perturbations," Combustion and Flame, 2010, doi:10.1016/j.combustflame.2010.04.006

$\checkmark$ Kim, K.T., Lee, J.G., Quay, B.D., Santavicca, D.A., "Reconstruction of the heat release response of partially premixed flames," Combustion Science and Technology, 2010, In Press.

$\checkmark$ Kim, K.T., Lee, J.G., Quay, B.D., Santavicca, D.A., "Experimental investigation of the nonlinear response of swirl stabilized flames to equivalence ratio oscillations," Journal of Engineering for Gas Turbines and Power, 2010, In Press. 


\section{List of Tables}

Table 1 : Analytical results for quasi-steady linear transfer function ........................................... 18

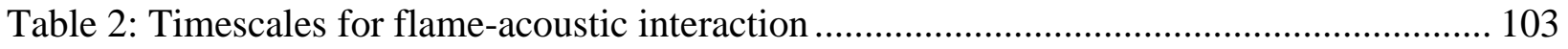

Table 3 : Relevant length scales for flame-acoustic interaction ................................................ 104

Table 4: Summary of physical processes influencing flame response at different regimes in the

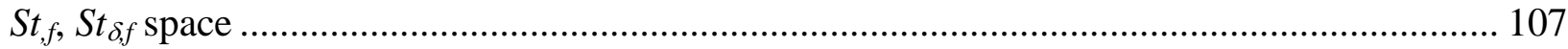

Table 5: Test conditions for forced flame response measurements.......................................... 163

Table 6: Comparison of two different flames: Flame "A" and "B". ........................................... 183

Table 7: Test conditions for forced flame response measurements............................................. 201

Table 8: Test conditions for forced flame response measurements.......................................... 214

\section{$\underline{\text { List of Figures }}$}

Figure 1: Schematic representations of investigated geometries. (top-left) Schematic showing one half of the instantaneous flame surface (solid red curve), the instantaneous coherent flame surface (solid green curve), and the nominal flame surface (dashed black line). (top-right) Schematic of geometry for bluff-body stabilized flame. (bottom-left) Schematic of geometry for the fuel/air ratio and pressure forcing investigation of a conical flame. (bottom-right) Schematic of geometry for effect of swirl on a velocity-forced center-body stabilized flame. ..................... 17

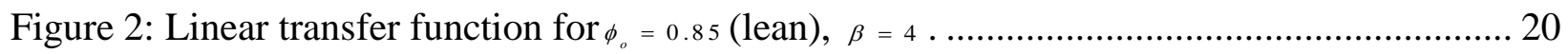

Figure 3: Quasi-steady and non quasi-steady response characteristics of a premixed flame

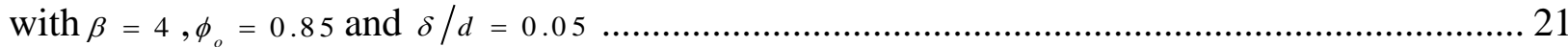

Figure 4: Variation of the non quasi steady transfer function correction for gas turbine conditions

Figure 5: (left) Variation of the linear transfer function with $S t_{2}$ for different values of equivalence ratio, $\beta=4$ (center) Differences between Quasi-steady and Non quasi-steady effects. (right) Differences between stretched and un-stretched flame response. ..................................... 23

Figure 6: Qualitative map illustrating regimes of dominance of various nonlinearity mechanisms at $\phi_{o}=0.85$. (1) Linear, (2) $s_{L^{-}} \phi$ non-linearity, (3) kinematic restoration and (4) "cross-over"

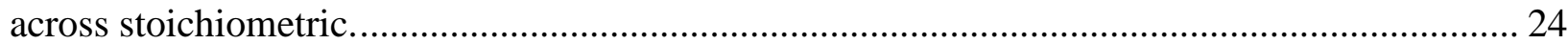

Figure 7: Variation of the transfer function with (a) Strouhal number for various excitation

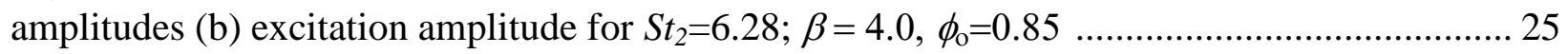


Figure 8: Typical flame shapes for characteristic swirl numbers of 0.0, 0.6 and 1.1 respectively.

Figure 9: (left) Schematic of investigated geometry showing one half of the instantaneous flame surface (solid red curve), the instantaneous coherent flame surface (solid green curve) and the nominal flame surface (broken black line). The flow is from bottom to top. (right) Spatial variation of local consumption speed normalized by the linearized value at three different turbulence intensities (solid curves) given by the value of $u^{\prime} / s_{L}$. Flame angle, $\gamma \sim 4^{\mathrm{o}} \ldots \ldots \ldots \ldots \ldots . . . . . .28$

Figure 10: Chemiluminescence intensity response characteristics for $\phi_{o}=0.7, \beta=4 \ldots \ldots \ldots \ldots \ldots . . .29$

Figure 11: (a) Summary of heat release response of premixed flames to harmonic disturbances for a premixed methane/air flame, (b) Flame transfer function comparisons of premixed flame response to velocity fluctuations (red), equivalence ratio fluctuations (green), pressure perturbations (blue). These figures are for nominally two dimensional V-shaped flames with $\phi_{o}=0.85$ and aspect ratio (i.e., nominal flame length/flame duct radius) of 4 . 30

Figure 12: Schematic of investigated geometry showing the instantaneous flame surface (solid red curve), the instantaneous coherent flame surface (solid black curve) and the nominal flame surface (broken black line). The flow is from bottom to top. 31

Figure 13: Variation of local consumption speed along the flame surface. $\mathrm{u}^{\prime} / \mathrm{s}_{\mathrm{L}}=0.4 . \beta=\cot \gamma$. Notice that the consumption speed is spatially varying along the flame surface due to the influence of non-locality. 36

Figure 14: Comparison of heat release-pressure oscillation and heat release-velocity perturbation transfer function (and some contributions) 40

Figure 15: High frequency response of the contributions to heat release due to velocity perturbations $\left(F_{u}\right)$ and that due to flame speed perturbations $\left(F_{p}=F-F_{u}\right)$. The total transfer function $(F)$ is also plotted. 40

Figure 16: Schematic of investigated geometry - axis-symmetric bluff-body stabilized flame. . 42

Figure 17: Schematic of the investigated geometry showing the ensemble averaged flame surface. 47

Figure 18: Variation of total transfer function magnitude and phase with Strouhal number for typical values of $K=$ (a)-(b) 0, (c)-(d) 1.0 and (e)-(f) 3.0. Also shown for reference in each case is the corresponding laminar transfer function. Flame angle $\gamma=45^{\circ}$. 53

Figure 19: Variation of the leading order turbulence correction term with Strouhal number (a) magnitude and (b) phase. Flame angle, $\gamma=45^{\circ}$ and flame length, $\Lambda=1$. 55

Figure 20: Schematic showing the influence of the wavelength of coherent forcing relative to the turbulent scales on burning area destruction. The solid curves on the left show schematically the resultant wrinkling if there were no kinematic restoration. The broken curves show schematically, the base-line coherent wrinkling. The small arrows show the direction of flame surface propagation. 56 
Figure 21: Variation of the (a) Magnitude and (b) phase relative to laminar response of the leading order turbulence correction for different flame angles $\gamma, K=1.0, \Lambda=1 \ldots \ldots \ldots \ldots \ldots \ldots \ldots . . . .56$

Figure 22: (a) Variation of local consumption and displacement speeds from the non-local model and (b) schematic showing the reference area on the unforced mean surface used to define $f_{c}(r), \varepsilon_{T}=0.05, \gamma=45^{\circ}$ and $\Lambda=1$. 58

Figure 23: Variation of normalized local consumption/displacement speeds from the theory of Lipatnikov and Chomiak. Turbulent mixing time $T=50, S_{o} / s_{L}=0.13, \gamma=45^{\circ}$ and flame length, $\Lambda=1$.

Figure 24: Comparison of transfer function magnitude and phase from the two modeling approaches and the theoretical analysis (a)-(b) $K=0.3$, (c)-(d) $K=0.5$, (e)-(f) $K=1.5, \varepsilon_{T}=0.05$ and $\gamma=45^{\circ}$

Figure 25: Physical mechanisms causing heat release oscillations due to fluctuations in reactant equivalence ratio 61

Figure 26: Chemiluminescence transfer function gain (left) and phase (right, with respect to velocity perturbations) for velocity-coupled flame response, for $\square_{o}=0.75, u_{o}=25 \mathrm{~m} / \mathrm{s}, 5 \%$ perturbation amplitude 62

Figure 27: Variation of $\mathrm{CO}_{2} *$ chemiluminescence intensity/fuel flow rate with mean equivalence ratio 68

Figure 28: Schematic of the flame geometry - (a) axisymmetric V-flame and (b) axisymmetric conical flame 68

Figure 29: Contributions to $F_{Q}$ by area and effective equivalence ratio fluctuations for (a) Vflame (b) conical flame at $1 \mathrm{~atm}, 300 \mathrm{~K}, \phi_{o}=0.6, \beta=4$. 70

Figure 30: Contributions to $F_{C}$ by area and effective equivalence ratio fluctuations for (a) Vflame (b) conical flame at $1 \mathrm{~atm}, 300 \mathrm{~K}, \phi_{o}=0.6, \beta=4$. 71

Figure 31: Comparison between $F_{C}$ and $F_{Q}$ for (a) V-flame (b) Conical flame at $1 \mathrm{~atm}, 300 \mathrm{~K}$. $\phi_{o}=0.6, \beta=4$ 72

Figure 32 : Variation of various sensitivities with mean equivalence ratio at (a) 1 atm, $300 \mathrm{~K}$ (b) 5 atm, $300 \mathrm{~K}$. 73

Figure 33 : Variation of various sensitivities with mean equivalence ratio at 5 atm, $600 \mathrm{~K} \ldots \ldots . .73$

Figure 34: Fundamental processes controlling the heat release response of premixed flames to equivalence ratio oscillations 75

Figure 35: Linear transfer function for (a) $\phi_{o}=0.85$ (lean) (b) $\phi_{o}=1.28$ (rich), $\beta=4$. 84

Figure 36: Variation of the linear transfer function with $S t_{2}$ for different values of equivalence ratio. $\beta=4$. 85 
Figure 37: (a) Non quasi-steady versus quasi-steady flame response for $\phi_{o}=0.85, \beta=4, \delta=0.1 R$

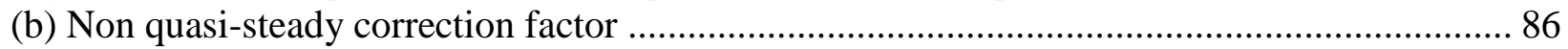

Figure 38: Effect of Flame Stretch on a lean $\mathrm{CH}_{4} /$ air flame, $\phi_{o}=0.85, \beta=4, \delta=0.1 R, M a=1-$ (a) Effect on Flame speed contribution to heat release. (b) Effect on burning area contribution to heat release. 87

Figure 39: Comparison between unstretched, quasi-steady (solid blue), stretched, quasi-steady (solid green) and unstretched, non quasi-steady (solid red) global heat release responses of a

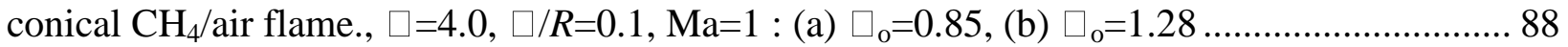

Figure 40: Physical mechanisms causing heat release oscillations due to velocity fluctuations .. 89 Figure 41: Physical mechanisms causing heat release oscillations due to fluctuations in acoustic pressure 91

Figure 42: Strouhal number dependence of transfer function for (a) $K=1$ (b) $K=M_{o} . M_{o}=0.1$, $\beta=4, \delta=0.01$. The $s t_{f}=\beta / K \delta$ limit (see Eq.(203)) is denoted by vertical dash-dot lines. 97

Figure 43: Gain of equivalence ratio coupled response and velocity-coupled response. $\phi_{o}=0.85, \delta=0.01, \beta=4, K=1$. 101

Figure 44: Magnitude of the heat release transfer function of a $2 \mathrm{D}$ wedge flame and its constituent contributions. $M_{o}=0.1,(\gamma-1) \theta=3, \delta=0.01, \beta=4, K=M_{o}$. The $s t_{f}=\beta / K \delta$ limit (see Eq.(203)) is denoted by vertical dash-dot lines 102

Figure 45: Summary of heat release response of premixed flames to pressure/velocity disturbances. 108

Figure 46: (a) Flame interactions with transverse excitations; (b) Longitudinal forcing; (c) Transverse forcing. 110

Figure 47: Amplitude variation along the flame for different values of $\sigma=\Omega / \omega$, see Eq.(285), with downstream radial distance at (a) $\theta=0$, (b) $\theta=\pi / 2$; (c) Phase variation for different values of $\sigma=\Omega / \omega$., see Eq.(286), with downstream radial distance at $\theta=0, \pi$.

Figure 48: Flame shape visualizations for $\psi=14^{\circ}, \Omega / \omega=0.00$ at $\mathrm{t}=0.3$; (a) 3D flame surface, (b) Flame branches at $\theta=0, \pi$. 122

Figure 49: Flame shape visualizations for $\psi=14^{\circ}, \Omega / \omega=0.60$ at $\mathrm{t}=0.3$; (a) 3D flame surface, (b) Flame branches at $\theta=0, \pi$; black : $\Omega / \omega=0.60$, gray : $\Omega / \omega=0.00$.

Figure 50: Flame shape visualizations for $\psi=14^{\circ}, \Omega / \omega=0.95$ at $\mathrm{t}=0.3$; (a) 3D flame surface, (b) Flame branches at $\theta=0, \pi$; black : $\Omega / \omega=0.95$, gray : $\Omega / \omega=0.00$.

Figure 51: Flame shape visualizations $\psi=14^{\circ}, \Omega / \omega=1.00$ at $\mathrm{t}=0.3$; (a) 3D flame surface, (b) Flame branches at $\theta=0, \pi$; black : $\Omega / \omega=1.00$, gray : $\Omega / \omega=0.00$.

Figure 52: Flame shape visualizations for $\psi=14^{\circ}, \Omega / \omega=1.10$ at $\mathrm{t}=0.3$; (a) 3D flame surface, (b) Flame branches at $\theta=0, \pi$; black : $\Omega / \omega=1.10$, gray : $\Omega / \omega=0.00$. 124 
Figure 53: Flame shape visualizations for $\psi=14^{\circ}, \Omega / \omega=2.10$ at $\mathrm{t}=0.3$; (a) 3D flame surface, (b) Flame branches at $\theta=0, \pi$; black : $\Omega / \omega=2.10$, gray : $\Omega / \omega=0.00$.

Figure 54: Flame Transfer function gain for a nominally axisymmetric flame in a non-compact transverse disturbance field. The non-compactness is controlled by the Mach Number...... 126

Figure 55: Time sequence evolution of velocity perturbation field for (red, green, blue in order) a (a) traveling wave, black arrow shows direction of wave and (b) standing wave 136

Figure 56: Normalized fluctuating flame area (Eq.(291)) for a nominally axisymmetric flame in a non-compact disturbance field (Eq.(323) and Eq.(329)). The ratio of flame size to wavelength is controlled by the Mach number, but also varies along the $\mathrm{x}$-axis with Strouhal number. 137

Figure 57: Schematic of the two dimensional axisymmetric swirl flow configuration used in the CFD study. The wall boundaries are indicated by white lines. The inlet is on the left section and the outlet is on the right. The axis is the base of the figure. 139

Figure 58: Steady state solution contours for unforced case, used as initial reference state for forced cases, showing (a) axial velocity $(\mathrm{X})$ and (b) radial velocity (Y). 140

Figure 59: Snapshot of the flow field contours at a time instant during the acoustic forcing for a case with only axial forcing, showing (a) axial velocity and (b) radial velocity. Contour levels same as their respective in Figure 58. 140

Figure 60: Snapshot of the flow field contours at a time instant during the acoustic forcing for a case with both axial and azimuthal forcing, showing (a) axial velocity and (b) radial velocity. Contour levels same as their respective in Figure 58. 141

Figure 61: Snapshot of the flow field contours at a time instant during the acoustic forcing for a case with only azimuthal forcing, showing (a) axial velocity and (b) radial velocity. Contour levels same as their respective in Figure $58 . .$. 141

Figure 62: Decay of resolved turbulent kinetic energy................................................. 143

Figure 63: $\mathrm{OH}^{*}, \mathrm{CH}^{*}$, and $\mathrm{CO} 2 *$ chemiluminescence spectra showing spectra..................... 145

Figure 64: Relationship between the $\mathrm{CO}_{2}{ }^{*}$ chemiluminescence intensity at (a) $307 \mathrm{~nm}$ and 365 $\mathrm{nm}$ and (b) $430 \mathrm{~nm}$ and $365 \mathrm{~nm}$. 145

Figure 65: Actual equivalence ratio versus the measured $\mathrm{OH}^{*} / \mathrm{CH}^{*}$ chemiluminescence intensity. Red data corresponds to the raw data; while the blue data corresponds to the background subtracted data. 146

Figure 66: Equivalence ratio fluctuation measurements for a (a) $100 \mathrm{~Hz}$ forced oscillation with a $10 \%$ velocity fluctuation, (b) $250 \mathrm{~Hz}$ forcd oscillation with an $8 \%$ velocity fluctuation. ( $\mathrm{V}=30$ $\mathrm{m} / \mathrm{s}, \phi=0.7)$. 147

Figure 67: The error in the equivalence ratio fluctuation measurement as a function of fluctuation frequency and the magnitude of the forced velocity fluctuation. 147 
Figure 68: Normalized rms velocity fluctuation versus modulation frequency with $0 \%$ bypass measured in the performance test rig.

Figure 69: Schematic drawing of the mixing section in the combustor test rig.

Figure 70: Normalized velocity fluctuation versus modulation frequency with $0 \%$ bypass measured in the combustor test rig.

Figure 71: $\mathrm{OH}^{*}, \mathrm{CH}^{*}$, and $\mathrm{CO}_{2} *$ chemiluminescence intensity, inlet velocity, and combustor pressure versus time for a $100 \mathrm{~Hz}$ forcing frequency. 152

Figure 72: Power spectra of $\mathrm{OH}^{*}, \mathrm{CH}^{*}$, and $\mathrm{CO}_{2} *$ chemiluminescence intensity, inlet velocity, and combustor pressure fluctuations with a forcing frequency of $100 \mathrm{~Hz}$. 152

Figure 73: The dependence of normalized global heat release fluctuation (as indicated by the $\mathrm{OH}^{*}$ chemiluminescence intensity) on the forcing velocity amplitude. Normalized fluctuation in the rate of heat release, as indicated by $\mathrm{OH}^{*}$ chemiluminescence, versus the normalized velocity fluctuation for an inlet temperature of $200^{\circ} \mathrm{C}$, a mean nozzle velocity of $60 \mathrm{~m} / \mathrm{s}$ and an equivalence ratio of 0.6 over a range of forcing frequencies from 100 to $450 \mathrm{~Hz}$ with natural gas fuel. 153

Figure 74: (a) Gain and (b) Phase of the flame transfer function based as a function of forcing velocity amplitude. 154

Figure 75: (a) Gain and (b) Phase of the flame transfer function, and (c) Convection time delay at a constant inlet velocity fluctuation magnitudes of $15 \%$. 155

Figure 76: (a) Gain and (b) Phase of the flame transfer function, and (c) Convection time delay at a constant inlet velocity fluctuation magnitudes of $11,15,20$, and $25 \%$. 156

Figure 77: Normalized combustor pressure fluctuation and corresponding frequency of selfexcited instabilities as a function of the combustor length at an inlet temperature of $200^{\circ} \mathrm{C}$, a mean inlet velocity of $60 \mathrm{~m} / \mathrm{s}$, and an equivalence ration of 0.6 . The graphs on the left are for $100 \%$ natural gas and the graphs on the right are for a 55\%/45\% natural gas-hydrogen fuel mixture. 157

Figure 78: Gain and phase of the flame transfer function vs. forcing frequency at $\mathrm{V}^{\prime} / \mathrm{V}_{\text {mean }}=$ 0.100 . Inlet conditions: $\mathrm{T}_{\mathrm{in}}=200{ }^{\circ} \mathrm{C}, \mathrm{V}_{\text {mean }}=60 \mathrm{~m} / \mathrm{s}, \Phi=0.60$, premixed, and $\mathrm{X}_{\mathrm{H} 2}=0.00,0.15$. Note that the gain of FTF is well fitted by a second-order oscillator model (lines). 158

Figure 79: (A) Phase-synchronized $\mathrm{CH}^{*}$ chemiluminescence imaging at modulation frequency of $200 \mathrm{~Hz}$ and $\mathrm{V}^{\prime} / \mathrm{V}_{\text {mean }}=25 \%$. (B) line-of-sight integrated, background-corrected $\mathrm{CH}^{*}$ images at $\varphi$ $=60,150,240,330^{\circ}$. Inlet conditions: $\mathrm{T}_{\mathrm{in}}=200^{\circ} \mathrm{C}, \mathrm{V}_{\text {mean }}=60 \mathrm{~m} / \mathrm{s}, \Phi=0.60$, premixed, and $\mathrm{X}_{\mathrm{H} 2}$ $=0.00$. 158

Figure 80: (A) Phase-synchronized $\mathrm{CH}^{*}$ chemiluminescence imaging at $f=200 \mathrm{~Hz}$ and $\mathrm{V}^{\prime} / \mathrm{V}_{\text {mean }}$ $=25 \%$, (B) line-of-sight integrated $\mathrm{CH}^{*}$ images at $\varphi=90^{\circ}, 150^{\circ}, 210^{\circ}$, and $270^{\circ}$. Inlet conditions: $\mathrm{T}_{\mathrm{in}}=200^{\circ} \mathrm{C}, \mathrm{V}_{\text {mean }}=60 \mathrm{~m} / \mathrm{s}, \Phi=0.60$, premixed, and $\mathrm{X}_{\mathrm{H} 2}=0.30$. 158 
Figure 81: Prediction of the normalized heat release response of a partially premixed flame at a modulation frequency of $100 \mathrm{~Hz}$ and amplitude of $V^{\prime} / V_{\text {mean }}=10.0 \%, \Phi^{\prime} / \Phi_{\text {mean }}=2.9 \%$. Inlet conditions: $T_{i n}=200{ }^{\circ} \mathrm{C}, V_{\text {mean }}=60 \mathrm{~m} / \mathrm{s}, \Phi_{\text {mean }}=0.60$, and $x_{H 2}=0.00$.

Figure 82: Phase-synchronized $\mathrm{CH}^{*}$ chemiluminescence images at a modulation frequency of $200 \mathrm{~Hz}$. (A) in-phase interference, (B) out-of-phase interference. Inlet conditions: $T_{\text {in }}=200{ }^{\circ} \mathrm{C}$, $V_{\text {mean }}=60 \mathrm{~m} / \mathrm{s}, \Phi_{\text {mean }}=0.60, \mathrm{x}_{H_{2}}=0.60, f=200 \mathrm{~Hz}$, and $\mathrm{V}^{\prime} / \mathrm{V}_{\text {mean }}=42 \%$. 161

Figure 83: (A) Normalized heat release response and (B) gain of the flame transfer function plotted as a function of the forcing amplitude. Inlet conditions: $\mathrm{T}_{\text {in }}=200{ }^{\circ} \mathrm{C}, \mathrm{V}_{\text {mean }}=60 \mathrm{~m} / \mathrm{s}$, $\Phi_{\text {mean }}=0.60, \mathrm{X}_{\mathrm{H} 2}=0.30, \mathrm{PM}, \mathrm{PPM}\left(\mathrm{L}_{\mathrm{f}, \mathrm{inj}}=152 \mathrm{~mm}\right)$, and $f=200 \mathrm{~Hz}$. 164

Figure 84: (A) Normalized heat release response and (B) gain of the flame transfer function plotted as a function of the forcing amplitude. Inlet conditions: $\mathrm{T}_{\text {in }}=200{ }^{\circ} \mathrm{C}, \mathrm{V}_{\text {mean }}=60 \mathrm{~m} / \mathrm{s}$, $\Phi_{\text {mean }}=0.60, \mathrm{X}_{\mathrm{H} 2}=0.00, \mathrm{PM}, \mathrm{PPM}\left(\mathrm{L}_{\mathrm{f}, \text { inj }}=6 "\right), f=100 \mathrm{~Hz}$. 164

Figure 85: (A) Phase-synchronized $\mathrm{CH}^{*}$ chemiluminescence imaging at a modulation frequency of $200 \mathrm{~Hz}$ and $\mathrm{V}^{\prime} / \mathrm{V}_{\text {mean }}=41 \%(\mathrm{PM})$. (B) Bottom row shows line-of-sight integrated, background-corrected $\mathrm{CH}^{*}$ chemiluminescence images at $\varphi=60,150,240$, and $330^{\circ}$. Inlet conditions: $\mathrm{T}_{\text {in }}=200{ }^{\circ} \mathrm{C}, \mathrm{V}_{\text {mean }}=60 \mathrm{~m} / \mathrm{s}, \Phi_{\text {mean }}=0.60, \mathrm{X}_{\mathrm{H} 2}=0.30, \mathrm{PM}$, and $f=200 \mathrm{~Hz}$. 165

Figure 86: Phase-synchronized $\mathrm{CH}^{*}$ chemiluminescence imaging at a modulation frequency of $200 \mathrm{~Hz}$ and $\mathrm{V}^{\prime} / \mathrm{V}_{\text {mean }}=42 \%(\mathrm{PPM})$. Top row (A) shows individually-scaled images to highlight the flame structure. Middle row (B) shows images normalized by maximum intensity. Bottom row (C) shows line-of-sight integrated, background-corrected $\mathrm{CH}^{*}$ chemiluminescence images at $\varphi=60,150,240$, and $330^{\circ}$. Inlet conditions: $\mathrm{T}_{\text {in }}=200{ }^{\circ} \mathrm{C}, \mathrm{V}_{\text {mean }}=60 \mathrm{~m} / \mathrm{s}, \Phi_{\text {mean }}=0.60, \mathrm{X}_{\mathrm{H} 2}=$ $0.30, \operatorname{PPM}\left(\mathrm{L}_{\mathrm{f}, \mathrm{inj}}=6\right.$ "'), and $f=200 \mathrm{~Hz}$. 166

Figure 87: Global $\mathrm{CH}^{*}$ chemiluminescence signal variations over two cycles of oscillations at a modulation frequency of $200 \mathrm{~Hz}$ and $\mathrm{V}^{\prime} / \mathrm{V}_{\text {mean }}=42 \%$. Inlet conditions: $\mathrm{T}_{\text {in }}=200{ }^{\circ} \mathrm{C}, \mathrm{V}_{\text {mean }}=60$ $\mathrm{m} / \mathrm{s}, \Phi_{\text {mean }}=0.60, \mathrm{X}_{\mathrm{H} 2}=0.30,\left(\mathrm{~L}_{\mathrm{f}, \text { inj }}=6 "\right)$, and $f=200 \mathrm{~Hz}$.

Figure 88: Phase-synchronized $\mathrm{CH}^{*}$ chemiluminescence imaging at a modulation frequency of $200 \mathrm{~Hz}$ and $\mathrm{V}^{\prime} / \mathrm{V}_{\text {mean }}=42 \%$ (PPM). Top row (A) shows auto-scaled intensity images. Middle row (B) shows images normalized by maximum intensity. Bottom row (C) shows line-of-sight integrated, background-corrected $\mathrm{CH}^{*}$ chemiluminescence images at $\varphi=60,150,240,330^{\circ}$. Inlet conditions: $\mathrm{T}_{\mathrm{in}}=200{ }^{\circ} \mathrm{C}, \mathrm{V}_{\text {mean }}=60 \mathrm{~m} / \mathrm{s}, \Phi=0.60, \mathrm{X}_{\mathrm{H} 2}=0.60, \operatorname{PPM}\left(\mathrm{L}_{\mathrm{f}, \text { inj }}=6 ”\right)$, and $f=$ $200 \mathrm{~Hz}$. 167

Figure 89: (A) Normalized heat release response $\left(\mathrm{CH}^{*} / \mathrm{CH}^{*}\right.$ mean $)$ and (B) gain of flame transfer function as a function of the magnitude of inlet velocity perturbation. Inlet conditions: $T_{\text {in }}=200$ ${ }^{\circ} \mathrm{C}, \mathrm{V}_{\text {mean }}=60 \mathrm{~m} / \mathrm{s}, \Phi_{\text {mean }}=0.60, \mathrm{X}_{\mathrm{H} 2}=0.60, f=200,300 \mathrm{~Hz}$, and $\mathrm{L}_{\mathrm{f}, \mathrm{inj}}=6 . "$ 168

Figure 90: Phase-synchronized $\mathrm{CH}^{*}$ chemiluminescence imaging at modulation frequencies of (A) $200 \mathrm{~Hz}$ (individually scaled) and (B) $300 \mathrm{~Hz}$ (normalized by maximum intensity). Inlet conditions: $\mathrm{T}_{\mathrm{in}}=200{ }^{\circ} \mathrm{C}, \mathrm{V}_{\text {mean }}=60 \mathrm{~m} / \mathrm{s}, \Phi_{\text {mean }}=0.60, \mathrm{X}_{\mathrm{H} 2}=0.60, \mathrm{PPM}\left(\mathrm{L}_{\mathrm{f}, \text { inj }}=6\right.$ "), and $\mathrm{V}^{\prime} / \mathrm{V}_{\text {mean }}=42 \%$. 169 
Figure 91: Time signals of inlet velocity, equivalence ratio, and $\mathrm{CH}^{*}$ chemiluminescence during a cycle of oscillation at a modulation frequency of $200 \mathrm{~Hz}$ and amplitude of $\mathrm{V}^{\prime} / \mathrm{V}_{\text {mean }}=42 \%$. Inlet conditions: $\mathrm{T}_{\mathrm{in}}=200{ }^{\circ} \mathrm{C}, \mathrm{V}_{\text {mean }}=60 \mathrm{~m} / \mathrm{s}, \Phi_{\text {mean }}=0.60, \mathrm{X}_{\mathrm{H} 2}=0.60$, and PPM $\left(\mathrm{L}_{\mathrm{f}, \text { inj }}=6\right.$ ").

Figure 92: Phase-synchronized $\mathrm{CH}^{*}$ chemiluminescence imaging at a modulation frequency of $200 \mathrm{~Hz}$. Right column shows individually-scaled images to highlight the flame structure. Left column is images normalized by their maximum intensity. Inlet conditions: $\mathrm{T}_{\text {in }}=200{ }^{\circ} \mathrm{C}, \mathrm{V}_{\text {mean }}=$ $60 \mathrm{~m} / \mathrm{s}, \Phi_{\text {mean }}=0.60, \mathrm{X}_{\mathrm{H} 2}=0.60, \operatorname{PPM}\left(\mathrm{L}_{\mathrm{f}, \text { inj }}=6 ”\right), f=200 \mathrm{~Hz}$, and $\mathrm{V}^{\prime} / \mathrm{V}_{\text {mean }}=42 \%$.

Figure 93: Time signals of inlet velocity, equivalence ratio, and $\mathrm{CH}^{*}$ chemiluminescence during a cycle of oscillation at a modulation frequency of $300 \mathrm{~Hz}$ and $\mathrm{V}^{\prime} / \mathrm{V}_{\text {mean }}=42 \%$. Inlet conditions: $\mathrm{T}_{\text {in }}=200^{\circ} \mathrm{C}, \mathrm{V}_{\text {mean }}=60 \mathrm{~m} / \mathrm{s}, \Phi_{\text {mean }}=0.60, \mathrm{X}_{\mathrm{H} 2}=0.60$, and PPM $\left(\mathrm{L}_{\mathrm{f}, \text { inj }}=6 "\right)$. 172

Figure 94: Phase-synchronized $\mathrm{CH}^{*}$ chemiluminescence imaging at a modulation frequency of $300 \mathrm{~Hz}$. White lines denote equivalence ratio oscillations, and yellow lines denote velocity perturbations. Inlet conditions: $\mathrm{T}_{\mathrm{in}}=200{ }^{\circ} \mathrm{C}, \mathrm{V}_{\text {mean }}=60 \mathrm{~m} / \mathrm{s}, \Phi_{\text {mean }}=0.60, \mathrm{X}_{\mathrm{H} 2}=0.60, \mathrm{PPM}\left(\mathrm{L}_{\mathrm{f}}\right.$, inj $=6 "$ '), $f=300 \mathrm{~Hz}$, and $\mathrm{V}^{\prime} / \mathrm{V}_{\text {mean }}=42 \%$. 173

Figure 95: Calculation of the normalized $\mathrm{CH}^{*}$ chemiluminescence intensity (heat release) of a partially premixed flame at a modulation frequency of $100 \mathrm{~Hz}$ and amplitude of $\mathrm{V} / \mathrm{V}_{\text {mean }}=$ $10.0 \%, \Phi^{\prime} / \Phi_{\text {mean }}=2.9 \%$. Inlet conditions: $\mathrm{T}_{\text {in }}=200^{\circ} \mathrm{C}, \mathrm{V}_{\text {mean }}=60 \mathrm{~m} / \mathrm{s}, \Phi_{\text {mean }}=0.60$, and $\mathrm{X}_{\mathrm{H} 2}=$ 0.00 .

Figure 96: Calculation of the normalized $\mathrm{CH}^{*}$ chemiluminescence intensity (heat release) of a partially premixed flame at a modulation frequency of $100 \mathrm{~Hz}$ and amplitude of $\mathrm{V} / \mathrm{V}_{\text {mean }}=$ $7.0 \%, \Phi^{\prime} / \Phi$ mean $=1.8 \%$. Inlet conditions: $\mathrm{T}_{\text {in }}=200{ }^{\circ} \mathrm{C}, \mathrm{V}_{\text {mean }}=60 \mathrm{~m} / \mathrm{s}, \Phi_{\text {mean }}=0.60$, and $\mathrm{X}_{\mathrm{H} 2}=$ 0.00 . 175

Figure 97: Dependence of the amplitude of equivalence ratio oscillations upon the amplitude of inlet velocity perturbations at a modulation frequency of $100 \mathrm{~Hz}$. Inlet conditions: $\mathrm{T}_{\text {in }}=200{ }^{\circ} \mathrm{C}$, $\mathrm{V}_{\text {mean }}=60 \mathrm{~m} / \mathrm{s}, \Phi_{\text {mean }}=0.60$, and $\mathrm{X}_{\mathrm{H} 2}=0.00$. 176

Figure 98: (left) Spectrum of flame sheet fluctuations at different downstream locations $(50 \mathrm{~m} / \mathrm{s}$, $644 \mathrm{~K})$. The $x$-coordinate is the downstream distance with $x=0$ located at the bluff body trailing edge and $f_{o}$ is the acoustic forcing frequency. The flame response is enveloped at the forcing frequency as well as the first sub-harmonic and the first two harmonics. (right) Sample mean velocity fields obtained from PIV with flame brush and mean flame position overlaid. $(100 \mathrm{~m} / \mathrm{s}$, $755 \mathrm{~K})$ 177

Figure 99: Velocity validation study with flame edge as input : (a) amplitude comparison, (b) corresponding phase comparison; Flame edge validation study with velocity as input : (c) amplitude comparison, (d) corresponding phase comparison. 178

Figure 100: Velocity validation study with flame edge as input : (a) amplitude comparison, (b) corresponding phase comparison; Flame edge validation study with velocity as input : (c) amplitude comparison, (d) corresponding phase comparison. 
Figure 101: Experimental set-up for swirler acoustic impedance measurements. Bottom figure includes dimensions given in millimeters. 179

Figure 102: The phase-difference between the velocity fluctuation upstream and downstream of the swirler location, both with and without the swirler. 180

Figure 103: (a) Gain and (b) phase of swirler transfer function (velocity) as a function of Strouhal number when the upstream pressure oscillation amplitude is kept constant, $1 \sim 2 \% .180$

Figure 104: Acoustic impedance in complex domain upstream and downstream of the swirler 181

Figure 105: Deconvoluted stable flame images ( $\mathrm{CH}^{*}$ chemiluminescence). Operating conditions: $\mathrm{T}_{\mathrm{in}}=200{ }^{\circ} \mathrm{C}, \mathrm{V}_{\text {mean }}=60 \mathrm{~m} / \mathrm{s}, \Phi=0.55,0.60,0.65,0.70$, premixed, $\mathrm{X}_{\mathrm{H} 2}=0.00,0.15,0.30,0.45$, 0.60 . 182

Figure 106: Coordinates of maximum $\mathrm{CH}^{*}$ chemiluminescence intensity locations and flame angle $(\alpha)$ versus the flame length $\left(\mathrm{L}_{\mathrm{CH}^{*} \max }\right)$. 182

Figure 107: Comparison of normalized heat release response and gain of Flame " $\mathrm{A}$ " and "B": $f=$ $100 \mathrm{~Hz}$ and $250 \mathrm{~Hz}$. 183

Figure 108: Theoretical fits (second order model) of gain of FTF. Operating conditions: $\mathrm{T}_{\text {in }}=$ $200{ }^{\circ} \mathrm{C}, \mathrm{V}_{\text {mean }}=60,70,80 \mathrm{~m} / \mathrm{s}, \Phi=0.55,0.60,0.65,0.70$, premixed, $\mathrm{X}_{\mathrm{H} 2}=0.00,0.15,0.30 . .184$

Figure 109: Dependence of the Strouhal number $(\mathrm{St})$ upon $\mathrm{X}_{\mathrm{H} 2}$. 185

Figure 110: Linear fits of phase difference of FTF. Operating conditions: $\mathrm{T}_{\text {in }}=200{ }^{\circ} \mathrm{C}, \mathrm{V}_{\text {mean }}=$ $60,70,80 \mathrm{~m} / \mathrm{s}, \Phi=0.55,0.60,0.65,0.70$, premixed, $\mathrm{X}_{\mathrm{H} 2}=0.00,0.15,0.30$. 186

Figure 111: The dependence of damping coefficient $(\xi)$ and convection time delay $(\tau)$ upon the normalized flame length $\left(\mathrm{L}_{\mathrm{CH}^{*} \text { max }} / \mathrm{R}\right)$. Operating conditions: $\mathrm{T}_{\text {in }}=200{ }^{\circ} \mathrm{C}, \mathrm{V}_{\text {mean }}=60,70,80$ $\mathrm{m} / \mathrm{s}, \Phi=0.55,0.60,0.65,0.70$, premixed, $\mathrm{X}_{\mathrm{H} 2}=0.00,0.15,0.30 \ldots$ 186

Figure 112: The dependence of phase difference and gain of FTF upon the Strouhal number (St). Operating conditions: $\mathrm{T}_{\text {in }}=200^{\circ} \mathrm{C}, \mathrm{V}_{\text {mean }}=60,70,80 \mathrm{~m} / \mathrm{s}, \Phi=0.55,0.60,0.65,0.70$, premixed, $\mathrm{X}_{\mathrm{H} 2}=0.00,0.15,0.30$. 187

Figure 113: Schematic of the experimental setup used for studying the (a) Bunsen flame and (b) swirl flame. 188

Figure 114: Sample instantaneous (a) Mie-scattering image used for the PIV analysis $\left(f_{o}=200 \mathrm{~Hz}\right.$, $u_{A}^{\prime} / u_{o}=0.2,0^{\circ}$ phase with respect to the forcing signal) and (b) OH PLIF image of the unforced swirl flame $(R e=21,000)$. 190

Figure 115: Sample images showing the variation of the ensemble averaged progress variable ( $\bar{c}=0$ indicates reactants, while $\bar{c}=1$ indicates products) for the (a) forced Bunsen flame and (b) forced swirl flame. 191

Figure 116: Evolution of the flame brush as given by the progress variable contours of $\bar{c}=0.3$ (green line) and $\bar{c}=0.7$ (red line) of an unforced swirl flame at $R e=21,000$. 192 
Figure 117: Contour plots of the progress variables $\bar{c}=0.3$ (green line) and $\bar{c}=0.7$ (red line) showing the development of the flame brush at $R e=21,000, f_{o}=130 \mathrm{~Hz}$ and $u^{\prime} / u_{o}=0.6 \ldots \ldots .193$

Figure 118: Variation of the normalized flame brush thickness $\left(\delta_{t} / D_{a n}\right)$ along the normalized flame coordinate $\left(s / D_{a n}\right)$ at $R e=21,000, f_{o}=130 \mathrm{~Hz}$ and $u ' u_{o}=0.6$. 194

Figure 119: Centerline turbulence kinetic energy spectrum for Bunsen flame. 195

Figure 120: Overlay of several extracted flame edges showing flame brush development for the unforced case. 195

Figure 121: Mean axial velocity contours for (a) the unforced case and (b) forced case at $u$ ' $/ u_{o}$ $=0.2$ and phase angle of $90^{\circ}$. 196

Figure 122: Sample ensemble averaged images and progress variable contours of $\bar{c}=0.3$ (green line) and $\bar{c}=0.7$ (red line) illustrating the development of the flame brush at $R e=10,200, f_{o}=200$ $\mathrm{Hz}$ and $u^{\prime} / u_{o}=0.15$. 197

Figure 123: Variation of the normalized flame brush thickness $\left(\delta_{t} / D\right)$ along the normalized flame coordinate $(s / D)$. 197

Figure 124: Variation of axial velocity gradients at different radial locations. 198

Figure 125: Variation of flame brush thickness, acoustic velocity and their gradients along axial distance for lean methane......

Figure 126: Variation of flame brush thickness, acoustic velocity and their gradients along axial distance for lean propane. 200

Figure 127: (a) Normalized heat release response $\left(\mathrm{CH}^{*} / \mathrm{CH}^{*}\right.$ mean $)$, (b) gain, and (c) phase of flame transfer function as a function of the magnitude of inlet velocity perturbation. $\mathrm{T}_{\mathrm{in}}=200{ }^{\circ} \mathrm{C}$,

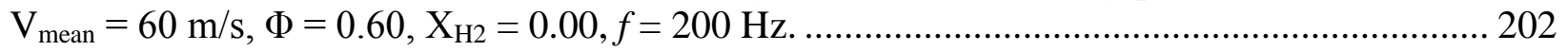

Figure 128: Phase-synchronized $\mathrm{CH}^{*}$ chemiluminescence imaging at modulation frequency of $200 \mathrm{~Hz}$ : (a) $\mathrm{V}^{\prime} / \mathrm{V}_{\text {mean }}=9 \%$ for linear regime (L) and (b) $\mathrm{V}^{\prime} / \mathrm{V}_{\text {mean }}=23 \%$ for transition regime (TR). 203

Figure 129: Phase-synchronized $\mathrm{CH}^{*}$ chemiluminescence imaging at modulation frequency of $200 \mathrm{~Hz}$ and $\mathrm{V}^{\prime} / \mathrm{V}_{\text {mean }}=25 \%$ for the first nonlinear regime (NL1). Bottom row shows line-ofsight integrated, background-corrected $\mathrm{CH}^{*}$ images at $\varphi=60,150,240,330^{\circ}$.... 204

Figure 130: Phase-synchronized $\mathrm{CH}^{*}$ chemiluminescence imaging at modulation frequency of $200 \mathrm{~Hz}$ and $\mathrm{V}^{\prime} / \mathrm{V}_{\text {mean }}=59 \%$ for the second nonlinear regime (NL2). Bottom row shows line-ofsight integrated, background-corrected $\mathrm{CH}^{*}$ images at $\varphi=60,150,240,330^{\circ}$. 204

Figure 131: Coordinates of maximum $\mathrm{CH}^{*}$ intensity location at phase of $\varphi=270^{\circ}$ (maximum intensity) for inlet velocity magnitude of $\mathrm{V}^{\prime} / \mathrm{V}_{\text {mean }}=9,23,25$, and $59 \%$. 205

Figure 132: (a) Phase-synchronized $\mathrm{CH}^{*}$ chemiluminescence imaging at $f=200 \mathrm{~Hz}$ and $\mathrm{V}^{\prime} / \mathrm{V}_{\text {mean }}$ $=26 \%$, (b) line-of-sight integrated $\mathrm{CH}^{*}$ images at $\varphi=90^{\circ}, 150^{\circ}, 210^{\circ}$, and $270^{\circ}$, (c) normalized 
heat release response, and (d) gain of flame transfer function as a function of modulation amplitude. Operating conditions: $\mathrm{T}_{\mathrm{in}}=200{ }^{\circ} \mathrm{C}, \mathrm{V}_{\text {mean }}=60 \mathrm{~m} / \mathrm{s}, \Phi=0.60$, and $\mathrm{X}_{\mathrm{H} 2}=0.30 \ldots \ldots .206$

Figure 133: $\mathrm{CH}^{*}$ chemiluminescence intensity and flame angle variation over one period of oscillation. Operating conditions: $\mathrm{T}_{\text {in }}=200{ }^{\circ} \mathrm{C}, \mathrm{V}_{\text {mean }}=60 \mathrm{~m} / \mathrm{s}, \Phi=0.60, \mathrm{X}_{\mathrm{H} 2}=0.30 f=200 \mathrm{~Hz}$, and $\mathrm{V}^{\prime} / \mathrm{V}_{\text {mean }}=26 \%$. 207

Figure 134: Normalized heat release response and phase of flame transfer function as a function of forcing amplitude. Operating conditions: $\mathrm{T}_{\mathrm{in}}=200{ }^{\circ} \mathrm{C}, \mathrm{V}_{\text {mean }}=60 \mathrm{~m} / \mathrm{s}, \Phi=0.60$, and $\mathrm{X}_{\mathrm{H} 2}=$ 0.30 208

Figure 135: Phase-synchronized $\mathrm{CH}^{*}$ chemiluminescence imaging at $f=300 \mathrm{~Hz}$ and $\mathrm{V} / \mathrm{V}_{\text {mean }}=$ $31 \%$. Operating conditions: $\mathrm{T}_{\mathrm{in}}=200{ }^{\circ} \mathrm{C}, \mathrm{V}_{\text {mean }}=60 \mathrm{~m} / \mathrm{s}, \Phi=0.60$, and $\mathrm{X}_{\mathrm{H} 2}=0.30 \ldots \ldots \ldots \ldots .208$

Figure 136: Gain and phase difference of FTF vs. forcing frequency at a constant forcing amplitude, $\mathrm{V}^{\prime} / \mathrm{V}_{\text {mean }}=0.100$. Operating conditions: $\mathrm{T}_{\text {in }}=200{ }^{\circ} \mathrm{C}, \mathrm{V}_{\text {mean }}=60 \mathrm{~m} / \mathrm{s}, \Phi=0.60$, and $\mathrm{X}_{\mathrm{H} 2}=0.00,0.30$. 209

Figure 137: Self-excited instability frequencies and the normalized combustor pressure oscillation magnitude plotted as a function of the combustor length. $T_{\text {in }}=200{ }^{\circ} \mathrm{C}, \mathrm{V}_{\text {mean }}=60 \mathrm{~m} / \mathrm{s}$, $\Phi=0.60, \mathrm{X}_{\mathrm{H} 2}=0.00$, and $\mathrm{L}_{\mathrm{c}}=30 " \sim 60 "$. 210

Figure 138: Phase-synchronized (self-excited) flame imaging at $\mathrm{L}_{\mathrm{c}}=54$ " and $f=234 \mathrm{~Hz}$. $\mathrm{V}^{\prime} / \mathrm{V}_{\text {mean }}=0.165, \mathrm{P}_{\mathrm{c}}{ }^{\prime} / \mathrm{P}_{\mathrm{c} \text {, mean }}=0.037$. Bottom row shows line-of-sight integrated, backgroundcorrected $\mathrm{CH}^{*}$ images at $\varphi=0,90,180,270^{\circ}$. 210

Figure 139: Phase-synchronized (self-excited) flame imaging at $\mathrm{L}_{\mathrm{c}}=60$ " and $f=218 \mathrm{~Hz}$. $\mathrm{V}^{\prime} / \mathrm{V}_{\text {mean }}=0.548, \mathrm{P}_{\mathrm{c}}{ }^{\prime} / \mathrm{P}_{\mathrm{c} \text {, mean }}=0.130$. Bottom row shows line-of-sight integrated, backgroundcorrected $\mathrm{CH}^{*}$ images at $\varphi=0,90,180,270^{\circ}$. 211

Figure 140: Phase-synchronized $\mathrm{CH}^{*}$ chemiluminescence imaging at modulation frequency of $218 \mathrm{~Hz}$ and $\mathrm{V}^{\prime} / \mathrm{V}_{\text {mean }}=57 \%\left(\mathrm{P}_{\mathrm{c}}{ }^{\prime} / \mathrm{P}_{\mathrm{c}}\right.$, mean $\left.=0.031\right)$. Bottom row shows line-of-sight integrated, background-corrected $\mathrm{CH}^{*}$ images at $\varphi=0,90,180,270^{\circ}$. 211

Figure 141: Normalized combustor pressure amplitude and $\mathrm{CH}^{*}$ chemiluminescence intensity for forced response and self-excited instability at $218 \mathrm{~Hz}$. Operating conditions: $\mathrm{T}_{\text {in }}=200{ }^{\circ} \mathrm{C}, \mathrm{V}_{\text {mean }}$ $=60 \mathrm{~m} / \mathrm{s}, \Phi=0.60$, and $\mathrm{X}_{\mathrm{H} 2}=0.00$. 212

Figure 142: (A) The normalized $\mathrm{CH}^{*}$ chemiluminescence intensity and (B) the phase of the flame transfer function at a modulation frequency of $100 \mathrm{~Hz}$. Inlet conditions: $\mathrm{T}_{\text {in }}=200{ }^{\circ} \mathrm{C}$, $\mathrm{V}_{\text {mean }}=60 \mathrm{~m} / \mathrm{s}$, and $\Phi_{\text {mean }}=0.60$. 215

Figure 143: Instantaneous $\mathrm{CH}^{*}$ chemiluminescence image at a modulation frequency of $100 \mathrm{~Hz}$ and $\Phi / \bar{\Phi}=4.3 \%$. Inlet conditions: $\mathrm{T}_{\text {in }}=200^{\circ} \mathrm{C}, \mathrm{V}_{\text {mean }}=60 \mathrm{~m} / \mathrm{s}$, and $\Phi_{\text {mean }}=0.55 \ldots \ldots \ldots \ldots . .215$

Figure 144: (A) The normalized $\mathrm{CH}^{*}$ chemiluminescence intensity and (B) the phase of the flame transfer function at a modulation frequency of $150 \mathrm{~Hz}$. Inlet conditions: $\mathrm{T}_{\text {in }}=200{ }^{\circ} \mathrm{C}$, $\mathrm{V}_{\text {mean }}=60 \mathrm{~m} / \mathrm{s}$, and $\Phi_{\text {mean }}=0.60$. 216 
Figure 145: Dependence of the normalized $\mathrm{CH}^{*}$ chemiluminescence intensity upon modulation frequency. Inlet conditions: $\mathrm{T}_{\mathrm{in}}=200{ }^{\circ} \mathrm{C}, \mathrm{V}_{\text {mean }}=60 \mathrm{~m} / \mathrm{s}$, and $\Phi_{\text {mean }}=0.60 \ldots$

Figure 146: Dependence of the response of a flame upon mean equivalence ratio at a modulation frequency of $100 \mathrm{~Hz}$. Inlet conditions: $\mathrm{T}_{\text {in }}=200{ }^{\circ} \mathrm{C}, \mathrm{V}_{\text {mean }}=60 \mathrm{~m} / \mathrm{s}$, and $\Phi_{\text {mean }}=0.60,0.65 \ldots 218$

Figure 147: Dependence of the response of a flame upon mean equivalence ratio at a modulation frequency of $150 \mathrm{~Hz}$. Inlet conditions: $\mathrm{T}_{\text {in }}=200{ }^{\circ} \mathrm{C}, \mathrm{V}_{\text {mean }}=60 \mathrm{~m} / \mathrm{s}$, and $\Phi_{\text {mean }}=0.60,0.65 \ldots 218$

Figure 148: (A) Gain and (B) phase of the flame transfer function in frequency domain. Inlet conditions: $\mathrm{T}_{\text {in }}=200{ }^{\circ} \mathrm{C}, \mathrm{V}_{\text {mean }}=60 \mathrm{~m} / \mathrm{s}$, and $\Phi_{\text {mean }}=0.60$....... 219

Figure 149: Phase-resolved $\mathrm{CH}^{*}$ chemiluminescence imaging during a period of oscillation at a modulation frequency of $200 \mathrm{~Hz}$ and amplitudes of $(\mathrm{A})_{\Phi} / \bar{\Phi}=2.2 \%$ and $(\mathrm{B})_{\Phi} / \bar{\Phi}=3.2 \%$. Inlet conditions: $\mathrm{T}_{\text {in }}=200{ }^{\circ} \mathrm{C}, \mathrm{V}_{\text {mean }}=60 \mathrm{~m} / \mathrm{s}$, and $\Phi_{\text {mean }}=0.60$.

Figure 150: Time signal of $\mathrm{CH}^{*}$ chemiluminescence intensity at a modulation frequency of 200 $\mathrm{Hz}$ and amplitude of $\Phi^{\prime} / \bar{\Phi}=2.2 \%$. Inlet conditions: $\mathrm{T}_{\text {in }}=200{ }^{\circ} \mathrm{C}, \mathrm{V}_{\text {mean }}=60 \mathrm{~m} / \mathrm{s}$, and $\Phi_{\text {mean }}=$ 0.60

Figure 151: Phase-resolved $\mathrm{CH}^{*}$ chemiluminescence imaging during a period of oscillation at a modulation frequency of $400 \mathrm{~Hz}$ and $\Phi^{\prime} / \bar{\Phi}=2.3 \%$. Inlet conditions: $\mathrm{T}_{\text {in }}=200{ }^{\circ} \mathrm{C}, \mathrm{V}_{\text {mean }}=60$ $\mathrm{m} / \mathrm{s}$, and $\Phi_{\text {mean }}=0.55$.

Figure 152: The dependence of the maximum $\mathrm{CH}^{*}$ chemiluminescence intensity locations $\left(\mathrm{L}_{\mathrm{CH}^{*}}\right.$ max) and $\mathrm{CH}^{*}$ chemiluminescence intensity upon the phase during a period of oscillation at a modulation frequency of $400 \mathrm{~Hz}$ and $\Phi / \bar{\Phi}=2.3 \%$. Inlet conditions: $\mathrm{T}_{\text {in }}=200{ }^{\circ} \mathrm{C}, \mathrm{Vmean}=60$ $\mathrm{m} / \mathrm{s}$, and $\Phi_{\text {mean }}=0.55$.

Figure 153: Two input/one output model 225

Figure 154: Dependence of the amplitude of equivalence ratio oscillations upon the amplitude of inlet velocity perturbations at a modulation frequency of 100 and $200 \mathrm{~Hz}$. Inlet conditions: $\mathrm{T}_{\text {in }}=$ $200{ }^{\circ} \mathrm{C}, \mathrm{V}_{\text {mean }}=60 \mathrm{~m} / \mathrm{s}, \Phi_{\text {mean }}=0.60$, and $\mathrm{X}_{\mathrm{H} 2}=0.00$. 226

Figure 155: Constructive and destructive reconstruction of the response of a partially premixed flame. 228

Figure 156: Phase difference between acoustic velocity and equivalence ratio at the combustor inlet as a function of modulation frequency for two fuel injection locations of $\mathrm{L}_{\mathrm{f}, \mathrm{inj}}=152 \mathrm{~mm}$ and $203 \mathrm{~mm}$. Inlet conditions: $\mathrm{T}_{\text {in }}=200^{\circ} \mathrm{C}, \mathrm{V}_{\text {mean }}=60 \mathrm{~m} / \mathrm{s}, \Phi_{\text {mean }}=0.60, \mathrm{X}_{\mathrm{H} 2}=0.60$, and $\mathrm{V}^{\prime} / \mathrm{V}_{\text {mean }}=$ $\min \sim \max$. 228

Figure 157: Phase-synchronized $\mathrm{CH}^{*}$ chemiluminescence images at a modulation frequency of $200 \mathrm{~Hz}$ and $\mathrm{V}^{\prime} / \mathrm{V}_{\text {mean }}=42 \%$. The inverted gray scale is linear from black to white, denoting the highest and the lowest chemiluminescence intensity. Inlet conditions: $\mathrm{T}_{\text {in }}=200{ }^{\circ} \mathrm{C}, \mathrm{V}_{\text {mean }}=60$ $\mathrm{m} / \mathrm{s}, \Phi=0.60, \mathrm{X}_{\mathrm{H} 2}=0.60$, and $\mathrm{L}_{\mathrm{f}, \mathrm{inj}}=152 \mathrm{~mm}$. 229 
Figure 158: Gain of the flame transfer function plotted as a function of the forcing amplitude. Inlet conditions: $\mathrm{T}_{\mathrm{in}}=200{ }^{\circ} \mathrm{C}, \mathrm{V}_{\text {mean }}=60 \mathrm{~m} / \mathrm{s}, \Phi_{\text {mean }}=0.60, \mathrm{X}_{\mathrm{H} 2}=0.60, \mathrm{~L}_{\mathrm{f}, \text { inj }}=152 \mathrm{~mm}$, and $f=$ $200 \mathrm{~Hz}$. 230

Figure 159: Phase-synchronized $\mathrm{CH}^{*}$ chemiluminescence images at a modulation frequency of $300 \mathrm{~Hz} \mathrm{~V} / \mathrm{V}_{\text {mean }}=27 \%$. The inverted gray scale is linear from black to white, denoting the highest and the lowest chemiluminescence intensity. Inlet conditions: $\mathrm{T}_{\text {in }}=200{ }^{\circ} \mathrm{C}, \mathrm{V}_{\text {mean }}=60$ $\mathrm{m} / \mathrm{s}, \Phi_{\text {mean }}=0.60, \mathrm{X}_{\mathrm{H} 2}=0.60$, and $\mathrm{L}_{\mathrm{f}, \mathrm{inj}}=203 \mathrm{~mm}$.

Figure 160: The dependence of the gain of the flame transfer function on modulation frequency for a range of magnitude of inlet velocity oscillations, $\mathrm{V}^{\prime} / \mathrm{V}_{\text {mean }}=0.03 \sim 0.50$. Inlet conditions: $\mathrm{T}_{\mathrm{in}}=200{ }^{\circ} \mathrm{C}, \mathrm{V}_{\text {mean }}=60 \mathrm{~m} / \mathrm{s}, \Phi_{\text {mean }}=0.60, \mathrm{X}_{\mathrm{H} 2}=0.60$, and $\mathrm{L}_{\mathrm{f}, \mathrm{inj}}=203 \mathrm{~mm}$. 232

Figure 161: The dependence of the gain of the flame transfer function on magnitude of inlet velocity oscillations at a modulation frequency of $300 \mathrm{~Hz}$ and for fuel injection locations of $\mathrm{L}_{\mathrm{f}, \text { inj }}$ $=152 \mathrm{~mm}$ and $203 \mathrm{~mm}$. Inlet conditions: $\mathrm{T}_{\text {in }}=200{ }^{\circ} \mathrm{C}, \mathrm{V}_{\text {mean }}=60 \mathrm{~m} / \mathrm{s}, \Phi_{\text {mean }}=0.60, \mathrm{X}_{\mathrm{H} 2}=0.60$, and $\mathrm{V}^{\prime} / \mathrm{V}_{\text {mean }}=\min \sim \max$. 232

Figure 162: Variation of the response amplitude of perturbation about the mean flame for a conical flame with $\beta=4.0, M a=1, \delta / R=0.1$ at a forcing Strouhal number of $S t=10$. 234

Figure 163: (A) X-Y coordinates of maximum $\mathrm{CH}^{*}$ chemiluminescence intensity locations and (B) Flame angle as a function of effective flame length, $\mathrm{L}_{\mathrm{CH}^{*} \max }$ for $30^{\circ}$ and $45^{\circ}$ swirlers. Inlet conditions: $\mathrm{T}_{\text {in }}=200,300{ }^{\circ} \mathrm{C}, \mathrm{V}_{\text {mean }}=60,70,80,90,100 \mathrm{~m} / \mathrm{s}$, perfectly premixed, $\mathrm{X}_{\mathrm{H} 2}=0.00$, $0.15,0.30,0.45,0.60$, and $\Phi=0.55,0.60,0.65,0.70$. 236

Figure 164: Line-of-sight integrated $\mathrm{CH}^{*}$ chemiluminescence images for $(\mathrm{A}) \alpha_{\mathrm{sw}}=30^{\circ}$ and (B) $\alpha_{\mathrm{sw}}=45^{\circ}$. Inlet conditions: $\mathrm{T}_{\mathrm{in}}=200^{\circ} \mathrm{C}, \mathrm{V}_{\text {mean }}=60 \mathrm{~m} / \mathrm{s}, \mathrm{X}_{\mathrm{H} 2}=0.60$, and $\Phi=0.70$. 236

Figure 165: Dependence of steady-state $\mathrm{CH}^{*}$ chemiluminescence images upon swirl angle $\left(\alpha_{\mathrm{sw}}=\right.$ $30^{\circ}$ and $45^{\circ}$ ): (A) $\mathrm{V}_{\text {mean }}=90 \mathrm{~m} / \mathrm{s}$ and (B) $\mathrm{V}_{\text {mean }}=100 \mathrm{~m} / \mathrm{s}$. Inlet conditions: $\mathrm{T}_{\text {in }}=300{ }^{\circ} \mathrm{C}, \mathrm{V}_{\text {mean }}=$ $90,100 \mathrm{~m} / \mathrm{s}, \mathrm{X}_{\mathrm{H} 2}=0.00$, and $\Phi=0.55,0.60,0.65,0.70$. 237

Figure 166: Dependence of effective flame length $\left(\mathrm{L}_{\mathrm{CH} * \max }\right)$ upon mean equivalence ratio for two swirl angles of $\alpha_{\mathrm{sw}}=30^{\circ}$ and $45^{\circ}$ : (A) $\mathrm{V}_{\text {mean }}=90 \mathrm{~m} / \mathrm{s}$ and (B) $\mathrm{V}_{\text {mean }}=100 \mathrm{~m} / \mathrm{s}$. Inlet conditions: $\mathrm{T}_{\text {in }}=300^{\circ} \mathrm{C}, \mathrm{V}_{\text {mean }}=90,100 \mathrm{~m} / \mathrm{s}, \mathrm{X}_{\mathrm{H} 2}=0.00$, and $\Phi=0.55,0.60,0.65,0.70$. 238

Figure 167: Flame transfer function gain and phase as a function of modulation frequency. Inlet conditions: $\mathrm{T}_{\text {in }}=300{ }^{\circ} \mathrm{C}, \mathrm{V}_{\text {mean }}=100 \mathrm{~m} / \mathrm{s}, \mathrm{X}_{\mathrm{H} 2}=0.00, \Phi=0.55,0.60,0.65,0.70$, and $\alpha_{\mathrm{sw}}=45^{\circ}$.

Figure 168: Dependence of flame transfer functions upon swirl angle. Inlet conditions: $\mathrm{T}_{\text {in }}=300$ ${ }^{\circ} \mathrm{C}, \mathrm{V}_{\text {mean }}=100 \mathrm{~m} / \mathrm{s}, \mathrm{X}_{\mathrm{H} 2}=0.00, \Phi=0.55$, and $\alpha_{\mathrm{sw}}=30^{\circ}, 45^{\circ}$ 240

Figure 169: The maximum gain of the flame transfer functions plotted against the normalized effective flame length for swirl angles of $30^{\circ}$ and $45^{\circ}$. Inlet conditions: $\mathrm{T}_{\text {in }}=200,300{ }^{\circ} \mathrm{C}, \mathrm{V}_{\text {mean }}$ $=60,70,80,90,100 \mathrm{~m} / \mathrm{s}, \Phi=0.55,0.60,0.65,70$, and $\mathrm{X}_{\mathrm{H} 2}=0.00,0.15,0.30$. 240 
Figure 170: The convection times as a function of the normalized effective flame length for swirl angles of $30^{\circ}$ and $45^{\circ}$. Inlet conditions: $\mathrm{T}_{\text {in }}=200,300^{\circ} \mathrm{C}, \mathrm{V}_{\text {mean }}=60,70,80,90,100 \mathrm{~m} / \mathrm{s}, \Phi=$ $0.55,0.60,0.65,70$, and $\mathrm{X}_{\mathrm{H} 2}=0.00,0.15,0.30$. 241 